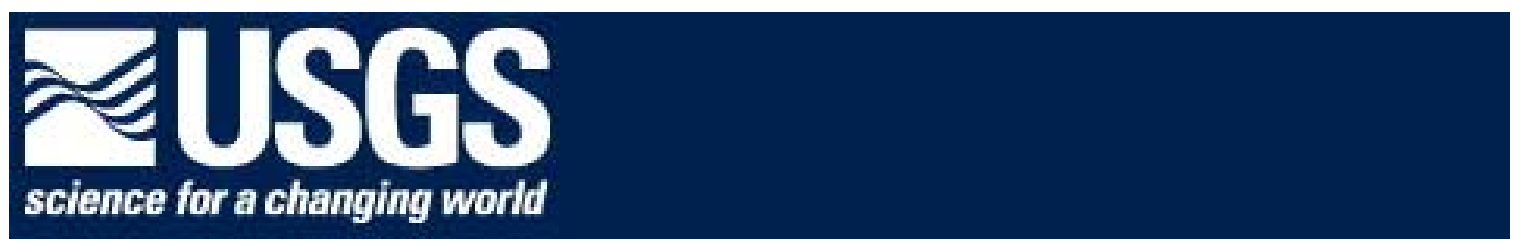

U.S. GEOLGICICAL SURVEY OPEN-FILE REPORT OF-00-131

\title{
U.S. GEOLOGICAL SURVEY COLLABORATION WITH INTERNATIONAL AUTHORS, 1987-1998
}

\section{By Valentina Markusova ${ }^{1}$ and Paul P. Hearn ${ }^{2}$ INTRODUCTION}

The use of science and technology indicators for making decisions on publicly funded research remains of major interest to the U.S. Government. As the recently published National Science Foundation (NSF) biannual report, Science and Engineering Indicators, 1998, stated, globalization is one of the distinguishing features of modern science. If research activity is to be evaluated properly, it is necessary to examine the publications resulting from such activity. To achieve this goal, the NSF report used statistical data derived from the Science Citation Index (SCI), which is a database produced on CD-ROM by the Institute for Scientific Information, based in Philadelphia, Pa.

The SCI is a unique index providing multidisciplinary coverage of journal literature and offering a variety of access points to these data. Issues of each journal included in the database are indexed cover to cover, including substantive items and all cited references. Advertisements and news notices are omitted. The SCI also includes monograph series, which are indexed in the same manner as journals. In general, each annual index includes about 700,000 items and about 15 million references from 3,400 leading journals published in 63 countries.

According to the NSF report, half of the articles published in SCI journals in 1995 had multiple authors; almost 30 percent of those articles involved international collaboration. This trend was evident in all fields as a steadily growing fraction of most nations' papers involved co-authors from different countries. Between 1981 and 1995, the publication of articles increased by 20 percent, that of co-authored articles by 80 percent, and that of internationally co-authored articles by 200 percent. For almost every nation having strong international co-authorship ties, the number of articles involving U.S. authors rose markedly between 1981 and 1995. Concurrently, however, as many nations broadened the scope of their international collaborations, the U.S. share of the world's internationally co-authored articles declined.

The SCI database was also used to evaluate USGS authorship of international collaborative papers. The "USGS Database of Collaborative Papers with Foreign Countries" (USGS-Papers) includes more than 1,200 papers published by USGS researchers and their colleagues from foreign countries from 1987 to 1998. This time span was chosen owing to the increase of collaborations observed in many scientific studies. The search is included on the SCI CD-ROM by address: US-Geol-Survey. The results of the search by year from 1987 to 1998 are shown in table 1.

\footnotetext{
${ }^{1}$ Director, All Russia Institute for Scientific and Technical Information, Ulitsa Usievicha 20, Moscow 125315, Russia.

${ }^{2}$ U.S. Geological Survey, 12201 Sunrise Valley Drive, Reston, VA 20192.
} 
Table 1. USGS international collaborative papers for 1987-1998

\begin{tabular}{|l|l|l|l|l|l|l|l|l|l|l|l|l|}
\hline Year & $\mathbf{8 7}$ & $\mathbf{8 ~ 8}$ & $\mathbf{8 9}$ & $\mathbf{9 0}$ & $\mathbf{9 1}$ & $\mathbf{9 2}$ & $\mathbf{9 3}$ & $\mathbf{9 4}$ & $\mathbf{9 5}$ & $\mathbf{9 6}$ & $\mathbf{9 7}$ & $\mathbf{9 8}$ \\
\hline $\begin{array}{l}\text { Total } \\
\text { USGS } \\
\text { papers }\end{array}$ & 796 & 671 & 592 & 701 & 746 & 668 & 625 & 662 & 695 & 667 & 658 & 723 \\
\hline $\begin{array}{l}\text { ool } \\
\text { papers }\end{array}$ & 80 & 52 & 72 & 81 & 128 & 109 & 102 & 115 & 122 & 124 & 106 & 128 \\
\hline
\end{tabular}

The table shows no decline in USGS international collaborative papers. 


\section{LISTING OF COLLABORATIVE PAPERS BY YEAR}

\section{7}

1. Authors: Schlee-JS Hinz-K

Title: Seismic Stratigraphy and Facies of Continental-Slope and Rise Seaward of Baltimore Canyon Trough Source: AAPG BULLETIN-AMERICAN ASSOCIATION OF PETROLEUM GEOLOGISTS, 1987, Vol 71, Iss 9, pp 10461067

Addresses:

US GEOL SURVEY, WOODS-HOLE, MA 02543, USA

BUNDESANSTALT GEOWISSENSCH \& ROHSTOFFE, HANOVER, FED-REP-GER

2. Authors: Eberl-DD Lee-M Nadeau-PH Northrop-HR Srodon-J

Title: Sericite from the Silverton Caldera, Colorado -

Correlation Among Structure, Composition, Origin, and Particle Thickness

Source: AMERICAN MINERALOGIST 1987, Vol 72, Iss 9-10, pp 914-934

Addresses:

US GEOL SURVEY, FED CTR, MAIL STOP 404, DENVER, CO 80225, USA

CASE WESTERN RESERVE UNIV, DEPT EARTH SCI, CLEVELAND, OH 44106, USA

MACAULAY INST SOIL RES, ABERDEEN AB9 2QJ, SCOTLAND

POLISH ACAD SCI, INST GEOL SCI, PL-31002 CRACOW, POLAND

3. Authors: Dunn-PJ Erd-RC Peacor-DR Ramik-RA

Title: Franciscanite and Orebroite, 2 New Minerals from

California and Sweden, Related to Redefined Welinite

Source: AMERICAN MINERALOGIST

1986, Vol 71, Iss 11-1, pp 1522-1526

Addresses:

SMITHSONIAN INST, DEPT MINERAL SCI, WASHINGTON, DC 20560, USA

UNIV MICHIGAN, DEPT GEOL SCI, ANN-ARBOR, MI 48109, USA

US GEOL SURVEY, MENLO-PK, CA 94025, USA

ROYAL ONTARIO MUSEUM, DEPT MINERAL \& GEOL, TORONTO M5S 2C6, ONTARIO, CANADA

4. Authors: Plafker-G Agar-R Asker-AH Hanif-M

Title: Surface Effects and Tectonic Setting of the 13 December 1982 North Yemen Earthquake

Source: BULLETIN OF THE SEISMOLOGICAL SOCIETY OF AMERICA 1987, Vol 77, Iss 6, pp 2018-2037

Addresses:

US GEOL SURVEY, 345 MIDDLEFIELD RD, MS 904, MENLO-PK, CA 94025, USA

MINIST PETR \& MINERAL RESOURCES, JEDDAH, SAUDI-ARABIA

YOMINCO, SAANA, YEMEN-ARAB-REP

5. Authors: Langer-CJ Bollinger-GA Merghelani-HM

Title: Aftershocks of the 13 December 1982 North Yemen

Earthquake - Conjugate Normal Faulting in an Extensional Setting

Source: BULLETIN OF THE SEISMOLOGICAL SOCIETY OF AMERICA 1987, Vol 77, Iss 6, pp 2038-2055

Addresses:

US GEOL SURVEY, DENVER FED CTR, POB 25046, MS 966, DENVER, CO 80225, USA

DEPUTY MINIST MINERAL RESOURCES, MINIST PETR \& MINERAL

RESOURCES, JEDDAH, SAUDI-ARABIA 
VIRGINIA POLYTECH INST \& STATE UNIV, DEPT GEOL SCI,

BLACKSBURG, VA 24061, USA

6. Authors: Nishenko-SP Singh-SK

Title: Conditional Probabilities for the Recurrence of Large and Great Interplate Earthquakes Along the Mexican

Subduction Zone

Source: BULLETIN OF THE SEISMOLOGICAL SOCIETY OF AMERICA

1987, Vol 77, Iss 6, pp 2095-2114

Language: English

Addresses:

US GEOL SURVEY, DENVER FED CTR, NATL EARTHQUAKE INFORMAT CTR, MS 967, DENVER, CO 80225, USA

NATL AUTONOMOUS UNIV MEXICO, INST GEOFIS, MEXICO CITY 04510, DF, MEXICO

7. Authors: Nishenko-SP Singh-SK

Title: The Acapulco-Ometepec, Mexico, Earthquakes of 1907-1982 - Evidence for a Variable Recurrence History

Source: BULLETIN OF THE SEISMOLOGICAL SOCIETY OF AMERICA 1987, Vol 77, Iss 4, pp 1359-1367

Addresses:

US GEOL SURVEY, DENVER FED CTR, NATL EARTHQUAKE INFORMAT CTR, DENVER, CO 80225, USA

NATL AUTONOMOUS UNIV MEXICO, INST GEOFIS, MEXICO CITY 04510, DF, MEXICO

8. Authors: Boore-DM Atkinson-GM

Title: Stochastic Prediction of Ground Motion and Spectral

Response Parameters at Hard-Rock Sites in Eastern North-America

Source: BULLETIN OF THE SEISMOLOGICAL SOCIETY OF AMERICA 1987, Vol 77, Iss 2, pp 440-467

Addresses:

US GEOL SURVEY, MS 977, MENLO-PK, CA 94025, USA

GEOL SURVEY CANADA, DIV GEOPHYS, OTTAWA K1A 0Y3, ONTARIO,

CANADA

9. Authors: Choy-GL Kind-R

Title: Rupture Complexity of a Moderate-Sized (MB-6.0)

Earthquake - Broad-Band Body-Wave Analysis of the North Yemen Earthquake of 13 December 1982

Source: BULLETIN OF THE SEISMOLOGICAL SOCIETY OF AMERICA 1987, Vol 77, Iss 1, pp 28-46

Addresses:

US GEOL SURVEY, DENVER FED CTR, NATL EARTHQUAKE INFORMAT CTR, POB 25046, MS 967, DENVER, CO 80225, USA

SEISMOL ZENT OBSERV GRAEFENBERG, D-8520 ERLANGEN, FED-REP-GER

10. Authors: Storey-BC Ford-AB Rowley-PD Wever-HE

Title: Report on Antarctic Fieldwork the Geology of the Central Black Coast, Eastern Palmer Land

Source: BRITISH ANTARCTIC SURVEY BULLETIN 1987, Iss 77, pp 145-155

Addresses:

NERC, BRITISH ANTARCTIC SURVEY, HIGH CROSS, MADINGLEY RD,

CAMBRIDGE CB3 OET, ENGLAND

US GEOL SURVEY, DENVER, CO 80225, USA

US GEOL SURVEY, MENLO-PK, CA 94024, USA

11. Authors: Smith-PE Farquhar-RM Tatsumoto-M

Title: Zircon Lu HF Systematics and the Evolution of the

Archean Crust in the Southern Superior-Province, Canada 
Source: CONTRIBUTIONS TO MINERALOGY AND PETROLOGY

1987, Vol 97, Iss 1, pp 93-104

Addresses:

UNIV TORONTO, DEPT PHYS, TORONTO M5S 1A1, ONTARIO, CANADA

US GEOL SURVEY, FED CTR, DENVER, CO 80225, USA

12. Authors: Piper-DZ Kolodny-Y

Title: The Stable Isotopic Composition of a Phosphorite Deposit - Delta-C-13, Delta-S-34 and Delta-O-18

Source: DEEP-SEA RESEARCH PART A-OCEANOGRAPHIC RESEARCH PAPERS

1987, Vol 34, Iss 5-6, pp 897-911

Addresses:

US GEOL SURVEY, MS-902, 345 MIDDLEFIELD RD, MENLO-PK, CA

94025, USA

HEBREW UNIV JERUSALEM, INST EARTH SCI, DEPT GEOL, IL-91904 JERUSALEM, ISRAEL

13. Authors: Kobayashi-K Aubouin-J Boulegue-J Cadet-JP Dubois-J Jolivet-L Kanazawa-T Kasahara-J Koizumi-K Lallemand-S Nakamura-Y Pautot-G Suyehiro-K Tani-S

Tokuyama-H Vonhuene-R Yamazaki-T

Title: Normal Faulting of the Daiichi-Kashima Seamount in the Japan Trench Revealed by the Kaiko-I Cruise, Leg-3

Source: EARTH AND PLANETARY SCIENCE LETTERS 1987, Vol 83, Iss 1-4, pp 257-266

Addresses:

UNIV TOKYO, OCEAN RES INST, 1-15-1 MINAMI DAI, NAKANO KU, TOKYO 164, JAPAN

UNIV ORLEANS, DEPT SCI TERRE, CNRS, GEOL DYNAM LAB 215,

F-45067 ORLEANS 2, FRANCE

UNIV PARIS 06, DEPT GEOTECTON, CNRS, UA 215, F-75252 PARIS 05, FRANCE

UNIV PARIS 06, CNRS, GEOCHIM \& MET LAB 196, F-75252 PARIS 05, FRANCE

ECOLE NORM SUPER, CNRS, GEOL LAB 215, F-75231 PARIS 05, FRANCE

MARITIME SAFETY AGCY, DEPT HYDROG, CHUO KU, TOKYO 104, JAPAN

GEOL SURVEY JAPAN, TSUKUBA, IBARAKI 305, JAPAN

UNIV PARIS 11, CNRS, GEOPHYS LAB 730, F-91405 ORSAY, FRANCE

CTR OCEANOL BRETAGNE, IFREMER, DEPT GEOSCI MARINE, F-29273 BREST, FRANCE

UNIV TOKYO, INST GEOPHYS, BUNKYO KU, TOKYO 113, JAPAN

CHIBA UNIV, FAC SCI, DEPT EARTH SCI, CHIBA 260, JAPAN

US GEOL SURVEY, OFF MARINE GEOL, MENLO-PK, CA 94025, USA

UNIV TOKYO, DEPT LIBERAL ARTS, MEGURO KU, TOKYO 153, JAPAN

UNIV TOKYO, EARTHQUAKE RES INST, BUNKYO KU, TOKYO 113, JAPAN

14. Authors: Cadet-JP Aubouin-J Boulegue-J Deplus-C Dubois-J Jolivet-L Kanazawa-T Kasahara-J Kobayashi-K

Koizumi-K Lallemand-S Nakamura-Y Pautot-G

Suyehiro-K Tani-S Tokuyama-H Vonhuene-R Yamazaki-T

Title: The Japan Trench and Its Juncture with the Kuril Trench - Cruise Results of the Kaiko Project, Leg-3

Source: EARTH AND PLANETARY SCIENCE LETTERS

1987, Vol 83, Iss 1-4, pp 267-284

Addresses:

UNIV ORLEANS, DEPT SCI TERRE, CNRS, GEOL DYNAM LAB 215,

F-45046 ORLEANS, FRANCE

UNIV PARIS 06, DEPT GEOTECTON, CNRS, UA 215, F-75252 PARIS 05, FRANCE

UNIV PARIS 06, CNRS, GEOCHIM \& MET LAB 196, F-75252 PARIS 05, FRANCE

MARITIME SAFETY AGCY, DEPT HYDROGR, CHUO KU, TOKYO 104, JAPAN

GEOL SURVEY JAPAN, TSUKUBA, IBARAKI 305, JAPAN

UNIV TOKYO, OCEAN RES INST, NAKANO KU, TOKYO 164, JAPAN

UNIV PARIS 11, CNRS, GEOPHYS \& GEODYNAM INTERNE LAB 730,

F-91405 ORSAY, FRANCE 
US GEOL SURVEY, OFF MARINE GEOL, MENLO-PK, CA 94025, USA

UNIV TOKYO, INST GEOPHYS, BUNKYO KU, TOKYO 113, JAPAN

UNIV TOKYO, FAC LIBERAL ARTS, TOKYO 153, JAPAN

UNIV TOKYO, EARTHQUAKE RES INST, BUNKYO KU, TOKYO 113, JAPAN

CTR OCEANOL BRETAGNE, IFREMER, F-29273 BREST, FRANCE

ECOLE NORM SUPER, CNRS, GEOL LAB 215, F-75231 PARIS 05, FRANCE

CHIBA UNIV, FAC SCI, DEPT EARTH SCI, CHIBA 260, JAPAN

15. Authors: Leventhal-JS Grauch-RI Lichte-FE Threlkeld-CN

Title: Unusual Organic-Matter Associated with Uranium from the Claude Deposit, Cluff Lake, Canada

Source: ECONOMIC GEOLOGY

1987, Vol 82, Iss 5, pp 1169-1176

Addresses:

US GEOL SURVEY, BOX 25046, MAIL STOP 916, FED CTR, DENVER, CO 80225, USA

SASKATCHEWAN GEOL SURVEY, REGINA S4P 4V4, SASKATCHEWAN, CANADA

16. Authors: Shanks-WC Jennings-DS Jilson-GA Modene-JS

Ryan-BD Woodruff-LG

Title: Sulfur and Lead Isotope Studies of Stratiform Zn-Pb-Ag Deposits, Anvil Range, Yukon - Basinal Brine Exhalation and Anoxic Bottom-Water Mixing

Source: ECONOMIC GEOLOGY

1987, Vol 82, Iss 3, pp 600-634

Addresses:

US GEOL SURVEY, NATL CTR, MAIL STOP 954, RESTON, VA 22092, USA

CYPRUS ANVIL MIN, VANCOUVER V6C 2G8, BC, CANADA

NO DYNASTY EXPLORAT LTD, VANCOUVER V6C 2G8, BC, CANADA

COMINCO AMER INC, SPOKANE, WA 99216, USA

CROWS NEST RESOURCES LTD, CALGARY T2P 2M7, ALBERTA, CANADA

17. Authors: Zepp-RG Braun-AM Hoigne-J Leenheer-JA

Title: Photoproduction of Hydrated Electrons from Natural

Organic Solutes in Aquatic Environments

Source: ENVIRONMENTAL SCIENCE \& TECHNOLOGY

1987, Vol 21, Iss 5, pp 485-490

Addresses:

US EPA, ENVIRONM RES LAB, ATHENS, GA 30613, USA

ECOLE POLYTECH FED LAUSANNE, INST CHIM PHYS, CH-1015 LAUSANNE,SWITZERLAND

SWISS FED INST WATER RESOURCES \& WATER POLLUT CONTROL, CH-8600 DUBENDORF, SWITZERLAND

US GEOL SURVEY, DENVER FED CTR, LAKEWOOD, CO 80403, USA

18. Authors: Grossman-JN Wasson-JT

Title: Compositional Evidence Regarding the Origins of Rims on Semarkona Chondrules

Source: GEOCHIMICA ET COSMOCHIMICA ACTA

1987, Vol 51, Iss 11, pp 3003-3011

Addresses:

US GEOL SURVEY, NATL CTR, MAIL STOP 990, RESTON, VA 22092, USA

UNIV CALIF LOS ANGELES, DEPT CHEM \& BIOCHEM, LOS-ANGELES, CA 90024, USA

UNIV CALIF LOS ANGELES, DEPT EARTH \& SPACE SCI, LOS-ANGELES, CA 90024, USA

UNIV CALIF LOS ANGELES, DEPT GEOPHYS \& PLANETARY PHYS,

LOS-ANGELES, CA 90024, USA

19. Authors: Harrison-TM Aleinikoff-JN Compston-W

Title: Observations and Controls on the Occurrence of Inherited Zircon in Concord-Type Granitoids, New-Hampshire Source: GEOCHIMICA ET COSMOCHIMICA ACTA

1987, Vol 51, Iss 9, pp 2549-2558 
Addresses:

SUNY ALBANY, DEPT GEOL SCI, ALBANY, NY 12222, USA

US GEOL SURVEY, DENVER FED CTR, ISOTOPE GEOL BRANCH, DENVER, CO 80225, USA

AUSTRALIAN NATL UNIV, RES SCH EARTH SCI, CANBERRA, ACT 2601, AUSTRALIA

20. Authors: Robie-RA Barton-MD Hemingway-BS Zhao-B

Title: Heat-Capacity and Thermodynamic Properties of Andradite

Garnet, Ca3Fe2Si3O12, Between 10-K and 1000-K and

Revised Values for Delta-Fgm-O (298.15-K) of

Hedenbergite and Wollastonite

Source: GEOCHIMICA ET COSMOCHIMICA ACTA

1987, Vol 51, Iss 8, pp 2219-2224

Addresses:

US GEOL SURVEY, RESTON, VA 22092, USA

UNIV CALIF LOS ANGELES, DEPT EARTH \& SPACE SCI, LOS-ANGELES, CA 90024, USA

ACAD SINICA, INST GEOCHEM, GUIYANG, PEOPLES-R-CHINA

21. Authors: Criss-RE Gregory-RT Taylor-HP

Title: Kinetic-Theory of Oxygen Isotopic Exchange Between Minerals and Water

Source: GEOCHIMICA ET COSMOCHIMICA ACTA 1987, Vol 51, Iss 5, pp 1099-1108

Addresses:

US GEOL SURVEY, RESTON, VA 22092, USA

MONASH UNIV, DEPT EARTH SCI, CLAYTON, VIC 3168, AUSTRALIA

CALTECH, DIV GEOL \& PLANETARY SCI, PASADENA, CA 91125, USA

22. Authors: Wilson-MR Hoeve-J Kyser-TK Mehnert-HH

Title: Changes in the H-O-Ar Isotope Composition of Clays

During Retrograde Alteration

Source: GEOCHIMICA ET COSMOCHIMICA ACTA 1987, Vol 51, Iss 4, pp 869-878

Addresses:

UNIV SASKATCHEWAN, DEPT GEOL SCI, SASKATOON S7N 0W0, SASKATCHEWAN, CANADA

US GEOL SURVEY, DENVER FED CTR, ISOTOPE GEOL BRANCH, DENVER, CO 80225, USA

SASKATCHEWAN RES COUNCIL, SASKATOON 0W0, SASKATCHEWAN, CANADA

23. Authors: Colman-SM Choquette-AF Huntley-DJ Miller-GH Rosholt-JN

Title: Dating the Upper Cenozoic Sediments in Fisher Valley, Southeastern Utah

Source: GEOLOGICAL SOCIETY OF AMERICA BULLETIN 1986, Vol 97, Iss 12, pp 1422-1431

Addresses:

US GEOL SURVEY, FED CTR, DENVER, CO 80225, USA

UNIV COLORADO, INST ARCT \& ALPINE RES, BOULDER, CO 80309, USA

SIMON FRASER UNIV, DEPT PHYS, BURNABY V5A 1S6, BC, CANADA

24. Authors: Barrett-PJ Elston-DP Harwood-DM Mckelvey-BC Webb-PN

Title: Mid-Cenozoic Record of Glaciation and Sea-Level Change on the Margin of the Victoria Land Basin, Antarctica Source: GEOLOGY 1987, Vol 15, Iss 7, pp 634-637

Addresses:

VICTORIA UNIV WELLINGTON, RES SCH EARTH SCI, ANTARCTIC RES CTR, WELLINGTON, NEW-ZEALAND US GEOL SURVEY, FLAGSTAFF, AZ 86001, USA 
OHIO STATE UNIV, INST POLAR STUDIES, COLUMBUS, OH 43210, USA

OHIO STATE UNIV, DEPT GEOL \& MINERAL, COLUMBUS, OH 43210, USA

UNIV NEW ENGLAND, DEPT GEOL, ARMIDALE, NSW 2601, AUSTRALIA

25. Authors: Muhs-DR Kyser-TK

Title: Stable Isotope Compositions of Fossil Mollusks from

Southern-California - Evidence for a Cool Last

Interglacial Ocean

Source: GEOLOGY

1987, Vol 15, Iss 2, pp 119-122

Addresses:

US GEOL SURVEY, MS 424, DENVER, CO 80225, USA

UNIV SASKATCHEWAN, DEPT GEOL SCI, SASKATOON S7N 0W0,

SASKATCHEWAN, CANADA

26. Authors: Spencer-C Green-A Luetgert-J

Title: More Seismic Evidence on the Location of Grenville

Basement Beneath the Appalachians of Quebec-Maine

Source: GEOPHYSICAL JOURNAL OF THE ROYAL ASTRONOMICAL SOCIETY 1987, Vol 89, Iss 1, pp 177-182

Addresses:

GEOL SURVEY CANADA, LITHOSPHERE \& CANADIAN SHIELD DIV, 1

OBSERV CRESCENT, OTTAWA K1A 0Y3, ONTARIO, CANADA

US GEOL SURVEY, SEISMOL BRANCH, MENLO-PK, CA 94025, USA

27. Authors: Luetgert-JH Klemperer-SL Mann-CE

Title: Wide-Angle Deep Crustal Reflections in the Northern Appalachians

Source: GEOPHYSICAL JOURNAL OF THE ROYAL ASTRONOMICAL SOCIETY 1987, Vol 89, Iss 1, pp 183-188

Addresses:

US GEOL SURVEY, 345 MIDDLEFIELD RD, MENLO-PK, CA 94025, USA

STANFORD UNIV, DEPT GEOPHYS, STANFORD, CA 94305, USA

BULLARD LABS, DEPT EARTH SCI, CAMBRIDGE CB3 OEZ, ENGLAND

28. Authors: Hall-SH Olhoeft-GR

Title: Nonlinear Complex Resistivity of Some Nickel Sulfides from Western Australia

Source: GEOPHYSICAL PROSPECTING 1986, Vol 34, Iss 8, pp 1255-1276

Addresses:

UNIV QUEENSLAND, DEPT GEOL \& MINERAL, ST LUCIA, QLD 4067, AUSTRALIA

US GEOL SURVEY, DENVER, CO 80225, USA

29. Authors: Goldman-MM Fitterman-DV

Title: Direct Time-Domain Calculation of the Transient-Response for a Rectangular Loop over a 2-Layer Medium Source: GEOPHYSICS 1987, Vol 52, Iss 7, pp 997-1006

Addresses:

INST PETR RES \& GEOPHYS, POB 1717, HOLON, ISRAEL

US GEOL SURVEY, FED CTR, DENVER, CO 80225, USA 
30. Authors: Schulz-E Whitney-JW

Title: Upper Pleistocene and Holocene Lakes in the an Nafud, Saudi-Arabia

Source: HYDROBIOLOGIA

1986, Vol 143, Iss DEC, pp 175-190

Addresses:

GEOG INST, AM HUBLAND, D-8700 WURZBURG, FED-REP-GER

US GEOL SURVEY, FED CTR, DENVER, CO 80225, USA

31. Authors: Poag-CW Cousin-M Goldberg-D Hart-D Miller-KD Mountain-GS Nakamura-Y Palmer-A SchiffelbeinPA Tarafa-M Thein-JE Valentine-PC Watts-AB Wilkins-RH

Title: Site-612

Source: INITIAL REPORTS OF THE DEEP SEA DRILLING PROJECT 1987, Vol 95, Iss APR, pp 31-153

Addresses:

US GEOL SURVEY, WOODS-HOLE, MA 02543, USA

COLUMBIA UNIV, LAMONT DOHERTY GEOL OBSERV, PALISADES, NY

10964, USA

PLYMOUTH POLYTECH, DEPT GEOL SCI, PLYMOUTH PL4 8AA, DEVON,ENGLAND

UNIV TOKYO, EARTHQUAKE CHEM LAB, TOKYO 113, JAPAN

PRINCETON UNIV, DEPT GEOL \& GEOPHYS SCI, PRINCETON, NJ 08544,USA

UNIV CALIF SAN DIEGO, SCRIPPS INST OCEANOG, DEEP SEA DRILLING PROJECT, LA-JOLLA, CA 92093, USA

CUNY QUEENS COLL, DEPT EARTH \& ENVIRONM SCI, FLUSHING, NY

11367, USA

WOODS HOLE OCEANOG INST MIT JOINT PROGRAM OCEANOG, DEPT CHEM, WOODS-HOLE, MA 02543, USA

UNIV PIERRE \& MARIE CURIE, GEODYNAM SOUS MARINE LAB, F-06230 VILLEFRANCHE MER, FRANCE UNIV BONN, INST GEOL, D-5300 BONN 1, FED-REP-GER

MIT, CAMBRIDGE, MA 02139, USA

32. Authors: Poag-CW Cousin-M Goldberg-D Hart-D Miller-KD Mountain-GS Nakamura-Y Palmer-A SchiffelbeinPA Tarafa-M Thein-JE Valentine-PC Watts-AB Wilkins-RH

Title: Site-613

Source: INITIAL REPORTS OF THE DEEP SEA DRILLING PROJECT 1987, Vol 95, Iss APR, pp 155-241

Addresses:

US GEOL SURVEY, WOODS-HOLE, MA 02543, USA

COLUMBIA UNIV, LAMONT DOHERTY GEOL OBSERV, PALISADES, NY

10964, USA

PLYMOUTH POLYTECH, DEPT GEOL SCI, PLYMOUTH PL4 8AA, DEVON,ENGLAND

UNIV TOKYO, EARTHQUAKE CHEM LAB, TOKYO 113, JAPAN

PRINCETON UNIV, DEPT GEOL \& GEOPHYS SCI, PRINCETON, NJ 08544, USA

UNIV CALIF SAN DIEGO, SCRIPPS INST OCEANOG, DEEP SEA DRILLING PROJECT, LA-JOLLA, CA 92093,

USA

CUNY QUEENS COLL, DEPT EARTH \& ENVIRONM SCI, FLUSHING, NY

11367, USA

WOODS HOLE OCEANOG INST MIT JOINT PROGRAM OCEANOG, DEPT CHEM, WOODS-HOLE, MA 02543, USA

MIT, CAMBRIDGE, MA 02139, USA

UNIV BONN, INST GEOL, D-5300 BONN 1, FED-REP-GER 
33. Authors: Evans-HT Tourne-CM Tourne-GF Weakley-TJR

Title: X-Ray Crystallographic and W-183 Nuclear Magnetic-

Resonance Structural Studies of the

(M4(H2O)2(Xw9O34)2)10- Heteropolyanions ( $\mathrm{M}=\mathrm{Co}(\mathrm{II})$ or $\mathrm{Zn}, \mathrm{X}=\mathrm{P}$ or As)

Source: JOURNAL OF THE CHEMICAL SOCIETY-DALTON TRANSACTIONS 1986, Iss 12, pp 2699-2705

Addresses:

US GEOL SURVEY, NATL CTR 959, RESTON, VA 22092, USA

UNIV MONTPELLIER 2, CHIM SOLIDES LAB, F-34060 MONTPELLIER, FRANCE

DUNDEE UNIV, DEPT CHEM, DUNDEE DD1 YNH, SCOTLAND

34. Authors: Gladwin-MT Francis-M Gwyther-RL Hart-R Johnston-MJS

Title: Borehole Tensor Strain-Measurements in California

Source: JOURNAL OF GEOPHYSICAL RESEARCH-SOLID EARTH AND PLANETS 1987, Vol 92, Iss NB8, pp 7981-7988

Addresses:

UNIV QUEENSLAND, DEPT PHYS, ST LUCIA, QLD 4067, AUSTRALIA

US GEOL SURVEY, MENLO-PK, CA 94025, USA

35. Authors: Mankinen-EA Coe-RS Gromme-CS Larson-EE Prevot-M

Title: The Steens Mountain (Oregon) Geomagnetic Polarity

Transition .3. Its Regional Significance

Source: JOURNAL OF GEOPHYSICAL RESEARCH-SOLID EARTH AND PLANETS 1987, Vol 92, Iss NB8, pp 8057-8076

Addresses:

US GEOL SURVEY, MS 937, 345 MIDDLEFIELD RD, MENLO-PK, CA 94025, USA

UNIV COLORADO, DEPT GEOL SCI, BOULDER, CO 80309, USA

UNIV MONTPELLIER 2, F-34060 MONTPELLIER, FRANCE

CNRS, CTR GEOL \& GEOPHYS, F-34033 MONTPELLIER, FRANCE

UNIV CALIF SANTA CRUZ, EARTH SCI BOARD, SANTA-CRUZ, CA 95064, USA

36. Authors: Shedlock-KM Baranowski-J Hu-XL Xiao-WW

Title: The Tangshan Aftershock Sequence

Source: JOURNAL OF GEOPHYSICAL RESEARCH-SOLID EARTH AND PLANETS 1987, Vol 92, Iss NB3, pp 2791-2803

Addresses:

US GEOL SURVEY, DENVER FED CTR, BOX 25046, MS 966, DENVER, CO 80225, USA

COLUMBIA UNIV, LAMONT DOHERTY GEOL OBSERV, PALISADES, NY

10964, USA

STATE SEISMOL BUR, INST GEOPHYS, BEIJING, PEOPLES-R-CHINA

HEBEI PROV SEISMOL BUR, SHIJIAZHUANG, PEOPLES-R-CHINA

Record 371 of 796.

37. Authors: Yukutake-T Cain-JC

Title: Solar-Cycle Variations in the Annual Mean-Values of the Geomagnetic Components of Observatory Data Source: JOURNAL OF GEOMAGNETISM AND GEOELECTRICITY 1987, Vol 39, Iss 1, pp 19-46

Addresses:

UNIV TOKYO, EARTHQUAKE RES INST, BUNKYO KU, TOKYO 113, JAPAN

US GEOL SURVEY, DENVER, CO 80225, USA 
38. Authors: Pojeta-J Norford-BS

Title: A Bohemian-Type Silurian (Wenlockian) Pelecypod Faunule from Arctic Canada

Source: JOURNAL OF PALEONTOLOGY

1987, Vol 61, Iss 3, pp 508-520

Addresses:

US GEOL SURVEY, WASHINGTON, DC 20560, USA

GEOL SURVEY CANADA, CALGARY T2L 2A7, ALBERTA, CANADA

39. Authors: Plusquellec-Y Sando-WJ

Title: The Microstructure of Michelinia-Meekana Girty, 1910

Source: JOURNAL OF PALEONTOLOGY

1987, Vol 61, Iss 1, pp 10-13

Addresses:

LAB PALEONTOL \& STRATIG PALEOZ, F-29283 BREST, FRANCE

US GEOL SURVEY, US NATL MUSEUM NAT HIST, WASHINGTON, DC 20560,USA

40. Authors: Wnuk-C Pfefferkorn-HW

Title: A Pennsylvanian-Age Terrestrial Storm Deposit - Using Plant Fossils to Characterize the History and Process of

Sediment Accumulation

Source: JOURNAL OF SEDIMENTARY PETROLOGY

1987, Vol 57, Iss 2, pp 212-221

Addresses:

US GEOL SURVEY, 956 NATL CTR, RESTON, VA 22092, USA

UNIV HEIDELBERG, INST GEOL PALAONTOL, D-6900 HEIDELBERG, FED-REP-GER

41. Authors: Wenk-HR Bechler-E Erskine-BG Matthies-S Takeshita-T

Title: Pure Shear and Simple Shear Calcite Textures -

Comparison of Experimental, Theoretical and Natural Data

Source: JOURNAL OF STRUCTURAL GEOLOGY

1987, Vol 9, Iss 5-6, pp 731-745

Addresses:

UNIV CALIF BERKELEY, DEPT GEOL \& GEOPHYS, BERKELEY, CA 94720, USA

US GEOL SURVEY, MENLO-PK, CA 94025, USA

ACAD SCI GDR, ZENT INST KERNFORSCH, DDR-8051 DRESDEN, GER-DEM-REP

INST UNIV TECHNOL, TROYES, FRANCE

42. Authors: Sigurdsson-H Devine-JD Evans-WC Presser-TS Pringle-MKW Tchoua-FM

Title: Origin of the Lethal Gas Burst from Lake Monoun, Cameroun

Source: JOURNAL OF VOLCANOLOGY AND GEOTHERMAL RESEARCH

1987, Vol 31, Iss 1-2, pp 1-16

Addresses:

UNIV RHODE ISL, GRAD SCH OCEANOG, KINGSTON, RI 02881, USA

UNIV YAOUNDE, DEPT SCI TERRE, YAOUNDE, CAMEROON

US GEOL SURVEY, MENLO-PK, CA 94025, USA

43. Authors: Sasada-M Belkin-HE Roedder-E

Title: Fluid Inclusions from Drill Hole Dw-5, Hohi Geothermal Area, Japan - Evidence of Boiling and Procedure for

Estimating $\mathrm{CO} 2$ Content

Source: JOURNAL OF VOLCANOLOGY AND GEOTHERMAL RESEARCH

1986, Vol 30, Iss 3-4, pp 231-251

Addresses:

GEOL SURVEY JAPAN, TSUKUBA, IBARAKI 305, JAPAN

US GEOL SURVEY, RESTON, VA 22092, USA 
44. Authors: Greene-HG Mckee-CO Tiffin-DL

Title: Structural Deformation and Sedimentation in an Active Caldera, Rabaul, Papua-New-Guinea

Source: JOURNAL OF VOLCANOLOGY AND GEOTHERMAL RESEARCH 1986, Vol 30, Iss 3-4, pp 327-356

Addresses:

US GEOL SURVEY, MENLO-PK, CA 94025, USA

UN ESCAP CCOP SOPAC, SUVA, FIJI

RABAUL VOLCANOL OBSERV, RABAUL, PAPUA-N-GUINEA

45. Authors: Iversen-N Klug-MJ Oremland-RS

Title: Big Soda Lake (Nevada) .3. Pelagic Methanogenesis and Anaerobic Methane Oxidation

Source: LIMNOLOGY AND OCEANOGRAPHY 1987, Vol 32, Iss 4, pp 804-814

Addresses:

INST WATER SOIL \& ENVIRONM TECHNOL, AALBORG, DENMARK

US GEOL SURVEY, MENLO-PK, CA 94025, USA

MICHIGAN STATE UNIV, KELLOGG BIOL STN, HICKORY-CORNERS, MI 49060, USA

46. Authors: Keller-G Dhondt-SL Gilmore-JS Molina-E Oliver-PQ Orth-CJ Shoemaker-EM

Title: Late Eocene Impact Microspherules - Stratigraphy, Age and Geochemistry

Source: METEORITICS

1987, Vol 22, Iss 1, pp 25-60

Addresses:

PRINCETON UNIV, DEPT GEOL \& GEOPHYS SCI, PRINCETON, NJ 08544,USA

UNIV CALIF LOS ALAMOS NATL LAB, LOS-ALAMOS, NM 87545, USA

US GEOL SURVEY, FLAGSTAFF, AZ 86001, USA

UNIV ZARAGOZA, DEPT PALEONTOL, E-50009 SARAGOSSA, SPAIN

47. Authors: Macdonald-R Mattey-DP Mcgarvie-DW Sigurdsson-H Smith-RL Sparks-RSJ

Title: The 1875 Eruption of Askja Volcano, Iceland - Combined Fractional Crystallization and Selective Contamination in the Generation of Rhyolitic Magma

Source: MINERALOGICAL MAGAZINE

1987, Vol 51, Iss 360, pp 183-202

Addresses:

UNIV LANCASTER, DEPT ENVIRONM SCI, LANCASTER LA1 4YQ, ENGLAND

UNIV CAMBRIDGE, DEPT EARTH SCI, CAMBRIDGE CB2 3EQ, ENGLAND

UNIV RHODE ISL, GRAD SCH OCEANOG, NARRAGANSETT, RI 02882, USA

OPEN UNIV, DEPT EARTH SCI, MILTON KEYNES MK7 6AA,

BUCKINGHAMSHIRE, ENGLAND

US GEOL SURVEY, SACRAMENTO, CA 95821, USA

48. Authors: Haynes-CV Elzaghloul-ES Haas-H Johnson-DL

Mehringer-PJ Muller-AB Swedan-A Wyerman-TA

Title: Evidence for the 1st Nuclear-Age Recharge of Shallow Groundwater, Arbain-Desert, Egypt

Source: NATIONAL GEOGRAPHIC RESEARCH 1987, Vol 3, Iss 4, pp 431-438

Addresses:

UNIV ARIZONA, DEPT ANTHROPOL, TUCSON, AZ 85721, USA

UNIV ARIZONA, DEPT GEOSCI, TUCSON, AZ 85721, USA

WASHINGTON STATE UNIV, DEPT ANTHROPOL, PULLMAN, WA 99164, USA

UNIV ILLINOIS, DEPT GEOG, URBANA, IL 61801, USA

SO METHODIST UNIV, RADIOCARBON DATING LAB, DALLAS, TX 75275,USA

SCI APPLICAT INT CORP, MCLEAN, VA, USA

GEOL SURVEY EGYPT, CAIRO, EGYPT

US GEOL SURVEY, RESTON, VA 22092, USA 
49. Authors: Chivas-AR Barnes-I Evans-WC Lupton-JE Stone-JO

Title: Liquid Carbon-Dioxide of Magmatic Origin and Its Role in Volcanic-Eruptions

Source: NATURE 1987, Vol 326, Iss 6113, pp 587-589

Addresses:

AUSTRALIAN NATL UNIV, RES SCH EARTH SCI, GPO BOX 4, CANBERRA, ACT 2601, AUSTRALIA

US GEOL SURVEY, DIV WATER RESOURCES, MENLO-PK, CA 94025, USA

UNIV CALIF SANTA BARBARA, INST MARINE SCI, SANTA-BARBARA, CA 93106, USA

UNIV CALIF SANTA BARBARA, DEPT GEOL SCI, SANTA-BARBARA, CA 93106, USA

50. Authors: Leenheer-JA Malcolm-RL Wilson-MA

Title: Presence and Potential Significance of Aromatic-Ketone

Groups in Aquatic Humic Substances

Source: ORGANIC GEOCHEMISTRY

1987, Vol 11, Iss 4, pp 273-280

Addresses:

US GEOL SURVEY, FED CTR, DENVER, CO 80225, USA

CSIRO, DIV FOSSIL FUELS, RYDE, NSW 2113, AUSTRALIA

51.Authors: Simoneit-BRT Cox-RE Grimalt-JO Hatcher-PG

Nissenbaum-A Wang-TG

Title: Cyclic Terpenoids of Contemporary Resinous Plant

Detritus and of Fossil Woods, Ambers and Coals

Source: ORGANIC GEOCHEMISTRY

1986, Vol 10, Iss 4-6, pp 877-889

Addresses:

OREGON STATE UNIV, COLL OCEANOG, CORVALLIS, OR 97331, USA

US GEOL SURVEY, NATL CTR RESTON, RESTON, VA 22092, USA

UNIV S PACIFIC, SCH NAT RESOURCES, SUVA, FIJI

WEIZMANN INST SCI, IL-76100 REHOVOT, ISRAEL

52.Authors:Palacas-JG Anders-DE Monopolis-D Nicolaou-CA

Title: Geochemical Correlation of Surface and Subsurface Oils, Western Greece

Source: ORGANIC GEOCHEMISTRY

1986, Vol 10, Iss 1-3, pp 417-423

Addresses:

US GEOL SURVEY, BOX 25046, DENVER FED CTR, DENVER, CO 80225,USA

PUBL PETR CORP GREECE, GR-15124 ATHENS, GREECE

53. Authors: Spicer-RA Wolfe-JA

Title: Plant Taphonomy of Late Holocene Deposits in Trinity(Clair Engle) Lake, Northern California

Source: PALEOBIOLOGY 1987, Vol 13, Iss 2, pp 227-245

Addresses:

GOLDSMITHS COLL, DEPT LIFE SCI, CREEK RD, LONDON SE8 3BU, ENGLAND

US GEOL SURVEY, PALEONTOL \& STRATIG BRANCH, DENVER, CO 80225, USA

54. Authors: Spicer-RA Nichols-DJ Wolfe-JA

Title: Alaskan Cretaceous-Tertiary Floras and Arctic Origins

Source: PALEOBIOLOGY

1987, Vol 13, Iss 1, pp 73-83 
Addresses:

GOLDSMITHS COLL, DEPT LIFE SCI, CREEK RD, LONDON SE8 3BU, ENGLAND

US GEOL SURVEY, PALEONTOL \& STRATIG BRANCH, DENVER, CO 80225,USA

55.Authors: Bowen-DQ Fullerton-DS Fulton-RJ Richmond-GM Sibrava-V Velichko-AA

Title: Correlation of Quaternary Glaciations in the Northern Hemisphere

Source: QUATERNARY SCIENCE REVIEWS 1986, Vol 5, pp 509-510

Addresses:

UNIV LONDON, ROYAL HOLLOWAY \& BEDFORD NEW COLL, DEPT GEOG,EGHAM TW20 0EX, SURREY, ENGLAND

US GEOL SURVEY, DENVER, CO 80225, USA

GEOL SURVEY CANADA, OTTAWA K1A 0E8, ONTARIO, CANADA

UNESCO, DIV EARTH SCI, F-75700 PARIS, FRANCE

56.Authors: Westgate-JA Carson-RJ Easterbrook-DJ Naeser-ND

Title: Lake Tapps Tephra - An Early Pleistocene Stratigraphic Marker in the Puget Lowland, Washington

Source: QUATERNARY RESEARCH 1987, Vol 28, Iss 3, pp 340-355

Addresses:

UNIV TORONTO, DEPT GEOL, SCARBOROUGH CAMPUS, SCARBOROUGH M1C 1A4, ONTARIO, CANADA WESTERN WASHINGTON STATE UNIV, DEPT GEOL, BELLINGHAM, WA

98225, USA

US GEOL SURVEY, DENVER, CO 80225, USA

WHITMAN COLL, DEPT GEOL, WALLA-WALLA, WA 99362, USA

57. Authors: Forester-RM Bradbury-JP Delorme-LD

Title: Mid-Holocene Climate in Northern Minnesota

Source: QUATERNARY RESEARCH

1987, Vol 28, Iss 2, pp 263-273

Addresses:

US GEOL SURVEY, MS 919, DENVER, CO 80225, USA

NWRI, BURLINGTON L7R 4A6, ONTARIO, CANADA

58. Authors: Minkin-JA Blank-H Chao-ECT Dulong-FT

Title: Proton Microprobe Analysis of Trace-Element Variations in Vitrinites in the Same and Different Coal Beds Source:

SCANNING MICROSCOPY 1987, Vol 1, Iss 2, pp 503-513

Addresses:

US GEOL SURVEY, 12201 SUNRISE VALLEY DR, NATL CTR, MAIL STOP 954, RESTON, VA 22092, USA

MAX PLANCK INST NUCL PHYS, D-6900 HEIDELBERG 1, FED-REP-GER

59.Authors: Kogel-I Hatcher-PG Hempfling-R Schulten-HR

Title: Decomposition in Forest Humus Layers Studied by Cpmas C-13 NMR, Pyrolysis-Field Ionization-Mass Spectrometry and $\mathrm{CuO}$ Oxidation

Source: SCIENCE OF THE TOTAL ENVIRONMENT

1987, Vol 62, Iss APR, pp 111-113

Addresses:

UNIV BAYREUTH, LEHRSTUHL BODENKUNDE, D-8580 BAYREUTH,

FED-REP-GER

US GEOL SURVEY, RESTON, VA 22092, USA

FACHHSCH FRESENIUS WIESBADEN, WIESBADEN, FED-REP-GER

60.Authors: Brouwers-EM Ager-TA Carter-LD Clemens-WA Sliter-WV Spicer-RA

Title: Dinosaurs on the North Slope, Alaska - High-Latitude, Latest Cretaceous Environments 


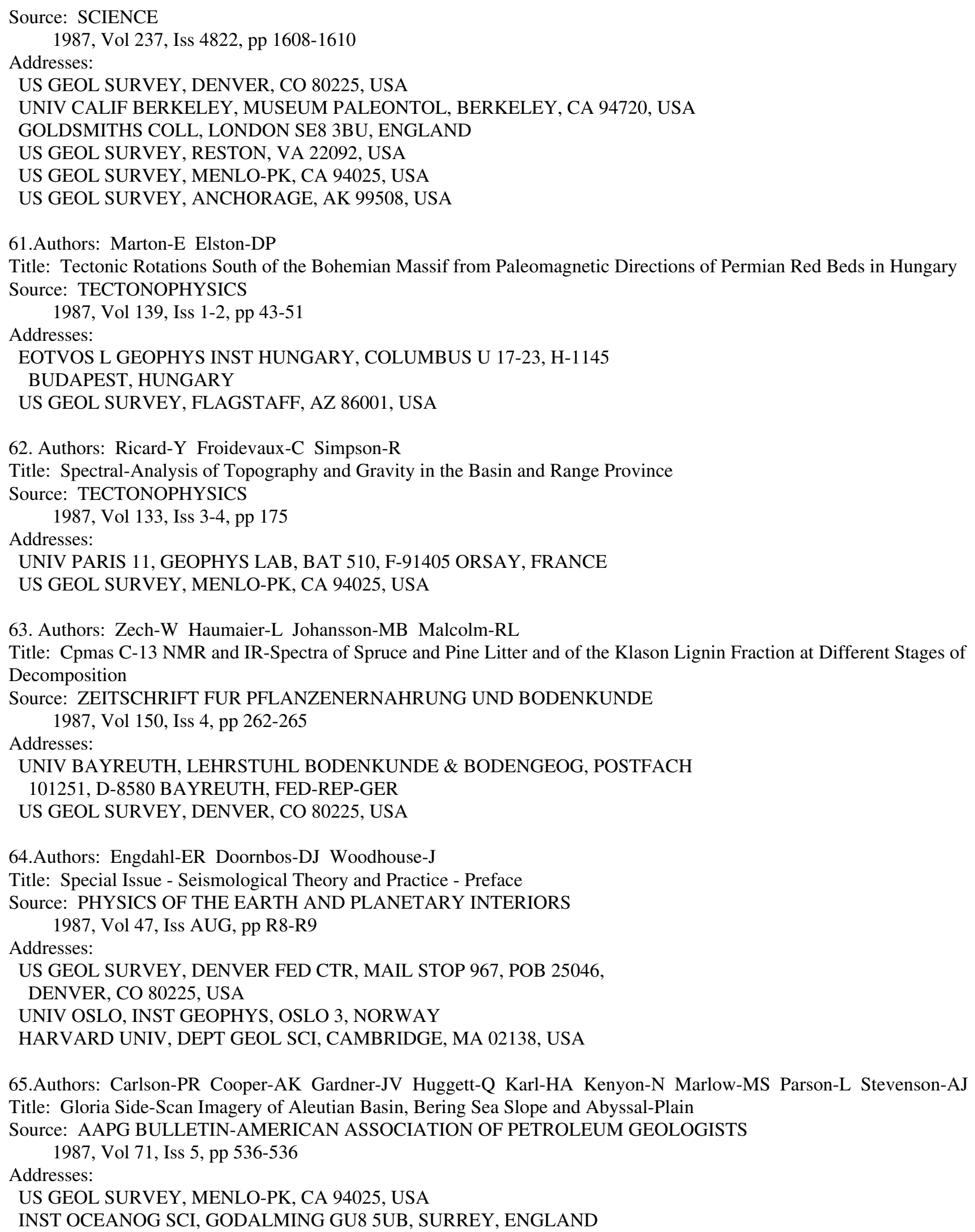


66. Authors: Cooper-AK Davey-FJ

Title: Structural Framework and Hydrocarbon Potential of Ross Sea, Antarctica

Source: AAPG BULLETIN-AMERICAN ASSOCIATION OF PETROLEUM GEOLOGISTS 1987, Vol 71, Iss 5, pp 543-543

Addresses:

US GEOL SURVEY, MENLO-PK, CA 94025, USA

DSIR, WELLINGTON, NEW-ZEALAND

67. Authors: Dillon-WP Edgar-NT Parson-LM Scanlon-KM

Title: Pattern of Growth Folding at Northern Caribbean Plate Boundary North of Western Hispaniola, As Disclosed by

Long-Range Side-Scan Sonar

Source: AAPG BULLETIN-AMERICAN ASSOCIATION OF PETROLEUM GEOLOGISTS 1987, Vol 71, Iss 5, pp 548-548

Addresses:

US GEOL SURVEY, WOODS-HOLE, MA 02543, USA

US GEOL SURVEY, RESTON, VA 22092, USA

INST OCEANOG SCI, GODALMING GU8 5UB, SURREY, ENGLAND

INST OCEANOG SCI, GODALMING GU8 5UB, SURREY, ENGLAND

68. Authors: Gautier-DL Fuentes-JCN Schenk-CJ

Title: Interbedded Alluvium, Tidal Muds and Coarse Littoral Deposits Near San-Felipe, Baja-California - Implications for the Sedimentary Record

Source: AAPG BULLETIN-AMERICAN ASSOCIATION OF PETROLEUM GEOLOGISTS 1987, Vol 71, Iss 5, pp 559-559

Addresses:

ESCUELA SUPER CIENCIAS MARINAS, ENSENADA, BAJA CALIFORNIA, MEXICO

US GEOL SURVEY, DENVER, CO 80225, USA

69. Authors: Mcgregor-BA Kenyon-NH Parson-LM Rothwell-RG Twichell-DC

Title: Morphology of Sea-Floor in Gulf of Mexico

Source: AAPG BULLETIN-AMERICAN ASSOCIATION OF PETROLEUM GEOLOGISTS 1987, Vol 71, Iss 5, pp 591-591

Addresses:

US GEOL SURVEY, RESTON, VA 22092, USA

US GEOL SURVEY, WOODS-HOLE, MA 02543, USA

INST OCEANOG SCI, GODALMING GU8 5UB, SURREY, ENGLAND

70. Authors: Richmond-BM Hein-JR

Title: Holocene Carbonate Sedimentation in Aitutaki Lagoon, Cook-Islands, South-Pacific

Source: AAPG BULLETIN-AMERICAN ASSOCIATION OF PETROLEUM GEOLOGISTS 1987, Vol 71, Iss 5, pp 606-607

Addresses:

US GEOL SURVEY, MENLO-PK, CA 94025, USA

US GEOL SURVEY, SUVA, FIJI

71.Authors: Ross-RJ Hintze-LF James-NP Poole-FG

Title: Evolution of Early Middle Ordovician Carbonate Platform, Basin Ranges of USA

Source: AAPG BULLETIN-AMERICAN ASSOCIATION OF PETROLEUM GEOLOGISTS 1987, Vol 71, Iss 5, pp 607-607

Addresses:

COLORADO STATE UNIV, FT-COLLINS, CO 80523, USA

BRIGHAM YOUNG UNIV, PROVO, UT 84602, USA

QUEENS UNIV, KINGSTON K7L 3N6, ONTARIO, CANADA

US GEOL SURVEY, DENVER, CO 80225, USA 
72. Authors: Williamson-PE Exon-NF Falvey-DA Lock-J Lockwood-K Marlow-MS Obrien-GW Scherl-AS Swift-MG Title: Basin Development and Petroleum Potential of Offshore Otway Basin, Australia

Source: AAPG BULLETIN-AMERICAN ASSOCIATION OF PETROLEUM GEOLOGISTS 1987, Vol 71, Iss 5, pp 627-628

Addresses:

BUR MINERAL RESOURCES GEOL \& GEOPHYS, CANBERRA, AUSTRALIA

US GEOL SURVEY, MENLO-PK, CA 94025, USA

73. Authors: Oremland-RS Miller-LG Robinson-SW Whiticar-MJ

Title: Identification of Sources of Methane in Mono Lake, California

Source: ABSTRACTS OF PAPERS OF THE AMERICAN CHEMICAL SOCIETY 1987, Vol 193, Iss APR, pp 60-

Addresses:

US GEOL SURVEY, MENLO-PK, CA 94025, USA

FED INST GEOSCI \& NAT RESOURCES, D-300051, FED-REP-GER

74. Authors: Peacock-S Crampin-S Fletcher-JB

Title: Temporal Variations in Shear-Wave Splitting in the Anza Seismic Gap

Source: GEOPHYSICAL JOURNAL OF THE ROYAL ASTRONOMICAL SOCIETY 1987, Vol 89, Iss 1, pp 463-463

Addresses:

US GEOL SURVEY, MENLO-PK, CA 94025, USA

BGS, EDINBURGH, SCOTLAND

75. Authors: Silva-RWS Hoover-DB Sampaio-EES

Title: Regional AMT Survey in the North-Central Part of Bahia State, Brazil

Source: GEOPHYSICS 1987, Vol 52, Iss 3, pp 386-386

Addresses:

UNIV FED BAHIA, SALVADOR, BA, BRAZIL

US GEOL SURVEY, WASHINGTON, DC 20242, USA

76. Authors: Mcgregor-BA Kenyon-NH Mattick-RS Schlee-JS

Twichell-DC

Title: Characterization of Sea-Floor in the Gulf of Mexico

Source: GEOPHYSICS 1987, Vol 52, Iss 3, pp 390-390

Addresses:

INST OCEANOG SCI, GODALMING GU8 5UB, SURREY, ENGLAND

US GEOL SURVEY, WASHINGTON, DC 20242, USA

77. Authors: Nishenko-SP Singh-SK

Title: Relocation of the Great Mexican Earthquake of 14 January 1903

Source: BULLETIN OF THE SEISMOLOGICAL SOCIETY OF AMERICA 1987, Vol 77, Iss 1, pp 256-259

Addresses:

US GEOL SURVEY, NATL EARTHQUAKE INFORMAT CTR, DENVER FED CTR, POB 25046, MS 967, DENVER, CO 80225, USA

UNIV NACL AUTONOMA MEXICO, INST GEOFIS, MEXICO CITY 94510, DF,MEXICO

78. Authors: Mckee-EH Rytuba-JJ Xu-K

Title: Geochronology of the Xihuashan Composite Granitic Body and Tungsten Mineralization, Jiangxi Province, South China

Source: ECONOMIC GEOLOGY

1987, Vol 82, Iss 1, pp 218-223 
Addresses:

US GEOL SURVEY, 345 MIDDLEFIELD RD, MAIL STOP 941, MENLO-PK, CA 94025, USA

NANJING UNIV, NANCHING, PEOPLES-R-CHINA

79. Authors: Suess-E Bourgois-J Cruzado-JD Dewever-P Eglinton-G Emeis-KC Garrison-R Greenberg-M Herrera-E Hill-P Ibaraki-M Kastner-M Kemp-AES Kvenvolden-K Langridge-R Lindsleygriffin-N Martini-E Masters-J Mccabe-R Ocola-L Resig-J Sanchez-AW Schrader-H

Thournburg-TM Vonhuene-R Wefer-G Yamano-M

Title: Plate Convergence and Coastal Upwelling - A History of the Peru Continental-Margin from ODP Leg 112 Results Source: COMPTES RENDUS DE L ACADEMIE DES SCIENCES SERIE II-MECANIQUE PHYSIQUE CHIMIE SCIENCES DE L UNIVERS SCIENCES DE LA TERRE 1987, Vol 305, Iss 11, pp 961-967

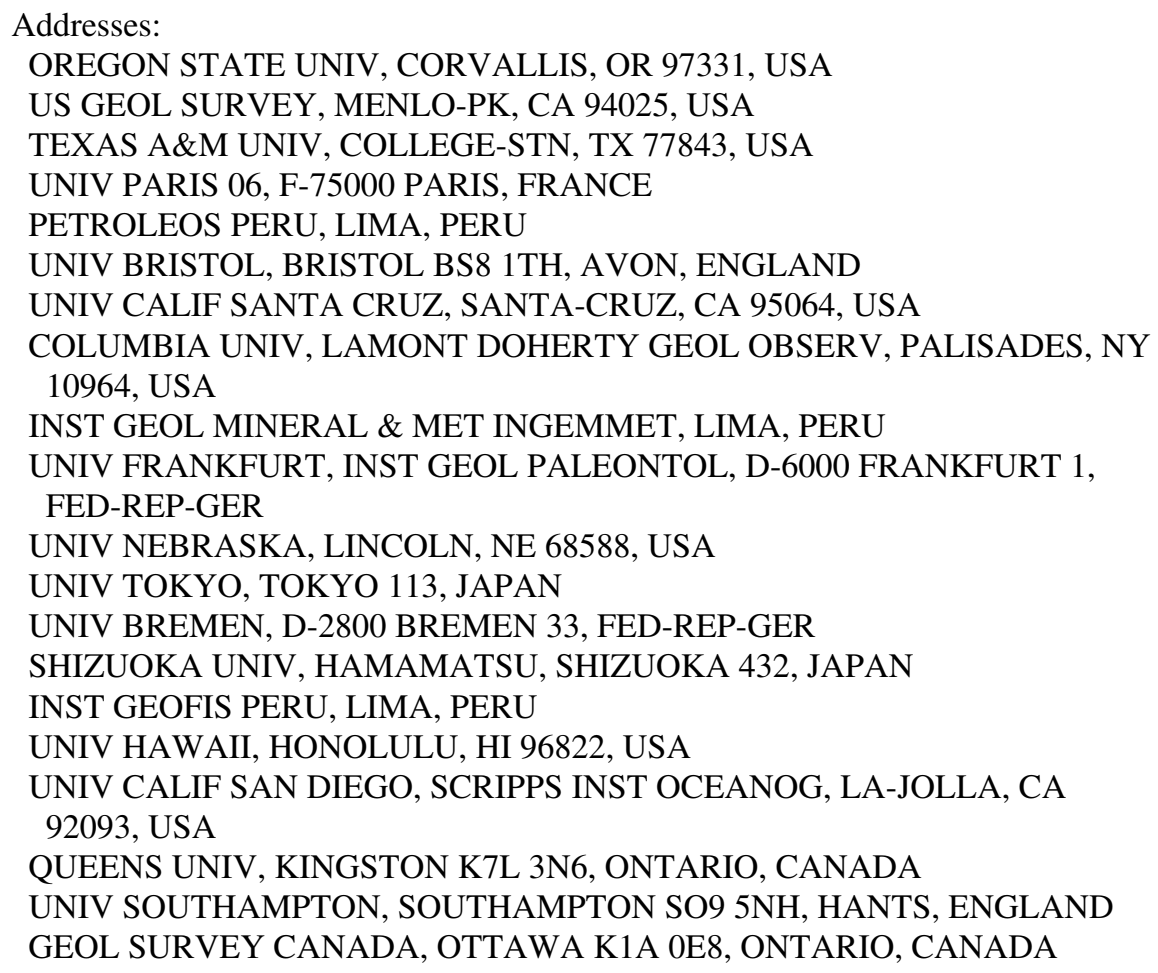

80. Authors: Thouret-JC Calvache-ML Cendrero-A Janda-RJ Pierson-TC

Title: the 13 November 1985 Eruption at Nevado-Del-Ruiz

Colombia) Relationships Between the Eruptive Dynamism, the Ice-Melting and the Genesis of Catastrophic Lahars Source: COMPTES RENDUS DE L ACADEMIE DES SCIENCES SERIE II-MECANIQUE PHYSIQUE CHIMIE SCIENCES DE L UNIVERS SCIENCES DE LA TERRE 1987, Vol 305, Iss 6, pp 505-509

Addresses:

UNIV GRENOBLE 1, INST GEOG ALPINE, CNRS, UA 344, 17 RUE

MAURICE GIGNOUX, F-38031 GRENOBLE, FRANCE

US GEOL SURVEY, CASCADES VOLCANO OBSERV, VANCOUVER, WA, USA

UNIV CANTABRIA, DEPT GEOL, SANTANDER, SPAIN

\section{8}

1. Authors: Nelson-CH Maldonado-A

Title: Factors Controlling Depositional Patterns of Ebro

Turbidite Systems, Mediterranean-Sea 
Source: AAPG BULLETIN-AMERICAN ASSOCIATION OF PETROLEUM GEOLOGISTS 1988, Vol 72, Iss 6, pp 698-716

Addresses:

US GEOL SURVEY, MENLO-PK, CA 94025, USA

CSIC, INST CIENCIAS MAR, E-08003 BARCELONA, SPAIN

2. Authors: Bredehoeft-JD Djevanshir-RD Belitz-KR

Title: Lateral Fluid-Flow in a Compacting Sand-Shale Sequence South Caspian Basin

Source: AAPG BULLETIN-AMERICAN ASSOCIATION OF PETROLEUM GEOLOGISTS 1988, Vol 72, Iss 4, pp 416-424

Addresses:

US GEOL SURVEY, 345 MIDDLEFIELD RD, MENLO-PK, CA 94025, USA

ACAD SCI AZSSR, INST DEEP OIL \& GAS DEPOSITS, BAKU 370143, AZERBAIDZHAN, USSR

3. Authors: Belkin-HE Cavarretta-G Devivo-B Tecce-F

Title: Hydrothermal Phlogopite and Anhydrite from the Sh2 Well, Sabatini Volcanic District, Latium, Italy - Fluid Inclusions and Mineral Chemistry

Source: AMERICAN MINERALOGIST 1988, Vol 73, Iss 7-8, pp 775-797

Addresses:

US GEOL SURVEY, 959 NATL CTR, RESTON, VA 22092, USA

UNIV ROME LA SAPIENZA, DEPARTIMENTO SCI TERRE, CNR, CTR STUDIO

GEOL ITALIA CENT, I-00185 ROME, ITALY

DIPARTIMENTO GEOFIS \& VULCANOL, I-80138 NAPOLI, ITALY

4. Authors: Brown-AV Page-NJ Love-AH

Title: Geology and Platinum-Group-Element Geochemistry of the Serpentine Hill Complex, Dundas Trough, Western Tasmania

Source: CANADIAN MINERALOGIST 1988, Vol 26, Iss MAR, pp 161-175

Addresses:

GEOL SURVEY TASMANIA, POB 56, ROSNY PK, TAS 7018, AUSTRALIA

US GEOL SURVEY, MENLO-PK, CA 94025, USA

US GEOL SURVEY, LAKEWOOD, CO 80225, USA

5. Authors: Broecker-WS Wanninkhof-R Mathieu-G Peng-TH Stine-S Robinson-S Herczeg-A Stuiver-M

Title: The Radiocarbon Budget for Mono Lake - An Unsolved Mystery

Source: EARTH AND PLANETARY SCIENCE LETTERS 1988, Vol 88, Iss 1-2, pp 16-26

Addresses:

COLUMBIA UNIV, LAMONT DOHERTY GEOL OBSERV, PALISADES, NY 10964, USA

OAK RIDGE NATL LAB, DIV ENVIRONM SCI, OAK-RIDGE, TN 37830, USA

UNIV CALIF BERKELEY, DEPT GEOG, BERKELEY, CA 94720, USA

US GEOL SURVEY, MENLO-PK, CA 94025, USA

AUSTRALIAN NATL UNIV, CANBERRA, ACT 2601, AUSTRALIA

UNIV WASHINGTON, DEPT GEOL SCI, SEATTLE, WA 98195, USA

6. Authors: Bourgois-J Pautot-G Bandy-W Boinet-T Chotin-P Huchon-P Delepinay-BM Monge-F Monlau-J Pelletier-B Sosson-M Vonhuene-R 
Title: Seabeam and Seismic-Reflection Imaging of the Tectonic

Regime of the Andean Continental-Margin Off Peru (4-

Degrees-S to 10-Degrees-S)

Source: EARTH AND PLANETARY SCIENCE LETTERS

1988, Vol 87, Iss 1-2, pp 111-126

Addresses:

UNIV PIERRE \& MARIE CURIE, DEPT GEOTECTON, CNRS, UNITE 215,

INSU, T26-00, E1, 4 PL JUSSIEU, F-75252 PARIS, FRANCE

IFREMER, CTR BREST, F-29273 BREST, FRANCE

ECOLE NORMALE SUPER, DEPT GEOL, INSU, CNRS, UNITE 215, F-75252

PARIS, FRANCE

INST GEOFIS PERU, LIMA, PERU

TEXAS A\&M UNIV, DEPT GEOPHYS, COLLEGE-STN, TX 77843, USA

US GEOL SURVEY, MENLO-PK, CA 94025, USA

ORSTOM, NOUMEA, NEW-CALEDONIA

7. Authors: Wilson-MA Batts-BD Hatcher-PG

Title: Molecular Composition and Mobility of Torbanite

Precursors - Implications for the Structure of Coal

Source: ENERGY \& FUELS

1988, Vol 2, Iss 5, pp 668-672

Addresses:

CSIRO, DIV COAL TECHNOL, POB 136, N RYDE, NSW 2113, AUSTRALIA

MACQUARIE UNIV, SCH CHEM, N RYDE, NSW 2109, AUSTRALIA

US GEOL SURVEY, RESTON, VA 22092, USA

8. Authors: Capel-PD Giger-W Reichert-P Wanner-O

Title: Accidental Input of Pesticides into the Rhine River

Source: ENVIRONMENTAL SCIENCE \& TECHNOLOGY 1988, Vol 22, Iss 9, pp 992-997

Addresses:

US GEOL SURVEY, ST-PAUL, MN 55101, USA

SWISS FED INST WATER RESOURCES \& WATER POLLUT CONTROL, CH-8600

DUBENDORF, SWITZERLAND

9. Authors: Heliotis-FD Karandinos-MG Whiton-JC

Title: Air-Pollution and the Decline of the Fir Forest in

Parnis-National-Park, Near Athens, Greece

Source: ENVIRONMENTAL POLLUTION 1988, Vol 54, Iss 1, pp 29-40

Addresses:

GEORGE MASON UNIV, DEPT BIOL, FAIRFAX, VA 22030, USA

ATHENS UNIV AGR SCI, ECOL \& ENVIRONM SCI SECT, ATHENS, GREECE

US GEOL SURVEY, RESTON, VA 22092, USA

10. Authors: Hatcher-PG Lerch-HE Kotra-RK Verheyen-TV

Title: Pyrolysis GC-MS of a Series of Degraded Woods and

Coalified Logs That Increase in Rank from Peat to

Subbituminous Coal

Source: FUEL

1988, Vol 67, Iss 8, pp 1069-1075

Addresses:

US GEOL SURVEY, 923 NATL CTR, RESTON, VA 22092, USA

COAL CORP VICTORIA, VIC 3842, AUSTRALIA 
11. Authors: Greene-HG Exon-NF

Title: Acoustic Stratigraphy and Hydrothermal Activity Within EPI Submarine Caldera, Vanuatu, New Hebrides Arc

Source: GEO-MARINE LETTERS 1988, Vol 8, Iss 3, pp 121-129

Addresses:

US GEOL SURVEY, MENLO-PK, CA 94025, USA

BUR MINERAL RESOURCES GEOL \& GEOPHYS, CANBERRA, ACT 2601, AUSTRALIA

12. Authors: Uchupi-E Schwab-WC Ballard-RD Cheminee-JL Francheteau-J Hekinian-R Blackman-DK Sigurdsson-H

Title: An Angus Argo Study of the Neovolcanic Zone Along the East Pacific Rise from the Clipperton Fracture-Zone to 12-Degrees-N

Source: GEO-MARINE LETTERS 1988, Vol 8, Iss 3, pp 131-138

Addresses:

WOODS HOLE OCEANOG INST, WOODS-HOLE, MA 02543, USA

BROWN UNIV, PROVIDENCE, RI 02883, USA

US GEOL SURVEY, WOODS-HOLE, MA 02543, USA

INST PHYS GLOBE PARIS, F-75230 PARIS 05, FRANCE

CTR BREST, INST FRANCAIS RECH \& EXPLOITAT MER, DEPT GEOSCI MARINES, F-29273 BREST, FRANCE

UNIV RHODE ISL, KINGSTON, RI 02881, USA

13. Authors: Trincardi-F Normark-WR

Title: Sediment Waves on the Tiber Prodelta Slope - Interaction of Deltaic Sedimentation and Currents Along the Shelf

Source: GEO-MARINE LETTERS 1988, Vol 8, Iss 3, pp 149-157

Addresses:

IST GEOL MARINA, VIA ZAMBONI 65, I-40127 BOLOGNA, ITALY

US GEOL SURVEY, MENLO-PK, CA 94025, USA

14. Authors: Oremland-RS Whiticar-MJ Strohmaier-FE Kiene-RP

Title: Bacterial Ethane Formation from Reduced, Ethylated

Sulfur-Compounds in Anoxic Sediments

Source: GEOCHIMICA ET COSMOCHIMICA ACTA 1988, Vol 52, Iss 7, pp 1895-1904

Addresses:

US GEOL SURVEY, MENLO-PK, CA 94025, USA

FED INST GEOSCI \& NAT RESOURCES, D-3000 HANNOVER 51, FED-REP-GER

UNIV MIAMI, ROSENSTIEL SCH MARINE \& ATMOSPHER SCI, MIAMI, FL 33149 , USA

15. Authors: Kennedy-WJ Cobban-WA

Title: Litophragmatoceras-Incomptum Gen Et Sp-Nov (Cretaceous Ammonoidea), a Cryptic Micromorph from the Upper Cenomanian of Arizona

Source: GEOLOGICAL MAGAZINE 1988, Vol 125, Iss 5, pp 535-539 
Addresses:

UNIV OXFORD MUSEUM, GEOL COLLECT, PARKS RD, OXFORD OX1 3PW, ENGLAND

US GEOL SURVEY, DENVER FED CTR, PALEONTOL \& STRATIG BRANCH, DENVER, CO 80225, USA

16. Authors: Silberling-NJ Nichols-KM Bradshaw-JD Blome-CD

Title: Limestone and Chert in Tectonic Blocks from the Esk Head Subterrane, South-Island, New-Zealand

Source: GEOLOGICAL SOCIETY OF AMERICA BULLETIN 1988, Vol 100, Iss 8, pp 1213-1223

Addresses:

US GEOL SURVEY, DENVER, CO 80225, USA

UNIV CANTERBURY, DEPT GEOL, CHRISTCHURCH 1, NEW-ZEALAND

17. Authors: Gardner-MC Bergman-SC Cushing-GW Mackevett-EM Plafker-G Campbell-RB Dodds-CJ Mcclelland-WC Mueller-PA

Title: Pennsylvanian Pluton Stitching of Wrangellia and the Alexander Terrane, Wrangell Mountains, Alaska

Source: GEOLOGY 1988, Vol 16, Iss 11, pp 967-971

Addresses:

ARCO OIL \& GAS CO, $2300 \mathrm{~W}$ PLANO PKWY, PLANO, TX 75075, USA

US GEOL SURVEY, MENLO-PK, CA 94025, USA

GEOL SURVEY CANADA, VANCOUVER V6B 1R8, BC, CANADA

UNIV ARIZONA, DEPT GEOSCI, TUCSON, AZ 85721, USA

UNIV FLORIDA, DEPT GEOL, GAINESVILLE, FL 32611, USA

18. Authors: Green-AG Milkereit-B Davidson-A Spencer-C Hutchinson-DR Cannon-WF Lee-MW Agena-WF Behrendt-JC Hinze-WJ

Title: Crustal Structure of the Grenville Front and Adjacent Terranes Source: GEOLOGY 1988, Vol 16, Iss 9, pp 788-792

Addresses:

GEOL SURVEY CANADA, OTTAWA K1A 0Y3, ONTARIO, CANADA

US GEOL SURVEY, WOODS-HOLE, MA 02543, USA

US GEOL SURVEY, DENVER, CO 80225, USA

PURDUE UNIV, W-LAFAYETTE, IN 47906, USA

19. Authors: Holcomb-RT Moore-JG Lipman-PW Belderson-RH

Title: Voluminous Submarine Lava Flows from Hawaiian Volcanos

Source: GEOLOGY 1988, Vol 16, Iss 5, pp 400-404

Addresses:

UNIV WASHINGTON, SCH OCEANOG WB-10, US GEOL SURVEY, SEATTLE, WA 98195, USA

US GEOL SURVEY, MENLO-PK, CA 94025, USA

US GEOL SURVEY, DENVER, CO 80225, USA

INST OCEANOG SCI, WORMLEY GU8 5UB, ENGLAND

20. Authors: Parrish-JT Spicer-RA

Title: Late Cretaceous Terrestrial Vegetation - A Near-Polar

Temperature Curve 
Source: GEOLOGY

1988, Vol 16, Iss 1, pp 22-25

Addresses:

US GEOL SURVEY, DENVER FED CTR, DENVER, CO 80225, USA

UNIV LONDON, GOLDSMITHS COLL, DEPT LIFE SCI, LONDON SE8 3BU, ENGLAND

21. Authors: Stuart-WF Green-AW

Title: Range Indexes of Geomagnetic-Activity

Source: JOURNAL OF ATMOSPHERIC AND TERRESTRIAL PHYSICS

1988, Vol 50, Iss 6, pp 501-506

Addresses:

BRITISH GEOL SOC, W MAINS RD, EDINBURGH EH9 3LA, SCOTLAND

US GEOL SURVEY, DENVER, CO 80225, USA

22. Authors: Lipman-PW Normark-WR Moore-JG Wilson-JB Gutmacher-CE

Title: The Giant Submarine Alika Debris-Slide, Mauna-Loa, Hawaii

Source: JOURNAL OF GEOPHYSICAL RESEARCH-SOLID EARTH AND PLANETS 1988, Vol 93, Iss NB5, pp 4279-4299

Addresses:

US GEOL SURVEY, DENVER FED CTR, MS 903, DENVER, CO 80225, USA

US GEOL SURVEY, MENLO-PK, CA 94025, USA

INST OCEANOG SCI, GODALMING GU8 5UB, SURREY, ENGLAND

23. Authors: Perrin-M Elston-DP Moussinepouchkine-A

Title: Paleomagnetism of Proterozoic and Cambrian Strata, Adrarde-Mauritanie, Cratonic West-Africa

Source: JOURNAL OF GEOPHYSICAL RESEARCH-SOLID EARTH AND PLANETS 1988, Vol 93, Iss NB3, pp 2159-2178

Addresses:

UNIV MONTPELLIER 2, CTR GEOL \& GEOPHYS, F-34060 MONTPELLIER, FRANCE

US GEOL SURVEY, FLAGSTAFF, AZ 86001, USA

24. Authors: Engdahl-ER Gubbins-D

Title: Simultaneous Travel Time Inversion for Earthquake Location and Subduction Zone Structure in the Central Aleutian Islands

Source: JOURNAL OF GEOPHYSICAL RESEARCH-SOLID EARTH AND PLANETS 1987, Vol 92, Iss NB13, pp 3855-3862

Addresses:

US GEOL SURVEY, DENVER FED CTR, NATL EARTHQUAKE INFORMAT CTR, POB 25046, MAIL STOP 967, DENVER, CO 80225, USA

UNIV CAMBRIDGE, DEPT EARTH SCI, BULLARD LABS, CAMBRIDGE CB3

OEZ, ENGLAND

25. Authors: Brett-R Evans-HT Gibson-EK Hedenquist-JW

Wandless-MV Sommer-MA

Title: Mineralogical Studies of Sulfide Samples and Volatile Concentrations of Basalt Glasses from the Southern Juande-Fuca Ridge

Source: JOURNAL OF GEOPHYSICAL RESEARCH-SOLID EARTH AND PLANETS 1987, Vol 92, Iss NB11, pp 1373-1379 
Addresses:

US GEOL SURVEY, 959 NATL CTR, RESTON, VA 22092, USA

NASA, LYNDON B JOHNSON SPACE CTR, HOUSTON, TX 77058, USA

LOCKHEED ELECTR CO INC, HOUSTON, TX 77058, USA

DSIR, GEOTHERMAL RES CTR, TAUPO, NEW-ZEALAND

26. Authors: Macdonald-R Davies-GR Bliss-CM Leat-PT Bailey-DK

Smith-RL

Title: Geochemistry of High-Silica Peralkaline Rhyolites,

Naivasha, Kenya Rift-Valley

Source: JOURNAL OF PETROLOGY

1987, Vol 28, Iss 6, pp 979-1008

Addresses:

UNIV LANCASTER, DEPT ENVIRONM SCI, LANCASTER LA1 4YQ, ENGLAND

UNIV LEEDS, DEPT EARTH SCI, LEEDS LS2 9JT, W YORKSHIRE, ENGLAND

UNIV LONDON IMPERIAL COLL SCI \& TECHNOL, DEPT GEOL, LONDON SW7 2BP, ENGLAND

UNIV READING, DEPT GEOL, READING RG6 2AB, BERKS, ENGLAND

US GEOL SURVEY, SACRAMENTO, CA 95821, USA

27. Authors: Sneyd-DS Mcsween-HY Sugiura-N Strangway-DW Nord-GL

Title: Origin of Petrofabrics and Magnetic-Anisotropy in Ordinary Chondrites

Source: METEORITICS 1988, Vol 23, Iss 2, pp 139-149

Addresses:

UNIV BRITISH COLUMBIA, VANCOUVER V6T 2B3, BC, CANADA

UNIV TENNESSEE, DEPT GEOL SCI, KNOXVILLE, TN 37996, USA

UNIV TOKYO, INST GEOPHYS, TOKYO 113, JAPAN

US GEOL SURVEY, RESTON, VA 22092, USA

28. Authors: Bischoff-JL Julia-R Mora-R

Title: Uranium-Series Dating of the Mousterian Occupation at Abric-Romani, Spain

Source: NATURE

1988, Vol 332, Iss 6159, pp 68-70

Addresses:

US GEOL SURVEY, 345 MIDDLEFIELD RD, MENLO-PK, CA 94025, USA

INST GEOL JAIME ALMERA, E-08028 BARCELONA, SPAIN

MUSEU HIST CIUTAT, E-17004 GERONA, SPAIN

29. Authors: Boyd-R Mcdade-JM Millard-HT Page-NJ

Title: Platinum Metal Geochemistry of the Bruvann Nickel CopperDeposit, Rana, North Norway

Source: NORSK GEOLOGISK TIDSSKRIFT 1987, Vol 67, Iss 3, pp 205-213

Addresses:

GEOL SURVEY NORWAY, POB 3006, N-7002 TRONDHEIM, NORWAY

US GEOL SURVEY, DENVER FED CTR, DENVER, CO 80225, USA

US GEOL SURVEY, MENLO-PK, CA 94025, USA

30. Authors: Wilson-MA Collin-PJ Malcolm-RL Perdue-EM

Cresswell-P

Title: Low-Molecular Weight Species in Humic and Fulvic Fractions

Source: ORGANIC GEOCHEMISTRY

1988, Vol 12, Iss 1, pp 7-12 
Addresses:

CSIRO, DIV FOSSIL FUELS, POB 136, N RYDE, NSW 2113, AUSTRALIA

US GEOL SURVEY, DIV WATER RES, DENVER, CO 80225, USA

GEORGIA INST TECHNOL, SCH GEOPHYS SCI, ATLANTA, GA 30332, USA

COMALCO RES, THOMASTOWN, VIC 3074, AUSTRALIA

31. Authors: Saito-T Barron-JA Sakamoto-M

Title: An Early Late Oligocene Age Indicated by Diatoms for a

Primitive Desmostylian Mammal Behemotops from Eastern

Hokkaido, Japan

Source: PROCEEDINGS OF THE JAPAN ACADEMY SERIES B-PHYSICAL AND BIOLOGICAL SCIENCES

1988, Vol 64, Iss 9, pp 269-273

Addresses:

YAMAGATA UNIV, DEPT EARTH SCI, YAMAGATA 990, JAPAN

US GEOL SURVEY, MENLO-PK, CA 94025, USA

32. Authors: Fanning-CM Flint-RB Parker-AJ Ludwig-KR Blissett-AH

Title: Refined Proterozoic Evolution of the Gawler Craton,

South-Australia, Through U-Pb Zircon Geochronology

Source: PRECAMBRIAN RESEARCH

1988, Vol 40-1, Iss OCT, pp 363-386

Addresses:

AUSTRALIAN MINERAL DEV LABS, POB 114, EASTWOOD 5063, AUSTRALIA

GEOL SURVEY S AUSTRALIA, EASTWOOD 5063, AUSTRALIA

US GEOL SURVEY, BRANCH ISOTOPE GEOL, DENVER, CO 80225, USA

33. Authors: Koesterer-ME Frost-CD Frost-BR Hulsebosch-TP

Bridgwater-D Worl-RG

Title: Development of the Archean Crust in the Medina Mountain Area, Wind River Range, Wyoming (USA)

Source: PRECAMBRIAN RESEARCH 1987, Vol 37, Iss 4, pp 287-304

Addresses:

UNIV WYOMING, DEPT GEOL \& GEOPHYS, LARAMIE, WY 82071, USA

COPENHAGEN UNIV, GEOL MUSEUM, DK-1350 COPENHAGEN K, DENMARK

US GEOL SURVEY, DENVER FED CTR, DENVER, CO 80225, USA

34. Authors: Gradstein-FM Agterberg-FP Aubry-MP Berggren-WA

Flynn-JJ Hewitt-R Kent-DV Klitgord-KD Miller-KG

Obradovitch-J Ogg-JG Prothero-DR Westerman-GEG

Title: Sea-Level History

Source: SCIENCE 1988, Vol 241, Iss 4865, pp 599-601

Addresses:

FISHERIES \& OCEANS CANADA, BEDFORD INST OCEANOG, GEOL SURVEY CANADA, DARTMOUTH B2Y 4A2, NS, CANADA

GEOL SURVEY CANADA, OTTAWA K1A 0E8, ONTARIO, CANADA

UNIV LYON 1, CTR PALEONTOL STRATIG \& PALEOECOL, F-6962 1

VILLEURBANNE, FRANCE

WOODS HOLE OCEANOG INST, DEPT GEOL \& GEOPHYS, WOODS-HOLE, MA 02543, USA

RUTGERS STATE UNIV, DEPT GEOL SCI, NEW-BRUNSWICK, NJ 08903, USA

MCMASTER UNIV, DEPT GEOL, HAMILTON L8S 4M1, ONTARIO, CANADA

COLUMBIA UNIV, LAMONT DOHERTY GEOL OBSERV, PALISADES, NY

10964, USA 
US GEOL SURVEY, WOODS-HOLE, MA 02543, USA

US GEOL SURVEY, DENVER, CO 80225, USA

OCCIDENTAL COLL, DEPT GEOL, LOS-ANGELES, CA 90041, USA

PURDUE UNIV, DEPT GEOSCI, W-LAFAYETTE, IN 47907, USA

35. Authors: Sternberg-RW Kranck-K Cacchione-DA Drake-DE

Title: Suspended Sediment Transport Under Estuarine Tidal

Channel Conditions

Source: SEDIMENTARY GEOLOGY

1988, Vol 57, Iss 3-4, pp 257-272

Addresses:

UNIV WASHINGTON, SCH OCEANOG, SEATTLE, WA 98195, USA

US GEOL SURVEY, MENLO-PK, CA 94025, USA

FISHERIES \& OCEANS CANADA, BEDFORD INST OCEANOG, DEPT

FISHERIES \& OCEANS PHYS \& CHEM SCI, DARTMOUTH B2Y 4A2, NS,

CANADA

36. Authors: Kogel-I Hempfling-R Zech-W Hatcher-PG Schulten-HR

Title: Chemical-Composition of the Organic-Matter in Forest

Soils .1. Forest Litter

Source: SOIL SCIENCE

1988, Vol 146, Iss 2, pp 124-136

Addresses:

UNIV BAYREUTH, LEHRSTUHL BODENKUNDE \& BODENGEOG, POSTFACH 101251, D-8580 BAYREUTH, FED-REP-GER

FACHHSCH FRESENIUS, SPURENANALYTIK ABT, D-6200 WIESBADEN, FED-REP-GER

US GEOL SURVEY, RESTON, VA 22092, USA

37. Authors: Li-CS Bockheim-JG Leide-JE Wentz-DA

Title: Potential for Buffering of Acidic Precipitation by

Mineral Weathering in a Forested Entisol

Source: SOIL SCIENCE SOCIETY OF AMERICA JOURNAL 1988, Vol 52, Iss 4, pp 1148-1154

Addresses:

UNIV WISCONSIN, DEPT SOIL SCI, 1525 OBSERV DR, MADISON, WI 53705, USA

CHINESE ACAD SCI, ECOENVIRONM SCI RES CTR, BEIJING,

PEOPLES-R-CHINA

US GEOL SURVEY, DIV WATER RESOURCES, MADISON, WI 53719, USA

38. Authors: Friedmann-H Aric-K Gutdeutsch-R King-CY Altay-C Sav-H

Title: Radon Measurements for Earthquake Prediction Along the North Anatolian Fault Zone - A Progress Report

Source: TECTONOPHYSICS 1988, Vol 152, Iss 3-4, pp 209-214

Addresses:

VIENNA UNIV, INST RADIUMFORSCHUNG \& KERNPHYS, BOLTZMANNGASSE 3, A-1090 VIENNA, AUSTRIA

MADEN TETKIK ARAMA, MINERAL RES \& EXPLORAT INST TURKEY, ANKARA, TURKEY

VIENNA UNIV, INST METEOROL \& GEOPHYS, A-1090 VIENNA, AUSTRIA

US GEOL SURVEY, MENLO-PK, CA 94025, USA 
39. Authors: Shapiro-AM Cvetkovic-VD

Title: Stochastic-Analysis of Solute Arrival Time in Heterogeneous Porous-Media

Source: WATER RESOURCES RESEARCH 1988, Vol 24, Iss 10, pp 1711-1718

Addresses:

US GEOL SURVEY, DIV WATER RESOURCES, 431 NATL CTR, RESTON, VA 22092, USA

ROYAL INST TECHNOL, S-10044 STOCKHOLM 70, SWEDEN

40. Authors: Kogelknabner-I Zech-W Hatcher-PG

Title: Chemical-Composition of the Organic-Matter in Forest

Soils - The Humus Layer

Source: ZEITSCHRIFT FUR PFLANZENERNAHRUNG UND BODENKUNDE 1988, Vol 151, Iss 5, pp 331-340

Addresses:

UNIV BAYREUTH, LEHRSTUHL BODENKUNDE \& BODENGEOG, D-8580

BAYREUTH, FED-REP-GER

US GEOL SURVEY, RESTON, VA 22092, USA

41. Authors: Stauffer-PH Peng-LC

Title: The Upper Paleozoic Pebbly Mudstone Facies of Peninsular Thailand and Western Malaysia - Continental-Margin Deposits of Paleoeurasia - Discussion

Source: GEOLOGISCHE RUNDSCHAU 1987, Vol 76, Iss 3, pp 945-948

Addresses:

US GEOL SURVEY, MS 951, 345 MIDDLEFIELD RD, MENLO-PK, CA 94025, USA

UNIV MALAYA, DEPT GEOG, KUALA LUMPUR 2211, MALAYSIA

42. Authors: Brouwers-EM Spicer-RA Clemens-WA

Title: Arctic Dinosaurs and Terminal Cretaceous Extinctions - Response

Source: SCIENCE 1988, Vol 239, Iss 4835, pp 11-11

Addresses:

US GEOL SURVEY, DENVER, CO 80225, USA

GOLDSMITHS COLL, LONDON SE8 3BU, ENGLAND

UNIV CALIF BERKELEY, MUSEUM PALEONTOL, BERKELEY, CA 94720, USA

43. Authors: Field-ME Gardner-JV Alonso-B

Title: Facies Architecture and Processes of Post-Messinian Prograding Basin Fill, Valencia Trough, Western Mediterranean

Source: AAPG BULLETIN-AMERICAN ASSOCIATION OF PETROLEUM GEOLOGISTS 1988, Vol 72, Iss 8, pp 1002-1002

Addresses:

US GEOL SURVEY, MENLO-PK, CA 94025, USA

UNIV BARCELONA, BARCELONA 7, SPAIN

44. Authors: Nelson-CH Maldonado-A

Title: Ebro Margin Sedimentary System in the Western Mediterranean-Sea, from Delta to Deep-Sea

Source: AAPG BULLETIN-AMERICAN ASSOCIATION OF PETROLEUM GEOLOGISTS 1988, Vol 72, Iss 8, pp 1016-1016 
Addresses:

US GEOL SURVEY, MENLO-PK, CA 94025, USA

CSIC, INST CIENCIAS MAR, BARCELONA, SPAIN

45. Authors: Trincardi-F Normark-WR

Title: Depositional Characteristics of Suvero Submarine Slide, Paola Slope Basin, Eastern Tyrrhenian Margin

Source: AAPG BULLETIN-AMERICAN ASSOCIATION OF PETROLEUM GEOLOGISTS 1988, Vol 72, Iss 8, pp 1027-1027

Addresses:

IST GEOL MARINA, BOLOGNA, ITALY

US GEOL SURVEY, MENLO-PK, CA 94025, USA

46. Authors: Aggarwal-PK Adams-S Gunter-WD Kharaka-YK

Title: The Effect of Pressure on Aqueous Equilibria

Source: ABSTRACTS OF PAPERS OF THE AMERICAN CHEMICAL SOCIETY 1988, Vol 196, Iss SEP, pp 51-

Addresses:

BATTELLE MEM INST, COLUMBUS, OH 43201, USA

ALBERTA RES COUNCIL, EDMONTON T6G 2C2, ALBERTA, CANADA

US GEOL SURVEY, MENLO-PK, CA 94025, USA

47. Authors: Crampin-S Booth-D Evans-R Peacock-S Fletcher-JB

Title: Precursory Shear-Wave Splitting at Anza - The Prospect for Earthquake Prediction

Source: GEOPHYSICAL JOURNAL-OXFORD 1988, Vol 92, Iss 3, pp 525-525

Addresses:

BRITISH GEOL SURVEY, EDINBURGH, SCOTLAND

UNIV READING, DEPT GEOL, READING RG6 2AH, BERKS, ENGLAND

US GEOL SURVEY, MENLO-PK, CA 94025, USA

48. Authors: Hildreth-W Moorbath-S

Title: Crustal Contributions to Arc Magmatism in the Andes of Central Chile

Source: CONTRIBUTIONS TO MINERALOGY AND PETROLOGY 1988, Vol 98, Iss 4, pp 455-489

Addresses:

US GEOL SURVEY, MENLO-PK, CA 94025, USA

UNIV OXFORD, DEPT EARTH SCI, OXFORD OX1 3PR, ENGLAND

49. Authors: Jones-BF Galan-E

Title: Sepiolite and Palygorskite

Source: REVIEWS IN MINERALOGY 1988, Vol 19, pp 631-674

Addresses:

US GEOL SURVEY, 432 NATL CTR, RESTON, VA 22092, USA

UNIV SEVILLE, DEPT GEOL, SEVILLE, SPAIN

50. Authors: Foucher-JP Dupont-J Bouysse-P Charlou-JL

Davagnier-M Eissen-JP Fouquet-Y Gueneley-S

Harmegnies-F Lafoy-Y Lapouille-A Maze-JP Morton-J

Ondreas-H Ruellan-E Sibuet-JC

Title: The Valu FA Ridge in the Southern Lau Basin (Southwest Pacific) 
Source: COMPTES RENDUS DE L ACADEMIE DES SCIENCES SERIE IIMECANIQUE PHYSIQUE CHIMIE SCIENCES DE L UNIVERS SCIENCES DE LA TERRE 1988, Vol 307, Iss 6, pp 609-616

Addresses:

INST FRANCAIS RECH \& EXPLOITAT MER CB, GTS OCEANOL \& GEODYNAM, BP 70, F-29263 PLOUZANE, FRANCE

UNIV NICE, CNRS, URA GEODYNAM, F-06565 VALBONNE, FRANCE

ORSTOM, GIS OCEANOL \& GEODYNAM, NOUMEA, NEW-CALEDONIA

BUR RECH GEOL \& MINIERES, F-45060 ORLEANS, FRANCE

UNIV BREST OCCIDENTALE, GIS OCEANOL \& GEODYNAM, F-29287 BREST,

FRANCE

US GEOL SURVEY, MENLO-PK, CA 94025, USA

51. Authors: Culver-SJ Pojeta-J Repetski-JE Robineau-B

Title: Stratigraphic Significance of Early Cambrian Shelly

Microfossils in the Taoudeni Basin at the Guinea-Senegal

Border

Source: COMPTES RENDUS DE L ACADEMIE DES SCIENCES SERIE IIMECANIQUE PHYSIQUE CHIMIE SCIENCES DE L UNIVERS

SCIENCES DE LA TERRE

1988, Vol 307, Iss 6, pp 651-656

Addresses:

OLD DOMINION UNIV, DEPT GEOL, NORFOLK, VA 23508, USA

US GEOL SURVEY, WASHINGTON, DC 20242, USA

UNIV DAKAR, +DEPT GEOL, DAKAR, SENEGAMBIA

52. Authors: Delumley-H Delumley-MA Beltrao-M Yokoyama-Y

Labeyrie-J Danon-J Delibrias-G Falgueres-C

Bischoff-JL

Title: Discovery of Flaked Tools Associated with Middle

Pleistocene Fauna in the Toca-da-Esperanca, Bahia State,

Brazil

Source: COMPTES RENDUS DE L ACADEMIE DES SCIENCES SERIE IIMECANIQUE PHYSIQUE CHIMIE SCIENCES DE L UNIVERS

SCIENCES DE LA TERRE

1988, Vol 306, Iss 3, pp 241-247

Addresses:

INST PALEONTOL HUMAINE, MUSEUM NATL HIST NAT, PREHIST LAB, CNRS, UNITE 184, 1 RUE RENE PANHARD, F-75013 PARIS, FRANCE

UNIV FED RIO DE JANEIRO, MUSEU NACL, DEPT ANTROPOL, SETOR

ARQUEOL, RIO DE JANEIRO, RJ, BRAZIL

CNRS, CEA, CTR FAIBLES RADIOACTIVITES, F-91190 GIF SUR YVETTE, FRANCE

CONSELHO NACL PESQUISAS, BR-22290 RIO DE JANEIRO, RJ, BRAZIL

US GEOL SURVEY, PACIFIC MARINE GEOL BRANCH, MENLO-PK, CA 94025, USA

\section{9}

1. Authors: Eberl-DD Srodon-J

Title: Ostwald Ripening and Interparticle-Diffraction Effects

for Illite Crystals

Source: AMERICAN MINERALOGIST

1988, Vol 73, Iss 11-1, pp 1335-1345 
Addresses:

US GEOL SURVEY, DENVER FED CTR, MAIL STOP 404, DENVER, CO

80225, USA

POLISH ACAD SCI, INST GEOL SCI, PL-31002 KRAKOW, POLAND

2. Authors: Oremland-RS Kiene-RP Mathrani-I Whiticar-MJ

Boone-DR

Title: Description of an Estuarine Methylotrophic Methanogen

Which Grows on Dimethyl Sulfide

Source: APPLIED AND ENVIRONMENTAL MICROBIOLOGY

1989, Vol 55, Iss 4, pp 994-1002

Addresses:

US GEOL SURVEY, 345 MIDDLEFIELD RD, MENLO-PK, CA 94025, USA

FED INST GEOSCI \& NAT RESOURCES, D-3000 HANNOVER 51,

FED-REP-GER

UNIV GEORGIA, INST MARINE, SAPELO-ISL, GA 31327, USA

UNIV CALIF LOS ANGELES, SCH PUBL HLTH, LOS-ANGELES, CA 90024, USA

OREGON GRAD CTR, DEPT ENVIRONM SCI \& ENGN, BEAVERTON, OR 97006, USA

3. Authors: Veevers-JJ Eittreim-SL

Title: Reconstruction of Antarctica and Australia At Breakup (95+/-5 Ma) and Before Rifting (160-Ma)

Source: AUSTRALIAN JOURNAL OF EARTH SCIENCES 1988, Vol 35, Iss 3, pp 355-362

Addresses:

MACQUARIE UNIV, SCH EARTH SCI, AUSTRALIAN PLATE RES GRP, RYDE, NSW 2109, AUSTRALIA

US GEOL SURVEY, MENLO-PK, CA 94025, USA

4. Authors: Smith-TJ Hudson-JH Robblee-MB Powell-GVN Isdale-PJ

Title: Fresh-Water Flow from the Everglades to Florida Bay - A

Historical Reconstruction Based on Fluorescent Banding

in the Coral Solenastrea-Bournoni

Source: BULLETIN OF MARINE SCIENCE

1989, Vol 44, Iss 1, pp 274-282

Addresses:

US GEOL SURVEY, FISHER ISL STN, MIAMI-BEACH, FL 33139, USA

EVERGLADES NATL PK, S FLORIDA RES CTR, HOMESTEAD, FL 33030, USA

AUSTRALIAN INST MARINE SCI, TOWNSVILLE, QLD 4810, AUSTRALIA

NATL AUDUBON SOC, RES DEPT, TAVERNIER, FL 33070, USA

5. Authors: Slack-JF Coad-PR

Title: Multiple Hydrothermal and Metamorphic Events in the Kidd-

Creek Volcanogenic Massive Sulfide Deposit, Timmins,

Ontario - Evidence from Tourmalines and Chlorites

Source: CANADIAN JOURNAL OF EARTH SCIENCES 1989, Vol 26, Iss 4, pp 694-715

Addresses:

US GEOL SURVEY, NATL CTR, MS 954, RESTON, VA 22092, USA

KIDD CREEK MINES LTD, TIMMINS P4N 7K1, ONTARIO, CANADA

6. Authors: Ludwig-KR Turi-B

Title: Paleozoic Age of the Capo-Spartivento Orthogneiss,

Sardinia, Italy 
Source: CHEMICAL GEOLOGY

1989, Vol 79, Iss 2, pp 147-153

Addresses:

US GEOL SURVEY, DENVER, CO 80225, USA

UNIV ROME LA SAPIENZA, DEPT EARTH SCI, I-01100 ROME, ITALY

7. Authors: Gregory-RT Criss-RE Taylor-HP

Title: Oxygen Isotope Exchange Kinetics of Mineral Pairs in Closed and Open Systems - Applications to Problems of Hydrothermal Alteration of Igneous Rocks and Precambrian Iron Formations

Source: CHEMICAL GEOLOGY 1989, Vol 75, Iss 1-2, pp 1-42

Addresses:

MONASH UNIV, DEPT EARTH SCI, CLAYTON, VIC 3168, AUSTRALIA

CALTECH, DIV GEOL \& PLANETARY SCI, PASADENA, CA 91125, USA

US GEOL SURVEY, RESTON, VA 22091, USA

8. Authors: Aleinikoff-JN Williams-IS Compston-W Stuckless-JS

Worl-RG

Title: Evidence for an Early Archean Component in the Middle to

Late Archean Gneisses of the Wind River Range, West-

Central Wyoming - Conventional and Ion Microprobe U-Pb

Data

Source: CONTRIBUTIONS TO MINERALOGY AND PETROLOGY 1989, Vol 101, Iss 2, pp 198-206

Addresses:

US GEOL SURVEY, BOX 25046, DENVER, CO 80225, USA

AUSTRALIAN NATL UNIV, RES SCH EARTH SCI, CANBERRA, ACT 2600 , AUSTRALIA

9. Authors: Burchfiel-BC Molnar-P Zhao-ZY Liang-K Wang-SJ

Huang-MM Sutter-J

Title: Geology of the Ulugh-Muztagh Area, Northern Tibet

Source: EARTH AND PLANETARY SCIENCE LETTERS 1989, Vol 94, Iss 1-2, pp 57-70

Addresses:

MIT, DEPT EARTH ATMOSPHER \& PLANETARY SCI, CAMBRIDGE, MA 02139, USA

XINJIANG PROV BUR GEOL, REG GEOL SURVEYING TEAM, QITAI, PEOPLES-R-CHINA

ACAD SINICA, INST GEOG, URUMQI, PEOPLES-R-CHINA

US GEOL SURVEY, RESTON, VA 22092, USA

10. Authors: Eisenreich-SJ Capel-PD Robbins-JA Bourbonniere-R

Title: Accumulation and Diagenesis of Chlorinated Hydrocarbons in Lacustrine Sediments

Source: ENVIRONMENTAL SCIENCE \& TECHNOLOGY 1989, Vol 23, Iss 9, pp 1116-1126

Addresses:

UNIV MINNESOTA, DEPT CIVIL \& MINERAL ENGN, MINNEAPOLIS, MN 55455, USA

US GEOL SURVEY, DIV WATER RESOURCES, ST-PAUL, MN 55101, USA

NOAA, GREAT LAKES ENVIRONM RES LAB, ANN-ARBOR, MI 48104, USA

ENVIRONM CANADA, CANADA CTR INLAND WATERS, NATL WATER RES

INST, BURLINGTON L7R 4A6, ONTARIO, CANADA 
11. Authors: Ludwig-KR Vollmer-R Turi-B Simmons-KR Perna-G

Title: Isotopic Constraints on the Genesis of Base-Metal Ores in Southern and Central Sardinia

Source: EUROPEAN JOURNAL OF MINERALOGY 1989, Vol 1, Iss 5, pp 657-666

Addresses:

US GEOL SURVEY, BOX 25046, DENVER, CO 80225, USA

UNIV LEEDS, DEPT EARTH SCI, LEEDS LS2 9JT, W YORKSHIRE, ENGLAND

UNIV BOLOGNA, IST ITALIANO SPELEOL, I-40126 BOLOGNA, ITALY

UNIV ROME LA SAPIENZA, DIPARTIMENTO SCI TERRA, I-00100 ROME, ITALY

12. Authors: Walker-RJ Shirey-SB Hanson-GN Rajamani-V Horan-MF

Title: Re-Os, Rb-Sr, and O Isotopic Systematics of the Archean

Kolar Schist Belt, Karnataka, India

Source: GEOCHIMICA ET COSMOCHIMICA ACTA

1989, Vol 53, Iss 11, pp 3005-3013

Addresses:

US GEOL SURVEY, MS 981, RESTON, VA 22092, USA

JAWAHARLAL NEHRU UNIV, SCH ENVIRONM SCI, NEW DELHI 110067 ,

INDIA

CARNEGIE INST WASHINGTON, DEPT TERR MAGNETISM, WASHINGTON, DC 20015, USA

SUNY STONY BROOK, DEPT EARTH \& SPACE SCI, STONY-BROOK, NY 11794, USA

13. Authors: Nordstrom-DK Olsson-T Carlsson-L Fritz-P

Title: Introduction to the Hydrogeochemical Investigations Within the International Stripa Project

Source: GEOCHIMICA ET COSMOCHIMICA ACTA 1989, Vol 53, Iss 8, pp 1717-1726

Addresses:

US GEOL SURVEY, 345 MIDDLEFIELD RD, MENLO-PK, CA 94025, USA

SWEDISH GEOL AB, S-4119 GOTHENBURG, SWEDEN

GESELL STRAHLEN \& UMWELTFORSCH MBH, INST RADIOHYDROMETRIE, D-8042 NEUHERBERG, FED-REP-GER

GEOSYST AB, S-75008 UPPSALA, SWEDEN

14. Authors: Nordstrom-DK Lindblom-S Donahoe-RJ Barton-CC

Title: Fluid Inclusions in the Stripa Granite and Their

Possible Influence on the Groundwater Chemistry

Source: GEOCHIMICA ET COSMOCHIMICA ACTA 1989, Vol 53, Iss 8, pp 1741-1755

Addresses:

US GEOL SURVEY, 345 MIDDLEFIELD RD, MENLO-PK, CA 94025, USA

US GEOL SURVEY, LAKEWOOD, CO 80225, USA

UNIV ALABAMA, DEPT GEOL, TUSCALOOSA, AL 35487, USA

UNIV STOCKHOLM, DEPT GEOL, ORE RES GRP, S-10691 STOCKHOLM, SWEDEN

15. Authors: Smith-PE Farquhar-RM Tatsumoto-M

Title: Assessment of the U-Th-Pb System in 2 Archean

Metabasalts - Deciphering the Complex Histories of

Sulfides and Silicates Using Acid Leaching Methods 


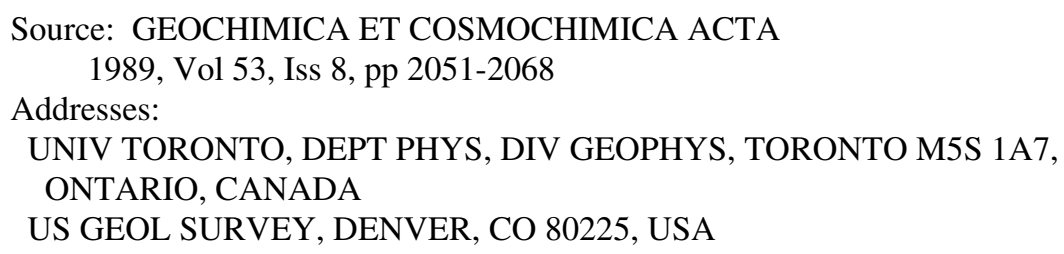

16. Authors: Hatcher-PG Schnitzer-M Vassallo-AM Wilson-MA

Title: The Chemical-Structure of Highly Aromatic Humic Acids in 3 Volcanic Ash Soils As Determined by Dipolar Dephasing NMR-Studies

Source: GEOCHIMICA ET COSMOCHIMICA ACTA 1989, Vol 53, Iss 1, pp 125-130

Addresses:

US GEOL SURVEY, MS 923, RESTON, VA 22092, USA

CSIRO, DIV COAL TECHNOL, N RYDE, NSW 2113, AUSTRALIA

AGR CANADA, LAND RESOURCE RES CTR, OTTAWA K1A 0C6, ONTARIO, CANADA

17. Authors: Kennedy-WJ Cobban-WA

Title: Mid-Turonian Ammonite Faunas from Northern Mexico

Source: GEOLOGICAL MAGAZINE 1988, Vol 125, Iss 6, pp 593-612

Addresses:

UNIV OXFORD MUSEUM, PARKS RD, OXFORD OX1 3PW, ENGLAND

US GEOL SURVEY, DENVER, CO 80225, USA

18. Authors: Tarduno-JA Sliter-WV Bralower-TJ Mcwilliams-M Premolisilva-I Ogg-JG

Title: M-Sequence Reversals Recorded in DSDP Sediment Cores from the Western Mid-Pacific Mountains and Magellan Rise

Source: GEOLOGICAL SOCIETY OF AMERICA BULLETIN 1989, Vol 101, Iss 10, pp 1306-1316

Addresses:

STANFORD UNIV, DEPT GEOPHYS, STANFORD, CA 94305, USA

US GEOL SURVEY, MENLO-PK, CA 94025, USA

FLORIDA INT UNIV, DEPT GEOL, MIAMI, FL 33199, USA

UNIV MILAN, DEPARTIMENTO SCI TERRE, I-20122 MILAN, ITALY

PURDUE UNIV, DEPT EARTH \& ATMOSPHER SCI, W-LAFAYETTE, IN 47907, USA

19. Authors: Elliott-WC Aronson-JL Millard-HT Gierlowskikordesch-E

Title: The Origin of the Clay-Minerals At the Cretaceous Tertiary Boundary in Denmark

Source: GEOLOGICAL SOCIETY OF AMERICA BULLETIN 1989, Vol 101, Iss 5, pp 702-710

Addresses:

CASE WESTERN RESERVE UNIV, DEPT GEOL SCI, CLEVELAND, OH 44106, USA

FREE UNIV BERLIN, INST PALAEONTOL, D-1000 BERLIN 33, FED-REP-GER

US GEOL SURVEY, DENVER FED CTR, DENVER, CO 80225, USA 
20. Authors: Dolan-J Beck-C Ogawa-Y

Title: Upslope Deposition of Extremely Distal Turbidites - An

Example from the Tiburon Rise, West-Central Atlantic

Source: GEOLOGY

1989, Vol 17, Iss 11, pp 990-994

Addresses:

US GEOL SURVEY, MS 999, MENLO-PK, CA 94025, USA

UNIV SAVOIE, DEPT GEOSCI, F-77011 CAMBERY, FRANCE

KYUSHU UNIV 33, DEPT GEOL, FUKUOKA 812, JAPAN

21. Authors: Cerveny-PF Naeser-CW Kelemen-PB Lieberman-JE Zeitler-PK

Title: Zircon Fission-Track Ages from the Gasherbrum Diorite, Karakoram Range, Northern Pakistan

Source: GEOLOGY

1989, Vol 17, Iss 11, pp 1044-1048

Addresses:

DARTMOUTH COLL, DEPT EARTH SCI, HANOVER, NH 03755, USA

US GEOL SURVEY, CTR FED, DENVER, CO 80225, USA

WOODS HOLE OCEANOG INST, WOODS-HOLE, MA 02543, USA

UNIV BERN, INST MINERAL PETROG, CH-3012 BERN, SWITZERLAND

LEHIGH UNIV, DEPT GEOL SCI, BETHLEHEM, PA 18015, USA

22. Authors: Collot-JY Fisher-MA

Title: Formation of Fore-Arc Basins by Collision Between

Seamounts and Accretionary Wedges - An Example from the

New Hebrides Subduction Zone

Source: GEOLOGY

1989, Vol 17, Iss 10, pp 930-933

Addresses:

US GEOL SURVEY, 345 MIDDLEFIELD RD, MENLO-PK, CA 94025, USA

ORSTOM, NOUMEA, NEW-CALEDONIA

23. Authors: Li-XH Tatsumoto-M Premo-WR Gui-XT

Title: Age and Origin of the Tanghu Granite, Southeast China Results from U-Pb Single Zircon and Nd Isotopes

Source: GEOLOGY 1989, Vol 17, Iss 5, pp 395-399

Addresses:

ACAD SINICA, INST GEOCHEM, GUIYANG, PEOPLES-R-CHINA

US GEOL SURVEY, DENVER FED CTR, DENVER, CO 80225, USA

24. Authors: Turner-RJW Madrid-RJ Miller-EL

Title: Roberts Mountains Allochthon - Stratigraphic Comparison with Lower Paleozoic Outer Continental-Margin Strata of the Northern Canadian Cordillera

Source: GEOLOGY 1989, Vol 17, Iss 4, pp 341-344

Addresses:

GEOL SURVEY CANADA, 100 PENDER ST, VANCOUVER V6B 1R8, BC, CANADA

US GEOL SURVEY, MENLO-PK, CA 94025, USA

STANFORD UNIV, DEPT GEOL, STANFORD, CA 94305, USA 
25. Authors: Karl-HA Hampton-MA Kenyon-NH

Title: Lateral Migration of Cascadia Channel in Response to Accretionary Tectonics

Source: GEOLOGY 1989, Vol 17, Iss 2, pp 144-147

Addresses:

US GEOL SURVEY, MENLO-PK, CA 94025, USA

INST OCEANOG SCI, WORMLEY GU8 5UB, SURREY, ENGLAND

26. Authors: Rouer-O Lapierre-H Mascle-G Coulon-C Albers-J

Title: Geodynamic Implications of Devonian Silicic Arc Magmatism in the Sierra-Nevada and Klamath Mountains, California

Source: GEOLOGY 1989, Vol 17, Iss 2, pp 177-180

Addresses:

UNIV ORLEANS, GEOL STRUCT LAB, BP 6759, F-45067 ORLEANS, FRANCE UNIV NANCY 1, PETROL LAB, F-54506 VANDOEUVRE NANCY, FRANCE

UNIV AIX MARSEILLE 3, FAC SCI ST JEROME, PETROL LAB, F-13397

MARSEILLE, FRANCE

US GEOL SURVEY, MENLO-PK, CA 94025, USA

27. Authors: Barnes-PW Lien-R

Title: Icebergs Rework Shelf Sediments to 500-M Off Antarctica

Source: GEOLOGY 1988, Vol 16, Iss 12, pp 1130-1133

Addresses:

US GEOL SURVEY, MENLO-PK, CA 94025, USA

REIDAR LIEN AS, N-7079 FLATAS, NORWAY

28. Authors: Cain-JC Wang-ZG Kluth-C Schmitz-DR

Title: Derivation of a Geomagnetic Model to $\mathrm{N}=63$

Source: GEOPHYSICAL JOURNAL-OXFORD 1989, Vol 97, Iss 3, pp 431-441

Addresses:

FLORIDA STATE UNIV, DEPT GEOL, TALLAHASSEE, FL 32306, USA

STATE SEISMOL BUR, INST GEOPHYS, BEIJING, PEOPLES-R-CHINA

US GEOL SURVEY, GEOPHYS BRANCH, DENVER, CO 80225, USA

29. Authors: Newman-GA Anderson-WL Hohmann-GW

Title: Effect of Conductive Host Rock on Borehole Transient Electromagnetic Responses

Source: GEOPHYSICS 1989, Vol 54, Iss 5, pp 598-608

Addresses:

UNIV COLOGNE, INST GEOPHYS \& METEOROL, D-5000 COLOGNE 41, FED-REP-GER

US GEOL SURVEY, DENVER FED CTR, DENVER, CO 80225, USA

UNIV UTAH, DEPT GEOL \& GEOPHYS, SALT-LAKE-CITY, UT 84112, USA

30. Authors: Hatcher-PG Lerch-HE Verheyen-TV

Title: Organic Geochemical Studies of the Transformation of Gymnospermous Xylem During Peatification and Coalification to Subbituminous Coal

Source: INTERNATIONAL JOURNAL OF COAL GEOLOGY 1989, Vol 13, Iss 1-4, pp 65-97 
Addresses:

US GEOL SURVEY, RESTON, VA 22092, USA

COAL CORP VICTORIA, CHURCHILL, VIC 3842, AUSTRALIA

31. Authors: Hatcher-PG Wilson-MA Vassallo-AM Lerch-HE

Title: Studies of Angiospermous Wood in Australian Brown Coal by Nuclear Magnetic-Resonance and Analytical Pyrolysis New Insights into the Early Coalification Process

Source: INTERNATIONAL JOURNAL OF COAL GEOLOGY 1989, Vol 13, Iss 1-4, pp 99-126

Addresses:

US GEOL SURVEY, RESTON, VA 22092, USA

CSIRO, DIV COAL TECHNOL, RYDE, NSW 2113, AUSTRALIA

32. Authors: Marcomini-A Capel-PD Lichtensteiger-T Brunner-PH Giger-W

Title: Behavior of Aromatic Surfactants and PCBs in SludgeTreated Soil and Landfills

Source: JOURNAL OF ENVIRONMENTAL QUALITY 1989, Vol 18, Iss 4, pp 523-528

Addresses:

FED INST WATER RESOURCES \& WATER POLLUT CONTROL, EAWAG, CH-8600 DUBENDORF, SWITZERLAND

UNIV VENICE, DEPT ENVIRONM SCI, I-30123 VENICE, ITALY

US GEOL SURVEY, DIV WATER RESOURCES, ST-PAUL, MN 55101, USA

33. Authors: Liu-LB Roeloffs-E Zheng-XY

Title: Seismically Induced Water Level Fluctuations in the Wali Well, Beijing, China

Source: JOURNAL OF GEOPHYSICAL RESEARCH-SOLID EARTH AND PLANETS 1989, Vol 94, Iss NB7, pp 9453-9462

Addresses:

STATE SEISMOL BUR, CTR ANAL \& PREDICT, BEIJING, PEOPLES-R-CHINA

US GEOL SURVEY, MENLO-PK, CA 94025, USA

34. Authors: Drake-DE Cacchione-DA Gardner-JV Mcculloch-DS

Title: Morphology and Growth History of Delgada Fan Implications for the Neogene Evolution of Point Arena Basin and the Mendocino Triple Junction

Source: JOURNAL OF GEOPHYSICAL RESEARCH-SOLID EARTH AND PLANETS 1989, Vol 94, Iss NB3, pp 3139-3158

Addresses:

US GEOL SURVEY, 345 MIDDLEFIELD RD, MS 999, MENLO-PK, CA 94025, USA

INST OCEANOG SCI, GOLALMING GU8 54B, ENGLAND

35. Authors: Vonhuene-R Bourgois-J Miller-J Pautot-G

Title: A Large Tsunamogenic Landslide and Debris Flow Along the Peru Trench

Source: JOURNAL OF GEOPHYSICAL RESEARCH-SOLID EARTH AND PLANETS 1989, Vol 94, Iss NB2, pp 1703-1714

Addresses:

US GEOL SURVEY, 345 MIDDLEFIELD RD, MS-999, MENLO-PK, CA

94025, USA

INST FRANCAIS RECH EXPLORAT MER, CTR BREST, F-29273 BREST, FRANCE 
US GEOL SURVEY, DENVER FED CTR, DENVER, CO 80225, USA

UNIV PARIS 06, DEPT GEOTECTON, F-75230 PARIS 05, FRANCE

36. Authors: Ehhalt-DH Davidson-JA Cantrell-CA Friedman-I Tyler-S

Title: The Kinetic Isotope Effect in the Reaction of $\mathrm{H}-2$ with Oh

Source: JOURNAL OF GEOPHYSICAL RESEARCH-ATMOSPHERES 1989, Vol 94, Iss ND7, pp 9831-9836

Addresses:

KFA JULICH GMBH, INST ATMOSPHAR CHEM, POSTFACH 1913, D-5170

JULICH 1, FED-REP-GER

NATL CTR ATMOSPHER RES, BOULDER, CO 80307, USA

US GEOL SURVEY, DENVER, CO 80225, USA

37. Authors: Avon-L Bredehoeft-JD

Title: An Analysis of Trichloroethylene Movement in Groundwater At Castle-Air-Force-Base, California

Source: JOURNAL OF HYDROLOGY 1989, Vol 110, Iss 1-2, pp 23-50

Addresses:

US GEOL SURVEY, MENLO-PK, CA 94025, USA

ELECTROWATT ENGN SERV LTD, ZURICH, SWITZERLAND

38. Authors: Devivo-B Belkin-HE Barbieri-M Chelini-W Lattanzi-P Lima-A Tolomeo-L

Title: The Campi Flegrei (Italy) Geothermal System - A Fluid Inclusion Study of the Mofete and San-Vito Fields

Source: JOURNAL OF VOLCANOLOGY AND GEOTHERMAL RESEARCH 1989, Vol 36, Iss 4, pp 303-326

Addresses:

DIPARTIMENTO GEOFIS \& VULCANOL, LARGO S MARCELLINO 10, I-80138

NAPOLI, ITALY

UNIV LA SAPIENZA, DIPARTIMENTO SCI TERRA, I-00100 ROMA, ITALY

AGIP SPA, I-20097 SAN DONATO MILANE, ITALY

DIPARTIMENTO SCI TERRA, I-50121 FIRENZE, ITALY

US GEOL SURVEY, RESTON, VA 22092, USA

39. Authors: Mcknight-DM Thorn-KA Wershaw-RL Bracewell-JM Robertson-GW

Title: Rapid Changes in Dissolved Humic Substances in Spirit Lake and South Fork Castle Lake, Washington

Source: LIMNOLOGY AND OCEANOGRAPHY 1988, Vol 33, Iss 6, pp 1527-1541

Addresses:

US GEOL SURVEY, DIV WATER RESOURCES, 5293 WARD RD, ARVADA, CO 80002, USA

MACAULEY INST SOIL RES, ABERDEEN AB9 2Q5, SCOTLAND

40. Authors: Lough-RG Valentine-PC Potter-DC Auditore-PJ Bolz-GR Neilson-JD Perry-RI

Title: Ecology and Distribution of Juvenile Cod and Haddock in Relation to Sediment Type and Bottom Currents on Eastern Georges-Bank

Source: MARINE ECOLOGY-PROGRESS SERIES 1989, Vol 56, Iss 1-2, pp 1-12 
Addresses:

NATL MARINE FISHERIES SERV, CTR NE FISHERIES, WOODS-HOLE, MA 02543, USA

FISHERIES \& OCEANS CANADA, DIV MARINE FISH, ST ANDREWS E0G 2XO, NB, CANADA

US GEOL SURVEY, WOODS-HOLE, MA 02543, USA

41. Authors: Masson-DG Cacchione-DA Drake-DE

Title: Tectonic Evolution of Gorda Ridge Inferred from Sidescan Sonar Images

Source: MARINE GEOPHYSICAL RESEARCHES 1989, Vol 10, Iss 3-4, pp 191-204

Addresses:

INST OCEANOG SCI, BROOK RD, GODALMING GU8 5UB, SURREY, ENGLAND

US GEOL SURVEY, MENLO-PK, CA 94025, USA

42. Authors: Hampton-MA

Title: Geotechnical Properties of Sediment on the Kodiak Continental-Shelf and Upper Slope, Gulf of Alaska

Source: MARINE GEOTECHNOLOGY 1989, Vol 8, Iss 2, pp 159-180

Addresses:

US GEOL SURVEY, MENLO-PK, CA 94025, USA

43. Authors: Hampton-MA Karl-HA Kenyon-NH

Title: Sea-Floor Drainage Features of Cascadia Basin and the Adjacent Continental-Slope, Northeast Pacific-Ocean

Source: MARINE GEOLOGY 1989, Vol 87, Iss 2-4, pp 249-272

Addresses:

US GEOL SURVEY, MENLO-PK, CA 94025, USA

INST OCEANOG SCI, WORMLEY GU8 5UB, SURREY, ENGLAND

44. Authors: Hatcher-PG Lerch-HE Bates-AL Verheyen-TV

Title: Solid-State C-13 Nuclear Magnetic-Resonance Studies of Coalified Gymnosperm Xylem Tissue from Australian Brown Coals

Source: ORGANIC GEOCHEMISTRY 1989, Vol 14, Iss 2, pp 145-155

Addresses:

US GEOL SURVEY, RESTON, VA 22092, USA

COAL CORP VICTORIA, CHURCHILL, VIC, AUSTRALIA

45. Authors: Grimalt-JO Simoneit-BRT Hatcher-PG Nissenbaum-A

Title: The Molecular Composition of Ambers

Source: ORGANIC GEOCHEMISTRY 1988, Vol 13, Iss 4-6, pp 677-690

Addresses:

CSIC, CID, DEPT ENVIRONM CHEM, JORDI GIRONA 18, E-08034

BARCELONA, SPAIN

OREGON STATE UNIV, COLL OCEANOG, PETR RES GRP, CORVALLIS, OR 97331, USA

US GEOL SURVEY, NATL CTR, RESTON, VA 22092, USA

WEIZMANN INST SCI, IL-76100 REHOVOT, ISRAEL 
46. Authors: Wilson-MA Hatcher-PG

Title: Detection of Tannins in Modern and Fossil Barks and in

Plant Residues by High-Resolution Solid-State C-13

Nuclear Magnetic-Resonance

Source: ORGANIC GEOCHEMISTRY

1988, Vol 12, Iss 6, pp 539-546

Addresses:

CSIRO, DIV COAL TECHNOL, POB 136, N RYDE, NSW 2113, AUSTRALIA

US GEOL SURVEY, RESTON, VA 22092, USA

47. Authors: Ballance-PF Barron-JA Blome-CD Bukry-D Cawood-PA

Chaproniere-GCH Frisch-R Herzer-RH Nelson-CS

Quinterno-P Ryan-H Scholl-DW Stevenson-AJ

Tappin-DG Vallier-TL

Title: Late Cretaceous Pelagic Sediments, Volcanic Ash and

Biotas from Near the Louisville Hotspot, Pacific Plate,

Paleolatitude Approximately-42-Degrees-S

Source: PALAEOGEOGRAPHY PALAEOCLIMATOLOGY PALAEOECOLOGY 1989, Vol 71, Iss 3-4, pp 281-299

Addresses:

UNIV AUCKLAND, DEPT GEOL, AUCKLAND, NEW-ZEALAND

NEW ZEALAND GEOL SURVEY, LOWER HUTT, NEW-ZEALAND

MINIST LANDS \& SURVEYS, NUKUALOFA, TONGA

US GEOL SURVEY, MENLO-PK, CA 94025, USA

US GEOL SURVEY, DENVER FED CTR, DENVER, CO 80225, USA

UNIV CALIF SAN DIEGO, SCRIPPS INST OCEANOG, US MINERALS

MANAGEMENT SERV, LA-JOLLA, CA 92093, USA

MEM UNIV NEWFOUNDLAND, DEPT EARTH SCI, ST JOHNS A1B 3X5,

NEWFOUNDLAND, CANADA

BUR MINERAL RESOURCES GEOL \& GEOPHYS, CANBERRA, ACT 2601, AUSTRALIA

UNIV WAIKATO, DEPT EARTH SCI, HAMILTON, NEW-ZEALAND

MUSEUM PALEONTOL, BERKELEY, CA 94720, USA

48. Authors: Wilson-MA Sawyer-J Hatcher-PG Lerch-HE

Title: 1,3,5-Hydroxybenzene Structures in Mosses

Source: PHYTOCHEMISTRY 1989, Vol 28, Iss 5, pp 1395-1400

Addresses:

CSIRO, DIV COAL TECHNOL, POB 136, N RYDE, NSW 2113, AUSTRALIA

QUEENSLAND HERBARIUM, BRISBANE, AUSTRALIA

US GEOL SURVEY, RESTON, VA 22092, USA

49. Authors: Grimalt-JO Simoneit-BRT Hatcher-PG

Title: Chemical Affinities Between the Solvent Extractable and the Bulk Organic-Matter of Fossil Resin Associated with an Extinct Podocarpaceae

Source: PHYTOCHEMISTRY 1989, Vol 28, Iss 4, pp 1167-1171

Addresses:

CSIC, CID, DEPT ENVIRONM CHEM, JORDI GIRONA 18, E-08034

BARCELONA, SPAIN

OREGON STATE UNIV, COLL OCEANOG, PETR RES GRP, CORVALLIS, OR 97331, USA

US GEOL SURVEY, NATL CTR, RESTON, VA 22092, USA 
50. Authors: Kogelknabner-I Hatcher-PG

Title: Characterization of Alkyl Carbon in Forest Soils by

Cpmas C-13 NMR-Spectroscopy and Dipolar Dephasing

Source: SCIENCE OF THE TOTAL ENVIRONMENT

1989, Vol 81-2, Iss JUN, pp 169-177

Addresses:

UNIV BAYREUTH, LEHRSTUHL BODENKUNDE \& BODENGEOG, POSTFACH

101251, D-8580 BAYREUTH, FED-REP-GER

US GEOL SURVEY, RESTON, VA 22092, USA

51. Authors: Chen-Y Inbar-Y Hadar-Y Malcolm-RL

Title: Chemical-Properties and Solid-State Cpmas C-13-NMR of

Composted Organic-Matter

Source: SCIENCE OF THE TOTAL ENVIRONMENT

1989, Vol 81-2, Iss JUN, pp 201-208

Addresses:

HEBREW UNIV JERUSALEM, FAC AGR, SEAGRAM CTR SOIL \& WATER SCI, POB 12, IL-76100 REHOVOT, ISRAEL

HEBREW UNIV JERUSALEM, FAC AGR, DEPT PLANT PATHOL \& MICROBIOL, IL-76100 REHOVOT, ISRAEL

US GEOL SURVEY, DIV WATER RESOURCES, ARVADA, CO, USA

52. Authors: Miall-AD Turnerpeterson-CE

Title: Variations in Fluvial Style in the Westwater Canyon

Member, Morrison Formation (Jurassic), San-Juan Basin,

Colorado Plateau

Source: SEDIMENTARY GEOLOGY

1989, Vol 63, Iss 1-2, pp 21-

Addresses:

UNIV TORONTO, DEPT GEOL, TORONTO M5S 1A1, ONTARIO, CANADA

US GEOL SURVEY, DENVER FED CTR, DENVER, CO 80225, USA

53. Authors: Morton-AC Borg-G Hansley-PL Haughton-PDW

Krinsley-DH Trusty-P

Title: The Origin of Faceted Garnets in Sandstones -

Dissolution or Overgrowth

Source: SEDIMENTOLOGY

1989, Vol 36, Iss 5, pp 927-942

Addresses:

BRITISH GEOL SURVEY, STRATIG \& SEDIMENTOL RES GRP, KEYWORTH

NG12 5GG, NOTTS, ENGLAND

BUNDESANSTALT GEOWISSENSCH \& ROHSTOFFE, D-3000 HANOVER 51, FED-REP-GER

US GEOL SURVEY, DENVER FED CTR, SEDIMENTARY PROC BRANCH, DENVER, CO 80225, USA

UNIV GLASGOW, DEPT GEOL, GLASGOW G12 8QQ, SCOTLAND

ARIZONA STATE UNIV, DEPT GEOL, TEMPE, AZ 85287, USA

54. Authors: Ballance-PF Scholl-DW Vallier-TL Stevenson-AJ Ryan-H Herzer-RH

Title: Subduction of a Late Cretaceous Seamount of the Louisville Ridge At the Tonga Trench - A Model of Normal and Accelerated Tectonic Erosion 


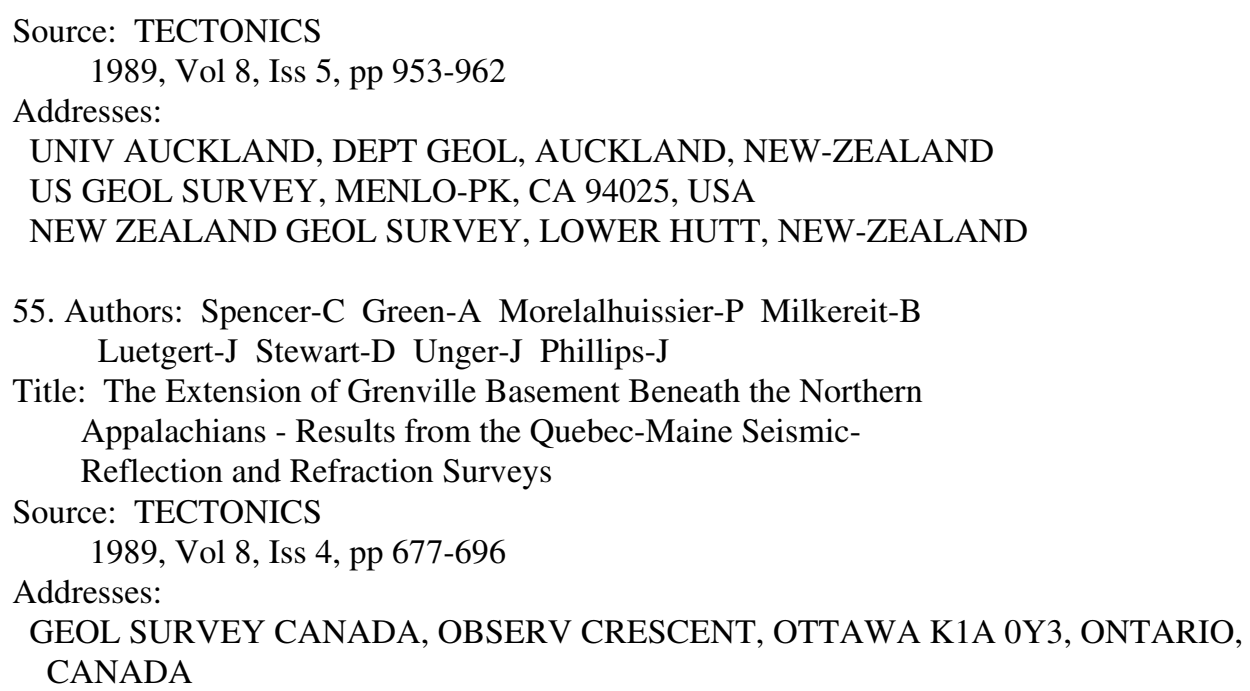

56. Authors: Cannon-WF Green-AG Hutchinson-DR Lee-M Milkereit-B Behrendt-JC Halls-HC Green-JC Dickas-AB Morey-GB Sutcliffe-R Spencer-C

Title: The North-American Midcontinent Rift Beneath LakeSuperior from Glimpce Seismic-Reflection Profiling

Source: TECTONICS 1989, Vol 8, Iss 2, pp 305-332

Addresses:

US GEOL SURVEY, RESTON, VA 22092, USA

GEOL SURVEY CANADA, OTTAWA K1A 0E8, ONTARIO, CANADA

US GEOL SURVEY, WOODS-HOLE, MA 02543, USA

US GEOL SURVEY, DENVER, CO 80225, USA

UNIV TORONTO, TORONTO M5S 1A1, ONTARIO, CANADA

UNIV MINNESOTA, DULUTH, MN 55812, USA

UNIV WISCONSIN, SUPERIOR, WI 54880, USA

MINNESOTA GEOL SURVEY, ST-PAUL, MN 55114, USA

ONTARIO GEOL SURVEY, TORONTO M5S 1B3, ONTARIO, CANADA

57. Authors: Rossman-DL Castanada-GC Bacuta-GC

Title: Geology of the Zambales Ophiolite, Luzon, Philippines

Source: TECTONOPHYSICS 1989, Vol 168, Iss 1-3, pp 1-

Addresses:

US GEOL SURVEY, MENLO-PK, CA 94025, USA

PHILIPPINE BUR MINES \& GEOSCI PETROLAB, QUEZON CITY, PHILIPPINES

58. Authors: Lallemand-S Culotta-R Vonhuene-R

Title: Subduction of the Daiichi Kashima Seamount in the Japan Trench Source: TECTONOPHYSICS 1989, Vol 160, Iss 1-4, pp 231-

Addresses:

UNIV PIERRE \& MARIE CURIE, DEPT GEOTECTON, T26-E1, 4 PL JUSSIEU, F-75252 PARIS 5, FRANCE 
US GEOL SURVEY, OFF PACIFIC MARINE GEOL, MENLO-PK, CA 94025, USA

59. Authors: Wolock-DM Hornberger-GM Beven-KJ Campbell-WG

Title: The Relationship of Catchment Topography and Soil

Hydraulic Characteristics to Lake Alkalinity in the

Northeastern United-States

Source: WATER RESOURCES RESEARCH

1989, Vol 25, Iss 5, pp 829-837

Addresses:

US GEOL SURVEY, DIV WATER RESOURCES, 810 BEAR TAVERN RD, SUITE

206, W-TRENTON, NJ 08628, USA

NORTHROP SERV INC, ENVIRONM SCI, CORVALLIS, OR 97330, USA

UNIV VIRGINIA, DEPT ENVIRONM SCI, CHARLOTTESVILLE, VA 22903, USA

UNIV LANCASTER, DEPT ENVIRONM SCI, LANCASTER LA1 4YQ, ENGLAND

60. Authors: Eberl-DD Srodon-J Lee-M Nadeau-PH

Title: Sericite from the Silverton Caldera, Colorado - Reply

Source: AMERICAN MINERALOGIST

1988, Vol 73, Iss 11-1, pp 1475-1477

Addresses:

US GEOL SURVEY, DENVER FED CTR, MAIL STOP 404, DENVER, CO 80225, USA

POLISH ACAD SCI, INST GEOL SCI, PL-31002 KRAKOW, POLAND

CASE WESTERN RESERVE UNIV, DEPT EARTH SCI, CLEVELAND, OH 44106, USA

61. Authors: Johnston-MJS Parrot-M

Title: Seismoelectromagnetic Effects - Foreword

Source: PHYSICS OF THE EARTH AND PLANETARY INTERIORS 1989, Vol 57, Iss 1-2, pp R8-R8

Addresses:

US GEOL SURVEY, MENLO-PK, CA 94025, USA

CNRS, PHYS \& CHEM ENVIRONM LAB, F-45071 ORLEANS 02, FRANCE

62. Authors: Tuttle-ML Goldhaber-MB Rice-CA Hutton-AC

Title: Sulfur Geochemistry of Lacustrine Oil Shales from the

Green River Formation (USA) and Rundle Formation

(Australia)

Source: ABSTRACTS OF PAPERS OF THE AMERICAN CHEMICAL SOCIETY 1989, Vol 197, Iss APR, pp 9-

Addresses:

US GEOL SURVEY, DENVER, CO 80225, USA

UNIV WOLLONGONG, DEPT GEOL \& CHEM, WOLLONGONG, NSW 2500, AUSTRALIA

63. Authors: Hatcher-PG Wilson-MA

Title: The Effect of Hydration on Solid-State C-13 NMR-Spectra of Fulvic-Acids

Source: ABSTRACTS OF PAPERS OF THE AMERICAN CHEMICAL SOCIETY 1989, Vol 197, Iss APR, pp 83-

Addresses:

US GEOL SURVEY, RESTON, VA 22092, USA

CSIRO, DIV COAL TECHNOL, N RYDE, NSW 2113, AUSTRALIA 
64. Authors: Gulson-BL Meier-AL Church-SE Mizon-KJ

Title: A Comparison of Lead-Isotope Measurements on Exploration-

Type Samples Using Inductively Coupled Plasma and

Thermal Ionization Mass-Spectrometry

Source: JOURNAL OF GEOCHEMICAL EXPLORATION

1989, Vol 32, Iss 1-3, pp 311-313

Addresses:

CSIRO, DIV EXPLORAT GEOSCI, POB 136, N RYDE, NSW 2113,

AUSTRALIA

US GEOL SURVEY, GEOCHEM BRANCH, BOX 25046, CTR FED, MS-973,

DENVER, CO 80205, USA

65. Authors: Izawa-E Cunningham-CG

Title: Hydrothermal Breccia Pipes and Gold Mineralization in the Iwashita Orebody, Iwato Deposit, Kyushu, Japan

Source: ECONOMIC GEOLOGY 1989, Vol 84, Iss 3, pp 715-724

Addresses:

KYUSHU UNIV, DEPT MINING, HIGASHI KU, FUKUOKA 812, JAPAN

US GEOL SURVEY, OFF MINERAL RESOURCES, RESTON, VA 22092, USA

66. Authors: Silberman-ML Giles-DA Graubard-C

Title: Characteristics of Gold Deposits in Northern Sonora, Mexico - A Preliminary-Report

Source: ECONOMIC GEOLOGY 1988, Vol 83, Iss 8, pp 1966-1974

Addresses:

US GEOL SURVEY, DENVER, CO 80225, USA

CONTRATISTA TORMEX, CHIHUAHUA, MEXICO

67. Authors: Cunningham-CG Ashley-RP Chou-IM Zushu-H Chaoyuan-W Li-WK

Title: Newly Discovered Sedimentary Rock-Hosted Disseminated Gold Deposits in the Peoples-Republic of China

Source: ECONOMIC GEOLOGY 1988, Vol 83, Iss 7, pp 1462-1467

Addresses:

US GEOL SURVEY, 959 NATL CTR, RESTON, VA 22092, USA

US GEOL SURVEY, MENLO-PK, CA 94025, USA

MINIST GEOL \& MINERAL RESOURCES, DEPT REG GEOL \& MINERAL RESOURCES, BEIJING, PEOPLES-R-CHINA

BUR GEOL \& MINERAL RESOURCES GUIZHOU PROVINCE, GUIYANG, PEOPLES-R-CHINA

SHENYANG INST GEOL \& MINERAL RESOURCES, SHENYANG, PEOPLES-R-CHINA

68. Authors: Zoback-ML Zoback-MD Adams-J Assumpcao-M Bell-S

Bergman-EA Blumling-P Brereton-NR Denham-D Ding-J

Fuchs-K Gay-N Gregersen-S Gupta-HK Gvishiani-A

Jacob-K Klein-R Knoll-P Magee-M Mercier-JL

Muller-BC Paquin-C Rajendran-K Stephansson-O

Suarez-G Suter-M Udias-A Xu-ZH Zhizhin-M

Title: Global Patterns of Tectonic Stress

Source: NATURE

1989, Vol 341, Iss 6240, pp 291-298 
Addresses:

US GEOL SURVEY, MENLO-PK, CA 94025, USA

STANFORD UNIV, DEPT GEOPHYS, STANFORD, CA 94305, USA

GEOL SURVEY CANADA, DIV GEOPHYS, OTTAWA K1A 0Y3, ONTARIO, CANADA

UNIV SAO PAULO, INST ASTRON \& GEOFIS, BR-01051 SAO PAULO, SP, BRAZIL

INST SEDIMENTARY \& PETR GEOL, CALGARY T2L 2A7, ALBERTA, CANADA

MIT, CAMBRIDGE, MA 02139, USA

NATL GENOSSENSCHAFTLAGERUNG RADIOAKTIVERABFALLE, CH-5401

BADEN, SWITZERLAND

BRITISH GEOL SURVEY, KEYWORTH NC12 56G, NOTTS, ENGLAND

NATL AUTONOMOUS UNIV MEXICO, INST GEOFIS, MEXICO CITY 04510 ,

DF, MEXICO

GEODET INST, DK-2920 CHARLOTTENLUND, DENMARK

ROCK MECH LAB, AUCKLAND PK 2006, SOUTH-AFRICA

UNIV PARIS 11, GEOL DYNAM INTERNE LAB, F-91405 ORSAY, FRANCE

COLUMBIA UNIV, LAMONT DOHERTY GEOL OBSERV, PALISADES, NY

10964, USA

STATE SEISMOL BUR, INST CRUSTAL DYNAM, BEIJING, PEOPLES-R-CHINA

LULEA UNIV TECHNOL, DIV ROCK MECH, S-95187 LULEA, SWEDEN

COCHIN UNIV SCI \& TECHNOL, COCHIN 682022, INDIA

BRITISH PETR RES INT, SUNBURY TW16 7LN, MIDDX, ENGLAND

NATL AUTONOMOUS UNIV MEXICO, INST GEOL, MEXICO CITY 04510, DF, MEXICO

UNIV KARLSRUHE, INST GEOPHYS, D-7500 KARLSRUHE 21, FED-REP-GER

ACAD SCI USSR, INST PHYS EARTH, MOSCOW 123810, USSR

STATE SEISMOL BUR, INST GEOPHYS, BEIJING, PEOPLES-R-CHINA

UNIV S CAROLINA, DEPT GEOL, COLUMBIA, SC 29208, USA

ZENT INST PHYS ERDE, DDR-1561 POTSDAM, GER-DEM-REP

AUSTRALIAN SEISMOL CTR, CANBERRA, ACT 2601, AUSTRALIA

UNIV PARIS 11, GEOPHYS \& GEODYNAM INTERNE LAB, F-91405 ORSAY, FRANCE

UNIV COMPLUTENSE MADRID, FAC C FIS, CATEDRA GEOFIS, E-28040

MADRID, SPAIN

69. Authors: Becker-K Sakai-H Adamson-AC Alexandrovich-J Alt-JC

Anderson-RN Bideau-D Gable-R Herzig-PM Houghton-S

Ishizuka-H Kawahata-H Kinoshita-H Langseth-MG

Lovell-MA Malpas-J Masuda-H Merrill-RB Morin-RH

Mottl-MJ Pariso-JE Pezard-P Phillips-J Sparks-J

Uhlig-S

Title: Drilling Deep into Young Oceanic-Crust, Hole-504B, Costa-

Rica Rift

Source: REVIEWS OF GEOPHYSICS

1989, Vol 27, Iss 1, pp 79-102

Addresses:

UNIV MIAMI, ROSENSTIEL SCH MARINE \& ATMOSPHER SCI, DIV MARINE

GEOL \& GEOPHYS, MIAMI, FL 33149, USA

TEXAS A\&M UNIV, OCEAN DRILLING PROGRAM, COLLEGE-STN, TX 77840, USA

INST FRANCAIS RECH EXPLOITAT LA MER, CTR BREST, F-29273 BREST, FRANCE

AACHEN UNIV TECHNOL, INST MINERAL \& ECON GEOL, D-5100 AACHEN

1, FED-REP-GER 
COLUMBIA UNIV, LAMONT DOHERTY GEOL OBSERV, PALISADES, NY

10964, USA

WASHINGTON UNIV, DEPT EARTH \& PLANETARY SCI, ST-LOUIS, MO

63130, USA

COLUMBIA UNIV, LAMONT DOHERTY GEOL OBSERV, BOREHOLE RES GRP,

PALISADES, NY 10964, USA

BUR RECH GEOL \& MINIERES, F-45060 ORLEANS, FRANCE

UNIV NOTTINGHAM, DEPT GEOL, NOTTINGHAM NG7 2RD, ENGLAND

MEM UNIV NEWFOUNDLAND, DEPT EARTH SCI, ST JOHNS A1B 3X5,

NEWFOUNDLAND, CANADA

OPEN UNIV, DEPT EARTH SCI, MILTON KEYNES MK7 6AA, BUCKS,

ENGLAND

UNIV GIESSEN, DEPT GEOSCI, D-6300 GIESSEN, FED-REP-GER

CHIBA UNIV, DEPT EARTH SCI, CHIBA 260, JAPAN

KOCHI UNIV, DEPT GEOL, KOCHI 780, JAPAN

US GEOL SURVEY, DENVER FED CTR, BOREHOLE GEOPHYS RES PROJECT, DENVER, CO 80225, USA

UNIV TORONTO, DEPT GEOL, TORONTO M5S 1A1, ONTARIO, CANADA

UNIV TEXAS, INST GEOPHYS, AUSTIN, TX 78759, USA

UNIV TOKYO, OCEAN RES INST, NAKANO KU, TOKYO 164, JAPAN

UNIV WASHINGTON, SCH OCEANOG, SEATTLE, WA 98195, USA

UNIV HAWAII, HAWAII INST GEOPHYS, HONOLULU, HI 96822, USA

UNIV MASSACHUSETTS, MERRILL SCI CTR, DEPT GEOL \& GEOG,

AMHERST, MA 01003, USA

70. Authors: Smith-BA Soderblom-LA Banfield-D Barnet-C

Basilevksy-AT Beebe-RF Bollinger-K Boyce-JM

Brahic-A Briggs-GA Brown-RH Chyba-C Collins-SA

Colvin-T Cook-AF Crisp-D Croft-SK Cruikshank-D

Cuzzi-JN Danielson-GE Davies-ME Dejong-E Dones-L

Godfrey-D Goguen-J Grenier-I Haemmerle-VR Hammel-H

Hansen-CJ Helfenstein-CP Howell-C Hunt-GE

Ingersoll-AP Johnson-TV Kargel-J Kirk-R Kuehn-DI

Limaye-S Masursky-H Mcewen-A Morrison-D Owen-T

Owen-W Pollack-JB Porco-CC Rages-K Rogers-P

Rudy-D Sagan-C Schwartz-J Shoemaker-EM Showalter-M

Sicardy-B Simonelli-D Spencer-J Sromovsky-LA

Stoker-C Strom-RG Suomi-VE Synott-SP Terrile-RJ

Thomas-P Thompson-WR Verbiscer-A Veverka-J

Title: Voyager-2 At Neptune - Imaging Science Results

Source: SCIENCE

1989, Vol 246, Iss 4936, pp 1422-1449

Addresses:

UNIV ARIZONA, TUCSON, AZ 85721, USA

US GEOL SURVEY, FLAGSTAFF, AZ 86001, USA

CALTECH, PASADENA, CA 91125, USA

NEW MEXICO STATE UNIV, LAS-CRUCES, NM 88003, USA

VI VERNADSKII COSMOCHEM INST, MOSCOW, USSR

CALTECH, JET PROP LAB, PASADENA, CA 91109, USA

NASA, WASHINGTON, DC 20546, USA

OBSERV PARIS, F-92190 MEUDON, FRANCE

UNIV TORONTO, TORONTO M5S 1A1, ONTARIO, CANADA

UNIV WISCONSIN, MADISON, WI 53706, USA

CORNELL UNIV, ITHACA, NY 14853, USA

NASA, AMES RES CTR, MOFFETT-FIELD, CA 94035, USA

STANFORD UNIV, STANFORD, CA 94305, USA 
SUNY STONY BROOK, STONY-BROOK, NY 11794, USA

LOGICA LTD, LONDON W1A 4SE, ENGLAND

MYCOL INC, SUNNYVALE, CA 94087, USA

UNIV HAWAII MANOA, INST ASTRON, HONOLULU, HI 96822, USA

RAND CORP, SANTA-MONICA, CA 90406, USA

NATL OPT ASTRON OBSERV, TUCSON, AZ 85726, USA

71. Authors: Robinson-PT Vonherzen-RP Cannat-M Adamson-AC

Becker-K Bloomer-SH Dick-HJB Emmermann-RFK Gard-G

Goldberg-D Hebert-R Hertogen-JHG Hoskins-H

Iturrino-G Kassenaar-DC Kempton-PD Kikawa-E

Kirby-SH Meyer-PS Natland-JH Ozawa-K Pariso-JH

Scott-JH Stakes-DS Swift-SA

Title: Deformed and Metamorphosed Gabbros from the Atlantis-II

Fracture-Zone (Southwest Indian-Ocean)

Source: COMPTES RENDUS DE L ACADEMIE DES SCIENCES SERIE IIMECANIQUE PHYSIQUE CHIMIE SCIENCES DE L UNIVERS

SCIENCES DE LA TERRE

1989, Vol 308, Iss 2, pp 215-220

Addresses:

DALHOUSIE UNIV, CTR MARINE GEOL, HALIFAX B3H 3J5, NS, CANADA

WOODS HOLE OCEANOG INST, WOODS-HOLE, MA 02543, USA

UNIV BRETAGNE OCCIDENTALE, GIS OCEANOL \& GEODYNAM, F-29287

BREST, FRANCE

TEXAS A\&M UNIV, ODP, COLLEGE-STN, TX 77840, USA

UNIV MIAMI, ROSENSTIEL SCH MARINE \& ATMOSPHER SCI, MIAMI, FL 33149, USA

INST GEOWISSENSCH \& LITHOSPHARENFORSCH, D-6300 GIESSEN, FED-REP-GER

UNIV STOCKHOLM, DEPT GEOL, S-10691 STOCKHOLM, SWEDEN

COLUMBIA UNIV, LAMONT DOHERTY GEOL OBSERV, PALISADES, NY

10964, USA

UNIV LAVAL, DEPT GEOL, QUEBEC CITY G1K 7P4, QUEBEC, CANADA

UNIV LEUVEN, B-3030 LEUVEN, BELGIUM

UNIV S CAROLINA, DEPT GEOL, COLUMBIA, SC 29208, USA

OPEN UNIV, DEPT EARTH SCI, MILTON KEYNES MK7 6AA, BUCKS, ENGLAND

UNIV WATERLOO, DEPT EARTH SCI, WATERLOO N2L 3G1, ONTARIO,

CANADA

UNIV TOKYO, INST GEOL, TOKYO 113, JAPAN

US GEOL SURVEY, MENLO-PK, CA 94025, USA

UNIV WASHINGTON, SCH OCEANOG, SEATTLE, WA 98195, USA

UNIV CALIF SAN DIEGO, SCRIPPS INST OCEANOG, LA-JOLLA, CA 92093, USA

PURDUE UNIV, DEPT EARTH \& ATMOSPHER SCI, W-LAFAYETTE, IN 47907, USA

72. Authors: Daniel-J Gerard-M Mauffret-A Boulanger-D Cantin-B

Collot-JY Durand-J Fisher-M Greene-HG Michaux-P

Pelletier-B Pezzimenti-A Renard-V Schaming-M

Tissot-JD

Title: Compressive Deformation of an Intra-Arc Basin in a Ridge

Arc Collision Context - The Aoba Basin, New-Hebrides

Island-Arc 
Source: COMPTES RENDUS DE L ACADEMIE DES SCIENCES SERIE IIMECANIQUE PHYSIQUE CHIMIE SCIENCES DE L UNIVERS SCIENCES DE LA TERRE 1989, Vol 308, Iss 2, pp 239-245

Addresses:

ORSTOM, BP 5, NOUMEA, NEW-CALEDONIA

ORSTOM, F-93140 BONDY, FRANCE

UNIV PIERRE \& MARIE CURIE, DEPT GEOL DYNAM, F-75252 PARIS 05,

FRANCE

US GEOL SURVEY, MENLO-PK, CA 94025, USA

INST FRANCAIS RECH EXPLOITAT MER, CTR BREST, F-29263 PLOUZANE, FRANCE

INST PHYS GLOBE STRASBOURG, F-67000 STRASBOURG, FRANCE

\section{0}

1. Authors: Foreman-MGG Walters-RA

Title: A Finite-Element Tidal Model for the Southwest Coast of Vancouver Island

Source: ATMOSPHERE-OCEAN 1990, Vol 28, Iss 3, pp 261-287

Addresses:

FISHERIES \& OCEANS CANADA, INST OCEAN SCI, POB 6000, SIDNEY V8L 4B2, BC, CANADA

US GEOL SURVEY, TACOMA, WA 98416,

2. Authors: Tipping-E Reddy-MM Hurley-MA

Title: Modeling Electrostatic and Heterogeneity Effects on Proton Dissociation from Humic Substances

Source: ENVIRONMENTAL SCIENCE \& TECHNOLOGY 1990, Vol 24, Iss 11, pp 1700-1705

Addresses:

WINDERMERE LAB, INST FRESHWATER ECOL, AMBLESIDE LA22 0LP, CUMBRIA, ENGLAND

US GEOL SURVEY, DENVER FED CTR, LAKEWOOD, CO 80225, USA

3. Authors: Kamineni-DC Stone-D Peterman-ZE

Title: Early Proterozoic Deformation in the Western Superior Province, Canadian Shield

Source: GEOLOGICAL SOCIETY OF AMERICA BULLETIN 1990, Vol 102, Iss 12, pp 1623-1634

Addresses:

ATOM ENERGY CANADA LTD, PINAWA R0E 1L0, MANITOBA, CANADA ONTARIO GEOL SURVEY, TORONTO M7A 1W4, ONTARIO, CANADA US GEOL SURVEY, ISOTOPE GEOL BRANCH, DENVER, CO 80225, USA

4. Authors: Szabo-BJ Kyser-TK

Title: Ages and Stable-Isotope Compositions of Secondary Calcite and Opal in Drill Cores from Tertiary Volcanic-

Rocks of the Yucca Mountain Area, Nevada

Source: GEOLOGICAL SOCIETY OF AMERICA BULLETIN 1990, Vol 102, Iss 12, pp 1714-1719

Addresses:

US GEOL SURVEY, DENVER FED CTR, BOX 25046, MS 963, DENVER, CO 80225, USA 
UNIV SASKATCHEWAN, DEPT GEOL SCI, SASKATOON S7N 0W0, SASKATCHEWAN, CANADA

5. Authors: Johnston-MJS Linde-AT Gladwin-MT

Title: Near-Field High-Resolution Strain-Measurements Prior to the October 18, 1989, Loma-Prieta MS 7.1 Earthquake

Source: GEOPHYSICAL RESEARCH LETTERS 1990, Vol 17, Iss 10, pp 1777-1780

Addresses:

US GEOL SURVEY, MENLO-PK, CA 94025, USA

CARNEGIE INST WASHINGTON, WASHINGTON, DC 20015, USA

UNIV QUEENSLAND, DEPT PHYS, ST LUCIA, QLD 4067, AUSTRALIA

6. Authors: Tuttle-M Cowie-P Tinsley-J Benett-M Berrill-J

Title: Liquefaction and Foundation Failure of Chevron Oil and Gasoline Tanks At Moss-Landing, California

Source: GEOPHYSICAL RESEARCH LETTERS 1990, Vol 17, Iss 10, pp 1797-1800

Addresses:

COLUMBIA UNIV, LAMONT DOHERTY GEOL OBSERV, PALISADES, NY 10964, USA

US GEOL SURVEY, MENLO-PK, CA 94025, USA

UNIV CANTERBURY, DEPT CIVIL ENGN, CHRISTCHURCH 1, NEW-ZEALAND

7. Authors: Hickson-CJ Peterson-DW

Title: Special Symposium Commemorating the 10th Anniversary of the Eruption of Mount St Helens, May 18, 1980

Source: GEOSCIENCE CANADA 1990, Vol 17, Iss 3, pp 125-125

Addresses:

GEOL SURVEY CANADA, $100 \mathrm{~W}$ PENDER ST, VANCOUVER V6B 1R8, BC, CANADA

US GEOL SURVEY, MENLO-PK, CA 94025, USA

8. Authors: Moore-TA Stanton-RW Pocknall-DT Glores-RM

Title: Maceral and Palynomorph Facies from 2 Tertiary PeatForming Environments in the Powder River Basin, UnitedStates

Source: INTERNATIONAL JOURNAL OF COAL GEOLOGY 1990, Vol 15, Iss 4, pp 293-316

Addresses:

UNIV KENTUCKY, DEPT GEOL SCI, COAL RES GRP, LEXINGTON, KY 40506, USA

US GEOL SURVEY, RESTON, VA 22092, USA

NEW ZEALAND GEOL SURVEY, LOWER HUTT, NEW-ZEALAND

US GEOL SURVEY, DENVER, CO 80225, USA

9. Authors: Mcgarr-A Bicknell-J Churcher-J Spottiswoode

Title: Comparison of Ground Motion from Tremors and Explosions in Deep Gold-Mines

Source: JOURNAL OF GEOPHYSICAL RESEARCH-SOLID EARTH AND PLANETS 1990, Vol 95, Iss NB13, pp 1777-1792

Addresses:

US GEOL SURVEY, 345 MIDDLEFIELD RD, MS 977, MENLO-PK, CA 94025, USA 
CHAMBER MINES S AFRICA, RES ORG, ROCK MECH LAB, AUCKLAND PK 2006, SOUTH-AFRICA

10. Authors: Leeman-WP Smith-DR Hildreth-W Palacz-Z Rogers-N

Title: Compositional Diversity of Late Cenozoic Basalts in a

Transect Across the Southern Washington Cascades -

Implications for Subduction Zone Magmatism

Source: JOURNAL OF GEOPHYSICAL RESEARCH-SOLID EARTH AND PLANETS 1990, Vol 95, Iss NB12, pp 9561-9582

Addresses:

RICE UNIV, KEITH WIESS GEOL LABS, HOUSTON, TX 77251, USA

US GEOL SURVEY, MENLO-PK, CA 94025, USA

OPEN UNIV, DEPT EARTH SCI, MILTON KEYNES MK7 GAA, ENGLAND

TRINITY UNIV, DEPT GEOL, SAN-ANTONIO, TX 78284, USA

11. Authors: Stimac-JA Pearce-TH Donnellynolan-JM Hearn-BC

Title: The Origin and Implications of Undercooled Andesitic

Inclusions in Rhyolites, Clear Lake Volcanics, California

Source: JOURNAL OF GEOPHYSICAL RESEARCH-SOLID EARTH AND PLANETS 1990, Vol 95, Iss NB11, pp 7729-7746

Addresses:

QUEENS UNIV, DEPT GEOL SCI, KINGSTON K7L 3N6, ONTARIO, CANADA

US GEOL SURVEY, MENLO-PK, CA 94025, USA

US GEOL SURVEY, RESTON, VA 22092, USA

12. Authors: Vonrad-U Riech-V Wissmann-G Stevenson-AJ Morton-JL Sinha-MC

Title: Sea-Floor Reflectivity, Sediment Distribution, and

Magnetic-Anomalies of the Lau Basin (SW Pacific, So35/48

Cruises)

Source: MARINE MINING

1990, Vol 9, Iss 2, pp 157-

Addresses:

BUNDESANSTALT GEOWISSENSCH \& ROHSTOFFE, D-3000 HANNOVER 51, FED-REP-GER

US GEOL SURVEY, MENLO-PK, CA 94025, USA

UNIV CAMBRIDGE, DEPT EARTH SCI, BULLARD LABS, CAMBRIDGE CB3 0E2, ENGLAND

13. Authors: Richet-P Robie-RA Rogez-J Hemingway-BS Courtial-P Tequi-C

Title: Thermodynamics of Open Networks - Ordering and Entropy in Naalsio4 Glass, Liquid, and Polymorphs

Source: PHYSICS AND CHEMISTRY OF MINERALS 1990, Vol 17, Iss 5, pp 385-394

Addresses:

INST PHYS GLOBE, 4 PL JUSSIEU, F-75252 PARIS 05, FRANCE

US GEOL SURVEY, RESTON, VA 22092, USA

CNRS, CTR THERMODYNAM \& MICROCALORIMETRIE, F-13003 MARSEILLE, FRANCE

14. Authors: Cobban-WA Kennedy-WJ

Title: Upper Cenomanian Ammonites from the Woodbridge Clay

Member of the Raritan Formation in New-Jersey

Source: JOURNAL OF PALEONTOLOGY

1990, Vol 64, Iss 5, pp 845-846 
Addresses:

US GEOL SURVEY, FED CTR, BOX 25046, MAIL STOP 919, DENVER, CO

80225, USA

UNIV OXFORD MUSEUM, OXFORD OX1 3PR, ENGLAND

15. Authors: Glynn-PD Reardon-EJ

Title: Solid-Solution Aqueous-Solution Equilibria -

Thermodynamic Theory and Representation

Source: AMERICAN JOURNAL OF SCIENCE

1990, Vol 290, Iss 2, pp 164-201

Addresses:

US GEOL SURVEY, 432 NATL CTR, RESTON, VA 22092, USA

UNIV WATERLOO, DEPT EARTH SCI, WATERLOO N2L 3G1, ONTARIO,

CANADA

16. Authors: Wershaw-RL Pinckney-DJ Llaguno-EC Vicentebeckett-V

Title: NMR Characterization of Humic-Acid Fractions from

Different Philippine Soils and Sediments

Source: ANALYTICA CHIMICA ACTA 1990, Vol 232, Iss 1, pp 31-42

Addresses:

US GEOL SURVEY, DENVER FED CTR, DENVER, CO 80225, USA

UNIV PHILIPPINES, INST CHEM, QUEZON CITY, PHILIPPINES

17. Authors: Carten-RB Tayeb-JM

Title: Formation of Volcanic-Exhalative Nickel-Sulfide Deposits

At a Late Proterozoic Spreading Ridge in the Proto-

Arabian Shield

Source: CANADIAN JOURNAL OF EARTH SCIENCES

1990, Vol 27, Iss 6, pp 742-757

Addresses:

US GEOL SURVEY, AMER POST OFF, NEW-YORK, NY 09697, USA

DIRECTORATE GEN MINERAL RESOURCES, JEDDAH 21431, SAUDI-ARABIA

18. Authors: Eberl-DD Velde-B

Title: Beyond the Kubler Index

Source: CLAY MINERALS

1989, Vol 24, Iss 4, pp 571-577

Addresses:

US GEOL SURVEY, FED CTR, MAIL STOP 404, DENVER, CO 80225, USA

ECOLE NORM SUPER, GEOL LAB, F-75230 PARIS 05, FRANCE

19. Authors: Fodor-RV Sial-AN Mukasa-SB Mckee-EH

Title: Petrology, Isotope Characteristics, and K-Ar Ages of the Maranhao, Northern Brazil, Mesozoic Basalt Province

Source: CONTRIBUTIONS TO MINERALOGY AND PETROLOGY 1990, Vol 104, Iss 5, pp 555-567

Addresses:

N CAROLINA STATE UNIV, DEPT MARINE EARTH \& ATMOSPHER SCI,

RALEIGH, NC 27695, USA

UNIV FED PERNAMBUCO, DEPT GEOL, BR-50000 RECIFE, PE, BRAZIL

UNIV MICHIGAN, DEPT GEOL SCI, ANN-ARBOR, MI 48109, USA

US GEOL SURVEY, MENLO-PK, CA 94025, USA 
20. Authors: Stern-CR Frey-FA Futa-K Zartman-RE Peng-ZC

Kyser-TK

Title: Trace-Element and $\mathrm{Sr}, \mathrm{Nd}, \mathrm{Pb}$, and $\mathrm{O}$ Isotopic Composition of Pliocene and Quaternary Alkali Basalts of the

Patagonian Plateau Lavas of Southernmost South-America

Source: CONTRIBUTIONS TO MINERALOGY AND PETROLOGY 1990, Vol 104, Iss 3, pp 294-308

Addresses:

UNIV COLORADO, DEPT GEOL SCI, BOULDER, CO 80309, USA

US GEOL SURVEY, DENVER FED CTR, ISOTOPE BRANCH, DENVER, CO 80225, USA

UNIV SASKATCHEWAN, DEPT GEOL, SASKATOON S7N 0W0, SASKATCHEWAN, CANADA

MIT, DEPT EARTH ATMOSPHERE \& PLANETARY SCI, CAMBRIDGE, MA

02139, USA

21. Authors: Sirkin-L Szabo-BJ Padilla-G Pedrin-S Diaz-ER

Title: Uranium-Series Ages of Marine Terraces, La-Paz

Peninsula, Baja-California-sur, Mexico

Source: CORAL REEFS 1990, Vol 9, Iss 1, pp 25-30

Addresses:

ADELPHI UNIV, DEPT EARTH SCI, GARDEN-CITY, NY 11530, USA

US GEOL SURVEY, DENVER FED CTR, DENVER, CO 80225, USA

CTR INVEST BIOL BAJA CALIF, LA PAZ 23060, BAJA CALIF SUR, MEXICO

22. Authors: Genin-A Noble-M Lonsdale-PF

Title: Tidal Currents and Anticyclonic Motions on 2 North

Pacific Seamounts

Source: DEEP-SEA RESEARCH PART A-OCEANOGRAPHIC RESEARCH PAPERS 1989, Vol 36, Iss 12, pp 1803-1815

Addresses:

HEBREW UNIV JERUSALEM, H STEINTZ MARINE BIOL LAB, POB 469, ELAT, ISRAEL

UNIV CALIF SAN DIEGO, SCRIPPS INST OCEANOG, LA-JOLLA, CA 92093, USA

US GEOL SURVEY, MENLO-PK, CA 94025, USA

23. Authors: Bongiovanni-G Celebi-M Clemente-P

Title: The Flaminio Obelisk in Rome - Vibrational Characteristics As Part of Preservation Efforts

Source: EARTHQUAKE ENGINEERING \& STRUCTURAL DYNAMICS 1990, Vol 19, Iss 1, pp 107-118

Addresses:

ENERGIA NUCL \& ENERGIA ALTERNAT, ROME, ITALY

US GEOL SURVEY, MENLO-PK, CA 94025, USA

Authors: Sparks-NHC Mann-S Bazylinski-DA Lovely-DR Jannasch-HW Frankel-RB

Title: Structure and Morphology of Magnetite AnaerobicallyProduced by a Marine Magnetotactic Bacterium and a Dissimilatory Iron-Reducing Bacterium

Source: EARTH AND PLANETARY SCIENCE LETTERS 1990, Vol 98, Iss 1, pp 14-22 
Addresses:

UNIV BATH, SCH CHEM, BATH BA2 7AY, AVON, ENGLAND

VIRGINIA POLYTECH INST \& STATE UNIV, DEPT ANAEROB MICROBIOL,

BLACKSBURG, VA 24061, USA

US GEOL SURVEY, RESTON, VA 22092, USA

WOODS HOLE OCEANOG INST, DEPT BIOL, WOODS-HOLE, MA 02543, USA

CALIF POLYTECH STATE UNIV SAN LUIS OBISPO, DEPT PHYS,

SAN-LUIS-OBISPO, CA 93407, USA

24. Authors: Gerlach-TM Taylor-BE

Title: Carbon Isotope Constraints on Degassing of Carbon-

Dioxide from Kilauea Volcano

Source: GEOCHIMICA ET COSMOCHIMICA ACTA 1990, Vol 54, Iss 7, pp 2051-2058

Addresses:

US GEOL SURVEY, DENVER FED CTR, BOX 25046, MS-903, DENVER, CO 80225, USA

GEOL SURVEY CANADA, OTTAWA K1A 0E8, ONTARIO, CANADA

25. Authors: Jensenius-J Burruss-RC

Title: Hydrocarbon-Water Interactions During Brine Migration -

Evidence from Hydrocarbon Inclusions in Calcite Cements

from Danish North-Sea-Oil Fields

Source: GEOCHIMICA ET COSMOCHIMICA ACTA

1990, Vol 54, Iss 3, pp 705-713

Addresses:

UNIV COPENHAGEN, INST PETR, DK-1350 COPENHAGEN, DENMARK

US GEOL SURVEY, PETR GEOL BRANCH, DENVER, CO 80225, USA

26. Authors: Glynn-PD Reardon-EJ Plummer-LN Busenberg-E

Title: Reaction Paths and Equilibrium End-Points in Solid-

Solution Aqueous-Solution Systems

Source: GEOCHIMICA ET COSMOCHIMICA ACTA 1990, Vol 54, Iss 2, pp 267-282

Addresses:

US GEOL SURVEY, 432 NATL CTR, RESTON, VA 22092, USA

UNIV WATERLOO, DEPT EARTH SCI, WATERLOO N2L 3G1, ONTARIO,

CANADA

27. Authors: Vonhuene-R Lallemand-S

Title: Tectonic Erosion Along the Japan and Peru Convergent Margins

Source: GEOLOGICAL SOCIETY OF AMERICA BULLETIN 1990, Vol 102, Iss 6, pp 704-720

Addresses:

US GEOL SURVEY, MS 999, 345 MIDDLEFIELD RD, MENLO-PK, CA

94025, USA

GEOMAR, KIEL, FED-REP-GER

UNIV PARIS 06, PARIS, FRANCE

28. Authors: Johnson-EA Liu-S Zhang-YL

Title: Depositional-Environments and Tectonic Controls on the Coal-Bearing Lower to Middle Jurassic Yanan Formation, Southern Ordos Basin, China

Source: GEOLOGY

1989, Vol 17, Iss 12, pp 1123-1126 
Addresses:

US GEOL SURVEY, DENVER FED CTR, BOX 25046, MS 939, DENVER, CO 80225, USA

MINIST COAL IND, GEOL BUR, BEIJING, PEOPLES-R-CHINA

29. Authors: Lombardi-S Reimer-GM

Title: Radon and Helium in Soil Gases in the Phlegraean Fields, Central Italy

Source: GEOPHYSICAL RESEARCH LETTERS 1990, Vol 17, Iss 6, pp 849-852

Addresses:

UNIV ROME LA SAPIENZA, DEPT SCI TERRA, I-00185 ROME, ITALY

US GEOL SURVEY, DENVER FED CTR, DENVER, CO 80225, USA

30. Authors: Spruill-TB Candela-L

Title: 2 Approaches to Design of Monitoring Networks

Source: GROUND WATER 1990, Vol 28, Iss 3, pp 430-442

Addresses:

US GEOL SURVEY, 230 COLLINS RD, BOISE, ID 83702, USA

UNIV POLITECN CATALUNYA, DEPT INGN TERRENO, BARCELONA, SPAIN

31. Authors: Hartzell-S Iida-M

Title: Source Complexity of the 1987 Whittier Narrows, California, Earthquake from the Inversion of Strong Motion Records

Source: JOURNAL OF GEOPHYSICAL RESEARCH-SOLID EARTH AND PLANETS 1990, Vol 95, Iss NB8, pp 2475-2485

Addresses:

US GEOL SURVEY, PASADENA, CA, USA

UNIV TOKYO, EARTHQUAKE RES INST, TOKYO 133, JAPAN

32. Authors: Hutchinson-DR White-RS Cannon-WF Schulz-KJ

Title: Keweenaw Hot-Spot - Geophysical Evidence for a 1.1 Ga Mantle Plume Beneath the Midcontinent Rift System

Source: JOURNAL OF GEOPHYSICAL RESEARCH-SOLID EARTH AND PLANETS 1990, Vol 95, Iss NB7, pp 869-884

Addresses:

US GEOL SURVEY, WOODS-HOLE, MA 02543, USA

US GEOL SURVEY, RESTON, VA 22092, USA

UNIV CAMBRIDGE, BULLARD LABS, CAMBRIDGE 3B3 0EZ, ENGLAND

33. Authors: Crampin-S Booth-DC Evans-R Peacock-S Fletcher-JB

Title: Changes in Shear-Wave Splitting At Anza Near the Time of the North Palm Springs Earthquake

Source: JOURNAL OF GEOPHYSICAL RESEARCH-SOLID EARTH AND PLANETS 1990, Vol 95, Iss NB7, pp 1197-1212

Addresses:

BRITISH GEOL SURVEY, MURCHISON HOUSE, W MAINS RD, EDINBURGH

EH9 3LA, SCOTLAND

UNIV READING, POSTGRAD RES INST SEDIMENT, READING RG6 2AB, BERKS, ENGLAND

US GEOL SURVEY, MENLO-PK, CA 94025, USA 
34. Authors: Choy-GL Bowman-JR

Title: Rupture Process of a Multiple Main Shock Sequence Analysis of Teleseismic, Local, and Field Observations of the Tennant Creek, Australia, Earthquakes of January 22, 1988

Source: JOURNAL OF GEOPHYSICAL RESEARCH-SOLID EARTH AND PLANETS 1990, Vol 95, Iss NB5, pp 6867-6882

Addresses:

US GEOL SURVEY, DENVER FED CTR, NATL EARTHQUAKE INFORMAT CTR, BOX 25046, MS 967, DENVER, CO 80225, USA

AUSTRALIAN NATL UNIV, RES SCH EARTH SCI, CANBERRA, ACT 2601, AUSTRALIA

35. Authors: Macfadden-BJ Anaya-F Perez-H Naeser-CW Zeitler-PK Campbell-KE

Title: Late Cenozoic Paleomagnetism and Chronology of Andean Basins of Bolivia - Evidence for Possible Oroclinal Bending

Source: JOURNAL OF GEOLOGY 1990, Vol 98, Iss 4, pp 541-555

Addresses:

SERV GEOL BOLIVIA, LAPAZ, BOLIVIA

US GEOL SURVEY, ISOTOPE GEOL BRANCH, DENVER, CO 80225, USA

NAT HIST MUSEUM LOS ANGELES, LOS-ANGELES, CA 90007, USA

CORNELL UNIV, INST STUDY CONTINENTS, ITHACA, NY 14853, USA

LEHIGH UNIV, DEPT GEOL SCI, BETHLEHEM, PA 18015, USA

36. Authors: Hodge-SM Wright-DL Bradley-JA Jacobel-RW Skou-N Vaughn-B

Title: Determination of the Surface and Bed Topography in Central Greenland

Source: JOURNAL OF GLACIOLOGY 1990, Vol 36, Iss 122, pp 17-30

Addresses:

UNIV PUGET SOUND, US GEOL SURVEY, ICE \& CLIMATE PROJECT,

TACOMA, WA 98416, USA

US GEOL SURVEY, DENVER, CO 80225, USA

ST OLAF COLL, NORTHFIELD, MN 55057, USA

TECH UNIV DENMARK, DK-2800 LYNGBY, DENMARK

37. Authors: Christophersen-N Neal-C Hooper-RP Vogt-RD Andersen-S

Title: Modeling Streamwater Chemistry As a Mixture of Soilwater End-Members - A Step Towards 2nd-Generation Acidification Models

Source: JOURNAL OF HYDROLOGY 1990, Vol 116, Iss 1-4, pp 307-320

Addresses:

CTR IND RES, POB 124 BLINDERN, N-0314 OSLO 3, NORWAY

US GEOL SURVEY, DORAVILLE, GA 30360, USA

ENVIRONM ANAL INC, N-1315 NESOYA, NORWAY

INST GEORESOURCES \& POLLUT RES, N-1432 AS, NORWAY

INST HYDROL, WALLINGFORD OX10 8BB, OXON, ENGLAND 
38. Authors: Hooper-RP Christophersen-N Peters-NE

Title: Modeling Streamwater Chemistry As a Mixture of Soilwater

End-Members - An Application to the Panola Mountain

Catchment, Georgia, USA

Source: JOURNAL OF HYDROLOGY

1990, Vol 116, Iss 1-4, pp 321-343

Addresses:

US GEOL SURVEY, DIV WATER RESOURCES, DORAVILLE, GA 30360, USA

CTR IND RES, DIV ENVIRONM CHEM, OSLO, NORWAY

39. Authors: Wolock-DM Hornberger-GM Musgrove-TJ

Title: Topographic Effects on Flow Path and Surface-Water

Chemistry of the Llyn-Brianne Catchments in Wales

Source: JOURNAL OF HYDROLOGY

1990, Vol 115, Iss 1-4, pp 243-259

Addresses:

US GEOL SURVEY, 810 BEAR TAVERN RD, W-TRENTON, NJ 08628, USA

UNIV VIRGINIA, DEPT ENVIRONM SCI, CHARLOTTESVILLE, VA 22903,

USA

INST HYDROL, WALLINGFORD OX10 8BB, OXON, ENGLAND

40. Authors: Revesz-K Woods-PH

Title: A Method to Extract Soil-Water for Stable Isotope Analysis

Source: JOURNAL OF HYDROLOGY

1990, Vol 115, Iss 1-4, pp 397-406

Addresses:

US GEOL SURVEY, 431 NATL CTR, RESTON, VA 22092, USA

CTR RES GROUNDWATER PROC, ADELAIDE, SA 5064, AUSTRALIA

41. Authors: Sohn-IG Rochacampos-AC

Title: Late Paleozoic (Gondwanan) Ostracodes in the Corumbatai

Formation, Parana Basin, Brazil

Source: JOURNAL OF PALEONTOLOGY

1990, Vol 64, Iss 1, pp 116-128

Addresses:

US GEOL SURVEY, WASHINGTON, DC 20242, USA

UNIV SAO PAULO, INST GEOSCI, SAO PAULO, SP, BRAZIL

42. Authors: Sawka-WN Chappell-BW Kistler-RW

Title: Granitoid Compositional Zoning by Side-Wall Boundary-

Layer Differentiation - Evidence from the Palisade Crest

Intrusive Suite, Central Sierra-Nevada, California

Source: JOURNAL OF PETROLOGY

1990, Vol 31, Iss 3, pp 519-553

Addresses:

AUSTRALIAN NATL UNIV, DEPT GEOL, CANBERRA, ACT 2601, AUSTRALIA

UNIV CALIF LAWRENCE LIVERMORE NATL LAB, DEPT EARTH SCI,

LIVERMORE, CA 94550, USA

US GEOL SURVEY, MENLO-PK, CA 94025, USA

43. Authors: Macdonald-R Mcgarvie-DW Pinkerton-H Smith-RL

Palacz-ZA

Title: Petrogenetic Evolution of the Torfajokull Volcanic

Complex, Iceland .1. Relationship Between the Magma Types

Source: JOURNAL OF PETROLOGY

1990, Vol 31, Iss 2, pp 429-459 
Addresses:

UNIV LANCASTER, DIV ENVIRONM SCI, LANCASTER LA1 4YQ, ENGLAND

US GEOL SURVEY, SACRAMENTO, CA 95821, USA

OPEN UNIV, DEPT EARTH SCI, MILTON KEYNES MK7 6AA, BUCKS, ENGLAND

44. Authors: Mcgarvie-DW Macdonald-R Pinkerton-H Smith-RL

Title: Petrogenetic Evolution of the Torfajokull Volcanic

Complex, Iceland .2. The Role of Magma Mixing

Source: JOURNAL OF PETROLOGY

1990, Vol 31, Iss 2, pp 461-481

Addresses:

UNIV LANCASTER, DIV ENVIRONM SCI, LANCASTER LA1 4YQ, ENGLAND

US GEOL SURVEY, SACRAMENTO, CA 95821, USA

45. Authors: Roure-F Howell-DG Muller-C Moretti-I

Title: Late Cenozoic Subduction Complex of Sicily

Source: JOURNAL OF STRUCTURAL GEOLOGY 1990, Vol 12, Iss 2, pp 259-266

Addresses:

INST FRANCAIS PETR, F-92506 RUEIL MALMAISON, FRANCE

US GEOL SURVEY, MENLO-PK, CA 94025, USA

46. Authors: Pierson-TC Janda-RJ Thouret-JC Borrero-CA

Title: Perturbation and Melting of Snow and Ice by the 13

November 1985 Eruption of Nevado-Del-Ruiz, Colombia, and

Consequent Mobilization, Flow and Deposition of Lahars

Source: JOURNAL OF VOLCANOLOGY AND GEOTHERMAL RESEARCH

1990, Vol 41, Iss 1-4, pp 17-66

Addresses:

US GEOL SURVEY, CASCADES VOLCANO OBSERV, VANCOUVER, WA 98661, USA

UNIV GRENOBLE 1, CNRS, UA 344, MONTAGNE ALPINE LAB, F-38041

GRENOBLE, FRANCE

UNIV CALDAS, FAC GEOL \& MINAS, MANIZALES, COLOMBIA

47. Authors: Banks-NG Carvajal-C Mora-H Tryggvason-E

Title: Deformation Monitoring At Nevado-Del-Ruiz, Colombia -

October 1985 - March 1988

Source: JOURNAL OF VOLCANOLOGY AND GEOTHERMAL RESEARCH 1990, Vol 41, Iss 1-4, pp 269-295

Addresses:

US GEOL SURVEY, CASCADES VOLCANO OBSERV, 5400 MACARTHUR BLVD, VANCOUVER, WA 98661, USA

INGEOMINAS, OBSERV VULCANOL COLOMBIA, MANIZALES, COLOMBIA

NORDIC VOLCANOL INST, REYKJAVIK, ICELAND

48. Authors: Sturchio-NC Keith-TEC Muehlenbachs-K

Title: Oxygen and Carbon Isotope Ratios of Hydrothermal

Minerals from Yellowstone Drill Cores

Source: JOURNAL OF VOLCANOLOGY AND GEOTHERMAL RESEARCH 1990, Vol 40, Iss 1, pp 23-37

Addresses:

ARGONNE NATL LAB, LMT-205, ARGONNE, IL 60439, USA

US GEOL SURVEY, MENLO-PK, CA 94025, USA

UNIV ALBERTA, DEPT GEOL, EDMONTON T6G 2E3, ALBERTA, CANADA 
49. Authors: Jany-I Scanlon-KM Mauffret-A

Title: Geological Interpretation of Combined Seabeam, Gloria and Seismic Data from Anegada Passage (Virgin-Islands, North Caribbean)

Source: MARINE GEOPHYSICAL RESEARCHES 1990, Vol 12, Iss 3, pp 173-196

Addresses:

UNIV PARIS 06, DEPT GEOL OCEAN, 4 PL JUSSIEU, F-75252 PARIS

05, FRANCE

US GEOL SURVEY, ATLANTIC GEOL BRANCH, WOODS-HOLE, MA 02543, USA

50. Authors: Leung-I Guo-WX Friedman-I Gleason-J

Title: Natural Occurrence of Silicon-Carbide in a

Diamondiferous Kimberlite from Fuxian

Source: NATURE

1990, Vol 346, Iss 6282, pp 352-354

Addresses:

CUNY HERBERT H LEHMAN COLL, DEPT GEOL \& GEOG, BRONX, NY 10468, USA

US GEOL SURVEY, DENVER, CO 80225, USA

SEVEN HUNDRED ONE DIAMOND MINE, SHANDONG, PEOPLES-R-CHINA

51. Authors: Srivastava-SP Schouten-H Roest-WR Klitgord-KD

Kovacs-LC Verhoef-J Macnab-R

Title: Iberian Plate Kinematics - A Jumping Plate Boundary

Between Eurasia and Africa

Source: NATURE

1990, Vol 344, Iss 6268, pp 756-759

Addresses:

GEOL SURVEY CANADA, ATLANTIC GEOSCI CTR, BEDFORD INST OCEANOG, DARTMOUTH B2Y 4A2, NS, CANADA

WOODS HOLE OCEANOG INST, DEPT GEOL \& GEOPHYS, WOODS-HOLE, MA 02543, USA

US GEOL SURVEY, WOODS-HOLE, MA 02543, USA

USN, RES LAB, WASHINGTON, DC 20375, USA

52. Authors: Slack-JF Palmer-MR Stevens-BPJ

Title: Boron Isotope Evidence for the Involvement of Non-Marine

Evaporites in the Origin of the Broken-Hill Ore-Deposits

Source: NATURE

1989, Vol 342, Iss 6252, pp 913-916

Addresses:

US GEOL SURVEY, NATL CTR, MS 954, RESTON, VA 22092, USA

MIT, DEPT EARTH \& PLANETARY SCI, CAMBRIDGE, MA 02139, USA

GEOL SURVEY NEW S WALES, BROKEN HILL, NSW 2880, AUSTRALIA

53. Authors: Clayton-JL Spencer-CW Koncz-I Szalay-A

Title: Origin and Migration of Hydrocarbon Gases and CarbonDioxide, Bekes Basin, Southeastern Hungary

Source: ORGANIC GEOCHEMISTRY

1990, Vol 15, Iss 3, pp 233-247

Addresses:

US GEOL SURVEY, DENVER FED CTR, BOX 25046, MS977, DENVER, CO

80225, USA

HUNGARIAN HYDROCARBON IND SCI IND ASSOC, H-2443 


\author{
SZAZHALOMBATTA, HUNGARY \\ HUNGARIAN OIL \& GAS TRUST, H-5001 SZOLNOK, HUNGARY
}

54. Authors: Thompson-RS Toolin-LJ Forester-RM Spencer-RJ

Title: Accelerator-Mass Spectrometer (AMS) Radiocarbon Dating of Pleistocene Lake-Sediments in the Great-Basin

Source: PALAEOGEOGRAPHY PALAEOCLIMATOLOGY PALAEOECOLOGY 1990, Vol 78, Iss 3-4, pp 301-313

Addresses:

US GEOL SURVEY, BOX 25046, MS 919, DENVER, CO 80225, USA

UNIV CALGARY, DEPT GEOL \& GEOPHYS, CALGARY T2N 1N4, ALBERTA, CANADA

UNIV ARIZONA, NATL SCI FDN ACCELERATOR FACIL, TUCSON, AZ 85721, USA

55. Authors: Rio-D Thunell-R Sprovieri-R Bukry-D Destefano-E Howell-M Raffi-I Sancetta-C Sanfilippo-A

Title: Stratigraphy and Depositional History of the Pliocene Bianco Section, Calabria, Southern Italy

Source: PALAEOGEOGRAPHY PALAEOCLIMATOLOGY PALAEOECOLOGY 1989, Vol 76, Iss 1-2, pp 85-105

Addresses:

UNIV PARMA, IST GEOL, I-43100 PARMA, ITALY

UNIV S CAROLINA, DEPT GEOL SCI, COLUMBIA, SC 29208, USA

UNIV PALERMO, IST GEOL, I-90134 PALERMO, ITALY

US GEOL SURVEY, MENLO-PK, CA 94025, USA

COLUMBIA UNIV, LAMONT DOHERTY GEOL OBSERV, PALISADES, NY

10964, USA

UNIV CALIF SAN DIEGO, SCRIPPS INST OCEANOG, LA-JOLLA, CA

92093, USA

56. Authors: Kennedy-WJ Cobban-WA

Title: Cenomanian Micromorphic Ammonites from the Western Interior of the USA

Source: PALAEONTOLOGY 1990, Vol 33, Iss MAY, pp 379-422

Addresses:

UNIV OXFORD MUSEUM, GEOL COLLECT, PARKS RD, OXFORD OX1 3PW, ENGLAND

US GEOL SURVEY, DENVER, CO 80225, USA

57. Authors: King-J Loveday-I Schuster-RL

Title: The 1985 Bairaman Landslide Dam and Resulting Debris Flow, Papua-New-Guinea

Source: QUARTERLY JOURNAL OF ENGINEERING GEOLOGY 1989, Vol 22, Iss 4, pp 257-270

Addresses:

GEOL SURVEY PAPUA NEW GUINEA, POB 778, PORT MORESBY, PAPUA-N-GUINEA

US GEOL SURVEY, GOLDEN, CO, USA

58. Authors: Kobayashi-Y Harp-EL Kagawa-T

Title: Simulation of Rockfalls Triggered by Earthquakes

Source: ROCK MECHANICS AND ROCK ENGINEERING

1990, Vol 23, Iss 1, pp 1-20 
Addresses:

KYOTO UNIV, INST GEOPHYS, KYOTO 606, JAPAN

US GEOL SURVEY, MENLO-PK, CA 94025, USA

OSAKA SOIL TEST LAB, OSAKA, JAPAN

59. Authors: Xie-GH Wang-JW Wei-KJ Liu-CQ Tatsumoto-M Basn-AR

Title: REE and $\mathrm{Sr}, \mathrm{Nd}, \mathrm{Pb}$ Isotopic Geochemistry of Huangyishan

Basalt, Kuandian, Liaoning, Northeast China

Source: SCIENCE IN CHINA SERIES B-CHEMISTRY LIFE SCIENCES \& EARTH SCIENCES 1990, Vol 33, Iss 6, pp 747-756

Addresses:

ACAD SINICA, INST GEOCHEM, GUIYANG 550002, PEOPLES-R-CHINA

US GEOL SURVEY, DENVER, CO 80225, USA

UNIV ROCHESTER, DEPT GEOL SCI, ROCHESTER, NY 14624, USA

60. Authors: Rubin-DM Ikeda-H

Title: Flume Experiments on the Alignment of Transverse,

Oblique, and Longitudinal Dunes in Directionally Varying

Flows

Source: SEDIMENTOLOGY

1990, Vol 37, Iss 4, pp 673-684

Addresses:

US GEOL SURVEY, MENLO-PK, CA 94025, USA

UNIV TSUKUBA, ENVIRONM RES CTR, SAKURA, IBARAKI 305, JAPAN

61. Authors: Kovach-RL Andreasen-GE Gettings-ME Elkaysi-K

Title: Geophysical Investigations in Jordan

Source: TECTONOPHYSICS

1990, Vol 180, Iss 1, pp 61-69

Addresses:

STANFORD UNIV, DEPT GEOPHYS, STANFORD, CA 94305, USA

URBANNA MARINE CORP, URBANNA, VA 23175, USA

US GEOL SURVEY, TUCSON, AZ 85705, USA

NAT RESOURCES AUTHOR, AMMAN, JORDAN

62. Authors: Milkereit-B Green-AG Lee-MW Agena-WF Spencer-C

Title: Prestack and Poststack Migration of Glimpce Reflection Data

Source: TECTONOPHYSICS 1990, Vol 173, Iss 1-4, pp 1-13

Addresses:

GEOL SURVEY CANADA, 1 OBSERV CTR, OTTAWA K1A 0Y3, ONTARIO, CANADA

US GEOL SURVEY, DENVER FED CTR, DENVER, CO 80225, USA

63. Authors: Behrendt-JC Hutchinson-DR Lee-M Thornber-CR

Trehu-A Cannon-W Green-A

Title: Glimpce Seismic-Reflection Evidence of Deep-Crustal and

Upper-Mantle Intrusions and Magmatic Underplating

Associated with the Midcontinent Rift System of North-

America

Source: TECTONOPHYSICS

1990, Vol 173, Iss 1-4, pp 595-

Addresses:

US GEOL SURVEY, DENVER FED CTR, BOX 25046, MS 964, DENVER, CO 80225, USA 
OREGON STATE UNIV, COLL OCEANOG, US GEOL SURVEY, CORVALLIS, OR 97331, USA

GEOL SURVEY CANADA, EARTH PHYS BRANCH, OTTAWA K1A 0Y3, ONTARIO, CANADA

US GEOL SURVEY, RESTON, VA 22092, USA

US GEOL SURVEY, WOODS-HOLE, MA 02543, USA

64. Authors: Green-A Milkereit-B Percival-J Davidson-A

Parrish-R Cook-F Geis-W Cannon-W Hutchinson-D

West-G Clowes-R

Title: Origin of Deep Crustal Reflections - Seismic Profiling

Across High-Grade Metamorphic Terranes in Canada

Source: TECTONOPHYSICS

1990, Vol 173, Iss 1-4, pp 627-638

Addresses:

GEOL SURVEY CANADA, OTTAWA K1A OY3, ONTARIO, CANADA

UNIV CALGARY, CALGARY T2N 1N4, ALBERTA, CANADA

US GEOL SURVEY, RESTON, VA 22092, USA

US GEOL SURVEY, WOODS-HOLE, MA 02543, USA

UNIV TORONTO, TORONTO M5S 1A7, ONTARIO, CANADA

UNIV BRITISH COLUMBIA, VANCOUVER V6T 1W5, BC, CANADA

65. Authors: Fuller-CC Davis-JA Cain-DJ Lamothe-PJ Fries-TL Fernandez-G Vargas-JA Murillo-MM

Title: Distribution and Transport of Sediment-Bound Metal

Contaminants in the Rio-Grande-de-Tarcoles, Costa-Rica (Central-America)

Source: WATER RESEARCH

1990, Vol 24, Iss 7, pp 805-812

Addresses:

US GEOL SURVEY, 345 MIDDLEFIELD RD, MAIL STOP 465, MENLO-PK, CA 94025, USA

UNIV COSTA RICA, CTR INVEST CIENCIAS MAR \& LIMNOL, SAN JOSE, COSTA-RICA

66. Authors: Cvetkovic-VD Shapiro-AM

Title: Mass Arrival of Sorptive Solute in Heterogeneous Porous-Media

Source: WATER RESOURCES RESEARCH 1990, Vol 26, Iss 9, pp 2057-2067

Addresses:

ROYAL INST TECHNOL, BRINELLVAGEN 32, S-10044 STOCKHOLM 70, SWEDEN

US GEOL SURVEY, RESTON, VA 22092, USA

67. Authors: Perlinger-JA Eisenreich-SJ Capel-PD Carr-PW Park-JH

Title: Adsorption of a Homologous Series of Alkylbenzenes to

Mineral Oxides At Low Organic-Carbon Content Using

Headspace Analysis

Source: WATER SCIENCE AND TECHNOLOGY

1990, Vol 22, Iss 6, pp 7-14

Addresses:

UNIV MINNESOTA, DEPT CIVIL \& MINERAL ENGN, MINNEAPOLIS, MN 55455, USA

UNIV MINNESOTA, DEPT CHEM, MINNEAPOLIS, MN 55455, USA

US GEOL SURVEY, DIV WATER RESOURCES, ST-PAUL, MN 55101, USA

YEUNGNAM UNIV, DEPT CHEM, GYONGSAN 713749, SOUTH-KOREA 
68. Authors: Annandale-GW Demissie-M Gilroy-EJ Cohn-TA

Nordin-CF Mcbean-EA Fitzpatrick-WP Kirby-WH

Glysson-GD Wahl-K Alnassri-S

Title: Uncertainty in Suspended Sediment Transport Curves - Discussion

Source: JOURNAL OF HYDRAULIC ENGINEERING-ASCE 1990, Vol 116, Iss 1, pp 140-151

Addresses:

ILLINOIS STATE WATER SURVEY, SURFACE WATER SECT, CHAMPAIGN, IL 61820, USA

US GEOL SURVEY, RESTON, VA 22092, USA

COLORADO STATE UNIV, DEPT CIVIL ENGN, FT-COLLINS, CO 80523, USA

US GEOL SURVEY, DENVER FED CTR, LAKEWOOD, CO 80225, USA

UNIV WATERLOO, DEPT CIVIL ENGN, WATERLOO N2L 3G1, ONTARIO,

CANADA

UNIV LIVERPOOL, DEPT CIVIL ENGN, LIVERPOOL L69 3BX, ENGLAND

69. Authors: Urabe-T Cygan-GL

Title: Special Issue - Deep Crust Fluid-Rock Interaction - Preface

Source: GEOCHEMICAL JOURNAL 1989, Vol 23, Iss 6, pp 269-270

Addresses:

GEOL SURVEY JAPAN, 1-1-3 HIGASHI, TSUKUBA, IBARAKI 305, JAPAN

US GEOL SURVEY, RESTON, VA 22092, USA

70. Authors: Rochette-P Nord-GL

Title: Application of Optical and Magnetic Techniques in Rock Magnetism - Foreward

Source: GEOPHYSICAL RESEARCH LETTERS 1990, Vol 17, Iss 6, pp 765-765

Addresses:

UNIV JOSEPH FOURIER, GRENOBLE, FRANCE

US GEOL SURVEY, RESTON, VA 22092, USA

71. Authors: Kennedy-WJ Cobban-WA

Title: Cenomanian Ammonite Faunas from the Woodbine Formation and Lower Part of the Eagle-Ford-Group, Texas

Source: PALAEONTOLOGY 1990, Vol 33, Iss FEB, pp 75-154

Addresses:

UNIV OXFORD MUSEUM, GEOL COLLECT, PARKS RD, OXFORD OX1 3PW, ENGLAND

US GEOL SURVEY, PALEONTOL \& STRATIG BRANCH, DENVER, CO 80225, USA

72. Authors: Kogelknabner-I Hatcher-PG Deleeuw-JW

Title: Aliphatic Components of Forest Soil Organic-Matter As

Determined by Solid-State C-13 NMR and Analytical Pyrolysis

Source: ABSTRACTS OF PAPERS OF THE AMERICAN CHEMICAL SOCIETY 1989, Vol 198, Iss SEP, pp 52-

Addresses:

UNIV BAYREUTH, LEHRSTUHL BODENKUNDE, D-8580 BAYREUTH, FED-REP-GER

US GEOL SURVEY, RESTON, VA 22092, USA

DELFT UNIV TECHNOL, DELFT, NETHERLANDS 
73. Authors: Tipping-E Reddy-MM

Title: Modeling Electrostatic and Heterogeneity Effects on Proton Dissociation of Fulvic-Acids

Source: ABSTRACTS OF PAPERS OF THE AMERICAN CHEMICAL SOCIETY 1989, Vol 198, Iss SEP, pp 76-

Addresses:

FRESHWATER BIOL ASSOC, AMBLESIDE LA22 OLP, CUMBRIA, ENGLAND

US GEOL SURVEY, DENVER FED CTR, DIV WATER RESOURCES, LAKEWOOD, CO 80225, USA

74. Authors: Breit-GN Meunier-JD

Title: Fluid Inclusion Delta-18-O and Sr-87/Sr-86 Evidence for the Origin of Fault-Controlled Copper Mineralization, Lisbon Valley, Utah, and Slick Rock District, Colorado

Source: ECONOMIC GEOLOGY AND THE BULLETIN OF THE SOCIETY OF ECONOMIC GEOLOGISTS 1990, Vol 85, Iss 4, pp 884-891

Addresses:

US GEOL SURVEY, DENVER FED CTR, BOX 25046, MAIL STOP 916, DENVER, CO 80225, USA

CTR RECH ECOL GEOL URANIUM, CNRS, SCI GRP, F-54501 VANDOEUVRE NANCY, FRANCE

CTR RECH GEOL URANIUM, F-54501 VANDOEUVRE NANCY, FRANCE

75. Authors: Kennedy-WJ Cobban-WA

Title: Rhamphidoceras-Saxatilis N-Gen and Sp a Micromorph Ammonite from the Lower Turonian of Trans-Pecos Texas

Source: JOURNAL OF PALEONTOLOGY 1990, Vol 64, Iss 4, pp 666-668

Addresses:

UNIV OXFORD MUSEUM, PARKS RD, OXFORD OX13PW, ENGLAND

US GEOL SURVEY, DENVER FED CTR, DENVER, CO 80225, USA

76. Authors: Genise-JF Bown-TM

Title: The Constructor of the Ichnofossil Chubutolithes

Source: JOURNAL OF PALEONTOLOGY 1990, Vol 64, Iss 3, pp 482-483

Addresses:

MUSEO ARGENTINO CIENCIAS NAT BERNARDINO RIVADAVIA, DIV

ENTOMOL, RA-1405 BUENOS AIRES, ARGENTINA

US GEOL SURVEY, PALEONTOL \& SRATIG BRANCH, DENVER, CO 80225, USA

77. Authors: Aggarwal-PK Gunter-WD Kharaka-YK

Title: Effect of Pressure on Aqueous Equilibria

Source: ACS SYMPOSIUM SERIES 1990, Vol 416, pp 87-101

Addresses:

BATTELLE MEM INST, 505 KING AVE, COLUMBUS, OH 43201, USA US GEOL SURVEY, DIV WATER RESOURCES, MENLO-PK, CA 94025, USA ALBERTA RES COUNCIL, OIL SANDS \& HYDROCARBON RECOVERY, EDMONTON T6H 5X2, ALBERTA, CANADA

78. Authors: Perkins-EH Kharaka-YK Gunter-WD Debraal-JD

Title: Geochemical Modeling of Water-Rock Interactions Using Solmineq-88 
Source: ACS SYMPOSIUM SERIES

1990, Vol 416, pp 117-127

Addresses:

ALBERTA RES COUNCIL, OIL SANDS \& HYDROCARBON RECOVERY, POB 8330, POSTAL STN F, EDMONTON T6H 5X2, ALBERTA, CANADA

US GEOL SURVEY, WATER RESOURCES DIV, MENLO-PK, CA 94025, USA

79. Authors: Cochonat-P Lenat-JF Bachelery-P Boivin-P

Cornaglia-B Deniel-C Labazuy-P Ledrezen-E Lipman-P

Ollier-G Savoye-B Vincent-P Voisset-M

Title: Gravity Events As a Primary Process in the Construction of a Submarine Volcano-Sedimentary System (Fournaise Volcano, Reunion-Island)

Source: COMPTES RENDUS DE L ACADEMIE DES SCIENCES SERIE IIMECANIQUE PHYSIQUE CHIMIE SCIENCES DE L UNIVERS SCIENCES DE LA TERRE 1990, Vol 311, Iss 6, pp 679-686

Addresses:

INST FRANCAIS RECH EXPLOITAT MER, CTR BREST, BP 70, F-29280 PLOUZANE, FRANCE

US GEOL SURVEY, DENVER, CO 80225, USA

UNIV CLERMONT FERRAND, F-63038 CLERMONT FERRAND, FRANCE

UNIV REUNION, F-97490 ST CLOTILDE, REUNION

INST PHYS GLOBE, F-75232 PARIS 16, FRANCE

80. Authors: Fryer-P Pearce-J Lagabrielle-Y Stokking-L Ali-J Arculus-RJ Balloti-D Burke-MM Ciampo-G Haggerty-J Aston-RB Heling-D Hobart-M Ishii-T Johnson-LE Maekawa-H Marlow-M Mccoy-FW Milner-G Mottl-MJ Murton-BJ Phipps-SP Rigsby-C Saboda-KL Sizun-JP Stabell-B Vanderlann-SR Xu-Y

Title: Fore-Arc Mantle and Boninites - Main Results of ODP Leg125 in the Mariana and the Izu-Bonin Fore-Arc Regions

Source: COMPTES RENDUS DE L ACADEMIE DES SCIENCES SERIE IIMECANIQUE PHYSIQUE CHIMIE SCIENCES DE L UNIVERS SCIENCES DE LA TERRE 1990, Vol 310, Iss 9, pp 1247-1254

Addresses:

UNIV HAWAII, HAWAII INST GEOPHYS, DIV PLANETARY GEOSCI, 2525

CORREA RD, HONOLULU, HI 96822, USA

UNIV NEWCASTLE UPON TYNE, DEPT GEOL, NEWCASTLE TYNE NE1 7RU, TYNE \& WEAR, ENGLAND

GDRGEDO, URA 1278, F-29287 BREST, FRANCE

TEXAS A\&M UNIV SYST, OCEAN DRILLING PROGRAM, COLLEGE-STN, TX 77840, USA

UNIV SOUTHAMPTON, DEPT OCEANOG, SOUTHAMPTON SO9 5NH, HANTS, ENGLAND

UNIV MICHIGAN, DEPT GEOL SCI, ANN-ARBOR, MI 48109, USA

UNIV MIAMI, ROSENSTIEL SCH MARINE \& ATMOSPHER SCI, MIAMI, FL 33149, USA

DALHOUSIE UNIV, DEPT GEOL, HALIFAX B3H 4H6, NS, CANADA

KOBE UNIV, FAC SCI, DEPT EARTH SCI, KOBE 657, JAPAN

UNIV OSLO, DEPT GEOL, N-0316 OSLO 3, NORWAY

UNIV WESTERN AUSTRALIA, NEDLANDS, AUSTRALIA

UNIV CALIF SANTA BARBARA, DEPT GEOL SCI, SANTA-BARBARA, CA 93106, USA 
CALTECH, DIV GEOL \& PLANETARY SCI, PASADENA, CA 91125, USA

INST SEDIMENTFORSCH, D-6900 HEIDELBERG 1, FED-REP-GER

FLORIDA STATE UNIV, DEPT GEOL, TALLAHASSEE, FL 32306, USA

UNIV CALIF SANTA CRUZ, DEPT EARTH SCI, SANTA-CRUZ, CA 95064, USA

UNIV PENN, DEPT GEOL, PHILADELPHIA, PA 19104, USA

US GEOL SURVEY, OFF MARINE GEOL, MENLO-PK, CA 94025, USA

OPEN UNIV, DEPT EARTH SCI, MILTON KEYNES MK7 6AA, BUCKS,

ENGLAND

UNIV TULSA, DEPT GEOSCI, TULSA, OK 74104, USA

DIPARTIMENTO SCI TERRA LARGO, I-80138 NAPLES, ITALY

DALHOUSIE UNIV, CTR MARINE GEOL, HALIFAX B3H 3J5, NS, CANADA

LAMONT DOHERTY GEOL OBSERV, PALISADES, NY 10964, USA

81. Authors: Collot-JY Pelletier-B Boulin-J Daniel-J Eissen-JP

Fisher-MA Greene-HG Lallemand-S Monzier-M

Title: 1st Results of Dives During the Subpso 1 Cruise in the

Collision Zone Between the Dentrecasteaux Ridges and the

New Hebrides Island-Arc

Source: COMPTES RENDUS DE L ACADEMIE DES SCIENCES SERIE II-

MECANIQUE PHYSIQUE CHIMIE SCIENCES DE L UNIVERS

SCIENCES DE LA TERRE

1989, Vol 309, Iss 19, pp 1947-1954

Addresses:

ORSTOM, BP A5, NOUMEA, NEW-CALEDONIA

US GEOL SURVEY, MENLO-PK, CA 94025, USA

UNIV MARSEILLE 3, GEOL STRUCT LAB, F-13397 MARSEILLE, FRANCE

UNIV PARIS 06, GEOL STRUCT LAB, F-75252 PARIS, FRANCE

\section{1}

1. Authors: Barker-CE

Title: Implications for Organic Maturation Studies of Evidence

for a Geologically Rapid Increase and Stabilization of

Vitrinite Reflectance at Peak Temperature - Cerro-Prieto

Geothermal System, Mexico

Source: AAPG BULLETIN-AMERICAN ASSOCIATION OF PETROLEUM GEOLOGISTS 1991, Vol 75, Iss 12, pp 1852-1863

Addresses:

US-GEOL-SURVEY, DENVER FED CTR, BOX 25046, MS 972, DENVER, CO

80225, USA

UNIV-ADELAIDE, ADELAIDE, SA 5001, AUSTRALIA

2. Authors: Miller-JJ Lee-MW Vonhuene-R

Title: An Analysis of a Seismic-Reflection from the Base of a

Gas Hydrate Zone, Offshore Peru

Source: AAPG BULLETIN-AMERICAN ASSOCIATION OF PETROLEUM GEOLOGISTS

1991, Vol 75, Iss 5, pp 910-924

Addresses:

US-GEOL-SURVEY, DENVER FED CTR, MS 960, BOX 25046, DENVER, CO 80225, USA

GEOMAR, MARINE GEOSCI RES CTR, W-2300 KIEL 14, GERMANY 
3. Authors: Rothwell-RG Kenyon-NH Mcgregor-BA

Title: Sedimentary Features of the South Texas Continental-

Slope as Revealed by Side-Scan Sonar and High-Resolution

Seismic Data

Source: AAPG BULLETIN-AMERICAN ASSOCIATION OF PETROLEUM GEOLOGISTS 1991, Vol 75, Iss 2, pp 298-312

Addresses:

INST-OCEANOG-SCI, DEACON LAB, BROOK RD, GODALMING GU8-5UB, SURREY, ENGLAND

US-GEOL-SURVEY, NATL CTR, RESTON, VA 22092, USA

4. Authors: Bradshaw-MA Mccartan-L

Title: Paleoecology and Systematics of Early Devonian Bivalves from the Horlick Formation, Ohio Range, Antarctica

Source: ALCHERINGA 1991, Vol 15, Iss 1-2, pp 1-42

Addresses:

CANTERBURY-MUSEUM, ROLLESTON AVE, CHRISTCHURCH, NEW-ZEALAND

US-GEOL-SURVEY, RESTON, VA 22092, USA

5. Authors: Webster-JD Duffield-WA

Title: Volatiles and Lithophile Elements in Taylor Creek Rhyolite - Constraints from Glass Inclusion Analysis

Source: AMERICAN MINERALOGIST 1991, Vol 76, Iss 9-10, pp 1628-1645

Addresses:

UNIV-EDINBURGH, GRANT INST GEOL, EDINBURGH EH9-3JW, SCOTLAND

US-GEOL-SURVEY, FLAGSTAFF, AZ 86001, USA

6. Authors: Brew-DA Karl-SM Barnes-DF Jachens-RC Ford-AB Horner-R

Title: A Northern Cordilleran Ocean Continent Transect - Sitka Sound, Alaska, to Atlin Lake, British-Columbia

Source: CANADIAN JOURNAL OF EARTH SCIENCES 1991, Vol 28, Iss 6, pp 840-853

Addresses:

US-GEOL-SURVEY, MS 904, MENLO-PK, CA 94025, USA

US-GEOL-SURVEY, ANCHORAGE, AK 99508, USA

GEOL-SURVEY-CANADA, PACIFIC GEOSCI CTR, SIDNEY V8L-4B2, BC, CANADA

7. Authors: Roberts-AC Ercit-TS Erd-RC Oscarson-RL

Title: Szymanskiite, $\mathrm{Hg}-1(16)+(\mathrm{Ni}, \mathrm{Mg}) 6(\mathrm{CO} 3) 12(\mathrm{OH}) 12(\mathrm{H} 3 \mathrm{O})-$

$1(8)+.3 \mathrm{H} 2 \mathrm{O}$, a New Mineral Species from the Clear Creek Claim, San-Benito County, California

Source: CANADIAN MINERALOGIST 1990, Vol 28, Iss DEC, pp 703-707

Addresses:

GEOL-SURVEY-CANADA, 601 BOOTH ST, OTTAWA K1A-0E8, ONTARIO, CANADA

CANADIAN-MUSEUM-NAT, MINERAL SCI SECT, OTTAWA K1P-6P4, ONTARIO, CANADA

US-GEOL-SURVEY, MENLO-PK, CA 94025, USA 
8. Authors: Vallier-TL Jenner-GA Frey-FA Gill-JB Davis-AS

Volpe-AM Hawkins-JW Morris-JD Cawood-PA Morton-JL

Scholl-DW Rautenschlein-M White-WM Williams-RW

Stevenson-AJ White-LD

Title: Subalkaline Andesite from Valu FA Ridge, a Back-Arc

Spreading Center in Southern Lau Basin - Petrogenesis,

Comparative Chemistry, and Tectonic Implications

Source: CHEMICAL GEOLOGY

1991, Vol 91, Iss 3, pp 227-256

Addresses:

US-GEOL-SURVEY, 345 MIDDLEFIELD RD, MENLO-PK, CA 94025, USA

MIT, CAMBRIDGE, MA 02139, USA

UNIV-CALIF-SANTA-CRUZ, SANTA-CRUZ, CA 95064, USA

LOS-ALAMOS-NATL-LAB, LOS-ALAMOS, NM 87544, USA

UNIV-CALIF-SAN-DIEGO, SCRIPPS INST OCEANOG, LA-JOLLA, CA

92093, USA

MAX-PLANCK-INST, MAINZ, GERMANY

CARNEGIE-INST-WASHINGTON, WASHINGTON, DC 20005, USA

MAX-PLANCK-INST, ITHACA, NY, USA

MEM-UNIV-NEWFOUNDLAND, ST-JOHNS A1C-5S7, NEWFOUNDLAND, CANADA

CORNELL-UNIV, ITHACA, NY 14853, USA

9. Authors: Philpotts-J Tatsumoto-M Li-XH Wang-KY

Title: Some Nd and Sr Isotopic Systematics for the REE-Enriched

Deposit at Bayan Obo, China

Source: CHEMICAL GEOLOGY

1991, Vol 90, Iss 3-4, pp 177-188

Addresses:

US-GEOL-SURVEY, RESTON, VA 22092, USA

US-GEOL-SURVEY, DENVER, CO 80225, USA

CHINESE-ACAD-SCI, INST GEOL, BEIJING, PEOPLES-R-CHINA

ACAD-SINICA, INST GEOCHEM, KUANZHOU, PEOPLES-R-CHINA

10. Authors: Robie-RA Hemingway-BS Gillet-P Reynard-B

Title: On the Entropy of Glaucophane Na2Mg3A12Si8O22(OH)2

Source: CONTRIBUTIONS TO MINERALOGY AND PETROLOGY

1991, Vol 107, Iss 4, pp 484-486

Addresses:

US-GEOL-SURVEY, RESTON, VA 22092, USA

UNIV-RENNES-1, INST GEOL, F-35042 RENNES, FRANCE

11. Authors: Scowen-PAH Roeder-PL Helz-RT

Title: Reequilibration of Chromite Within Kilauea Iki Lava Lake, Hawaii

Source: CONTRIBUTIONS TO MINERALOGY AND PETROLOGY 1991, Vol 107, Iss 1, pp 8-20

Addresses:

QUEENS-UNIV, DEPT GEOL SCI, KINGSTON K7L-3N6, ONTARIO, CANADA

US-GEOL-SURVEY, RESTON, VA 22092, USA

12. Authors: Basu-AR Wang-JW Huang-WK Xie-GH Tatsumoto-M

Title: Major Element, $\mathrm{REE}$, and $\mathrm{Pb}, \mathrm{Nd}$ and $\mathrm{Sr}$ Isotopic

Geochemistry of Cenozoic Volcanic-Rocks of Eastern China

- Implications for Their Origin from Suboceanic-Type

Mantle Reservoirs

Source: EARTH AND PLANETARY SCIENCE LETTERS

1991, Vol 105, Iss 1-3, pp 149-169 
Addresses:

UNIV-ROCHESTER, DEPT GEOL SCI, ROCHESTER, NY 14627, USA ACAD-SINICA, INST GEOCHEM, GUIYANG, PEOPLES-R-CHINA

US-GEOL-SURVEY, DENVER, CO 80225, USA

13. Authors: Walker-RJ Morgan-JW Naldrett-AJ Li-C Fassett-JD

Title: Re-Os Isotope Systematics of Ni-Cu Sulfide Ores, Sudbury Igneous Complex, Ontario - Evidence for a Major Crustal Component

Source: EARTH AND PLANETARY SCIENCE LETTERS 1991, Vol 105, Iss 4, pp 416-429

Addresses:

UNIV-MARYLAND, DEPT GEOL, COLLEGE-PK, MD 20742, USA

US-GEOL-SURVEY, RESTON, VA 22092, USA

UNIV-TORONTO, DEPT GEOL, TORONTO M5S-1A1, ONTARIO, CANADA

NATL-INST-STAND-\&-TECHNOL, GAITHERSBURG, MD 20899, USA

14. Authors: Velde-B Dubois-J Moore-D Touchard-G

Title: Fractal Patterns of Fractures in Granites

Source: EARTH AND PLANETARY SCIENCE LETTERS 1991, Vol 104, Iss 1, pp 25-35

Addresses:

ECOLE-NORM-SUPER, CNRS, UR 1316, DEPT GEOL, 24 RUE LHOMOND, F-75231 PARIS 05, FRANCE

INST-PHYS-GLOBE, OBSERV VOLCANOL, F-75252 PARIS, FRANCE

UNIV-POITIERS, MECAN FLUIDES LAB, F-89022 POITIERS, FRANCE

US-GEOL-SURVEY, MENLO-PK, CA 94025, USA

15. Authors: Staudigel-H Park-KH Pringle-M Rubenstone-JL

Smith-WHF Zindler-A

Title: The Longevity of the South-Pacific Isotopic and Thermal Anomaly

Source: EARTH AND PLANETARY SCIENCE LETTERS 1991, Vol 102, Iss 1, pp 24-44

Addresses:

UNIV-CALIF-SAN-DIEGO, SCRIPPS INST OCEANOG, A-015, LA-JOLLA, CA 92093, USA

KOREA-SCI-CTR, SEOUL 135619, SOUTH-KOREA

COLUMBIA-UNIV, LAMONT DOHERTY GEOL OBSERV, PALISADES, NY 10964, USA

US-GEOL-SURVEY, ISOTOPE BRANCH, MENLO-PK, CA 94025, USA

16. Authors: Kissling-E Lahr-JC

Title: Tomographic Image of the Pacific Slab Under Southern Alaska

Source: ECLOGAE GEOLOGICAE HELVETIAE 1991, Vol 84, Iss 2, pp 297-315

Addresses:

SWISS-FED-INST-TECHNOL, INST GEOPHYS, CH-8093 ZURICH, SWITZERLAND

US-GEOL-SURVEY, MENLO-PK, CA 94025, USA

17. Authors: Ruan-HC Hua-RM Cox-DP

Title: Copper Deposition by Fluid Mixing in Deformed Strata

Adjacent to a Salt Diapir, Dongchuan Area, Yunnan

Province, China 
Source: ECONOMIC GEOLOGY AND THE BULLETIN OF THE SOCIETY OF ECONOMIC GEOLOGISTS

1991, Vol 86, Iss 7, pp 1539-1545

Addresses:

NANJING-UNIV, NANJING, PEOPLES-R-CHINA

US-GEOL-SURVEY, MENLO-PK, CA 94025, USA

18. Authors: Sillitoe-RH Mckee-EH Vila-T

Title: Reconnaissance K-Ar Geochronology of the Maricunga GoldSilver Belt, Northern Chile

Source: ECONOMIC GEOLOGY AND THE BULLETIN OF THE SOCIETY OF ECONOMIC GEOLOGISTS

1991, Vol 86, Iss 6, pp 1261-1270

Addresses:

27-W-HILL-PK, HIGHGATE VILLAGE, LONDON N6-6ND, ENGLAND

US-GEOL-SURVEY, MENLO-PK, CA 94025, USA

MINERA-ANGLO-AMER-CHILE-LTD, SANTIAGO, CHILE

19. Authors: Bussell-MA Alpers-CN Petersen-U Shepherd-TJ

Bermudez-C Baxter-AN

Title: The Ag-Mn-Pb-Zn Vein, Replacement, and Skarn Deposits of

Uchucchacua, Peru - Studies of Structure, Mineralogy,

Metal Zoning, Sr Isotopes, and Fluid Inclusions

Source: ECONOMIC GEOLOGY AND THE BULLETIN OF THE SOCIETY OF ECONOMIC GEOLOGISTS

1990, Vol 85, Iss 7, pp 1348-1383

Addresses:

THAMES-POLYTECH, SCH EARTH SCI, WALBURGH HOUSE, BIGLAND ST,

LONDON E1-2NG, ENGLAND

US-GEOL-SURVEY, MENLO-PK, CA 94025, USA

HARVARD-UNIV, DEPT EARTH \& PLANETARY SCI, CAMBRIDGE, MA 02138 , USA

BRITISH-GEOL-SURVEY, NOTTINGHAM NG12-5GG, ENGLAND

CIA-MINAS-BUENAVENTURA-SA, LIMA 13, PERU

20. Authors: Vidal-CE Injoqueespinoza-J Sidder-GB Mukasa-SB

Title: Amphibolitic Cu-Fe Skarn Deposits in the Central Coast of Peru

Source: ECONOMIC GEOLOGY AND THE BULLETIN OF THE SOCIETY OF ECONOMIC GEOLOGISTS 1990, Vol 85, Iss 7, pp 1447-1461

Addresses:

PERUBAR-SA, JUAN ARONA 830, OFICINA 901, LIMA 27, PERU

CHEVRON-MINERAL-CORP-CHILE, SANTIAGO, CHILE

US-GEOL-SURVEY, DENVER FED CTR, DENVER, CO 80225, USA

UNIV-MICHIGAN, DEPT GEOL SCI, ANN-ARBOR, MI 48109, USA

21. Authors: Delosrios-HC Noble-DC Mckee-EH

Title: Geologic Setting and Epithermal Silver Veins of the Arcata-District, Southern Peru

Source: ECONOMIC GEOLOGY AND THE BULLETIN OF THE SOCIETY OF ECONOMIC GEOLOGISTS

1990, Vol 85, Iss 7, pp 1473-1490

Addresses:

MAURICIO-HOCHSCHILD-\&-CIA-LTD-SA, AVE REPUBL PANAMA 3055, LIMA

27, PERU 
UNIV-NEVADA, MACKAY SCH MINES, RENO, NV 89557, USA

US-GEOL-SURVEY, MENLO-PK, CA 94025, USA

22. Authors: Clark-AH Tosdal-RM Farrar-E Plazolles-A

Title: Geomorphologic Environment and Age of Supergene

Enrichment of the Cuajone, Quellaveco, and Toquepala

Porphyry Copper-Deposits, Southeastern Peru

Source: ECONOMIC GEOLOGY AND THE BULLETIN OF THE SOCIETY OF ECONOMIC GEOLOGISTS 1990, Vol 85, Iss 7, pp 1604-1628

Addresses:

QUEENS-UNIV, DEPT GEOL SCI, KINGSTON K7L-3N6, ONTARIO, CANADA

US-GEOL-SURVEY, MENLO-PK, CA 94025, USA

SO-PERU-COPPER-CORP, TACNA, PERU

23. Authors: Meade-RH Rayol-JM Daconceicao-SC Natividade-JRG

Title: Backwater Effects in the Amazon River Basin of Brazil

Source: ENVIRONMENTAL GEOLOGY AND WATER SCIENCES 1991, Vol 18, Iss 2, pp 105-114

Addresses:

US-GEOL-SURVEY, MS 413, BOX 25046, DENVER, CO 80225, USA

CO-PESQUISAS-RECURSOS-MINERAIS, BR-69000 MANAUS, AMAZONAS, BRAZIL

CO-PESQUISAS-RECURSOS-MINERAIS, BR-66000 BELEM, PARA, BRAZIL

DEPT-NACL-AGUAS-\&-ENERGIA-ELET, BR-66000 BELEM, PARA, BRAZIL

24. Authors: Tequi-C Robie-RA Hemingway-BS Neuville-DR Richet-P

Title: Melting and Thermodynamic Properties of Pyrope (Mg3Al2Si3O12)

Source: GEOCHIMICA ET COSMOCHIMICA ACTA 1991, Vol 55, Iss 4, pp 1005-1010

Addresses:

INST-PHYS-GLOBE, 4 PL JUSSIEU, F-75252 PARIS 05, FRANCE

US-GEOL-SURVEY, NATL CTR 959, RESTON, VA 22092, USA

25. Authors: Vidic-N Pavich-M Lobnik-F

Title: Statistical-Analyses of Soil Properties on a Quaternary

Terrace Sequence in the Upper Sava River Valley,

Slovenia, Yugoslavia

Source: GEODERMA

1991, Vol 51, Iss 1-4, pp 189-211

Addresses:

EDVARD-KARDELJ-UNIV, FAC BIOTECH, DEPT AGRON, YU-61000

LJUBLJANA, YUGOSLAVIA

US-GEOL-SURVEY, RESTON, VA 22092, USA

26. Authors: Woodward-D Menges-CM

Title: Application of Uphole Data from Petroleum Seismic

Surveys to Groundwater Investigations, Abu-Dhabi (United-

Arab-Emirates)

Source: GEOEXPLORATION

1991, Vol 27, Iss 1-2, pp 193-212

Addresses:

US-GEOL-SURVEY, TACOMA, WA 98402, USA

US-GEOL-SURVEY, AL-AIN, U-ARAB-EMIRATES 
27. Authors: Kennedy-WJ Cobban-WA

Title: Upper Cretaceous (Upper Santonian) Boehmoceras Fauna from the Gulf-Coast Region of the United-States

Source: GEOLOGICAL MAGAZINE 1991, Vol 128, Iss 2, pp 167-189

Addresses:

UNIV-MUSEUM-OXFORD, PARKS RD, OXFORD OX1-3PW, ENGLAND

US-GEOL-SURVEY, DENVER, CO 80225, USA

28. Authors: Masson-DG Scanlon-KM

Title: The Neotectonic Setting of Puerto-Rico

Source: GEOLOGICAL SOCIETY OF AMERICA BULLETIN 1991, Vol 103, Iss 1, pp 144-154

Addresses:

INST-OCEANOG-SCI, GODALMING GU8-5UB, SURREY, ENGLAND

US-GEOL-SURVEY, WOODS-HOLE, MA 02543, USA

29. Authors: England-P Wells-RE

Title: Neogene Rotations and Quasi-Continuous Deformation of the Pacific-Northwest Continental-Margin

Source: GEOLOGY 1991, Vol 19, Iss 10, pp 978-981

Addresses:

UNIV-OXFORD, DEPT EARTH SCI, PARKS RD, OXFORD OX1-3PR, ENGLAND

US-GEOL-SURVEY, MENLO-PK, CA 94025, USA

30. Authors: Twichell-DC Paull-CK Parson-LM

Title: Terraces on the Florida Escarpment - Implications for Erosional Processes

Source: GEOLOGY 1991, Vol 19, Iss 9, pp 897-900

Addresses:

US-GEOL-SURVEY, WOODS-HOLE, MA 02543, USA

UNIV-N-CAROLINA, CHAPEL-HILL, NC 27599, USA

INST-OCEANOG-SCI, WORMLEY GU8-5UB, SURREY, ENGLAND

31. Authors: Johnson-LE Fryer-P Taylor-B Silk-M Jones-DL Sliter-WV Itaya-T Ishii-T

Title: New Evidence for Crustal Accretion in the Outer Mariana Fore Arc - Cretaceous Radiolarian Cherts and Midocean Ridge Basalt-Like Lavas

Source: GEOLOGY 1991, Vol 19, Iss 8, pp 811-814

Addresses:

UNIV-HAWAII, SCH OCEAN \& EARTH SCI TECHNOL, 2525 CORREA RD, HONOLULU, HI 96822, USA

UNIV-CALIF-BERKELEY, DEPT GEOL \& GEOPHYS, BERKELEY, CA 94720, USA

US-GEOL-SURVEY, PALEONTOL \& STRATIG BRANCH, MENLO-PK, CA 94025, USA

OKAYAMA-UNIV-SCI, HIRUZEN RES INST, OKAYAMA 700, JAPAN

UNIV-TOKYO, OCEAN RES INST, NAKANI KU, TOKYO 164, JAPAN

32. Authors: Durrheim-RJ Mooney-WD

Title: Archean and Proterozoic Crustal Evolution - Evidence from Crustal Seismology 


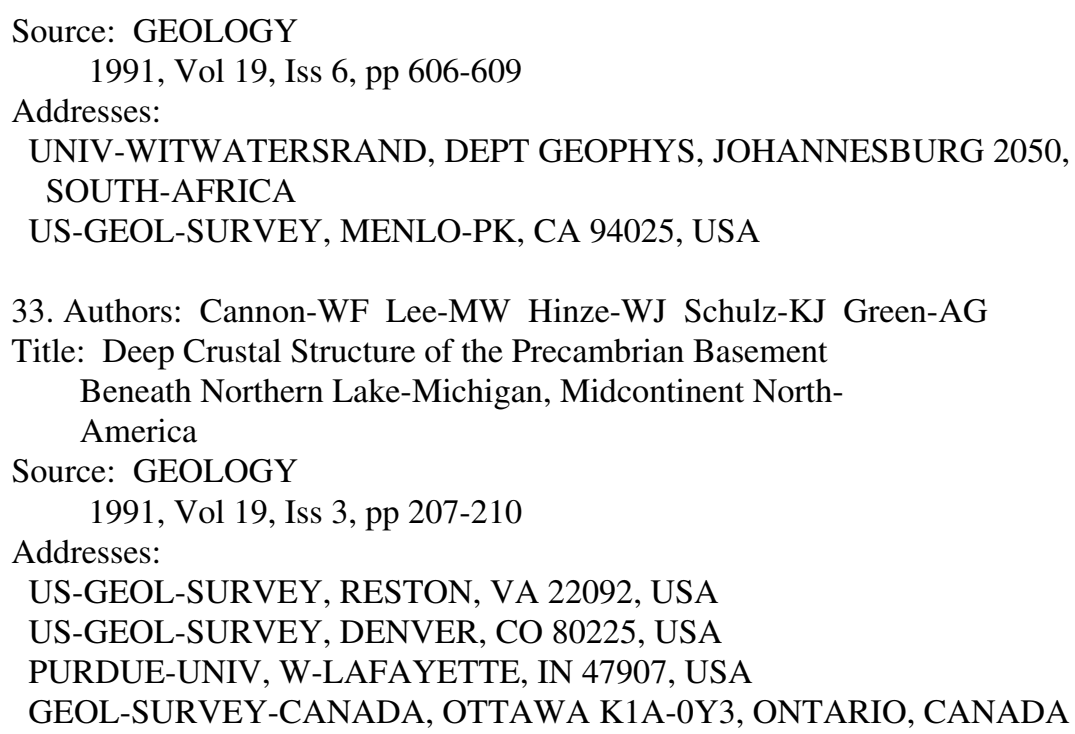

34. Authors: Vanderhilst-RD Engdahl-ER

Title: On Isc pp and pp Data and Their Use in Delay-Time

Tomography of the Caribbean Region

Source: GEOPHYSICAL JOURNAL INTERNATIONAL 1991, Vol 106, Iss 1, pp 169-188

Addresses:

UNIV-UTRECHT, DEPT THEORET GEOPHYS, 3508-TA UTRECHT, NETHERLANDS

US-GEOL-SURVEY, DENVER FED CTR, NATL EARTHQUAKE INFORMAT CTR, DENVER, CO 80225, USA

35. Authors: Kennett-BLN Engdahl-ER

Title: Traveltimes for Global Earthquake Location and Phase Identification

Source: GEOPHYSICAL JOURNAL INTERNATIONAL 1991, Vol 105, Iss 2, pp 429-465

Addresses:

AUSTRALIAN-NATL-UNIV, RES SCH EARTH SCI, GPO BOX 4, CANBERRA, ACT 2601, AUSTRALIA

US-GEOL-SURVEY, DENVER FED CTR, NATL EARTHQUAKE INFORMAT CTR, DENVER, CO 80225, USA

36. Authors: Dibona-M Boatwright-J

Title: Single-Station Decomposition of Seismograms for Subevent Time Histories

Source: GEOPHYSICAL JOURNAL INTERNATIONAL 1991, Vol 105, Iss 1, pp 103-117

Addresses:

INST-NAZL-GEOFIS, I-00161 ROME, ITALY

US-GEOL-SURVEY, MENLO-PK, CA 94025, USA

37. Authors: Ward-PL Pitt-AM Endo-E

Title: Seismic Evidence for Magma in the Vicinity of Mt Katmai, Alaska Source: GEOPHYSICAL RESEARCH LETTERS 1991, Vol 18, Iss 8, pp 1537-1540 
Addresses:

US-GEOL-SURVEY, MAIL STOP 977, 345 MIDDLEFIELD RD, MENLO-PK,

CA 94025, USA

US-GEOL-SURVEY, JEDDAH 21431, SAUDI-ARABIA

38. Authors: Lockner-D Hickman-S Kuksenko-V Ponomarev-A

Sidorin-A Byerlee-J Khakaev-B

Title: Laboratory-Determined Permeability of Cores from the

Kola Superdeep Well, USSR

Source: GEOPHYSICAL RESEARCH LETTERS

1991, Vol 18, Iss 5, pp 881-884

Addresses:

US-GEOL-SURVEY, 345 MIDDLEFIELD RD, MS 977, MENLO-PK, CA

94025, USA

MINIST-GEOL-USSR, OBEDINENIE NEDRA, YAROSLAVL 150003, USSR

USSR-ACAD-SCI, AF IOFFE PHYS TECH INST, LENINGRAD 194021, USSR

USSR-ACAD-SCI, INST PHYS EARTH, MOSCOW 123810, USSR

39. Authors: Trehu-A Morelalhuissier-P Meyer-R Hajnal-Z Karl-J

Mereu-R Sexton-J Shay-J Chan-WK Epili-D

Jefferson-T Shih-XR Wendling-S Milkereit-B Green-A

Hutchinson-D

Title: Imaging the Midcontinent Rift Beneath Lake-Superior

Using Large Aperture Seismic Data

Source: GEOPHYSICAL RESEARCH LETTERS

1991, Vol 18, Iss 4, pp 625-628

Addresses:

OREGON-STATE-UNIV, OCEAN ADM BLDG 104, CORVALLIS, OR 97331, USA

UNIV-WISCONSIN, DEPT GEOPHYS, MADISON, WI 53705, USA

UNIV-SASKATCHEWAN, DEPT GEOL SCI, SASKATOON S7N-0W0, SASKATCHEWAN, CANADA

UNIV-WISCONSIN, DEPT PHYS, OSHKOSH, WI 54901, USA

UNIV-WESTERN-ONTARIO, DEPT GEOPHYS, LONDON N6A-5B7, ONTARIO, CANADA

SO-ILLINOIS-UNIV, DEPT GEOL, CARBONDALE, IL 62901, USA

US-GEOL-SURVEY, WOODS-HOLE, MA 02543, USA

GEOL-SURVEY-CANADA, OTTAWA K1A-0Y3, ONTARIO, CANADA

40. Authors: Hatcher-PG Lerch-HE Verheyen-TV

Title: Organic Geochemical Studies of the Transformation of Gymnospermous Xylem During Peatification and

Coalification to Subbituminous Coal

Source: INTERNATIONAL JOURNAL OF COAL GEOLOGY

1990, Vol 16, Iss 1-3, pp 193-196

Addresses:

US-GEOL-SURVEY, RESTON, VA 22092, USA

COAL-CORP-VICTORIA, CHURCHILL 3842, VIC, AUSTRALIA

41. Authors: Hatcher-PG Wilson-MA Vassalo-M Lerch-HE

Title: Studies of Angiospermous Woods in Australian Brown Coal by Nuclear-Magnetic-Resonance and Analytical Pyrolysis -

New Insight into Early Coalification

Source: INTERNATIONAL JOURNAL OF COAL GEOLOGY

1990, Vol 16, Iss 1-3, pp 205-207 
Addresses:

US-GEOL-SURVEY, RESTON, VA 22092, USA

CSIRO, DIV COAL TECHNOL, N-RYDE, NSW 2113, AUSTRALIA

42. Authors: Gibson-TG Barbin-V Poignant-A Sztrakos-K

Title: Early Evolution of Tubulogenerina During the Paleogene of Europe

Source: JOURNAL OF FORAMINIFERAL RESEARCH 1991, Vol 21, Iss 4, pp 299-312

Addresses:

US-GEOL-SURVEY, 970 NATL CTR, RESTON, VA 22092, USA

UNIV-PARIS-06, MICROPALEONTOL LAB, F-75252 PARIS, FRANCE

UNIV-BERN, INST GEOL, CH-3012 BERN, SWITZERLAND

NATL-HIST-MUSEUM-GENEVA, CH-1211 GENEVA 6, SWITZERLAND

UNIV-PARIS-06, URA 1315, F-75252 PARIS, FRANCE

43. Authors: Perrin-M Prevot-M Mankinen-EA

Title: Low Intensity of the Geomagnetic-Field in Early Jurassic Time

Source: JOURNAL OF GEOPHYSICAL RESEARCH-SOLID EARTH AND PLANETS 1991, Vol 96, Iss NB9, pp 4197-4210

Addresses:

CNRS, CTR GEOL \& GEOPHYS, F-34095 MONTPELLIER 5, FRANCE

US-GEOL-SURVEY, ISOTOPE GEOL BRANCH, MENLO-PK, CA 94025, USA

UNIV-MONTPELLIER-2, F-34095 MONTPELLIER 5, FRANCE

44. Authors: Bowman-JR Dewey-JW

Title: Relocation of Teleseismically Recorded Earthquakes Near

Tennant Creek, Australia - Implications for Midplate

Seismogenesis

Source: JOURNAL OF GEOPHYSICAL RESEARCH-SOLID EARTH AND PLANETS 1991, Vol 96, Iss NB7, pp 1973-1979

Addresses:

AUSTRALIAN-NATL-UNIV, RES SCH EARTH SCI, GPO BOX 4, CANBERRA, ACT 2601, AUSTRALIA

US-GEOL-SURVEY, DENVER FED CTR, NATL EARTHQUAKE INFORMAT CTR, DENVER, CO 80225, USA

45. Authors: Gardner-JV Field-ME Lee-H Edwards-BE Masson-DG Kenyon-N Kidd-RB

Title: Ground-Truthing 6.5-KHz Side Scan Sonographs - What Are We Really Imaging

Source: JOURNAL OF GEOPHYSICAL RESEARCH-SOLID EARTH AND PLANETS 1991, Vol 96, Iss NB4, pp 5955-5974

Addresses:

US-GEOL-SURVEY, 345 MIDDLEFIELD RD, MS999, MENLO-PK, CA 94025, USA

UNIV-WALES-COLL-CARDIFF, DEPT GEOL, CARDIFF, WALES

INST-OCEANOG-SCI, WORMLEY, SURREY, ENGLAND

46. Authors: Crampin-S Booth-DC Evans-R Peacock-S Fletcher-JB

Title: Comment on Quantitative Measurements of Shear-Wave

Polarizations at the Anza Seismic Network, Southern

California - Implications for Shear-Wave Splitting and

Earthquake Prediction by Aster,Richard,C.,

Shearer,Peter,M., and Berger,John

Source: JOURNAL OF GEOPHYSICAL RESEARCH-SOLID EARTH AND PLANETS

1991, Vol 96, Iss NB4, pp 6403-6414 
Addresses:

BRITISH-GEOL-SURVEY, MURCHISON HOUSE, W MAINS RD, EDINBURGH

EH9-3LA, SCOTLAND

US-GEOL-SURVEY, MENLO-PK, CA 94025, USA

UNIV-READING, POSTGRAD RES INST SEDIMENTOL, READING RG6-2AB, BERKS, ENGLAND

47. Authors: Cordell-L Zorin-YA Keller-GR

Title: The Decompensative Gravity-Anomaly and Deep-Structure of the Region of the Rio-Grande Rift

Source: JOURNAL OF GEOPHYSICAL RESEARCH-SOLID EARTH AND PLANETS 1991, Vol 96, Iss NB4, pp 6557-6568

Addresses:

US-GEOL-SURVEY, DENVER FED CTR, POB 25046, DENVER, CO 80225, USA

UNIV-TEXAS, DEPT GEOL SCI, EL-PASO, TX 79968, USA

ACAD-SCI-USSR, INST EARTHS CRUST, IRKUTSK 664033, USSR

48. Authors: Collot-JY Fisher-MA

Title: The Collision Zone Between the North Dentrecasteaux

Ridge and the New Hebrides Island-Arc .1. Sea Beam

Morphology and Shallow Structure

Source: JOURNAL OF GEOPHYSICAL RESEARCH-SOLID EARTH AND PLANETS 1991, Vol 96, Iss NB3, pp 4457-4478

Addresses:

ORSTOM, GEODYNAM LAB, BP 48, F-06230 VILLEFRANCHE-MER, FRANCE

US-GEOL-SURVEY, MENLO-PK, CA 94025, USA

49. Authors: Fisher-MA Collot-JY Geist-EL

Title: The Collision Zone Between the North Dentrecasteaux

Ridge and the New Hebrides Island-Arc .2. Structure from

Multichannel Seismic Data

Source: JOURNAL OF GEOPHYSICAL RESEARCH-SOLID EARTH AND PLANETS 1991, Vol 96, Iss NB3, pp 4479-4495

Addresses:

US-GEOL-SURVEY, MS 999, 345 MIDDLEFIELD RD, MENLO-PK, CA

94025, USA

ORSTOM, GEODYNAM LAB, F-06230 VILLEFRANCHE-MER, FRANCE

50. Authors: Dvorak-JJ Berrino-G

Title: Recent Ground Movement and Seismic Activity in Campi

Flegrei, Southern Italy - Episodic Growth of a Resurgent

Dome

Source: JOURNAL OF GEOPHYSICAL RESEARCH-SOLID EARTH AND PLANETS 1991, Vol 96, Iss NB2, pp 2309-2323

Addresses:

US-GEOL-SURVEY, HAWAIIAN VOLCANO OBSERV, HAWAII-NATL-PK, HI, USA

OSSERV-VESUVIANO, NAPLES, ITALY

51. Authors: Theobald-PK Eppinger-RG Turner-RL Shen-SQ

Title: The Effect of Scale on the Interpretation of Geochemical Anomalies

Source: JOURNAL OF GEOCHEMICAL EXPLORATION

1991, Vol 40, Iss 1-3, pp 9-23 
Addresses:

US-GEOL-SURVEY, DENVER FED CTR, BOX 25046, MS 973, DENVER, CO 80225, USA

UNIV-NEVADA, MACKAY SCH MINES, US GEOL SURVEY, RENO, NV 89557, USA

MINIST-GEOL-\&-MINERAL-RESOURCES, BEIJING, PEOPLES-R-CHINA

52. Authors: Devivo-B Ayuso-RA Belkin-HE Lima-A Messina-A Viscardi-A

Title: Rock Chemistry and Fluid Inclusion Studies as Exploration Tools for Ore-Deposits in the Sila Batholith, Southern Italy

Source: JOURNAL OF GEOCHEMICAL EXPLORATION 1991, Vol 40, Iss 1-3, pp 291-310

Addresses:

DIPARTIMENTO-GEOFIS-\&-VULCANOL, LARGO S MARCELLINO 10, I-80138 NAPLES, ITALY

US-GEOL-SURVEY, RESTON, VA 22092, USA

INST-SCI-TERRA, I-98166 MESSINA, ITALY

53. Authors: Scott-KM Rabone-G Chaffee-MA

Title: Weathering and Its Effect upon Geochemical Dispersion at the Polymetallic Wagga Tank Deposit, NSW, Australia

Source: JOURNAL OF GEOCHEMICAL EXPLORATION 1991, Vol 40, Iss 1-3, pp 413-426

Addresses:

CSIRO, DIV EXPLORAT GEOSCI, POB 136, N-RYDE, NSW 2113, AUSTRALIA

HOMESTAKE-AUSTRALIA-LTD, NORWOOD, SA 5067, AUSTRALIA

US-GEOL-SURVEY, DENVER FED CTR, DENVER, CO 80225, USA

54. Authors: Trincardi-F Field-ME

Title: Geometry, Lateral Variation, and Preservation of Downlapping Regressive Shelf Deposits - Eastern Tyrrhenian Sea Margin, Italy

Source: JOURNAL OF SEDIMENTARY PETROLOGY 1991, Vol 61, Iss 5, pp 775-790

Addresses:

IST-GEOL-MARINA, VIA ZAMBONI 65, I-40127 BOLOGNA, ITALY

US-GEOL-SURVEY, MENLO-PK, CA 94025, USA

55. Authors: Polgari-M Okita-PM Hein-JR

Title: Stable Isotope Evidence for the Origin of the Urkut Manganese Ore Deposit, Hungary

Source: JOURNAL OF SEDIMENTARY PETROLOGY 1991, Vol 61, Iss 3, pp 384-393

Addresses:

HUNGARIAN-ACAD-SCI, GEOCHEM RES LAB, BUDAORSI UT 45, H-1112

BUDAPEST, HUNGARY

US-GEOL-SURVEY, RESTON, VA 22092, USA

US-GEOL-SURVEY, MENLO-PK, CA 94025, USA 
56. Authors: Dvorak-JJ Gasparini-P

Title: History of Earthquakes and Vertical Ground Movement in

Campi Flegrei Caldera, Southern Italy - Comparison of

Precursory Events to the AD.1538 Eruption of Monte Nuovo

and of Activity Since 1968

Source: JOURNAL OF VOLCANOLOGY AND GEOTHERMAL RESEARCH

1991, Vol 48, Iss 1-2, pp 77-92

Addresses:

US-GEOL-SURVEY, HAWAIIAN VOLCANO OBSERV, HAWAII-NATL-PK, HI 96718, USA

NAPLES-UNIV, DIPARTIMENTO GEOFIS \& VULCANOL, I-80138 NAPLES, ITALY

57. Authors: Heiken-G Ramos-N Duffield-W Musgrave-J Wohletz-K

Priest-S Aldrich-J Flores-W Ritchie-A Goff-F

Eppler-D Escobar-C

Title: Geology of the Platanares Geothermal Area, Departamentode-Copan, Honduras

Source: JOURNAL OF VOLCANOLOGY AND GEOTHERMAL RESEARCH 1991, Vol 45, Iss 1-2, pp 41-58

Addresses:

LOS-ALAMOS-NATL-LAB, MS 964, LOS-ALAMOS, NM 87544, USA

EMPRESA-NACL-ENERGIA-ELECT, TEGUCIGALPA, HONDURAS

COLL-CHARLESTON, CHARLESTON, SC 29424, USA

US-GEOL-SURVEY, FLAGSTAFF, AZ 86001, USA

58. Authors: Goff-F Goff-SJ Kelkar-S Shevenell-L Truesdell-AH

Musgrave-J Rufenacht-H Flores-W

Title: Exploration Drilling and Reservoir Model of the

Platanares Geothermal System, Honduras, Central-America

Source: JOURNAL OF VOLCANOLOGY AND GEOTHERMAL RESEARCH 1991, Vol 45, Iss 1-2, pp 101-123

Addresses:

LOS-ALAMOS-NATL-LAB, DIV EARTH \& SPACE SCI, LOS-ALAMOS, NM

87545, USA

SWISSBORING-LTD, GUATEMALA-CITY, GUATEMALA

EMPRESA-NACL-ENERGIA-ELECT, UNIDAD PROYECTO GEOTERM, TEGUCIGALPA, HONDURAS

US-GEOL-SURVEY, IGNEOUS \& GEOTHERMAL PROC BRANCH, MENLO-PK, CA 94025, USA

59. Authors: Feder-GL Radovanovic-Z Finkelman-RB

Title: Relationship Between Weathered Coal Deposits and the Etiology of Balkan Endemic Nephropathy

Source: KIDNEY INTERNATIONAL 1991, Vol 40, Iss S34, pp S9-S11

Addresses:

US-GEOL-SURVEY, DIV WATER RESOURCES, RESTON, VA 22092, USA

US-GEOL-SURVEY, DIV GEOL, RESTON, VA 22092, USA

UNIV-BELGRADE, SCH MED, INST EPIDEMIOL, BELGRADE, YUGOSLAVIA

60. Authors: Morris-SC Savoy-LE Harris-AG

Title: An Enigmatic Organism from the Exshaw Formation

(Devonian-Carboniferous), Alberta, Canada

Source: LETHAIA

1991, Vol 24, Iss 2, pp 139-152 
Addresses:

UNIV-CAMBRIDGE, DEPT EARTH SCI, CAMBRIDGE CB2-3EQ, ENGLAND

US-GEOL-SURVEY, RESTON, VA 22092, USA

MT-HOLYOKE-COLL, DEPT GEOG \& GEOL, S-HADLEY, MA 01075, USA

61. Authors: Oliver-WA Chlupac-I

Title: Defining the Devonian - 1979-89

Source: LETHAIA 1991, Vol 24, Iss 1, pp 119-122

Addresses:

US-GEOL-SURVEY, SMITHSONIAN INST, E 305 NAT HIST, WASHINGTON, DC 20560, USA

USTREDNI-USTAV-GEOL, CS-11821 PRAGUE 1, CZECHOSLOVAKIA

62. Authors: Drew-LJ Qingrun-M Weijun-S

Title: The Bayan Obo Iron Rare-Earth Niobium Deposits, InnerMongolia, China

Source: LITHOS 1990, Vol 26, Iss 1-2, pp 43-65

Addresses:

US-GEOL-SURVEY, RESTON, VA 22092, USA

TIANJIN-GEOL-RES-ACAD, TIANJIN, PEOPLES-R-CHINA

63. Authors: Cooper-JAG Flores-RM

Title: Shoreline Deposits and Diagenesis Resulting from 2 Late Pleistocene Highstands Near +5 and +6 Meters, Durban, South-Africa

Source: MARINE GEOLOGY

1991, Vol 97, Iss 3-4, pp 325-343

Addresses:

CSIR, DIV WATER TECHNOL, ESTUARINE \& FRESHWATER POLLUT

PROGRAMME, POB 17001, CONGELLA 4013, SOUTH-AFRICA

US-GEOL-SURVEY, DENVER FED CTR, COAL GEOL BRANCH, DENVER, CO 80225, USA

64. Authors: Schwab-WC Danforth-WW Scanlon-KM Masson-DG

Title: A Giant Submarine Slope Failure on the Northern Insular Slope of Puerto-Rico

Source: MARINE GEOLOGY 1991, Vol 96, Iss 3-4, pp 237-246

Addresses:

US-GEOL-SURVEY, QUISSETT CAMPUS, WOODS-HOLE, MA 02543, USA

INST-OCEANOG-SCI, WORMLEY GU8-5UB, SURREY, ENGLAND

65. Authors: Maldonado-A Nelson-CH

Title: The Ebro Margin Study, Northwestern Mediterranean-Sea An Introduction

Source: MARINE GEOLOGY 1990, Vol 95, Iss 3-4, pp 157-163

Addresses:

CSIC, INST CIENCIAS MAR, PASEO NACL S-N, E-08039 BARCELONA, SPAIN

US-GEOL-SURVEY, MENLO-PK, CA 94025, USA 
66. Authors: Cacchione-DA Drake-DE Losada-MA Medina-R

Title: Bottom-Boundary-Layer Measurements on the Continental-

Shelf Off the Ebro River, Spain

Source: MARINE GEOLOGY 1990, Vol 95, Iss 3-4, pp 179-192

Addresses:

US-GEOL-SURVEY, MS-999, 345 MIDDLEFIELD RD, MENLO-PK, CA

94025, USA

UNIV-CANTABRIA, ESCUELA TECH SUPER INGN CAMINOS, E-39005

SANTANDER, SPAIN

67. Authors: Palanques-A Drake-DE

Title: Distribution and Dispersal of Suspended Particulate

Matter on the Ebro Continental-Shelf, Northwestern

Mediterranean-Sea

Source: MARINE GEOLOGY

1990, Vol 95, Iss 3-4, pp 193-206

Addresses:

INST-CIENCIAS-MAR, PASEO NACL S-N, E-08039 BARCELONA, SPAIN

US-GEOL-SURVEY, MENLO-PK, CA 94025, USA

68. Authors: Gardner-JV Dean-WE Alonso-B

Title: Inorganic Geochemistry of Surface Sediments of the Ebro

Shelf and Slope, Northwestern Mediterranean

Source: MARINE GEOLOGY

1990, Vol 95, Iss 3-4, pp 225-245

Addresses:

US-GEOL-SURVEY, MENLO-PK, CA 94025, USA

US-GEOL-SURVEY, DENVER, CO 80225, USA

CSIC, INST CIENCIAS MAR, E-08003 BARCELONA, SPAIN

69. Authors: Alonso-B Field-ME Gardner-JV Maldonado-A

Title: Sedimentary Evolution of the Pliocene and Pleistocene

Ebro Margin, Northeastern Spain

Source: MARINE GEOLOGY

1990, Vol 95, Iss 3-4, pp 313-331

Addresses:

CSIC, INST CIENCIAS MAR, PASEO NACL S-N, E-08039 BARCELONA, SPAIN

US-GEOL-SURVEY, MENLO-PK, CA 94025, USA

70. Authors: Diaz-JI Nelson-CH Barber-JH Giro-S

Title: Late Pleistocene and Holocene Sedimentary Facies on the Ebro Continental-Shelf

Source: MARINE GEOLOGY 1990, Vol 95, Iss 3-4, pp 333-352

Addresses:

CSIC, INST CIENCIAS MAR, PASEO NACL S-N, E-08039 BARCELONA, SPAIN

US-GEOL-SURVEY, MENLO-PK, CA 94025, USA

71. Authors: Baraza-J Lee-HJ Kayen-RE Hampton-MA

Title: Geotechnical Characteristics and Slope Stability on the Ebro Margin, Western Mediterranean

Source: MARINE GEOLOGY

1990, Vol 95, Iss 3-4, pp 379-393 
Addresses:

CSIC, INST CIENCIAS MAR, PAVEO NACL S-N, E-08039 BARCELONA, SPAIN

US-GEOL-SURVEY, MENLO-PK, CA 94025, USA

72. Authors: Nelson-CH Maldonado-A

Title: Factors Controlling Late Cenozoic Continental-Margin

Growth from the Ebro Delta to the Western Mediterranean

Deep-Sea

Source: MARINE GEOLOGY

1990, Vol 95, Iss 3-4, pp 419-440

Addresses:

US-GEOL-SURVEY, 345 MIDDLEFIELD RD, MENLO-PK, CA 94025, USA

INST-CIENCIAS-MAR, E-08039 BARCELONA, SPAIN

73. Authors: Hein-JR Schulz-MS Kang-JK

Title: Insular and Submarine Ferromanganese Mineralization of the Tonga-Lau Region

Source: MARINE MINING 1990, Vol 9, Iss 3, pp 305-354

Addresses:

US-GEOL-SURVEY, MS-999, MENLO-PK, CA 94025, USA

KOREA-OCEAN-RES-\&-DEV-INST, SEOUL 425600, SOUTH-KOREA

74. Authors: Brouwers-EM Jorgensen-NO Cronin-TM

Title: Climatic Significance of the Ostracode Fauna from the

Pliocene Kap Kobenhavn Formation, North Greenland

Source: MICROPALEONTOLOGY

1991, Vol 37, Iss 3, pp 245-267

Addresses:

US-GEOL-SURVEY, DENVER FED CTR, DENVER, CO 80225, USA

UNIV-COPENHAGEN, INST HIST GEOL \& PALAEONTOL, DK-1350

COPENHAGEN, DENMARK

US-GEOL-SURVEY, NATL CTR, RESTON, VA 22092, USA

75. Authors: Cunningham-CG Arribas-A Rytuba-JJ Arribas-A

Title: Mineralized and Unmineralized Calderas in Spain .1.

Evolution of the Los Frailes Caldera

Source: MINERALIUM DEPOSITA 1990, Vol 25, Iss S, pp S21-S28

Addresses:

US-GEOL-SURVEY, 959 NATL CTR, RESTON, VA 22092, USA

UNIV-MICHIGAN, DEPT GEOL SCI, ANN-ARBOR, MI 48109, USA

US-GEOL-SURVEY, MENLO-PK, CA 94025, USA

COMMISS-EUROPEAN-COMMUNITIES, B-1049 BRUSSELS, BELGIUM

76. Authors: Rytuba-JJ Arribas-A Cunningham-CG Mckee-EH

Podwysocki-MH Smith-JG Kelly-WC Arribas-A

Title: Mineralized and Unmineralized Calderas in Spain .2.

Evolution of the Rodalquilar Caldera Complex and

Associated Gold-Alunite Deposits

Source: MINERALIUM DEPOSITA 1990, Vol 25, Iss S, pp S29-S35

Addresses:

US-GEOL-SURVEY, 345 MIDDLEFIELD RD, MENLO-PK, CA 94025, USA

UNIV-MICHIGAN, DEPT GEOL SCI, ANN-ARBOR, MI 48109, USA 
US-GEOL-SURVEY, RESTON, VA 22092, USA

COMMISS-EUROPEAN-COMMUNITIES, B-1049 BRUSSELS, BELGIUM

77. Authors: Pamic-J Lanphere-MA

Title: Alpine A-Type Granites from the Collisional Area of the

Northernmost Dinarides and Pannonian Basin, Yugoslavia

Source: NEUES JAHRBUCH FUR MINERALOGIE-ABHANDLUNGEN 1991, Vol 162, Iss 2, pp 215-236

Addresses:

INST-GEOL, SACHSOVA 2, YU-41000 ZAGREB, YUGOSLAVIA

US-GEOL-SURVEY, MENLO-PK, CA 94025, USA

78. Authors: Foord-EE Hlava-PF Fitzpatrick-JJ Erd-RC Hinton-RW

Title: Maxwellite and Squawcreekite 2 New Mineral from the Black Range Tin District, Catron County, New-Mexico, USA

Source: NEUES JAHRBUCH FUR MINERALOGIE-MONATSHEFTE 1991, Iss 8, pp 363-384

Addresses:

US-GEOL-SURVEY, DENVER FED CTR, MS 905, BOX 25046, DENVER, CO 80225, USA

UNIV-EDINBURGH, DEPT GEOL \& GEOPHYS, EDINBURGH GH9-3JW, SCOTLAND

US-GEOL-SURVEY, MENLO-PK, CA 94025, USA

SANDIA-NATL-LABS, DIV 1822, ALBUQUERQUE, NM 87185, USA

79. Authors: Nagy-B Gauthierlafaye-F Holliger-P Davis-DW

Mossman-DJ Leventhal-JS Rigali-MJ Parnell-J

Title: Organic-Matter and Containment of Uranium and

Fissiogenic Isotopes at the Oklo Natural Reactors

Source: NATURE

1991, Vol 354, Iss 6353, pp 472-475

Addresses:

UNIV-ARIZONA, DEPT GEOSCI, ORGAN GEOCHEM LAB, TUCSON, AZ 85721, USA

CNRS, CTR GEOCHIM SURFACE, F-67084 STRASBOURG, FRANCE

US-GEOL-SURVEY, DENVER FED CTR, DENVER, CO 80225, USA

QUEENS-UNIV-BELFAST, DEPT GEOL, BELFAST BT7-1NN, ANTRIM, NORTH-IRELAND

CEN, F-38041 GRENOBLE, FRANCE

ROYAL-ONTARIO-MUSEUM, DEPT GEOL, TORONTO M5S-2C6, ONTARIO, CANADA

MT-ALLISON-UNIV, DEPT GEOL, SACKVILLE E0A-3C0, NB, CANADA

80. Authors: Prodehl-C Mechie-J Kaminski-W Fuchs-K Grosse-C

Hoffmann-H Stangl-R Stellrecht-R Khan-MA

Maguire-PKH Kirk-W Keller-GR Githui-A Baker-M

Mooney-W Criley-E Luetgert-J Jacob-B Thybo-H

Demartin-M Scarascia-S Hirn-A Bowman-JR Nyambok-I

Gaciri-S Patel-J Dindi-E Griffiths-DH King-RF

Mussett-AE Braile-LW Thompson-G Olsen-K Harder-S

Vees-R Gajewski-D Schulte-A Obel-J Mwango-F

Mukinya-J Riaroh-D

Title: Large-Scale Variation in Lithospheric Structure Along

and Across the Kenya Rift

Source: NATURE

1991, Vol 354, Iss 6350, pp 223-227 


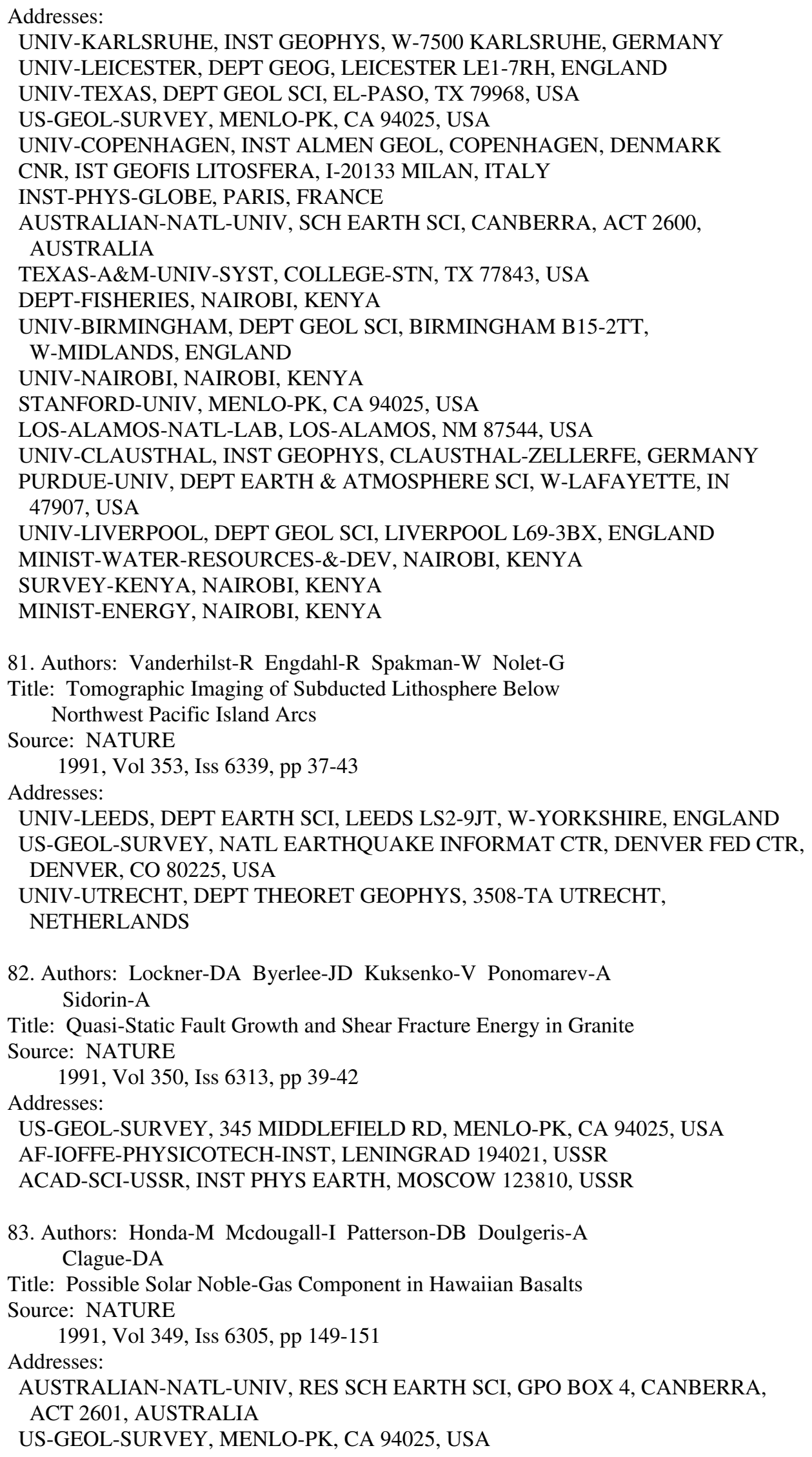

81. Authors: Vanderhilst-R Engdahl-R Spakman-W Nolet-G

Title: Tomographic Imaging of Subducted Lithosphere Below

Northwest Pacific Island Arcs

Source: NATURE

1991, Vol 353, Iss 6339, pp 37-43

Addresses:

UNIV-LEEDS, DEPT EARTH SCI, LEEDS LS2-9JT, W-YORKSHIRE, ENGLAND

US-GEOL-SURVEY, NATL EARTHQUAKE INFORMAT CTR, DENVER FED CTR, DENVER, CO 80225, USA

UNIV-UTRECHT, DEPT THEORET GEOPHYS, 3508-TA UTRECHT, NETHERLANDS

82. Authors: Lockner-DA Byerlee-JD Kuksenko-V Ponomarev-A Sidorin-A

Title: Quasi-Static Fault Growth and Shear Fracture Energy in Granite

Source: NATURE 1991, Vol 350, Iss 6313, pp 39-42

Addresses:

US-GEOL-SURVEY, 345 MIDDLEFIELD RD, MENLO-PK, CA 94025, USA

AF-IOFFE-PHYSICOTECH-INST, LENINGRAD 194021, USSR

ACAD-SCI-USSR, INST PHYS EARTH, MOSCOW 123810, USSR

83. Authors: Honda-M Mcdougall-I Patterson-DB Doulgeris-A Clague-DA

Title: Possible Solar Noble-Gas Component in Hawaiian Basalts

Source: NATURE 1991, Vol 349, Iss 6305, pp 149-151

Addresses:

AUSTRALIAN-NATL-UNIV, RES SCH EARTH SCI, GPO BOX 4, CANBERRA, ACT 2601, AUSTRALIA

US-GEOL-SURVEY, MENLO-PK, CA 94025, USA 
84. Authors: Vourvopoulos-G Brahana-JV Nolte-E Korschinek-G Priller-A Dockhorn-B

Title: Cl-36 Measurements and the Hydrology of an Acid Injection Site

Source: NUCLEAR INSTRUMENTS \& METHODS IN PHYSICS RESEARCH SECTION B-BEAM INTERACTIONS WITH MATERIALS AND ATOMS 1990, Vol 52, Iss 3-4, pp 451-454

Addresses:

WESTERN-KENTUCKY-UNIV, DEPT PHYS \& ASTRON, BOWLING-GREEN, KY 42101, USA

US-GEOL-SURVEY, NASHVILLE, TN 37203, USA

UNIV-MUNICH, FAC PHYS, W-8046 GARCHING, GERMANY

85. Authors: Hatcher-PG Wilson-MA

Title: The Effect of Sample Hydration on C-13 CPMAS NMR-Spectra of Fulvic-Acids

Source: ORGANIC GEOCHEMISTRY 1991, Vol 17, Iss 3, pp 293-299

Addresses:

US-GEOL-SURVEY, RESTON, VA 22092, USA

CSIRO, DIV COAL TECHNOL, N-RYDE, NSW 2113, AUSTRALIA

86. Authors: Bates-AL Hatcher-PG Lerch-HE Cecil-CB Neuzil-SG Supardi

Title: Studies of a Peatified Angiosperm Log Cross-Section from Indonesia by Nuclear-Magnetic-Resonance Spectroscopy and Analytical Pyrolysis

Source: ORGANIC GEOCHEMISTRY 1991, Vol 17, Iss 1, pp 37-45

Addresses:

US-GEOL-SURVEY, NATL CTR, RESTON, VA 22092, USA

DIRECTORATE-MINERAL-RESOURCES, BANDUNG, INDONESIA

87. Authors: Miller-GH Wendorf-F Ernst-R Schild-R Close-AE Friedman-I Schwarcz-HP

Title: Dating Lacustrine Episodes in the Eastern Sahara by the Epimerization of Isoleucine in Ostrich Eggshells

Source: PALAEOGEOGRAPHY PALAEOCLIMATOLOGY PALAEOECOLOGY 1991, Vol 84, Iss 1-4, pp 175-189

Addresses:

UNIV-COLORADO, INSTAAR, CTR GEOCHRONOL RES, BOULDER, CO 80309, USA

UNIV-COLORADO, DEPT GEOL SCI, BOULDER, CO 80309, USA

SO-METHODIST-UNIV, DEPT ANTHROPOL, DALLAS, TX 75275, USA

US-GEOL-SURVEY, ISOTOPE GEOL BRANCH, DENVER, CO 80225, USA

MCMASTER-UNIV, DEPT GEOL, HAMILTON L8S-4M1, ONTARIO, CANADA

88. Authors: Zoback-ML Magee-M

Title: Stress Magnitudes in the Crust - Constraints from Stress Orientation and Relative Magnitude Data

Source: PHILOSOPHICAL TRANSACTIONS OF THE ROYAL SOCIETY OF LONDON SERIES A-PHYSICAL SCIENCES AND ENGINEERING 1991, Vol 337, Iss 1645, pp 181-194

Addresses:

UNIV-KARLSRUHE, INST GEOPHYS, HERTZSTR 16, W-7500 KARLSRUHE 21, GERMANY 
US-GEOL-SURVEY, MENLO-PK, CA 94025, USA

STANFORD-UNIV, DEPT GEOPHYS, STANFORD, CA 94305, USA

89. Authors: Ehlers-M Greenlee-D Smith-T Star-J

Title: Integration of Remote-Sensing and Gis - Data and Data Access

Source: PHOTOGRAMMETRIC ENGINEERING AND REMOTE SENSING 1991, Vol 57, Iss 6, pp 669-675

Addresses:

INT-INST-AEROSP-SURVEY-\&-EARTH-SCI, ITC, DEPT GEOINFORMAT, ENSCHEDE, NETHERLANDS

US-GEOL-SURVEY, EROS DATA CTR, SIOUX-FALLS, SD 57198, USA

UNIV-CALIF-SANTA-BARBARA, NATL CTR GEOG INFORMAT \& ANAL, SANTA-BARBARA, CA 93106, USA

90. Authors: Gladenkov-YB Barinov-KB Basilian-AE Cronin-TM

Title: Stratigraphy and Paleoceanography of Pliocene Deposits of Karaginsky Island, Eastern Kamchatka, USSR

Source: QUATERNARY SCIENCE REVIEWS 1991, Vol 10, Iss 2-3, pp 239-245

Addresses:

ACAD-SCI-USSR, INST GEOL, MOSCOW V-71, USSR

US-GEOL-SURVEY, RESTON, VA 22092, USA

91. Authors: Edwards-LE Mudie-PJ Devernal-A

Title: Pliocene Paleoclimatic Reconstruction Using

Dinoflagellate Cysts - Comparison of Methods

Source: QUATERNARY SCIENCE REVIEWS

1991, Vol 10, Iss 2-3, pp 259-274

Addresses:

US-GEOL-SURVEY, 970 NATL CTR, RESTON, VA 22092, USA

GEOL-SURVEY-CANADA, ATLANTIC GEOSCI CTR, DARTMOUTH B2Y-4A2, NS, CANADA

UNIV-QUEBEC, GEOTOP, GEOCHIM ISOTOP \& GEOCHRONOL LAB, MONTREAL H3C-3P8, QUEBEC, CANADA

92. Authors: Kendrick-GW Wyrwoll-KH Szabo-BJ

Title: Pliocene-Pleistocene Coastal Events and History Along the Western Margin of Australia

Source: QUATERNARY SCIENCE REVIEWS 1991, Vol 10, Iss 5, pp 419-439

Addresses:

WESTERN-AUSTRALIAN-MUSEUM, DEPT EARTH \& PLANETARY SCI C, FRANCIS ST, PERTH, WA 6000, AUSTRALIA

UNIV-WESTERN-AUSTRALIA, DEPT GEOG, NEDLANDS, WA 6009, AUSTRALIA

US-GEOL-SURVEY, DENVER FED CTR, LAKEWOOD, CO 80225, USA

93. Authors: Aitchison-TC Leese-M Michczynska-DJ Mook-WG

Otlet-RL Ottaway-BS Pazdur-MF Vanderplicht-J

Reimer-PJ Robinson-SW Scott-EM Stuiver-M Weninger-B

Title: A Comparison of Methods Used for the Calibration of Radiocarbon-Dates

Source: RADIOCARBON 1989, Vol 31, Iss 3, pp 846-864

Addresses:

UNIV-GLASGOW, DEPT STAT, GLASGOW G12-8QW, SCOTLAND

BRITISH-MUSEUM, RES LAB, LONDON WC1B-3DG, ENGLAND 
SILESIA-TECH-UNIV, INST PHYS, RADIOCARBON LAB, PL-44100

GLIWICE, POLAND

CENT-V-ISOTOPEN-ONDERZOEK, 9718-CM GRONINGEN, NETHERLANDS

UNIV-BRADFORD, DEPT ARCHAEOL SCI, BRADFORD BD7-1DP,

W-YORKSHIRE, ENGLAND

UNIV-WASHINGTON, DEPT GEOL SCI, SEATTLE, WA 98195, USA

UNIV-WASHINGTON, QUATERNARY RES CTR, SEATTLE, WA 98195, USA

US-GEOL-SURVEY, MENLO-PK, CA 94025, USA

UNIV-FRANKFURT, INST UR \& FRUHEGESCH, W-6000 FRANKFURT, GERMANY

94. Authors: Tarduno-JA Sliter-WV Kroenke-L Leckie-M Mayer-H Mahoney-JJ Musgrave-R Storey-M Winterer-EL

Title: Rapid Formation of Ontong Java Plateau by Aptian Mantle Plume Volcanism

Source: SCIENCE

1991, Vol 254, Iss 5030, pp 399-403

Addresses:

SCRIPPS-CLIN-\&-RES-FDN, LA-JOLLA, CA 92037, USA

US-GEOL-SURVEY, MENLO-PK, CA 94025, USA

UNIV-HAWAII, DEPT GEOL \& GEOPHYS, HONOLULU, HI 96822, USA

UNIV-MASSACHUSETTS, DEPT GEOL \& GEOG, AMHERST, MA 01003, USA

UNIV-TASMANIA, DEPT GEOL, HOBART, TAS 7001, AUSTRALIA

UNIV-LEICESTER, DEPT GEOL, LEICESTER LE1-7RH, ENGLAND

95. Authors: Belton-MJS Gierasch-PJ Smith-MD Helfenstein-P

Schinder-PJ Pollack-JB Rages-KA Ingersoll-AP

Klaasen-KP Veverka-J Anger-CD Carr-MH Chapman-CR

Davies-ME Fanale-FP Greeley-R Greenberg-R Head-JW

Morrison-D Neukum-G Pilcher-CB

Title: Images from Galileo of the Venus Cloud Deck

Source: SCIENCE 1991, Vol 253, Iss 5027, pp 1531-1536

Addresses:

NATL-OPT-ASTRON-OBSERV, TUCSON, AZ 85719, USA

CORNELL-UNIV, ITHACA, NY 14853, USA

NASA, AMES RES CTR, MOFFETT-FIELD, CA 94035, USA

CALTECH, PASADENA, CA 91125, USA

CALTECH, JET PROP LAB, PASADENA, CA 91109, USA

ITRES-RES-LTD, CALGARY T2E-7H7, ALBERTA, CANADA

US-GEOL-SURVEY, MENLO-PK, CA 94025, USA

SCI-APPLICAT-INT-CORP, INST CHAPMAN PLANETARY SCI, TUCSON, AZ

85719, USA

NASA-HEADQUARTERS, WASHINGTON, DC 20546, USA

INST-GEOPHYS, HONOLULU, HI 96822, USA

RAND-CORP, SANTA-MONICA, CA 90406, USA

BROWN-UNIV, PROVIDENCE, RI 02912, USA

DFVLR, W-8031 OBERPFAFFENHOFEN, GERMANY

ARIZONA-STATE-UNIV, TEMPE, AZ 85281, USA

UNIV-ARIZONA, TUCSON, AZ 85721, USA

96. Authors: Carlson-RW Baines-KH Encrenaz-T Taylor-FW

Drossart-P Kamp-LW Pollack-JB Lellouch-E

Collard-AD Calcutt-SB Grinspoon-D Weissman-PR

Smythe-WD Ocampo-AC Danielson-GE Fanale-FP

Johnson-TV Kieffer-HH Matson-DL Mccord-TB

Soderblom-LA 
Title: Galileo Infrared Imaging Spectroscopy Measurements at Venus

Source: SCIENCE

1991, Vol 253, Iss 5027, pp 1541-1548

Addresses:

CALTECH, JET PROP LAB, PASADENA, CA 91109, USA

OBSERV-PARIS, F-92195 MEUDON, FRANCE

UNIV-OXFORD, OXFORD OX1-3PU, ENGLAND

NASA, AMES RES CTR, MOFFETT-FIELD, CA 94035, USA

CALTECH, DIV GEOL \& PLANETARY SCI, PASADENA, CA 91109, USA

UNIV-HAWAII, HONOLULU, HI 96822, USA

US-GEOL-SURVEY, FLAGSTAFF, AZ 86001, USA

97. Authors: Pettengill-GH Ford-PG Johnson-WTK Raney-RK

Soderblom-LA

Title: Magellan - Radar Performance and Data Products

Source: SCIENCE 1991, Vol 252, Iss 5003, pp 260-265

Addresses:

MIT, CAMBRIDGE, MA 02139, USA

CANADA-CTR-REMOTE-SENSING, OTTAWA K1A-0Y7, ONTARIO, CANADA

CALTECH, JET PROP LAB, PASADENA, CA 91109, USA

US-GEOL-SURVEY, FLAGSTAFF, AZ 86001, USA

98. Authors: Head-JW Campbell-DB Elachi-C Guest-JE Mckenzie-DP

Saunders-RS Schaber-GG Schubert-G

Title: Venus Volcanism - Initial Analysis from Magellan Data

Source: SCIENCE 1991, Vol 252, Iss 5003, pp 276-288

Addresses:

BROWN-UNIV, DEPT GEOL SCI, PROVIDENCE, RI 02912, USA

UNIV-COLL-LONDON, UNIV LONDON OBSERV, LONDON NW7-2QS, ENGLAND

UNIV-CAMBRIDGE, DEPT EARTH SCI, CAMBRIDGE CB3-0EZ, ENGLAND

CORNELL-UNIV, DEPT ASTRON, ITHACA, NY 14853, USA

CALTECH, JET PROP LAB, PASADENA, CA 91109, USA

US-GEOL-SURVEY, FLAGSTAFF, AZ 86001, USA

UNIV-CALIF-LOS-ANGELES, INST GEOPHYS \& PLANETARY PHYS, DEPT

EARTH \& SPACE SCI, LOS-ANGELES, CA 90024, USA

99. Authors: Phillips-RJ Arvidson-RE Boyce-JM Campbell-DB

Guest-JE Schaber-GG Soderblom-LA

Title: Impact Craters on Venus - Initial Analysis from Magellan

Source: SCIENCE

1991, Vol 252, Iss 5003, pp 288-297

Addresses:

SO-METHODIST-UNIV, DEPT GEOL SCI, DALLAS, TX 75275, USA

UNIV-LONDON-OBSERV, LONDON NW7-2QS, ENGLAND

WASHINGTON-UNIV, MCDONNELL CTR SPACE SCI, DEPT EARTH \&

PLANETARY SCI, ST-LOUIS, MO 63130, USA

NASA, DIV SOLAR SYST EXPLORAT, WASHINGTON, DC 20546, USA

CORNELL-UNIV, DEPT ASTRON, ITHACA, NY 14853, USA

US-GEOL-SURVEY, FLAGSTAFF, AZ 86001, USA

100. Authors: Pirc-S Mcneal-JM Lenarcic-T Prohic-E Svrkota-R

Title: Geochemical Mapping of Carbonate Terrains 
Source: TRANSACTIONS OF THE INSTITUTION OF MINING AND METALLURGY SECTION B-APPLIED EARTH SCIENCE 1991, Vol 100, Iss MAY-, pp B74-B87

Addresses:

EDVARD-KARDELJ-UNIV, GEOCHEM MINEAL \& ECOM GEOL, YU-61000 LJUBLJANA, YUGOSLAVIA US-GEOL-SURVEY, ENVIRONM CHEM SECT, RESTON, VA 22092, USA

ADELPHI-UNIV, GARDEN-CITY, NY 11530, USA

101. Authors: Clark-SHB Gallagher-MJ Poole-FG

Title: World Barite Resources - A Review of Recent Production Patterns and a Genetic Classification

Source: TRANSACTIONS OF THE INSTITUTION OF MINING AND METALLURGY SECTION B-APPLIED EARTH SCIENCE 1990, Vol 99, Iss SEP-, pp B125-B132

Addresses:

US-GEOL-SURVEY, BRANCH EASTERN MINERAL RESOURCES, RESTON, VA 22092, USA

BRITISH-GEOL-SURVEY, LONDON, ENGLAND

US-GEOL-SURVEY, BRANCH CENT MINERAL RESOURCES, DENVER, CO 80225, USA

102. Authors: Behrendt-JC Lemasurier-WE Cooper-AK Tessensohn-F Trehu-A Damaske-D

Title: Geophysical Studies of the West Antarctic Rift System

Source: TECTONICS 1991, Vol 10, Iss 6, pp 1257-1273

Addresses:

US-GEOL-SURVEY, MS 964, BOX 25046 FED CTR, DENVER, CO 80225, USA

US-GEOL-SURVEY, MENLO-PK, CA 94035, USA

FED-INST-GEOSCI-\&-NAT-RESOURCES, W-3000 HANNOVER 51, GERMANY

UNIV-COLORADO, DEPT GEOL, DENVER, CO 80204, USA

OREGON-STATE-UNIV, COLL OCEANOG, CORVALLIS, OR 97331, USA

103. Authors: Fisher-MA Collot-JY Geist-EL

Title: Structure of the Collision Zone Between Bougainville

Guyot and the Accretionary Wedge of the New-Hebrides

Island-Arc, Southwest Pacific

Source: TECTONICS

1991, Vol 10, Iss 5, pp 887-903

Addresses:

US-GEOL-SURVEY, MS 999, 345 MIDDLEFIELD RD, MENLO-PK, CA 94025, USA

ORSTOM, GEODYNAM LAB, F-06230 VILLEFRANCHE, FRANCE

104. Authors: Bouchard-M Pavich-MJ

Title: Characteristics and Significance of Pre-Wisconsinan Saprolites in the Northern Appalachians - Discussion Reply

Source: ZEITSCHRIFT FUR GEOMORPHOLOGIE 1991, Vol 35, Iss 3, pp 373-377

Addresses:

UNIV-QUEBEC, DEPT GEOG, CP 8888, SUCC A, MONTREAL H3C-3P8, QUEBEC, CANADA

US-GEOL-SURVEY, RESTON, VA 22092, USA 
105. Authors: Lyons-PC Cohen Callcott-T Adolphi-J Teichmuller-M

Title: Peat and Coal - Origin, Facies, and Coalification - Open

Discussion

Source: INTERNATIONAL JOURNAL OF COAL GEOLOGY 1990, Vol 16, Iss 1-3, pp 137-138

Addresses:

US-GEOL-SURVEY, RESTON, VA 22092, USA

UNIV-GREIFSWALD, O-2200 GREIFSWALD, GERMANY

106. Authors: Woolley-A Ross-M

Title: Alkaline Igneous Rocks and Carbonatites - Introduction

Source: LITHOS 1990, Vol 26, Iss 1-2, pp 1-1

Addresses:

NATL-HIST-MUSEUM, DEPT MINERAL, CROMWELL RD, LONDON SW7-5BD, ENGLAND

US-GEOL-SURVEY, RESTON, VA 22092, USA

107. Authors: Nelson-CH Maldonado-A

Title: The Ebro Continental-Margin, Northwestern MediterraneanSea - Preface

Source: MARINE GEOLOGY 1990, Vol 95, Iss 3-4, pp R3-R3

Addresses:

US-GEOL-SURVEY, 345 MIDDLEFIELD RD, MAIL STOP 999, MENLO-PK, CA 94025, USA

CSIC, INST CIENCIAS MAR, E-08039 BARCELONA, SPAIN

108.Authors: Honda-M Mcdougall-I Patterson-DB Doulgeris-A

Title: Terrestrial Primordial Neon - Reply

Source: NATURE 1991, Vol 352, Iss 6334, pp 388-388

Addresses:

AUSTRALIAN-NATL-UNIV, RES SCH EARTH SCI, GPO BOX 4, CANBERRA, ACT 2601, AUSTRALIA

US-GEOL-SURVEY, MENLO-PK, CA 94025, USA

109. Authors: Cook-HE Gatosvseey-YA Ponoeearenko-SB

Styehtsyehnka-IV Styehtsyennka-VP Zhemchuzhnikov-V

Zoran-AE

Title: Carbonate Platform Evolution, Upper Paleozoic, Southern Kazakhstan, USSR - A Surface Analog for the Super Giant Tengiz Oil-Field, Western Kazakhstan

Source: AAPG BULLETIN-AMERICAN ASSOCIATION OF PETROLEUM GEOLOGISTS 1991, Vol 75, Iss 8, pp 1408-1408

Addresses:

US-GEOL-SURVEY, MENLO-PK, CA 94025, USA

ACAD-SCI-KASSR, INST GEOL SCI, ALMA-ATA 480021, KAZAKHSTAN, USSR

MINIST-GEOL-ALMA-ATA, ALMA-ATA, KAZAKHSTAN, USSR

110. Authors: Masson-DG Huggett-QJ Weaver-PPE Kidd-RB Gardner-JV

Title: Quaternary Sedimentary Processes on the Northwestern African Continental-Margin - An Integrated Study Using Side-Scan Sonar, High-Resolution Profiling, and Core Data 
Source: AAPG BULLETIN-AMERICAN ASSOCIATION OF PETROLEUM GEOLOGISTS 1991, Vol 75, Iss 8, pp 1416-1416

Addresses:

INST-OCEANOG-SCI, WORMLEY, SURREY, ENGLAND

UNIV-WALES-COLL-CARDIFF, CARDIFF CF1-1XL, S-GLAM, WALES

US-GEOL-SURVEY, MENLO-PK, CA 94025, USA

111. Authors: Clayton-JL Konca-I

Title: Application of Organic Geochemistry to PetroleumExploration in Bekes Basin, Hungary

Source: AAPG BULLETIN-AMERICAN ASSOCIATION OF PETROLEUM GEOLOGISTS 1991, Vol 75, Iss 3, pp 554-554

Addresses:

US-GEOL-SURVEY, DENVER, CO 80225, USA

HUNGARIAN-HYDROCARBON-IND-SCI-IND-ASSOC, SZAZHALOMBATTA, HUNGARY

112. Authors: Cook-HE Taylor-ME Zhemchuzhnikov-SV Apollonov-MK Ergaliev-GK Sargaskaev-ZS

Title: Comparative Development of the Western United-States and Southern Kazakhstan, Soviet-Union - 2 Early Paleozoic

Carbonate Passive Margins

Source: AAPG BULLETIN-AMERICAN ASSOCIATION OF PETROLEUM GEOLOGISTS 1991, Vol 75, Iss 2, pp 360-360

Addresses:

US-GEOL-SURVEY, MENLO-PK, CA 94025, USA

US-GEOL-SURVEY, DENVER, CO 80225, USA

ACAD-SCI-KASSR, INST GEOL SCI, ALMA-ATA 480021, KAZAKHSTAN, USSR

ACAD-SCI-USSR, INST GEOL, MOSCOW V-71, USSR

113. Authors: Ross-RJ James-NP Hintze-LF Ketner-KB

Title: Early Middle Ordovician (Whiterockian) Paleogeography of Basin Ranges

Source: AAPG BULLETIN-AMERICAN ASSOCIATION OF PETROLEUM GEOLOGISTS 1991, Vol 75, Iss 2, pp 378-378

Addresses:

COLORADO-SCH-MINES, GOLDEN, CO 80401, USA

QUEENS-UNIV, KINGSTON K7L-3N6, ONTARIO, CANADA

BRIGHAM-YOUNG-UNIV, PROVO, UT 84602, USA

US-GEOL-SURVEY, DENVER, CO 80225, USA

114. Authors: Taylor-ME Cook-HE Melnikova-L

Title: Biofacies Evidence for Late Cambrian Low-Paleolatitude

Oceans, Western United-States and Central-Asia

Source: AAPG BULLETIN-AMERICAN ASSOCIATION OF PETROLEUM GEOLOGISTS 1991, Vol 75, Iss 2, pp 383-383

Addresses:

US-GEOL-SURVEY, DENVER, CO 80225, USA

US-GEOL-SURVEY, MENLO-PK, CA 94025, USA

ACAD-SCI-USSR, INST PALEONTOL, MOSCOW V-71, USSR

115. Authors: Krabbenhoft-DP Bowser-CJ Gat-JR Kendall-C

Title: Stable Isotope Techniques in Lake Hydrology

Source: ABSTRACTS OF PAPERS OF THE AMERICAN CHEMICAL SOCIETY

1991, Vol 201, Iss APR, pp 51- 
Addresses:

US-FOREST-SERV, MADISON, WI 53705, USA

UNIV-WISCONSIN, DEPT GEOL \& GEOPHYS, MADISON, WI 53706, USA

WEIZMANN-INST-SCI, IL-76100 REHOVOT, ISRAEL

US-GEOL-SURVEY, MENLO-PK, CA 94025, USA

116. Authors: Davis-JA Kent-DB Anderson-LCD Waite-TD

Title: Chemical Processes Affecting the Transport of $\mathrm{Cr}(\mathrm{VI})$ in Groundwater

Source: ABSTRACTS OF PAPERS OF THE AMERICAN CHEMICAL SOCIETY 1991, Vol 201, Iss APR, pp 80-

Addresses:

AUSTRALIAN-NUCL-SCI-\&-TECHNOL-ORGANISAT, SUTHERLAND, NSW, AUSTRALIA

US-GEOL-SURVEY, MENLO-PK, CA 94025, USA

117. Authors: Hildreth-W Moorbath-S

Title: Crustal Contributions to Arc Magmatism in the Andes of Central Chile - Comment

Source: CONTRIBUTIONS TO MINERALOGY AND PETROLOGY 1991, Vol 108, Iss 1-2, pp 247-252

Addresses:

US-GEOL-SURVEY, MENLO-PK, CA 94025, USA

UNIV-OXFORD, DEPT EARTH SCI, OXFORD OX1-3PR, ENGLAND

118. Authors: Cunningham-CG Mcnamee-J Vasquez-JP Ericksen-GE

Title: A Model of Volcanic Dome-Hosted Precious Metal Deposits in Bolivia

Source: ECONOMIC GEOLOGY AND THE BULLETIN OF THE SOCIETY OF ECONOMIC GEOLOGISTS 1991, Vol 86, Iss 2, pp 415-421

Addresses:

US-GEOL-SURVEY, 959 NATL CTR, RESTON, VA 22092, USA

COMPANIA-MINERA-SUR-SA, EDIFICIO PETR, LA-PAZ, BOLIVIA

UN, DEPT TECH COOPERAT DEV, SANTA-CRUZ, BOLIVIA

119. Authors: Mckeown-FA Hamilton-RM Diehl-SF Glick-EE

Title: Diapiric Origin of the Blytheville and Pascola Arches in the Reelfoot Rift, East-Central United-States - Relation to New Madrid Seismicity - Reply

Source: GEOLOGY 1991, Vol 19, Iss 6, pp 668-669

Addresses:

US-GEOL-SURVEY, DENVER, CO 80225, USA

UN, GENEVA, SWITZERLAND

120. Authors: Sandberg-CA Gutschick-RC Petersen-MS Poole-FG Ziegler-W

Title: Delle Phosphatic Member - An Anomalous Phosphatic Interval in the Mississippian (Osagean-Meramecian) Shelf Sequence of Central Utah - Comment

Source: GEOLOGY 1991, Vol 19, Iss 4, pp 410-411 
Addresses:

US-GEOL-SURVEY, DENVER FED CTR, BOX 25046, DENVER, CO 80225,

USA

US-GEOL-SURVEY, MEDFORD, OR 97504, USA

BRIGHAM-YOUNG-UNIV, DEPT GEOL, PROVO, UT 84602, USA

FORSCH-INST-SENCHENBERG, W-6000 FRANKFURT 1, GERMANY

121. Authors: Genise-JF Bown-TM

Title: A Reassessment of the Ichnofossil ChubutolithesGaimanensis Bown and Ratcliffe - Reply

Source: JOURNAL OF PALEONTOLOGY 1991, Vol 65, Iss 4, pp 705-706

Addresses:

MUSEO-ARGENTINO-CIENCIAS-NAT-BERNARDINO-RIVADAVIA, DEPT

ENTOMOL, BUENOS-AIRES, ARGENTINA

US-GEOL-SURVEY, PALEONTOL \& STRATIG BRANCH, DENVER, CO 80225 , USA

122. Authors: Lirer-L Rolandi-G Rubin-M

Title: C-14 Age of the Museum Breccia (Campi Flegrei) and Its Relevance for the Origin of the Campanian Ignimbrite

Source: JOURNAL OF VOLCANOLOGY AND GEOTHERMAL RESEARCH 1991, Vol 48, Iss 1-2, pp 223-227

Addresses:

DIPARTIMENTO-GEOFIS-\&-VULCANOL, LARGO S MARCELLINO 10, I-80138

NAPLES, ITALY

US-GEOL-SURVEY, 971 NATL CTR, RESTON, VA 22092, USA

123. Authors: Houghton-BF Lloyd-EF Wilson-CJN Lanphere-MA

Title: K-Ar Ages from the Western Dome Belt and Associated Rhyolitic Lavas in the Maroa Taupo Area, Taupo Volcanic Zone, New-Zealand

Source: NEW ZEALAND JOURNAL OF GEOLOGY AND GEOPHYSICS 1991, Vol 34, Iss 1, pp 99-101

Addresses:

DSIR, POB 499, WELLINGTON, NEW-ZEALAND

UNIV-CAMBRIDGE, DEPT GEOL, CAMBRIDGE, ENGLAND

US-GEOL-SURVEY, MENLO-PK, CA 94025, USA

124. Authors: Vonhuene-R Scholl-DW

Title: Observations at Convergent Margins Concerning Sediment Subduction, Subduction Erosion, and the Growth of Continental-Crust

Source: REVIEWS OF GEOPHYSICS 1991, Vol 29, Iss 3, pp 279-316

Addresses:

GEOMAR, W-2300 KIEL 14, GERMANY

US-GEOL-SURVEY, MENLO-PK, CA 94025, USA

125. Authors: Ayuso-RA Bevier-ML

Title: Regional Differences in $\mathrm{Pb}$ Isotopic Compositions of Feldspars in Plutonic Rocks of the Northern Appalachian Mountains, USA and Canada - A Geochemical Method of Terrane Correlation

Source: TECTONICS

1991, Vol 10, Iss 1, pp 191- 
Addresses:

US-GEOL-SURVEY, NATL CTR 954, RESTON, VA 22092, USA

GEOL-SURVEY-CANADA, OTTAWA K18-0E8, ONTARIO, CANADA

126. Authors: Collot-JY Greene-HG Stokking-L Akimoto-K Ask-MVS

Baker-PE Briqueu-L Chabernaud-T Coltorti-M

Fisher-MA Goud-M Hasenaka-T Hobart-M Krammer-A

Leonard-J Martin-JB Martinezrodrigez-JJ Menger-S

Meschede-M Pelletier-B Perembo-RCB Quinn-TM

Roperch-P Reid-P Riedel-WR Staerker-TS Taylor-FW

Zhao-X

Title: Preliminary-Results of ODP Leg 134 in the Collision Zone

Between the New-Hebrides Island-Arc and the

Dentrecasteaux Zone

Source: COMPTES RENDUS DE L ACADEMIE DES SCIENCES SERIE IIMECANIQUE PHYSIQUE CHIMIE SCIENCES DE L UNIVERS

SCIENCES DE LA TERRE

1991, Vol 313, Iss 5, pp 539-546

Addresses:

ORSTOM, BP 48, F-06230 VILLEFRANCHE-MER, FRANCE

US-GEOL-SURVEY, MENLO-PK, CA 94025, USA

ODP, COLLEGE-STN, TX 77845, USA

127. Authors: Green-AU Worthington-EU Pilipenko-VA Herzog-GB

Kurneva-NA

Title: Effect of Magnetospheric Alfven Resonance on the

Spectrum of Packets of Pc3-4 Pulsations at Mid Latitudes

Source: GEOMAGNETIZM I AERONOMIYA

1991, Vol 31, Iss 4, pp 619-624

Addresses:

ACAD-SCI-USSR, INST EARTH PHYS, MOSCOW V-71, USSR

US-GEOL-SURVEY, DENVER, CO 80225, USA

128. Authors: Zorin-YA Cordell-L

Title: Crust Stretching at the Baikal Rift-Zone from Gravity-Data

Source: IZVESTIYA AKADEMII NAUK SSSR FIZIKA ZEMLI 1991, Iss 5, pp 3-11

Addresses:

ACAD-SCI-USSR, INST EARTH CRUST, IRKUTSK 664003, USSR

US-GEOL-SURVEY, DENVER FED CTR, DENVER, CO 80225, USA

\section{2}

1. Authors: Pasternak-MP Taylor-RD Jeanloz-R Bohlen-SR

Title: Magnetic-Ordering Transition in Mg0.9Fe0.1Sio3 Ortho-Pyroxene

Source: AMERICAN MINERALOGIST

1992, Vol 77, Iss 9-10, pp 901-903

Addresses:

LOS-ALAMOS-NATL-LAB, DIV PHYS, LOS-ALAMOS, NM 87545, USA

UNIV-CALIF-BERKELEY, DEPT GEOL \& GEOPHYS, BERKELEY, CA 94720, USA

US-GEOL-SURVEY, MENLO-PK, CA 94025, USA

TEL-AVIV-UNIV, SCH PHYS \& ASTRON, IL-69978 TEL-AVIV, ISRAEL 
2. Authors: Foord-EE Martin-RF Fitzpatrick-JJ Taggart-JE

Crock-JG

Title: Boromuscovite, a New Member of the Mica Group, from the Little 3 Mine Pegmatite, Ramona District, San-Diego

County, California

Source: AMERICAN MINERALOGIST

1991, Vol 76, Iss 11-12, pp 1998-2002

Addresses:

US-GEOL-SURVEY, DENVER FED CTR, MS 905, BOX 25046, DENVER, CO 80225, USA

MCGILL-UNIV, DEPT GEOL SCI, MONTREAL H3A-2A7, QUEBEC, CANADA

3. Authors: Taylor-HE Garbarino-JR Murphy-DM Beckett-R

Title: Inductively Coupled Plasma Mass-Spectrometry as an Element-Specific Detector for Field-Flow Fractionation

Particle Separation

Source: ANALYTICAL CHEMISTRY

1992, Vol 64, Iss 18, pp 2036-2041

Addresses:

US-GEOL-SURVEY, DENVER FED CTR, DIV WATER RESOURCES, BOX 25046, MS 458, DENVER, CO 80225, USA

MONASH-UNIV, DEPT CHEM, MELBOURNE, VIC 3004, AUSTRALIA

MONASH-UNIV, CTR WATER RESOURCES, MELBOURNE, VIC 3004, AUSTRALIA

4. Authors: Foreman-MGG Baptista-AM Walters-RA

Title: Tidal Model Studies of Particle Trajectories Around a Shallow Coastal Bank

Source: ATMOSPHERE-OCEAN

1992, Vol 30, Iss 1, pp 43-69

Addresses:

INST-OCEAN-SCI, SIDNEY V8L-4B2, BC, CANADA

OREGON-GRAD-INST-SCI-\&-TECHNOL, BEAVERTON, OR, USA

US-GEOL-SURVEY, TACOMA, WA, USA

5. Authors: Zientek-ML Pardiarto-B Simandjuntak-HRW Wikrama-A Oscarson-RL Meier-AL Carlson-RR

Title: Placer and Lode Platinum-Group Minerals in South Kalimantan, Indonesia - Evidence for Derivation from Alaskan-Type Ultramafic Intrusions

Source: AUSTRALIAN JOURNAL OF EARTH SCIENCES 1992, Vol 39, Iss 3, pp 405-417

Addresses:

US-GEOL-SURVEY, W 920 RIVERSIDE AVE, SPOKANE, WA 99201, USA DIRECTORATE-MINERAL-RESOURCES, BANDUNG 40122, INDONESIA

PT-ANEKA-TAMBANG, BOGOR, INDONESIA

US-GEOL-SURVEY, MENLO-PK, CA 94025, USA

US-GEOL-SURVEY, DENVER, CO 80225, USA

6. Authors: Raven-KG Novakowski-KS Yager-RM Heystee-RJ

Title: Supernormal Fluid Pressures in Sedimentary-Rocks of Southern Ontario - Western New-York-State

Source: CANADIAN GEOTECHNICAL JOURNAL 1992, Vol 29, Iss 1, pp 80-93 
Addresses:

RAVEN-BECK-ENVIRONM-LTD, 265 CARLING AVE, SUITE 208, OTTAWA

K1S-2E1, ONTARIO, CANADA

ENVIRONM-CANADA, NATL WATER RES INST, BURLINGTON L7R-4A6,

ONTARIO, CANADA

US-GEOL-SURVEY, ITHACA, NY 14850, USA

ONTARIO-HYDRO, TORONTO M5G-1X6, ONTARIO, CANADA

7. Authors: Zelt-BC Ellis-RM Clowes-RM Kanasewich-ER Asudeh-I

Luetgert-JH Hajnal-Z Ikami-A Spence-GD Hyndman-RD

Title: Crust and Upper Mantle Velocity Structure of the

Intermontane Belt, Southern Canadian Cordillera

Source: CANADIAN JOURNAL OF EARTH SCIENCES 1992, Vol 29, Iss 7, pp 1530-1548

Addresses:

UNIV-BRITISH-COLUMBIA, DEPT GEOPHYS \& ASTRON, VANCOUVER

V6T-1Z4, BC, CANADA

UNIV-ALBERTA, DEPT PHYS, EDMONTON T6G-2J1, ALBERTA, CANADA

GEOL-SURVEY-CANADA, DIV CONTINENTAL GEOSCI, OTTAWA K1A-0Y3, ONTARIO, CANADA

US-GEOL-SURVEY, MENLO-PK, CA 94025, USA

UNIV-SASKATCHEWAN, DEPT GEOL SCI, SASKATOON S7N-0W0,

SASKATCHEWAN, CANADA

NAGOYA-UNIV, DEPT EARTH SCI, NAGOYA, AICHI 464, JAPAN

UNIV-VICTORIA, DEPT PHYS \& ASTRON, VICTORIA V8N-3P6, BC, CANADA

GEOL-SURVEY-CANADA, PACIFIC GEOSCI CTR, SIDNEY V8L-3X9, BC,

CANADA

8. Authors: Czamanske-GK Kunilov-VE Zientek-ML Cabri-LJ

Likhachev-AP Calk-LC Oscarson-RL

Title: A Proton-Microprobe Study of Magmatic Sulfide Ores from the Norilsk-Talnakh District, Siberia

Source: CANADIAN MINERALOGIST 1992, Vol 30, Iss JUN, pp 249-287

Addresses:

US-GEOL-SURVEY, 345 MIDDLEFIELD RD, MENLO-PK, CA 94025, USA

NORILSK-NICKEL-STATE-CONCERN, NORILSK 663300, RUSSIA

US-GEOL-SURVEY, SPOKANE, WA 99201, USA

CANADA-CTR-MINERAL-\&-ENERGY-TECHNOL, OTTAWA K1A-0G1, ONTARIO, CANADA

GEOL-PROSPECTING-BASE-\&-PRECIOUS-MET-CENT-RES-INST, MOSCOW

113545, RUSSIA

9. Authors: Kettler-RM Rye-RO Kessler-SE Meyers-PA Polanco-J Russell-N

Title: Gold Deposition by Sulfidation of Ferrous Fe in the

Lacustrine Sediments of the Pueblo Viejo District

(Dominican-Republic) - The Effect of Fe-C-S Diagenesis

on Later Hydrothermal Mineralization in a Maar Diatreme

Complex

Source: CHEMICAL GEOLOGY

1992, Vol 99, Iss 1-3, pp 29-50

Addresses:

UNIV-NEBRASKA, DEPT GEOG, LINCOLN, NE 68588, USA

ROSARIO-DOMINICANA-SA, SANTO-DOMINGO, DOMINICAN-REP 
US-GEOL-SURVEY, LAKEWOOD, CO 80225, USA

UNIV-MICHIGAN, DEPT GEOL SCI, ANN-ARBOR, MI 48109, USA

Source: CONTRIBUTIONS TO MINERALOGY AND PETROLOGY

10. Authors: Genin-A Paull-CK Dillon-WP

Title: Anomalous Abundances of Deep-Sea Fauna on a Rocky Bottom

Exposed to Strong Currents

Source: DEEP-SEA RESEARCH PART A-OCEANOGRAPHIC RESEARCH PAPERS 1992, Vol 39, Iss 2A, pp 293+

Addresses:

HEBREW-UNIV-JERUSALEM, H STEINITZ MARINE BIOL LAB, POB 469, ELAT, ISRAEL

UNIV-N-CAROLINA, DEPT GEOL, CHAPEL-HILL, NC 27599, USA

US-GEOL-SURVEY, WOODS-HOLE, MA 02543, USA

11. Authors: Tatsumoto-M Basu-AR Huang-WK Wang-JW Xie-GH

Title: $\mathrm{Sr}, \mathrm{Nd}$, and $\mathrm{Pb}$ Isotopes of Ultramafic Xenoliths in Volcanic-Rocks of Eastern China - Enriched Components EMI and Emii in Subcontinental Lithosphere

Source: EARTH AND PLANETARY SCIENCE LETTERS 1992, Vol 113, Iss 1-2, pp 107-128

Addresses:

US-GEOL-SURVEY, ISOTOPE SECT, BOX 25046, MS 963, DENVER, CO 80225, USA

UNIV-ROCHESTER, DEPT GEOL SCI, ROCHESTER, NY 14627, USA

ACAD-SINICA, INST GEOCHEM, GUIZHOU, PEOPLES-R-CHINA

12. Authors: Hemley-JJ Cygan-GL Fein-JB Robinson-GR Dangelo-WM

Title: Hydrothermal Ore-Forming Processes in the Light of Studies in Rock-Buffered Systems .1. Iron-Copper-Zinc-

Lead Sulfide Solubility Relations

Source: ECONOMIC GEOLOGY AND THE BULLETIN OF THE SOCIETY OF ECONOMIC GEOLOGISTS 1992, Vol 87, Iss 1, pp 1-22

Addresses:

US-GEOL-SURVEY, RESTON, VA 22092, USA

UNIV-EDINBURGH, GRANT INST GEOL, EDINBURGH EH9-8JW, SCOTLAND

13.Authors: Thorn-KA Arterburn-JB Mikita-MA

Title: N-15 and C-13 NMR Investigation of HydroxylamineDerivatized Humic Substances

Source: ENVIRONMENTAL SCIENCE \& TECHNOLOGY 1992, Vol 26, Iss 1, pp 107-116

Addresses:

US-GEOL-SURVEY, DENVER FED CTR, DIV WATER RESOURCES, MAIL STOP 408, BOX 25046, DENVER, CO 80225, USA

SWISS-FED-INST-TECHNOL, ORGAN CHEM LAB, CH-8092 ZURICH, SWITZERLAND

CALIF-STATE-UNIV-BAKERSFIELD, DEPT CHEM, BAKERSFIELD, CA 93311, USA

14. Authors: Devivo-B Ayuso-RA Belkin-HE Lima-A Messina-A

Russo-S Viscardi-A

Title: Whole-Rock Geochemistry and Fluid Inclusions as

Exploration Tools for Mineral-Deposits Assessment in the

Serre Batholith, Calabria, Southern Italy 
Source: EUROPEAN JOURNAL OF MINERALOGY

1992, Vol 4, Iss 5, pp 1035-1051

Addresses:

DIPARTIMENTO-GEOFIS-\&-VULCANOL, LARGO S MARCELLINO 10, I-80138

NAPLES, ITALY

IST-SCI-TERRA, I-98166 MESSINA, ITALY

US-GEOL-SURVEY, RESTON, VA 22092, USA

15. Authors: Vaggelli-G Belkin-HE Devivo-B Trigila-R

Title: Silicate-Melt Inclusions in Recent Vesuvius Lavas (AD 1631-1944) .1. Petrography and Microthermometry

Source: EUROPEAN JOURNAL OF MINERALOGY 1992, Vol 4, Iss 5, pp 1113-1124

Addresses:

DIPARTIMENTO-SCI-TERRA, VIA G LA PIRA 4, I-50121 FLORENCE,

ITALY

DIPARTIMENTO-GEOFIS-\&-VULCANOL, I-80138 NAPLES, ITALY

DIPARTIMENTO-SCI-TERRA, I-00185 ROME, ITALY

US-GEOL-SURVEY, RESTON, VA 22092, USA

16 Authors: Baraza-J Ercilla-G Lee-HJ

Title: Geotechnical Properties and Preliminary Assessment of

Sediment Stability on the Continental-Slope of the

Northwestern Alboran Sea

Source: GEO-MARINE LETTERS

1992, Vol 12, Iss 2-3, pp 150-156

Addresses:

CSIC, INST CIENCIAS MAR, PASEO NACL S-N, E-08039 BARCELONA,

SPAIN

US-GEOL-SURVEY, MENLO-PK, CA 94025, USA

17. Authors: Scanlon-KM Masson-DG

Title: Fe-Mn Nodule Field Indicated by Gloria, North of the Puerto-Rico Trench

Source: GEO-MARINE LETTERS

1992, Vol 12, Iss 4, pp 208-213

Addresses:

US-GEOL-SURVEY, WOODS-HOLE, MA 02543, USA

INST-OCEANOG-SCI, GODALMING GU8-5UB, SURREY, ENGLAND

18. Authors: Fein-JB Hemley-JJ Dangelo-WM Komninou-A

Sverjensky-DA

Title: Experimental-Study of Iron-Chloride Complexing in Hydrothermal Fluids

Source: GEOCHIMICA ET COSMOCHIMICA ACTA 1992, Vol 56, Iss 8, pp 3179-3190

Addresses:

UNIV-EDINBURGH, DEPT GEOL \& GEOPHYS, W MAINS RD, EDINBURGH

EH9-3JW, SCOTLAND

US-GEOL-SURVEY, RESTON, VA 22092, USA

JOHNS-HOPKINS-UNIV, DEPT EARTH \& PLANETARY SCI, BALTIMORE, MD 21218, USA 
19. Authors: Burruss-RC Ging-TG Eppinger-RG Samson-IM

Title: Laser-Excited Fluorescence of Rare-Earth Elements in

Fluorite - Initial Observations with a Laser Raman

Microprobe

Source: GEOCHIMICA ET COSMOCHIMICA ACTA 1992, Vol 56, Iss 7, pp 2713-2723

Addresses:

US-GEOL-SURVEY, BOX 25046, MS 973, DENVER, CO 80225, USA

UNIV-WINDSOR, DEPT GEOL, WINDSOR N9B-3P4, ONTARIO, CANADA

20. Authors: Peter-JM Shanks-WC

Title: Sulfur, Carbon, and Oxygen Isotope Variations in

Submarine Hydrothermal Deposits of Guaymas Basin, Gulf

of California, USA

Source: GEOCHIMICA ET COSMOCHIMICA ACTA

1992, Vol 56, Iss 5, pp 2025-2040

Addresses:

UNIV-TORONTO, DEPT GEOL, MARINE GEOL RES LAB, TORONTO M5S-3B1, ONTARIO, CANADA

US-GEOL-SURVEY, NATL CTR, RESTON, VA 22092, USA

21. Authors: Lyons-PC Outerbridge-WF Triplehorn-DM Evans-HT

Congdon-RD Capiro-M Hess-JC Nash-WP

Title: An Appalachian Isochron - A Kaolinized Carboniferous Air-

Fall Volcanic-Ash Deposit (Tonstein)

Source: GEOLOGICAL SOCIETY OF AMERICA BULLETIN 1992, Vol 104, Iss 11, pp 1515-1527

Addresses:

US-GEOL-SURVEY, MS 956, NATL CTR, RESTON, VA 22092, USA

UNIV-ALASKA, DEPT GEOL \& GEOPHYS, FAIRBANKS, AK 99701, USA

UNIV-HEIDELBERG, GEOCHRONOL LAB, W-6900 HEIDELBERG, GERMANY

UNIV-UTAH, DEPT GEOL \& GEOPHYS, SALT-LAKE-CITY, UT 84112, USA

22. Authors: Pogue-KR Wardlaw-BR Harris-AG Hussain-A

Title: Paleozoic and Mesozoic Stratigraphy of the Peshawar

Basin, Pakistan - Correlations and Implications

Source: GEOLOGICAL SOCIETY OF AMERICA BULLETIN 1992, Vol 104, Iss 8, pp 915-927

Addresses:

OREGON-STATE-UNIV, DEPT GEOSCI, CORVALLIS, OR 97331, USA

US-GEOL-SURVEY, RESTON, VA 22092, USA

GEOL-SURVEY-PAKISTAN, PESHAWAR, PAKISTAN

23. Authors: Coates-AG Jackson-JBC Collins-LS Cronin-TM

Dowsett-HJ Bybell-LM Jung-P Obando-JA

Title: Closure of the Isthmus of Panama - The Near-Shore Marine

Record of Costa-Rica and Western Panama

Source: GEOLOGICAL SOCIETY OF AMERICA BULLETIN

1992, Vol 104, Iss 7, pp 814-828

Addresses:

SMITHSONIAN-TROP-RES-INST, BOX 2072, BALBOA, PANAMA

GEORGE-WASHINGTON-UNIV, DEPT GEOL, WASHINGTON, DC 20052, USA

UNIV-MICHIGAN, MUSEUM PALEONTOL, ANN-ARBOR, MI 48109, USA

RECOPE-SA, SAN-JOSE, COSTA-RICA

US-GEOL-SURVEY, RESTON, VA 22092, USA

NAT-HIST-MUSEUM, CH-4051 BASEL, SWITZERLAND 
24. Authors: Barnes-CG Petersen-SW Kistler-RW Prestvik-T Sundvoll-B

Title: Tectonic Implications of Isotopic Variation Among Jurassic and Early Cretaceous Plutons, Klamath Mountains

Source: GEOLOGICAL SOCIETY OF AMERICA BULLETIN 1992, Vol 104, Iss 1, pp 117-126

Addresses:

TEXAS-TECH-UNIV, DEPT GEOSCI, LUBBOCK, TX 79409, USA

US-GEOL-SURVEY, MENLO-PK, CA 94025, USA

GEOL-INST, N-7034 TRONDHEIM, NORWAY

UNIV-OSLO, MINERAL GEOL MUSEUM, N-0562 OSLO 5, NORWAY

25. Authors: Milkereit-B Forsyth-DA Green-AG Davidson-A

Hanmer-S Hutchinson-DR Hinze-WJ Mereu-RF

Title: Seismic Images of a Grenvillian Terrane Boundary

Source: GEOLOGY 1992, Vol 20, Iss 11, pp 1027-1030

Addresses:

GEOL-SURVEY-CANADA, OTTAWA K1A-0Y3, ONTARIO, CANADA

US-GEOL-SURVEY, WOODS-HOLE, MA 02543, USA

PURDUE-UNIV, W-LAFAYETTE, IN 47906, USA

UNIV-WESTERN-ONTARIO, LONDON N6A-5B7, ONTARIO, CANADA

26. Authors: Kroner-A Pallister-JS Fleck-RJ

Title: Age of Initial Oceanic Magmatism in the Late Proterozoic Arabian Shield

Source: GEOLOGY 1992, Vol 20, Iss 9, pp 803-806

Addresses:

UNIV-MAINZ, INST GEOWISSENSCH, POSTFACH 3980, W-6500 MAINZ, GERMANY

US-GEOL-SURVEY, DENVER, CO 80225, USA

US-GEOL-SURVEY, MENLO-PK, CA 94025, USA

27. Authors: Twichell-DC Schwab-WC Nelson-CH Kenyon-NH Lee-HJ

Title: Characteristics of a Sandy Depositional Lobe on the Outer Mississippi Fan from Seamarc Ia Sidescan Sonar Images

Source: GEOLOGY 1992, Vol 20, Iss 8, pp 689-692

Addresses:

US-GEOL-SURVEY, WOODS-HOLE, MA 02543, USA

US-GEOL-SURVEY, MENLO-PK, CA 94025, USA

INST-OCEANOG-SCI, WORMLEY GU8-5UB, SURREY, ENGLAND

28. Authors: Nelson-CH Twichell-DC Schwab-WC Lee-HJ Kenyon-NH

Title: Upper Pleistocene Turbidite Sand Beds and Chaotic Silt

Beds in the Channelized, Distal, Outer-Fan Lobes of the Mississippi Fan

Source: GEOLOGY 1992, Vol 20, Iss 8, pp 693-696

Addresses:

US-GEOL-SURVEY, MENLO-PK, CA 94025, USA

US-GEOL-SURVEY, WOODS-HOLE, MA 02543, USA

INST-OCEANOG-SCI, WORMLEY GU8-5UB, SURREY, ENGLAND 
29. Authors: White-JDL White-DL Vallier-T Stanley-GD Ash-SR

Title: Middle Jurassic Strata Link Wallowa, Olds Ferry, and

Izee Terranes in the Accreted Blue Mountains Island-Arc,

Northeastern Oregon

Source: GEOLOGY

1992, Vol 20, Iss 8, pp 729-732

Addresses:

DALHOUSIE-UNIV, CTR MARINE GEOL, HALIFAX B3H-3J5, NS, CANADA

US-GEOL-SURVEY, MENLO-PK, CA 94025, USA

UNIV-MONTANA, DEPT GEOL, MISSOULA, MT 59812, USA

WEBER-STATE-COLL, DEPT GEOL, OGDEN, UT 84408, USA

30. Authors: Hutchinson-DR Golmshtok-AJ Zonenshain-LP Moore-TC Scholz-CA Klitgord-KD

Title: Depositional and Tectonic Framework of the Rift Basins of Lake Baikal from Multichannel Seismic Data

Source: GEOLOGY 1992, Vol 20, Iss 7, pp 589+

Addresses:

US-GEOL-SURVEY, WOODS-HOLE, MA 02543, USA

DUKE-UNIV, MARINE LAB, BEAUFORT, NC 28516, USA

GELENDZHIK-OCEANOL-INST, SO BRANCH, GELENDZHIK, RUSSIA

UNIV-MICHIGAN, ANN-ARBOR, MI 48109, USA

MOSCOW-OCEANOL-INST, MOSCOW, RUSSIA

31. Authors: Quick-JE Sinigoi-S Negrini-L Demarchi-G Mayer-A

Title: Synmagmatic Deformation in the Underplated Igneous

Complex of the Ivrea-Verbano Zone

Source: GEOLOGY

1992, Vol 20, Iss 7, pp 613-616

Addresses:

US-GEOL-SURVEY, DENVER FED CTR, MS 903, DENVER, CO 80225, USA

UNIV-TRIESTE, IST MINERAL \& PETROG, I-34100 TRIESTE, ITALY

32. Authors: Pringle-MS Mcwilliams-M Houghton-BF Lanphere-MA

Wilson-CJN

Title: Ar-40/Ar-39 Dating of Quaternary Feldspar - Examples

from the Taupo Volcanic Zone, New-Zealand

Source: GEOLOGY

1992, Vol 20, Iss 6, pp 531-534

Addresses:

US-GEOL-SURVEY, MENLO-PK, CA 94025, USA

STANFORD-UNIV, DEPT GEOPHYS, STANFORD, CA 94305, USA

DSIR, ROTORUA, NEW-ZEALAND

UNIV-CAMBRIDGE, DEPT EARTH SCI, CAMBRIDGE CB3-0EZ, ENGLAND

33. Authors: Mueller-PA Wooden-JL Nutman-AP

Title: 3.96 Ga Zircons from an Archean Quartzite, Beartooth

Mountains, Montana

Source: GEOLOGY

1992, Vol 20, Iss 4, pp 327-330 
Addresses:

UNIV-FLORIDA, DEPT GEOL, GAINESVILLE, FL 32611, USA

US-GEOL-SURVEY, MENLO-PK, CA 94025, USA

AUSTRALIAN-NATL-UNIV, RES SCH EARTH SCI, CANBERRA, ACT 2600, AUSTRALIA

34. Authors: Aitchison-JC Ireland-TR Blake-MC Flood-PG

Title: 530 Ma Zircon Age for Ophiolite from the New-England

Orogen - Oldest Rocks Known from Eastern Australia

Source: GEOLOGY

1992, Vol 20, Iss 2, pp 125-128

Addresses:

UNIV-SYDNEY, DEPT GEOL \& GEOPHYS, SYDNEY, NSW 2006, AUSTRALIA

AUSTRALIAN-NATL-UNIV, RES SCH EARTH SCI, CANBERRA, ACT 2601, AUSTRALIA

US-GEOL-SURVEY, MENLO-PK, CA 94025, USA

UNIV-NEW-ENGLAND, DEPT GEOL \& GEOPHYS, ARMIDALE, NSW 2351, AUSTRALIA

35. Authors: Mcdougall-I Brown-FH Cerling-TE Hillhouse-JW

Title: A Reappraisal of the Geomagnetic Polarity Time Scale to 4 Ma Using Data from the Turkana Basin, East-Africa

Source: GEOPHYSICAL RESEARCH LETTERS

1992, Vol 19, Iss 23, pp 2349-2352

Addresses:

AUSTRALIAN-NATL-UNIV, RES SCH EARTH SCI, GPO BOX 4, CANBERRA, ACT 2601, AUSTRALIA

US-GEOL-SURVEY, MENLO-PK, CA 94025, USA

UNIV-UTAH, DEPT GEOL \& GEOPHYS, SALT-LAKE-CITY, UT 84112, USA

36. Authors: Jeanloz-R Oneill-B Pasternak-MP Taylor-RD Bohlen-SR

Title: Mossbauer-Spectroscopy of Mg0.9Fe0.1Sio3 Perovskite

Source: GEOPHYSICAL RESEARCH LETTERS

1992, Vol 19, Iss 21, pp 2135-2138

Addresses:

UNIV-CALIF-BERKELEY, DEPT GEOL \& GEOPHYS, BERKELEY, CA 94720, USA

TEL-AVIV-UNIV, SCH PHYS \& ASTRON, IL-69978 TEL-AVIV, ISRAEL

LOS-ALAMOS-NATL-LAB, DIV PHYS, LOS-ALAMOS, NM 87545, USA

US-GEOL-SURVEY, MENLO-PK, CA 94025, USA

37. Authors: Barrientos-SE Plafker-G Lorca-E

Title: Postseismic Coastal Uplift in Southern Chile

Source: GEOPHYSICAL RESEARCH LETTERS

1992, Vol 19, Iss 7, pp 701-704

Addresses:

UNIV-CHILE, DEPT GEOL \& GEOFIS, CASILLA 2777, SANTIAGO, CHILE

SERV-HIDROGRAF-\&-OCEANOG-ARMADA, VALPARAISO, CHILE

US-GEOL-SURVEY, MENLO-PK, CA 94025, USA

38. Authors: Gable-R Morin-RH Becker-K

Title: Geothermal State of DSDP Hole-395A and Hole-534A -

Results from the Dianaut Program

Source: GEOPHYSICAL RESEARCH LETTERS

1992, Vol 19, Iss 5, pp 505-508 
Addresses:

BUR-RECH-GEOL-\&-MINIERES, AVE DE CONCYR, BP 6009, F-56060

ORLEANS 2, FRANCE

US-GEOL-SURVEY, DENVER, CO 80225, USA

UNIV-MIAMI, ROSENSTIEL SCH MARINE \& ATMOSPHER SCI, MIAMI, FL 33149 , USA

39. Authors: Linde-AT Gladwin-MT Johnston-MJS

Title: The Loma-Prieta Earthquake, 1989 and Earth Strain Tidal

Amplitudes - An Unsuccessful Search for Associated

Changes

Source: GEOPHYSICAL RESEARCH LETTERS

1992, Vol 19, Iss 3, pp 317-320

Addresses:

CARNEGIE-INST-WASHINGTON, DEPT TERR MAGNETISM, 5241 BROAD

BRANCH RD NW, WASHINGTON, DC 20015, USA

UNIV-QUEENSLAND, DEPT PHYS, BRISBANE, QLD 4072, AUSTRALIA

US-GEOL-SURVEY, MENLO-PK, CA 94025, USA

40. Authors: Poddar-M Anderson-WL

Title: Transient Electromagnetic Modeling of Shallow A-Type

Sections with 3-D Inhomogeneities

Source: GEOPHYSICS

1992, Vol 57, Iss 6, pp 774-780

Addresses:

NATL-GEOPHYS-RES-INST, UPPAL RD, HYDERABAD 500007,

ANDHRA-PRADESH, INDIA

US-GEOL-SURVEY, DENVER FED CTR, DENVER, CO 80225, USA

41. Authors: Carr-MH Wanke-H

Title: Earth and Mars - Water Inventories as Clues to Accretional Histories

Source: ICARUS 1992, Vol 98, Iss 1, pp 61-71

Addresses:

US-GEOL-SURVEY, 345 MIDDLEFIELD RD, MS-946, MENLO-PK, CA 94025, USA

MAX-PLANCK-INST-CHEM, KOSMOCHEM ABT, W-6500 MAINZ, GERMANY

42. Authors: Meade-RH Koehnken-L

Title: Distribution of the River Dolphin, Tonina Inia-

Geoffrensis, in the Orinoco River Basin of Venezuela and

Colombia

Source: INTERCIENCIA

1991, Vol 16, Iss 6, pp 300-312

Addresses:

US-GEOL-SURVEY, DENVER FED CTR, MAIL STOP 413, BOX 25046,

DENVER, CO 80225, USA

DEPT-ENVIRONM-PLANNING, HOBART, TAS 7001, AUSTRALIA

43. Authors: Tindall-JA Hemmen-K Dowd-JF

Title: An Improved Method for Field Extraction and Laboratory

Analysis of Large, Intact Soil Cores

Source: JOURNAL OF ENVIRONMENTAL QUALITY

1992, Vol 21, Iss 2, pp 259-263 
Addresses:

US-GEOL-SURVEY, DENVER FED CTR, DIV WATER RESOURCES, MS 413, POB 25046, DENVER, CO 80225, USA

POLYTECH-SW, PLYMOUTH, ENGLAND

UNIV-GEORGIA, DEPT GEOL, ATHENS, GA 30602, USA

44. Authors: Hutchinson-DR Lee-MW Behrendt-J Cannon-WF Green-AG

Title: Variations in the Reflectivity of the Moho Transition

Zone Beneath the Midcontinent Rift System of North-

America - Results from True Amplitude Analysis of

Glimpce Data

Source: JOURNAL OF GEOPHYSICAL RESEARCH-SOLID EARTH 1992, Vol 97, Iss B4, pp 4721-4737

Addresses:

US-GEOL-SURVEY, QUISSETT CAMPUS, WOODS-HOLE, MA 02543, USA

US-GEOL-SURVEY, DENVER FED CTR, DENVER, CO 80225, USA

US-GEOL-SURVEY, RESTON, VA 22092, USA

GEOL-SURVEY-CANADA, OTTAWA K1A-0Y3, ONTARIO, CANADA

45. Authors: Liao-JJ Savage-WZ Amadei-B

Title: Gravitational Stresses in Anisotropic Ridges and Valleys with Small Slopes

Source: JOURNAL OF GEOPHYSICAL RESEARCH-SOLID EARTH 1992, Vol 97, Iss B3, pp 3325-3336

Addresses:

NATL-CHIAO-TUNG-UNIV, DEPT CIVIL ENGN, 1001 TA HSUEI RD, HSINCHU 30050, TAIWAN

UNIV-COLORADO, DEPT CIVIL ENGN, BOULDER, CO 80309, USA

US-GEOL-SURVEY, DENVER, CO 80225, USA

46. Authors: Cameron-KL Robinson-JV Niemeyer-S Nimz-GJ

Kuentz-DC Harmon-RS Bohlen-SR Collerson-KD

Title: Contrasting Styles of Pre-Cenozoic and Midtertiary

Crustal Evolution in Northern Mexico - Evidence from

Deep Crustal Xenoliths from La-Olivina

Source: JOURNAL OF GEOPHYSICAL RESEARCH-SOLID EARTH 1992, Vol 97, Iss B12, pp 17353-17376

Addresses:

UNIV-CALIF-SANTA-CRUZ, BOARD EARTH SCI, SANTA-CRUZ, CA 95064, USA

NERC, ISOTOPE GEOSCI LAB, KEYWORTH NG12-5GG, ENGLAND

FUGRO-MCCLELLAND-W-INC, SACRAMENTO, CA 95815, USA

US-GEOL-SURVEY, MENLO-PK, CA 94025, USA

UNIV-QUEENSLAND, DEPT EARTH SCI, ST-LUCIA, QLD 4067, AUSTRALIA

LAWRENCE-LIVERMORE-NATL-LAB, LIVERMORE, CA 94550, USA

47. Authors: Marlow-MS Johnson-LE Pearce-JA Fryer-PB

Pickthorn-LBG Murton-BJ

Title: Upper Cenozoic Volcanic-Rocks in the Mariana Fore-Arc

Recovered from Drilling at Ocean Drilling Program Site-

781 - Implications for Fore-Arc Magmatism

Source: JOURNAL OF GEOPHYSICAL RESEARCH-SOLID EARTH 1992, Vol 97, Iss B11, pp 15085-15097

Addresses:

US-GEOL-SURVEY, 345 MIDDEFIELD RD, MENLO-PK, CA 94025, USA

INST-OCEANOG-SCI, WORMLEY GU8-5UB, SURREY, ENGLAND 
UNIV-HAWAII, HONOLULU, HI 96822, USA

UNIV-DURHAM, DEPT GEOL SCI, DURHAM DH1-3LE, ENGLAND

48. Authors: Tarduno-JA Lowrie-W Sliter-WV Bralower-TJ Heller-F

Title: Reversed Polarity Characteristic Magnetizations in the Albian Contessa Section, Umbrian Apennines, Italy Implications for the Existence of a Midcretaceous Mixed Polarity Interval

Source: JOURNAL OF GEOPHYSICAL RESEARCH-SOLID EARTH 1992, Vol 97, Iss B1, pp 241-271

Addresses:

UNIV-CALIF-SAN-DIEGO, SCRIPPS INST OCEANOG, DIV GEOL RES, LA-JOLLA, CA 92093, USA

SWISS-FED-INST-TECHNOL, INST GEOPHYS, CH-8093 ZURICH, SWITZERLAND

US-GEOL-SURVEY, MENLO-PK, CA 94025, USA

UNIV-N-CAROLINA, DEPT GEOL, CHAPEL-HILL, NC 27599, USA

49. Authors: Stein-RS Briole-P Ruegg-JC Tapponnier-P Gasse-F

Title: Contemporary, Holocene, and Quaternary Deformation of the Asal Rift, Djibouti - Implications for the Mechanics of Slow Spreading Ridges

Source: JOURNAL OF GEOPHYSICAL RESEARCH-SOLID EARTH 1991, Vol 96, Iss B13, pp 21789-21806

Addresses:

US-GEOL-SURVEY, 345 MIDDLEFIELD RD, MS 977, MENLO-PK, CA 94025, USA

UNIV-PARIS-11, HYDROL \& GEOCHIM ISOTOP LAB, F-91405 ORSAY,

FRANCE

INST-PHYS-GLOBE, F-75252 PARIS 05, FRANCE

50. Authors: Luria-M Boatman-JF Harris-J Ray-J Straube-T

Chin-J Gunter-RL Herbert-G Gerlach-TM Vanvalin-CC

Title: Atmospheric Sulfur-Dioxide at Mauna-Loa, Hawaii

Source: JOURNAL OF GEOPHYSICAL RESEARCH-ATMOSPHERES 1992, Vol 97, Iss D5, pp 6011-6022

Addresses:

NOAA, ENVIRONM RES LAB, AIR RESOURCES LAB, AEROSOL RES SECT, BOULDER, CO 80303, USA

HEBREW-UNIV-JERUSALEM, DEPT ENVIRONM SCI, JERUSALEM, ISRAEL NOAA, ENVIRONM RES LAB, CLIMATE MONITORING \& DIAGNOST LAB, BOULDER, CO 80303, USA

NOAA, ENVIRONM RES LAB, CLIMATE MONITORING \& DIAGNOST LAB, MAUNA LOA OBSERV, HILO, HI, USA

US-GEOL-SURVEY, DENVER FED CTR, DENVER, CO 80225, USA

51. Authors: Kanemasu-ET Verma-SB Smith-EA Fritschen-LJ

Wesely-M Field-RT Kustas-WP Weaver-H Stewart-JB

Gurney-R Panin-G Moncrieff-JB

Title: Surface Flux Measurements in Fife - An Overview

Source: JOURNAL OF GEOPHYSICAL RESEARCH-ATMOSPHERES 1992, Vol 97, Iss D17, pp 18547-18555

Addresses:

UNIV-GEORGIA, DEPT AGRON, GRIFFIN, GA 30223, USA

UNIV-NEBRASKA, DEPT AGR METEOROL, LINCOLN, NE 68583, USA

FLORIDA-STATE-UNIV, DEPT METEOROL, TALLAHASSEE, FL 32307, USA 
UNIV-WASHINGTON, COLL FOREST RESOURCES, SEATTLE, WA 98195, USA

ARGONNE-NATL-LAB, DIV ENVIRONM RES, ARGONNE, IL 60439, USA

UNIV-DELAWARE, COLL MARINE STUDIES, NEWARK, DE 19717, USA

USDA-ARS, BELTSVILLE AGR RES CTR, BELTSVILLE, MD 20705, USA

US-GEOL-SURVEY, FED CTR, DENVER, CO 80201, USA

UK-INST-HYDROL, WALLINGFORD, ENGLAND

UNIV-READING, READING RG6-2AH, BERKS, ENGLAND

UNIV-EDINBURGH, INST ECOL \& RESOURCE MANAGEMENT, EDINBURGH

EH8-9YL, MIDLOTHIAN, SCOTLAND

ACAD-SCI, INST ATMOSPHER PHYS, MOSCOW, RUSSIA

52. Authors: Smith-EA Hsu-AY Crosson-WL Field-RT Fritschen-LJ

Gurney-RJ Kanemasu-ET Kustas-WP Nie-D

Shuttleworth-WJ Stewart-JB Verma-SB Weaver-HL

Wesely-ML

Title: Area-Averaged Surface Fluxes and Their Time-Space

Variability over the Fife Experimental Domain

Source: JOURNAL OF GEOPHYSICAL RESEARCH-ATMOSPHERES

1992, Vol 97, Iss D17, pp 18599-18622

Addresses:

FLORIDA-STATE-UNIV, DEPT METEOROL, TALLAHASSEE, FL 32306, USA

FLORIDA-STATE-UNIV, SUPERCOMP COMPUTAT RES INST, TALLAHASSEE, FL 32306, USA

NASA, GODDARD SPACE FLIGHT CTR, GREENBELT, MD 20771, USA

UNIV-DELAWARE, COLL MARINE, NEWARK, DE 19718, USA

UNIV-WASHINGTON, COLL FOREST RESOURCES, SEATTLE, WA 98195, USA

UNIV-GEORGIA, DEPT AGRON, GRIFFIN, GA 30223, USA

USDA-ARS, BELTSVILLE AGR RES CTR, BELTSVILLE, MD 20705, USA

KANSAS-STATE-UNIV-AGR-\&-APPL-SCI, EVAPOTRANSPIRAT LAB,

MANHATTAN, KS 66506, USA

UNIV-NEBRASKA, CTR AGR METEOROL \& CLIMATOL, LINCOLN, NE 68588, USA

ARGONNE-NATL-LAB, DIV ENVIRONM RES, ARGONNE, IL 60439, USA

US-GEOL-SURVEY, DENVER, CO 80225, USA

INST-HYDROL, WALLINGFORD OX10-8BB, OXON, ENGLAND

53. Authors: Campbell-WH Schiffmacher-ER Arora-BR

Title: Quiet Geomagnetic-Field Representation for All Days and Latitudes

Source: JOURNAL OF GEOMAGNETISM AND GEOELECTRICITY 1992, Vol 44, Iss 6, pp 459-480

Addresses:

US-GEOL-SURVEY, MAIL STOP 968, BOX 25046, DENVER, CO 80225, USA

INDIAN-INST-GEOMAGNETISM, BOMBAY 400005, INDIA

54. Authors: Sacks-LA Herman-JS Konikow-LF Vela-AL

Title: Seasonal Dynamics of Groundwater-Lake Interactions at

Donana National-Park, Spain

Source: JOURNAL OF HYDROLOGY

1992, Vol 136, Iss 1-4, pp 123-154

Addresses:

US-GEOL-SURVEY, RESTON, VA 22092, USA

UNIV-VIRGINIA, DEPT ENVIRONM SCI, CHARLOTTESVILLE, VA 22903, USA

UNIV-COMPLUTENSE-MADRID, FAC CIENCIAS GEOL, CATEDRA

GEODINAMICA, E-28040 MADRID, SPAIN 
55. Authors: Cobban-WA Kennedy-WJ

Title: Campanian Ammonites from the Upper Cretaceous Gober

Chalk of Lamar County, Texas

Source: JOURNAL OF PALEONTOLOGY

1992, Vol 66, Iss 3, pp 440-454

Addresses:

US-GEOL-SURVEY, MAIL STOP 919, BOX 25046 FED CTR, DENVER, CO

80225, USA

UNIV-OXFORD-MUSEUM, GEOL COLLECT, OXFORD OX1-3PW, ENGLAND

56. Authors: Mozley-PS Carothers-WW

Title: Elemental and Isotopic Composition of Siderite in the

Kuparuk Formation, Alaska - Effect of Microbial Activity

and Water Sediment Interaction on Early Pore-Water

Chemistry

Source: JOURNAL OF SEDIMENTARY PETROLOGY

1992, Vol 62, Iss 4, pp 681-692

Addresses:

UNIV-BERN, INST GEOL, CH-3012 BERN, SWITZERLAND

US-GEOL-SURVEY, MENLO-PK, CA 94025, USA

57. Authors: Sheppard-DS Truesdell-AH Janik-CJ

Title: Geothermal Gas Compositions in Yellowstone-National-Park, USA

Source: JOURNAL OF VOLCANOLOGY AND GEOTHERMAL RESEARCH 1992, Vol 51, Iss 1-2, pp 79-93

Addresses:

DSIR, PRIVATE BAG, PETONE, NEW-ZEALAND

US-GEOL-SURVEY, MENLO-PK, CA 94025, USA

58. Authors: Houghton-BF Weaver-SD Wilson-CJN Lanphere-MA

Title: Evolution of a Quaternary Peralkaline Volcano - Mayor-

Island, New-Zealand

Source: JOURNAL OF VOLCANOLOGY AND GEOTHERMAL RESEARCH 1992, Vol 51, Iss 3, pp 217-236

Addresses:

DSIR, GEOL \& GEOPHYS, POB 499, ROTORUA, NEW-ZEALAND

UNIV-CANTERBURY, DEPT GEOL, CHRISTCHURCH 1, NEW-ZEALAND

US-GEOL-SURVEY, MENLO-PK, CA 94025, USA

UNIV-CAMBRIDGE, DEPT EARTH SCI, CAMBRIDGE, ENGLAND

59. Authors: Hey-RN Sinton-JM Kleinrock-MC Yonover-RN

Macdonald-KC Miller-SP Searle-RC Christie-DM

Atwater-TM Sleep-NH Johnson-HP Neal-CA

Title: Alvin Investigation of an Active Propagating Rift

System, Galapagos 95.5-Degrees-W

Source: MARINE GEOPHYSICAL RESEARCHES

1992, Vol 14, Iss 3, pp 207-226

Addresses:

UNIV-HAWAII, SCH OCEAN \& EARTH SCI \& TECHNOL, HONOLULU, HI

96822, USA

UNIV-CALIF-SANTA-BARBARA, INST MARINE SCI, SANTA-BARBARA, CA 93106, USA

UNIV-CALIF-SANTA-BARBARA, DEPT GEOL SCI, SANTA-BARBARA, CA

93106, USA

UNIV-DURHAM, DEPT GEOL SCI, DURHAM DH1-3LE, ENGLAND

OREGON-STATE-UNIV, COLL OCEANOG, CORVALLIS, OR 97331, USA 
STANFORD-UNIV, DEPT GEOPHYS, STANFORD, CA 94305, USA

UNIV-WASHINGTON, SCH OCEANOG, SEATTLE, WA 98195, USA

US-GEOL-SURVEY, ANCHORAGE, AK 99508, USA

60. Authors: Trincardi-F Field-ME

Title: Collapse and Flow of Lowstand Shelf-Margin Deposits - An

Example from the Eastern Tyrrhenian Sea, Italy

Source: MARINE GEOLOGY

1992, Vol 105, Iss 1-4, pp 77-94

Addresses:

IST-GEOL-MARINA, VIA ZAMBONI 65, I-40127 BOLOGNA, ITALY

US-GEOL-SURVEY, MENLO-PK, CA 94025, USA

61. Authors: Chiocci-FL Normark-WR

Title: Effect of Sea-Level Variation on Upper-Slope

Depositional Processes Offshore of Tiber Delta,

Tyrrhenian Sea, Italy

Source: MARINE GEOLOGY

1992, Vol 104, Iss 1-4, pp 109-122

Addresses:

CNR, CTR STUDIO GEOL TECN, VIA EUDOSSIANA 18, I-00184 ROME,

ITALY

US-GEOL-SURVEY, MENLO-PK, CA 94025, USA

62. Authors: Cooper-AK Barrett-PJ Hinz-K Traube-V Leitchenkov-G

Stagg-HMJ

Title: Cenozoic Prograding Sequences of the Antarctic

Continental-Margin - A Record of Glacioeustatic and

Tectonic Events

Source: MARINE GEOLOGY

1991, Vol 102, Iss 1-4, pp 175-213

Addresses:

US-GEOL-SURVEY, 345 MIDDLEFIELD RD, MENLO-PK, CA 94025, USA

VICTORIA-UNIV-WELLINGTON, WELLINGTON, NEW-ZEALAND

BUNDESANSTALT-GEOWISSENSCH-\&-ROHSTOFFE, W-3000 HANNOVER 51, GERMANY

MINIST-GEOL, ST-PETERSBURG 190121, RUSSIA

BUR-MINERAL-RESOURCES, DIV MARINE GEOSCI, CANBERRA, AUSTRALIA

63. Authors: Sharpton-VL Dalrymple-GB Marin-LE Ryder-G

Schuraytz-BC Urrutiafucugauchi-J

Title: New Links Between the Chicxulub Impact Structure and the Cretaceous Tertiary Boundary

Source: NATURE 1992, Vol 359, Iss 6398, pp 819-821

Addresses:

LUNAR-\&-PLANETARY-INST, 3600 BAY AREA BLVD, HOUSTON, TX 77058, USA

US-GEOL-SURVEY, MENLO-PK, CA 94025, USA

NATL-AUTONOMOUS-UNIV-MEXICO, INST GEOFIS, MEXICO-CITY 04510, DF, MEXICO

64. Authors: Foulger-GR Jahn-CH Seeber-G Einarsson-P Julian-BR

Heki-K

Title: Post-Rifting Stress-Relaxation at the Divergent Plate

Boundary in Northeast Iceland 
Source: NATURE

1992, Vol 358, Iss 6386, pp 488-490

Addresses:

UNIV-DURHAM, DEPT GEOL SCI, DURHAM DH1-3LE, ENGLAND

UNIV-ICELAND, INST SCI, REYKJAVIK, ICELAND

UNIV-HANNOVER, INST ERDMESSUNG, W-3000 HANNOVER 1, GERMANY

US-GEOL-SURVEY, MENLO-PK, CA 94025, USA

65. Authors: Pallister-JS Hoblitt-RP Reyes-AG

Title: A Basalt Trigger for the 1991 Eruptions of Pinatubo Volcano

Source: NATURE 1992, Vol 356, Iss 6368, pp 426-428

Addresses:

US-GEOL-SURVEY, DENVER FED CTR, BOX 25046, MS 903, DENVER, CO 80225, USA

US-GEOL-SURVEY, DAVID A JOHNSTON CASCADES VOLCANO OBSERV, VANCOUVER, WA 98661, USA

PHILIPPINE-NATL-OIL-CO, MANILA, PHILIPPINES

66. Authors: Rogers-KL Larson-EE Smith-G Katzman-D Smith-GR

Cerling-T Wang-Y Baker-RG Lohmann-KC Repenning-CA

Patterson-P Mackie-G

Title: Pliocene and Pleistocene Geologic and Climatic Evolution in the San-Luis Valley of South-Central Colorado

Source: PALAEOGEOGRAPHY PALAEOCLIMATOLOGY PALAEOECOLOGY 1992, Vol 94, Iss 1-4, pp 55-86

Addresses:

ADAMS-STATE-COLL, DEPT BIOL, ALAMOSA, CO 81102, USA

UNIV-MICHIGAN, MUSEUM PALEONTOL, ANN-ARBOR, MI 48104, USA

UNIV-COLORADO, DEPT GEOL SCI, BOULDER, CO 80309, USA

UNIV-NEW-MEXICO, DEPT GEOL, ALBUQUERQUE, NM 87131, USA

US-GEOL-SURVEY, PALEONTOL \& STRATIG BRANCH, DENVER, CO 80225, USA

UNIV-IOWA, DEPT GEOL, IOWA-CITY, IA 52242, USA

UNIV-UTAH, DEPT GEOL, SALT-LAKE-CITY, UT 84112, USA

UNIV-GUELPH, DEPT ZOOL, GUELPH N1G-2W1, ONTARIO, CANADA

UNIV-MICHIGAN, DEPT GEOL SCI, ANN-ARBOR, MI 48104, USA

67. Authors: Gray-SC Hein-JR Hausmann-R Radtke-U

Title: Geochronology and Subsurface Stratigraphy of Pukapuka and Rakahanga Atolls, Cook Islands - Late Quaternary Reef Growth and Sea-Level History

Source: PALAEOGEOGRAPHY PALAEOCLIMATOLOGY PALAEOECOLOGY 1992, Vol 91, Iss 3-4, pp 377-394

Addresses:

US-GEOL-SURVEY, MENLO-PK, CA 94025, USA

UNIV-DUSSELDORF, INST GEOG, W-4000 DUSSELDORF 1, GERMANY

68. Authors: Delaeter-JR Heumann-KG Barber-RC Cesario-JW Coplen-TB Dietze-HJ Gramlich-JW Hertz-HS Krouse-HR Lamberty-A Murphy-TJ Rosman-KJR Seyfried-MP

Shima-M Wade-K Debievre-P Greenwood-NN Peiser-HS

Rao-NK

Title: Reporting of Nitrogen-Isotope Abundances - (Technical Report)

Source: PURE AND APPLIED CHEMISTRY

1992, Vol 64, Iss 6, pp 907-908 
Addresses:

US-GEOL-SURVEY, RESTON, VA 22092, USA

UNIV-CALGARY, CALGARY T2N-1N4, ALBERTA, CANADA

69. Authors: Sancetta-C Lyle-M Heusser-L Zahn-R Bradbury-JP

Title: Late-Glacial to Holocene Changes in Winds, Upwelling, and Seasonal Production of the Northern California Current System

Source: QUATERNARY RESEARCH 1992, Vol 38, Iss 3, pp 359-370

Addresses:

COLUMBIA-UNIV, LAMONT DOHERTY GEOL OBSERV, PALISADES, NY 10964, USA

GEOMAR, W-2300 KIEL 14, GERMANY

US-GEOL-SURVEY, DENVER FED CTR, DENVER, CO 80225, USA

70. Authors: Falgueres-C Delumley-H Bischoff-JL

Title: U-Series Dates for Stalagmitic Flowstone-E (Riss Wurm Interglaciation) at Grotte du Lazaret, Nice, France

Source: QUATERNARY RESEARCH 1992, Vol 38, Iss 2, pp 227-233

Addresses:

INST-PALEONTOL-HUMAINE, 1 RUE RENE PANHARD, F-75013 PARIS,

FRANCE

US-GEOL-SURVEY, MENLO-PK, CA 94025, USA

71. Authors: Campbell-IH Czamanske-GK Fedorenko-VA Hill-RI

Stepanov-V

Title: Synchronism of the Siberian Traps and the PermianTriassic Boundary

Source: SCIENCE 1992, Vol 258, Iss 5089, pp 1760-1763

Addresses:

AUSTRALIAN-NATL-UNIV, RES SCH EARTH SCI, CANBERRA, ACT 2600, AUSTRALIA

US-GEOL-SURVEY, MENLO-PK, CA 94025, USA

CENT-RES-INST-GEOL-PROSPECTING-BASE-\&-PRECIOUS-MET, MOSCOW 113545, RUSSIA

72. Authors: Stein-RS King-GCP Lin-J

Title: Change in Failure Stress on the Southern San-Andreas Fault System Caused by the 1992 Magnitude $=7.4$ Lander Earthquake

Source: SCIENCE 1992, Vol 258, Iss 5086, pp 1328-1332

Addresses:

US-GEOL-SURVEY, MAIL STOP 977, MENLO-PK, CA 94025, USA

INST-PHYS-GLOBE, F-67084 STRASBOURG, FRANCE

WOODS-HOLE-OCEANOG-INST, WOODS-HOLE, MA 02543, USA

73. Authors: Dowsett-HJ Cronin-TM Poore-RZ Thompson-RS Whatley-RC Wood-AM

Title: Micropaleontological Evidence for Increased Meridional Heat-Transport in the North-Atlantic Ocean During the Pliocene 
Source: SCIENCE

1992, Vol 258, Iss 5085, pp 1133-1135

Addresses:

US-GEOL-SURVEY, RESTON, VA 22092, USA

UNIV-COLL-WALES, ABERYSTWYTH SY23-3DB, DYFED, WALES

US-GEOL-SURVEY, DENVER FED CTR, DENVER, CO 80225, USA

RAND-CORP, SANTA-MONICA, CA 90406, USA

74. Authors: Belton-MJS Veverka-J Thomas-P Helfenstein-P

Simonelli-D Chapman-C Davies-ME Greeley-R

Greenberg-R Head-J Murchie-S Klaasen-K Johnson-TV

Mcewen-A Morrison-D Neukum-G Fanale-F Anger-C

Carr-M Pilcher-C

Title: Galileo Encounter with 951 Gaspra - 1st Pictures of an Asteroid

Source: SCIENCE 1992, Vol 257, Iss 5077, pp 1647-1652

Addresses:

CORNELL-UNIV, DEPT ASTRON, ITHACA, NY 14853, USA

NATL-OPT-ASTRON-OBSERV, TUCSON, AZ 85719, USA

PLANETARY-SCI-INST, TUCSON, AZ 85719, USA

RAND-CORP, SANTA-MONICA, CA 90406, USA

ARIZONA-STATE-UNIV, DEPT GEOL, TEMPE, AZ 85287, USA

UNIV-ARIZONA, LUNAR \& PLANETARY LAB, TUCSON, AZ 85721, USA

BROWN-UNIV, DEPT GEOL, PROVIDENCE, RI 02912, USA

JET-PROP-LAB, PASADENA, CA 91109, USA

NASA, AMES RES CTR, MOFFETT-FIELD, CA 94035, USA

NASA-HEADQUARTERS, WASHINGTON, DC 20546, USA

US-GEOL-SURVEY, MENLO-PK, CA 94025, USA

INST-SPACE-\&-TERR-SCI, CONCORD L4K-3C8, ONTARIO, CANADA

DFVLR, W-8031 OBERPFAFFENHOFEN, GERMANY

UNIV-HAWAII, INST GEOPHYS, HONOLULU, HI 96822, USA

US-GEOL-SURVEY, FLAGSTAFF, AZ 86001, USA

75. Authors: Kruger-MB Jeanloz-R Pasternak-MP Taylor-RD

Snyder-BS Stacy-AM Bohlen-SR

Title: Antiferromagnetism in Pressure-Amorphized Fe2SiO4

Source: SCIENCE 1992, Vol 255, Iss 5045, pp 703-705

Addresses:

UNIV-CALIF-BERKELEY, DEPT PHYS, BERKELEY, CA 94720, USA

US-GEOL-SURVEY, MENLO-PK, CA 94025, USA

LOS-ALAMOS-NATL-LAB, DIV PHYS, LOS-ALAMOS, NM 87545, USA

TEL-AVIV-UNIV, SCH PHYS \& ASTRON, IL-69978 TEL-AVIV, ISRAEL

UNIV-CALIF-BERKELEY, DEPT CHEM, BERKELEY, CA 94720, USA

UNIV-CALIF-BERKELEY, DEPT GEOL \& GEOPHYS, BERKELEY, CA 94720,

USA

76. Authors: Belton-MJS Head-JW Pieters-CM Greeley-R Mcewen-AS

Neukum-G Klaasen-KP Anger-CD Carr-MH Chapman-CR

Davies-ME Fanale-FP Gierasch-PJ Greenberg-R

Ingersoll-AP Johnson-T Paczkowski-B Pilcher-CB

Veverka-J

Title: Lunar Impact Basins and Crustal Heterogeneity - New

Western Limb and Far Side Data from Galileo

Source: SCIENCE

1992, Vol 255, Iss 5044, pp 570-576 
Addresses:

NATL-OPT-ASTRON-OBSERV, TUCSON, AZ 85719, USA

CALTECH, PASADENA, CA 91125, USA

ITRES-RES-LTD, CALGARY T2E-7H7, ALBERTA, CANADA

NASA-HEADQUARTERS, WASHINGTON, DC 20546, USA

BROWN-UNIV, PROVIDENCE, RI 02912, USA

ARIZONA-STATE-UNIV, TEMPE, AZ 85287, USA

US-GEOL-SURVEY, FLAGSTAFF, AZ 86001, USA

DFVLR, W-8031 OBERPFAFFENHOFEN, GERMANY

RAND-CORP, SANTA-MONICA, CA 90406, USA

CORNELL-UNIV, ITHACA, NY 14853, USA

UNIV-ARIZONA, TUCSON, AZ 85721, USA

CALTECH, JET PROP LAB, PASADENA, CA 91109, USA

SCI-APPLICAT-INT-CORP, INST PLANETARY SCI, TUCSON, AZ 85719, USA

US-GEOL-SURVEY, MENLO-PK, CA 94025, USA

UNIV-HAWAII, HONOLULU, HI 96822, USA

77. Authors: Kachanoski-RG Tanji-KK Rollins-LT Whittig-LD

Fujii-R

Title: Dissolution Kinetics of CaCO3 - Carkin-1, a Computer-Model

Source: SOIL SCIENCE 1992, Vol 153, Iss 1, pp 13-24

Addresses:

UNIV-GUELPH, DEPT LAND RESOURCE SCI, GUELPH N1G-2W1, ONTARIO, CANADA

UNIV-CALIF-DAVIS, DAVIS, CA 95616, USA

US-GEOL-SURVEY, DIV WATER RESOURCES, SACRAMENTO, CA, USA

78. Authors: Achauer-U Maguire-PKH Mechie-J Green-WV Prodehl-C

Fuchs-K Khan-MA Keller-GR Mooney-W Jacob-B

Thybo-H Nyambok-IO Patel-J Braile-LW Thompson-G

Gajewski-D Meyer-RP Riaroh-D

Title: Some Remarks on the Structure and Geodynamics of the Kenya Rift

Source: TECTONOPHYSICS

1992, Vol 213, Iss 1-2, pp 257-268

Addresses:

UNIV-LEICESTER, DEPT GEOL, LEICESTER LE1-7RH, ENGLAND

UNIV-WISCONSIN, DEPT GEOL \& GEOPHYS, MADISON, WI 53706, USA

UNIV-KARLSRUHE, TH, INST GEOPHYS, W-7500 KARLSRUHE, GERMANY

OBSERV-GRENOBLE, GEOPHYS INTERNE \& TECTONOPHYS, BP 53X,

F-38041 GRENOBLE, FRANCE

UNIV-TEXAS, DEPT GEOL SCI, EL-PASO, TX 79968, USA

US-GEOL-SURVEY, MENLO-PK, CA 94025, USA

DUBLIN-INST-ADV-STUDIES, GEOPHYS SECT, DUBLIN 4, IRELAND

UNIV-COPENHAGEN, INST ALMEN GEOL, DK-1168 COPENHAGEN, DENMARK

UNIV-CLAUSTHAL, INST GEOPHYS, CLAUSTHAL-ZELLERFE, GERMANY

STANFORD-UNIV, MENLO-PK, CA 94025, USA

MINIST-ENERGY, NAIROBI, KENYA

UNIV-NAIROBI, NAIROBI, KENYA

PURDUE-UNIV, DEPT EARTH \& ATMOSPHER SCI, W-LAFAYETTE, IN

47907, USA

UNIV-CALIF-LOS-ANGELES, LOS-ANGELES, CA 90024, USA 
79. Authors: Collot-JY Lallemand-S Pelletier-B Eissen-JP

Glacon-G Fisher-MA Greene-HG Boulin-J Daniel-J

Monzier-M

Title: Geology of the Dentrecasteaux-New Hebrides Arc Collision

Zone - Results from a Deep Submersible Survey

Source: TECTONOPHYSICS

1992, Vol 212, Iss 3-4, pp 213-241

Addresses:

ORSTOM, BP 48, F-06230 VILLEFRANCHE-MER, FRANCE

UNIV-PROVENCE, STRATIG \& PALEOECOL LAB, F-13000 MARSEILLE, FRANCE

UNIV-MARSEILLE-ST-JEROME-3, GEOL STRUCT LAB, F-13397

MARSEILLE, FRANCE

UNIV-PARIS-06, GEOL STRUCT LAB, F-75252 PARIS, FRANCE

ORSTOM, NOUMEA, NEW-CALEDONIA

US-GEOL-SURVEY, MENLO-PK, CA 94025, USA

80. Authors: Zorin-Y Cordell-L

Title: Crustal Extension in the Baikal Rift-Zone

Source: TECTONOPHYSICS

1991, Vol 198, Iss 1, pp 117-121

Addresses:

RUSSIAN-ACAD-SCI, INST EARTHS CRUST, IRKUTSK 664003, RUSSIA

US-GEOL-SURVEY, DENVER FED CTR, DENVER, CO 80225, USA

81. Authors: Severson-RC Gough-LP Vandenboom-G

Title: Base-Line Element Concentrations in Soils and Plants,

Wattenmeer National-Park, North and East Frisian

Islands, Federal-Republic-of-Germany

Source: WATER AIR AND SOIL POLLUTION

1992, Vol 61, Iss 1-2, pp 169-184

Addresses:

US-GEOL-SURVEY, BOX 25046, MS 973, DENVER, CO 80225, USA

BUNDESANSTALT-GEOWISSENSCHAFTEN-\&-ROHSTOFFE, W-3000 HANNOVER

51, GERMANY

82. Authors: Engesgaard-P Kipp-KL

Title: A Geochemical Transport Model for Redox-Controlled

Movement of Mineral Fronts in Groundwater-Flow Systems -

A Case of Nitrate Removal by Oxidation of Pyrite

Source: WATER RESOURCES RESEARCH

1992, Vol 28, Iss 10, pp 2829-2843

Addresses:

TECH-UNIV-DENMARK, GROUND WATER RES CTR, INST HYDRODYNAM \& HYDRAUL ENGN, BLDG 115, DK-2800 LYNGBY, DENMARK

US-GEOL-SURVEY, DENVER FED CTR, DIV WATER RESOURCES, DENVER, CO 80225, USA

83. Authors: Hooper-RP Christophersen-N

Title: Predicting Episodic Stream Acidification in the

Southeastern United-States - Combining a Long-Term

Acidification Model and the End-Member Mixing Concept

Source: WATER RESOURCES RESEARCH

1992, Vol 28, Iss 7, pp 1983-1990 
Addresses:

US-GEOL-SURVEY, PEACHTREE BUSINESS CTR, 3039 AMWITER RD, SUITE 130, ATLANTA, GA 30360, USA

UNIV-OSLO, DEPT INFORMAT, N-0316 OSLO 3, NORWAY

US-GEOL-SURVEY, DIV WATER RESOURCES, DORAVILLE, GA, USA

84. Authors: Dagan-G Cvetkovic-V Shapiro-A

Title: A Solute Flux Approach to Transport in Heterogeneous

Formations .1. The General Framework

Source: WATER RESOURCES RESEARCH

1992, Vol 28, Iss 5, pp 1369-1376

Addresses:

US-GEOL-SURVEY, DIV WATER RESOURCES, RESTON, VA 22092, USA

TEL-AVIV-UNIV, FAC ENGN, IL-69978 TEL-AVIV, ISRAEL

ROYAL-INST-TECHNOL, DEPT WATER RESOURCES ENGN, S-10044

STOCKHOLM 70, SWEDEN

85. Authors: Cvetkovic-V Shapiro-AM Dagan-G

Title: A Solute Flux Approach to Transport in Heterogeneous

Formations .2. Uncertainty Analysis

Source: WATER RESOURCES RESEARCH

1992, Vol 28, Iss 5, pp 1377-1388

Addresses:

ROYAL-INST-TECHNOL, DEPT WATER RESOURCES ENGN, S-10044

STOCKHOLM 70, SWEDEN

TEL-AVIV-UNIV, DIV WATER RESOURCES, IL-69978 TEL-AVIV, ISRAEL

US-GEOL-SURVEY, DIV WATER RESOURCES, RESTON, VA 22092, USA

86. Authors: Gaal-G Schultz-K

Title: Precambrian Metallogeny Related to Plate-Tectonics - Preface

Source: PRECAMBRIAN RESEARCH

1992, Vol 58, Iss 1-4, pp R7-R9

Addresses:

HUNGARIAN-GEOL-INST, STEFANIA 14, POB 106, H-1143 BUDAPEST, HUNGARY

GEOL-SURVEY-FINLAND, ESPOO, FINLAND

US-GEOL-SURVEY, EASTERN MINERAL RESOURCES, RESTON, VA 22092, USA

87. Authors: Ishiwatari-R Lowe-L Mcknight-D Shinozuka-N Yonebayashi-K

Title: Advances in Humic Substances Research - A Collection of Papers from the 5th International Meeting of the International-Humic-Substances-Society, Nagoya, Japan, 6-

10 August 1990 - Preface

Source: SCIENCE OF THE TOTAL ENVIRONMENT

1992, Vol 118, Iss MAY, pp R11-R11

Addresses:

TOKYO-METROPOLITAN-UNIV, FAC SCI, DEPT CHEM, TOKYO 158, JAPAN

UNIV-BRITISH-COLUMBIA, DEPT SOIL SCI, VANCOUVER V6T-2A2, BC,

CANADA

US-GEOL-SURVEY, DENVER FED CTR, DENVER, CO 80225, USA 
88. Authors: Mikumo-T Aki-K Ohnaka-M Ruff-LJ Spudich-PHP

Title: Special Issue - Earthquake Source Physics and Earthquake

Precursors - Papers from International-Symposium on

Earthquake Source Physics and Earthquake Precursors,

Held in Tokyo, Japan, November 19-22, 1990 - Preface

Source: TECTONOPHYSICS

1992, Vol 211, Iss 1-4, pp R7-R10

Addresses:

KYOTO-UNIV, DISASTER PREVENT RES INST, EARTHQUAKE PREDICT RES

CTR, UJI, KYOTO 611, JAPAN

UNIV-SO-CALIF, DEPT GEOL SCI, LOS-ANGELES, CA 90089, USA

UNIV-TOKYO, EARTHQUAKE RES INST, BUNKYO KU, TOKYO 113, JAPAN

UNIV-MICHIGAN, DEPT GEOL SCI, ANN-ARBOR, MI 48109, USA

US-GEOL-SURVEY, MENLO-PK, CA 94025, USA

89. Authors: Glynn-PD Reardon-EJ

Title: Solid-Solution Aqueous-Solution Equilibria -

Thermodynamic Theory and Representation - Reply

Source: AMERICAN JOURNAL OF SCIENCE

1992, Vol 292, Iss 3, pp 215-225

Addresses:

US-GEOL-SURVEY, 432 NATL CTR, RESTON, VA 22092, USA

UNIV-WATERLOO, DEPT EARTH SCI, WATERLOO N2L-3G1, ONTARIO,

CANADA

90. Authors: Chiou-CT Lee-JF Boyd-SA

Title: The Surface-Area of Soil Organic-Matter - Reply

Source: ENVIRONMENTAL SCIENCE \& TECHNOLOGY 1992, Vol 26, Iss 2, pp 404-406

Addresses:

US-GEOL-SURVEY, DENVER FED CTR, DIV WATER RESOURCES, BOX 25046, MS 408, DENVER, CO 80225, USA

NATL-CENT-UNIV, GRAD INST ENVIRONM ENGN, CHUNGLI 32054, TAIWAN

MICHIGAN-STATE-UNIV, DEPT CROP \& SOIL SCI, E-LANSING, MI

48824, USA

91. Authors: Alpers-CN Nordstrom-DK Thompson-JM Lund-M

Title: Cyclic Precipitation and Dissolution of $\mathrm{Zn}-\mathrm{Cu}-\mathrm{Bearing}$

Melanterite Controlling the Composition of Acid-Mine

Drainage from Iron Mountain, California

Source: ABSTRACTS OF PAPERS OF THE AMERICAN CHEMICAL SOCIETY

1992, Vol 204, Iss AUG, pp 39-GEOC

Addresses:

US-GEOL-SURVEY, SACRAMENTO, CA 95825, USA

US-GEOL-SURVEY, BOULDER, CO 80303, USA

US-GEOL-SURVEY, MENLO-PK, CA 94025, USA

MCGILL-UNIV, DEPT GEOL SCI, MONTREAL H3A-2A7, QUEBEC, CANADA

92. Authors: Webster-JG Nordstrom-DK

Title: Transport and Natural Attenuation of $\mathrm{Cu}, \mathrm{Zn}$ and as in the Leviathan Bryant Creek Drainage System

Source: ABSTRACTS OF PAPERS OF THE AMERICAN CHEMICAL SOCIETY 1992, Vol 204, Iss AUG, pp 67-GEOC

Addresses:

CRI-GEOSPHERE, AUCKLAND, NEW-ZEALAND

US-GEOL-SURVEY, BOULDER, CO 80303, USA 
93. Authors: Anderson-LD Kent-DB Davis-JA Waite-TD

Title: Batch Experiments Characterizing the Reduction of $\mathrm{Cr}(\mathrm{VI})$

Using Suboxic Material from a Mildly Reducing Sand and

Gravel Aquifer

Source: ABSTRACTS OF PAPERS OF THE AMERICAN CHEMICAL SOCIETY 1992, Vol 203, Iss APR, pp 20-ENVR

Addresses:

AUSTRALIAN-NUCL-SCI-\&-TECHNOL-ORG, SUTHERLAND, NSW, AUSTRALIA

US-GEOL-SURVEY, MENLO-PK, CA 94025, USA

94. Authors: Field-JA Barber-LB

Title: Fate of Alkylbenzenesulfonate Surfactants During Rapid Infiltration of Sewage Effluent to a Shallow Groundwater Aquifer

Source: ABSTRACTS OF PAPERS OF THE AMERICAN CHEMICAL SOCIETY 1992, Vol 203, Iss APR, pp 298-ENVR

Addresses:

SWISS-FED-INST-WATER-RESOURCES-\&-WATER-POLLUT-CONTROL, DUBENDORF, SWITZERLAND

US-GEOL-SURVEY, DENVER FED CTR, DENVER, CO 80225, USA

95. Authors: Bohor-BF Krogh-TE Kamo-SL

Title: U-Pb Isotopic Ages of the K/T Impact Event and Its

Target Rocks from Shocked Zircons

Source: METEORITICS

1992, Vol 27, Iss 3, pp 205-205

Addresses:

US-GEOL-SURVEY, DENVER, CO 80225, USA

ROYAL-ONTARIO-MUSEUM, GEOCHRON LAB, TORONTO M5S-2C6, ONTARIO, CANADA

96. Authors: Hartung-JB Kunk-MR Reimold-WU Miller-RM Grieve-RAF

Title: Roter Kamm Crater Age - 3.5 to 4.0 Ma

Source: METEORITICS 1991, Vol 26, Iss 4, pp 342-343

Addresses:

US-GEOL-SURVEY, NATL CTR, RESTON, VA 22092, USA

UNIV-WITWATERSRAND, DEPT GEOL, JOHANNESBURG 2001, SOUTH-AFRICA

GEOL-SURVEY-NAMIBIA, WINDHOEK 9000, NAMIBIA

ENERGY-MINES-\&-RESOURCES-CANADA, GEOL SURVEY CANADA, OTTAWA, ONTARIO, CANADA

97. Authors: Wooden-JL Czamanske-GK Bouse-RM Likhachev-AP

Kunilov-VE Lyulko-V

Title: Pb Isotope Data Indicate a Complex, Mantle Origin for the Norilsk-Talnakh Ores, Siberia

Source: ECONOMIC GEOLOGY AND THE BULLETIN OF THE SOCIETY OF ECONOMIC GEOLOGISTS 1992, Vol 87, Iss 4, pp 1153-1165

Addresses:

US-GEOL-SURVEY, 345 MIDDLEFIELD RD, MENLO-PK, CA 94025, USA

CENT-RES-INST-GEOL-PROSPECTING-BASE-\&-PRECIOUS-MET, MOSCOW

113545, RUSSIA

NORILSK-NICKEL-STATE-CONCERN, NORILSK 663300, RUSSIA 


\section{NORILSK-EXPEDIT, NORILSK 663300, RUSSIA}

98. Authors: Chung-CF Singer-DA Menzie-WD

Title: Predicting Sizes of Undiscovered Mineral-Deposits - An

Example Using Mercury Deposits in California

Source: ECONOMIC GEOLOGY AND THE BULLETIN OF THE SOCIETY OF ECONOMIC GEOLOGISTS 1992, Vol 87, Iss 4, pp 1174-1179

Addresses:

GEOL-SURVEY-CANADA, 601 BOOTH ST, OTTAWA K1A-0E8, ONTARIO, CANADA

US-GEOL-SURVEY, MENLO-PK, CA 94025, USA

US-GEOL-SURVEY, RESTON, VA 22092, USA

99. Authors: Chung-CF Jefferson-CW Singer-DA

Title: A Quantitative Link Among Mineral-Deposit Modeling, Geoscience Mapping, and Exploration-Resource Assessment

Source: ECONOMIC GEOLOGY AND THE BULLETIN OF THE SOCIETY OF ECONOMIC GEOLOGISTS 1992, Vol 87, Iss 1, pp 194-197

Addresses:

GEOL-SURVEY-CANADA, 601 BOOTH ST, OTTAWA K1A-0E8, ONTARIO, CANADA

US-GEOL-SURVEY, MENLO-PK, CA 94025, USA

100. Authors: Glynn-PD Reardon-EJ Plummer-LN Busenberg-E

Title: Reaction Paths and Equilibrium End-Points in SolidSolution Aqueous-Solution Systems - Reply

Source: GEOCHIMICA ET COSMOCHIMICA ACTA 1992, Vol 56, Iss 6, pp 2559-2572

Addresses:

US-GEOL-SURVEY, 432 NATL CTR, RESTON, VA 22092, USA

UNIV-WATERLOO, DEPT EARTH SCI, WATERLOO N2L-3G1, ONTARIO, CANADA

101. Authors: Kennedy-WJ Cobban-WA Scott-GR

Title: Ammonite Correlation of the Uppermost Campanian of Western-Europe, the United-States Gulf-Coast, Atlantic Seaboard and Western Interior, and the Numerical Age of the Base of the Maastrichtian

Source: GEOLOGICAL MAGAZINE 1992, Vol 129, Iss 4, pp 497-500

Addresses:

UNIV-MUSEUM-OXFORD, GEOL COLLECT, PARKS RD, OXFORD OX1-3PW, ENGLAND

US-GEOL-SURVEY, DENVER FED CTR, DENVER, CO 80225, USA

102. Authors: Durrheim-RJ Mooney-WD

Title: Archean and Proterozoic Crustal Evolution - Evidence from Crustal Seismology - Reply

Source: GEOLOGY 1992, Vol 20, Iss 7, pp 665-665

Addresses:

UNIV-WITWATERSRAND, DEPT GEOPHYS, JOHANNESBURG 2050, SOUTH-AFRICA

US-GEOL-SURVEY, MENLO-PK, CA 94025, USA 
103. Authors: Cobban-WA Kennedy-WJ

Title: The Last Western Interior Baculites from the Fox Hills

Formation of South-Dakota

Source: JOURNAL OF PALEONTOLOGY

1992, Vol 66, Iss 4, pp 682-684

Addresses:

US-GEOL-SURVEY, BOX 25046, MAIL STOP 919, FED CTR, DENVER, CO

80225, USA

UNIV-OXFORD-MUSEUM, GEOL COLLECT, OXFORD OX1-3PW, ENGLAND

104. Authors: Bayliss-P Chen-KQ Criddle-AJ Desborough-GA

Title: Mineral Nomenclature - Sulrhodite

Source: MINERALOGICAL MAGAZINE 1992, Vol 56, Iss 382, pp 125-126

Addresses:

UNIV-CALGARY, DEPT GEOL \& GEOPHYS, CALGARY T2N-1N4, ALBERTA, CANADA

CHINESE-ACAD-GEOL-SCI, INST MINERAL DEPOSITS, BEIJING, PEOPLES-R-CHINA

NAT-HIST-MUSEUM, DEPT MINERAL, LONDON SW7-2BD, ENGLAND

US-GEOL-SURVEY, DENVER, CO 80225, USA

105. Authors: Cobban-WA Kennedy-WJ

Title: Campanian Trachyscaphites-Spiniger Ammonite Fauna in North-East Texas

Source: PALAEONTOLOGY 1992, Vol 35, Iss FEB, pp 63-93

Addresses:

US-GEOL-SURVEY, DENVER, CO 80225, USA

UNIV-MUSEUM, OXFORD, ENGLAND

106. Authors: Belton-MJS Klaasen-KP Clary-MC Anderson-JL Anger-CD Carr-MH Chapman-CR Davies-ME Greeley-R Anderson-D Bolef-LK Townsend-TE Greenberg-R

Head-JW Neukum-G Pilcher-CB Veverka-J Gierasch-PJ

Fanale-FP Ingersoll-AP Masursky-H Morrison-D

Pollack-JB

Title: The Galileo Solid-State Imaging Experiment

Source: SPACE SCIENCE REVIEWS 1992, Vol 60, Iss 1-4, pp 413-455

Addresses:

NATL-OPT-ASTRON-OBSERV, TUCSON, AZ, USA

JET-PROP-LAB, PASADENA, CA 91109, USA

ITRES-RES-LTD, CALGARY, ALBERTA, CANADA

YORK-UNIV, INST SPACE \& TERR SCI, N-YORK M3J-1P3, ONTARIO,

CANADA

US-GEOL-SURVEY, MENLO-PK, CA 94025, USA

SAIC, INST PLANETARY SCI, TUCSON, AZ, USA

RAND-CORP, SANTA-MONICA, CA 90406, USA

ARIZONA-STATE-UNIV, TEMPE, AZ 85287, USA

UNIV-ARIZONA, TUCSON, AZ 85721, USA

CALTECH, PASADENA, CA 91125, USA

DLR, OBERPFAFFENHOFEN, GERMANY

US-GEOL-SURVEY, FLAGSTAFF, AZ 86001, USA

INST-GEOPHYS, HONOLULU, HI, USA 
CORNELL-UNIV, ITHACA, NY 14853, USA

BROWN-UNIV, PROVIDENCE, RI 02912, USA

NASA, AMES RES CTR, MOFFETT-FIELD, CA 94035, USA

NASA, WASHINGTON, DC 20546, USA

107. Authors: Kosobokov-VG Heley-JH Keilisborok-VI Dewey-JW Khokhlov-AV

Title: The Test of an Intermediate-Term Earthquake Prediction Algorithm - The Design of Real-Time Monitoring and Retroactive Application

Source: DOKLADY AKADEMII NAUK 1992, Vol 325, Iss 1, pp 46-48

Addresses:

INT-INST-THEORY-EARTHQUAKE-PREDICT-\&-MATH-GEOPHYS, MOSCOW, RUSSIA

US-GEOL-SURVEY, NATL EARTHQUAKE INFORMAT CTR, DENVER, CO 80225, USA

108. Authors: Senderov-EE Hearn-PP Kuznetsova-TP Tobelko-KT

Title: The Structural State of Secondary Potassium Feldspars as a Function of Their Condition of Formation

Source: GEOKHIMIYA 1991, Iss 7, pp 958-971

Addresses:

VI-VERNADSKII-GEOCHEM-\&-ANAL-CHEM-INST, MOSCOW, RUSSIA

US-GEOL-SURVEY, RESTON, VA 22092, USA

109. Authors: Bogatikov-OA Gurbanov-AG Kovalenko-VI Koronovskiy-NV Lipman-P Tsvetkov-AA

Title: Upper Caldera Complex, Northern Caucasus

Source: IZVESTIYA AKADEMII NAUK SSSR SERIYA GEOLOGICHESKAYA 1992, Iss 1, pp 5-21

Addresses:

RUSSIAN-ACAD-SCI, INST ORE DEPOSIT GEOL PETROG MINERAL \& GEOCHEM, MOSCOW, RUSSIA

MOSCOW-MV-LOMONOSOV-STATE-UNIV, MOSCOW 117234, RUSSIA

US-GEOL-SURVEY, MENLO-PK, CA 94025, USA

\section{3}

1. Authors: Czamanske-GK Sisson-TW Campbell-JL Teesdale-WJ

Title: Micro-PIXE Analysis of Silicate Reference-Standards

Source: AMERICAN MINERALOGIST

1993, Vol 78, Iss 9-10, pp 893-903

Addresses:

US-GEOL-SURVEY, 345 MIDDLEFIELD RD, MENLO-PK, CA 94025, USA

UNIV-GUELPH, DEPT PHYS, GUELPH N1G-2W1, ONTARIO, CANADA

2. Authors: Alt-JC Shanks-WC Jackson-MC

Title: Cycling of Sulfur in Subduction Zones - The Geochemistry of Sulfur in the Mariana-Island Arc and Back-Arc Trough

Source: EARTH AND PLANETARY SCIENCE LETTERS 1993, Vol 119, Iss 4, pp 477-494

Addresses:

UNIV-MICHIGAN, DEPT GEOL SCI, 1006 CC LITTLE BLDG, ANN-ARBOR, MI 48109, USA 
US-GEOL-SURVEY, EASTERN MINERAL RESOURCES BRANCH, RESTON, VA 22092, USA

MCGILL-UNIV, DEPT EARTH \& PLANETARY SCI, MONTREAL H3A-2A7, QUEBEC, CANADA

3. Authors: Bohor-BF Betterton-WJ Krogh-TE

Title: Impact-Shocked Zircons - Discovery of Shock-Induced

Textures Reflecting Increasing Degrees of Shock

Metamorphism

Source: EARTH AND PLANETARY SCIENCE LETTERS 1993, Vol 119, Iss 3, pp 419-424

Addresses:

US-GEOL-SURVEY, BOX 25046, MS 972, DENVER, CO 80225, USA

ROYAL-ONTARIO-MUSEUM, JACK SATTERLY GEOCHRONOL LAB, TORONTO M5S-2C6, ONTARIO, CANADA

4. Authors: Krogh-TE Kamo-SL Bohor-BF

Title: Fingerprinting the K/T Impact Site and Determining the Time of Impact by U-Pb Dating of Single Shocked Zircons from Distal Ejecta

Source: EARTH AND PLANETARY SCIENCE LETTERS 1993, Vol 119, Iss 3, pp 425-429

Addresses:

ROYAL-ONTARIO-MUSEUM, JACK SATTERLY GEOCHRONOL LAB, 100 QUEENS

PK, TORONTO M5S-2C6, ONTARIO, CANADA

US-GEOL-SURVEY, DENVER, CO 80225, USA

5. Authors: Zweng-PL Mortensen-JK Dalrymple-GB

Title: Thermochronology of the Camflo Gold Deposit, Malartic, Quebec - Implications for Magmatic Underplating and the Formation of Gold-Bearing Quartz Veins

Source: ECONOMIC GEOLOGY AND THE BULLETIN OF THE SOCIETY OF ECONOMIC GEOLOGISTS 1993, Vol 88, Iss 6, pp 1700-1721

Addresses:

STANFORD-UNIV, DEPT APPL EARTH SCI, STANFORD, CA 94305, USA

GEOL-SURVEY-CANADA, OTTAWA K1A-0E8, ONTARIO, CANADA

US-GEOL-SURVEY, MENLO-PK, CA 94025, USA

6. Authors: Grew-ES Belakovskiy-DI Fleet-ME Yates-MG Mcgee-JJ Marquez-N

Title: Reedmergnerite and Associated Minerals from Peralkaline Pegmatite, Dara-I-Pioz, Southern Tien-Shan, Tajikistan

Source: EUROPEAN JOURNAL OF MINERALOGY 1993, Vol 5, Iss 5, pp 971-984

Addresses:

UNIV-MAINE, DEPT GEOL SCI, 5711 BOARDMAN HALL, ORONO, ME 04469, USA

RUSSIAN-ACAD-SCI, AE FERSMAN MINERAL MUSEUM, MOSCOW 117071, RUSSIA

UNIV-WESTERN-ONTARIO, DEPT GEOL, LONDON N6A-5B7, ONTARIO, CANADA

US-GEOL-SURVEY, NATL CTR, RESTON, VA 22092, USA

AEROSP-CORP, LOS-ANGELES, CA 90009, USA 
7. Authors: Voss-CI Andersson-J

Title: Regional Flow in the Baltic Shield During Holocene Coastal Regression

Source: GROUND WATER 1993, Vol 31, Iss 6, pp 989-1006

Addresses:

US-GEOL-SURVEY, NATL CTR 431, RESTON, VA 22092, USA

SWEDISH-NUCL-POWER-INSPECTORATE, S-10252 STOCKHOLM, SWEDEN

8. Authors: Pieters-CM Head-JW Sunshine-JM Fischer-EM Murchie-SL Belton-M Mcewen-A Gaddis-L Greeley-R Neukum-G Jaumann-R Hoffmann-H

Title: Crustal Diversity of the Moon - Compositional Analyses of Galileo Solid-State Imaging Data

Source: JOURNAL OF GEOPHYSICAL RESEARCH-PLANETS 1993, Vol 98, Iss E9, pp 17127-17148

Addresses:

BROWN-UNIV, DEPT GEOL SCI, PROVIDENCE, RI 02912, USA

NATL-OPT-ASTRON-OBSERV, KITT PEAK NATL OBSERV, TUCSON, AZ 85726, USA

US-GEOL-SURVEY, FLAGSTAFF, AZ 86001, USA

ARIZONA-STATE-UNIV, TEMPE, AZ 85287, USA

DLR, INST PLANETARY EXPLORAT, D-82230 OBERPFAFFENHOFEN, GERMANY

9. Authors: Head-JW Murchie-S Mustard-JF Pieters-CM Neukum-G Mcewen-A Greeley-R Nagel-E Belton-MJS

Title: Lunar Impact Basins - New Data for the Western Limb and Far Side (Orientale and South-Pole Aitken-Basins) from the 1st Galileo Flyby

Source: JOURNAL OF GEOPHYSICAL RESEARCH-PLANETS 1993, Vol 98, Iss E9, pp 17149-17181

Addresses:

BROWN-UNIV, DEPT GEOL SCI, PROVIDENCE, RI 02912, USA

NATL-OPT-ASTRON-OBSERV, TUCSON, AZ 85726, USA

DLR, INST PLANETARY EXPLORAT, D-12484 BERLIN, GERMANY

ARIZONA-STATE-UNIV, DEPT GEOL, TEMPE, AZ 85287, USA

US-GEOL-SURVEY, FLAGSTAFF, AZ 86001, USA

DLR, INST PLANETARY EXPLORAT, D-82230 OBERPFAFFENHOFEN, GERMANY

10. Authors: Greeley-R Kadel-SD Williams-DA Gaddis-LR Head-JW Mcewen-AS Murchie-SL Nagel-E Neukum-G Pieters-CM Sunshine-JM Wagner-R Belton-MJS

Title: Galileo Imaging Observations of Lunar Maria and Related Deposits

Source: JOURNAL OF GEOPHYSICAL RESEARCH-PLANETS 1993, Vol 98, Iss E9, pp 17183-17205

Addresses:

ARIZONA-STATE-UNIV, DEPT GEOL, TEMPE, AZ 85287, USA

NATL-OPT-ASTRON-OBSERV, TUCSON, AZ 85726, USA

US-GEOL-SURVEY, FLAGSTAFF, AZ 86001, USA

BROWN-UNIV, DEPT GEOL SCI, PROVIDENCE, RI 02912, USA

GERMAN-AEROSP-RES-ESTAB, INST PLANETARY EXPLORAT, BERLIN, GERMANY

GERMAN-AEROSP-RES-ESTAB, INST PLANETARY EXPLORAT, OBERPFAFFENHOFEN, GERMANY 
11. Authors: Mcewen-AS Gaddis-LR Neukum-G Hoffman-H Pieters-CM

Head-JW

Title: Galileo Observations of Post-Imbrium Lunar Craters

During the 1st Earth-Moon Flyby

Source: JOURNAL OF GEOPHYSICAL RESEARCH-PLANETS

1993, Vol 98, Iss E9, pp 17207-17231

Addresses:

US-GEOL-SURVEY, 2255 N GEMINI DR, FLAGSTAFF, AZ 86001, USA

GERMAN-AEROSP-RES, INST PLANETARY EXPLORAT, BERLIN, GERMANY

BROWN-UNIV, DEPT GEOL SCI, PROVIDENCE, RI 02912, USA

12. Authors: Tenbrink-US Benavraham-Z Bell-RE Hassouneh-M

Coleman-DF Andreasen-G Tibor-G Coakley-B

Title: Structure of the Dead-Sea Pull-Apart Basin from Gravity Analyses

Source: JOURNAL OF GEOPHYSICAL RESEARCH-SOLID EARTH 1993, Vol 98, Iss B12, pp 21877-21894

Addresses:

US-GEOL-SURVEY, QUINSETT CAMPUS, WOODS-HOLE, MA 02543, USA

TEL-AVIV-UNIV, DEPT GEOPHYS \& PLANETARY SCI, IL-69978

TEL-AVIV, ISRAEL

COLUMBIA-UNIV, LAMONT DOHERTY GEOL OBSERV, PALISADES, NY

10964, USA

NAT-RESOURCES-AUTHOR, DIV GEOL, AMMAN, JORDAN

US-GEOL-SURVEY, RESTON, VA 22092, USA

13. Authors: Spotl-C Matter-A Brevart-O

Title: Diagenesis and Pore-Water Evolution in the Keuper

Reservoir, Paris Basin (France)

Source: JOURNAL OF SEDIMENTARY PETROLOGY

1993, Vol 63, Iss 5, pp 909-928

Addresses:

US-GEOL-SURVEY, 915 NATL CTR, 12201 SUNRISE VALLEY DR, RESTON, VA 22092, USA

UNIV-BERN, INST GEOL, CH-3012 BERN, SWITZERLAND

SOC-NATL-ELF-AQUITAINE, CTR SCI \& TECHN JEAN FEGER, F-64018

PAU, FRANCE

14. Authors: Lipman-PW Bogatikov-OA Tsvetkov-AA Gazis-C

Gurbanov-AG Hon-K Koronovsky-NV Kovalenko-VI

Marchev-P

Title: 2.8-Ma Ash-Flow Caldera at Chegem River in the Northern

Caucasus Mountains (Russia), Contemporaneous Granites,

and Associated Ore-Deposits

Source: JOURNAL OF VOLCANOLOGY AND GEOTHERMAL RESEARCH 1993, Vol 57, Iss 1-2, pp 85-124

Addresses:

US-GEOL-SURVEY, MS910, 345 MIDDLEFIELD RD, MENLO-PK, CA 94025, USA

RUSSIAN-ACAD-SCI, INST GEOL ORE DEPOSITS PETROG MINERAL \& GEOCHEM, MOSCOW 109017, RUSSIA

MOSCOW-MV-LOMONOSOV-STATE-UNIV, FAC GEOL, MOSCOW 119899, RUSSIA

BULGARIAN-ACAD-SCI, INST GEOL, BU-1113 SOFIA, BULGARIA

CALTECH, DIV GEOL \& PLANETARY SCI, PASADENA, CA 91125, USA

US-GEOL-SURVEY, DENVER FED CTR, DENVER, CO 80225, USA 
15. Authors: Ikeya-N Cronin-TM

Title: Quantitative-Analysis of Ostracoda and Water Masses Around Japan - Application to Pliocene and Pleistocene Paleoceanography

Source: MICROPALEONTOLOGY 1993, Vol 39, Iss 3, pp 263-281

Addresses:

SHIZUOKA-UNIV, INST GEOSCI, SHIZUOKA 422, JAPAN

US-GEOL-SURVEY, RESTON, VA 22092, USA

16. Authors: Barker-CE Pawlewicz-MJ

Title: An Empirical Determination of the Minimum Number of Measurements Needed to Estimate the Mean Random Vitrinite Reflectance of Disseminated Organic-Matter

Source: ORGANIC GEOCHEMISTRY 1993, Vol 20, Iss 6, pp 643-651

Addresses:

US-GEOL-SURVEY, MAIL STOP 960, FED CTR BOX 25046, LAKEWOOD, CO 80225, USA

UNIV-ADELAIDE, ADELAIDE, SA 5001, AUSTRALIA

17. Authors: Spudis-PD Ryder-G Taylor-GJ Mccormick-KA Keil-K Grieve-RAF

Title: Sources of Mineral Fragments in Impact Melt-15445 and Melt-15455 - Toward the Origin of Low-K Fra-Mauro Basalt

Source: PROCEEDINGS OF LUNAR AND PLANETARY SCIENCE 1991, Vol 21, pp 151-165

Addresses:

US-GEOL-SURVEY, ASTROGEOL BRANCH, 2255 N GEMINI DR, FLAGSTAFF, AZ 86001, USA

GEOL-SURVEY-CANADA, DIV GEOPHYS, OTTAWA K1A-0Y3, ON, CANADA

UNIV-HAWAII, SCH OCEAN EARTH SCI \& TECHNOL, DEPT GEOL \&

GEOPHYS, HONOLULU, HI 96822, USA

LUNAR-\&-PLANETARY-INST, HOUSTON, TX 77058, USA

18. Authors: Vanderhilst-RD Engdahl-ER Spakman-W

Title: Tomographic Inversion of P-Data and pp-Data for

Aspherical Mantle Structure Below the Northwest Pacific

Region

Source: GEOPHYSICAL JOURNAL INTERNATIONAL 1993, Vol 115, Iss 1, pp 264-302

Addresses:

UNIV-LEEDS, DEPT EARTH SCI, LEEDS LS2-9JT, W-YORKSHIRE, ENGLAND

US-GEOL-SURVEY, NATL EARTHQUAKE INFORMAT CTR, DENVER, CO 80225, USA

UNIV-UTRECHT, DEPT THEORET GEOPHYS, 3508-TA UTRECHT, NETHERLANDS

19. Authors: Clark-PU Clague-JJ Curry-BB Dreimanis-A Hicock-SR Miller-GH Berger-GW Eyles-N Lamothe-M Miller-BB

Mott-RJ Oldale-RN Stea-RR Szabo-JP Thorleifson-LH

Vincent-JS

Title: Initiation and Development of the Laurentide and Cordilleran Ice Sheets Following the Last Interglaciation

Source: QUATERNARY SCIENCE REVIEWS

1993, Vol 12, Iss 2, pp 79-114 
Addresses:

OREGON-STATE-UNIV, DEPT GEOSCI, CORVALLIS, OR 97331, USA

GEOL-SURVEY-CANADA, VANCOUVER V6B-1R8, BC, CANADA

ILLINOIS-STATE-GEOL-SURVEY, CHAMPAIGN, IL 61820, USA

UNIV-WESTERN-ONTARIO, DEPT GEOL, LONDON N6A-5B7, ONTARIO,

CANADA

UNIV-COLORADO, INST ARCTIC \& ALPINE RES, CTR GEOCHRONOL RES,

BOULDER, CO 80309, USA

UNIV-COLORADO, DEPT GEOL SCI, BOULDER, CO 80309, USA

WESTERN-WASHINGTON-UNIV, DEPT GEOL, BELLINGHAM, WA 98225, USA

UNIV-TORONTO, DEPT GEOL, SCARBOROUGH M1C-1A4, ON, CANADA

DEPT-NAT-RESOURCES, HALIFAX B3J-2T9, NS, CANADA

UNIV-AKRON, DEPT GEOL, AKRON, OH 44325, USA

GEOL-SURVEY-CANADA, OTTAWA K1A-0E8, ONTARIO, CANADA

UNIV-QUEBEC, DEPT SCI TERRE, MONTREAL H3C-3P8, QUEBEC, CANADA

KENT-STATE-UNIV, DEPT GEOL, KENT, OH 44242, USA

US-GEOL-SURVEY, WOODS-HOLE, MA 02543, USA

20. Authors: Hawthorne-FC Ungaretti-L Oberti-R Bottazzi-P

Czamanske-GK

Title: Li - An Important Component in Igneous Alkali Amphiboles

Source: AMERICAN MINERALOGIST

1993, Vol 78, Iss 7-8, pp 733-745

Addresses:

CNR, CTR STUDIO CRISTALLOCHIM \& CRISTALLOG, VIA BASSI 4, I-27100 PAVIA, ITALY

US-GEOL-SURVEY, MENLO-PK, CA 94025, USA

21. Authors: Voncken-JHL Vanroermund-HLM Vandereerden-AMJ

Jansen-JBH Erd-RC

Title: Holotype Buddingtonite - An Ammonium Feldspar Without Zeolitic $\mathrm{H} 2 \mathrm{O}$

Source: AMERICAN MINERALOGIST

1993, Vol 78, Iss 1-2, pp 204-209

Addresses:

FAC-EARTH-SCI-UTRECHT, DEPT CHEM GEOL, 3508-TA UTRECHT, NETHERLANDS

US-GEOL-SURVEY, IGNEOUS \& GEOTHERMAL PROC BRANCH, MENLO-PK, CA 94025, USA

22. Authors: Cocco-M Boatwright-J

Title: The Envelopes of Acceleration Time Histories

Source: BULLETIN OF THE SEISMOLOGICAL SOCIETY OF AMERICA 1993, Vol 83, Iss 4, pp 1095-1114

Addresses:

IST-NAZL-GEOFIS, VIA DI VIGNA MURATA 605, I-00193 ROME, ITALY

US-GEOL-SURVEY, MENLO-PK, CA 94025, USA

23. Authors: Harlow-DH White-RA Rymer-MJ Alvarez-S

Title: The San-Salvador Earthquake of 10 October 1986 and Its

Historical Context

Source: BULLETIN OF THE SEISMOLOGICAL SOCIETY OF AMERICA 1993, Vol 83, Iss 4, pp 1143-1154

Addresses:

US-GEOL-SURVEY, OFF EARTHQUAKES VOLCANOES \& ENGN, 345

MIDDLEFIELD RD, MS 977, MENLO-PK, CA 94025, USA 
MINIST-OBRAS-PUBL, CTR INVEST GEOTECN, DEPT SISMOL,

SAN-SALVADOR, EL-SALVADOR

24. Authors: Foulger-GR Julian-BR

Title: Non-Double-Couple Earthquakes at the Hengill-Grensdalur

Volcanic Complex, Iceland - Are They Artifacts of

Crustal Heterogeneity

Source: BULLETIN OF THE SEISMOLOGICAL SOCIETY OF AMERICA 1993, Vol 83, Iss 1, pp 38-52

Addresses:

UNIV-DURHAM, DEPT GEOL SCI, SCI LABS, DURHAM DH1-3LE, ENGLAND

US-GEOL-SURVEY, MENLO-PK, CA 94025, USA

25. Authors: Dennison-WC Orth-RJ Moore-KA Stevenson-JC Carter-V Kollar-S Bergstrom-PW Batiuk-RA

Title: Assessing Water-Quality with Submersed Aquatic Vegetation

Source: BIOSCIENCE

1993, Vol 43, Iss 2, pp 86-94

Addresses:

COLL-WILLIAM-\&-MARY, VIRGINIA INST MARINE SCI,

GLOUCESTER-POINT, VA 23602, USA

UNIV-QUEENSLAND, DEPT BOT, ST-LUCIA, QLD 4072, AUSTRALIA

UNIV-MARYLAND, HORN POINT ENVIRONM LAB, CAMBRIDGE, MD 21613, USA

HARFORD-COMMUNITY-COLL, BEL-AIR, MD 21014, USA

COMP-SCI-CORP, ANNAPOLIS, MD 21403, USA

US-GEOL-SURVEY, RESTON, VA 22092, USA

US-EPA, CHESAPEAKE BAY PROGRAM OFF, ANNAPOLIS, MD 21403, USA

26. Authors: Churcher-CS Morgan-AV Carter-LD

Title: Arctodus-Simus from the Alaskan Arctic Slope

Source: CANADIAN JOURNAL OF EARTH SCIENCES 1993, Vol 30, Iss 5, pp 1007-1013

Addresses:

UNIV-TORONTO, DEPT ZOOL, TORONTO M5S-1A1, ONTARIO, CANADA

UNIV-WATERLOO, DEPT EARTH SCI, WATERLOO N2L-3G1, ONTARIO,

CANADA

US-GEOL-SURVEY, ANCHORAGE, AK 99508, USA

27. Authors: Foord-EE Birmingham-SD Demartin-F Pilati-T

Gramaccioli-CM Lichte-FE

Title: Thortveitite and Associated SC-Bearing Minerals from

Ravalli County, Montana

Source: CANADIAN MINERALOGIST

1993, Vol 31, Iss JUN, pp 337-346

Addresses:

US-GEOL-SURVEY, DENVER FED CTR, MS 905, DENVER, CO 80225, USA

BOULDER-SCI-CO, MEAD, CO 80542, USA

UNIV-MILAN, IST CHIM STRUTTURIST INORGAN, I-20133 MILAN, ITALY

CNR, CTR STUDIO RELAZIONI STRUTTURA \& REATTIVITA CHIM, I-20133

MILAN, ITALY

UNIV-MILAN, DIPARTIMENTO SCI TERRA, I-20133 MILAN, ITALY 
28. Authors: Whitney-G Velde-B

Title: Changes in Particle Morphology During Illitization - An Experimental-Study

Source: CLAYS AND CLAY MINERALS 1993, Vol 41, Iss 2, pp 209-218

Addresses:

US-GEOL-SURVEY, MS 904 BOX 25046, DENVER, CO 80225, USA

ECOLE-NORMALE-SUPER, F-75231 PARIS, FRANCE

29. Authors: Eberl-DD Velde-B Mccormick-T

Title: Synthesis of Illite-Smectite from Smectite at Earth

Surface Temperatures and High $\mathrm{pH}$

Source: CLAY MINERALS 1993, Vol 28, Iss 1, pp 49-60

Addresses:

US-GEOL-SURVEY, 3215 MARINE ST, BOULDER, CO 80303, USA

ECOLE-NORMALE-SUPER, DEPT GEOL, F-75231 PARIS 05, FRANCE

UNIV-COLORADO, DEPT GEOL SCI, BOULDER, CO 80309, USA

30. Authors: Lange-IM Nokleberg-WJ Newkirk-SR Aleinikoff-JN

Church-SE Krouse-HR

Title: Devonian Volcanogenic Massive Sulfide Deposits and Occurrences, Southern Yukon-Tanana Terrane, Eastern Alaska Range, Alaska

Source: ECONOMIC GEOLOGY AND THE BULLETIN OF THE SOCIETY OF ECONOMIC GEOLOGISTS 1993, Vol 88, Iss 2, pp 344-376

Addresses:

US-GEOL-SURVEY, MENLO-PK, CA 94025, USA

US-GEOL-SURVEY, DENVER, CO 80225, USA

UNIV-ARIZONA, DEPT GEOSCI, TUCSON, AZ 85721, USA

UNIV-CALGARY, DEPT PHYS, CALGARY T2N-1N4, ALBERTA, CANADA

31. Authors: Stamatakis-MG Hein-JR

Title: Origin of Barite in Tertiary Marine Sedimentary-Rocks from Lefkas Island, Greece

Source: ECONOMIC GEOLOGY AND THE BULLETIN OF THE SOCIETY OF ECONOMIC GEOLOGISTS 1993, Vol 88, Iss 1, pp 91-103

Addresses:

UNIV-ATHENS, DEPT GEOL, PANEPISTIMIOPOLIS ANO ILISSIA 15784, ATHENS, GREECE

US-GEOL-SURVEY, MENLO-PK, CA 94025, USA

32. Authors: Barbanti-A Bothner-MH

Title: A Procedure for Partitioning Bulk Sediments into Distinct Grain-Size Fractions for Geochemical Analysis

Source: ENVIRONMENTAL GEOLOGY 1993, Vol 21, Iss 1-2, pp 3-13

Addresses:

CNR, IST GEOL MARINA, VIA ZAMBONI 65, I-40126 BOLOGNA, ITALY

US-GEOL-SURVEY, WOODS-HOLE, MA 02543, USA 
33. Authors: Cheng-RT Casulli-V Gartner-JW

Title: Tidal, Residual, Intertidal Mudflat (Trim) Model and Its Applications to San-Francisco Bay, California

Source: ESTUARINE COASTAL AND SHELF SCIENCE 1993, Vol 36, Iss 3, pp 235-280

Addresses:

US-GEOL-SURVEY, MENLO-PK, CA 94025, USA

UNIV-TRENT, DEPT MATH, I-38050 TRENT, ITALY

34. Authors: Caffrey-JM Sloth-NP Kaspar-HF Blackburn-TH

Title: Effect of Organic Loading on Nitrification and Denitrification in a Marine Sediment Microcosm

Source: FEMS MICROBIOLOGY ECOLOGY 1993, Vol 12, Iss 3, pp 159-167

Addresses:

US-GEOL-SURVEY, 345 MIDDLEFIELD RD, MS 496, MENLO-PK, CA 94025, USA

AARHUS-UNIV, DEPT ECOL \& GENET, DK-8000 AARHUS, DENMARK

CAWTHORN-INST, NELSON, NEW-ZEALAND

35. Authors: Kvenvolden-KA Ginsburg-GD Soloviev-VA

Title: Worldwide Distribution of Subaquatic Gas Hydrates

Source: GEO-MARINE LETTERS 1993, Vol 13, Iss 1, pp 32-40

Addresses:

US-GEOL-SURVEY, 345 MIDDLEFIELD RD, MAIL STOP 999, MENLO-PK, CA 94025, USA

VNIIOKEANGEOL, 190121 ST-PETERSBURG, RUSSIA

36. Authors: Ginsburg-GD Soloviev-VA Cranston-RE Lorenson-TD Kvenvolden-KA

Title: Gas Hydrates from the Continental-Slope, Offshore Sakhalin Island, Okhotsk Sea

Source: GEO-MARINE LETTERS 1993, Vol 13, Iss 1, pp 41-48

Addresses:

RES-INST-GEOL-\&-MINERAL-RESOURCES-OCEAN, MAKLINA PR 1, 190121 ST-PETERSBURG, RUSSIA

GEOL-SURVEY-CANADA, ATLANTIC GEOSCI CTR, DARTMOUTH B2Y-4A2, NS, CANADA

US-GEOL-SURVEY, MENLO-PK, CA 94025, USA

37. Authors: Keir-RS Michel-RL

Title: Interface Dissolution Control of the C-14 Profile in Marine Sediment

Source: GEOCHIMICA ET COSMOCHIMICA ACTA 1993, Vol 57, Iss 15, pp 3563-3573

Addresses:

CHRISTIAN-ALBRECHTS-UNIV-KIEL, MARINE GEOWISSENSCH FORSCHUNGSZENTRUM, GEOMAR, WISCHHOFSTR 1-3, W-2300 KIEL 1, GERMANY

US-GEOL-SURVEY, RESTON, VA 22092, USA 
38. Authors: Wooden-JL Czamanske-GK Fedorenko-VA Arndt-NT

Chauvel-C Bouse-RM King-BSW Knight-RJ Siems-DF

Title: Isotopic and Trace-Element Constraints on Mantle and

Crustal Contributions to Siberian Continental Flood

Basalts, Norilsk Area, Siberia

Source: GEOCHIMICA ET COSMOCHIMICA ACTA

1993, Vol 57, Iss 15, pp 3677-3704

Addresses:

US-GEOL-SURVEY, 345 MIDDLEFIELD RD, MENLO-PK, CA 94025, USA

CENT-RES-INST-GEOL-PROSPECTING-BASE-\&-PRECIOUS-MET, MOSCOW 113545, RUSSIA

UNIV-RENNES-1, GEOSCI RENNES, F-35042 RENNES, FRANCE

US-GEOL-SURVEY, DENVER FED CTR, DENVER, CO 80225, USA

39. Authors: Richet-P Robie-RA Hemingway-BS

Title: Entropy and Structure of Silicate-Glasses and Melts

Source: GEOCHIMICA ET COSMOCHIMICA ACTA

1993, Vol 57, Iss 12, pp 2751-2766

Addresses:

INST-PHYS-GLOBE, CNRS, URA 734, 4 PL JUSSIEU, F-75252 PARIS,

FRANCE

US-GEOL-SURVEY, RESTON, VA 22092, USA

40. Authors: Honda-M Mcdougall-I Patterson-DB Doulgeris-A

Clague-DA

Title: Noble-Gases in Submarine Pillow Basalt Glasses from

Loihi and Kilauea, Hawaii - A Solar Component in the

Earth

Source: GEOCHIMICA ET COSMOCHIMICA ACTA 1993, Vol 57, Iss 4, pp 859-874

Addresses:

AUSTRALIAN-NATL-UNIV, RES SCH EARTH SCI, GPO BOX 4, CANBERRA, ACT 2601, AUSTRALIA

US-GEOL-SURVEY, HAWAII VOLCANO OBSERV, HAWAII-NATL-PK, HI 96718, USA

41. Authors: Kennedy-WJ Cobban-WA

Title: Maastrichtian Ammonites from the Corsicana Formation in Northeast Texas

Source: GEOLOGICAL MAGAZINE 1993, Vol 130, Iss 1, pp 57-67

Addresses:

UNIV-OXFORD-MUSEUM, PARKS RD, OXFORD OX1-3PW, ENGLAND

US-GEOL-SURVEY, DENVER FED CTR, PALEONTOL \& STRATIG BRANCH, DENVER, CO 80225, USA

42. Authors: Mueller-PA Shuster-RD Wooden-JL Erslev-EA Bowes-DR

Title: Age and Composition of Archean Crystalline Rocks from the Southern Madison Range, Montana - Implications for Crustal Evolution in the Wyoming Craton

Source: GEOLOGICAL SOCIETY OF AMERICA BULLETIN 1993, Vol 105, Iss 4, pp 437-446

Addresses:

UNIV-FLORIDA, DEPT GEOL, GAINESVILLE, FL 32611, USA

US-GEOL-SURVEY, MENLO-PK, CA 94025, USA 
COLORADO-STATE-UNIV, DEPT EARTH RESOURCES, FT-COLLINS, CO

80523, USA

UNIV-GLASGOW, DEPT GEOL \& APPL GEOL, GLASGOW G12-8QQ, SCOTLAND

43. Authors: Bloch-RB Vonhuene-R Hart-PE Wentworth-CM

Title: Style and Magnitude of Tectonic Shortening Normal to the

San-Andreas Fault Across Pyramid Hills and Kettleman

Hills South Dome, California

Source: GEOLOGICAL SOCIETY OF AMERICA BULLETIN

1993, Vol 105, Iss 4, pp 464-478

Addresses:

STANFORD-UNIV, DEPT APPL EARTH SCI, STANFORD, CA 94305, USA

GEOMAR, KIEL, GERMANY

US-GEOL-SURVEY, MENLO-PK, CA 94025, USA

44. Authors: King-G Sturdy-D Whitney-J

Title: The Landscape Geometry and Active Tectonics of Northwest

Greece

Source: GEOLOGICAL SOCIETY OF AMERICA BULLETIN

1993, Vol 105, Iss 2, pp 137-161

Addresses:

INST-PHYS-GLOBE, 5 RUE RENE DESCARTES, F-67084 STRASBOURG,

FRANCE

UNIV-LIVERPOOL, INST PREHIST SCI \& ARCHAEOL, LIVERPOOL L69-3BX, ENGLAND

US-GEOL-SURVEY, DENVER FED CTR, DENVER, CO 80225, USA

45. Authors: Hagstrum-JT Martinez-ML York-D

Title: Paleomagnetic and Ar-40/Ar-39 Evidence for

Remagnetization of Mesozoic Oceanic Rocks on the

Vizcaino Peninsula, Baja-California-sur, Mexico

Source: GEOPHYSICAL RESEARCH LETTERS

1993, Vol 20, Iss 17, pp 1831-1834

Addresses:

US-GEOL-SURVEY, 345 MIDDLEFIELD RD, MS 937, MENLO-PK, CA

94025, USA

CTR-INVEST-CIENT-\&-EDUC-SUPER-ENSENADA, ENSENADA,

BAJA-CALIFORNIA, MEXICO

UNIV-TORONTO, DEPT PHYS, TORONTO M5S-1A7, ONTARIO, CANADA

46. Authors: Pribnow-D Williams-CF Burkhardt-H

Title: Well Log-Derived Estimates of Thermal-Conductivity in

Crystalline Rocks Penetrated by the 4-km Deep KTB

Vorbohrung

Source: GEOPHYSICAL RESEARCH LETTERS

1993, Vol 20, Iss 12, pp 1155-1158

Addresses:

KTB-FELDLAB, W-8486 WINDISCHESCHENBAC, GERMANY

INST-ANGEW-GEOPHYS, BERLIN, GERMANY

US-GEOL-SURVEY, MENLO-PK, CA 94025, USA

47. Authors: Thomas-RE Khan-MR Khan-SA

Title: Coal Resources of the Sonda Coal Field, Sindh Province, Pakistan

Source: INTERNATIONAL JOURNAL OF COAL GEOLOGY

1993, Vol 23, Iss 1-4, pp 159-\& 
Addresses:

US-GEOL-SURVEY, NATL CTR, COAL GEOL BRANCH, MS956, RESTON, VA 22092, USA

GEOL-SURVEY-PAKISTAN, KARACHI, PAKISTAN

48. Authors: Eklundh-L Singh-A

Title: A Comparative-Analysis of Standardized and

Unstandardized Principal Components-Analysis in Remote-

Sensing

Source: INTERNATIONAL JOURNAL OF REMOTE SENSING 1993, Vol 14, Iss 7, pp 1359-1370

Addresses:

UNEP, GRID, POB 30552, NAIROBI, KENYA

US-GEOL-SURVEY, EROS DATA CTR, GRID SIOUX FALLS, SIOUX-FALLS, SD 57198, USA

49. Authors: Murphy-DM Garbarino-JR Taylor-HE Hart-BT Beckett-R

Title: Determination of Size and Element Composition

Distributions of Complex Colloids by Sedimentation Field-

Flow Fractionation Inductively-Coupled Plasma-Mass

Spectrometry

Source: JOURNAL OF CHROMATOGRAPHY

1993, Vol 642, Iss 1-2, pp 459-467

Addresses:

MONASH-UNIV, CTR WATER STUDIES, DEPT CHEM, 900 DANDENONG RD,

CAULFIELD E, MELBOURNE, VIC 3145, AUSTRALIA

US-GEOL-SURVEY, DIV WATER RESOURCES, BOULDER, CO, USA

50. Authors: Nelson-AR Kashima-K

Title: Diatom Zonation in Southern Oregon Tidal Marshes

Relative to Vascular Plants, Foraminifera, and Sea-Level

Source: JOURNAL OF COASTAL RESEARCH 1993, Vol 9, Iss 3, pp 673-697

Addresses:

US-GEOL-SURVEY, MS 966, POB 25046, DENVER, CO 80225, USA

KYUSHU-UNIV, COLL GEN EDUC, FUKUOKA 810, JAPAN

51. Authors: Green-AW Worthington-EW Baransky-LN Fedorov-EN

Kurneva-NA Pilipenko-VA Shvetzov-DN Bektemirov-AA

Philipov-GV

Title: Alfven Field Line Resonances at Low Latitudes $(\mathrm{L}=1.5)$

Source: JOURNAL OF GEOPHYSICAL RESEARCH-SPACE PHYSICS 1993, Vol 98, Iss A9, pp 15693-15699

Addresses:

US-GEOL-SURVEY, BOX 25046, MS 968, DENVER, CO 80225, USA

MOSCOW-PHYS-EARTH-INST, MOSCOW 123810, RUSSIA

KYRGYZIAN-ACAD-SCI, INST SEISMOL, BISHKEK 720060, KYRGYZSTAN

52. Authors: Campbell-WH Arora-BR Schiffmacher-ER

Title: External Sq Currents in the India-Siberia Region

Source: JOURNAL OF GEOPHYSICAL RESEARCH-SPACE PHYSICS 1993, Vol 98, Iss A3, pp 3741-3752

Addresses:

US-GEOL-SURVEY, MAIL STOP 968, BOX 25046, DENVER, CO 80225, USA

INDIAN-INST-GEOMAGNETISM, BOMBAY 400005, INDIA 
53. Authors: Suzukikamata-K Kamata-H Bacon-CR

Title: Evolution of the Caldera-Forming Eruption at Crater Lake, Oregon, Indicated by Component Analysis of Lithic Fragments

Source: JOURNAL OF GEOPHYSICAL RESEARCH-SOLID EARTH 1993, Vol 98, Iss B8, pp 14059-14074

Addresses:

US-GEOL-SURVEY, MENLO-PK, CA 94025, USA

GEOL-SURVEY-JAPAN, OSAKA OFF, CHUO KU, OSAKA 540, JAPAN

54. Authors: Heki-K Foulger-GR Julian-BR Jahn-CH

Title: Plate Dynamics Near Divergent Boundaries - Geophysical

Implications of Postrifting Crustal Deformation in $\mathrm{Ne}$ Iceland

Source: JOURNAL OF GEOPHYSICAL RESEARCH-SOLID EARTH 1993, Vol 98, Iss B8, pp 14279-14297

Addresses:

UNIV-DURHAM, DEPT GEOL SCI, DURHAM DH1-3HP, ENGLAND

US-GEOL-SURVEY, MENLO-PK, CA 94025, USA

UNIV-HANNOVER, INST ERDMESSUNG, W-3000 HANNOVER, GERMANY

55. Authors: Velde-B Moore-D Badri-A Ledesert-B

Title: Fractal and Length Analysis of Fractures During Brittle to Ductile Changes

Source: JOURNAL OF GEOPHYSICAL RESEARCH-SOLID EARTH 1993, Vol 98, Iss B7, pp 11935-11940

Addresses:

ECOLE-NORMALE-SUPER, DEPT GEOL, UR 1316, 24 RUE LHOMOND, F-75235 PARIS, FRANCE

US-GEOL-SURVEY, MENLO-PK, CA 94025, USA

UNIV-POITIERS, ALTERAT HYDROTHERMALES LAB, F-86022 POITIERS, FRANCE

UNIV-POITIERS, MECAN FLUIDES LAB, F-86022 POITIERS, FRANCE

56. Authors: Pantosti-D Schwartz-DP Valensise-G

Title: Paleoseismology Along the 1980 Surface Rupture of the Irpinia Fault - Implications for Earthquake Recurrence in the Southern Apennines, Italy

Source: JOURNAL OF GEOPHYSICAL RESEARCH-SOLID EARTH 1993, Vol 98, Iss B4, pp 6561-\&

Addresses:

IST-NAZL-GEOFIS, VIA VIGNA MURATA 605, I-00143 ROME, ITALY

US-GEOL-SURVEY, MENLO-PK, CA 94025, USA

57. Authors: Foreman-MGG Henry-RF Walters-RA Ballantyne-VA

Title: A Finite-Element Model for Tides and Resonance Along the North Coast of British-Columbia

Source: JOURNAL OF GEOPHYSICAL RESEARCH-OCEANS 1993, Vol 98, Iss C2, pp 2509-2531

Addresses:

FISHERIES-\&-OCEANS-CANADA, INST OCEAN SCI, POB 6000, SIDNEY V8L-4B2, BC, CANADA

US-GEOL-SURVEY, TACOMA, WA 98402, USA 
58. Authors: Smith-DB Theobald-PK Shen-SQ Ren-TX Hou-ZH

Title: The Hatu Gold Anomaly, Xinjiang-Uygur Autonomous Region, China - Testing the Hypothesis of Aeolian Transport of Gold

Source: JOURNAL OF GEOCHEMICAL EXPLORATION 1993, Vol 47, Iss 1-3, pp 201-216

Addresses:

US-GEOL-SURVEY, DENVER FED CTR, BOX 25046, MAIL STOP 973, DENVER, CO 80225, USA

MINIST-GEOL-\&-MINERAL-RESOURCES, ADVISORY COMM SCI \& TECHNOL, BEIJING, PEOPLES-R-CHINA

INST-GEOPHYS-\&-GEOCHEM-EXPLORAT, LANGFANG 102849, PEOPLES-R-CHINA

59. Authors: Nordstrom-DK Mcnutt-RH Puigdomenech-I Smellie-JAT Wolf-M

Title: Ground-Water Chemistry and Geochemical Modeling of Water Rock Interactions at the Osamu Utsumi Mine and the MorroDo-Ferro Analog Study Sites, Pocos-de-Caldas, MinasGerais, Brazil

Source: JOURNAL OF GEOCHEMICAL EXPLORATION 1992, Vol 45, Iss 1-3, pp 249-287

Addresses:

US-GEOL-SURVEY, DENVER FED CTR, MS-458, BOULDER POUCH, BOX 25046, DENVER, CO 80225, USA

MCMASTER-UNIV, DEPT GEOL, HAMILTON L8S-4K1, ONTARIO, CANADA

STUDSVIK-AB, S-61182 NYKOPING, SWEDEN

CONTERRA-AB, S-75106 UPPSALA, SWEDEN

GSF-MUNICH, INST HYDROL, W-8042 NEUHERBERG, GERMANY

60. Authors: Woo-MK Winter-TC

Title: The Role of Permafrost and Seasonal Frost in the Hydrology of Northern Wetlands in North-America

Source: JOURNAL OF HYDROLOGY 1993, Vol 141, Iss 1-4, pp 5-31

Addresses:

US-GEOL-SURVEY, DENVER FED CTR, BOX 25046, MAIL STOP 413, DENVER, CO 80225, USA

MCMASTER-UNIV, DEPT GEOG, HAMILTON L8S-4K1, ONTARIO, CANADA

61. Authors: Cobban-WA Kennedy-WJ

Title: Middle Campanian Ammonites and Inoceramids from the Wolfe-City Sand in Northeastern Texas

Source: JOURNAL OF PALEONTOLOGY 1993, Vol 67, Iss 1, pp 71-82

Addresses:

US-GEOL-SURVEY, DENVER FED CTR, BOX 25046, MAIL STOP 919, DENVER, CO 80225, USA

UNIV-OXFORD-MUSEUM, GEOL COLLECT, OXFORD OX1-3PW, ENGLAND

62. Authors: Kennedy-WJ Cobban-WA

Title: Campanian Ammonites from the Annona Chalk Near Yancy, Arkansas

Source: JOURNAL OF PALEONTOLOGY 1993, Vol 67, Iss 1, pp 83-97 
Addresses:

UNIV-OXFORD-MUSEUM, GEOL COLLECT, PARKS RD, OXFORD OX1-3PW, ENGLAND

US-GEOL-SURVEY, DENVER FED CTR, DENVER, CO 80225, USA

63. Authors: Sheppard-DS Janik-CJ Keith-TEC

Title: A Comparison of Gas Geochemistry of Fumaroles in the 1912 Ash-Flow Sheet and on Active Stratovolcanoes, Katmai-National-Park, Alaska

Source: JOURNAL OF VOLCANOLOGY AND GEOTHERMAL RESEARCH 1992, Vol 53, Iss 1-4, pp 185-197

Addresses:

DSIR, PETONE, NEW-ZEALAND

US-GEOL-SURVEY, MENLO-PK, CA 94025, USA

64. Authors: Vogel-RM Thomas-WO Mcmahon-TA

Title: Flood-Flow Frequency Model Selection in Southwestern United-States

Source: JOURNAL OF WATER RESOURCES PLANNING AND MANAGEMENT-ASCE 1993, Vol 119, Iss 3, pp 353-366

Addresses:

TUFTS-UNIV, DEPT CIVIL ENGN, MEDFORD, MA 02155, USA

US-GEOL-SURVEY, RESTON, VA 22092, USA

UNIV-MELBOURNE, PARKVILLE, VIC 3052, AUSTRALIA

65. Authors: Koeberl-C Hartung-JB Kunk-MJ Klein-J Matsuda-J

Nagao-K Reimold-WU Storzer-D

Title: The Age of the Roter Kamm Impact Crater, Namibia -

Constraints from Ar-40-Ar-39, K-Ar, Rb-Sr, Fission-

Track, and Be-10 Al-26 Studies

Source: METEORITICS

1993, Vol 28, Iss 2, pp 204-212

Addresses:

UNIV-VIENNA, INST GEOCHEM, DR KARL LUEGER RING 1, A-1010

VIENNA, AUSTRIA

IOWA-GEOL-SURV-BUR, IOWA-CITY, IA 52242, USA

US-GEOL-SURVEY, RESTON, VA 22092, USA

UNIV-PENN, DEPT PHYS, PHILADELPHIA, PA 19104, USA

OSAKA-UNIV, DEPT EARTH \& SPACE SCI, TOYONAKA, OSAKA 560, JAPAN

OKAYAMA-UNIV, INST STUDY EARTHS INTERIOR, TOTTORI 68201, JAPAN

UNIV-WITWATERSRAND, DEPT GEOL, ECON GEOL RES UNIT,

JOHANNESBURG 2050, SOUTH-AFRICA

MUSEUM-HIST-NAT, MINERAL LAB, F-75005 PARIS, FRANCE

66. Authors: Coleman-ML Hedrick-DB Lovley-DR White-DC Pye-K

Title: Reduction of Fe(III) in Sediments by Sulfate-Reducing

Bacteria

Source: NATURE

1993, Vol 361, Iss 6411, pp 436-438

Addresses:

UNIV-READING, POSTGRAD RES INST SEDIMENTOL, WHITEKNIGHTS, POB 227, READING RG6-2AB, BERKS, ENGLAND

BP-RES-\&-ENGN-CTR, SUNBURY TW16-7LN, MIDDX, ENGLAND

UNIV-TENNESSEE, CTR ENVIRONM BIOTECHNOL, KNOXVILLE, TN 37932, USA

US-GEOL-SURVEY, DIV WATER RESOURCES, RESTON, VA 22092, USA 
UNIV-TENNESSEE, DEPT MICROBIOL, KNOXVILLE, TN 37996, USA

OAK-RIDGE-NATL-LAB, DIV ENVIRONM SCI, OAK-RIDGE, TN 37831, USA

67. Authors: Ruppert-LF Moore-TA

Title: Differentiation of Volcanic Ash-Fall and Water-Borne

Detrital Layers in the Eocene Senakin Coal Bed, Tanjung

Formation, Indonesia

Source: ORGANIC GEOCHEMISTRY

1993, Vol 20, Iss 2, pp 233-247

Addresses:

US-GEOL-SURVEY, NATL CTR, MS 956, RESTON, VA 22092, USA

UNIV-CANTERBURY, DEPT GEOL, COAL RES GRP, CHRISTCHURCH, NEW-ZEALAND

68. Authors: Fowler-A Walder-J

Title: Creep Closure of Channels in Deforming Subglacial Till

Source: PROCEEDINGS OF THE ROYAL SOCIETY OF LONDON SERIES AMATHEMATICAL AND PHYSICAL SCIENCES 1993, Vol 441, Iss 1911, pp 17-31

Addresses:

MATH-INST, 24-26 ST GILES, OXFORD OX1-3LB, ENGLAND

US-GEOL-SURVEY, CASCADES VOLCANO OBSERV, VANCOUVER, WA 98661, USA

69. Authors: Brouwers-EM Dedeckker-P

Title: Late Maastrichtian and Danian Ostracode Faunas from

Northern Alaska - Reconstructions of Environment and

Paleogeography

Source: PALAIOS

1993, Vol 8, Iss 2, pp 140-154

Addresses:

US-GEOL-SURVEY, MAIL STOP 919, BOX 25046, FED CTR, DENVER, CO

80225, USA

AUSTRALIAN-NATL-UNIV, DEPT GEOL, CANBERRA, ACT 2601, AUSTRALIA

70. Authors: Cronin-TM Whatley-R Wood-A Tsukagoshi-A Ikeya-N

Brouwers-EM Briggs-WM

Title: Microfaunal Evidence for Elevated Pliocene Temperatures in the Arctic-Ocean

Source: PALEOCEANOGRAPHY 1993, Vol 8, Iss 2, pp 161-173

Addresses:

US-GEOL-SURVEY, MS 970 NATL CTR, RESTON, VA 22092, USA

UNIV-COLL-WALES-ABERYSTWYTH, INST EARTH STUDIES, ABERYSTWYTH SY23-1NE, DYFED, WALES

UNIV-TOKYO-MUSEUM, BUNKYO KU, TOKYO, JAPAN

SHIZUOKA-UNIV, INST GEOSCI, OYA, SHIZUOKA 422, JAPAN

US-GEOL-SURVEY, DENVER, CO 80225, USA

UNIV-COLORADO, INSTAAR, BOULDER, CO 80309, USA

71. Authors: Hein-JR Yeh-HW Gunn-SH Sliter-WV Benninger-LM Wang-CH

Title: 2 Major Cenozoic Episodes of Phosphogenesis Recorded in Equatorial Pacific Seamount Deposits

Source: PALEOCEANOGRAPHY

1993, Vol 8, Iss 2, pp 293-311 
Addresses:

US-GEOL-SURVEY, 345 MIDDLEFIELD RD, MS 999, MENLO-PK, CA

94025, USA

UNIV-HAWAII, HAWAII INST GEOPHYS, HONOLULU, HI 96822, USA

UNIV-CALIF-DAVIS, DEPT GEOL, DAVIS, CA 95616, USA

ACAD-SINICA, INST EARTH SCI, TAIPEI 115, TAIWAN

72. Authors: Higgins-SS Larsen-FE Bendel-RB Radamaker-GK

Bassman-JH Bidlake-WR Alwir-A

Title: Comparative Gas-Exchange Characteristics of Potted,

Glasshouse-Grown Almond, Apple, Fig, Grape, Olive, Peach

and Asian Pear

Source: SCIENTIA HORTICULTURAE

1992, Vol 52, Iss 4, pp 313-329

Addresses:

WASHINGTON-STATE-UNIV, DEPT HORT \& LANDSCAPE ARCHITECTURE,

PULLMAN, WA 99164, USA

UNIV-CONNECTICUT, CTR ENVIRONM HLTH, STORRS, CT 06269, USA

WASHINGTON-STATE-UNIV, DEPT NAT RESOURCE SCI, PULLMAN, WA

99164, USA

US-GEOL-SURVEY, TAMPA, FL 33634, USA

UNIV-JORDAN, FAC AGR, AMMAN, JORDAN

73. Authors: Tenbrink-US Bannister-S Beaudoin-BC Stern-TA

Title: Geophysical Investigations of the Tectonic Boundary

Between East and West Antarctica

Source: SCIENCE 1993, Vol 261, Iss 5117, pp 45-50

Addresses:

US-GEOL-SURVEY, WOODS-HOLE, MA 02543, USA

INST-GEOL-\&-NUCL-SCI, WELLINGTON, NEW-ZEALAND

STANFORD-UNIV, DEPT GEOPHYS, STANFORD, CA 94305, USA

VICTORIA-UNIV-WELLINGTON, RES SCH EARTH SCI, WELLINGTON, NEW-ZEALAND

74. Authors: Nelson-CH Baraza-J Maldonado-A

Title: Mediterranean Undercurrent Sandy Contourites, Gulf of Cadiz, Spain

Source: SEDIMENTARY GEOLOGY 1993, Vol 82, Iss 1-4, pp 103-131

Addresses:

US-GEOL-SURVEY, MENLO-PK, CA 94025, USA

CIENCIAS-MAR, E-08039 BARCELONA, SPAIN

75. Authors: Arndt-NT Czamanske-GK Wooden-JL Fedorenko-VA

Title: Mantle and Crustal Contributions to Continental Flood

Volcanism

Source: TECTONOPHYSICS

1993, Vol 223, Iss 1-2, pp 39-52

Addresses:

UNIV-RENNES-1, F-35010 RENNES, FRANCE

CENT-RES-INST-GEOL-PROSPECTING-BASE-\&-PRECIOUS-METALS, MOSCOW 113545 , RUSSIA

US-GEOL-SURVEY, MENLO-PK, CA 94025, USA 
76. Authors: Vonhuene-R Scholl-DW

Title: The Return of Sialic Material to the Mantle Indicated by

Terrigeneous Material Subducted at Convergent Margins

Source: TECTONOPHYSICS

1993, Vol 219, Iss 1-3, pp 163-175

Addresses:

CHRISTIAN-ALBRECHTS-UNIV-KIEL, RES INST, GEOMAR, WISCHOFST

1-3, W-2300 KIEL 14, GERMANY

US-GEOL-SURVEY, MENLO-PK, CA 94025, USA

77. Authors: Console-R Digiovambattista-R Favali-P Presgrave-BW Smriglio-G

Title: Seismicity of the Adriatic Microplate

Source: TECTONOPHYSICS 1993, Vol 218, Iss 4, pp 343-354

Addresses:

IST-NAZL-GEOFIS, VIA VILLA RICOTTI 42, I-00161 ROME, ITALY

US-GEOL-SURVEY, NEIC, DENVER, CO 80225, USA

78. Authors: Konikow-LF Arevalo-JR

Title: Advection and Diffusion in a Variable-Salinity Confining Layer

Source: WATER RESOURCES RESEARCH

1993, Vol 29, Iss 8, pp 2747-2761

Addresses:

US-GEOL-SURVEY, DIV WATER RESOURCES, 431 NATL CTR, RESTON, VA 22092, USA

UNIV-COMPLUTENSE-MADRID, DEPT GEODINAM, MADRID 3, SPAIN

79. Authors: Schmidt-JC Rubin-DM Ikeda-H

Title: Flume Simulation of Recirculating Flow and Sedimentation

Source: WATER RESOURCES RESEARCH 1993, Vol 29, Iss 8, pp 2925-2939

Addresses:

UTAH-STATE-UNIV, COLL NAT RESOURCES, DEPT GEOG \& EARTH RESOURCES, WATERSHED SCI UNIT, LOGAN, UT 84322, USA

US-GEOL-SURVEY, MENLO-PK, CA 94025, USA

UNIV-TSUKUBA, ENVIRONM RES CTR, TSUKUBA, IBARAKI 305, JAPAN

80. Authors: Enzel-Y Ely-LL House-PK Baker-VR Webb-RH

Title: Paleoflood Evidence for a Natural Upper Bound to Flood Magnitudes in the Colorado River Basin

Source: WATER RESOURCES RESEARCH 1993, Vol 29, Iss 7, pp 2287-2297

Addresses:

HEBREW-UNIV-JERUSALEM, INST EARTH SCI, DEPT PHYS GEOG, IL-91904 JERUSALEM, ISRAEL

US-GEOL-SURVEY, TUCSON, AZ 85705, USA

UNIV-ARIZONA, DEPT GEOSCI, ARIZONA LAB PALEOHYDROL \&

HYDROCLIMATOL ANAL, TUCSON, AZ 85721, USA

81. Authors: Harjono-H Sebrier-M Diament-M

Title: Seismicity of the Sundra Strait - Evidence for Crustal Extension and Volcanologic Implications (Vol 10, Pg 17, 1991) 


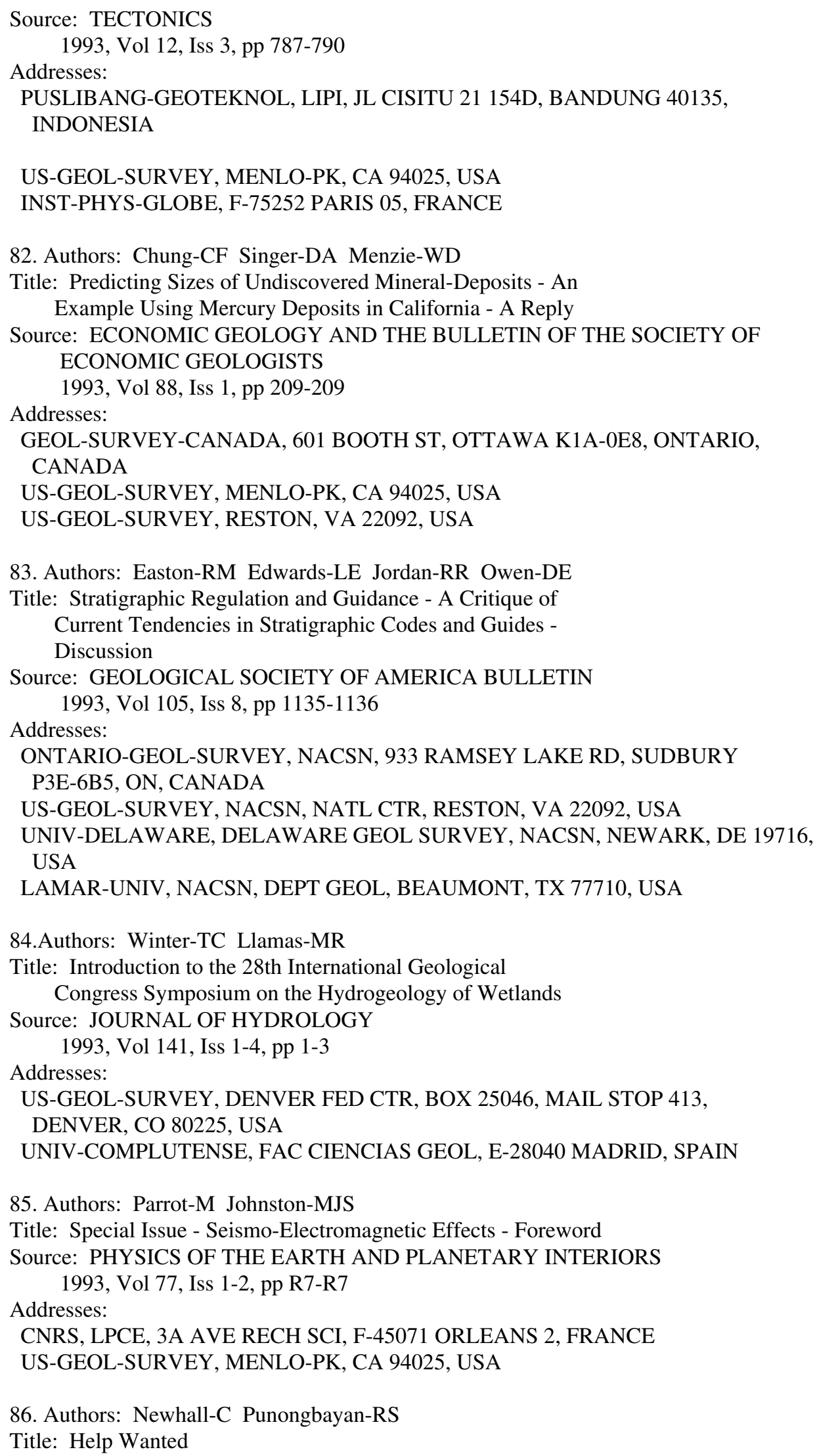

85. Authors: Parrot-M Johnston-MJS

Title: Special Issue - Seismo-Electromagnetic Effects - Foreword

Source: PHYSICS OF THE EARTH AND PLANETARY INTERIORS 1993, Vol 77, Iss 1-2, pp R7-R7

Addresses:

CNRS, LPCE, 3A AVE RECH SCI, F-45071 ORLEANS 2, FRANCE

US-GEOL-SURVEY, MENLO-PK, CA 94025, USA

86. Authors: Newhall-C Punongbayan-RS

Title: Help Wanted 
Source: NATURE

1993, Vol 364, Iss 6438, pp 568-568

Addresses:

US-GEOL-SURVEY, MAIL STOP 959, RESTON, VA 22092, USA

PHILIPPINE-INST-VOLCANOL-\&-SEISMOL, QUEZON, PHILIPPINES

87. Authors: Rice-DD Ekweozor-CM

Title: Occurrence and Origin of Natural Gases, Niger-Delta, Nigeria

Source: ABSTRACTS OF PAPERS OF THE AMERICAN CHEMICAL SOCIETY 1993, Vol 206, Iss AUG, pp 53-GEOC

Addresses:

US-GEOL-SURVEY, DFC, DENVER, CO 80225, USA

UNIV-IBADAN, IBADAN, NIGERIA

88. Authors: Waite-TD Payne-TE Davis-JA

Title: Uranium Sorption to Natural Substrates - Insights

Provided by Isotope Exchange, Selective Extraction and

Surface Complexation Modeling Approaches

Source: ABSTRACTS OF PAPERS OF THE AMERICAN CHEMICAL SOCIETY 1993, Vol 205, Iss MAR, pp 92-GEOC

Addresses:

AUSTRALIAN-NUCL-SCI-\&-TECHNOL-ORG, MENAI, NSW 2234, AUSTRALIA

US-GEOL-SURVEY, DIV WATER RESOURCES, MENLO-PK, CA 94025, USA

89. Authors: Smith-KS Ranville-JF Macalady-DL

Title: Influence of Fulvic-Acid on Trace-Metal Sorption Onto

Ferric Oxyhydroxysulfate Particles in Acidic Systems

Source: ABSTRACTS OF PAPERS OF THE AMERICAN CHEMICAL SOCIETY 1993, Vol 205, Iss MAR, pp 96-GEOC

Addresses:

US-GEOL-SURVEY, DENVER FED CTR, DENVER, CO 80225, USA

COLORADO-SCH-MINES, DEPT CHEM \& GEOCHEM, GOLDEN, CO 80401, USA

MONASH-UNIV, CTR WATER STUDIES, MELBOURNE, VIC 3145, AUSTRALIA

90. Authors: Marsh-JD Ashwood-TL Hicks-DS

Title: Geochemical Factors Influencing the Occurrence of cm-244 and Am-241 in Groundwater at a Shallow Waste Burial Site at Oak-Ridge-National-Laboratory

Source: ABSTRACTS OF PAPERS OF THE AMERICAN CHEMICAL SOCIETY 1993, Vol 205, Iss MAR, pp 131-GEOC

Addresses:

US-GEOL-SURVEY, MENLO-PK, CA 94025, USA

ANSTO, SUTHERLAND, NSW, AUSTRALIA

91. Authors: Davis-JA Payne-TE Waite-TD Kohler-M Kent-DB

Title: Modeling the Effects of $\mathrm{pH}$ and Complexing Ligands on the Adsorption and Mobility of U(VI)

Source: ABSTRACTS OF PAPERS OF THE AMERICAN CHEMICAL SOCIETY 1993, Vol 205, Iss MAR, pp 132-GEOC

Addresses:

US-GEOL-SURVEY, MENLO-PK, CA 94025, USA

ANSTO, SUTHERLAND, NSW, AUSTRALIA 
92. Authors: Columba-M Cunningham-CG

Title: Geologic Model for the Mineral-Deposits of the La-Joya District, Oruro, Bolivia

Source: ECONOMIC GEOLOGY AND THE BULLETIN OF THE SOCIETY OF ECONOMIC GEOLOGISTS 1993, Vol 88, Iss 3, pp 701-708

Addresses:

EMPRESA-MINERA-INTI-RAYMI-SA, CORNETA MAMANI 1989, PISO 2, CASILLA 9576-9676, LA-PAZ, BOLIVIA

US-GEOL-SURVEY, RESTON, VA 22092, USA

93. Authors: Young-JB Aichele-H Presgrave-BW

Title: Region Name Conventions in the Flinn-Engdahl Regionalization Scheme

Source: GEOPHYSICAL JOURNAL INTERNATIONAL 1993, Vol 114, Iss 2, pp 411-413

Addresses:

MINIST-DEF, READING RG7-4RS, BERKS, ENGLAND

SEISMOL-ZENT-OBSERV, W-8520 ERLANGEN, GERMANY

US-GEOL-SURVEY, DENVER FED CTR, DENVER, CO 80225, USA

94. Authors: Forte-AM Peltier-WR Dziewonski-AM Woodward-RL

Title: Dynamic Surface-Topography - A New Interpretation Based upon Mantle Flow Models Derived from Seismic Tomography Reply

Source: GEOPHYSICAL RESEARCH LETTERS 1993, Vol 20, Iss 15, pp 1665-1666

Addresses:

HARVARD-UNIV, DEPT EARTH \& PLANET SCI, 20 OXFORD ST, CAMBRIDGE, MA 02138, USA

US-GEOL-SURVEY, ALBUQUERQUE SEISMOL LAB, ALBUQUERQUE, NM 87115, USA

UNIV-TORONTO, DEPT PHYS, TORONTO M5S-1A7, ONTARIO, CANADA

95. Authors: Kwok-YK Beyer-LA

Title: Gravity Due to a Body with Rotational Symmetry About a Vertical Axis

Source: GEOPHYSICS 1993, Vol 58, Iss 2, pp 298-306

Addresses:

HONG-KONG-UNIV-SCI-\&-TECHNOL, DEPT MATH, CLEAR WATER BAY RD, HONG-KONG, HONG-KONG

US-GEOL-SURVEY, PALO-ALTO, CA 94304, USA

96. Authors: Brenner-IB Taylor-HE

Title: A Critical-Review of Inductively Coupled Plasma-Mass

Spectrometry for Geoanalysis, Geochemistry, and Hydrology .1. Analytical Performance

Source: CRITICAL REVIEWS IN ANALYTICAL CHEMISTRY 1992, Vol 23, Iss 5, pp 355-367

Addresses:

GEOL-SURVEY-ISRAEL, 30 MALKHE ISRAEL ST, IL-95501 JERUSALEM, ISRAEL

US-GEOL-SURVEY, DENVER FED CTR, DENVER, CO 80255, USA 
97. Authors: Slack-JF Palmer-MR Stevens-BPJ Barnes-RG

Title: Origin and Significance of Tourmaline-Rich Rocks in the Broken-Hill District, Australia

Source: ECONOMIC GEOLOGY AND THE BULLETIN OF THE SOCIETY OF ECONOMIC GEOLOGISTS 1993, Vol 88, Iss 3, pp 505-541

Addresses:

US-GEOL-SURVEY, NATL CTR, MAIL STOP 954, RESTON, VA 22092, USA

UNIV-BRISTOL, DEPT GEOL, BRISTOL BS8-1RJ, ENGLAND

GEOL-SURVEY-NEW-S-WALES, BROKEN-HILL, NSW 2880, AUSTRALIA

GEOL-SURVEY-NEW-S-WALES, ARMIDALE, NSW 2350, AUSTRALIA

98. Authors: Kennedy-WJ Cobban-WA

Title: Lower Campanian (Upper Cretaceous) Ammonites from the Merchantville Formation of New-Jersey, Maryland, and Delaware

Source: JOURNAL OF PALEONTOLOGY 1993, Vol 67, Iss 5, pp 828-849

Addresses:

UNIV-OXFORD-MUSEUM, PARKS RD, OXFORD 0X1-3PW, ENGLAND

US-GEOL-SURVEY, DENVER FED CTR, DENVER, CO 80225, USA

99. Authors: Kennedy-WJ Cobban-WA

Title: Ammonites from the Saratoga Chalk (Upper Cretaceous), Arkansas

Source: JOURNAL OF PALEONTOLOGY 1993, Vol 67, Iss 3, pp 404-434

Addresses:

UNIV-OXFORD-MUSEUM, PARKS RD, OXFORD OX1-3PW, ENGLAND

US-GEOL-SURVEY, DENVER FED CTR, PALEONTOL \& STRATIG BRANCH, DENVER, CO 80225, USA

100. Authors: Normark-WR Posamentier-H Mutti-E

Title: Turbidite Systems - State-of-the-Art and Future-Directions

Source: REVIEWS OF GEOPHYSICS 1993, Vol 31, Iss 2, pp 91-116

Addresses:

US-GEOL-SURVEY, MENLO-PK, CA 94025, USA

UNIV-PARMA, IST GEOL, I-43100 PARMA, ITALY

ARCO-EXPLORAT-\&-PROD-TECHNOL, PLANO, TX 75075, USA

101. Authors: Arefyev-SS Pletnev-KG Tatevosyan-RE Borisov-BA Aptekman-JY Vasilyev-VY Delitsyn-LL Romanov-AA Osher-BV Parini-IE Afimyina-TV Shilova-NE Shumilina-LS Javakhishvili-Z Cisternas-A Haessler-A Rivera-L Dorbath-L King-G Fuenzalida-A Owen-T Mccormack-D Baker-C Langer-CJ Mayerroza-D Smith-P

Title: The Racha Earthquake of 1991 - Results of Field Seismological Observations

Source: FIZIKA ZEMLI

1993, Iss 3, pp 12-23

Addresses:

OY-SHMIDT-INST-EARTH-PHYS, MOSCOW, RUSSIA

TBILISI-GEOPHYS-INST, TBILISI, REP-OF-GEORGIA

INST-PHYS-GLOBE, F-67084 STRASBOURG, FRANCE

BULLARD-LABS, CAMBRIDGE, ENGLAND 
US-GEOL-SURVEY, DENVER, CO 80225, USA

INST-GEOPHYS, ZURICH, SWITZERLAND

102. Authors: Silantev-SA Basylev-BA Klitgord-KD Casey-JF

Kuzmin-MI Lomakin-IE Sborshchikov-ID

Title: 3rd Layer Composition of North-Atlantic (40-51 Nl)

Oceanic-Crust

Source: GEOKHIMIYA

1992, Iss 12, pp 1415-1435

Addresses:

VI-VERNADSKII-GEOCHEM-\&-ANALYT-CHEM-INST, MOSCOW, RUSSIA

US-GEOL-SURVEY, WASHINGTON, DC 20242, USA

UNIV-HOUSTON, HOUSTON, TX 77004, USA

RUSSIAN-ACAD-SCI, SIBERIAN DIV, INST GEOCHEM, IRKUTSK, RUSSIA

POLAR-INST-FISHERIES-\&-OCEANOG, MURMANSK, RUSSIA

RUSSIAN-ACAD-SCI, INST OCEANOL, MOSCOW, RUSSIA

\section{4}

1. Authors: Percival-JA Peterman-ZE

Title: Rb-Sr Biotite and Whole-Rock Data from the Kapuskasing

Uplift and Their Bearing on the Cooling and Exhumation

History

Source: CANADIAN JOURNAL OF EARTH SCIENCES 1994, Vol 31, Iss 7, pp 1172-1181

Addresses:

GEOL-SURVEY-CANADA, 601 BOOTH ST, OTTAWA K1A-0E8, ON, CANADA

US-GEOL-SURVEY, DENVER FED CTR, DENVER, CO 80225, USA

2. Authors: Gleason-JD Miller-CF Wooden-JL Bennett-VC

Title: Petrogenesis of the Highly Potassic 1.42 Ga Barrel

Spring Pluton, Southeastern California, with

Implications for Midproterozoic Magma Genesis in the

Southwestern USA

Source: CONTRIBUTIONS TO MINERALOGY AND PETROLOGY 1994, Vol 118, Iss 2, pp 182-197

Addresses:

US-GEOL-SURVEY, MS 937, MENLO-PK, CA 94025, USA

AUSTRALIAN-NATL-UNIV, RES SCH EARTH SCI, CANBERRA, ACT 2601, AUSTRALIA

VANDERBILT-UNIV, DEPT GEOL, NASHVILLE, TN 37235, USA

3. Authors: Ludwig-KR Titterington-DM

Title: Calculation of (230)Th/U Isochrons, Ages, and Errors

Source: GEOCHIMICA ET COSMOCHIMICA ACTA 1994, Vol 58, Iss 22, pp 5031-5042

Addresses:

US-GEOL-SURVEY, MAIL STOP 963, DENVER, CO 80225, USA

UNIV-GLASGOW, DEPT STAT, GLASGOW G12-8QW, SCOTLAND

4. Authors: Walker-RJ Morgan-JW Horan-MF Czamanske-GK

Krogstad-EJ Fedorenko-VA Kunilov-VE

Title: Re-Os Isotopic Evidence for an Enriched-Mantle Source

for the Norilsk-Type, Ore-Bearing Intrusions, Siberia

Source: GEOCHIMICA ET COSMOCHIMICA ACTA

1994, Vol 58, Iss 19, pp 4179-4197 
Addresses:

UNIV-MARYLAND, DEPT GEOL, ISOTOPE GEOCHEM LAB, COLLEGE-PK, MD

20742, USA

US-GEOL-SURVEY, MENLO-PK, CA 94025, USA

US-GEOL-SURVEY, RESTON, VA 22092, USA

CENT-RES-INST-GEOL-PROSPECTING-BASE-\&-PRECIOUS-ME, MOSCOW

113545, RUSSIA

NORILSK-NICKEL-CONCERN, NORILSK 663300, RUSSIA

5. Authors: Evans-WC White-LD Tuttle-ML Kling-GW Tanyileke-G

Michel-RL

Title: 6 Years of Change at Lake Nyos, Cameroon, Yield Clues to the Past and Cautions for the Future

Source: GEOCHEMICAL JOURNAL 1994, Vol 28, Iss 3, pp 139-162

Addresses:

US-GEOL-SURVEY, MENLO-PK, CA 94025, USA

US-GEOL-SURVEY, DENVER, CO 80225, USA

INST-GEOL-\&-MIN-RES, YAOUNDE, CAMEROON

UNIV-MICHIGAN, DEPT BIOL, ANN-ARBOR, MI 48109, USA

US-GEOL-SURVEY, RESTON, VA 22092, USA

6. Authors: Obradovic-J Hein-JR Djurdjevic-J

Title: Diagenesis of Diatomite from the Kolubara Coal Basin,

Barosevac, Serbia

Source: GEOLOGICAL JOURNAL 1994, Vol 29, Iss 3, pp 209-217

Addresses:

UNIV-BELGRADE, FAC MIN \& GEOL, DJUSINA 7, BELGRADE 11000, YUGOSLAVIA

US-GEOL-SURVEY, MENLO-PK, CA 94025, USA

7. Authors: Bischoff-JL Julia-R Shanks-WC Rosenbauer-RJ

Title: Karstification Without Carbonic-Acid - Bedrock

Dissolution by Gypsum-Driven Dedolomitization

Source: GEOLOGY

1994, Vol 22, Iss 11, pp 995-998

Addresses:

US-GEOL-SURVEY, 345 MIDDLEFIELD RD, MENLO-PK, CA 94025, USA

INST-GEOL-JAUME-ALMERA, E-08028 BARCELONA, SPAIN

US-GEOL-SURVEY, DENVER FED CTR, DENVER, CO 80225, USA

8. Authors: Quick-JE Sinigoi-S Mayer-A

Title: Emplacement Dynamics of a Large Mafic Intrusion in the Lower Crust, Ivrea-Verbano Zone, Northern Italy

Source: JOURNAL OF GEOPHYSICAL RESEARCH-SOLID EARTH 1994, Vol 99, Iss B11, pp 21559-21573

Addresses:

US-GEOL-SURVEY, DENVER FED CTR, MAIL STOP 903, BOX 25046, DENVER, CO 80225, USA

UNIV-TRIESTE, IST MINERAL \& PETROG, I-34100 TRIESTE, ITALY

9. Authors: Sinigoi-S Quick-JE Clemensknott-D Mayer-A

Demarchi-G Mazzucchelli-M Negrini-L Rivalenti-G

Title: Chemical Evolution of a Large Mafic Intrusion in the Lower Crust, Ivrea-Verbano Zone, Northern Italy 
Source: JOURNAL OF GEOPHYSICAL RESEARCH-SOLID EARTH

1994, Vol 99, Iss B11, pp 21575-21590

Addresses:

UNIV-TRIESTE, IST MINERAL \& PETROG, PIAZZALE EUROPA 1, I-34100

TRIESTE, ITALY

US-GEOL-SURVEY, DENVER FED CTR, DENVER, CO 80225, USA

CALTECH, DIV GEOL \& PLANETARY SCI, PASADENA, CA 91125, USA

UNIV-MODENA, IST MINERAL \& PETROG, I-41100 MODENA, ITALY

10. Authors: Vonhuene-R Klaeschen-D Cropp-B

Title: Tectonic Structure Across the Accretionary and Erosional

Parts of the Japan Trench Margin

Source: JOURNAL OF GEOPHYSICAL RESEARCH-SOLID EARTH 1994, Vol 99, Iss B11, pp 22349-22361

Addresses:

CHRISTIAN-ALBRECHTS-UNIV-KIEL, MARINE GEOSCI RES CTR, GEOMAR, WISCHLOFSTR 1-3, D-24148 KIEL, GERMANY

US-GEOL-SURVEY, DENVER, CO 80225, USA

11. Authors: Kissling-E Ellsworth-WL Eberhartphillips-D

Kradolfer-U

Title: Initial Reference Models in Local Earthquake Tomography

Source: JOURNAL OF GEOPHYSICAL RESEARCH-SOLID EARTH 1994, Vol 99, Iss B10, pp 19635-19646

Addresses:

ETH-HOENGGERBERG, INST GEOPHYS, CH-8093 ZURICH, SWITZERLAND

US-GEOL-SURVEY, MENLO-PK, CA 94025, USA

INST-GEOL-\&-NUCL-SCI, WELLINGTON, NEW-ZEALAND

12. Authors: Campbell-WH Arora-BR Schiffmacher-ER

Title: Polar-Cap Field Response to IMF B-Y Sector Changes on Quiet Days at a Longitude Line of Observatories

Source: JOURNAL OF GEOMAGNETISM AND GEOELECTRICITY 1994, Vol 46, Iss 9, pp 735-746

Addresses:

US-GEOL-SURVEY, MAIL STOP 968, BOX 25046, DENVER, CO 80225, USA

INDIAN-INST-GEOMAGNETISM, BOMBAY 400005, MAHARASHTRA, INDIA

13. Authors: Hutton-JT Prescott-JR Bowman-JR Dunham-MNE

Crone-AJ Machette-MN Twidale-CR

Title: Thermoluminescence Dating of Australian Palaeo-Earthquakes

Source: QUATERNARY SCIENCE REVIEWS

1994, Vol 13, Iss 2, pp 143-147

Addresses:

UNIV-ADELAIDE, DEPT PHYS \& MATH PHYS, ADELAIDE 5005, AUSTRALIA

AUSTRALIAN-GEOL-SURV-ORG, CANBERRA, ACT, AUSTRALIA

UNIV-ADELIADE, DEPT GEOL \& GEOPHYS, ADELAIDE, AUSTRALIA

US-GEOL-SURVEY, DENVER, CO 80225, USA

14. Authors: King-CY Minissale-A

Title: Seasonal Variability of Soil-Gas Radon Concentration in Central California

Source: RADIATION MEASUREMENTS

1994, Vol 23, Iss 4, pp 683-692 
Addresses:

US-GEOL-SURVEY, MENLO-PK, CA 94025, USA

CNR, CTR STUDIO MINERAL \& GEOCHEM SEDIMENTI, I-50121 FLORENCE, ITALY

15. Authors: Nozette-S Rustan-P Pleasance-LP Horan-DM Regeon-P

Shoemaker-EM Spudis-PD Acton-CH Baker-DN

Blamont-JE Buratti-BJ Corson-MP Davies-ME

Duxbury-TC Eliason-EM Jakosky-BM Kordas-JF Lewis-IT Lichtenberg-CL Lucey-PG Malaret-E

Massie-MA Resnick-JH Rollins-CJ Park-HS Mcewen-AS

Priest-RE Pieters-CM Reisse-RA Robinson-MS

Simpson-RA Smith-DE Sorenson-TC Breugge-RWV

Zuber-MT

Title: The Clementine Mission to the Moon - Scientific Overview

Source: SCIENCE

1994, Vol 266, Iss 5192, pp 1835-1839

Addresses:

BALLIST-MISSILE-DEF-ORG, WASHINGTON, DC, USA

LAWRENCE-LIVERMORE-NATL-LAB, LIVERMORE, CA 94550, USA

USN, RES LAB, WASHINGTON, DC 20375, USA

US-GEOL-SURVEY, FLAGSTAFF, AZ 86001, USA

LUNAR-\&-PLANETARY-INST, HOUSTON, TX 77058, USA

CALTECH, JET PROP LAB, PASADENA, CA 91109, USA

UNIV-COLORADO, LASP, BOULDER, CO 80309, USA

CTR-NATL-ETUD-SPATIALES, PARIS, FRANCE

BROWN-UNIV, PROVIDENCE, RI 02912, USA

SCI-INQUIRIES, CATONSVILLE, MD 21228, USA

JOHNS-HOPKINS-UNIV, BALTIMORE, MD 21218, USA

NASA, GODDARD SPACE FLIGHT CTR, GREENBELT, MD 20771, USA

RAND-CORP, SANTA-MONICA, CA 90407, USA

ALLIED-SIGNAL-AEROSP-CO, ALEXANDRIA, VA 22314, USA

STANFORD-UNIV, STANFORD, CA 94305, USA

APPL-COHERENT-TECHNOL, HERNDON, VA 22070, USA

SCI-APPLICAT-INT-CORP, WASHINGTON, DC 20024, USA

RES-SUPPORT-INSTRUMENTS, ALEXANDRIA, VA 22314, USA

UNIV-HAWAII-MANOA, HONOLULU, HI 96822, USA

PACIFIC-ADV-TECHNOL, SOLVANG, CA 94550, USA

16. Authors: Trehu-AM Asudeh-I Brocher-TM Luetgert-JH Mooney-WD

Nabelek-JL Nakamura-Y

Title: Crustal Architecture of the Cascadia Fore-Arc

Source: SCIENCE 1994, Vol 266, Iss 5183, pp 237-243

Addresses:

OREGON-STATE-UNIV, DEPT OCEAN \& ATMOSPHER SCI, OCEANOG ADM

BLDG 104, CORVALLIS, OR 97331, USA

GEOL-SURVEY-CANADA, OTTAWA K1A-0Y3, ON, CANADA

US-GEOL-SURVEY, MENLO-PK, CA 94025, USA

UNIV-TEXAS, INST GEOPHYS, AUSTIN, TX 78759, USA

17. Authors: Prodehl-C Mechie-J Achauer-U Keller-GR Khan-MA

Mooney-WD Gaciri-SJ Obel-JD

Title: The Krisp-90 Seismic Experiment - A Technical Review

Source: TECTONOPHYSICS

1994, Vol 236, Iss 1-4, pp 33-60 
Addresses:

UNIV-KARLSRUHE, INST GEOPHYS, HERTZSTR 16, D-76187 KARLSRUHE, GERMANY

UNIV-TEXAS, DEPT GEOL SCI, EL-PASO, TX 79968, USA

UNIV-LEICESTER, DEPT GEOL, LEICESTER LE1-7RH, LEICS, ENGLAND

US-GEOL-SURVEY, MENLO-PK, CA 94025, USA

UNIV-NAIROBI, DEPT GEOL, NAIROBI, KENYA

SURVEY-KENYA, NAIROBI, KENYA

Authors: Jacob-AWB Vees-R Braile-LW Criley-E

Title: Optimization of Wide-Angle Seismic Signal-to-Noise

Ratios and P-Wave Transmission in Kenya

Source: TECTONOPHYSICS

1994, Vol 236, Iss 1-4, pp 61-79

Addresses:

DUBLIN-INST-ADV-STUDIES, 5 MERRION SQ, DUBLIN 2, IRELAND

TECH-UNIV-CLAUSTHAL, INST GEOPHYS, W-3392

CLAUSTHAL-ZELLERFELD, GERMANY

PURDUE-UNIV, DEPT EARTH \& ATMOSPHER SCI, W-LAFAYETTE, IN 47907, USA

US-GEOL-SURVEY, MENLO-PK, CA 94025, USA

18. Authors: Mechie-J Keller-GR Prodehl-C Gaciri-S Braile-LW

Mooney-WD Gajewski-D Sandmeier-KJ

Title: Crustal Structure Beneath the Kenya Rift from Axial Profile Data

Source: TECTONOPHYSICS

1994, Vol 236, Iss 1-4, pp 179-200

Addresses:

UNIV-KARLSRUHE, INST GEOPHYS, D-76187 KARLSRUHE, GERMANY

UNIV-TEXAS, DEPT GEOL SCI, EL-PASO, TX 79968, USA

UNIV-NAIROBI, DEPT GEOL, NAIROBI, KENYA

PURDUE-UNIV, DEPT GEOSCI, W-LAFAYETTE, IN 47907, USA

US-GEOL-SURVEY, OFF EARTHQUAKE RES, MENLO-PK, CA 94025, USA

TECH-UNIV-CLAUSTHAL, INST GEOPHYS, W-3392

CLAUSTHAL-ZELLERFELD, GERMANY

19. Authors: Keller-GR Mechie-J Braile-LW Mooney-WD Prodehl-C

Title: Seismic Structure of the Uppermost Mantle Beneath the Kenya Rift

Source: TECTONOPHYSICS

1994, Vol 236, Iss 1-4, pp 201-216

Addresses:

UNIV-TEXAS, DEPT GEOL SCI, EL-PASO, TX 79968, USA

UNIV-KARLSRUHE, INST GEOPHYS, D-76187 KARLSRUHE, GERMANY

PURDUE-UNIV, DEPT GEOSCI, W-LAFAYETTE, IN 47907, USA

US-GEOL-SURVEY, OFF EARTQUAKE RES, MENLO-PK, CA 94025, USA

20. Authors: Keller-GR Prodehl-C Mechie-J Fuchs-K Khan-MA

Maguire-PKH Mooney-WD Achauer-U Davis-PM Meyer-RP

Braile-LW Nyambok-IO Thompson-GA

Title: The East-African Rift System in the Light of Krisp-90

Source: TECTONOPHYSICS

1994, Vol 236, Iss 1-4, pp 465-483

Addresses:

UNIV-TEXAS, DEPT GEOL SCI, EL-PASO, TX 79968, USA

UNIV-KARLSRUHE, INST GEOPHYS, D-76187 KARLSRUHE, GERMANY

UNIV-LEICESTER, DEPT GEOL, LEICESTER LE1-7RH, LEICS, ENGLAND

US-GEOL-SURVEY, OFF EARTHQUAKE RES, MENLO-PK, CA 94025, USA 
UNIV-STRASBOURG, INST PHYS GLOBE, F-67084 STRASBOURG, FRANCE UNIV-CALIF-LOS-ANGELES, DEPT EARTH \& SPACE SCI, LOS-ANGELES, CA 90024, USA

UNIV-WISCONSIN, DEPT GEOL \& GEOPHYS, MADISON, WI 53706, USA

PURDUE-UNIV, DEPT EARTH \& ATMOSPHER SCI, W-LAFAYETTE, IN 47907, USA

STANFORD-UNIV, DEPT GEOPHYS, STANFORD, CA 94305, USA

UNIV-NAIROBI, DEPT GEOL, NAIROBI, KENYA

21. Authors: Finn-C Kimura-G Suyehiro-K

Title: Introduction to the Special Section Northeast Japan - A

Case-History of Subduction

Source: JOURNAL OF GEOPHYSICAL RESEARCH-SOLID EARTH 1994, Vol 99, Iss B11, pp 22137-22145

Addresses:

US-GEOL-SURVEY, DENVER FED CTR, MS 964, DENVER, CO 80225, USA

UNIV-OSAKA-PREFECTURE, COLL INTEGRATED ARTS \& SCI, DEPT EARTH SCI, SAKAI, OSAKA 593, JAPAN

UNIV-TOKYO, OCEAN RES INST, NAKANO KU, TOKYO 164, JAPAN

22. Authors: Colman-JA Edwards-P

Title: Extending Limits of Pond Production - Response

Source: AQUACULTURE

1994, Vol 127, Iss 2-3, pp 277-278

Addresses:

US-GEOL-SURVEY, 28 LORD RD, SUITE 280, MARLBOROUGH, MA, USA

ASIAN-INST-TECHNOL, BANGKOK 10501, THAILAND

23. Authors: Brown-KM Bekins-B Clennell-B Dewhurst-D

Westbrook-GK

Title: Heterogeneous Hydrofracture Development and Accretionary

Fault Dynamics - Reply

Source: GEOLOGY

1994, Vol 22, Iss 11, pp 1053-1054

Addresses:

UNIV-CALIF-SAN-DIEGO, SCRIPPS INST OCEANOG, LA-JOLLA, CA 92093, USA

US-GEOL-SURVEY, MENLO-PK, CA 94025, USA

UNIV-LEEDS, DEPT EARTH SCI, LEEDS LS2-9JT, W-YORKSHIRE, ENGLAND

UNIV-NEWCASTLE-UPON-TYNE, FOSSIL FUELS \& ENVIRONM GEOCHEM

POSTGRAD INST, NEWCASTLE-UPON-TYNE NE1-7RU, TYNE-\&-WEAR, ENGLAND

UNIV-BIRMINGHAM, BIRMINGHAM B15-2TT, W-MIDLANDS, ENGLAND

24. Authors: Clayton-JL Koncz-I

Title: Petroleum Geochemistry of the Zala Basin, Hungary

Source: AAPG BULLETIN-AMERICAN ASSOCIATION OF PETROLEUM GEOLOGISTS 1994, Vol 78, Iss 1, pp 1-22

Addresses:

US-GEOL-SURVEY, DENVER FED CTR, BOX 25044, DENVER, CO 80225, USA

HUNG-ARIAN-OIL-\&-GAS-CORP, H-8801 NAGYKANIZSA, HUNGARY

25. Authors: Mccoy-TJ Steele-IM Keil-K Leonard-BF Endress-M

Title: Chladniite, Na2Camg7(PO4)6 - A New Mineral from the

Carlton (Iiicd) Iron Meteorite 
Source: AMERICAN MINERALOGIST

1994, Vol 79, Iss 3-4, pp 375-380

Addresses:

UNIV-HAWAII-MANOA, SCH OCEAN \& EARTH SCI \& TECHNOL, HAWAII CTR

VOLCANOL, HONOLULU, HI 96822, USA

UNIV-CHICAGO, DEPT GEOPHYS SCI, CHICAGO, IL 60637, USA

US-GEOL-SURVEY, DENVER, CO 80225, USA

UNIV-MUNSTER, INST PLANETOL, W-4400 MUNSTER, GERMANY

26. Authors: Jibson-RW Prentice-CS Borissoff-BA Rogozhin-EA

Langer-CJ

Title: Some Observations of Landslides Triggered by the 29-

April-1991 Racha Earthquake, Republic-of-Georgia

Source: BULLETIN OF THE SEISMOLOGICAL SOCIETY OF AMERICA 1994, Vol 84, Iss 4, pp 963-973

Addresses:

US-GEOL-SURVEY, DENVER, CO 80225, USA

US-GEOL-SURVEY, MENLO-PK, CA 94025, USA

ACAD-SCI-MOSCOW, INST PHYS EARTH, MOSCOW 123810, RUSSIA

27. Authors: Mori-J Filson-J Cranswick-E Borcherdt-R

Amirbekian- $\mathrm{R}$ Aharonian-V Hachverdian-L

Title: Measurements of P and S-Wave Fronts from the Dense 3-

Dimensional Array at Garni, Armenia

Source: BULLETIN OF THE SEISMOLOGICAL SOCIETY OF AMERICA 1994, Vol 84, Iss 4, pp 1089-1096

Addresses:

US-GEOL-SURVEY, PASADENA, CA 91106, USA

US-GEOL-SURVEY, DENVER, CO 80225, USA

US-GEOL-SURVEY, MENLO-PK, CA 94025, USA

NATL-SURVEY-SEISM-PROTECT, YEREVAN, ARMENIA

ARMENIAN-ACAD-SCI, YEREVAN, ARMENIA

28. Authors: Hough-SE Benzion-Y Leary-P

Title: Fault-Zone Waves Observed at the Southern Joshua-Tree Earthquake Rupture Zone

Source: BULLETIN OF THE SEISMOLOGICAL SOCIETY OF AMERICA 1994, Vol 84, Iss 3, pp 761-767

Addresses:

US-GEOL-SURVEY, PASADENA, CA 91106, USA

HARVARD-UNIV, CAMBRIDGE, MA 02138, USA

UNIV-EDINBURGH, EDINBURGH EH8-9YL, MIDLOTHIAN, SCOTLAND

29. Authors: Johnston-MJS Mueller-RJ Sasai-Y

Title: Magnetic-Field Observations in the Near-Field the 28

June $1992 \mathrm{M}(\mathrm{W})$ 7.3 Landers, California, Earthquake

Source: BULLETIN OF THE SEISMOLOGICAL SOCIETY OF AMERICA 1994, Vol 84, Iss 3, pp 792-798

Addresses:

US-GEOL-SURVEY, MENLO-PK, CA 94025, USA

UNIV-TOKYO, EARTHQUAKE RES INST, TOKYO 113, JAPAN

30. Authors: King-GCP Stein-RS Lin-J

Title: Static Stress Changes and the Triggering of Earthquakes

Source: BULLETIN OF THE SEISMOLOGICAL SOCIETY OF AMERICA 1994, Vol 84, Iss 3, pp 935-953 
Addresses:

INST-PHYS-GLOBE, F-67084 STRASBOURG, FRANCE

WOODS-HOLE-OCEANOG-INST, WOODS-HOLE, MA 02543, USA

US-GEOL-SURVEY, MENLO-PK, CA 94025, USA

31. Authors: Mendoza-C Hartzell-S Monfret-T

Title: Wide-Band Analysis of the 3 March 1985 Central Chile

Earthquake - Overall Source Process and Rupture History

Source: BULLETIN OF THE SEISMOLOGICAL SOCIETY OF AMERICA 1994, Vol 84, Iss 2, pp 269-283

Addresses:

US-GEOL-SURVEY, DENVER FED CTR, POB 25046, MS967, DENVER, CO 80225, USA

MISSION-ORSTOM, SANTIAGO 1, CHILE

32. Authors: Spudich-P Iida-M

Title: The Seismic Coda, Site Effects, and Scattering in Alluvial Basins Studied Using Aftershocks of the 1986

North Palm-Springs, California, Earthquake as Source Arrays

Source: BULLETIN OF THE SEISMOLOGICAL SOCIETY OF AMERICA 1993, Vol 83, Iss 6, pp 1721-1743

Addresses:

US-GEOL-SURVEY, 345 MIDDLEFIELD RD, MENLO-PK, CA 94025, USA

UNIV-TOKYO, EARTHQUAKE RES INST, TOKYO 113, JAPAN

33. Authors: Seitzinger-SP Nielsen-LP Caffrey-J Christensen-PB

Title: Denitrification Measurements in Aquatic Sediments - A Comparison of 3 Methods

Source: BIOGEOCHEMISTRY 1993, Vol 23, Iss 3, pp 147-167

Addresses:

ACAD-NAT-SCI-PHILADELPHIA, DIV ENVIRONM RES, 1900 BENJAMIN

FRANKLIN PKWY, PHILADELPHIA, PA 19103, USA

UNIV-AARHUS, DEPT ECOL \& GENET, DK-8000 AARHUS, DENMARK

US-GEOL-SURVEY, MS 496, MENLO-PK, CA 94025, USA

NATL-ENVIRONM-RES-INST, DIV FRESHWATER ECOL, DK-8600

SILKEBORG, DENMARK

34. Authors: Forsyth-DA Milkereit-B Davidson-A Hanmer-S

Hutchinson-DR Hinze-WJ Mereu-R

Title: Seismic Images of a Tectonic Subdivision of the

Grenville-Orogen Beneath Lakes Ontario and Erie

Source: CANADIAN JOURNAL OF EARTH SCIENCES

1994, Vol 31, Iss 2, pp 229-242

Addresses:

GEOL-SURVEY-CANADA, OTTAWA K1A-0Y3, ON, CANADA

US-GEOL-SURVEY, WOODS-HOLE, MA 02543, USA

PURDUE-UNIV, W-LAFAYETTE, IN 47907, USA

UNIV-WESTERN-ONTARIO, LONDON N6A-5B7, ONTARIO, CANADA 
35. Authors: Forsyth-DA Milkereit-B Zelt-CA White-DJ Easton-RM Hutchinson-DR

Title: Deep-Structure Beneath Lake-Ontario - Crustal-Scale Grenville Subdivisions

Source: CANADIAN JOURNAL OF EARTH SCIENCES 1994, Vol 31, Iss 2, pp 255-270

Addresses:

GEOL-SURVEY-CANADA, OTTAWA K1A-0Y3, ON, CANADA

MINIST-NO-DEV-\&-MINES, SUDBURY P3E-6B5, ON, CANADA

US-GEOL-SURVEY, WOODS-HOLE, MA 02543, USA

36. Authors: Grauch-RI Lindahl-I Evans-HT Burt-DM

Fitzpatrick-JJ Foord-EE Graff-PR Hysingjord-J

Title: Hogtuvaite, a New Beryllian Member of the Aenigmatite Group from Norway, with New X-Ray Data on Aenigmatite

Source: CANADIAN MINERALOGIST 1994, Vol 32, Iss JUN, pp 439-448

Addresses:

US-GEOL-SURVEY, 973 DENVER FED CTR, DENVER, CO 80225, USA

NORGES-GEOL-UNDERSOKELSE, N-7002 TRONDHEIM, NORWAY

US-GEOL-SURVEY, RESTON, VA 22092, USA

ARIZONA-STATE-UNIV, DEPT GEOL, TEMPE, AZ 85281, USA

37. Authors: Roberts-AC Szymanski-JT Erd-RC Criddle-AJ Bonardi-M

Title: Deanesmithite, Hg21+hg32+cr6+o5S2, a New Mineral Species from the Clear Creek Claim, San Benito County, California

Source: CANADIAN MINERALOGIST 1993, Vol 31, Iss DEC, pp 787-793

Addresses:

GEOL-SURVEY-CANADA, 601 BOOTH ST, OTTAWA K1A-0E8, ONTARIO, CANADA

CANADA-CTR-MINERAL-\&-ENERGY-TECHNOL, ENERGY MINES \& RESOURCES

CANADA, OTTAWA K1A-0G1, ON, CANADA

US-GEOL-SURVEY, MENLO-PK, CA 94025, USA

NAT-HIST-MUSEUM, DEPT MINERAL, LONDON SW7-5BD, ENGLAND

38. Authors: Jones-BF Hanor-JS Evans-WR

Title: Sources of Dissolved Salts in the Central Murray Basin, Australia

Source: CHEMICAL GEOLOGY 1994, Vol 111, Iss 1-4, pp 135-154

Addresses:

US-GEOL-SURVEY, MS 432 NATL CTR, RESTON, VA 22092, USA

LOUISIANA-STATE-UNIV, DEPT GEOL \& GEOPHYS, BATON-ROUGE, LA 70803, USA

AUSTRALIAN-GEOL-SURVEY-ORG, ENVIRONM GEOSCI \& GROUNDWATER PROGRAM, CANBERRA, ACT 2601, AUSTRALIA

39. Authors: Ayuso-RA Messina-A Devivo-B Russo-S Woodruff-LG Sutter-JF Belkin-HE

Title: Geochemistry and Argon Thermochronology of the Variscan Sila Batholith, Southern Italy - Source Rocks and Magma Evolution

Source: CONTRIBUTIONS TO MINERALOGY AND PETROLOGY 1994, Vol 117, Iss 1, pp 87-109 
Addresses:

US-GEOL-SURVEY, RESTON, VA 22092, USA

IST-SCI-TERRA, I-98166 MESSINA, ITALY

DIPARTIMENTO-GEOFIS-\&-VULCANOL, NAPLES, ITALY

40. Authors: Tauxe-L Gee-J Gallet-Y Pick-T Bown-T

Title: Magnetostratigraphy of the Willwood Formation, Bighorn

Basin, Wyoming - New Constraints on the Location of

Paleocene Eocene Boundary

Source: EARTH AND PLANETARY SCIENCE LETTERS

1994, Vol 125, Iss 1-4, pp 159-172

Addresses:

UNIV-CALIF-SAN-DIEGO, SCRIPPS INST OCEANOG, LA-JOLLA, CA

92093, USA

UNIV-UTRECHT, FORT HOOFDDIJK PALEOMAGNET LAB, 3584-CD UTRECHT,

NETHERLANDS

LAMONT-DOHERTY-EARTH-OBS, PALISADES, NY, USA

US-GEOL-SURVEY, DENVER, CO 80225, USA

UNIV-PARIS-06, INST PHYS GLOBE, F-75230 PARIS 05, FRANCE

41. Authors: Peck-JA King-JW Colman-SM Kravchinsky-VA

Title: A Rock-Magnetic Record from Lake Baikal, Siberia -

Evidence for Late Quaternary Climate-Change

Source: EARTH AND PLANETARY SCIENCE LETTERS

1994, Vol 122, Iss 1-2, pp 221-238

Addresses:

UNIV-RHODE-ISL, GRAD SCH OCEANOG, NARRAGANSETT, RI 02882, USA

E-SIBERIAN-GEOL-GEOPHYS-\&-MINERAL-RESOURCES-RES-INST, IRKUTSK

664026, RUSSIA

US-GEOL-SURVEY, WOODS-HOLE, MA 02543, USA

42. Authors: Takada-H Farrington-JW Bothner-MH Johnson-CG

Tripp-BW

Title: Transport of Sludge-Derived Organic Pollutants to Deep-

Sea Sediments at Deep-Water Dump Site-106

Source: ENVIRONMENTAL SCIENCE \& TECHNOLOGY

1994, Vol 28, Iss 6, pp 1062-1072

Addresses:

WOODS-HOLE-OCEANOG-INST, WOODS-HOLE, MA 02543, USA

TOKYO-UNIV-AGR-\&-TECHNOL, FAC AGR, FUCHU, TOKYO 183, JAPAN

US-GEOL-SURVEY, WOODS-HOLE, MA 02543, USA

43. Authors: Krumme-ML Smith-RL Egestorff-J Thiem-SM Tiedje-JM

Timmis-KN Dwyer-DF

Title: Behavior of Pollutant-Degrading Microorganisms in

Aquifers - Predictions for Genetically-Engineered

Organisms

Source: ENVIRONMENTAL SCIENCE \& TECHNOLOGY

1994, Vol 28, Iss 6, pp 1134-1138

Addresses:

NATL-RES-CTR-BIOTECHNOL, DEPT MICROBIOL, MOLEC MICROBIAL ECOL GRP, BRAUNSCHWEIG, GERMANY

US-GEOL-SURVEY, DIV WATER RESOURCES, BOULDER-CREEK, CO 80303, USA

MICHIGAN-STATE-UNIV, DEPT MICROBIOL, E-LANSING, MI 48824, USA 
44. Authors: Harkey-GA Lydy-MJ Kukkonen-J Landrum-PF

Title: Feeding Selectivity and Assimilation of PAH and PCB in Diporeia spp

Source: ENVIRONMENTAL TOXICOLOGY AND CHEMISTRY 1994, Vol 13, Iss 9, pp 1445-1455

Addresses:

GREAT-LAKES-ENVIRONM-RES-LAB, 2200 COMMONWEALTH BLVD,

ANN-ARBOR, MI 48105, USA

CLEMSON-UNIV, INST WILDLIFE \& ENVIRONM TOXICOL, PENDLETON, SC 29670, USA

US-GEOL-SURVEY, DIV WATER RESOURCES, INDIANAPOLIS, IN 46278, USA

UNIV-JOENSUU, DEPT BIOL, SF-80101 JOENSUU, FINLAND

45. Authors: Slack-JF Stevens-BPJ

Title: Clastic Metasediments of the Early Proterozoic BrokenHill Group, New-South-Wales, Australia - Geochemistry, Provenance, and Metallogenic Significance

Source: GEOCHIMICA ET COSMOCHIMICA ACTA 1994, Vol 58, Iss 17, pp 3633-3652

Addresses:

US-GEOL-SURVEY, NATL CTR, MS 954, RESTON, VA 22092, USA

GEOL-SURVEY-NEW-S-WALES, BROKEN-HILL, NSW 2880, AUSTRALIA

46. Authors: Hinkley-TK Lecloarec-MF Lambert-G

Title: Fractionation of Families of Major, Minor, and TraceMetals Across the Melt Vapor Interface in Volcanic Exhalations

Source: GEOCHIMICA ET COSMOCHIMICA ACTA 1994, Vol 58, Iss 15, pp 3255-3263

Addresses:

US-GEOL-SURVEY, MS 903, DENVER, CO 80225, USA

CEA, CNRS, MIXTE LAB, CTR FAIBLES RADIOACT, F-91190 GIF-SUR-YVETTE, FRANCE

47. Authors: Ridley-WI Perfit-MR Jonasson-IR Smith-MF

Title: Hydrothermal Alteration in Oceanic Ridge Volcanics - A

Detailed Study at the Galapagos Fossil Hydrothermal Field

Source: GEOCHIMICA ET COSMOCHIMICA ACTA 1994, Vol 58, Iss 11, pp 2477-2494

Addresses:

US-GEOL-SURVEY, DENVER FED CTR, BRANCH GEOCHEM, DENVER, CO 80225, USA

UNIV-FLORIDA, DEPT GEOL, GAINESVILLE, FL 32611, USA

GEOL-SURVEY-CANADA, OTTAWA K1A-0E8, ONTARIO, CANADA

48. Authors: Murowchick-JB Coveney-RM Grauch-RI Eldridge-CS

Shelton-KL

Title: Cyclic Variations of Sulfur Isotopes in Cambrian Stratabound Ni-Mo-(PGE-Au) Ores of Southern China

Source: GEOCHIMICA ET COSMOCHIMICA ACTA 1994, Vol 58, Iss 7, pp 1813-1823

Addresses:

UNIV-MISSOURI, DEPT GEOSCI, KANSAS-CITY, MO 64110, USA

US-GEOL-SURVEY, DENVER FED CTR, DENVER, CO 80225, USA 
AUSTRALIAN-NATL-UNIV, RES SCH EARTH SCI, CANBERRA, ACT 2600,

AUSTRALIA

UNIV-MISSOURI, DEPT GEOL SCI, COLUMBIA, MO 65211, USA

49. Authors: Hein-JR Yeh-HW Gunn-SH Gibbs-AE Wang-CH

Title: Composition and Origin of Hydrothermal Ironstones from Central Pacific Seamounts

Source: GEOCHIMICA ET COSMOCHIMICA ACTA 1994, Vol 58, Iss 1, pp 179-189

Addresses:

US-GEOL-SURVEY, 345 MIDDLEFIELD RD, MENLO-PK, CA 94025, USA

UNIV-HAWAII, HAWAII INST GEOPHYS, HONOLULU, HI 96822, USA

ACAD-SINICA, INST EARTH SCI, TAIPEI 115, TAIWAN

50. Authors: Horan-MF Morgan-JW Grauch-RI Coveney-RM

Murowchick-JB Hulbert-LJ

Title: Rhenium and Osmium Isotopes in Black Shales and Ni-MoPGE-Rich Sulfide Layers, Yukon-Territory, Canada, and Hunan and Guizhou Provinces, China

Source: GEOCHIMICA ET COSMOCHIMICA ACTA 1994, Vol 58, Iss 1, pp 257-265

Addresses:

US-GEOL-SURVEY, 981 NATL CTR, RESTON, VA 22092, USA

US-GEOL-SURVEY, DENVER FED CTR, DENVER, CO 80225, USA

UNIV-MISSOURI, DEPT GEOSCI, KANSAS-CITY, MO 64110, USA

GEOL-SURVEY-CANADA, OTTAWA K1A-0E8, ONTARIO, CANADA

51. Authors: Hon-K Kauahikaua-J Denlinger-R Mackay-K

Title: Emplacement and Inflation of Pahoehoe Sheet Flows -

Observations and Measurements of Active Lava Flows on

Kilauea Volcano, Hawaii

Source: GEOLOGICAL SOCIETY OF AMERICA BULLETIN 1994, Vol 106, Iss 3, pp 351-370

Addresses:

US-GEOL-SURVEY, HAWAIIAN VOLCANO OBSERV, HAWAII-NATL-PK, HI 96718, USA

VICTORIA-UNIV-WELLINGTON, WELLINGTON, NEW-ZEALAND

52. Authors: Mueller-PA Heatherington-AL Wooden-JL Shuster-RD

Nutman-AP Williams-IS

Title: Precambrian Zircons from the Florida Basement - A

Gondwanan Connection

Source: GEOLOGY

1994, Vol 22, Iss 2, pp 119-122

Addresses:

UNIV-FLORIDA, DEPT GEOL, GAINESVILLE, FL 32611, USA

US-GEOL-SURVEY, MENLO-PK, CA 94025, USA

UNIV-NEBRASKA, DEPT GEOG \& GEOL, OMAHA, NE 68182, USA

AUSTRALIAN-NATL-UNIV, RES SCH EARTH SCI, CANBERRA, ACT, AUSTRALIA

53. Authors: Steltenpohl-MG Cymerman-Z Krogh-EJ Kunk-MJ

Title: Exhumation of Eclogitized Continental Basement During

Variscan Lithospheric Delamination and Gravitational

Collapse, Sudety Mountains, Poland 
Source: GEOLOGY

1993, Vol 21, Iss 12, pp 1111-1114

Addresses:

AUBURN-UNIV, DEPT GEOL, AUBURN, AL 36849, USA

STATE-INST-GEOL, PL-53122 WROCLAW, POLAND

UNIV-TROMSO, INST BIOL \& GEOL, N-9001 TROMSO, NORWAY

US-GEOL-SURVEY, RESTON, VA 22092, USA

54. Authors: Novarino-G Warren-A Kinner-NE Harvey-RW

Title: Protists from a Sewage-Contaminated Aquifer on Cape-Cod, Massachusetts

Source: GEOMICROBIOLOGY JOURNAL 1994, Vol 12, Iss 1, pp 23-36

Addresses:

NAT-HIST-MUSEUM, DEPT ZOOL, MICROBIOL GRP, CROMWELL RD, LONDON

SW7-5BD, ENGLAND

UNIV-NEW-HAMPSHIRE, DEPT CIVIL ENGN, ENVIRONM RES GRP, DURHAM, NH 03824, USA

US-GEOL-SURVEY, DIV WATER RESOURCES, BOULDER, CO, USA

55. Authors: Gat-JR Bowser-CJ Kendall-C

Title: The Contribution of Evaporation from the Great-Lakes to the Continental Atmosphere - Estimate Based on StableIsotope Data

Source: GEOPHYSICAL RESEARCH LETTERS 1994, Vol 21, Iss 7, pp 557-560

Addresses:

WEIZMANN-INST-SCI, DEPT ENVIRONM SCI \& ENERGY RES, IL-76100

REHOVOT, ISRAEL

UNIV-WISCONSIN, DEPT GEOL \& GEOPHYS, MADISON, WI 53706, USA

US-GEOL-SURVEY, DIV WATER RESOURCES, MENLO-PK, CA 94025, USA

56. Authors: Demiranda-FP Mccafferty-AE Taranik-JV

Title: Reconnaissance Geologic Mapping of a Portion of the Rain-

Forest Covered Guiana Shield, Northwestern Brazil, Using

Sir-B and Digital Aeromagnetic Data

Source: GEOPHYSICS

1994, Vol 59, Iss 5, pp 733-743

Addresses:

PETROLEO-BRASILEIRO-S-A-PETROBRAS-CENPES, ILHA FUNDAO, CIDADE

UNIV, BR-21949900 RIO-DE-JANEIRO, BRAZIL

UNIV-NEVADA-SYST, DESERT RES INST, RENO, NV 89512, USA

US-GEOL-SURVEY, DENVER, CO 80225, USA

57. Authors: Veverka-J Thomas-P Simonelli-D Belton-MJS Carr-M

Chapman-C Davies-ME Greeley-R Greenberg-R Head-J

Klaasen-K Johnson-TV Morrison-D Neukum-G

Title: Discovery of Grooves on Gaspra

Source: ICARUS 1994, Vol 107, Iss 1, pp 72-83

Addresses:

US-GEOL-SURVEY, DEPT ASTRON, MENLO-PK, CA 94025, USA

NATL-OPT-ASTRON-OBSERV, TUCSON, AZ 85719, USA

US-GEOL-SURVEY, MENLO-PK, CA 94025, USA

PLANETARY-SCI-INST, TUCSON, AZ 85719, USA

RAND-CORP, SANTA-MONICA, CA 90406, USA 
ARIZONA-STATE-UNIV, DEPT GEOL, TEMPE, AZ 85287, USA

UNIV-ARIZONA, LUNAR \& PLANETARY LAB, TUCSON, AZ 85721, USA

BROWN-UNIV, DEPT GEOL, PROVIDENCE, RI 02912, USA

NASA, AMES RES CTR, MOFFETT-FIELD, CA 94035, USA

JET-PROP-LAB, PASADENA, CA 91109, USA

DEUTSCH-FORSCHUNGSANSTALT-LUFT-\&-RAUMFAHRT, W-8031

OBERPFAFFENHOFEN, GERMANY

58. Authors: Zhou-YP Ren-YL Tang-DZ Bohor-B

Title: Characteristics of Zircons from Volcanic Ash-Derived

Tonsteins in Late Permian Coal Fields of Eastern Yunnan,

China

Source: INTERNATIONAL JOURNAL OF COAL GEOLOGY

1994, Vol 25, Iss 3-4, pp 243-264

Addresses:

US-GEOL-SURVEY, BOX 25046, MS972, DENVER, CO 80225, USA

KUNMING-INST-COAL-SCI, KUNMING, PEOPLES-R-CHINA

COLORADO-SCH-MINES, DEPT GEOCHIM, GOLDEN, CO 80401, USA

59. Authors: Cortini-M Barton-CC

Title: Chaos in Geomagnetic Reversal Records - A Comparison

Between Earths Magnetic-Field Data and Model Disk Dynamo

Data

Source: JOURNAL OF GEOPHYSICAL RESEARCH-SOLID EARTH 1994, Vol 99, Iss B9, pp 18021-18033

Addresses:

US-GEOL-SURVEY, DENVER FED CTR, MS 940, BOX 25046, DENVER, CO 80225, USA

UNIV-NAPLES-FEDERICO-II, DIPARTIMENTO GEOFIS \& VULCANOL, I-80138 NAPLES, ITALY

60. Authors: Reches-Z Lockner-DA

Title: Nucleation and Growth of Faults in Brittle Rocks

Source: JOURNAL OF GEOPHYSICAL RESEARCH-SOLID EARTH 1994, Vol 99, Iss B9, pp 18159-18173

Addresses:

US-GEOL-SURVEY, 345 MIDDLEFIELD RD, MS 970, MENLO-PK, CA

94025, USA

HEBREW-UNIV-JERUSALEM, DEPT GEOL, IL-91904 JERUSALEM, ISRAEL

61. Authors: Durrheim-RJ Mooney-WD

Title: Evolution of the Precambrian Lithosphere - Seismological and Geochemical Constraints

Source: JOURNAL OF GEOPHYSICAL RESEARCH-SOLID EARTH 1994, Vol 99, Iss B8, pp 15359-15374

Addresses:

UNIV-WITWATERSRAND, DEPT GEOPHYS, JOHANNESBURG 2001, SOUTH-AFRICA

US-GEOL-SURVEY, MENLO-PK, CA 94025, USA

62. Authors: Got-JL Frechet-J Klein-FW

Title: Deep Fault Plane Geometry Inferred from Multiplet

Relative Relocation Beneath the South Flank of Kilauea

Source: JOURNAL OF GEOPHYSICAL RESEARCH-SOLID EARTH

1994, Vol 99, Iss B8, pp 15375-15386 
Addresses:

UNIV-SAVOIE, F-73376 LE-BOURGET-DU-LAC, FRANCE

OBSERV-GRENOBLE, GEOPHYS INTERNE \& TECTONOPHYS LAB, F-38041

GRENOBLE, FRANCE

US-GEOL-SURVEY, MENLO-PK, CA 94025, USA

63. Authors: Hughes-S Hall-J Luetgert-JH

Title: The Seismic Velocity Structure of the Newfoundland Appalachian Orogen

Source: JOURNAL OF GEOPHYSICAL RESEARCH-SOLID EARTH 1994, Vol 99, Iss B7, pp 13633-13653

Addresses:

MEM-UNIV-NEWFOUNDLAND, DEPT EARTH SCI, ST-JOHNS A1B-3X5, NEWFOUNDLAND, CANADA

US-GEOL-SURVEY, MENLO-PK, CA 94025, USA

64. Authors: Morrow-C Lockner-D Hickman-S Rusanov-M Rockel-T

Title: Effects of Lithology and Depth on the Permeability of

Core Samples from the Kola and KTB Drill Holes

Source: JOURNAL OF GEOPHYSICAL RESEARCH-SOLID EARTH 1994, Vol 99, Iss B4, pp 7263-7274

Addresses:

US-GEOL-SURVEY, MENLO-PK, CA 94025, USA

KONTINENTALES-TIEFBOHRPROGRAMM-BUNDESREPUBL-OBERFALZ, W-8486 WINDISCHESCHENBACH, GERMANY

NEDRA-ENTERPRISE, 184415 ZAPOLYARNYI, RUSSIA

65. Authors: Gladwin-MT Gwyther-RL Hart-RHG Breckenridge-KS

Title: Measurements of the Strain Field Associated with

Episodic Creep Events on the San-Andreas Fault at San-

Juan-Bautista, California

Source: JOURNAL OF GEOPHYSICAL RESEARCH-SOLID EARTH 1994, Vol 99, Iss B3, pp 4559-4565

Addresses:

UNIV-QUEENSLAND, DEPT PHYS, ST-LUCIA, QLD 4067, AUSTRALIA

US-GEOL-SURVEY, EARTHQUAKE GEOL \& GEOPHYS BRANCH, MENLO-PK, CA 94025, USA

66. Authors: Koski-RA Jonasson-IR Kadko-DC Smith-VK Wong-FL

Title: Compositions, Growth Mechanisms, and Temporal Relations of Hydrothermal Sulfide-Sulfate-Silica Chimneys at the Northern Cleft Segment, Juan-de-Fuca Ridge

Source: JOURNAL OF GEOPHYSICAL RESEARCH-SOLID EARTH 1994, Vol 99, Iss B3, pp 4813-4832

Addresses:

US-GEOL-SURVEY, MS 999, 345 MIDDLEFIELD RD, MENLO-PK, CA

94025, USA

UNIV-MIAMI, ROSENSTIEL SCH MARINE \& ATMOSPHER SCI, MIAMI, FL 33149, USA

GEOL-SURVEY-CANADA, OTTAWA K1A-0E8, ONTARIO, CANADA

67. Authors: Eittreim-SL Gnibidenko-H Helsley-CE Sliter-R

Mann-D Ragozin-N

Title: Oceanic Crustal Thickness and Seismic Character Along a

Central Pacific Transect 


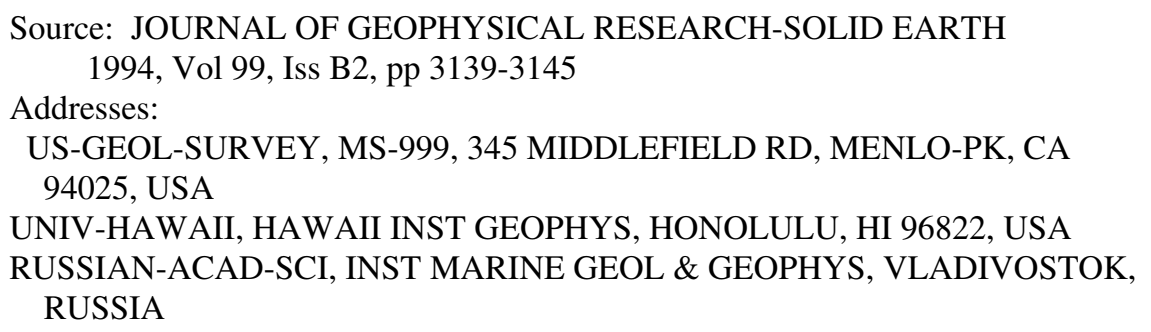

68. Authors: Laubacher-G Naeser-CW

Title: Fission-Track Dating of Granitic-Rocks from the Eastern Cordillera of Peru - Evidence for Late Jurassic and Cenozoic Cooling

Source: JOURNAL OF THE GEOLOGICAL SOCIETY 1994, Vol 151, Iss MAY, pp 473-483

Addresses:

INST-FRANCAIS-RECH-SCI-DEV-COOPERAT-ORSTOM, BP 5045, F-34032 MONTPELLIER 1, FRANCE

US-GEOL-SURVEY, DENVER, CO 80225, USA

69. Authors: Walder-JS Fowler-A

Title: Channelized Subglacial Drainage over a Deformable Bed Source: JOURNAL OF GLACIOLOGY 1994, Vol 40, Iss 134, pp 3-15

Addresses:

US-GEOL-SURVEY, CASCADES VOLCANO OBSERV, VANCOUVER, WA 98661, USA UNIV-OXFORD, INST MATH, OXFORD OX1-3LB, ENGLAND

70. Authors: Christophersen-N Neal-C Hooper-RP

Title: Modeling the Hydrochemistry of Catchments - A Challenge for the Scientific Method

Source: JOURNAL OF HYDROLOGY 1993, Vol 152, Iss 1-4, pp 1-12

Addresses:

UNIV-OSLO, DEPT INFORMAT, POB 1080, BLINDERN, N-0316 OSLO, NORWAY

US-GEOL-SURVEY, DIV WATER RESOURCES, ATLANTA, GA 30360, USA

INST-HYDROL, WALLINGFORD OX10-8BB, OXON, ENGLAND

71. Authors: Lapcevic-PA Novakowski-KS Paillet-FL

Title: Analysis of Flow in an Observation Well Intersecting a Single Fracture

Source: JOURNAL OF HYDROLOGY 1993, Vol 151, Iss 2-4, pp 229-239

Addresses:

ENVIRONM-CANADA, NATL WATER RES INST, 867 LAKESHORE RD, BURLINGTON L7R-4A6, ON, CANADA US-GEOL-SURVEY, DENVER FED CTR, DENVER, CO 80225, USA

72. Authors: Gubanov-AP Yochelson-EL

Title: A Wenlockian (Silurian) Gastropod Shell and Operculum from Siberia

Source: JOURNAL OF PALEONTOLOGY 1994, Vol 68, Iss 3, pp 486-491 
Addresses:

RUSSIAN-ACAD-SCI, INST GEOL, NOVOSIBIRSK 630090, RUSSIA

NATL-MUSEUM-NAT-HIST, DEPT PALEOBIOL, WASHINGTON, DC 20560, USA

US-GEOL-SURVEY, WASHINGTON, DC 20242, USA

73. Authors: Kennedy-WJ Cobban-WA

Title: Ammonite Fauna from the Wenonah Formation (Upper

Cretaceous) of New-Jersey

Source: JOURNAL OF PALEONTOLOGY

1994, Vol 68, Iss 1, pp 95-110

Addresses:

UNIV-OXFORD-MUSEUM, GEOL COLLECT, PARKS RD, OXFORD OX1-3PW, ENGLAND

US-GEOL-SURVEY, DENVER, CO 80225, USA

74. Authors: Nakada-S Bacon-CR Gartner-AE

Title: Origin of Phenocrysts and Compositional Diversity in Pre-

Mazama Rhyodacite Lavas, Crater Lake, Oregon

Source: JOURNAL OF PETROLOGY

1994, Vol 35, Iss 1, pp 127-162

Addresses:

US-GEOL-SURVEY, 345 MIDDLEFIELD RD, MENLO-PK, CA 94025, USA

KYUSHU-UNIV, FAC SCI 33, DEPT EARTH \& PLANETARY SCI, FUKUOKA

812, JAPAN

75. Authors: Spotl-C Houseknecht-DW Longstaffe-FJ

Title: Authigenic Chlorites in Sandstones as Indicators of High-

Temperature Diagenesis, Arkoma Foreland Basin, USA

Source: JOURNAL OF SEDIMENTARY RESEARCH SECTION A-SEDIMENTARY PETROLOGY AND PROCESSES

1994, Vol 64, Iss 3, pp 553-566

Addresses:

US-GEOL-SURVEY, RESTON, VA 22092, USA

UNIV-WESTERN-ONTARIO, DEPT EARTH SCI, LONDON N6A-5B7, ONTARIO, CANADA

76. Authors: Till-AB Yount-ME Bevier-ML

Title: The Geologic History of Redoubt Volcano, Alaska

Source: JOURNAL OF VOLCANOLOGY AND GEOTHERMAL RESEARCH 1994, Vol 62, Iss 1-4, pp 11-30

Addresses:

US-GEOL-SURVEY, 4200 UNIV DR, ANCHORAGE, AK, USA

UNIV-BRITISH-COLUMBIA, DEPT GEOL SCI, VANCOUVER V6T-1Z4, BC,

CANADA

77. Authors: Belkin-HE Devivo-B

Title: Fluid Inclusion Studies of Ejected Nodules from Plinian

Eruptions of Mt Somma-Vesuvius

Source: JOURNAL OF VOLCANOLOGY AND GEOTHERMAL RESEARCH

1993, Vol 58, Iss 1-4, pp 89-100

Addresses:

US-GEOL-SURVEY, MAIL STOP 959, RESTON, VA 22092, USA

UNIV-NAPOLI-FEDERICO-II, DIPARTIMENTO GEOFIS \& VULCANOL, I-80138 NAPLES, ITALY 
78. Authors: Belkin-HE Kilburn-CRJ Devivo-B

Title: Sampling and Major-Element Chemistry of the Recent (AD 1631-1944) Vesuvius Activity

Source: JOURNAL OF VOLCANOLOGY AND GEOTHERMAL RESEARCH 1993, Vol 58, Iss 1-4, pp 273-290

Addresses:

US-GEOL-SURVEY, MAIL STOP 959, RESTON, VA 22092, USA

OSSERV-VESUVIANO, CTR SORVEGLIANZA, I-80123 NAPLES, ITALY

UNIV-NAPOLI-FEDERICO-II, DIPARTIMENTO GEOFIS \& VULCANOL, I-80138 NAPLES, ITALY

79. Authors: Nurnberg-D Wollenburg-I Dethleff-D Eicken-H

Kassens-H Letzig-T Reimnitz-E Thiede-J

Title: Sediments in Arctic Sea-Ice - Implications for Entrainment, Transport and Release

Source: MARINE GEOLOGY 1994, Vol 119, Iss 3-4, pp 185-214

Addresses:

ALFRED-WEGENER-INST-POLAR-\&-MARINE-RES, COLUMBUSSTR, D-27515 BREMERHAVEN, GERMANY

GEOMAR, MARINE GEOSCI RES CTR, D-24148 KIEL, GERMANY

US-GEOL-SURVEY, MENLO-PK, CA 94025, USA

ENERGIESYST-NORD-GMBH, D-24103 KIEL, GERMANY

80. Authors: Reimnitz-E Dethleff-D Nurnberg-D

Title: Contrasts in Arctic Shelf Sea-Ice Regimes and Some Implications - Beaufort Sea Versus Laptev Sea

Source: MARINE GEOLOGY 1994, Vol 119, Iss 3-4, pp 215-225

Addresses:

US-GEOL-SURVEY, 345 MIDDLEFIELD RD, MENLO-PK, CA 94025, USA

GEOMAR, MARINE GEOSCI RES CTR, D-24148 KIEL, GERMANY

ALFRED-WEGENER-INST-POLAR-\&-MEEREFORSCH, D-27515 BREMERHAVEN, GERMANY

81. Authors: Cronin-TM Holtz-TR Whatley-RC

Title: Quaternary Paleoceanography of the Deep Arctic-Ocean Based on Quantitative-Analysis of Ostracoda

Source: MARINE GEOLOGY 1994, Vol 119, Iss 3-4, pp 305-332

Addresses:

US-GEOL-SURVEY, MAIL STOP 970 NATL CTR, RESTON, VA 22092, USA

UNIV-COLL-WALES, INST EARTH STUDIES, ABERYSTWYTH SY23-3DB, DYFED, WALES

82. Authors: Gronlie-A Naeser-CW Naeser-ND Mitchell-JG Sturt-BA Ineson-PR

Title: Fission-Track and K-Ar Dating of Tectonic Activity in a

Transect Across the More-Trondelag Fault Zone, Central

Norway

Source: NORSK GEOLOGISK TIDSSKRIFT 1994, Vol 74, Iss 1, pp 24-34

Addresses:

NORGES-GEOL-UNDERSOKELSE, N-7002 TRONDHEIM, NORWAY

US-GEOL-SURVEY, DENVER, CO 80225, USA

UNIV-NEWCASTLE-UPON-TYNE, DEPT PHYS, NEWCASTLE-UPON-TYNE 
NE1-7RU, TYNE-\&-WEAR, ENGLAND

UNIV-SHEFFIELD, EARTH SCI UNIT, SHEFFIELD S3-7HF, S-YORKSHIRE, ENGLAND

83. Authors: Miller-DS Crowley-KD Dokka-RK Galbraith-RF

Kowallis-BJ Naeser-CW

Title: Results of Interlaboratory Comparison of Fission-Track Ages for 1992 Fission-Track Workshop

Source: NUCLEAR TRACKS AND RADIATION MEASUREMENTS 1993, Vol 21, Iss 4, pp 565-573

Addresses:

RENSSELAER-POLYTECH-INST, DEPT GEOL, TROY, NY 12181, USA

MIAMI-UNIV, DEPT GEOL, OXFORD, OH 45056, USA

LOUISIANA-STATE-UNIV, DEPT NEUROCHIM, BATON-ROUGE, LA 70803, USA

UNIV-LONDON-UNIV-COLL, DEPT STAT SCI, LONDON WC1E-6BT, ENGLAND

BRIGHAM-YOUNG-UNIV, DEPT GEOL, PROVO, UT 84602, USA

US-GEOL-SURVEY, DENVER FED CTR, DENVER, CO 80225, USA

84. Authors: Normark-WR Piper-DJW

Title: Turbidite Sedimentation

Source: OCEANUS

1994, Vol 36, Iss 4, pp 107-110

Addresses:

US-GEOL-SURVEY, DENVER, CO 80225, USA

GEOL-SURVEY-CANADA, BEDFORD INST OCEANOG, OTTAWA K1A-0E8, ONTARIO, CANADA

85. Authors: King-JD Yang-JQ Pu-F

Title: Thermal History of the Periphery of the Junggar Basin, Northwestern China

Source: ORGANIC GEOCHEMISTRY 1994, Vol 21, Iss 3-4, pp 393-405

Addresses:

US-GEOL-SURVEY, DENVER FED CTR, BOX 25046, MS 977, DENVER, CO 80225, USA

RES-INST-EXPLORAT-\&-DEV, KARAMAY 834000, PEOPLES-R-CHINA

ACAD-SINICA, LANZHOU 730000, PEOPLES-R-CHINA

86. Authors: Cronin-TM Kitamura-A Ikeya-N Watanabe-M Kamiya-T

Title: Late Pliocene Climate-Change 3.4-2.3 Ma -

Paleoceanographic Record from the Yabuta Formation, Sea of Japan

Source: PALAEOGEOGRAPHY PALAEOCLIMATOLOGY PALAEOECOLOGY 1994, Vol 108, Iss 3-4, pp 437-455

Addresses:

US-GEOL-SURVEY, MS 970, RESTON, VA 22092, USA

KYOTO-UNIV, FAC SCI, DEPT GEOL \& MINERAL, KYOTO 606, JAPAN

SHIZUOKA-UNIV, INST GEOSCI, SHIZUOKA, JAPAN

GEOL-SURVEY-JAPAN, DEPT FUEL RESOURCES, TSUKUBA 305, JAPAN

KANAZAWA-UNIV, DEPT GEOL, KANAZAWA, ISHIKAWA 920, JAPAN

87. Authors: Lyons-PC Spears-DA Outerbridge-WF Congdon-RD

Evans-HT

Title: Euramerican Tonsteins - Overview, Magmatic Origin, and

Depositional-Tectonic Implications 
Source: PALAEOGEOGRAPHY PALAEOCLIMATOLOGY PALAEOECOLOGY 1994, Vol 106, Iss 1-4, pp 113-134

Addresses:

US-GEOL-SURVEY, MS 956 NATL CTR, RESTON, VA 22092, USA

UNIV-SHEFFIELD, DEPT EARTH SCI, SHEFFIELD S3-7HF, S-YORKSHIRE, ENGLAND

88. Authors: Oliver-WA Pedder-AEH

Title: Crises in the Devonian History of the Rugose Corals

Source: PALEOBIOLOGY 1994, Vol 20, Iss 2, pp 178-190

Addresses:

US-GEOL-SURVEY, SMITHSONIAN INST, NATL MUSEUM NAT HIST E205, WASHINGTON, DC 20560, USA

GEOL-SURVEY-CANADA, CALGARY T2L-2A7, AB, CANADA

89. Authors: Wood-AM Whatley-RC Cronin-TM Holtz-T

Title: Pliocene Paleotemperature Reconstruction for the Southern North-Sea Based on Ostracoda

Source: QUATERNARY SCIENCE REVIEWS 1993, Vol 12, Iss 9, pp 747-767

Addresses:

UNIV-WALES, INST EARTH STUDIES, ABERYSTWYTH SY23-3DB, WALES US-GEOL-SURVEY, RESTON, VA 22092, USA

90. Authors: Belton-MJS Chapman-CR Veverka-J Klaasen-KP Harch-A Greeley-R Greenberg-R Head-JW Mcewen-A Morrison-D

Thomas-PC Davies-ME Carr-MH Neukum-G Fanale-FP

Davis-DR Anger-C Gierasch-PJ Ingersoll-AP

Pilcher-CB

Title: First Images of Asteroid-243-IDA

Source: SCIENCE 1994, Vol 265, Iss 5178, pp 1543-1547

Addresses:

SCI-APPLICAT-INT-CORP, INST PLANETARY SCI, TUCSON, AZ 85705, USA

NATL-OPT-ASTRON-OBSERV, TUCSON, AZ 85719, USA

CORNELL-UNIV, DEPT ASTRON, ITHACA, NY 14853, USA

CALTECH, JET PROP LAB, PASADENA, CA 91109, USA

ARIZONA-STATE-UNIV, DEPT GEOL, TEMPE, AZ 85287, USA

UNIV-ARIZONA, LUNAR \& PLANETARY LAB, TUCSON, AZ 85721, USA

BROWN-UNIV, DEPT GEOL, PROVIDENCE, RI 02912, USA

US-GEOL-SURVEY, FLAGSTAFF, AZ 86001, USA

INST-SPACE-\&-TERR-SCI, CONCORD L4K-3C8, ON, CANADA

UNIV-HAWAII, INST GEOPHYS, HONOLULU, HI 96822, USA

RAND-CORP, SANTA-MONICA, CA 90406, USA

NASA, AMES RES CTR, MOFFETT-FIELD, CA 94035, USA

US-GEOL-SURVEY, MENLO-PK, CA 94025, USA

DEUTSCH-FORSCH-ANSTALT-LUFT-\&-RAUMFAHRT, INST PLANETARY EXPLORAT, BERLIN, GERMANY

CALTECH, PASADENA, CA 91125, USA

NASA, WASHINGTON, DC 20546, USA

91. Authors: Stein-RS King-GCP Lin-J

Title: Stress Triggering of the $1994 \mathrm{M}=6.7$ Northridge,

California, Earthquake by Its Predecessors 
Source: SCIENCE

1994, Vol 265, Iss 5177, pp 1432-1435

Addresses:

US-GEOL-SURVEY, MAIL STOP 977, MENLO-PK, CA 94025, USA

INST-PHYS-GLOBE, F-67084 STRASBOURG, FRANCE

WOODS-HOLE-OCEANOG-INST, WOODS-HOLE, MA 02543, USA

92. Authors: Belton-MJS Greeley-R Greenberg-R Mcewen-A

Klaasen-KP Head-JW Pieters-C Neukum-G Chapman-CR

Geissler-P Heffernan-C Breneman-H Anger-C Carr-MH

Davies-ME Fanale-FP Gierasch-PJ Ingersoll-AP

Johnson-TV Pilcher-CB Thompson-WR Veverka-J Sagan-C

Title: Galileo Multispectral Imaging of the North Polar and

Eastern Limb Regions of the Moon

Source: SCIENCE

1994, Vol 264, Iss 5162, pp 1112-1115

Addresses:

NATL-OPT-ASTRON-OBSERV, TUCSON, AZ 85719, USA

ARIZONA-STATE-UNIV, DEPT GEOL, TEMPE, AZ 85287, USA

UNIV-ARIZONA, LUNAR \& PLANETARY LAB, TUCSON, AZ 85721, USA

US-GEOL-SURVEY, FLAGSTAFF, AZ 86001, USA

CALTECH, JET PROP LAB, PASADENA, CA 91109, USA

BROWN-UNIV, DEPT GEOL, PROVIDENCE, RI 02912, USA

DEUTSCH-FORSCHUNGSANSTALT-LUFT-\&-RAUMFAHRT, W-8031

OBERPFAFFENHOFEN, GERMANY

PLANETARY-SCI-INST, TUCSON, AZ 85719, USA

UNIV-HAWAII-MANOA, INST GEOPHYS, HONOLULU, HI 96822, USA

INST-SPACE-\&-TERR-SCI, CONCORD L4K-3C8, ON, CANADA

CORNELL-UNIV, DEPT ASTRON, ITHACA, NY 14853, USA

RAND-CORP, SANTA-MONICA, CA 90406, USA

US-GEOL-SURVEY, MENLO-PK, CA 94025, USA

CALTECH, PASADENA, CA 91125, USA

NASA-HEADQUARTERS, WASHINGTON, DC 20546, USA

93. Authors: Weaver-HA Feldman-PD Ahearn-MF Arpigny-C Brown-RA

Helin-EF Levy-DH Marsden-BG Meech-KJ Larson-SM

Noll-KS Scotti-JV Sekanina-Z Shoemaker-CS

Shoemaker-EM Smith-TE Storrs-AD Yeomans-DK

Zellner-B

Title: Hubble-Space-Telescope Observations of Comet-P/Shoemaker-

Levy-9 (1993E)

Source: SCIENCE

1994, Vol 263, Iss 5148, pp 787-791

Addresses:

SPACE-TELESCOPE-SCI-INST, 3700 SAN MARTIN DR, BALTIMORE, MD

21218, USA

JOHNS-HOPKINS-UNIV, DEPT PHYS \& ASTRON, BALTIMORE, MD 21218, USA

UNIV-MARYLAND, DEPT ASTRON, COLLEGE-PK, MD 20742, USA

STATE-UNIV-LIEGE, INST ASTROPHYS, B-4000 LIEGE, BELGIUM

JET-PROP-LAB, PASADENA, CA 91109, USA

UNIV-ARIZONA, LUNAR \& PLANETARY LAB, TUCSON, AZ 85721, USA

HARVARD-SMITHSONIAN-CTR-ASTROPHYS, CAMBRIDGE, MA 02138, USA

UNIV-HAWAII, INST ASTRON, HONOLULU, HI 96822, USA

NO-ARIZONA-UNIV, DEPT PHYS \& ASTRON, FLAGSTAFF, AZ 86011, USA 
SPACE-TELESCOPE-SCI-INST, COMP SCI CORP, ASTRON PROGRAM,

BALTIMORE, MD 21218, USA

US-GEOL-SURVEY, FLAGSTAFF, AZ 86001, USA

94. Authors: Sitthithaworn-E Albino-GV Fyfe-WS

Title: Copper-Gold Porphyry and Skarn Mineralization at Phu-

Lon, Northern Thailand

Source: TRANSACTIONS OF THE INSTITUTION OF MINING AND

METALLURGY SECTION B-APPLIED EARTH SCIENCE

1993, Vol 102, Iss SEP-, pp B181-B191

Addresses:

US-GEOL-SURVEY, POB 1488, JEDDAH 21431, SAUDI-ARABIA

DEPT-MINERAL-RESOURCES-THAILAND, BANGKOK, THAILAND

UNIV-WESTERN-ONTARIO, LONDON N6A-3K7, ONTARIO, CANADA

95. Authors: Fuis-GS Clowes-RM

Title: Comparison of Deep-Structure Along 3 Transects of the Western North-American Continental-Margin

Source: TECTONICS

1993, Vol 12, Iss 6, pp 1420-1435

Addresses:

US-GEOL-SURVEY, 345 MIDDLEFIELD RD, MENLO-PK, CA 94025, USA

UNIV-BRITISH-COLUMBIA, DEPT GEOPHYS \& ASTRON, VANCOUVER

V6T-1Z4, BC, CANADA

96. Authors: Marillier-F Hall-J Hughes-S Louden-K Reid-I

Roberts-B Clowes-R Cote-T Fowler-J Guest-S Lu-H

Luetgert-J Quinlan-G Spencer-C Wright-J

Title: Lithoprobe East Onshore Offshore Seismic-Refraction

Survey - Constraints on Interpretation of Reflection

Data in the Newfoundland Appalachians

Source: TECTONOPHYSICS

1994, Vol 232, Iss 1-4, pp 43-58

Addresses:

FISHERIES-\&-OCEANS-CANADA, BEDFORD INST OCEANOG, ATLANTIC

GEOSCI CTR, POB 1006, DARTMOUTH B2Y-4A2, NS, CANADA

MEM-UNIV-NEWFOUNDLAND, DEPT EARTH SCI, ST-JOHNS A1B-3X5, NEWFOUNDLAND, CANADA

DALHOUSIE-UNIV, DEPT OCEANOG, HALIFAX B3H-4J1, NS, CANADA

UNIV-BRITISH-COLUMBIA, DEPT GEOPHYS \& ASTRON, VANCOUVER

V6T-1Z4, BC, CANADA

GEOL-SURVEY-CANADA, DIV CONTINENTAL GEOSCI, OTTAWA K1A-0Y3, ON, CANADA

IRIS, ARLINGTON, VA 22209, USA

QUEENS-UNIV, DEPT GEOL SCI, KINGSTON K7L-3N6, ONTARIO, CANADA

US-GEOL-SURVEY, MENLO-PK, CA 94025, USA

97. Authors: Hejzlar-J Szpakowska-B Wershaw-RL

Title: Comparison of Humic Substances Isolated from Peatbog

Water by Sorption on Deae-Cellulose and Amberlite XAD-2

Source: WATER RESEARCH

1994, Vol 28, Iss 9, pp 1961-1970

Addresses:

ACAD-SCI-CZECH-REPUBL, INST HYDROBIOL, NA SADKACH 7, CR-37005

CESKE-BUDEJOVICE, CZECH-REPUBLIC 
POLISH-ACAD-SCI, DEPT AGROBIOL \& FORESTRY, PL-60809 POZNAN, POLAND

US-GEOL-SURVEY, DENVER FED CTR, DENVER, CO 80225, USA

98. Authors: Kent-DB Davis-JA Anderson-LCD Rea-BA Waite-TD

Title: Transport of Chromium and Selenium in the Suboxic Zone of a Shallow Aquifer - Influence of Redox and Adsorption Reactions

Source: WATER RESOURCES RESEARCH 1994, Vol 30, Iss 4, pp 1099-1114

Addresses:

US-GEOL-SURVEY, DIV WATER RESOURCES, 345 MIDDLEFIELD RD, MENLO-PK, CA 94025, USA

AUSTRALIAN-NUCL-TECHNOL-\&-SCI-ORG, MENAI, NSW 2234, AUSTRALIA

99. Authors: Rossi-C Nimmo-JR

Title: Modeling of Soil-Water Retention from Saturation to Oven Dryness

Source: WATER RESOURCES RESEARCH 1994, Vol 30, Iss 3, pp 701-708

Addresses:

UNIV-GENOA, INST HYDRAUL, VIA MONTALLEGRO 1, I-16145 GENOA, ITALY

US-GEOL-SURVEY, DIV WATER RESOURCES, MENLO-PK, CA 94025, USA

100. Authors: Burr-DT Sudicky-EA Naff-RL

Title: Nonreactive and Reactive Solute Transport in 3-

Dimensional Heterogeneous Porous-Media - Mean

Displacement, Plume Spreading, and Uncertainty

Source: WATER RESOURCES RESEARCH

1994, Vol 30, Iss 3, pp 791-815

Addresses:

WATER-\&-EARTH-SCI-ASSOCIATES-LTD, ST PAUL PL, KINGSTON

K7M-7S4, ON, CANADA

US-GEOL-SURVEY, DENVER, CO 80225, USA

UNIV-WATERLOO, WATERLOO CTR GROUNDWATER RES, WATERLOO N2L-3G1, ONTARIO, CANADA

101. Authors: Demetriadeshah-TH Fuchs-M Kanemasu-ET Flitcroft-ID

Title: Further Discussions on the Relationship Between Cumulated Intercepted Solar-Radiation and Crop Growth

Source: AGRICULTURAL AND FOREST METEOROLOGY 1994, Vol 68, Iss 3-4, pp 231-242

Addresses:

ALABAMA-A\&M-UNIV, DEPT PLANT \& SOIL SCI, POB 1208, NORMAL, AL 35762, USA

AGR-RES-ORG, VOLCANI CTR, INST SOILS \& WATER, IL-50250

BET-DAGAN, ISRAEL

UNIV-GEORGIA, COLL AGR, DEPT AGRON, GRIFFIN, GA 30223, USA

US-GEOL-SURVEY, EROS DATA CTR, SIOUX-FALLS, SD 57198, USA

102. Authors: Chapellier-D Fitterman-D Destadelhofen-CM

Parasnis-DS Steeples-DW Valla-P

Title: Geophysics and Environment - International-Symposium

Held 14, 15 and 16 April 1993 at the University-of-

Lausanne, Switzerland - Preface 
Source: JOURNAL OF APPLIED GEOPHYSICS

1994, Vol 31, Iss 1-4, pp R7-R7

Addresses:

INST-GEOPHYS, BFSH2, 1015 DORIGNYNTO, LAUSANNE, SWITZERLAND

US-GEOL-SURVEY, DENVER, CO 80225, USA

LULEA-UNIV-TECHNOL, DEPT APPL GEOPHYS, S-97187 LULEA, SWEDEN

UNIV-KANSAS, DEPT GEOL, LAWRENCE, KS 66047, USA

BGRM, F-45090 ORLEANS, FRANCE

103. Authors: Okubo-Y Tamaki-K Honkura-Y

Title: Magnetic-Anomalies in Japan and Its Adjacent Regions - Preface

Source: JOURNAL OF GEOMAGNETISM AND GEOELECTRICITY 1994, Vol 46, Iss 6, pp 409-409

Addresses:

US-GEOL-SURVEY, WASHINGTON, DC 20242, USA

UNIV-TOKYO, OCEAN RES INST, TOKYO 113, JAPAN

TOKYO-INST-TECHNOL, TOKYO 152, JAPAN

104. Authors: Hill-D Gasparini-P Mcnutt-S Rymer-H

Title: Internal Structure of Volcanos and Geophysical

Precursors of Eruptions - Selected Papers from Napoli-91

- International-Conference on Active Volcanos and Risk

Mitigation (1841-1991, Jubilee of the Osservatorio-

Vesuviano), Held in Napoli, Italy, 27 August 1 September

1991, Sponsored by Iavcei and CNR - Preface

Source: JOURNAL OF VOLCANOLOGY AND GEOTHERMAL RESEARCH 1994, Vol 61, Iss 3-4, pp R3-R4

Addresses:

US-GEOL-SURVEY, 345 MIDDLEFIELD RD, MENLO-PK, CA 94025, USA

OPEN-UNIV, DEPT EARTH SCI, MILTON-KEYNES MK7-6AA, BUCKS,

ENGLAND

UNIV-ALASKA, INST GEOPHYS, ALASKA VOLCANO OBSERV, FAIRBANKS, AK 99755, USA

UNIV-NAPLES-FEDERICO-2, DEPT GEOPHYS \& VOLCANOL, I-8013

NAPLES, ITALY

105. Authors: Ogasawara-K Wolfe-JA Cronin-TM

Title: Special Issue - Cenozoic Climate and Paleogeographic

Changes in the Pacific Region - Preface

Source: PALAEOGEOGRAPHY PALAEOCLIMATOLOGY PALAEOECOLOGY 1994, Vol 108, Iss 3-4, pp 193-193

Addresses:

UNIV-TSUKUBA, INST GEOSCI, TENNODAI 1-1-1, TSUKUBA 305, JAPAN

US-GEOL-SURVEY, DENVER FED CTR, PALEONTOL \& STRATIG BRANCH, DENVER, CO 80225, USA

US-GEOL-SURVEY, NATL CTR, RESTON, VA 22092, USA

106. Authors: Kling-GW Evans-WC Tuttle-ML Tanyileke-G

Title: Degassing of Lake Nyos

Source: NATURE

1994, Vol 368, Iss 6470, pp 405-406

Addresses:

UNIV-MICHIGAN, DEPT BIOL, ANN-ARBOR, MI 48109, USA

US-GEOL-SURVEY, MENLO-PK, CA 94025, USA

US-GEOL-SURVEY, DENVER FED CTR, DENVER, CO 80225, USA

INST-GEOL-\&-MIN-RES, YAOUNDE, CAMEROON 
107. Authors: Koopmans-MP Lewan-MD Damste-JSS Deleeuw-JW

Title: Thermally-Induced Transformations of Organic Sulfur-

Compounds and Sulfur-Rich Geomacromolecules as Revealed

by Hydrous Pyrolysis

Source: ABSTRACTS OF PAPERS OF THE AMERICAN CHEMICAL SOCIETY 1994, Vol 208, Iss AUG, pp 63-GEOC

Addresses:

NETHERLANDS-INST-SEA-RES, 1790-AB DEN-BURG, NETHERLANDS

US-GEOL-SURVEY, DENVER, CO 80225, USA

108. Authors: Koopmans-MP Damste-JSS Lewan-MD Deleeuw-JW

Title: Distinct Precursors for Pristane and Phytane in the Marine-Environment Impact on the Rationale and Use of the Pr/pH Ratio

Source: ABSTRACTS OF PAPERS OF THE AMERICAN CHEMICAL SOCIETY 1994, Vol 208, Iss AUG, pp 97-GEOC

Addresses:

NETHERLANDS-INST-SEA-RES, 1790-AB DEN-BURG, NETHERLANDS

US-GEOL-SURVEY, DENVER, CO 80225, USA

109. Authors: Pomes-ML Thurman-EM

Title: Fractionation of Humic Material in Tall-Grass Prairie Soil Waters

Source: ABSTRACTS OF PAPERS OF THE AMERICAN CHEMICAL SOCIETY 1994, Vol 207, Iss MAR, pp 48-GEOC

Addresses:

US-GEOL-SURVEY, WRD, LAWRENCE, KS 66049, USA

110. Authors: Waite-TD Payne-TE Davis-JA

Title: Impact of Mineral Transformation on Elemental Mobility in Aquatic Environments

Source: ABSTRACTS OF PAPERS OF THE AMERICAN CHEMICAL SOCIETY 1994, Vol 207, Iss MAR, pp 232-ENVR

Addresses:

UNIV-NEW-S-WALES, DEPT WATER ENGN, KENSINGTON, NSW 2033, AUSTRALIA

AUSTRALIAN-NUCL-SCI-\&-TECHNOL-ORG, MENAI, NSW 2234, AUSTRALIA

US-GEOL-SURVEY, MENLO-PK, CA 94025, USA

111. Authors: Zhang-JS Passchier-CW Slack-JF Fliervoet-TF Deboorder-H

Title: Cryptocrystalline Permian Tourmalinites of Possible Metasomatic Origin in the Orobic Alps, Northern Italy

Source: ECONOMIC GEOLOGY AND THE BULLETIN OF THE SOCIETY OF ECONOMIC GEOLOGISTS 1994, Vol 89, Iss 2, pp 391-396

Addresses:

STATE-SEISMOL-BUR, INST GEOL, POB 634, 100029 BEIJING,

PEOPLES-R-CHINA

UNIV-UTRECHT, FAC EARTH SCI, 3508-TA UTRECHT, NETHERLANDS

US-GEOL-SURVEY, NATL CTR, MAIL STOP 954, RESTON, VA 22092, USA

112. Authors: Oremland-RS Boone-DR

Title: Methanolobus Taylorii Sp-Nov, a New Methylotrophic,

Estuarine Methanogen 


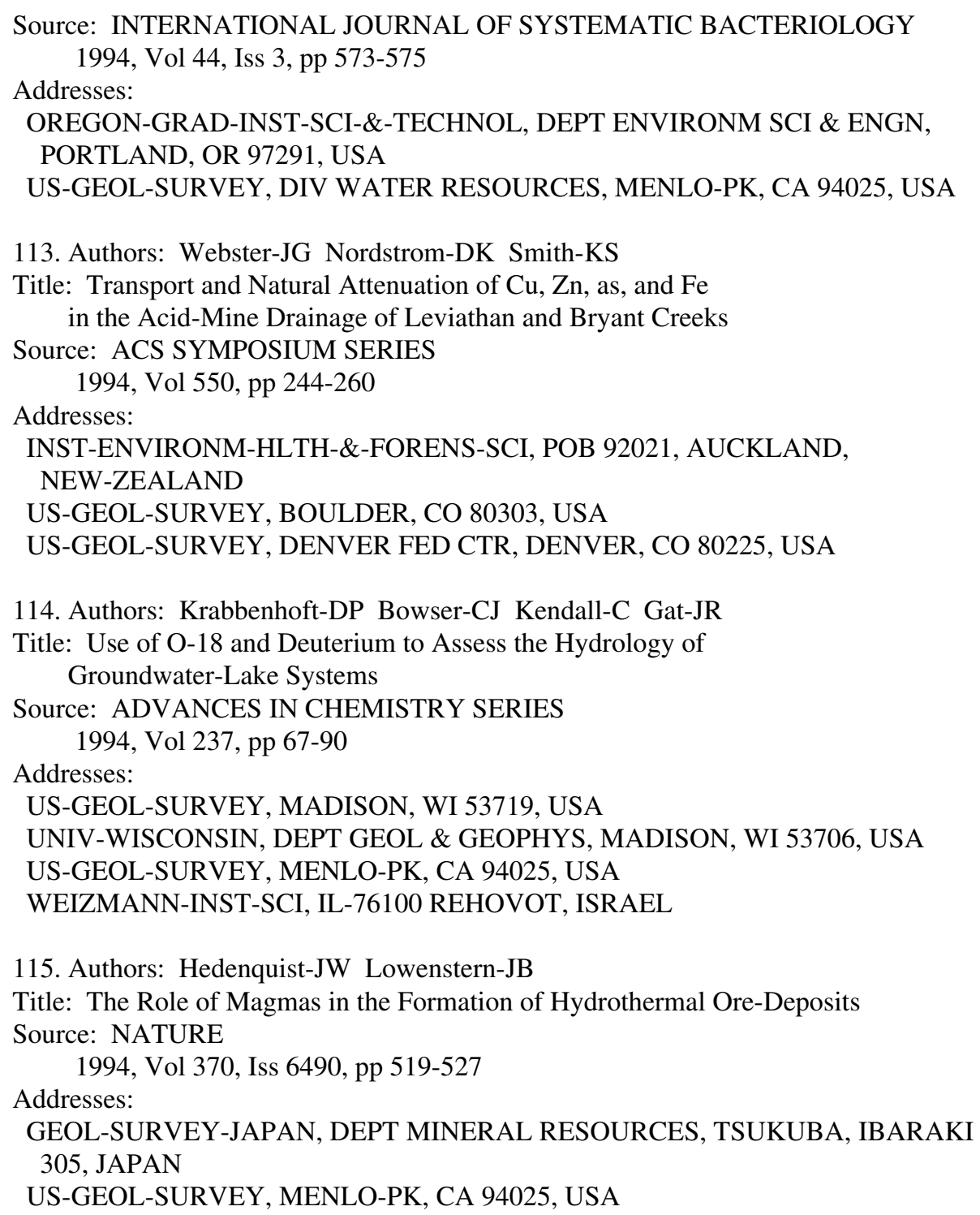

115. Authors: Hedenquist-JW Lowenstern-JB

Title: The Role of Magmas in the Formation of Hydrothermal Ore-Deposits

Source: NATURE 1994, Vol 370, Iss 6490, pp 519-527

Addresses:

GEOL-SURVEY-JAPAN, DEPT MINERAL RESOURCES, TSUKUBA, IBARAKI 305, JAPAN

US-GEOL-SURVEY, MENLO-PK, CA 94025, USA

\section{5}

1. Authors: Magoon-LB Sanchez-RMO

Title: Beyond the Petroleum System

Source: AAPG BULLETIN-AMERICAN ASSOCIATION OF PETROLEUM GEOLOGISTS 1995, Vol 79, Iss 12, pp 1731-1736

Addresses:

US-GEOL-SURVEY, MS 999, 345 MIDDLEFIELD RD, MENLO-PK, CA 94025, USA

PETR-MEXICANOS, PROLONGAC AMORES, MEXICO-CITY 03100, DF, MEXICO

2. Authors: Lyons-PC Millay-MA Zodrow-EL Cross-AT Gillis-KS

Title: Discovery of Permineralized Plant Fossils (Coal Balls) in the Bolsovian (EG, Westphalian C) (Middle Pennsylvanian, Upper Carboniferous), Stellarton Basin, Nova-Scotia, Canada

Source: CANADIAN JOURNAL OF BOTANY-REVUE CANADIENNE DE BOTANIQUE 1995, Vol 73, Iss 9, pp 1407-1416 
Addresses:

US-GEOL-SURVEY, MS 956 NATL CTR, RESTON, VA 22092, USA

OHIO-UNIV, DEPT ECOL \& PLANT BIOL, IRONTON, OH 45638, USA

UNIV-COLL-CAPE-BRETON, SYDNEY, NS B1P-6L2, CANADA

MICHIGAN-STATE-UNIV, DEPT GEOL SCI, E-LANSING, MI 48824, USA

NOVA-SCOTIA-DEPT-NAT-RESOURCES, STELLARTON, NS B0K-1S0, CANADA

3. Authors: Hickson-CJ Moore-JG Calk-L Metcalfe-P

Title: Intraglacial Volcanism in the Wells-Gray Clearwater

Volcanic Field, East-Central British-Columbia, Canada

Source: CANADIAN JOURNAL OF EARTH SCIENCES

1995, Vol 32, Iss 7, pp 838-851

Addresses:

GEOL-SURVEY-CANADA, 100 W PENDER ST, VANCOUVER, BC V6B-1R8,

CANADA

US-GEOL-SURVEY, MENLO-PK, CA 94025, USA

4. Authors: Lundberg-J Taggart-BE

Title: Dissolution Pipes in Northern Puerto-Rico - An Exhumed Paleokarst

Source: CARBONATES AND EVAPORITES

1995, Vol 10, Iss 2, pp 171-183

Addresses:

CARLETON-UNIV, DEPT GEOG, OTTAWA, ON K1S-5B6, CANADA

US-GEOL-SURVEY, CTR GSA, GUAYNABO, PR 00965, USA

5. Authors: Verplanck-PL Farmer-GL Mccurry-M Mertzman-S Snee-LW

Title: Isotopic Evidence on the Origin of Compositional

Layering in an Epizonal Magma Body

Source: EARTH AND PLANETARY SCIENCE LETTERS

1995, Vol 136, Iss 1-2, pp 31-41

Addresses:

UNIV-COLORADO, COOPERAT INST RES ENVIRONM SCI, BOULDER, CO

80309, USA

UNIV-COLORADO, DEPT GEOL SCI, BOULDER, CO 80309, USA

IDAHO-STATE-UNIV, DEPT GEOL, POCATELLO, ID 83209, USA

FRANKLIN-\&-MARSHALL-COLL, DEPT GEOSCI, LANCASTER 17604, ENGLAND

US-GEOL-SURVEY, DENVER, CO 80225, USA

6. Authors: Sharma-M Wasserburg-GJ Papanastassiou-DA Quick-JE

Sharkov-EV Lazko-EE

Title: High Nd-143/Nd-144 in Extremely Depleted Mantle Rocks

Source: EARTH AND PLANETARY SCIENCE LETTERS

1995, Vol 135, Iss 1-4, pp 101-114

Addresses:

CALTECH, DIV GEOL \& PLANETARY SCI, CHARLES ARMS LAB, LUNAT

ASYLUM, PASADENA, CA 91125, USA

US-GEOL-SURVEY, DENVER FED CTR, DENVER, CO 80225, USA

RUSSIAN-ACAD-SCI, IGEM, MOSCOW 109017, RUSSIA

7. Authors: Woodward-RL Molnar-P

Title: Lateral Heterogeneity in the Upper-Mantle and SS-S

Travel-Time Intervals for SS-Rays Reflected from the

Tibetan Plateau and Its Surroundings

Source: EARTH AND PLANETARY SCIENCE LETTERS

1995, Vol 135, Iss 1-4, pp 139-148 
Addresses:

US-GEOL-SURVEY, ALBUQUERQUE SEISMOL LAB, ALBUQUERQUE, NM, USA

MIT, DEPT EARTH ATMOSPHER \& PLANETARY SCI, CAMBRIDGE, MA 02139, USA

UNIV-OXFORD, DEPT EARTH SCI, OXFORD OX1-3PR, ENGLAND

8. Authors: Gazis-CA Lanphere-M Taylor-HP Gurbanov-A

Title: Ar-40/Ar-39 and O-18/O-16 Studies of the Chegem Ash-Flow

Caldera and the Eldjurta Granite - Cooling of 2 Late

Pliocene Igneous Bodies in the Greater-Caucasus

Mountains, Russia

Source: EARTH AND PLANETARY SCIENCE LETTERS

1995, Vol 134, Iss 3-4, pp 377-391

Addresses:

CALTECH, DIV GEOL \& PLANETARY SCI, PASADENA, CA 91125, USA

US-GEOL-SURVEY, MENLO-PK, CA 94025, USA

RUSSIAN-ACAD-SCI, INST ORE DEPOSIT GEOL PETROG MINERAL \&

GEOCHE, MOSCOW 109017, RUSSIA

9. Authors: Symons-DTA Sangster-DF Leach-DL

Title: A Tertiary Age from Paleomagnetism for Mississippi

Valley-Type Zinc-Lead Mineralization in Upper Silesia,

Poland

Source: ECONOMIC GEOLOGY AND THE BULLETIN OF THE SOCIETY OF ECONOMIC GEOLOGISTS

1995, Vol 90, Iss 4, pp 782-794

Addresses:

UNIV-WINDSOR, DEPT GEOL, WINDSOR, ON N9B-3P4, CANADA

GEOL-SURVEY-CANADA, OTTAWA, ON K1A-0E8, CANADA

US-GEOL-SURVEY, DENVER, CO 80225, USA

10. Authors: Shinohara-H Kazahaya-K Lowenstern-JB

Title: Volatile Transport in a Convecting Magma Column Implications for Porphyry Mo Mineralization

Source: GEOLOGY

1995, Vol 23, Iss 12, pp 1091-1094

Addresses:

GEOL-SURVEY-JAPAN, 1-1-3 HIGASHI, TSUKUBA, IBARAKI 305, JAPAN

US-GEOL-SURVEY, MENLO-PK, CA 94025, USA

11. Authors: Behrendt-JC Blankenship-DD Damaske-D Cooper-AK

Title: Glacial Removal of Late Cenozoic Subglacially Emplaced

Volcanic Edifices by the West Antarctic Ice-Sheet

Source: GEOLOGY

1995, Vol 23, Iss 12, pp 1111-1114

Addresses:

US-GEOL-SURVEY, BOX 25046, DENVER, CO 80225, USA

UNIV-TEXAS, INST GEOPHYS, AUSTIN, TX 78759, USA

GERMAN-FED-INST-GEOSCI-\&-NAT-RESOURCES, HANNOVER, GERMANY

US-GEOL-SURVEY, MENLO-PK, CA 94025, USA

12. Authors: Brewster-ML Annan-AP Greenhouse-JP Kueper-BH

Olhoeft-GR Redman-JD Sander-KA

Title: Observed Migration of a Controlled Dnapl Release by

Geophysical Methods 
Source: GROUND WATER

1995, Vol 33, Iss 6, pp 977-987

Addresses:

UNIV-WATERLOO, WATERLOO CTR GROUNDWATER RES, WATERLOO, ON N2L-3G1, CANADA

KOMEX-INT-LTD, CALGARY, AB T3B-0M6, CANADA

QUEENS-UNIV, DEPT CIVIL ENGN, KINGSTON, ON K7L-3N6, CANADA

US-GEOL-SURVEY, DENVER FED CTR, DENVER, CO 80225, USA

COLORADO-SCH-MINES, GOLDEN, CO 80401, USA

13. Authors: Komada-N Westrum-EF Hemingway-BS Zolotov-MY

Semenov-YV Khodakovsky-IL Anovitz-LM

Title: Thermodynamic Properties of Sodalite at Temperatures from $15 \mathrm{~K}$ to $1000 \mathrm{~K}$

Source: JOURNAL OF CHEMICAL THERMODYNAMICS 1995, Vol 27, Iss 10, pp 1119-1132

Addresses:

UNIV-MICHIGAN, DEPT CHEM, ANN-ARBOR, MI 48109, USA

US-GEOL-SURVEY, RESTON, VA 22092, USA

RUSSIAN-ACAD-SCI, VERNADSKY INST GEOCHEM \& ANALYT CHEM, MOSCOW, RUSSIA

UNIV-MICHIGAN, DEPT GEOL SCI, ANN-ARBOR, MI 48109, USA

14. Authors: Arora-BR Campbell-WH Schiffmacher-ER

Title: Upper-Mantle Electrical-Conductivity in the Himalayan Region

Source: JOURNAL OF GEOMAGNETISM AND GEOELECTRICITY 1995, Vol 47, Iss 7, pp 653-665

Addresses:

INDIAN-INST-GEOMAGNETISM, BOMBAY 400005, MAHARASHTRA, INDIA

US-GEOL-SURVEY, DENVER, CO 80225, USA

15. Authors: Trincardi-F Correggiari-A Field-ME Normark-WR

Title: Turbidite Deposition from Multiple Sources - Quaternary

Paola Basin (Eastern Tyrrhenian Sea)

Source: JOURNAL OF SEDIMENTARY RESEARCH SECTION B-STRATIGRAPHY

AND GLOBAL STUDIES

1995, Vol 65, Iss 4, pp 469-483

Addresses:

IST-GEOL-MARINA, I-40129 BOLOGNA, ITALY

US-GEOL-SURVEY, MENLO-PK, CA 94025, USA

16. Authors: Hequette-A Desrosiers-M Barnes-PW

Title: Sea-Ice Scouring on the Inner Shelf of the Southeastern

Canadian-Beaufort-Sea

Source: MARINE GEOLOGY

1995, Vol 128, Iss 3-4, pp 201-219

Addresses:

UNIV-LAVAL, CTR ETUD NORD, ST-FOY, PQ G1K-7P4, CANADA

US-GEOL-SURVEY, MENLO-PK, CA 94025, USA

17. Authors: Foord-EE Cerny-P Jackson-LL Sherman-DM Eby-RK

Title: Mineralogical and Geochemical Evolution of Micas from

Miarolitic Pegmatites of the Anorogenic Pikes-Peak

Batholith, Colorado

Source: MINERALOGY AND PETROLOGY

1995, Vol 55, Iss 1-3, pp 1-26 
Addresses:

US-GEOL-SURVEY, CENT MINERAL RESOURCES BRANCH, DENVER, CO 80225, USA

UNIV-MANITOBA, DEPT GEOL SCI, WINNIPEG, MB R3T-2N2, CANADA

US-GEOL-SURVEY, GEOCHEM BRANCH, DENVER, CO 80225, USA

US-GEOL-SURVEY, GEOPHYS BRANCH, DENVER, CO 80225, USA

TOPOMETR, BEDMINISTER, PA, USA

18. Authors: Nelson-AR Atwater-BF Bobrowsky-PT Bradley-LA

Clague-JJ Carver-GA Darienzo-ME Grant-WC

Krueger-HW Sparks-R Stafford-TW Stuiver-M

Title: Radiocarbon Evidence for Extensive Plate-Boundary

Rupture About 300 Years Ago at the Cascadia Subduction

Zone

Source: NATURE

1995, Vol 378, Iss 6555, pp 371-374

Addresses:

US-GEOL-SURVEY, MS 966, BOX 25046, DENVER, CO 80225, USA

UNION-COLL, INST ARCTIC \& ALPINE RES, BOULDER, CO 80309, USA

UNIV-WASHINGTON, DEPT GEOL SCI, US GEOL SURVEY, SEATTLE, WA 98195, USA

BRITISH-COLUMBIA-GEOL-SURVEY-BRANCH, VICTORIA, BC V8V-1X4,

CANADA

GEOL-SURVEY-CANADA, VANCOUVER, BC V6B-1R8, CANADA

HUMBOLDT-STATE-UNIV, DEPT GEOL, ARCATA, CA 95521, USA

PORTLAND-STATE-UNIV, DEPT GEOL, PORTLAND, OR 97207, USA

KRUEGER-ENTERPRISES-INC, DIV GEOCHRON LABS, CAMBRIDGE, MA 02138, USA

UNIV-WASHINGTON, QUATERNARY RES CTR, SEATTLE, WA 98195, USA

INST-GEOL-\&-NUCL-SCI-LTD, RAFTER RADIOCARBON LAB, NUCL SCI

GRP, LOWER-HUTT, NEW-ZEALAND

19. Authors: Tanaka-K Machette-MN Crone-AJ Bowman-JR

Title: ESR Dating of Aeolian Sand Near Tennant Creek, Northern-

Territory, Australia

Source: QUATERNARY SCIENCE REVIEWS

1995, Vol 14, Iss 4, pp 385-393

Addresses:

CENT-RES-INST-ELECT-POWER-IND, ABIKO 1646, CHIBA, JAPAN

US-GEOL-SURVEY, DENVER, CO 80225, USA

SCI-APPLICAT-INT-CORP, ARLINGTON, VA, USA

20. Authors: Dwyer-GS Cronin-TM Baker-PA Raymo-ME Buzas-JS Correge-T

Title: North-Atlantic Deep-Water Temperature-Change During Late

Pliocene and Late Quaternary Climatic Cycles

Source: SCIENCE

1995, Vol 270, Iss 5240, pp 1347-1351

Addresses:

DUKE-UNIV, DEPT GEOL, DURHAM, NC 27708, USA

US-GEOL-SURVEY, PALEONTOL \& STRATIG BRANCH, RESTON, VA 22092, USA

MIT, DEPT EARTH ATMOSPHER \& PLANETARY SCI, CAMBRIDGE, MA 02139, USA

UNIV-VERMONT, DEPT MATH \& STAT, BURLINGTON, VT 05051, USA

UNIV-BORDEAUX-1, DEPT GEOL \& OCEANOG, BORDEAUX, FRANCE 
21. Authors: Albino-GV Jalal-S Christensen-K

Title: Neoproterozoic Mesothermal Gold Mineralization at Sukhaybarat East Mine, Saudi-Arabia

Source: TRANSACTIONS OF THE INSTITUTION OF MINING AND METALLURGY SECTION B-APPLIED EARTH SCIENCE 1995, Vol 104, Iss SEP-, pp B157-B170

Addresses:

US-GEOL-SURVEY, POB 1488, JEDDAH 21431, SAUDI-ARABIA

22. Authors: Fernandez-IJ Lawrence-GB Son-YH

Title: Soil-Solution Chemistry in a Low-Elevation Spruce-Fir

Ecosystem, Howland, Maine

Source: WATER AIR AND SOIL POLLUTION

1995, Vol 84, Iss 1-2, pp 129-145

Addresses:

UNIV-MAINE, DEPT APPL ECOL \& ENVIRONM SCI, ORONO, ME 04469, USA

KOREA-UNIV, DEPT FOREST RESOURCES, SEOUL 136701, SOUTH-KOREA

US-GEOL-SURVEY, ALBANY, NY, USA

23. Authors: List-JH Terwindt-JHJ

Title: Large-Scale Coastal Behavior - Introduction

Source: MARINE GEOLOGY

1995, Vol 126, Iss 1-4, pp 1-3

Addresses:

US-GEOL-SURVEY, 384 WOODS HOLE RD, WOODS-HOLE, MA 02543, USA

UNIV-UTRECHT, INST MARINE \& ATMOSPHER RES UTRECHT, NETHERLANDS

CTR COASTAL RES, 3508-TC UTRECHT, NETHERLANDS

24. Authors: Woitsekhowskaya-MB Hemley-JJ

Title: Modeling Metal Transport and Deposition in Butte-Type Hydrothermal Systems

Source: ECONOMIC GEOLOGY AND THE BULLETIN OF THE SOCIETY OF ECONOMIC GEOLOGISTS 1995, Vol 90, Iss 5, pp 1329-1337

Addresses:

INST-MINERAL-\&-PETR, NOVOSIBIRSK 630090, RUSSIA

US-GEOL-SURVEY, RESTON, VA 22092, USA

25. Authors: Torigoyekita-N Misawa-K Tatsumoto-M

Title: U-Th-Pb and Sm-Nd Isotopic Systematics of the Goalpara

Ureilite - Resolution of Terrestrial Contamination -

Reply

Source: GEOCHIMICA ET COSMOCHIMICA ACTA 1995, Vol 59, Iss 19, pp 4087-4091

Addresses:

GEOL-SURVEY-JAPAN, HIGASHI 1-1-3, TSUKUBA, IBARAKI 305, JAPAN

KOBE-UNIV, DEPT EARTH \& PLANETARY SCI, NADA KU, KOBE 657, JAPAN

US-GEOL-SURVEY, MS 963, DENVER, CO 80225, USA

26. Authors: Young-JB Presgrave-BW

Title: The Political Subdivision of Southeast-Asia and

Northwest Africa in the Flinn-Engdahl Regionalization

Scheme

Source: GEOPHYSICAL JOURNAL INTERNATIONAL 1995, Vol 123, Iss 2, pp 623-627 
Addresses:

AWE-BLACKNEST, READING RG7-4RS, BERKS, ENGLAND

US-GEOL-SURVEY, NATL EARTHQUAKE INFORMAT CTR, DENVER FED CTR, DENVER, CO 80225, USA

27. Authors: Berggren-WA Hilgen-FJ Langereis-CG Kent-DV

Obradovich-JD Raffi-I Raymo-ME Shackleton-NJ

Title: Late Neogene Chronology - New Perspectives in High-

Resolution Stratigraphy

Source: GEOLOGICAL SOCIETY OF AMERICA BULLETIN 1995, Vol 107, Iss 11, pp 1272-1287

Addresses:

WOODS-HOLE-OCEANOG-INST, DEPT GEOL \& GEOPHYS, WOODS-HOLE, MA 02543, USA

UNIV-UTRECHT, INST EARTH SCI, 3584-CD UTRECHT, NETHERLANDS

COLUMBIA-UNIV, LAMONT DOHERTY EARTH OBSERV, PALISADES, NY

10964, USA

US-GEOL-SURVEY, ISOTOPE GEOL BRANCH, DENVER, CO 80225, USA

UNIV-G-DANNUNZIO, FAC SCI MM FF NN, CHIETI, ITALY

MIT, DEPT EARTH ATMOSPHER \& PLANETARY SCI, CAMBRIDGE, MA

02139, USA

UNIV-CAMBRIDGE, GODWIN LAB QUATERNARY RES, CAMBRIDGE CB2-3RS, ENGLAND

28. Authors: Cobban-WA Kennedy-WJ

Title: Maastrichtian Ammonites Chiefly from the Prairie Bluff

Chalk in Alabama and Mississippi

Source: JOURNAL OF PALEONTOLOGY

1995, Vol 69, Iss 5, pp 1-40

Addresses:

US-GEOL-SURVEY, DENVER FED CTR, BOX 25046, MAIL STOP 919, DENVER, CO 80225, USA

UNIV-OXFORD-MUSEUM, GEOL COLLECT, OXFORD OX1-3PW, ENGLAND

29. Authors: Wilson-CJN Houghton-BF Mcwilliams-MO Lanphere-MA

Weaver-SD Briggs-RM

Title: Volcanic and Structural Evolution of Taupo Volcanic

Zone, New-Zealand - A Review

Source: JOURNAL OF VOLCANOLOGY AND GEOTHERMAL RESEARCH

1995, Vol 68, Iss 1-3, pp 1-28

Addresses:

WAIRAKEI-RES-CTR, INST GEOL \& NUCL SCI, PRIVATE BAG 2000,

TAUPO, NEW-ZEALAND

STANFORD-UNIV, DEPT GEOPHYS, STANFORD, CA 94305, USA

US-GEOL-SURVEY, MENLO-PK, CA 94025, USA

UNIV-CANTERBURY, DEPT GEOL SCI, CHRISTCHURCH, NEW-ZEALAND

UNIV-WAIKATO, DEPT EARTH SCI, HAMILTON, NEW-ZEALAND

30. Authors: Barker-CE Pawlewicz-MJ

Title: Calculation of Vitrinite Reflectance from Thermal

Histories and Peak Temperatures - A Comparison of Methods

Source: ACS SYMPOSIUM SERIES

1994, Vol 570, pp 216-229

Addresses:

US-GEOL-SURVEY, BOX 25046, MS 971, DENVER, CO 80225, USA

UNIV-ADELAIDE, ADELAIDE, SA 5005, AUSTRALIA 
31. Authors: Oliver-WA Pedder-AEH Weiland-RJ Vanufford-AQ

Title: Middle Paleozoic Corals from the Southern Slope of the

Central Ranges of Irian-Jaya, Indonesia

Source: ALCHERINGA

1995, Vol 19, Iss 1-2, pp 1-15

Addresses:

US-GEOL-SURVEY, MRC 137, WASHINGTON, DC 20560, USA

US-NATL-MUSEUM-NAT-HIST, SMITHSONIAN INST, DEPT PALEOBIOL, WASHINGTON, DC 20560, USA

GEOL-SURVEY-CANADA, CALGARY, AB T2L-2A7, CANADA

UNIV-TEXAS, DEPT GEOL SCI, AUSTIN, TX 78712, USA

32. Authors: Takeda-H Ross-M

Title: Mica Polytypism - Identification and Origin

Source: AMERICAN MINERALOGIST

1995, Vol 80, Iss 7-8, pp 715-724

Addresses:

UNIV-TOKYO, FAC SCI, INST MINERAL, TOKYO 113, JAPAN

US-GEOL-SURVEY, NATL CTR 959, RESTON, VA 22092, USA

33. Authors: Marinsky-JA Reddy-MM Ephraim-JH Mathuthu-AS

Title: Computational Scheme for the Prediction of Metal-Ion Binding by a Soil Fulvic-Acid

Source: ANALYTICA CHIMICA ACTA 1995, Vol 302, Iss 2-3, pp 309-322

Addresses:

LINKOPING-UNIV, DEPT WATER \& ENVIRONM STUDIES, S-58183

LINKOPING, SWEDEN

SUNY-BUFFALO, DEPT CHEM, BUFFALO, NY 14214, USA

US-GEOL-SURVEY, DENVER FED CTR, LAKEWOOD, CO 80225, USA

UNIV-ZIMBABWE, DEPT CHEM, HARARE, ZIMBABWE

34. Authors: Atkinson-GM Hanks-TC

Title: A High-Frequency Magnitude Scale

Source: BULLETIN OF THE SEISMOLOGICAL SOCIETY OF AMERICA 1995, Vol 85, Iss 3, pp 825-833

Addresses:

CARLETON-UNIV, DEPT EARTH SCI, OTTAWA, ON K1S-5B6, CANADA

US-GEOL-SURVEY, MENLO-PK, CA 94025, USA

35. Authors: Hartzell-S Liu-PC

Title: Determination of Earthquake Source Parameters Using a

Hybrid Global Search Algorithm

Source: BULLETIN OF THE SEISMOLOGICAL SOCIETY OF AMERICA 1995, Vol 85, Iss 2, pp 516-524

Addresses:

US-GEOL-SURVEY, EARTHQUAKE \& LANDSLIDE HAZARDS BRANCH, BOX 25046, DENVER, CO 80225, USA

CHINESE-ACAD-SCI, INST GEOPHYS, BEIJING 100101, PEOPLES-R-CHINA 
36. Authors: Lamberty-A Lapitajs-G Vannevel-L Gotz-A Moody-JR

Erdmann-DE Debievre-P

Title: The Irmm International Measurement Evaluation Program

(Imep) - Imep-3-Trace Elements in Synthetic and Natural-

Water

Source: BIOLOGICAL TRACE ELEMENT RESEARCH

1994, Vol 43-5, Iss FAL, pp 571-583

Addresses:

IRMM, B-2440 GEEL, BELGIUM

UNIV-SAARBRUCKEN, SAARBRUCKEN, GERMANY

UNIV-ANTWERP, IRMM, ANTWERP, BELGIUM

NIST, GAITHERSBURG, MD 20899, USA

US-GEOL-SURVEY, DENVER, CO 80225, USA

37. Authors: Richter-DH Preece-SJ Mcgimsey-RG Westgate-JA

Title: Mount-Churchill, Alaska - Source of the Late Holocene White River Ash

Source: CANADIAN JOURNAL OF EARTH SCIENCES 1995, Vol 32, Iss 6, pp 741-748

Addresses:

US-GEOL-SURVEY, ALASKAN GEOL BRANCH, 4200 UNIV DR, ANCHORAGE, AK 99508, USA

MIAMI-UNIV, DEPT GEOL, OXFORD, OH 45056, USA

US-GEOL-SURVEY, ALASKA VOLCANO OBSERV, ANCHORAGE, AK 99508, USA

UNIV-TORONTO, DEPT GEOL, TORONTO, ON M5S-3B1, CANADA

38. Authors: Walossek-D Repetski-JE Muller-KJ

Title: An Exceptionally Preserved Parasitic Arthropod,

Heymonsicambria-Taylori N-Sp (Arthropoda-Incertae Sedis,

Pentastomida), from Cambrian-Ordovician Boundary Beds of

Newfoundland, Canada

Source: CANADIAN JOURNAL OF EARTH SCIENCES

1994, Vol 31, Iss 11, pp 1664-1671

Addresses:

UNIV-BONN, INST PALEONTOL, NUSSALLEE 8, D-53115 BONN, GERMANY

US-GEOL-SURVEY, NATL CTR, RESTON, VA 22092, USA

39. Authors: Roberts-AC Ercit-TS Groat-LA Criddle-AJ Erd-RC

Williams-RS

Title: Peterbaylissite, Hg-3(1+)(CO3)(Oh)Center-Dot-2H(2)O, a

New Mineral Species from the Clear-Creek Claim, San-

Benito County, California

Source: CANADIAN MINERALOGIST

1995, Vol 33, Iss FEB, pp 47-53

Addresses:

GEOL-SURVEY-CANADA, 601 BOOTH ST, OTTAWA, ON K1A-0E8, CANADA

CANADIAN-MUSEUM-NAT, DIV RES, OTTAWA, ON K1P-6P4, CANADA

UNIV-BRITISH-COLUMBIA, DEPT GEOL SCI, VANCOUVER, BC V6T-2B4,

CANADA

NAT-HIST-MUSEUM, DEPT MINERAL, LONDON SW7-5BD, ENGLAND

US-GEOL-SURVEY, MENLO-PK, CA 94025, USA

CANADIAN-CONSERVAT-INST, OTTAWA, ON K1A-0C8, CANADA 
40. Authors: Birch-WD Pring-A Foord-EE

Title: Selwynite, $\mathrm{NaK}(\mathrm{Be}, \mathrm{Al}) \mathrm{Zr}-2(\mathrm{PO} 4)(4)$ Center-Dot-2H(2)O, a

New Gainesite-Like Mineral from Wycheproof, Victoria,

Australia

Source: CANADIAN MINERALOGIST

1995, Vol 33, Iss FEB, pp 55-58

Addresses:

MUSEUM-VICTORIA, DEPT MINERAL \& PETR, 328 SWANSTON ST, MELBOURNE, VIC 3000, AUSTRALIA

S-AUSTRALIAN-MUSEUM, DEPT MINERAL, ADELAIDE, SA 5000, AUSTRALIA

US-GEOL-SURVEY, DENVER FED CTR, DENVER, CO 80225, USA

41. Authors: Calvo-JP Jones-BF Bustillo-M Fort-R Zarza-AMA

Kendall-C

Title: Sedimentology and Geochemistry of Carbonates from

Lacustrine Sequences in the Madrid Basin, Central Spain

Source: CHEMICAL GEOLOGY

1995, Vol 123, Iss 1-4, pp 173-191

Addresses:

UNIV-COMPLUTENSE-MADRID, DEPT PETROL \& GEOQUIM, E-28040

MADRID, SPAIN

US-GEOL-SURVEY, RESTON, VA 22092, USA

CSIC, INST GEOL ECON, E-28040 MADRID, SPAIN

42. Authors: Kowallis-BJ Christiansen-EH Deino-AL Kunk-MJ

Heaman-LM

Title: Age of the Cenomanian-Turonian Boundary in the Western Interior of the United-States

Source: CRETACEOUS RESEARCH

1995, Vol 16, Iss 1, pp 109-129

Addresses:

BRIGHAM-YOUNG-UNIV, DEPT GEOL, PROVO, UT 84602, USA

INST-HUMAN-ORIGINS, CTR GEOCHRONOL, BERKELEY, CA 94709, USA

US-GEOL-SURVEY, NATL CTR 981, RESTON, VA 22092, USA

ROYAL-ONTARIO-MUSEUM, DEPT GEOL, TORONTO, ON M5S-2C6, CANADA

43. Authors: Jackson-HR Grantz-A Reid-I May-SD Hart-PE

Title: Observations of Anomalous Oceanic-Crust in the Canada

Basin, Arctic-Ocean

Source: EARTH AND PLANETARY SCIENCE LETTERS

1995, Vol 134, Iss 1-2, pp 99-106

Addresses:

GEOL-SURVEY-CANADA, ATLANTIC GEOSCI CTR, BOX 1006, DARTMOUTH, NS B2Y-4A2, CANADA

US-GEOL-SURVEY, MENLO-PK, CA 94025, USA

44.Authors: Park-JK Buchan-KL Harlan-SS

Title: A Proposed Giant Radiating Dyke Swarm Fragmented by the

Separation of Laurentia and Australia Based on

Paleomagnetism of Ca.780 Ma Mafic Intrusions in Western

North-America

Source: EARTH AND PLANETARY SCIENCE LETTERS

1995, Vol 132, Iss 1-4, pp 129-139 
Addresses:

GEOL-SURVEY-CANADA, DIV CONTINENTAL GEOSCI, 601 BOOTH ST, OTTAWA, ON K1A-0E8, CANADA

US-GEOL-SURVEY, DENVER FED CTR, DENVER, CO 80225, USA

45. Authors: Sinigoi-S Quick-JE Mayer-A Demarchi-G

Title: Density-Controlled Assimilation of Underplated Crust, Ivrea-Verbano Zone, Italy

Source: EARTH AND PLANETARY SCIENCE LETTERS 1995, Vol 129, Iss 1-4, pp 183-191

Addresses:

UNIV-TRIESTE, IST MINERAL \& PETROG, P EUROPA 1, I-34100

TRIESTE, ITALY

US-GEOL-SURVEY, DENVER FED CTR, DENVER, CO 80225, USA

46. Authors: Brown-ET Stallard-RF Larsen-MC Raisbeck-GM Yiou-F

Title: Denudation Rates Determined from the Accumulation of in Situ-Produced Be-10 in the Luquillo Experimental Forest, Puerto-Rico

Source: EARTH AND PLANETARY SCIENCE LETTERS 1995, Vol 129, Iss 1-4, pp 193-202

Addresses:

CTR-SPECTROMETRIE-NUCL-\&-SPECTROMETRIE-MASSE, CNRS, IN2P3, BATIMENT 108, F-91405 ORSAY, FRANCE

US-GEOL-SURVEY, BOULDER, CO 80303, USA

47. Authors: King-G Oppenheimer-D Amelung-F

Title: Block Versus Continuum Deformation in the Western United-States

Source: EARTH AND PLANETARY SCIENCE LETTERS 1994, Vol 128, Iss 3-4, pp 55-64

Addresses:

INST-PHYS-GLOBE, 5 RUE RENE DESCARTES, F-67084 STRASBOURG, FRANCE

US-GEOL-SURVEY, MENLO-PK, CA, USA

48. Authors: Cunningham-CG Aparicio-H Murillo-F Jimenez-N Lizeca-JL Mckee-EH Ericksen-GE Tavera-F

Title: Relationship Between the Porco, Bolivia, Ag-Zn-Pb-Sn Deposit and the Porco Caldera

Source: ECONOMIC GEOLOGY AND THE BULLETIN OF THE SOCIETY OF ECONOMIC GEOLOGISTS 1994, Vol 89, Iss 8, pp 1833-1841

Addresses:

US-GEOL-SURVEY, 959 NATL CTR, RESTON, VA 22092, USA

CO-MINERA-SUR-SA, POTOSI, BOLIVIA

SERV-GEOL-BOLIVIA, LA-PAZ, BOLIVIA

US-GEOL-SURVEY, MENLO-PK, CA 94025, USA

49. Authors: Gray-JE Coolbaugh-MF

Title: Geology and Geochemistry of Summitville, Colorado - An Epithermal Acid Sulfate Deposit in a Volcanic Dome

Source: ECONOMIC GEOLOGY AND THE BULLETIN OF THE SOCIETY OF ECONOMIC GEOLOGISTS

1994, Vol 89, Iss 8, pp 1906-1923 
Addresses:

US-GEOL-SURVEY, DENVER FED CTR, DENVER, CO 80225, USA

CARSON-GOLD-CORP, VANCOUVER, BC V6C-2G8, CANADA

50. Authors: Kile-DE Chiou-CT Zhou-HD Li-H Xu-OY

Title: Partition of Nonpolar Organic Pollutants from Water to

Soil and Sediment Organic Matters

Source: ENVIRONMENTAL SCIENCE \& TECHNOLOGY

1995, Vol 29, Iss 5, pp 1401-1406

Addresses:

US-GEOL-SURVEY, DENVER FED CTR, DENVER, CO 80225, USA

MINIST-WATER-RESOURCES, WATER QUAL RES CTR, BEIJING 100044, PEOPLES-R-CHINA

NANJING-UNIV, DEPT ENVIRONM SCI \& ENGN, NANJING 210093, PEOPLES-R-CHINA

51. Authors: Brasier-M Cowie-J Taylor-M

Title: Decision on the Precambrian-Cambrian Boundary Stratotype

Source: EPISODES 1994, Vol 17, Iss 1-2, pp 3-8

Addresses:

UNIV-OXFORD, DEPT EARTH SCI, OXFORD OX1-3PR, ENGLAND

US-GEOL-SURVEY, DENVER, CO 80225, USA

52. Authors: Devivo-B Torok-K Ayuso-RA Lima-A Lirer-L

Title: Fluid Inclusion Evidence for Magmatic Silicate/Saline/CO2 Immiscibility and Geochemistry of Alkaline Xenoliths from Ventotene Island, Italy

Source: GEOCHIMICA ET COSMOCHIMICA ACTA 1995, Vol 59, Iss 14, pp 2941-2953

Addresses:

DIPARTIMENTO-GEOFIS-\&-VULCANOL, VIA MEZZOCANNONE 8, I-80134

NAPLES, ITALY

LORAND-EOTVOS-UNIV, DEPT PETROG \& GEOCHEM, H-1088 BUDAPEST, HUNGARY

US-GEOL-SURVEY, RESTON, VA 22092, USA

53. Authors: Torigoyekita-N Misawa-K Dalrymple-GB Tatsumoto-M

Title: Further Evidence for a Low U/Pb, Source in the Moon - U-

$\mathrm{Th}-\mathrm{Pb}, \mathrm{Sm}-\mathrm{Nd}$, and Ar-Ar Isotopic Systematics of Lunar

Meteorite Yamato-793169

Source: GEOCHIMICA ET COSMOCHIMICA ACTA 1995, Vol 59, Iss 12, pp 2621-2632

Addresses:

GEOL-SURVEY-JAPAN, 1-1-3 HIGASHI, TSUKUBA, IBARAKI 305, JAPAN

US-GEOL-SURVEY, DENVER, CO 80225, USA

US-GEOL-SURVEY, MENLO-PK, CA 94025, USA

54. Authors: Torigoyekita-N Tatsumoto-M Meeker-GP Yanai-K

Title: The $4.56 \mathrm{Ga}$ U-Pb Age of the Met-78008 Ureilite

Source: GEOCHIMICA ET COSMOCHIMICA ACTA 1995, Vol 59, Iss 11, pp 2319-2329

Addresses:

US-GEOL-SURVEY, DENVER, CO 80225, USA

NATL-INST-POLAR-RES, ITABASHI KU, TOKYO 173, JAPAN 
55. Authors: Dalrymple-GB Czamanske-GK Fedorenko-VA Simonov-ON

Lanphere-MA Likhachev-AP

Title: A Reconnaissance Ar-40/Ar-39 Geochronological Study of

Ore-Bearing and Related Rocks, Siberian Russia

Source: GEOCHIMICA ET COSMOCHIMICA ACTA 1995, Vol 59, Iss 10, pp 2071-2083

Addresses:

US-GEOL-SURVEY, 345 MIDDLEFIELD RD, MENLO-PK, CA 94025, USA

OREGON-STATE-UNIV, COLL OCEAN \& ATMOSPHER SCI, CORVALLIS, OR 97331, USA

TSNIGRI, MOSCOW 113545, RUSSIA

TAYMYRIAN-GEOL-COMM, NORILSK 663300, RUSSIA

56. Authors: Fournier-RO Kennedy-BM Aoki-M Thompson-JM

Title: Correlation of Gold in Siliceous Sinters with $\mathrm{He}-3 / \mathrm{He}-4$ in Hot-Spring Waters of Yellowstone-National-Park

Source: GEOCHIMICA ET COSMOCHIMICA ACTA 1994, Vol 58, Iss 24, pp 5401-5419

Addresses:

US-GEOL-SURVEY, MS-910, 345 MIDDLEFIELD RD, MENLO-PK, CA 94025, USA

UNIV-CALIF-BERKELEY, LAWRENCE BERKELEY LAB, DIV EARTH SCI, BERKELEY, CA 94720, USA

GEOL-SURVEY-JAPAN, TSUKUBA, IBARAKI 305, JAPAN

57. Authors: Waite-TD Davis-JA Payne-TE Waychunas-GA Xu-N

Title: Uranium(VI) Adsorption to Ferrihydrite - Application of a Surface Complexation Model

Source: GEOCHIMICA ET COSMOCHIMICA ACTA 1994, Vol 58, Iss 24, pp 5465-5478

Addresses:

AUSTRALIAN-NUCL-SCI-\&-TECHNOL-ORG, ENVIRONM SCI PROGRAM, MENAI, NSW 2234, AUSTRALIA

US-GEOL-SURVEY, DIV WATER RESOURCES, MENLO-PK, CA 94025, USA

STANFORD-UNIV, CTR MAT RES, STANFORD, CA 94305, USA

58. Authors: Behrmann-JH Lewis-SD Cande-SC Musgrave-R Bangs-N

Boden-P Brown-K Collombat-H Didenko-AN Didyk-BM

Froelich-PN Golovchenko-X Forsythe-R Kurnosov-V

Lindsleygriffin-N Marsaglia-K Osozawa-S Prior-D

Sawyer-D Scholl-D Spiegler-D Strand-K Takahashi-K

Torres-M Vegafaundez-M Vergara-H Wasada-A

Title: Tectonics and Geology of Spreading Ridge Subduction at the Chile Triple Junction - A Synthesis of Results from Leg-141 of the Ocean Drilling Program

Source: GEOLOGISCHE RUNDSCHAU 1994, Vol 83, Iss 4, pp 832-852

Addresses:

UNIV-GIESSEN, INST GEOWISSENSCH \& LITHOSPHARENFORSCH, SENCKENBERGSTR 3, D-35390 GIESSEN, GERMANY

US-GEOL-SURVEY, BRANCH PACIFIC MARINE GEOL, MENLO-PK, CA 94025, USA

TEXAS-A\&M-UNIV, OCEAN DRILLING PROGRAM, COLLEGE-STN, TX 77845, USA

UNIV-CALIF-SAN-DIEGO, SCRIPPS INST OCEANOG, LA-JOLLA, CA 92093, USA 
INST-GEOPHYS, AUSTIN, TX 78759, USA

UNIV-STOCKHOLM, DEPT GEOL \& GEOCHEM, S-10691 STOCKHOLM, SWEDEN

MISS-ORSTOM, QUITO, ECUADOR

RUSSIAN-ACAD-SCI, INST PHYS EARTH, MOSCOW 123810, RUSSIA

SERV-HIDROG-ARMADA, DEPT OCEANOG, VALPARAISO, CHILE

REFINERIA-PETR-CONCON-SA, EMPRESA NACL PETR, CONCEPCION, CHILE

JAPEX, RES CTR, CHIBA 260, JAPAN

UNIV-NEBRASKA, DEPT GEOL, LINCOLN, NE 68588, USA

UNIV-N-CAROLINA, DEPT GEOG \& EARTH SCI, CHARLOTTE, NC 28223,

USA

UNIV-OULU, DEPT GEOL, SF-90570 OULU, FINLAND

COLUMBIA-UNIV, LAMONT DOHERTY GEOL OBSERV, PALISADES, NY

10964, USA

RUSSIAN-ACAD-SCI, INST GEOL, MOSCOW 109017, RUSSIA

WOODS-HOLE-OCEANOG-INST, DEPT GEOL \& GEOPHYS, WOODS-HOLE, MA

02543, USA

RICE-UNIV, DEPT GEOL \& GEOPHYS, HOUSTON, TX 77251, USA

UNIV-TEXAS, DEPT GEOL SCI, EL-PASO, TX 79968, USA

UNIV-LIVERPOOL, DEPT EARTH SCI, LIVERPOOL L69-3BX, MERSEYSIDE,

ENGLAND

GEOMAR, W-2300 KIEL 1, GERMANY

TOHOKU-UNIV, FAC SCI, INST GEOL \& PALAEONTOL, AOBA KU, SENDAI, MIYAGI 980, JAPAN

UNIV-CATOLICA-NORTE, DEPT CIENCIAS GEOL, ANTOFAGASTA, CHILE

59. Authors: Gehrels-GE Dickinson-WR Ross-GM Stewart-JH

Howell-DG

Title: Detrital Zircon Reference for Cambrian to Triassic

Miogeoclinal Strata of Western North-America

Source: GEOLOGY

1995, Vol 23, Iss 9, pp 831-834

Addresses:

UNIV-ARIZONA, DEPT GEOSCI, TUCSON, AZ 85721, USA

GEOL-SURVEY-CANADA, CALGARY, AB T2L-2A7, CANADA

US-GEOL-SURVEY, MENLO-PK, CA 94025, USA

60. Authors: Quick-JE Sinigoi-S Mayer-A

Title: Emplacement of Mantle Peridotite in the Lower

Continental-Crust, Ivrea-Verbano Zone, Northwest Italy

Source: GEOLOGY

1995, Vol 23, Iss 8, pp 739-742

Addresses:

MAX-PLANCK-INST-CHEM, SAARSTR 23, POSTFACH 3060, W-6500 MAINZ, GERMANY

UNIV-TRIESTE, IST MINERAL \& PETROG, I-34100 TRIESTE, ITALY

US-GEOL-SURVEY, DENVER, CO 80225, USA

61. Authors: Dallimore-SR Collett-TS

Title: Intrapermafrost Gas Hydrates from a Deep Core-Hole in the Mackenzie Delta, Northwest-Territories, Canada

Source: GEOLOGY

1995, Vol 23, Iss 6, pp 527-530

Addresses:

GEOL-SURVEY-CANADA, 601 BOOTH ST, OTTAWA, ON K1A-0E8, CANADA

US-GEOL-SURVEY, DENVER FED CTR, DENVER, CO 80225, USA 
62. Authors: Houghton-BF Wilson-CJN Mcwilliams-MO Lanphere-MA

Weaver-SD Briggs-RM Pringle-MS

Title: Chronology and Dynamics of a Large Silicic Magmatic

System - Central Taupo Volcanic Zone, New-Zealand

Source: GEOLOGY

1995, Vol 23, Iss 1, pp 13-16

Addresses:

WAIRAKEI-RES-CTR, INST GEOL \& NUCL SCI, PRIVATE BAG 2000,

TAUPO, NEW-ZEALAND

STANFORD-UNIV, DEPT GEOPHYS, STANFORD, CA 94305, USA

US-GEOL-SURVEY, MENLO-PK, CA 94025, USA

UNIV-CANTERBURY, DEPT GEOL, CHRISTCHURCH 1, NEW-ZEALAND

UNIV-WAIKATO, DEPT EARTH SCI, HAMILTON, NEW-ZEALAND

63. Authors: Liu-PC Hartzell-S Stephenson-W

Title: Nonlinear Multiparameter Inversion Using a Hybrid Global

Search Algorithm - Applications in Reflection Seismology

Source: GEOPHYSICAL JOURNAL INTERNATIONAL 1995, Vol 122, Iss 3, pp 991-1000

Addresses:

CHINESE-ACAD-SCI, INST GEOPHYS, BOX 9701, BEIJING 100101,

PEOPLES-R-CHINA

US-GEOL-SURVEY, EARTHQUAKE \& LANDSLIDE HAZARDS BRANCH, DENVER, CO 80225, USA

64. Authors: Kennett-BLN Engdahl-ER Buland-R

Title: Constraints on Seismic Velocities in the Earth from Travel-Times

Source: GEOPHYSICAL JOURNAL INTERNATIONAL 1995, Vol 122, Iss 1, pp 108-124

Addresses:

AUSTRALIAN-NATL-UNIV, RES SCH EARTH SCI, CANBERRA, ACT 0200, AUSTRALIA

US-GEOL-SURVEY, DENVER FED CTR, NATL EARTHQUAKE INFORMAT CTR, DENVER, CO 80225, USA

65. Authors: Engdahl-ER Vanderhilst-RD Berrocal-J

Title: Imaging of Subducted Lithosphere Beneath South-America

Source: GEOPHYSICAL RESEARCH LETTERS 1995, Vol 22, Iss 16, pp 2317-2320

Addresses:

US-GEOL-SURVEY, NATL EARTHQUAKE INFORMAT CTR, DENVER, CO 80225, USA

AUSTRALIAN-NATL-UNIV, RES SCH EARTH SCI, CANBERRA, ACT, AUSTRALIA

UNIV-SAO-PAULO, INST GEOPHYS, SAO-PAULO, BRAZIL

66. Authors: Creager-KC Chiao-LY Winchester-JP Engdahl-ER

Title: Membrane Strain Rates in the Subducting Plate Beneath

South-America

Source: GEOPHYSICAL RESEARCH LETTERS

1995, Vol 22, Iss 16, pp 2321-2324

Addresses:

UNIV-WASHINGTON, GEOPHYS PROGRAM, SEATTLE, WA 98195, USA

NATL-TAIWAN-UNIV, INST OCEANOG, TAIPEI, TAIWAN

US-GEOL-SURVEY, DENVER FED CTR, NEIC, DENVER, CO 80225, USA 
67. Authors: Foulger-GR Miller-AD Julian-BR Evans-JR

Title: 3-Dimensional Upsilon (P), and Upsilon (P)/Upsilon (S)

Structure of the Hengill Triple Junction and Geothermal

Area, Iceland, and the Repeatability of Tomographic

Inversion

Source: GEOPHYSICAL RESEARCH LETTERS

1995, Vol 22, Iss 10, pp 1309-1312

Addresses:

UNIV-DURHAM, DEPT GEOL SCI, DURHAM DH1-3LE, ENGLAND

US-GEOL-SURVEY, SEISMOL BRANCH, MENLO-PK, CA 94025, USA

68. Authors: Evans-JR Julian-BR Foulger-GR Ross-A

Title: Shear-Wave Splitting from Local Earthquakes at the

Geysers Geothermal-Field, California

Source: GEOPHYSICAL RESEARCH LETTERS

1995, Vol 22, Iss 4, pp 501-504

Addresses:

US-GEOL-SURVEY, 345 MIDDLEFIELD RD, MS-977, MENLO-PK, CA

94025, USA

UNIV-DURHAM, DEPT GEOL SCI, DURHAM DH1-3LE, ENGLAND

Record 316 of 695.

69. Authors: Jorgensen-DG Petricola-M

Title: Research Borehole-Geophysical Logging in Determining

Geohydrologic Properties

Source: GROUND WATER 1995, Vol 33, Iss 4, pp 589-596

Addresses:

US-GEOL-SURVEY, AL-AIN, U-ARAB-EMIRATES

SCHLUMBERGER-MIDDLE-E, ABU-DHABI, U-ARAB-EMIRATES

70. Authors: Lyons-PC Orem-WH Mastalerz-M Zodrow-EL Viethredemann-A Bustin-RM

Title: C-13 NMR, Micro-FTIR and Fluorescence-Spectra, and Pyrolysis-Gas Chromatograms of Coalified Foliage of Late Carboniferous Medullosan Seed Ferns, Nova-Scotia, Canada

- Implications for Coalification and Chemotaxonomy

Source: INTERNATIONAL JOURNAL OF COAL GEOLOGY 1995, Vol 27, Iss 2-4, pp 227-248

Addresses:

US-GEOL-SURVEY, NATL CTR, MS 956, RESTON, VA 22092, USA

UNIV-BRITISH-COLUMBIA, DEPT GEOL SCI, VANCOUVER, BC V6T-1Z4, CANADA

UNIV-COLL-CAPE-BRETON, DEPT EARTH SCI, SYDNEY, NSW B1P-6L2,

AUSTRALIA

GEOL-LANDESAMT-NORDRHEIN-WESTFALEN, D-44710 KREFELD, GERMANY

71. Authors: Willard-DA Dimichele-WA Eggert-DL Hower-JC Rexroad-CB Scott-AC

Title: Paleoecology of the Springfield Coal Member (Desmoinesian, Illinois Basin) Near the Leslie Cemetery

Paleochannel, Southwestern Indiana

Source: INTERNATIONAL JOURNAL OF COAL GEOLOGY

1995, Vol 27, Iss 1, pp 59-98 
Addresses:

US-GEOL-SURVEY, NATL CTR, MS 970, RESTON, VA 22092, USA

NAT-MUSEUM-NAT-HIST, DEPT PALEOBIOL, WASHINGTON, DC 20560, USA

INDIANA-GEOL-SURVEY, BLOOMINGTON, IN 47405, USA

UNIV-KENTUCKY, CTR APPL ENERGY RES, LEXINGTON, KY 40511, USA

UNIV-LONDON-ROYAL-HOLLOWAY-\&-BEDFORD-NEW-COLL, DEPT GEOL, EGHAM TW20-0EX, SURREY, ENGLAND

72. Authors: Aiken-G Cotsaris-E

Title: Soil and Hydrology - Their Effect on Nom

Source: JOURNAL AMERICAN WATER WORKS ASSOCIATION 1995, Vol 87, Iss 1, pp 36-45

Addresses:

US-GEOL-SURVEY, DIV WATER RESOURCES, MARIN ST SCI CTR, 3215

MARINE ST, BOULDER, CO 80303, USA

STATE-WATER-LAB, DEPT ENGN \& WATER SUPPLY, SALISBURY, SA 5108, AUSTRALIA

73. Authors: Jennings-AE Nelson-AR Scott-DB Aravena-JC

Title: Marsh Foraminiferal Assemblages in the Valdivia Estuary,

South-Central Chile, Relative to Vascular Plants and Sea-

Level

Source: JOURNAL OF COASTAL RESEARCH

1995, Vol 11, Iss 1, pp 107-123

Addresses:

UNIV-COLORADO, INSTAAR, CAMPUS BOX 450, BOULDER, CO 80309, USA

UNIV-COLORADO, DEPT GEOL SCI, BOULDER, CO 80309, USA

US-GEOL-SURVEY, DENVER, CO 80225, USA

DALHOUSIE-UNIV, CTR MARINE GEOL, HALIFAX, NS B3H-3J5, CANADA

UNIV-CHILE, FAC CIENCIAS, DEPT BIOL, SECC BOT, SANTIAGO, CHILE

74. Authors: Helz-RT Banks-NG Heliker-C Neal-CA Wolfe-EW

Title: Comparative Geothermometry of Recent Hawaiian Eruptions

Source: JOURNAL OF GEOPHYSICAL RESEARCH-SOLID EARTH 1995, Vol 100, Iss B9, pp 17637-17657

Addresses:

US-GEOL-SURVEY, NATL CTR, MS959, RESTON, VA 22092, USA

US-GEOL-SURVEY, CASCADES VOLCANO OBSERV, VANCOUVER, WA 98661, USA

US-GEOL-SURVEY, HAWAIIAN VOLCANO OBSERV, HILO, HI 96718, USA

US-GEOL-SURVEY, ALASKAN VOLCANO OBSERV, ANCHORAGE, AK 99508,

75. Authors: Ryberg-T Fuchs-K Egorkin-AV Solodilov-L

Title: Observation of High-Frequency Teleseismic P-N on the

Long-Range Quartz Profile Across Northern Eurasia

Source: JOURNAL OF GEOPHYSICAL RESEARCH-SOLID EARTH

1995, Vol 100, Iss B9, pp 18151-18163

Addresses:

US-GEOL-SURVEY, MS977, 345 MIDDLEFIELD RD, MENLO-PK, CA 94025, USA

GEON, CTR REG GEOPHYS \& GEOECOL RES, MOSCOW 119034, RUSSIA

UNIV-KARLSRUHE, INST GEOPHYS, D-76187 KARLSRUHE, GERMANY

76. Authors: Craymer-MR Vanicek-P Castle-RO

Title: Estimation of Rod Scale Errors in Geodetic Leveling 
Source: JOURNAL OF GEOPHYSICAL RESEARCH-SOLID EARTH

1995, Vol 100, Iss B8, pp 15129-15145

Addresses:

GEOMAT-CANADA, DIV GEODET SURVEY, 615 BOOTH ST, OTTAWA, ON

K1A-0E9, CANADA

UNIV-NEW-BRUNSWICK, DEPT SURVEYING ENGN, FREDERICTON, NB

E3B-5A3, CANADA

US-GEOL-SURVEY, MENLO-PK, CA 94025, USA

77. Authors: Andreassen-K Hart-PE Grantz-A

Title: Seismic Studies of a Bottom Simulating Reflection

Related to Gas Hydrate Beneath the Continental-Margin of

the Beaufort Sea

Source: JOURNAL OF GEOPHYSICAL RESEARCH-SOLID EARTH 1995, Vol 100, Iss B7, pp 12659-12673

Addresses:

UNIV-TROMSO, INST BIOL \& GEOL, N-9037 TROMSO, NORWAY

US-GEOL-SURVEY, MENLO-PK, CA 94025, USA

78. Authors: Chiarabba-C Amato-A Evans-JR

Title: Variations on the Neht High-Resolution Tomography Method

- A Test of Technique and Results for Medicine-Lake

Volcano, Northern California

Source: JOURNAL OF GEOPHYSICAL RESEARCH-SOLID EARTH 1995, Vol 100, Iss B3, pp 4035-4052

Addresses:

IST-NAZL-GEOFIS, VIA VIGNA MURATA 605, I-00143 ROME, ITALY

US-GEOL-SURVEY, MENLO-PK, CA 94025, USA

79 Authors: Vernik-L Hickman-S Lockner-D Rusanov-M

Title: Ultrasonic Velocities in Cores from the Kola Superdeep

Well and the Nature of Subhorizontal Seismic Reflections

Source: JOURNAL OF GEOPHYSICAL RESEARCH-SOLID EARTH 1994, Vol 99, Iss B12, pp 24209-24219

Addresses:

STANFORD-UNIV, DEPT GEOPHYS, STANFORD, CA 94305, USA

US-GEOL-SURVEY, MENLO-PK, CA 94025, USA

NEDRA-ENTERPRISE, ZAPOLYARNYI 184415, RUSSIA

80. Authors: Beardsley-RC Candela-J Limeburner-R Geyer-WR

Lentz-SJ Castro-BM Cacchione-D Carneiro-N

Title: The M(2) Tide on the Amazon Shelf

Source: JOURNAL OF GEOPHYSICAL RESEARCH-OCEANS 1995, Vol 100, Iss C2, pp 2283-2319

Addresses:

WOODS-HOLE-OCEANOG-INST, DEPT PHYS OCEANOG, WOODS-HOLE, MA 02543, USA

UNIV-SAO-PAULO, INST OCEANOG, BR-05508 SAO-PAULO, BRAZIL

US-GEOL-SURVEY, MENLO-PK, CA 94025, USA

DIRECTORIA-HIDROGRAFIA-\&-NAVEGACAO, NITEROI, RJ, BRAZIL

81. Authors: Foreman-MGG Walters-RA Henry-RF Keller-CP

Dolling-AG

Title: A Tidal Model for Eastern Juan-de-Fuca Strait and the

Southern Strait of Georgia 
Source: JOURNAL OF GEOPHYSICAL RESEARCH-OCEANS

1995, Vol 100, Iss C1, pp 721-740

Addresses:

INST-OCEAN-SCI, DEPT FISHERIES \& OCEANS, POB 6000, SIDNEY, BC V8L-4B2, CANADA

CHANNEL-CONSULTING-LTD, VICTORIA, BC V8T-4L1, CANADA

UNIV-VICTORIA, DEPT GEOG, VICTORIA, BC V8W-3P5, CANADA

US-GEOL-SURVEY, TACOMA, WA 98402, USA

82. Authors: Adrain-JM Chatterton-BDE Blodgett-RB

Title: Silurian Trilobites from Southwestern Alaska

Source: JOURNAL OF PALEONTOLOGY

1995, Vol 69, Iss 4, pp 723-736

Addresses:

UNIV-WESTERN-ONTARIO, DEPT EARTH SCI, LONDON, ON N6A-5B7, CANADA

UNIV-ALBERTA, DEPT GEOL, EDMONTON, AB T6G-2E3, CANADA

US-GEOL-SURVEY, PALEONTOL \& STRATIG BRANCH, RESTON, VA 22092, USA

83. Authors: Gubanov-AP Blodgett-RB Lytochkin-VM

Title: Early Devonian (Pragian) Gastropods from Kyrgyzstan

(Central-Asia)

Source: JOURNAL OF PALEONTOLOGY

1995, Vol 69, Iss 3, pp 431-440

Addresses:

INST-GEOL, UNIV AVE 3, NOVOSIBIRSK 630090, RUSSIA

US-GEOL-SURVEY, PALEONTOL \& STRATIG BRANCH, RESTON, VA 22092,

USA

PKF-KYRTASH, BISHKEK 720039, KYRGYZSTAN

84. Authors: Popov-LY Blodgett-RB Anderson-AV

Title: First Occurrence of the Genus Bicarinatina (Brachiopoda, Inarticulata) from the Middle Devonian in North-America

(Alaska)

Source: JOURNAL OF PALEONTOLOGY

1994, Vol 68, Iss 6, pp 1214-1218

Addresses:

ALL-UNION-GEOL-RES-INST, SREDNII PROSPEKT 74, ST-PETERSBURG

199026, RUSSIA

US-GEOL-SURVEY, NATL CTR 970, RESTON, VA 22092, USA

UNIV-ALASKA, DEPT GEOL \& GEOPHYS, FAIRBANKS, AK 99775, USA

85. Authors: Barnes-CG Johnson-K Barnes-MA Prestvik-T

Kistler-RW Sundvoll-B

Title: The Grayback Pluton - Magmatism in a Jurassic Back-Arc Environment, Klamath Mountains, Oregon

Source: JOURNAL OF PETROLOGY

1995, Vol 36, Iss 2, pp 397-415

Addresses:

TEXAS-TECH-UNIV, DEPT GEOSCI, LUBBOCK, TX 79409, USA

UNIV-TRONDHEIM, NORWEGIAN INST TECHNOL, DEPT GEOL, N-7034

TRONDHEIM, NORWAY

US-GEOL-SURVEY, MENLO-PK, CA 94025, USA

UNIV-OSLO, MINERAL GEOL MUSEUM, N-0562 OSLO 5, NORWAY 
86. Authors: Loferski-PJ Arculus-RJ Czamanske-GK

Title: Rare-Earth Element Evidence for the Petrogenesis of the Banded Series of the Stillwater Complex, Montana, and Its Anorthosites

Source: JOURNAL OF PETROLOGY 1994, Vol 35, Iss 6, pp 1623-1649

Addresses:

US-GEOL-SURVEY, 954 NATL CTR, RESTON, VA 22092, USA

AUSTRALIAN-NATL-UNIV, DEPT GEOL, CANBERRA, ACT 0200, AUSTRALIA

US-GEOL-SURVEY, MENLO-PK, CA 94025, USA

87. Authors: Stout-MZ Nicholls-J Kuntz-MA

Title: Petrological and Mineralogical Variations in 2500-2000

Yr Bp Lava Flows, Craters of the Moon Lava-Field, Idaho

Source: JOURNAL OF PETROLOGY

1994, Vol 35, Iss 6, pp 1681-1715

Addresses:

UNIV-CALGARY, CALGARY, AB T2N-1N4, CANADA

US-GEOL-SURVEY, DENVER FED CTR, DENVER, CO 80225, USA

Record 458 of 695.

88. Authors: Vallance-JW Siebert-L Rose-WI Giron-JR Banks-NG

Title: Edifice Collapse and Related Hazards in Guatemala

Source: JOURNAL OF VOLCANOLOGY AND GEOTHERMAL RESEARCH 1995, Vol 66, Iss 1-4, pp 337-355

Addresses:

MICHIGAN-TECHNOL-UNIV, DEPT GEOL ENGN \& SCI, HOUGHTON, MI 49931, USA

SMITHSONIAN-INST, GLOBAL VOLCANISM PROGRAM, WASHINGTON, DC 20560, USA

INSIVUMEH, SECC VULCANOL, GUATEMALA-CITY, GUATEMALA

US-GEOL-SURVEY, VANCOUVER, WA 98661, USA

89. Authors: Bluth-GJS Casadevall-TJ Schnetzler-CC Doiron-SD

Walter-LS Krueger-AJ Badruddin-M

Title: Evaluation of Sulfur-Dioxide Emissions from Explosive Volcanism - The 1982-1983 Eruptions of Galunggung, Java, Indonesia

Source: JOURNAL OF VOLCANOLOGY AND GEOTHERMAL RESEARCH 1994, Vol 63, Iss 3-4, pp 243-256

Addresses:

UNIV-MARYLAND, DEPT GEOL, COLLEGE-PK, MD 20742, USA

US-GEOL-SURVEY, DENVER, CO 80225, USA

UNIV-MARYLAND, DEPT GEOG, COLLEGE-PK, MD 20742, USA

HUGHES-STX-CORP, LANHAM, MD, USA

NASA, GODDARD SPACE FLIGHT CTR, EARTH SCI DIRECTORATE,

GREENBELT, MD 20771, USA

DIREKTORAT-VULKANOLOGI, DEPT PERTAMBANGAN DAN ENERGI, BANDUNG, INDONESIA

90. Authors: Hekinian-R Stoffers-P Akermand-D Binard-N

Francheteau-J Devey-C Garbeschonberg-D

Title: Magmatic Evolution of the Easter Microplate-Crough

Seamount Region (South East Pacific)

Source: MARINE GEOPHYSICAL RESEARCHES

1995, Vol 17, Iss 4, pp 375-397 
Addresses:

IFREMER, CTR BREST, BP 70, F-29263 PLOUZANE, FRANCE

CHRISTIAN-ALBRECHTS-UNIV-KIEL, INST GEOL PALAONTOL, W-2300

KIEL, GERMANY

CHRISTIAN-ALBRECHTS-UNIV-KIEL, INST MINERAL PETROG, W-2300

KIEL, GERMANY

US-GEOL-SURVEY, MENLO-PK, CA 94025, USA

UNIV-BRETAGNE-OCCIDENTALE, F-29287 BREST, FRANCE

91. Authors: Dewever-P Bourgois-J Caulet-JP Fourtanier-E Barron-J Dumitrica-P

Title: Stratigraphic Significance of Siliceous Microfossils

Collected During Nautiperc Dives (Off Peru, 5-Degrees-6Degrees-S)

Source: MARINE MICROPALEONTOLOGY 1994, Vol 24, Iss 3-4, pp 287-305

Addresses:

UNIV-PARIS-06, INSU, CNRS, URA 1761, DEPT GEOL SEDIMENTAIRE, CASE 117, 4 PL JUSSIEU, F-75252 PARIS 05, FRANCE

MUSEUM-NATL-HIST-NAT, CNRS, F-75231 PARIS, FRANCE

CALIF-ACAD-SCI, DEPT INVERTEBRATE ZOOL \& GEOL, DIATOM COLLECT, SAN-FRANCISCO, CA 94118, USA

US-GEOL-SURVEY, MENLO-PK, CA 94025, USA

UNIL, INST GEOL \& PALEONTOL, CH-1015 LAUSANNE, SWITZERLAND

92. Authors: Luoma-SN Ho-YB Bryan-GW

Title: Fate, Bioavailability and Toxicity of Silver in

Estuarine Environments

Source: MARINE POLLUTION BULLETIN 1995, Vol 31, Iss 1-3, pp 44-54

Addresses:

US-GEOL-SURVEY, MAIL STOP 465, MENLO-PK, CA 94025, USA

UNIV-HONG-KONG, DEPT BOT, HONG-KONG, HONG-KONG

PLYMOUTH-MARINE-LAB, PLYMOUTH PL1-2PB, DEVON, ENGLAND

93. Authors: Mayer-H

Title: Cyclostratigraphic Calibration of Cretaceous Magnetic Polarity Events (Cismon, Southern Alps, Italy

Source: MATHEMATICAL GEOLOGY 1994, Vol 26, Iss 7, pp 783-798

Addresses:

UNIV-TUBINGEN, INST GEOL \& PALAONTOL, W-7400 TUBINGEN 1, GERMANY

US-GEOL-SURVEY, DENVER, CO 80225, USA

94. Authors: Chapman-CR Veverka-J Thomas-PC Klaasen-K

Belton-MJS Harch-A Mcewen-A Johnson-TV

Helfenstein-P Davies-ME Merline-WJ Denk-T

Title: Discovery and Physical-Properties of Dactyl, a Satellite of Asteroid 243-IDA

Source: NATURE 1995, Vol 374, Iss 6525, pp 783-785

Addresses:

SJI, INST PLANETARY SCI, 620 N 6TH AVE, TUCSON, AZ 85705, USA

CORNELL-UNIV, CTR RADIOPHYS \& SPACE RES, ITHACA, NY 14853, USA

CALTECH, JET PROP LAB, PASADENA, CA 91109, USA 
NATL-OPT-ASTRON-OBSERV, TUCSON, AZ 85719, USA

US-GEOL-SURVEY, FLAGSTAFF, AZ 86001, USA

RAND-CORP, SANTA-MONICA, CA 90406, USA

INST-PLANETENERKUNDUNG, D-12484 BERLIN, GERMANY

95. Authors: Koopmans-MP Damste-JSS Lewan-MD Deleeuw-JW

Title: Thermal-Stability of Thiophene Biomarkers as Studied by Hydrous Pyrolysis

Source: ORGANIC GEOCHEMISTRY 1995, Vol 23, Iss 6, pp 583-596

Addresses:

NETHERLANDS-INST-SEA-RES, DEPT MARINE BIOGEOCHEM, POB 59, 1790-AB DEN-BURG, NETHERLANDS

US-GEOL-SURVEY, DENVER FED CTR, DENVER, CO 80225, USA

96. Authors: Poag-CW Aubry-MP

Title: Upper Eocene Impactites of the Us East-Coast -

Depositional Origins, Biostratigraphic Framework, and

Correlation

Source: PALAIOS 1995, Vol 10, Iss 1, pp 16-43

Addresses:

US-GEOL-SURVEY, WOODS-HOLE, MA 02543, USA

CNRS, GEOL QUATERNAIRE LAB, F-13288 MARSEILLE, FRANCE

97. Authors: Bralower-TJ Zachos-JC Thomas-E Parrow-M Paull-CK Kelly-DC Silva-IP Sliter-WV Lohmann-KC

Title: Late Paleocene to Eocene Paleoceanography of the Equatorial Pacific-Ocean - Stable Isotopes Recorded at Ocean Drilling Program Site-865, Allison-Guyot

Source: PALEOCEANOGRAPHY 1995, Vol 10, Iss 4, pp 841-865

Addresses:

UNIV-N-CAROLINA, DEPT GEOL, CB 3315, CHAPEL-HILL, NC 27599, USA

UNIV-MICHIGAN, DEPT GEOL SCI, ANN-ARBOR, MI 48109, USA

UNIV-MILAN, DIPARTIMENTO SCI TERRA, I-20133 MILAN, ITALY

US-GEOL-SURVEY, PALEONTOL \& STRATIG BRANCH, MENLO-PK, CA 94025, USA

YALE-UNIV, DEPT GEOL \& GEOPHYS, NEW-HAVEN, CT 06511, USA

UNIV-CALIF-SANTA-CRUZ, EARTH SCI BOARD, SANTA-CRUZ, CA 95064, USA

WESLEYAN-UNIV, DEPT EARTH \& ENVIRONM SCI, MIDDLETOWN, CT 06457, USA

98. Authors: Cronin-TM Holtz-TR Stein-R Spielhagen-R Futterer-D Wollenburg-J

Title: Late Quaternary Paleoceanography of the Eurasian Basin, Arctic-Ocean

Source: PALEOCEANOGRAPHY

1995, Vol 10, Iss 2, pp 259-281

Addresses:

US-GEOL-SURVEY, RESTON, VA 22092, USA

ALFRED-WEGENER-INST-POLAR-\&-MARINE-RES, BREMERHAVEN, GERMANY

CHRISTIAN-ALBRECHTS-UNIV-KIEL, GEOMAR RES CTR MARINE SCI,

D-24148 KIEL, GERMANY 
99. Authors: Lantos-M Elston-DP

Title: Low-Amplitude to High-Amplitude Oscillations and Secular Variation in a $1.2 \mathrm{~km}$ Late Miocene Inclination Record

Source: PHYSICS OF THE EARTH AND PLANETARY INTERIORS 1995, Vol 90, Iss 1-2, pp 37-53

Addresses:

HUNGARIAN-GEOL-INST, STEFANIA UT 14, POB 106, H-1442 BUDAPEST, HUNGARY

US-GEOL-SURVEY, FLAGSTAFF, AZ 86001, USA

100. Authors: Payne-TE Davis-JA Waite-TD

Title: Uranium Retention by Weathered Schists - The Role of Iron Minerals

Source: RADIOCHIMICA ACTA 1994, Vol 66-7, pp 297-303

Addresses:

AUSTRALIAN-NUCL-SCI-\&-TECHNOL-ORG, PRIVATE MAIL BAG 1, MENAI, NSW 2234, AUSTRALIA

US-GEOL-SURVEY, DIV WATER RESOURCES, MENLO-PK, CA 94025, USA

UNIV-NEW-S-WALES, DEPT WATER ENGN, KENSINGTON, NSW 2033, AUSTRALIA

101. Authors: Weaver-HA Ahearn-MF Arpigny-C Boice-DC Feldman-PD Larson-SM Lamy-P Levy-DH Marsden-BG Meech-KJ Noll-KS Scotti-JV Sekanina-Z Shoemaker-CS

Shoemaker-EM Smith-TE Stern-SA Storrs-AD

Trauger-JT Yeomans-DK Zellner-B

Title: The Hubble-Space-Telescope (HST) Observing Campaign on Comet Shoemaker-Levy-9

Source: SCIENCE 1995, Vol 267, Iss 5202, pp 1282-1288

Addresses:

SPACE-TELESCOPE-SCI-INST, BALTIMORE, MD 21218, USA

UNIV-MARYLAND, DEPT ASTRON, COLLEGE-PK, MD 20742, USA

UNIV-LIEGE, INST ASTROPHYS, B-4000 LIEGE, BELGIUM

SW-RES-INST, DIV 15, SAN-ANTONIO, TX 78228, USA

JOHNS-HOPKINS-UNIV, DEPT PHYS \& ASTRON, BALTIMORE, MD 21218, USA

UNIV-ARIZONA, LUNAR \& PLANETARY LAB, TUCSON, AZ 85721, USA

LAB-ASTRON-SPATIALE-TRAVERSE-SIPHON, F-13376 MARSEILLE 12,

FRANCE

HARVARD-SMITHSONIAN-CTR-ASTROPHYS, CAMBRIDGE, MA 02138, USA

GEORGIA-SO-UNIV, DEPT PHYS, STATESBORO, GA 30460, USA

US-GEOL-SURVEY, FLAGSTAFF, AZ 86001, USA

SW-RES-INST, DIV 15, BOULDER EXTENS OFF, BOULDER, CO 80302, USA

JET-PROPULS-LAB, PASADENA, CA 91109, USA

UNIV-HAWAII, INST ASTRON, HONOLULU, HI 96822, USA

NO-ARIZONA-UNIV, DEPT PHYS \& ASTRON, FLAGSTAFF, AZ 86011, USA

102. Authors: Eittreim-SL Cooper-AK Wannesson-J

Title: Seismic Stratigraphic Evidence of Ice-Sheet Advances on the Wilkes Land Margin of Antarctica

Source: SEDIMENTARY GEOLOGY 1995, Vol 96, Iss 1-2, pp 131-156 
Addresses:

US-GEOL-SURVEY, BRANCH PACIFIC MARINE GEOL, 345 MIDDLEFIELD

RD, MENLO-PK, CA 94025, USA

INST-FRANCAIS-PETR, F-92506 RUEIL-MALMAISON, FRANCE

103. Authors: Coker-JE Steltenpohl-MG Andresen-A Kunk-MJ

Title: An Ar-40/Ar-39 Thermochronology of the Ofoten-Troms

Region - Implications for Terrane Amalgamation and

Extensional Collapse of the Northern Scandinavian

Caledonides

Source: TECTONICS

1995, Vol 14, Iss 2, pp 435-447

Addresses:

UNIV-OSLO, DEPT GEOL, OSLO 3, NORWAY

US-GEOL-SURVEY, RESTON, VA 22092, USA

AUBURN-UNIV, DEPT GEOL, AUBURN, AL 36849, USA

104. Authors: Vakarcs-G Vail-PR Tari-G Pogacsas-G Mattick-RE Szabo-A

Title: 3rd-Order Middle Miocene Early Pliocene Depositional

Sequences in the Prograding Delta-Complex of the

Pannonian Basin

Source: TECTONOPHYSICS

1994, Vol 240, Iss 1-4, pp 81-106

Addresses:

RICE-UNIV, DEPT GEOL \& GEOPHYS, HOUSTON, TX 77251, USA

MOL-RT-EXPLORAT-DEPT, H-1039 BUDAPEST, HUNGARY

US-GEOL-SURVEY, RESTON, VA 22092, USA

105. Authors: Vigliotti-L Langenheim-VE

Title: When Did Sardinia Stop Rotating - New Paleomagnetic Results

Source: TERRA NOVA 1995, Vol 7, Iss 4, pp 424-435

Addresses:

CNR, IST GEOL MARINA, I-40129 BOLOGNA, ITALY

US-GEOL-SURVEY, MENLO-PK, CA 94025, USA

106. Authors: Aravena-R Wassenaar-LI Plummer-LN

Title: Estimating C-14 Groundwater Ages in a Methanogenic Aquifer

Source: WATER RESOURCES RESEARCH

1995, Vol 31, Iss 9, pp 2307-2317

Addresses:

UNIV-WATERLOO, WATERLOO CTR GROUNDWATER RES, WATERLOO, ON

N2L-3G1, CANADA

US-GEOL-SURVEY, 432 NATL CTR, RESTON, VA 22092, USA

ENVIRONM-CANADA, NATL HYDROL RES INST, SASKATOON, SK S7N-3H5, CANADA

107. Authors: Mayer-H

Title: Flexural Extension of the Upper Continental-Crust in Collisional Foredeeps

Source: GEOLOGICAL SOCIETY OF AMERICA BULLETIN 1995, Vol 107, Iss 4, pp 499-501 
Addresses:

UNIV-COLORADO, INST ARCTIC \& ALPINE RES, BOULDER, CO 80309, USA

US-GEOL-SURVEY, DENVER, CO 80225, USA

UNIV-TUBINGEN, INST GEOL \& PALAONTOL, TUBINGEN, GERMANY

108. Authors: King-CY Koizumi-N Kitagawa-Y

Title: Hydrogeochemical Anomalies and the 1995 Kobe Earthquake

Source: SCIENCE 1995, Vol 269, Iss 5220, pp 38-39

Addresses:

US-GEOL-SURVEY, 345 MIDDLEFIELD RD, MENLO-PK, CA 94025, USA

KYOTO-UNIV, DISASTER PREVENT RES INST, KYOTO, JAPAN

109. Authors: Chabaux-F Cohen-AS Onions-RK Hein-JR

Title: U-238 U-234-Th-230 Chronometry of Fe-Mn Crusts - GrowthProcesses and Recovery of Thorium Isotopic-Ratios of Seawater

Source: GEOCHIMICA ET COSMOCHIMICA ACTA 1995, Vol 59, Iss 3, pp 633-638

Addresses:

UNIV-CAMBRIDGE, DEPT EARTH SCI, CAMBRIDGE CB2-3EQ, ENGLAND

US-GEOL-SURVEY, MENLO-PK, CA 94025, USA

110. Authors: Kile-DE Chiou-CT Wershaw-RL Zhou-H

Title: Differences in Organic-Matter Polarities Between Soils and Sediments as Measured by Nonpolar Solute PartitionCoefficients and Solid-State C-13 NMR

Source: ABSTRACTS OF PAPERS OF THE AMERICAN CHEMICAL SOCIETY 1995, Vol 210, Iss AUG, pp 77-ENVR

Addresses:

MINIST-WATER-RESOURCES, BEIJING 100044, PEOPLES-R-CHINA

US-GEOL-SURVEY, LAKEWOOD, CO 80225, USA

111. Authors: Thurman-EM Goolsby-DA Meyer-MT Cromwell-AE

Title: Evidence for Long-Range Atmospheric Transport and Degradation of Herbicides

Source: ABSTRACTS OF PAPERS OF THE AMERICAN CHEMICAL SOCIETY 1995, Vol 209, Iss APR, pp 58-ENVR

Addresses:

US-GEOL-SURVEY, LAWRENCE, KS 66049, USA

TECH-UNIV-MUNICH, W-8050 FREISING, GERMANY

112. Authors: Kita-NT Misawa-K Tatsumoto-M

Title: Lree-Rich Component in Ureilites - Further Evidence of Terrestrial Contamination

Source: METEORITICS 1995, Vol 30, Iss 5, pp 528-528

Addresses:

GEOL-SURVEY-JAPAN, TSUKUBA, IBARAKI 305, JAPAN

KOBE-UNIV, DEPT EARTH \& PLANETARY SCI, KOBE 657, JAPAN

US-GEOL-SURVEY, DENVER, CO 80225, USA

113. Authors: Koeberl-C Reimold-WU Brandt-D Poag-CW

Title: Chesapeake Bay Crater, Virginia - Confirmation of Impact Origin

Source: METEORITICS

1995, Vol 30, Iss 5, pp 528-529 
Addresses:

UNIV-VIENNA, INST GEOCHEM, A-1090 VIENNA, AUSTRIA

UNIV-WITWATERSRAND, DEPT GEOL, JOHANNESBURG 2050, SOUTH-AFRICA

US-GEOL-SURVEY, WOODS-HOLE, MA 02543, USA

114. Authors: Field-EH Clement-AC Jacob-KH Aharonian-V Hough-SE

Friberg-PA Babaian-TO Karapetian-SS Hovanessian-SM

Abramian-HA

Title: Earthquake Site-Response Study in Giumri (Formerly

Leninakan), Armenia, Using Ambient Noise Observations

Source: BULLETIN OF THE SEISMOLOGICAL SOCIETY OF AMERICA 1995, Vol 85, Iss 1, pp 349-353

Addresses:

COLUMBIA-UNIV, LAMONT DOHERTY EARTH OBSERV, PALISADES, NY

10964, USA

US-GEOL-SURVEY, PASADENA, CA 91106, USA

INST-GEOPHYS, GIUMRI, ARMENIA

115. Authors: Gray-JE Coolbaugh-MF Plumlee-GS Atkinson-WW

Title: Environmental Geology of the Summitville Mine, Colorado

Source: ECONOMIC GEOLOGY AND THE BULLETIN OF THE SOCIETY OF ECONOMIC GEOLOGISTS 1994, Vol 89, Iss 8, pp 2006-2014

Addresses:

US-GEOL-SURVEY, DENVER FED CTR, DENVER, CO 80225, USA

CARSON-GOLD-CORP, VANCOUVER, BC V6C-2G8, CANADA

UNIV-COLORADO, DEPT GEOL SCI, BOULDER, CO 80302, USA

116. Authors: Wang-WC Freemark-K

Title: The Use of Plants for Environmental Monitoring and Assessment

Source: ECOTOXICOLOGY AND ENVIRONMENTAL SAFETY 1995, Vol 30, Iss 3, pp 289-301

Addresses:

US-GEOL-SURVEY, 720 GRACERN RD, SUITE 129, COLUMBIA, SC 29210, USA

ENVIRONM-CANADA, NATL WILDLIFE RES CTR, CANADIAN WILDLIFE SERV, OTTAWA, ON K1A-0H3, CANADA

117. Authors: Kargel-JS Baker-VR Beget-JE Lockwood-JF Pewe-TL Shaw-JS Strom-RG

Title: Evidence of Ancient Continental-Glaciation in the Martian Northern Plains

Source: JOURNAL OF GEOPHYSICAL RESEARCH-PLANETS 1995, Vol 100, Iss E3, pp 5351-5368

Addresses:

US-GEOL-SURVEY, 2255 N GEMINI DR, FLAGSTAFF, AZ 86001, USA UNIV-ALASKA, DEPT GEOL \& GEOPHYS, FAIRBANKS, AK 99775, USA UNIV-ARIZONA, DEPT GEOSCI, TUCSON, AZ 85721, USA

SAHUARO-HIGH-SCH, TUCSON, AZ, USA

ARIZONA-STATE-UNIV, DEPT GEOL, TEMPE, AZ 85287, USA UNIV-ALBERTA, DEPT GEOG, EDMONTON, AB T6G-2H4, CANADA UNIV-ARIZONA, LUNAR \& PLANETARY LAB, TUCSON, AZ 85721, USA

UNIV-ARIZONA, DEPT PLANETARY SCI, TUCSON, AZ 85721, USA 
118. Authors: Hickman-S Sibson-R Bruhn-R

Title: Introduction to Special Section - Mechanical Involvement of Fluids in Faulting

Source: JOURNAL OF GEOPHYSICAL RESEARCH-SOLID EARTH 1995, Vol 100, Iss B7, pp 12831-12840

Addresses:

US-GEOL-SURVEY, MS 977, 345 MIDDLEFIELD RD, MENLO-PK, CA

94025, USA

UNIV-UTAH, DEPT GEOL \& GEOPHYS, SALT-LAKE-CITY, UT 84112, USA

UNIV-OTAGO, DEPT GEOL, DUNEDIN, NEW-ZEALAND

119. Authors: Eberhartphillips-D Stanley-WD Rodriguez-BD Lutter-WJ

Title: Surface Seismic and Electrical Methods to Detect Fluids Related to Faulting

Source: JOURNAL OF GEOPHYSICAL RESEARCH-SOLID EARTH 1995, Vol 100, Iss B7, pp 12919-12936

Addresses:

INST-GEOL-\&-NUCL-SCI, POB 1320, WELLINGTON, NEW-ZEALAND

UNIV-WISCONSIN, DEPT GEOL \& GEOPHYS, MADISON, WI 53706, USA

US-GEOL-SURVEY, DENVER FED CTR, DENVER, CO 80225, USA

US-GEOL-SURVEY, MENLO-PK, CA 94025, USA

120. Authors: Lambert-DD Walker-RJ Morgan-JW Shirey-SB Carlson-RW Zientek-ML Lipin-BR Koski-MS Cooper-RL

Title: Re-Os and Sm-Nd Isotope Geochemistry of the Stillwater Complex, Montana - Implications for the Petrogenesis of the J-M Reef

Source: JOURNAL OF PETROLOGY 1994, Vol 35, Iss 6, pp 1717-1753

Addresses:

MONASH-UNIV, VICTORIAN INST EARTH \& PLANETARY SCI, DEPT EARTH SCI, CLAYTON, VIC 3168, AUSTRALIA

UNIV-MARYLAND, DEPT GEOL, COLLEGE-PK, MD 20742, USA

US-GEOL-SURVEY, RESTON, VA 22092, USA

CARNEGIE-INST-WASHINGTON, DEPT TERR MAGNETISM, WASHINGTON, DC 20015, USA

US-GEOL-SURVEY, SPOKANE, WA 99201, USA

STILLWATER-MIN-CO, NYE, MT 59061, USA

LAMAR-UNIV, DEPT GEOL, BEAUMONT, TX 77710, USA

121. Authors: Schiffman-R Dean-A Chaney-RC Suchanek-J Vega-B

Mcfarland-T Schultheiss-PJ Gunn-D Keller-GH

Noorany-I Faghihi-M Shephard-L Lipkin-J Kinker-J

Bryant-WR Taylor-E Johns-M Silva-AJ Levy-WP

Siciliano-R Dadey-K Olsen-H

Title: Geotechnical Properties of Northwest Pacific Pelagic

Clays - Deep-Sea Drilling Project Leg-86, Hole-576A

(Reprinted from Initial Reports of the Deep Sea Drilling Project, Vol 86, 1985)

Source: MARINE GEORESOURCES \& GEOTECHNOLOGY 1994, Vol 12, Iss 4, pp 341-404

Addresses:

UNIV-COLORADO, BOULDER, CO 80302, USA

HUMBOLDT-STATE-UNIV, ARCATA, CA, USA

INST-OCEANOG-SCI, GODALMING GU8-5UB, SURREY, ENGLAND

OREGON-STATE-UNIV, CORVALLIS, OR, USA 
SAN-DIEGO-UNIV, SAN-DIEGO, CA, USA

SANDIA-NATL-LABS, ALBUQUERQUE, NM, USA

TEXAS-A\&M-UNIV, COLLEGE-STN, TX, USA

UNIV-RHODE-ISL, NARRAGANSETT, RI, USA

US-GEOL-SURVEY, DENVER, CO, USA

122. Authors: Dubrovskii-VA Sergeev-VN Fuis-GS

Title: Generalized Isostasy Condition

Source: DOKLADY AKADEMII NAUK

1995, Vol 342, Iss 1, pp 105-107

Addresses:

OY-SHMIDT-EARTH-PHYS-JOINT-INST, MOSCOW, RUSSIA

US-GEOL-SURVEY, WASHINGTON, DC 20242, USA

\section{6}

1. Authors: Mattick-RE Teleki-PG Phillips-RL Clayton-JL

David-G Pogacsas-G Bardocz-B Simon-E

Title: Structure, Stratigraphy, and Petroleum Geology of the

Little Plain Basin, Northwestern Hungary

Source: AAPG BULLETIN-AMERICAN ASSOCIATION OF PETROLEUM GEOLOGISTS

1996, Vol 80, Iss 11, pp 1780-1800

Addresses:

US-GEOL-SURVEY, NATL CTR, MS 909, RESTON, VA 22092, USA

TELEKI-\&-ASSOCIATES, HERNDON, VA 22071, USA

US-GEOL-SURVEY, MENLO-PK, CA 94303, USA

US-GEOL-SURVEY, DENVER, CO 80225, USA

MOL-PLC, HUNGARIAN OIL \& GAS CO, H-1117 BUDAPEST, HUNGARY

2.Authors: Howarth-RW Billen-G Swaney-D Townsend-A Jaworski-N Lajtha-K Downing-JA Elmgren-R Caraco-N Jordan-T

Berendse-F Freney-J Kudeyarov-V Murdoch-P Zhu-ZL

Title: Regional Nitrogen Budgets and Riverine N-and-P Fluxes

for the Drainages to the North-Atlantic Ocean - Natural

and Human Influences

Source: BIOGEOCHEMISTRY

1996, Vol 35, Iss 1, pp 75-139

Addresses:

CORNELL-UNIV, ITHACA, NY 14853, USA

FREE-UNIV-BRUSSELS, GRP MICROBIOL MILIEUX AQUAT, B-1050

BRUSSELS, BELGIUM

US-EPA, ENVIRONM RES LAB, NARRAGANSETT, RI 02882, USA

OREGON-STATE-UNIV, DEPT BOT \& PLANT PATHOL, CORVALLIS, OR 97331, USA

IOWA-STATE-UNIV, AMES, IA 50011, USA

UNIV-STOCKHOLM, DEPT SYST ECOL, S-10691 STOCKHOLM, SWEDEN

UNIV-STOCKHOLM, CTR MARINE RES, S-10691 STOCKHOLM, SWEDEN

INST-ECOSYST-STUDIES, MILLBROOK, NY 12545, USA

INST-SOIL-SCI-\&-PHOTOSYNTH, PUSHCHINO 142292, RUSSIA

DLO, CABO, NL-6700-AA WAGENINGEN, NETHERLANDS

CSIRO, DIV PLANT IND, CANBERRA, ACT 2601, AUSTRALIA

INST-SOIL-SCI, LAB MAT CYCLING PEDOSPHERE, NANJING,

PEOPLES-R-CHINA

US-GEOL-SURVEY, DIV WATER RESOURCES, ALBANY, NY 12201, USA

SMITHSONIAN-ENVIRONM-RES-CTR, EDGEWATER, MD 21037, USA 
3. Authors: Hawthorne-FC Oberti-R Ottolini-L Foord-EE

Title: Lithium-Bearing Fluor-Arfvedsonite from Hurricane Mountain, New-Hampshire - A Crystal-Chemical Study

Source: CANADIAN MINERALOGIST 1996, Vol 34, Iss OCT, pp 1015-1019

Addresses:

CNR, CTR STUDIO CRISTALLOCHIM \& CRISTALLOG, I-27100 PAVIA, ITALY

US-GEOL-SURVEY, DENVER FED CTR, LAKEWOOD, CO 80225, USA

4. Authors: Uncles-RJ Peterson-DH

Title: The Long-Term Salinity Field in San-Francisco Bay

Source: CONTINENTAL SHELF RESEARCH 1996, Vol 16, Iss 15, pp 2005-2039

Addresses:

PLYMOUTH-MARINE-LAB, CITADEL HILL, PLYMOUTH PL1-3DH, DEVON, ENGLAND

US-GEOL-SURVEY, MENLO-PK, CA 94025, USA

5. Authors: Griffin-WL Slack-JF Ramsden-AR Win-TT Ryan-CG

Title: Trace-Elements in Tourmalines from Massive Sulfide Deposits and Tourmalinites - Geochemical Controls and Exploration Applications

Source: ECONOMIC GEOLOGY AND THE BULLETIN OF THE SOCIETY OF ECONOMIC GEOLOGISTS 1996, Vol 91, Iss 4, pp 657-675

Addresses:

CSIRO, POB 136, 51 DELHI RD, N-RYDE, NSW 2113, AUSTRALIA

MACQUARIE-UNIV, SCH EARTH SCI, KEY CTR GEOCHEM EVOLUT \& METALLOGENY CONTINENTS, SYDNEY, NSW 2109, AUSTRALIA

US-GEOL-SURVEY, NATL CTR, RESTON, VA 22092, USA

6. Authors: Moore-DE Lockner-DA Summers-R Shengli-M Byerlee-JD

Title: Strength of Chrysotile-Serpentinite Gouge Under Hydrothermal Conditions - Can It Explain a Weak SanAndreas Fault

Source: GEOLOGY 1996, Vol 24, Iss 11, pp 1041-1044

Addresses:

US-GEOL-SURVEY, 345 MIDDLEFIELD RD, MENLO-PK, CA 94025, USA

STATE-SEISMOL-BUR, INST GEOL, BEIJING 100029, PEOPLES-R-CHINA

7. Authors: Kutzbach-JE Bartlein-PJ Foley-JA Harrison-SP Hostetler-SW Liu-Z Prentice-IC Webb-T

Title: Potential Role of Vegetation Feedback in the Climate Sensitivity of High-Latitude Regions - A Case-Study at 6000 Years Bp

Source: GLOBAL BIOGEOCHEMICAL CYCLES 1996, Vol 10, Iss 4, pp 727-736

Addresses:

UNIV-WISCONSIN, INST ENVIRONM STUDIES, CLIN RES CTR, $1225 \mathrm{~W}$

DAYTON, MADISON, WI 53706, USA

UNIV-OREGON, DEPT GEOG, EUGENE, OR 97403, USA

LUND-UNIV, S-22100 LUND, SWEDEN

US-GEOL-SURVEY, CORVALLIS, OR 97333, USA 
UNIV-WISCONSIN, DEPT ATOMOSPHER \& OCEAN SCI, MADISON, WI 53706, USA

LUND-UNIV, DEPT ECOL, GLOBAL SYST GRP, S-22362 LUND, SWEDEN

BROWN-UNIV, DEPT GEOL SCI, PROVIDENCE, RI 02912, USA

8. Authors: Dorbath-C Oppenheimer-D Amelung-F King-G

Title: Seismic Tomography and Deformation Modeling of the Junction of the San-Andreas and Calaveras Faults

Source: JOURNAL OF GEOPHYSICAL RESEARCH-SOLID EARTH 1996, Vol 101, Iss B12, pp 27917-27941

Addresses:

EOPG, URA 1356 CNRS, 5 RUE RENE DESCARTES, F-67084 STRASBOURG,

FRANCE

US-GEOL-SURVEY, MENLO-PK, CA 94025, USA

INST-FRANCAIS-RECH-SCI-DEV-COOPERAT, ORSTOM, PARIS, FRANCE

9. Authors: Bird-RT Searle-RC Paskevich-V Twichell-DC

Title: Merged Gloria Sidescan and Hydrosweep Pseudo-Sidescan -

Processing and Creation of Digital Mosaics

Source: MARINE GEOPHYSICAL RESEARCHES 1996, Vol 18, Iss 6, pp 651-661

Addresses:

UNIV-RHODE-ISL, GRAD SCH OCEANOG, NARRAGANSETT, RI 02882, USA

UNIV-DURHAM, DEPT GEOL SCI, DURHAM DH1-3LE, ENGLAND

US-GEOL-SURVEY, WOODS-HOLE, MA 02543, USA

10. Authors: Lyons-PC Palmer-CA Bustin-RM Vassallo-AM

Title: Special Issue - New Techniques in the Chemical-Analysis

of Coal - A Selection of Papers Presented at a

Symposium, Which Was Part of the 1995 International

Chemical Congress of Pacific Basin Societies, Held in

Honolulu, Hawaii, December 17-22, 1995 - Introduction

Source: INTERNATIONAL JOURNAL OF COAL GEOLOGY 1996, Vol 32, Iss 1-4, pp 1-3

Addresses:

US-GEOL-SURVEY, NATL CTR, MS 956, RESTON, VA 22092, USA

UNIV-BRITISH-COLUMBIA, DEPT GEOL SCI, VANCOUVER, BC V6T-1Z4, CANADA

CSIRO, DIV COAL \& ENERGY TECHNOL, N-RYDE, NSW 2113, AUSTRALIA

11. Authors: Maczura-G Moody-KJ Anderson-EM Kunka-MK

Title: Annual Minerals Review

Source: AMERICAN CERAMIC SOCIETY BULLETIN

1996, Vol 75, Iss 6, pp 93-\&

Addresses:

ALCOA, IND CHEM DIV, 6100 FAIRVIEW RD, SUITE 610, CHARLOTTE,

NC 28210, USA

ADV-REFRACTORY-TECHNOL-INC, BUFFALO, NY 14207, USA

F\&S-INT-INC, NEW-YORK, NY 10158, USA

OLD-HICKORY-CLAY-CO, HICKORY, KY 42051, USA

TAM-CERAM-INC, NIAGARA-FALLS, NY 14305, USA

BASSTECH-INT, ENGLEWOOD, NJ 07631, USA

US-GEOL-SURVEY, RESTON, VA 22092, USA

C-E-MINERALS, KING-OF-PRUSSIA, PA 19406, USA

GWALIA-CONSOLIDATED-LTD, PERTH, WA 6872, AUSTRALIA

UNIMIN-CORP, NEW-CANAAN, CT 06840, USA 
AMER-MINERAL-INC, KING-OF-PRUSSIA, PA, USA

LEHIGH-PORTLAND-CEMENT-CO, ALLENTOWN, PA 18195, USA

US-BORAX-INC, VALENCIA, CA 91355, USA

EAGLE-ZINC-CO, NEW-YORK, NY 10112, USA

KENTUCKY-TENNESSEE-CLAY-CO, MAYFIELD, KY 42066, USA

BAKER-REFRACTORIES, YORK, PA, USA

SEAFORTH-MINERAL-\&-ORE-CO-INC, CAVE-IN-ROCK, IL 62919, USA

RT-VANDERBILT-CO-INC, NORWALK, CT 06856, USA

AS-OLIVIN, AAHEIM, NORWAY

BOULDER-SCI-CO, BOULDER, CO, USA

ST-GOBAIN-NORTON-INC-CERAM-CORP, WORCESTER, MA 01615, USA

US-BUR-MINES, WASHINGTON, DC 20241, USA

ASBURY-GRAPHITE-MILLS-INC, ASBURY-PK, NJ 08802, USA

12. Authors: Ayuso-RA Barr-SM Longstaffe-FJ

Title: $\mathrm{Pb}$ and $\mathrm{O}$ Isotopic Constraints on the Source of Granitic-

Rocks from Cape-Breton Island, Nova-Scotia, Canada

Source: AMERICAN JOURNAL OF SCIENCE

1996, Vol 296, Iss 7, pp 789-817

Addresses:

US-GEOL-SURVEY, MAIL STOP 954, RESTON, VA 22092, USA

ACADIA-UNIV, DEPT GEOL, WOLFVILLE, NS BOP-1X0, CANADA

UNIV-WESTERN-ONTARIO, DEPT EARTH SCI, LONDON, ON N6A-5B7, CANADA

13. Authors: Cygan-GL Chou-IM Sherman-DM

Title: Reinvestigation of the Annite Equals Sanidine Plus

Magnetite Plus H-2 Reaction Using F(H-2)-Sensor Technique

Source: AMERICAN MINERALOGIST

1996, Vol 81, Iss 3-4, pp 475-484

Addresses:

US-GEOL-SURVEY, 959 NATL CTR, RESTON, VA 22092, USA

UNIV-BRISTOL, DEPT GEOL, BRISTOL BS8-1RJ, AVON, ENGLAND

14. Authors: Hawthorne-FC Oberti-R Ungaretti-L Ottolini-L

Grice-JD Czamanske-GK

Title: Fluor-Ferro-Leakeite, Nana2(Fe22+fe23+li)Si8O22F2, a New

Alkali Amphibole from the Canada Pinabete Pluton,

Questa, New-Mexico, USA

Source: AMERICAN MINERALOGIST

1996, Vol 81, Iss 1-2, pp 226-228

Addresses:

UNIV-MANITOBA, DEPT GEOL SCI, WINNIPEG, MB R3T-2N2, CANADA

CNR, CTR STUDIO CRISTALLOCHIM \& CRISTALLOG, I-27100 PAVIA,

ITALY

CANADIAN-MUSEUM-NAT, DIV RES, OTTAWA, ON K1P-6P4, CANADA

US-GEOL-SURVEY, MENLO-PK, CA 94025, USA

15. Authors: Peng-GY Lewis-J Lipin-B Mcgee-J Bao-PS Wang-XB

Title: Inclusions of Phlogopite and Phlogopite Hydrates in

Chromite from the Hongguleleng Ophiolite in Xinjiang,

Northwest China

Source: AMERICAN MINERALOGIST

1995, Vol 80, Iss 11-12, pp 1307-1316 
Addresses:

GEORGE-WASHINGTON-UNIV, DEPT GEOL, WASHINGTON, DC 20052, USA

US-GEOL-SURVEY, NATL CTR, RESTON, VA 22092, USA

CHINESE-ACAD-MED-SCI, INST GEOL, BEIJING 100037, PEOPLES-R-CHINA

16. Authors: Dowdle-PR Laverman-AM Oremland-RS

Title: Bacterial Dissimilatory Reduction of Arsenic(V) to Arsenic(III) in Anoxic Sediments

Source: APPLIED AND ENVIRONMENTAL MICROBIOLOGY

1996, Vol 62, Iss 5, pp 1664-1669

Addresses:

US-GEOL-SURVEY, MENLO-PK, CA 94025, USA

FREE-UNIV-AMSTERDAM, DEPT BIOL, 1081-HV AMSTERDAM, NETHERLANDS

17. Authors: Hall-GEM Bonhamcarter-GF Horowitz-AJ Lum-K Lemieux-C Quemerais-B Garbarino-JR

Title: The Effect of Using Different 0.45-Mu-M Filter Membranes on Dissolved Element Concentrations in Natural-Waters

Source: APPLIED GEOCHEMISTRY 1996, Vol 11, Iss 1-2, pp 243-249

Addresses:

GEOL-SURVEY-CANADA, 601 BOOTH ST, OTTAWA, ON K1A-0E8, CANADA US-GEOL-SURVEY, ATLANTA, GA 30360, USA

ENVIRONM-CANADA, CTR ST LAURENT, LONGUEUIL, PQ J4K-1A1, CANADA

US-GEOL-SURVEY, DENVER FED CTR, LAKEWOOD, CO 80225, USA

18. Authors: King-CY King-BS Evans-WC Zhang-W

Title: Spatial Radon Anomalies on Active Faults in California

Source: APPLIED GEOCHEMISTRY

1996, Vol 11, Iss 4, pp 497-510

Addresses:

US-GEOL-SURVEY, 345 MIDDLEFIELD RD, MENLO-PK, CA 94025, USA

STATE-SEISMOL-BUR, BEIJING, PEOPLES-R-CHINA

19. Authors: Caccavo-F Coates-JD Rossellomora-RA Ludwig-W Schleifer-KH Lovley-DR Mcinerney-MJ

Title: Geovibrio Ferrireducens, a Phylogenetically Distinct Dissimilatory Fe(III)-Reducing Bacterium

Source: ARCHIVES OF MICROBIOLOGY 1996, Vol 165, Iss 6, pp 370-376

Addresses:

MONTANA-STATE-UNIV, CTR BIOFILM ENGN, 409 COBLEIGH HALL, BOZEMAN, MT 59717, USA

US-GEOL-SURVEY, DIV WATER RESOURCES, RESTON, VA 22092, USA

TECH-UNIV-MUNICH, LEHRSTUHL MIKROBIOL, D-80290 MUNICH, GERMANY

UNIV-MASSACHUSETTS, DEPT MICROBIOL, AMHERST, MA 01003, USA

UNIV-OKLAHOMA, DEPT BOT \& MICROBIOL, NORMAN, OK 73019, USA

20. Authors: Reimnitz-E Eicken-H Martin-T

Title: Multiyear Fast Ice Along the Taymyr Peninsula, Siberia

Source: ARCTIC

1995, Vol 48, Iss 4, pp 359-367 
Addresses:

US-GEOL-SURVEY, 345 MIDDLEFIELD RD, MS 999, MENLO-PK, CA

94025, USA

ALFRED-WEGENER-INST-POLAR-\&-MARINE-RES, D-27515 BREMERHAVEN, GERMANY

21. Authors: Julian-BR Foulger-GR

Title: Earthquake Mechanisms from Linear-Programming Inversion of Seismic-Wave Amplitude Ratios

Source: BULLETIN OF THE SEISMOLOGICAL SOCIETY OF AMERICA 1996, Vol 86, Iss 4, pp 972-980

Addresses:

US-GEOL-SURVEY, 345 MIDDLEFIELD RD, MS977, MENLO-PK, CA 94025, USA

UNIV-DURHAM, DEPT GEOL SCI, DURHAM DH1-3LE, ENGLAND

22. Authors: Pridnya-MV Cherpakov-VV Paillet-FL

Title: Ecology and Pathology of European Chestnut (CastaneaSativa) in the Deciduous Forests of the Caucasus Mountains in Southern Russia

Source: BULLETIN OF THE TORREY BOTANICAL CLUB 1996, Vol 123, Iss 3, pp 213-222

Addresses:

CAUCASUS-STATE-BIOSPHERE-FOREST-PRESERVE, SOCI, RUSSIA

US-GEOL-SURVEY, DENVER FED CTR, DENVER, CO 80225, USA

23.Authors: Stasiuk-MV Barclay-J Carroll-MR Jaupart-C Ratte-JC Sparks-RSJ Tait-SR

Title: Degassing During Magma Ascent in the Mule Creek Vent (USA)

Source: BULLETIN OF VOLCANOLOGY 1996, Vol 58, Iss 2-3, pp 117-130

Addresses:

UNIV-PARIS-07, F-5252 PARIS 05, FRANCE

INST-PHYS-GLOBE, F-5252 PARIS 05, FRANCE

UNIV-BRISTOL, DEPT GEOL, BRISTOL BS8-1RJ, AVON, ENGLAND

US-GEOL-SURVEY, DENVER FED CTR, DENVER, CO 80225, USA

24. Authors: Parfitt-EA Wilson-L Neal-CA

Title: Factors Influencing the Height of Hawaiian Lava

Fountains - Implications for the Use of Fountain Height

as an Indicator of Magma Gas Content

Source: BULLETIN OF VOLCANOLOGY 1995, Vol 57, Iss 6, pp 440-450

Addresses:

BROWN-UNIV, DEPT GEOL SCI, PROVIDENCE, RI 02912, USA

UNIV-LANCASTER, DIV ENVIRONM SCI, LANCASTER LA1-4YQ, ENGLAND

US-GEOL-SURVEY, ALASKA VOLCANO OBSERV, ANCHORAGE, AK 99508, USA

25. Authors: Force-ER Richards-RP Scot-KM Valentine-PC Fishman-NS

Title: Mineral Intergrowths Replaced by Elbow-Twinned Rutile in Altered Rocks

Source: CANADIAN MINERALOGIST 1996, Vol 34, Iss JUN, pp 605-614

Addresses:

UNIV-ARIZONA, US GEOL SURVEY, GOULD SIMPSON BLDG, TUCSON, AZ 85721, USA 
MORPHOGENESIS-INC, OBERLIN, OH 44074, USA

CSIRO, DIV EXPLORAT \& MIN, N-RYDE, NSW 2113, AUSTRALIA

US-GEOL-SURVEY, WOODS-HOLE, MA 02543, USA

US-GEOL-SURVEY, DENVER FED CTR, DENVER, CO 80225, USA

26. Authors: Jiang-SY Palmer-MR

Title: Mn-Rich Ilmenite from the Sullivan Pb-Zn-Ag Deposit, British-Columbia

Source: CANADIAN MINERALOGIST 1996, Vol 34, Iss FEB, pp 29-36

Addresses:

UNIV-BRISTOL, DEPT GEOL, WILLS MEM BLDG, BRISTOL BS8-1RJ, AVON, ENGLAND

US-GEOL-SURVEY, NATL CTR, RESTON, VA 22092, USA

27. Authors: Roberts-AC Grice-JD Groat-LA Criddle-AJ Gault-RA Erd-RC Moffatt-EA

Title: Jensenite, Cu3Te6+o6-Center-Dot-2H(2)O, a New Mineral Species from the Centennial-Eureka Mine, Tintic District, Juab County, Utah

Source: CANADIAN MINERALOGIST 1996, Vol 34, Iss FEB, pp 49-54

Addresses:

GEOL-SURVEY-CANADA, 601 BOOTH ST, OTTAWA, ON K1A-0E8, CANADA

CANADIAN-MUSEUM-NAT, DIV RES, OTTAWA, ON K1P-6P4, CANADA

UNIV-BRITISH-COLUMBIA, DEPT GEOL SCI, VANCOUVER, BC V6T-1Z4, CANADA

NAT-HIST-MUSEUM, DEPT MINERAL, LONDON SW7-5BD, ENGLAND

US-GEOL-SURVEY, MENLO-PK, CA 94025, USA

CANADIAN-CONSERVAT-INST, OTTAWA, ON K1A-0M5, CANADA

28. Authors: Jiang-SY Palmer-MR Mcdonald-AM Slack-JF Leitch-CHB

Title: Feruvite from the Sullivan Pb-Zn-Ag Deposit, British-

Columbia

Source: CANADIAN MINERALOGIST

1996, Vol 34, Iss AUG, pp 733-740

Addresses:

UNIV-BRISTOL, DEPT GEOL, WILLS MEM BLDG, BRISTOL BS8-1RJ, AVON, ENGLAND

LAURENTIAN-UNIV, DEPT GEOL, SUDBURY, ON P3E-2C6, CANADA

US-GEOL-SURVEY, NATL CTR, RESTON, VA 22092, USA

GEOL-SURVEY-CANADA, VANCOUVER, BC V6B-1R8, CANADA

29. Authors: Amelin-YV Neymark-LA Ritsk-EY Nemchin-AA

Title: Enriched Nd-Sr-Pb Isotopic Signatures in the Dovyren

Layered Intrusion (Eastern Siberia, Russia), Evidence

for Source Contamination by Ancient Upper-Crustal

Material

Source: CHEMICAL GEOLOGY

1996, Vol 129, Iss 1-2, pp 39-69

Addresses:

RUSSIAN-ACAD-SCI, INST PRECAMBRIAN GEOL \& GEOCHRONOL, MAKAROVA

EMB 2, ST-PETERSBURG 199034, RUSSIA

ROYAL-ONTARIO-MUSEUM, GEOCHRONOL LAB, TORONTO, ON M5S-2C6, CANADA

US-GEOL-SURVEY, DENVER FED CTR, DENVER, CO 80225, USA

CURTIN-UNIV-TECHNOL, SCH APPL GEOL, PERTH, WA 6001, AUSTRALIA 
30. Authors: Zierenberg-RA Schiffman-P Jonasson-IR Tosdal-R

Pickthorn-W Mcclain-J

Title: Alteration of Basalt Hyaloclastite at the Off-Axis Sea

Cliff Hydrothermal Field, Gorda Ridge

Source: CHEMICAL GEOLOGY 1995, Vol 126, Iss 2, pp 77-99

Addresses:

US-GEOL-SURVEY, 345 MIDDLEFIELD RD, MS 901, MENLO-PK, CA 94025, USA

UNIV-CALIF-DAVIS, DEPT GEOL, DAVIS, CA 95616, USA

GEOL-SURVEY-CANADA, OTTAWA, ON K1A-0E8, CANADA

31. Authors: Powers-PS Chiarle-M Savage-WZ

Title: A Digital Photogrammetric Method for Measuring Horizontal Surficial Movements on the Slumgullion Earthflow, Hinsdale County, Colorado

Source: COMPUTERS \& GEOSCIENCES 1996, Vol 22, Iss 6, pp 651-663

Addresses:

US-GEOL-SURVEY, DENVER FED CTR, BOX 25046, MAIL STOP 966, DENVER, CO 80225, USA

CNR, ITALIAN NATL RES COUNCIL, IRPI, I-10132 TURIN, ITALY

32. Authors: Sinigoi-S Quick-JE Mayer-A Budahn-J

Title: Influence of Stretching and Density Contrasts on the Chemical Evolution of Continental Magmas - An Example from the Ivrea-Verbano Zone

Source: CONTRIBUTIONS TO MINERALOGY AND PETROLOGY 1996, Vol 123, Iss 3, pp 238-250

Addresses:

UNIV-TRIESTE, IST MINERAL \& PETROG, PIAZZALE EUROPA 1, I-34100

TRIESTE, ITALY

US-GEOL-SURVEY, DENVER FED CTR, DENVER, CO 80225, USA

33. Authors: Vonblanckenburg-F Onions-RK Belshaw-NS Gibb-A Hein-JR

Title: Global Distribution of Beryllium Isotopes in Deep-Ocean Water as Derived from Fe-Mn Crusts

Source: EARTH AND PLANETARY SCIENCE LETTERS 1996, Vol 141, Iss 1-4, pp 213-226

Addresses:

UNIV-OXFORD, DEPT EARTH SCI, PARKS RD, OXFORD OX1-3PR, ENGLAND

US-GEOL-SURVEY, MENLO-PK, CA 94025, USA

34. Authors: Baker-J Snee-L Menzies-M

Title: A Brief Oligocene Period of Flood Volcanism in Yemen Implications for the Duration and Rate of Continental Flood Volcanism at the Afro-Arabian Triple Junction

Source: EARTH AND PLANETARY SCIENCE LETTERS 1996, Vol 138, Iss 1-4, pp 39-55

Addresses:

UNIV-LONDON-ROYAL-HOLLOWAY-\&-BEDFORD-NEW-COLL, DEPT GEOL, EGHAM TW20-0EX, SURREY, ENGLAND

US-GEOL-SURVEY, BRANCH ISOTOPE GEOL, DENVER, CO 80225, USA 
35. Authors: Johnson-CA Cardellach-E Tritlla-J Hanan-BB

Title: Cierco Pb-Zn-Ag Vein Deposits - Isotopic and Fluid Inclusion Evidence for Formation During the Mesozoic Extension in the Pyrenees of Spain

Source: ECONOMIC GEOLOGY AND THE BULLETIN OF THE SOCIETY OF ECONOMIC GEOLOGISTS 1996, Vol 91, Iss 3, pp 497-506

Addresses:

US-GEOL-SURVEY, BOX 25046, MAIL STOP 963, DENVER, CO 80225, USA UNIV-AUTONOMA-BARCELONA, DEPT GEOL, BELLATERRA 08193, SPAIN UNIV-LEEDS, DEPT EARTH SCI, LEEDS LS2-9JT, W-YORKSHIRE, ENGLAND SAN-DIEGO-STATE-UNIV, DEPT GEOL SCI, SAN-DIEGO, CA 92182, USA

36. Authors: Leanderson-PJ Yoldash-M Johnson-PR Offield-TW

Title: Structure, Vein Paragenesis, and Alteration in the Al Wajh Gold District, Saudi-Arabia

Source: ECONOMIC GEOLOGY AND THE BULLETIN OF THE SOCIETY OF ECONOMIC GEOLOGISTS 1995, Vol 90, Iss 8, pp 2262-2273

Addresses:

MINIST-MINERAL-RESOURCES, JEDDAH, SAUDI-ARABIA

US-GEOL-SURVEY-MISSION, JEDDAH, SAUDI-ARABIA

US-GEOL-SURVEY, RESTON, VA 22092, USA

37. Authors: Rowan-LC Bowers-TL Crowley-JK Antonpacheco-C Gumiel-P Kingston-MJ

Title: Analysis of Airborne Visible Infrared Imaging Spectrometer (AVIRIS) Data of the Iron-Hill, Colorado, Carbonatite Alkalic Igneous Complex

Source: ECONOMIC GEOLOGY AND THE BULLETIN OF THE SOCIETY OF ECONOMIC GEOLOGISTS 1995, Vol 90, Iss 7, pp 1966-1982

Addresses:

US-GEOL-SURVEY, MAIL STOP 954, RESTON, VA 22092, USA

INST-TECNOL-GEOMINERO-ESPANA, E-28003 MADRID, SPAIN

38. Authors: Salmon-LG Cass-GR Kozlowski-R Hejda-A Spiker-EC Bates-AL

Title: Air Pollutant Intrusion into the Wieliczka Salt Mine

Source: ENVIRONMENTAL SCIENCE \& TECHNOLOGY 1996, Vol 30, Iss 3, pp 872-880

Addresses:

CALTECH, DEPT ENVIRONM ENGN SCI, PASADENA, CA 91125, USA

POLISH-ACAD-SCI, INST CATALYSIS \& SURFACE CHEM, KRAKOW, POLAND

US-GEOL-SURVEY, RESTON, VA 22092, USA

39. Authors: Horowitz-AJ Lum-KR Garbarino-JR Hall-GEM Lemieux-C

Demas-CR

Title: Problems Associated with Using Filtration to Define

Dissolved Trace-Element Concentrations in Natural-Water

Samples

Source: ENVIRONMENTAL SCIENCE \& TECHNOLOGY 1996, Vol 30, Iss 3, pp 954-963

Addresses:

US-GEOL-SURVEY, PEACHTREE BUSINESS CTR, 3039 AMWILER RD, ATLANTA, GA 30360, USA 
ENVIRONM-CANADA, CTR ST LAURENT, MONTREAL, PQ H2Y-2E7, CANADA

GEOL-SURVEY-CANADA, OTTAWA, ON K1A-0E8, CANADA

US-GEOL-SURVEY, BATON-ROUGE, LA 70816, USA

US-GEOL-SURVEY, BRANCH ANALYT SERV, ARVADA, CO 80002, USA

40. Authors: Kamo-SL Czamanske-GK Krogh-TE

Title: A Minimum U-Pb Age for Siberian Flood-Basalt Volcanism

Source: GEOCHIMICA ET COSMOCHIMICA ACTA

1996, Vol 60, Iss 18, pp 3505-3511

Addresses:

ROYAL-ONTARIO-MUSEUM, JACK SATTERLY GEOCHRONOL LAB, 100 QUEENS

PK, TORONTO, ON M5S-2C6, CANADA

US-GEOL-SURVEY, MENLO-PK, CA 94025, USA

41. Authors: Bau-M Koschinsky-A Dulski-P Hein-JR

Title: Comparison of the Partitioning Behaviors of Yttrium,

Rare-Earth Elements, and Titanium Between Hydrogenetic

Marine Ferromanganese Crusts and Seawater

Source: GEOCHIMICA ET COSMOCHIMICA ACTA

1996, Vol 60, Iss 10, pp 1709-1725

Addresses:

GEOFORSCHUNGSZENTRUM-POTSDAM, PB 43 LAGERSTATTENBILDUNG,

D-14473 POTSDAM, GERMANY

US-GEOL-SURVEY, MS 999, MENLO-PK, CA 94025, USA

FREE-UNIV-BERLIN, FR ROHSTOFF \& UMWELTGEOL, D-12249 BERLIN, GERMANY

42. Authors: Horan-MF Walker-RJ Fedorenko-VA Czamanske-GK

Title: Osmium and Neodymium Isotopic Constraints on the

Temporal and Spatial Evolution of Siberian Flood-Basalt

Sources

Source: GEOCHIMICA ET COSMOCHIMICA ACTA

1995, Vol 59, Iss 24, pp 5159-5168

Addresses:

US-GEOL-SURVEY, RESTON, VA 22092, USA

UNIV-MARYLAND, DEPT GEOL, COLLEGE-PK, MD 20742, USA

TSNIGRI, MOSCOW 113545, RUSSIA

US-GEOL-SURVEY, MENLO-PK, CA 94025, USA

43. Authors: Koschinsky-A Halbach-P Hein-JR Mangini-A

Title: Ferromanganese Crusts as Indicators for

Paleoceanographic Events in the Ne Atlantic

Source: GEOLOGISCHE RUNDSCHAU

1996, Vol 85, Iss 3, pp 567-576

Addresses:

FREE-UNIV-BERLIN, FACHRICHTUNG ROHSTOFF \& UMWELTGEOL, MALTESERSTR 74-100, D-12249 BERLIN, GERMANY

US-GEOL-SURVEY, MENLO-PK, CA 94025, USA

HEIDELBERGER-AKAD-WISSENSCH, D-69120 HEIDELBERG, GERMANY

44. Authors: Lipman-PW Dungan-MA Brown-LL Deino-A

Title: Recurrent Eruption and Subsidence at the Platoro-Caldera

Complex, Southeastern San-Juan Volcanic Field, Colorado -

New Tales from Old Tuffs

Source: GEOLOGICAL SOCIETY OF AMERICA BULLETIN

1996, Vol 108, Iss 8, pp 1039-1055 
Addresses:

US-GEOL-SURVEY, MS 910, 345 MIDDLEFIELD RD, MENLO-PK, CA

94025, USA

UNIV-GENEVA, DEPT MINERAL, CH-1211 GENEVA 4, SWITZERLAND

UNIV-MASSACHUSETTS, DEPT GEOSCI, AMHERST, MA 01003, USA

BERKELEY-GEOCHRONOL-CTR, BERKELEY, CA 94709, USA

45. Authors: Nelson-AR Jennings-AE Kashima-K

Title: An Earthquake History Derived from Stratigraphic and

Microfossil Evidence of Relative Sea-Level Change at

Coos-Bay, Southern Coastal Oregon

Source: GEOLOGICAL SOCIETY OF AMERICA BULLETIN 1996, Vol 108, Iss 2, pp 141-154

Addresses:

US-GEOL-SURVEY, BOX 25046, MS 966, DENVER, CO 80225, USA

UNIV-COLORADO, INST ARCTIC \& ALPINE RES, BOULDER, CO 80309, USA

KYUSHU-UNIV, DEPT EARTH \& PLANETARY SCI, FUKUOKA 810, JAPAN

46.Authors: Bayarsayhan-C Bayasgalan-A Enhtuvshin-B Hudnut-KW

Kurushin-RA Molnar-P Olziybat-M

Title: 1957 Gobi-Altay, Mongolia Earthquake as a Prototype for

Southern Californias Most Devastating Earthquake

Source: GEOLOGY

1996, Vol 24, Iss 7, pp 579-582

Addresses:

MONGOLIAN-ACAD-SCI, CTR SEIMOL \& GEOMAGNETISM, ULAANBAATAR

210351, MONGOL-PEO-REP

MONGOLIAN-ACAD-SCI, CTR INFORMAT \& REMOTE SENSING, ULAANBAATAR

210351, MONGOL-PEO-REP

US-GEOL-SURVEY, PASADENA, CA 91106, USA

RUSSIAN-ACAD-SCI, INST EARTH CRUST, IRKUTSK 664003, RUSSIA

MIT, DEPT EARTH ATMOSPHER \& PLANETARY SCI, CAMBRIDGE, MA

02139, USA

47. Authors: Wieczorek-GF Jager-S

Title: Triggering Mechanisms and Depositional Rates of

Postglacial Slope-Movement Processes in the Yosemite

Valley, California

Source: GEOMORPHOLOGY

1996, Vol 15, Iss 1, pp 17-31

Addresses:

US-GEOL-SURVEY, NATL CTR, MS 922, RESTON, VA 22092, USA

UNIV-HEIDELBERG, INST GEOG, D-69120 HEIDELBERG, GERMANY

48. Authors: Self-S Thordarson-T Keszthelyi-L Walker-GPL Hon-K Murphy-MT Long-P Finnemore-S

Title: A New Model for the Emplacement of Columbia River

Basalts as Large, Inflated Pahoehoe Lava Flow-Fields

Source: GEOPHYSICAL RESEARCH LETTERS

1996, Vol 23, Iss 19, pp 2689-2692

Addresses:

UNIV-HAWAII, SOEST, HAWAII CTR VOLCANOL, HONOLULU, HI 96822,

USA

UNIV-HAWAII, SOEST, DEPT GEOL \& GEOPHYS, HONOLULU, HI 96822 ,

USA 
INST-GEOL-\&-NUCL-SCI, WAIRAKEI RES CTR, TAUPO, NEW-ZEALAND

US-GEOL-SURVEY, DENVER FED CTR, DENVER, CO 80225, USA

BATTELLE-MEM-INST, PACIFIC NW LABS, RICHLAND, WA 99352, USA

49. Authors: Stark-MA Davis-SD

Title: Remotely Triggered Microearthquakes at the Geysers

Geothermal-Field, California

Source: GEOPHYSICAL RESEARCH LETTERS

1996, Vol 23, Iss 9, pp 945-948

Addresses:

PHILIPPINE-GEOTHERMAL-INC, UNOCAL GEOTHERMAL DIV, MANILA, PHILIPPINES

US-GEOL-SURVEY, MEMPHIS, TN, USA

MEMPHIS-STATE-UNIV, CTR EARTHQUAKE RES \& INFORMAT, MEMPHIS, TN

38152, USA

50. Authors: Julian-BR Ross-A Foulger-GR Evans-JR

Title: 3-Dimensional Seismic Image of a Geothermal Reservoir -

The Geysers, California

Source: GEOPHYSICAL RESEARCH LETTERS

1996, Vol 23, Iss 6, pp 685-688

Addresses:

US-GEOL-SURVEY, 345 MIDDLEFIELD RD, MS 977, MENLO-PK, CA

94025, USA

UNIV-DURHAM, DEPT GEOL SCI, DURHAM DH1-3LE, ENGLAND

51.Authors: Evans-JR Foulger-GR Julian-BR Miller-AD

Title: Crustal Shear-Wave Splitting from Local Earthquakes in the Hengill Triple Junction, Southwest Iceland

Source: GEOPHYSICAL RESEARCH LETTERS 1996, Vol 23, Iss 5, pp 455-458

Addresses:

US-GEOL-SURVEY, 345 MIDDLEFIELD RD, MS-977, MENLO-PK, CA

94025, USA

UNIV-DURHAM, DEPT GEOL SCI, DURHAM DH1-3LE, ENGLAND

52. Authors: Watson-K Rowan-LC Bowers-TL Antonpacheco-C Gumiel-P Miller-SH

Title: Lithologic Analysis from Multispectral Thermal Infrared

Data of the Alkalic Rock Complex at Iron-Hill, Colorado

Source: GEOPHYSICS

1996, Vol 61, Iss 3, pp 706-721

Addresses:

US-GEOL-SURVEY, MS 964, DENVER, CO 80228, USA

US-GEOL-SURVEY, RESTON, VA 22092, USA

INST-TECNOL-GEOMINERO-ESPANA, E-28003 MADRID, SPAIN

53. Authors: Kargel-JS Pozio-S

Title: The Volcanic and Tectonic History of Enceladus

Source: ICARUS

1996, Vol 119, Iss 2, pp 385-404

Addresses:

US-GEOL-SURVEY, FLAGSTAFF, AZ 86001, USA

REPARTO-PLANETOL, I-00185 ROME, ITALY 
54. Authors: Zodrow-EL Lyons-PC Millay-MA

Title: Geochemistry of Autochthonous and Hypautochthonous

Siderite Dolomite Coal-Balls (Foord Seam, Bolsovian,

Upper Carboniferous), Nova-Scotia, Canada

Source: INTERNATIONAL JOURNAL OF COAL GEOLOGY

1996, Vol 29, Iss 1-3, pp 199-216

Addresses:

UNIV-COLL-CAPE-BRETON, DEPT EARTH SCI, SYDNEY, NS B1P-6L2,

CANADA

US-GEOL-SURVEY, RESTON, VA 22092, USA

OHIO-UNIV, IRONTON, OH 45638, USA

55. Authors: Ruppert-L Finkelman-R Boti-E Milosavljevic-M

Tewalt-S Simon-N Dulong-F

Title: Origin and Significance of High-Nickel and Chromium

Concentrations in Pliocene Lignite of the Kosovo Basin,

Serbia

Source: INTERNATIONAL JOURNAL OF COAL GEOLOGY 1996, Vol 29, Iss 4, pp 235-258

Addresses:

US-GEOL-SURVEY, NATL CTR, MS 956, RESTON, VA 22092, USA

ELECTROECON-KOSOVO-INST-SCI-RES-\&-DEV, YU-38000 PRISHTINA, YUGOSLAVIA

56. Authors: Flores-RM Sykes-R

Title: Depositional Controls on Coal Distribution and Quality in the Eocene Brunner Coal Measures, Buller Coalfield, South Island, New-Zealand

Source: INTERNATIONAL JOURNAL OF COAL GEOLOGY 1996, Vol 29, Iss 4, pp 291-336

Addresses:

US-GEOL-SURVEY, BOX 25046, MS 972, DENVER, CO 80225, USA

INST-GEOL-\&-NUCL-SCI-LTD, LOWER-HUTT, NEW-ZEALAND

57. Authors: Ono-A Sakuma-F Arai-K Yamaguchi-Y Fujisada-H

Slater-PN Thome-KJ Palluconi-FD Kieffer-HH

Title: Preflight and in-Flight Calibration Plan for Aster

Source: JOURNAL OF ATMOSPHERIC AND OCEANIC TECHNOLOGY 1996, Vol 13, Iss 2, pp 321-335

Addresses:

NATL-RES-LAB-METROL, THERMOPHYS METROL DEPT, TSUKUBA, IBARAKI 305, JAPAN

SAGA-UNIV, SAGA 840, JAPAN

GEOL-SURVEY-JAPAN, TSUKUBA, IBARAKI 305, JAPAN

ELECTROTECH-LAB, TSUKUBA, IBARAKI 305, JAPAN

CALTECH, JET PROP LAB, PASADENA, CA, USA

US-GEOL-SURVEY, FLAGSTAFF, AZ 86001, USA

58. Authors: Komada-N Moecher-DP Westrum-EF Hemingway-BS

Zolotov-MY Semenov-YV Khodakovsky-IL

Title: Thermodynamic Properties of Scapolites at Temperatures Ranging from $10-\mathrm{K}$ to $1000-\mathrm{K}$

Source: JOURNAL OF CHEMICAL THERMODYNAMICS 1996, Vol 28, Iss 9, pp 941-973 
Addresses:

UNIV-MICHIGAN, DEPT CHEM, ANN-ARBOR, MI 48109, USA

US-GEOL-SURVEY, RESTON, VA 22092, USA

VI-VERNADSKII-INST-GEOCHEM-\&-ANALYT-CHEM, MOSCOW V334, RUSSIA

UNIV-MICHIGAN, DEPT GEOL SCI, ANN-ARBOR, MI 48109, USA

59.Authors: Hartzell-S Liu-PC Mendoza-C

Title: The 1994 Northridge, California, Earthquake -

Investigation of Rupture Velocity, Risetime, and High-

Frequency Radiation

Source: JOURNAL OF GEOPHYSICAL RESEARCH-SOLID EARTH 1996, Vol 101, Iss B9, pp 20091-20108

Addresses:

US-GEOL-SURVEY, DENVER FED CTR, BOX 25046, MS 966, DENVER, CO 80255, USA

CHINESE-ACAD-SCI, INST GEOPHYS, BEIJING, PEOPLES-R-CHINA

60. Authors: Alsina-D Woodward-RL Snieder-RK

Title: Shear-Wave Velocity Structure in North-America from

Large-Scale Wave-Form Inversions of Surface-Waves

Source: JOURNAL OF GEOPHYSICAL RESEARCH-SOLID EARTH 1996, Vol 101, Iss B7, pp 15969-15986

Addresses:

UNIV-UTRECHT, DEPT THERORET GEOPHYS, POB 80021, 3508-TA

UTRECHT, NETHERLANDS

US-GEOL-SURVEY, ALBUQUERQUE SEISMOL LAB, ALBUQUERQUE, NM 87115 , USA

61. Authors: Slack-PD Davis-PM Baldridge-WS Olsen-KH Glahn-A Achauer-U Spence-W

Title: The Upper-Mantle Structure of the Central Rio-Grande Rift Region from Teleseismic P-Wave and S-Wave Travel-

Time Delays and Attenuation

Source: JOURNAL OF GEOPHYSICAL RESEARCH-SOLID EARTH 1996, Vol 101, Iss B7, pp 16003-16023

Addresses:

UNIV-CALIF-LOS-ANGELES, DEPT EARTH \& SPACE SCI, LOS-ANGELES, CA 90024, USA

LOS-ALAMOS-NATL-LAB, DIV EARTH \& SPACE SCI, LOS-ALAMOS, NM 87545, USA

UNIV-KARLSRUHE, INST GEOPHYS, D-7500 KARLSRUHE, GERMANY

INST-PHYS-GLOBE, F-67984 STRASBOURG, FRANCE

US-GEOL-SURVEY, NATL EARTHQUAKE INFORMAT CTR, DENVER, CO 80225, USA

62. Authors: Koepenick-KW Brantley-SL Thompson-JM Rowe-GL Nyblade-AA Moshy-C

Title: Volatile Emissions from the Crater and Flank of OldoinyoLengai Volcano, Tanzania

Source: JOURNAL OF GEOPHYSICAL RESEARCH-SOLID EARTH 1996, Vol 101, Iss B6, pp 13819-13830

Addresses:

ICF-KAISER-ENGINEERS-INC, 1301 CONTINENTAL DR, SUITE 101, ABINGDON, MD 21009, USA

MINIST-WATER-ENERGY-\&-MINERALS, DODOMA, TANZANIA

US-GEOL-SURVEY, DIV WATER RESOURCES, COLUMBUS, OH 43212, USA 
US-GEOL-SURVEY, CASCADES VOLCANO OBSERV, VANCOUVER, WA 98661, USA

PENN-STATE-UNIV, DEPT GEOSCI, UNIVERSITY-PK, PA 16802, USA

63. Authors: Peck-JA King-JW Colman-SM Kravchinsky-VA

Title: An 84-Kyr Paleomagnetic Record from the Sediments of Lake-Baikal, Siberia

Source: JOURNAL OF GEOPHYSICAL RESEARCH-SOLID EARTH 1996, Vol 101, Iss B5, pp 11365-11385

Addresses:

UNIV-RHODE-ISL, GRAD SCH OCEANOG, NARRAGANSETT, RI 02882, USA

US-GEOL-SURVEY, WOODS-HOLE, MA 02543, USA

E-SIBERIAN-RES-INST-GEOL-GEOPHYS-\&-MINERAL-RESOUR, IRKUTSK, RUSSIA

64. Authors: Hodgkinson-KM Stein-RS King-GCP

Title: The 1954 Rainbow Mountain-Fairview Peak-Dixie Valley

Earthquakes - A Triggered Normal Faulting Sequence

Source: JOURNAL OF GEOPHYSICAL RESEARCH-SOLID EARTH 1996, Vol 101, Iss B11, pp 25459-25471

Addresses:

US-GEOL-SURVEY, 345 MIDDLEFIELD RD, MS 977, MENLO-PK, CA

94025, USA

INST-PHYS-GLOBE, F-75252 PARIS, FRANCE

65. Authors: Howard-KA Foster-DA

Title: Thermal and Unroofing History of a Thick, Tilted Basinand-Range Crustal Section in the Tortilla-Mountains, Arizona

Source: JOURNAL OF GEOPHYSICAL RESEARCH-SOLID EARTH 1996, Vol 101, Iss B1, pp 511-522

Addresses:

US-GEOL-SURVEY, BRANCH WESTERN REG GEOL, 345 MIDDLEFIELD RD, MS 975, MENLO-PK, CA 94025, USA

LA-TROBE-UNIV, SCH EARTH SCI, VICTORIAN INST EARTH \& PLANETARY SCI, BUNDOORA, VIC 3083, AUSTRALIA

66. Authors: Benavraham-Z Tenbrink-U Bell-R Reznikov-M

Title: Gravity-Field over the Sea-of-Galilee - Evidence for a

Composite Basin Along a Transform-Fault

Source: JOURNAL OF GEOPHYSICAL RESEARCH-SOLID EARTH 1996, Vol 101, Iss B1, pp 533-544

Addresses:

TEL-AVIV-UNIV, DEPT GEOPHYS \& PLANETARY SCI, IL-69978

TEL-AVIV, ISRAEL

COLUMBIA-UNIV, LAMONT DOHERTY GEOL OBSERV, PALISADES, NY

10964, USA

US-GEOL-SURVEY, WOODS-HOLE, MA 02543, USA

67. Authors: Mendoza-C Fukuyama-E

Title: The July 12, 1993, Hokkaido-Nansei-Oki, Japan,

Earthquake - Coseismic Slip Pattern from Strong-Motion

and Teleseismic Recordings

Source: JOURNAL OF GEOPHYSICAL RESEARCH-SOLID EARTH

1996, Vol 101, Iss B1, pp 791-801 
Addresses:

US-GEOL-SURVEY, BOX 25046, MS 967, DENVER, CO 80225, USA

NATL-RES-INST-EARTH-SCI-\&-DISASTER-PREVENT, TSUKUBA, IBARAKI 305, JAPAN

68. Authors: Stuart-WD Banks-PO Sasai-Y Liu-SW

Title: Piezomagnetic Field for Parkfield Fault Model

Source: JOURNAL OF GEOPHYSICAL RESEARCH-SOLID EARTH 1995, Vol 100, Iss B12, pp 24101-24110

Addresses:

CASE-WESTERN-RESERVE-UNIV, DEPT GEOL SCI, CLEVELAND, OH 44106, USA

UNIV-TOKYO, EARTHQUAKE RES INST, BUNKYO KU, TOKYO 113, JAPAN

US-GEOL-SURVEY, MS 977, MENLO-PK, CA 94025, USA

69. Authors: Moore-JG Hickson-CJ Calk-LC

Title: Tholeiitic-Alkalic Transition at Subglacial Volcanos, Tuya Region, British-Columbia, Canada

Source: JOURNAL OF GEOPHYSICAL RESEARCH-SOLID EARTH 1995, Vol 100, Iss B12, pp 24577-24592

Addresses:

US-GEOL-SURVEY, 345 MIDDLEFIELD RD, MS 910, MENLO-PK, CA 94025, USA

GEOL-SURVEY-CANADA, VANCOUVER, BC V6B-1R8, CANADA

70. Authors: Baransky-LN Green-AW Fedorov-EN Kurneva-NA Pilipenko-VA Worthington-EW

Title: Gradient and Polarization Methods of Ground-Based Monitoring of Magnetospheric Plasma

Source: JOURNAL OF GEOMAGNETISM AND GEOELECTRICITY 1995, Vol 47, Iss 12, pp 1293-1309

Addresses:

MOSCOW-PHYS-EARTH-INST, MOSCOW 123810, RUSSIA

US-GEOL-SURVEY, DENVER, CO 80225, USA

71. Authors: Darby-SE Thorne-CR Simon-A

Title: Numerical-Simulation of Widening and Bed Deformation of Straight Sand-Bed Rivers .2. Model Evaluation

Source: JOURNAL OF HYDRAULIC ENGINEERING-ASCE 1996, Vol 122, Iss 4, pp 194-202

Addresses:

UNIV-NOTTINGHAM, DEPT GEOG, NOTTINGHAM NG7-2RD, ENGLAND

US-GEOL-SURVEY, RALEIGH, NC 27607, USA

72. Authors: Schaffranek-RW Lai-CT

Title: Friction-Term Response to Boundary-Condition Type in Flow Models

Source: JOURNAL OF HYDRAULIC ENGINEERING-ASCE 1996, Vol 122, Iss 2, pp 73-81

Addresses:

US-GEOL-SURVEY, NATL CTR 430, 12201 SUNRISE VALLEY DR, RESTON, VA 22092, USA

NATL-TAIWAN-UNIV, DEPT CIVIL ENGN, TAIPEI 106, TAIWAN 
73. Authors: Coates-JD Coughlan-MF Colleran-E

Title: Simple Method for the Measurement of the

Hydrogenotrophic Methanogenic Activity of Anaerobic

Sludges

Source: JOURNAL OF MICROBIOLOGICAL METHODS 1996, Vol 26, Iss 3, pp 237-246

Addresses:

US-GEOL-SURVEY, DIV WATER RESOURCES, NATL CTR 430, 12201

SUNRISE VALLEY DR, MAILSTOP 430, RESTON, VA 22092, USA

NATL-UNIV-IRELAND-UNIV-COLL-GALWAY, DEPT MICROBIOL, GALWAY, IRELAND

74. Authors: Kennedy-WJ Cobban-WA

Title: Maastrichtian Ammonites from the Hornerstown Formation in New-Jersey

Source: JOURNAL OF PALEONTOLOGY 1996, Vol 70, Iss 5, pp 798-804

Addresses:

UNIV-OXFORD, GEOL COLLECT, UNIV MUSEUM, PARKS RD, OXFORD

OX1-3PW, ENGLAND

US-GEOL-SURVEY, DENVER, CO 80225, USA

75. Authors: Culver-SJ Repetski-JE Pojeta-J Hunt-D

Title: Early and Middle() Cambrian Metazoan and Protistan Fossils from West-Africa

Source: JOURNAL OF PALEONTOLOGY 1996, Vol 70, Iss 1, pp 1-4

Addresses:

NAT-HIST-MUSEUM, DEPT PALAEONTOL, CROMWELL RD, LONDON SW7-5BD, ENGLAND

US-GEOL-SURVEY, RESTON, VA 22092, USA

UNIV-MANCHESTER, DEPT GEOL, MANCHESTER M13-9PL, LANCS, ENGLAND

76.Authors: Allen-CM Wooden-JL Howard-KA Foster-DA Tosdal-RM

Title: Sources of the Early Cretaceous Plutons in the Turtle and West Riverside Mountains, California - Anomalous Cordilleran Interior Intrusions

Source: JOURNAL OF PETROLOGY 1995, Vol 36, Iss 6, pp 1675-1700

Addresses:

AUSTRALIAN-NATL-UNIV, DEPT GEOL, GPO BOX 4, CANBERRA, ACT 0200, AUSTRALIA

US-GEOL-SURVEY, MENLO-PK, CA 94025, USA

LA-TROBE-UNIV, SCH EARTH SCI, BUNDOORA, VIC 3083, AUSTRALIA

77. Authors: Schwab-WC Lee-HJ Twichell-DC Locat-J Nelson-CH

Mcarthur-WG Kenyon-NH

Title: Sediment Mass-Flow Processes on a Depositional Lobe, Outer Mississippi Fan

Source: JOURNAL OF SEDIMENTARY RESEARCH 1996, Vol 66, Iss 5, pp 916-927

Addresses:

US-GEOL-SURVEY, QUISSETT CAMPUS, WOODS-HOLE, MA 02543, USA

US-GEOL-SURVEY, MENLO-PK, CA 94025, USA 
UNIV-LAVAL, DEPT GEOL \& GEOL ENGN, ENGN GEOL RES GRP, ST-FOY, PQ G1K-7P4, CANADA

INST-OCEANOG-SCI, DEACON LAB, GODALMING GU8-5UB, SURREY, ENGLAND

78. Authors: Harris-PT Pattiaratchi-CB Keene-JB Dalrymple-RW Gardner-JV Baker-EK Cole-AR Mitchell-D Gibbs-P Schroeder-WW

Title: Late Quaternary Deltaic and Carbonate Sedimentation in the Gulf-of-Papua Foreland Basin - Response to Sea-Level Change

Source: JOURNAL OF SEDIMENTARY RESEARCH 1996, Vol 66, Iss 4, pp 801-819

Addresses:

UNIV-TASMANIA, AUSTRALIAN GEOL SURVEY ORG, ANTARCTIC CRC, GPO BOX 252C, HOBART, TAS 7001, AUSTRALIA

UNIV-WESTERN-AUSTRALIA, CTR WATER RES, NEDLANDS, WA 6009, AUSTRALIA

UNIV-SYDNEY, INST OCEAN SCI, SYDNEY, NSW 2006, AUSTRALIA

QUEENS-UNIV, DEPT GEOL SCI, KINGSTON, ON K7L-3N6, CANADA

US-GEOL-SURVEY, MENLO-PK, CA 94025, USA

NEW-S-WALES-STATE-FISHERIES-RES-INST, CRONULLA, NSW 2230, AUSTRALIA

UNIV-ALABAMA, MARINE SCI PROGRAM, DAUPHIN-ISL, AL 36528, USA

79. Authors: Spotl-C Kralik-M Kunk-MJ

Title: Authigenic Feldspar as an Indicator of Paleo-Rock Water Interactions in Permian Carbonates of the Northern Calcareous Alps, Austria

Source: JOURNAL OF SEDIMENTARY RESEARCH 1996, Vol 66, Iss 1, pp 139-146

Addresses:

INNSBRUCK-UNIV, INST GEOL \& PALAONTOL, INNRAIN 52, A-6020

INNSBRUCK, AUSTRIA

BUNDESFORSCH-\&-PRUFZENTRUM-ARSENAL, INST GEOTECH, A-1030

VIENNA, AUSTRIA

US-GEOL-SURVEY, RESTON, VA 22092, USA

80. Authors: Symonds-RB Mizutani-Y Briggs-PH

Title: Long-Term Geochemical Surveillance of Fumaroles at ShowaShinzan Dome, Usu Volcano, Japan

Source: JOURNAL OF VOLCANOLOGY AND GEOTHERMAL RESEARCH 1996, Vol 73, Iss 3-4, pp 177-211

Addresses:

US-GEOL-SURVEY, 5400 MACARTHUR BLVD, VANCOUVER, WA 98661, USA

TOYAMA-UNIV, DEPT ENVIRONM BIOL \& CHEM, GOFU KU, TOYAMA 930, JAPAN

US-GEOL-SURVEY, FED CTR, DENVER, CO 80225, USA

81. Authors: Gazis-C Taylor-HP Hon-K Tsvetkov-A

Title: Oxygen Isotopic and Geochemical Evidence for a ShortLived, High-Temperature Hydrothermal Event in the Chegem Caldera, Caucasus Mountains, Russia

Source: JOURNAL OF VOLCANOLOGY AND GEOTHERMAL RESEARCH 1996, Vol 73, Iss 3-4, pp 213-244 
Addresses:

CALTECH, DIV GEOL \& PLANETARY SCI, PASADENA, CA 91125, USA

US-GEOL-SURVEY, DENVER FED CTR, DENVER, CO 80225, USA

RUSSIAN-ACAD-SCI, INST ORE DEPOSIT GEOL PETROG MINERAL \&

GEOCHE, MOSCOW 109017, RUSSIA

82. Authors: Melching-CS Yoon-CG

Title: Key Sources of Uncertainty in Qual2E Model of Passaic-River

Source: JOURNAL OF WATER RESOURCES PLANNING AND MANAGEMENT-ASCE 1996, Vol 122, Iss 2, pp 105-113

Addresses:

US-GEOL-SURVEY, DIV WATER RESOURCES, 102 E MAIN, 4TH FLOOR,

URBANA, IL 61801, USA

KON-KUK-UNIV, DEPT AGR ENGN, SEOUL 133701, SOUTH-KOREA

83. Authors: Evans-JR Kaminski-MA Cronin-TM Futterer-DK

Title: Pleistocene Agglutinated Foraminifera from the Lomonosov Ridge and Amundsen Basin, Arctic Basin - Initial Report

on Piston Cores 2177-5 (KAL) and 2176-3 (KAL)

Source: MARINE MICROPALEONTOLOGY 1995, Vol 26, Iss 1-4, pp 245-253

Addresses:

UNIV-LONDON-UNIV-COLL, DEPT GEOL SCI, GOWER ST, LONDON

WC1E-6BT, ENGLAND

UNIV-LONDON-BIRKBECK-COLL, RES SCH, LONDON WC1E-6BT, ENGLAND

US-GEOL-SURVEY, RESTON, VA 22092, USA

ALFRED-WEGENER-INST-POLAR-\&-MARINE-RES, D-27515 BREMERHAVEN, GERMANY

84. Authors: Mullen-MW Mcneil-DH

Title: Biostratigraphic and Paleoclimatic Significance of a New Pliocene Foraminiferal Fauna from the Central ArcticOcean

Source: MARINE MICROPALEONTOLOGY 1995, Vol 26, Iss 1-4, pp 273-280

Addresses:

US-GEOL-SURVEY, 345 MIDDLEFIELD RD, MENLO-PK, CA 94025, USA

GEOL-SURVEY-CANADA, INST SEDIMENTARY \& PETR GEOL, CALGARY, AB T2L-2A7, CANADA

85. Authors: Spotl-C Houseknecht-DW Burns-SJ

Title: Diagenesis of an Overmature Gas-Reservoir - The Spiro Sand of the Arkoma Basin, USA

Source: MARINE AND PETROLEUM GEOLOGY 1996, Vol 13, Iss 1, pp 25-40

Addresses:

INNSBRUCK-UNIV, INST GEOL \& PALAONTOL, INNRAIN 52, A-6020

INNSBRUCK, AUSTRIA

US-GEOL-SURVEY, NATL CTR 915, RESTON, VA 22092, USA

UNIV-BERN, INST GEOL, CH-3012 BERN, SWITZERLAND

86. Authors: Stamatakis-MG Hall-A Hein-JR

Title: The Zeolite Deposits of Greece

Source: MINERALIUM DEPOSITA

1996, Vol 31, Iss 6, pp 473-481 
Addresses:

UNIV-LONDON, ROYAL HOLLOWAY \& BEDFORD NEW COLL, DEPT GEOL,

EGHAM TW20-0EX, SURREY, ENGLAND

US-GEOL-SURVEY, MENLO-PK, CA 94025, USA

NATL-UNIV-ATHENS, DEPT GEOL, GR-15785 ATHENS, GREECE

87. Authors: Cunningham-CG Zartman-RE Mckee-EH Rye-RO Naeser-CW Sanjines-O Ericksen-GE Tavera-F

Title: The Age and Thermal History of Cerro-Rico-de-Potosi, Bolivia

Source: MINERALIUM DEPOSITA

1996, Vol 31, Iss 5, pp 374-385

Addresses:

US-GEOL-SURVEY, 954 NATL CTR, RESTON, VA 22092, USA

US-GEOL-SURVEY, DENVER FED CTR, DENVER, CO 80225, USA

US-GEOL-SURVEY, MENLO-PK, CA 94025, USA

SERV-GEOL-BOLIVIA-GEOBOL, LA-PAZ, BOLIVIA

88. Authors: Birch-WD Pring-A Self-PG Keck-E Jensen-MC Foord-EE

Title: Meurigite, a New Fibrous Iron Phosphate Resembling

Kidwellite

Source: MINERALOGICAL MAGAZINE

1996, Vol 60, Iss 402, pp 787-793

Addresses:

MUSEUM-VICTORIA, DEPT MINERAL \& PETROL, 328 SWANSTON ST,

MELBOURNE, VIC 3000, AUSTRALIA

S-AUSTRALIAN-MUSEUM, DEPT MINERAL, ADELAIDE, SA 5000, AUSTRALIA

CSIRO, DIV SOILS, GLEN-OSMOND, SA 5064, AUSTRALIA

US-GEOL-SURVEY, DENVER FED CTR, DENVER, CO 80225, USA

89.Authors: Linde-AT Gladwin-MT Johnston-MJS Gwyther-RL Bilham-RG

Title: A Slow Earthquake Sequence on the San-Andreas Fault

Source: NATURE 1996, Vol 383, Iss 6595, pp 65-68

Addresses:

CARNEGIE-INST-WASHINGTON, DEPT TERR MAGNETISM, 5241 BROAD

BRANCH RD NW, WASHINGTON, DC 20015, USA

CSIRO, QUEENSLAND CTR ADV TECHNOL, PINJARRA, QLD 4069,

AUSTRALIA

US-GEOL-SURVEY, MENLO-PK, CA 94025, USA

UNIV-COLORADO, DEPT GEOL SCI, BOULDER, CO 80309, USA

90. Authors: Massonnet-D Thatcher-W Vadon-H

Title: Detection of Postseismic Fault-Zone Collapse Following the Landers Earthquake

Source: NATURE 1996, Vol 382, Iss 6592, pp 612-616

Addresses:

CTR-NATL-ETUD-SPATIALES, 18 AVE EDOUARD BELIN, F-31055

TOULOUSE, FRANCE

US-GEOL-SURVEY, MENLO-PK, CA 94025, USA

91. Authors: Elias-SA Short-SK Nelson-CH Birks-HH

Title: Life and Times of the Bering Land-Bridge

Source: NATURE

1996, Vol 382, Iss 6586, pp 60-63 
Addresses:

UNIV-COLORADO, INST ARCTIC \& ALPINE RES, CAMPUS BOX 450,

BOULDER, CO 80309, USA

US-GEOL-SURVEY, MENLO-PK, CA 94025, USA

UNIV-BERGEN, INST BOT, N-5007 BERGEN, NORWAY

92. Authors: Smith-MP Sansom-IJ Repetski-JE

Title: Histology of the First Fish

Source: NATURE 1996, Vol 380, Iss 6576, pp 702-704

Addresses:

UNIV-BIRMINGHAM, SCH EARTH SCI, BIRMINGHAM B15-2TT,

W-MIDLANDS, ENGLAND

US-GEOL-SURVEY, NATL CTR, RESTON, VA 22092, USA

93. Authors: Hobden-BJ Houghton-BF Lanphere-MA Nairn-IA

Title: Growth of the Tongariro Volcanic Complex - New Evidence from $\mathrm{K}$-Ar Age-Determinations

Source: NEW ZEALAND JOURNAL OF GEOLOGY AND GEOPHYSICS 1996, Vol 39, Iss 1, pp 151-154

Addresses:

UNIV-CANTERBURY, DEPT GEOL, PRIVATE BAG 4800, CHRISTCHURCH, NEW-ZEALAND

INST-GEOL-\&-NUCL-SCI-LTD, WAIRAKEI RES CTR, TAUPO, NEW-ZEALAND

US-GEOL-SURVEY, BRANCH ISOTOPE GEOL, MENLO-PK, CA 94305, USA

94. Authors: Peng-SP Flores-RM

Title: Modern Pearl River Delta and Permian Huainan Coalfield, China - A Comparative Sedimentary Facies Study

Source: ORGANIC GEOCHEMISTRY 1996, Vol 24, Iss 2, pp 159-179

Addresses:

CHINA-UNIV-MIN-\&-TECHNOL, BEIJING GRAD SCH, BEIJING 100083, PEOPLES-R-CHINA

US-GEOL-SURVEY, DENVER, CO 80225, USA

95. Authors: Young-JB Presgrave-BW Aichele-H Wiens-DA Flinn-EA

Title: The Flinn-Engdahl Regionalization Scheme - The 1995 Revision

Source: PHYSICS OF THE EARTH AND PLANETARY INTERIORS 1996, Vol 96, Iss 4, pp 223-297

Addresses:

AWE-BLACKNEST, READING RG7-4RS, BERKS, ENGLAND

US-GEOL-SURVEY, NATL EARTHQUAKE INFORMAT CTR, DENVER, CO 80225, USA

SEISMOL-ZENT-OBSERV, D-91054 ERLANGEN, GERMANY

WASHINGTON-UNIV, DEPT EARTH \& PLANETARY SCI, ST-LOUIS, MO 63130, USA

NASA, WASHINGTON, DC 20546, USA

96. Authors: Hartzell-S Liu-PC

Title: Calculation of Earthquake Rupture Histories Using a Hybrid Global Search Algorithm - Application to the 1992

Landers, California, Earthquake

Source: PHYSICS OF THE EARTH AND PLANETARY INTERIORS

1996, Vol 95, Iss 1-2, pp 79-99 
Addresses:

US-GEOL-SURVEY, BRANCH EARTHQUAKE \& LANDSLIDE HAZARDS, BOX 25046, MS 966, LAKEWOOD, CO 80225, USA

CHINESE-ACAD-SCI, INST GEOPHYS, BEIJING 100101, PEOPLES-R-CHINA

97.Authors:Mueller-PA Wooden-JL Mogk-DW Nutman-AP Williams-IS

Title: Extended History of a 3.5-Ga Trondhjemitic Gneiss, Wyoming Province, USA - Evidence from U-Pb Systematics in Zircon

Source: PRECAMBRIAN RESEARCH 1996, Vol 78, Iss 1-3, pp 41-52

Addresses:

UNIV-FLORIDA, DEPT GEOL, GAINESVILLE, FL 32611, USA

US-GEOL-SURVEY, MENLO-PK, CA 94025, USA

MONTANA-STATE-UNIV, DEPT EARTH SCI, BOZEMAN, MT 59717, USA

AUSTRALIAN-NATL-UNIV, RES SCH EARTH SCI, CANBERRA, ACT 2601, AUSTRALIA

98. Authors: Kuksenko-V Tomilin-N Damaskinskaya-E Lockner-D

Title: A 2-Stage Model of Fracture of Rocks

Source: PURE AND APPLIED GEOPHYSICS 1996, Vol 146, Iss 2, pp 253-263

Addresses:

RUSSIAN-ACAD-SCI, AF IOFFE PHYS TECH INST, POLYTECHNICHSKAYA 26, ST-PETERSBURG 194021, RUSSIA

US-GEOL-SURVEY, MENLO-PK, CA 94025, USA

99. Authors: Occhietti-S Balescu-S Lamothe-M Clet-M Cronin-T Ferland-P Pichet-P

Title: Late-Stage-5 Glacio-Isostatic Sea in the St-Lawrence Valley, Canada and United-States

Source: QUATERNARY RESEARCH 1996, Vol 45, Iss 2, pp 128-137

Addresses:

UNIV-QUEBEC, GEOTOP, CP 8888 CTR VILLE, MONTREAL, PQ H3C-3P8, CANADA

UNIV-QUEBEC, GEETERAP, LAB IUMINESCENCE LUX, MONTREAL, PQ H3C-3P8, CANADA CNRS, CTR GEOMORPHOL, F-14000 CAEN, FRANCE

US-GEOL-SURVEY, NATL CTR 970, RESTON, VA 22092, USA

100. Authors: Payne-TE Davis-JA Waite-TD

Title: Uranium Adsorption on Ferrihydrite - Effects of Phosphate and Humic-Acid

Source: RADIOCHIMICA ACTA 1996, Vol 74, pp 239-243

Addresses:

AUSTRALIAN-NUCL-SCI-\&-TECHNOL-ORG, PRIVATE MAIL BAG 1, MENAI, NSW 2234, AUSTRALIA

US-GEOL-SURVEY, DIV WATER RESOURCES, MENLO-PK, CA 94025, USA

UNIV-NEW-S-WALES, DEPT WATER ENGN, KENSINGTON, NSW 2033, AUSTRALIA

101. Authors: Slack-JF Passchier-CW Zhang-JS

Title: Metasomatic Tourmalinite Formation Along Basement-Cover Decollements, Orobic Alps, Italy 
Source: SCHWEIZERISCHE MINERALOGISCHE UND PETROGRAPHISCHE MITTEILUNGEN

1996, Vol 76, Iss 2, pp 193-207

Addresses:

US-GEOL-SURVEY, NATL CTR, MAIL STOP 954, RESTON, VA 22092, USA

UNIV-MAINZ, INST GEOWISSENSCH TEKTONOPHYS, D-55099 MAINZ, GERMANY

STATE-SEISMOL-BUR, INST GEOL, BEIJING 100029, PEOPLES-R-CHINA

102.Authors: Kirchner-JW Hooper-RP Kendall-C Neal-C Leavesley-G

Title: Testing and Validating Environmental-Models

Source: SCIENCE OF THE TOTAL ENVIRONMENT 1996, Vol 183, Iss 1-2, pp 33-47

Addresses:

UNIV-CALIF-BERKELEY, DEPT GEOL \& GEOPHYS, BERKELEY, CA 94720, USA

US-GEOL-SURVEY, ATLANTA, GA 30360, USA

US-GEOL-SURVEY, MENLO-PK, CA 94025, USA

INST-HYDROL, WALLINGFORD OX10-8BB, OXON, ENGLAND

US-GEOL-SURVEY, DENVER FED CTR, DENVER, CO 80225, USA

103. Authors: Chen-YS Smith-PE Evensen-NM York-D Lajoie-KR

Title: The Edge of Time - Dating Young Volcanic Ash Layers with the Ar-40-Ar-39 Laser Probe

Source: SCIENCE 1996, Vol 274, Iss 5290, pp 1176-1178

Addresses:

UNIV-TORONTO, DEPT PHYS, 60 ST GEORGE ST, TORONTO, ON M5S-1A7, CANADA

US-GEOL-SURVEY, MENLO-PK, CA 94025, USA

104. Authors: Belton-MJS Head-JW Ingersoll-AP Greeley-R Mcewen-AS Klaasen-KP Senske-D Pappalardo-R

Collins-G Vasavada-AR Sullivan-R Simonelli-D

Geissler-P Carr-MH Davies-ME Veverka-J Gierasch-PJ

Banfield-D Bell-M Chapman-CR Anger-C Greenberg-R

Neukum-G Pilcher-CB Beebe-RF Burns-JA Fanale-F

Ip-W Johnson-TV Morrison-D Moore-J Orton-GS

Thomas-P West-RA

Title: Galileo First Images of Jupiter and the Galilean Satellites

Source: SCIENCE 1996, Vol 274, Iss 5286, pp 377-385

Addresses:

NATL-OPT-ASTRON-OBSERV, 950 N CHERRY AVE, TUCSON, AZ 85719, USA

BROWN-UNIV, DEPT GEOL SCI, PROVIDENCE, RI 02912, USA

CALTECH, DEPT GEOL \& PLANETARY SCI, PASADENA, CA 91125, USA

ARIZONA-STATE-UNIV, DEPT GEOL, TEMPE, AZ 85287, USA

UNIV-ARIZONA, LUNAR \& PLANETARY LAB, TUCSON, AZ 85721, USA

CALTECH, JET PROP LAB, PASADENA, CA 91109, USA

CORNELL-UNIV, DEPT ASTRON, ITHACA, NY 14853, USA

US-GEOL-SURVEY, MENLO-PK, CA 94025, USA

NASA, AMES RES CTR, MOFFETT-FIELD, CA 94035, USA

ITTRES-LTD, CALGARY, AB T1Y-5Z6, CANADA

NASA, WASHINGTON, DC 20546, USA

SW-RES-INST, BOULDER, CO 80302, USA

NEW-MEXICO-STATE-UNIV, DEPT ASTRON, LAS-CRUCES, NM 88003, USA 


\section{MAX-PLANCK-INST-AERON, LINDAU, GERMANY \\ DEUTSCH-FORSCH-ANSTALT-LUFT-\&-RAUMFAHRT, INST PLANETARY EXPLORAT, BERLIN, GERMANY}

105. Authors: Carlson-R Smythe-W Baines-K Barbinis-E Becker-K

Burns-R Calcutt-S Calvin-W Clark-R Danielson-G

Davies-A Drossart-P Encrenaz-T Fanale-F Granahan-J

Hansen-G Herrera-P Hibbitts-C Hui-J Irwin-P

Johnson-T Kamp-L Kieffer-H Leader-F Lellouch-E

Lopesgautier-R Matson-D Mccord-T Mehlman-R

Ocampo-A Orton-G Roosserote-M Segura-M Shirley-J

Soderblom-L Stevenson-A Taylor-F Torson-J Weir-A

Weissman-P

Title: Near-Infrared Spectroscopy and Spectral Mapping of

Jupiter and the Galilean Satellites - Results from

Galileos Initial Orbit

Source: SCIENCE

1996, Vol 274, Iss 5286, pp 385-388

Addresses:

CALTECH, JET PROP LAB, 4800 OAK GROVE DR, PASADENA, CA 91109,

USA

UNIV-CALIF-LOS-ANGELES, INST GEOPHYS \& PLANETARY PHYS,

LOS-ANGELES, CA 90095, USA

UNIV-OXFORD, DEPT ATMOSPHER OCEAN \& PLANETARY SCI, OXFORD

OX1-3PU, ENGLAND

US-GEOL-SURVEY, FLAGSTAFF, AZ 86001, USA

CALTECH, DIV GEOL \& PLANETARY SCI, PASADENA, CA 91125, USA

US-GEOL-SURVEY, DENVER, CO 80225, USA

OBSERV-PARIS, F-92150 MEUDON, FRANCE

UNIV-HAWAII, HAWAII INST GEOPHYS \& PLANETOL, HONOLULU, HI

96822, USA

106. Authors: Koeberl-C Poag-CW Reimold-WU Brandt-D

Title: Impact Origin of the Chesapeake Bay Structure and the

Source of the North-American Tektites

Source: SCIENCE

1996, Vol 271, Iss 5253, pp 1263-1266

Addresses:

UNIV-VIENNA, INST GEOCHEM, ALTHANSTR 14, A-1090 VIENNA, AUSTRIA

UNIV-WITWATERSRAND, DEPT GEOL, JOHANNESBURG 2050, SOUTH-AFRICA

US-GEOL-SURVEY, WOODS-HOLE, MA 02543, USA

107. Authors: Miller-KG Mountain-GS

Title: Drilling and Dating New-Jersey Oligocene-Miocene

Sequences - Ice Volume, Global Sea-Level, and Exxon

Records

Source: SCIENCE

1996, Vol 271, Iss 5252, pp 1092-1095

Addresses:

RUTGERS-STATE-UNIV, DEPT GEOL SCI, PISCATAWAY, NJ 08855, USA

COLUMBIA-UNIV, LAMONT DOHERTY GEOL OBSERV, PALISADES, NY

10964, USA

TEXAS-A\&M-UNIV, COLLEGE-STN, TX 77845, USA

LUND-UNIV, S-22100 LUND, SWEDEN

INST-SCI-EVOLUT, MONTPELLIER 5, FRANCE

UNIV-S-CAROLINA, COLUMBIA, SC 29208, USA 
UNIV-S-FLORIDA, ST-PETERSBURG, FL 33701, USA

UNIV-TEXAS, ARLINGTON, TX 76019, USA

UNIV-TEXAS, AUSTIN, TX 78759, USA

UNIV-CALIF-SANTA-CRUZ, SANTA-CRUZ, CA 95064, USA

US-GEOL-SURVEY, MENLO-PK, CA 94025, USA

UNIV-COLOGNE, W-5000 COLOGNE, GERMANY

UNIV-NEWCASTLE-UPON-TYNE, NEWCASTLE-UPON-TYNE NE1-7RU,

TYNE-\&-WEAR, ENGLAND

E-CAROLINA-STATE-UNIV, GREENVILLE, NC 27858, USA

UNIV-LILLE, F-59655 VILLENEUVE-DASCQ, FRANCE

UNIV-OXFORD, OXFORD OX1-3PR, ENGLAND

NEW-JERSEY-GEOL-SURVEY, TRENTON, NJ 08625, USA

UNIV-FREIBURG, INST GEOL, D-79104 FREIBURG, GERMANY

CHIBA-UNIV, CHIBA 263, JAPAN

UNIV-TORONTO, TORONTO, ON M5S-3B1, CANADA

GEOL-SURVEY-JAPAN, TSUKUBA, IBARAKI 305, JAPAN

FREE-UNIV-AMSTERDAM, AMSTERDAM, NETHERLANDS

LOUISIANA-STATE-UNIV, BATON-ROUGE, LA 70803, USA

UNIV-WESTERN-AUSTRALIA, NEDLANDS, WA 6009, AUSTRALIA

US-GEOL-SURVEY, RESTON, VA 22092, USA

CUNY-QUEENS-COLL, FLUSHING, NY 11367, USA

108. Authors: Behrendt-JC Saltus-R Damaske-D Mccafferty-A

Finn-CA Blankenship-D Bell-RE

Title: Patterns of Late Cenozoic Volcanic and Tectonic Activity

in the West Antarctic Rift System Revealed by

Aeromagnetic Surveys

Source: TECTONICS

1996, Vol 15, Iss 3, pp 660-676

Addresses:

US-GEOL-SURVEY, MS 964, DENVER, CO 80225, USA

GERMAN-FED-INST-GEOSCI-\&-NAT-RESOURCES, HANNOVER, GERMANY

UNIV-TEXAS, INST GEOPHYS, AUSTIN, TX, USA

COLUMBIA-UNIV, LAMONT DOHERTY EARTH OBSERV, PALISADES, NY

10964, USA

109. Authors: Rabbel-W Mooney-WD

Title: Seismic Anisotropy of the Crystalline Crust - What Does

It Tell Us

Source: TERRA NOVA

1996, Vol 8, Iss 1, pp 16-21

Addresses:

CHRISTIAN-ALBRECHTS-UNIV-KIEL, INST GEOPHYS, OLSHAUSENSTR

40-60, D-24098 KIEL, GERMANY

US-GEOL-SURVEY, MENLO-PK, CA 94025, USA

110. Authors: Horowitz-AJ Lum-KR Garbarino-JR Hall-GEM Lemieux-C

Demas-CR

Title: The Effect of Membrane Filtration on Dissolved TraceElement Concentrations

Source: WATER AIR AND SOIL POLLUTION

1996, Vol 90, Iss 1-2, pp 281-294

Addresses:

US-GEOL-SURVEY, PEACHTREE BUSINESS CTR, 3039 AMWILER RD,

ATLANTA, GA 30360, USA

ENVIRONM-CANADA, CTR ST LAURENT, MONTREAL, PQ H3Y-2EY, CANADA 
US-GEOL-SURVEY, BRANCH ANALYT SERV, ARVADA, CO 80002, USA

GEOL-SURVEY-CANADA, OTTAWA, ON K1A-0E8, CANADA

US-GEOL-SURVEY, BATON-ROUGE, LA 70816, USA

111.Authors: Watt-BE Malcolm-RL Hayes-MHB Clark-NWE Chipman-JK

Title: Chemistry and Potential Mutagenicity of Humic Substances

in Waters from Different Watersheds in Britain and

Ireland

Source: WATER RESEARCH

1996, Vol 30, Iss 6, pp 1502-1516

Addresses:

UNIV-BIRMINGHAM, SCH CHEM, POB 363, BIRMINGHAM B15-2TT,

W-MIDLANDS, ENGLAND

UNIV-BIRMINGHAM, SCH BIOCHEM, BIRMINGHAM B15-2TT, W-MIDLANDS, ENGLAND

US-GEOL-SURVEY, DIV WATER RESOURCES, DENVER, CO 80225, USA

112.Authors: Rudolph-DL Kachanoski-RG Celia-MA Leblanc-DR

Stevens-JH

Title: Infiltration and Solute Transport Experiments in

Unsaturated Sand and Gravel, Cape-Cod, Massachusetts -

Experimental-Design and Overview of Results

Source: WATER RESOURCES RESEARCH

1996, Vol 32, Iss 3, pp 519-532

Addresses:

UNIV-WATERLOO, WATERLOO CTR GROUNDWATER RES, WATERLOO, ON

N2L-3G1, CANADA

PRINCETON-UNIV, DEPT CIVIL ENGN \& OPERAT RES, PRINCETON, NJ

08540, USA

US-GEOL-SURVEY, DIV WATER RESOURCES, MARLBOROUGH, MA 01752, USA

UNIV-GUELPH, DEPT LAND RESOURCE SCI, GUELPH, ON N1G-2W1, CANADA

ENVIRON, PRINCETON, NJ, USA

113. Authors: Hauhs-M Neal-C Hooper-R Christophersen-N

Title: Summary of a Workshop on Ecosystem Modeling - The End of an Era

Source: SCIENCE OF THE TOTAL ENVIRONMENT 1996, Vol 183, Iss 1-2, pp 1-5

Addresses:

UNIV-BAYREUTH, BITOK, D-95440 BAYREUTH, GERMANY

INST-HYDROL, WALLINGFORD OX11-8BB, OXON, ENGLAND

US-GEOL-SURVEY, ATLANTA, GA 30360, USA

UNIV-OSLO, DEPT INFORMAT, N-0316 OSLO, NORWAY

114.Authors: Horowitz-AJ Lum-KR Garbarino-JR Hall-GEM Lemieux-C Demas-CR

Title: Problems Associated with Using Filtration to Define

Dissolved Trace-Element Concentrations in Natural-Water

Samples

Source: ENVIRONMENTAL SCIENCE \& TECHNOLOGY

1996, Vol 30, Iss 11, pp 3398-3400

Addresses:

US-GEOL-SURVEY, PEACHTREE BUSINESS CTR, 3039 AMWILER RD,

ATLANTA, GA 30360, USA

ENVIRONM-CANADA, CTR ST LAURENT, MONTREAL, PQ H2Y-2E7, CANADA

US-GEOL-SURVEY, BRANCH ANALYT SERV, ARVADA, CO 80002, USA 
GEOL-SURVEY-CANADA, OTTAWA, ON K1A-0E8, CANADA

US-GEOL-SURVEY, BATON-ROUGE, LA 70816, USA

115. Authors: Takada-H Satoh-F Bothner-M Tripp-BT Farrington-J

Title: Anthropogenic Markers - Molecular Tools to Identify the

Source(S) and Transport-Pathway of Pollutants

Source: ABSTRACTS OF PAPERS OF THE AMERICAN CHEMICAL SOCIETY

1996, Vol 212, Iss AUG, pp 31-ENVR

Addresses:

TOKYO-UNIV-AGR-\&-TECHNOL, FAC AGR, FUCHU, TOKYO 183, JAPAN

US-GEOL-SURVEY, WOODS-HOLE, MA 02543, USA

WOODS-HOLE-OCEANOG-INST, WOODS-HOLE, MA 02543, USA

116. Authors: Cloern-JE Grenz-C Vidergarlucas-L

Title: An Empirical-Model of the Phytoplankton Chlorophyll-

Carbon Ratio - The Conversion Factor Between

Productivity and Growth-Rate

Source: LIMNOLOGY AND OCEANOGRAPHY

1995, Vol 40, Iss 7, pp 1313-1321

Addresses:

US-GEOL-SURVEY, MS 496, 345 MIDDLEFIELD RD, MENLO-PK, CA

94025, USA

UNIV-AIX-MARSEILLE-2, CTR OCEANOL MARSEILLE, F-13007

MARSEILLE, FRANCE

STANFORD-UNIV, DEPT CIVIL ENGN, STANFORD, CA 94305, USA

117. Authors: Sherlock-RL Tosdal-RM Lehrman-NJ Graney-JR Losh-S Jowett-EC Kesler-SE

Title: Origin of the Mclaughlin Mine Sheeted Vein Complex Metal Zoning, Fluid Inclusion, and Isotopic Evidence

Source: ECONOMIC GEOLOGY AND THE BULLETIN OF THE SOCIETY OF ECONOMIC GEOLOGISTS

1995, Vol 90, Iss 8, pp 2156-2181

Addresses:

UNIV-WATERLOO, DEPT EARTH SCI, WATERLOO, ON N2L-3G1, CANADA

UNIV-WATERLOO, WATERLOO CTR GROUND WATER RES, WATERLOO, ON

N2L-3G1, CANADA

US-GEOL-SURVEY, MENLO-PK, CA 94025, USA

HOMESTAKE-MIN-CO-INC, SPARKS, NV 89431, USA

UNIV-MICHIGAN, DEPT GEOL SCI, ANN-ARBOR, MI 48109, USA

CORNELL-UNIV, DEPT GEOL SCI, ITHACA, NY 14853, USA

118. Authors: Mueller-S Choy-GL Spence-W

Title: Inelastic Models of Lithospheric Stress .1. Theory and Application to Outer-Rise Plate Deformation

Source: GEOPHYSICAL JOURNAL INTERNATIONAL 1996, Vol 125, Iss 1, pp 39-53

Addresses:

US-GEOL-SURVEY, NATL EARTHQUAKE INFORMAT CTR, DENVER FED CTR, BOX 25046, MS 967, DENVER, CO 80225, USA

UNIV-TOKYO, EARTHQUAKE RES INST, BUNKYO KU, TOKYO 113, JAPAN

119. Authors: Mueller-S Spence-W Choy-GL

Title: Inelastic Models of Lithospheric Stress .2. Implications

for Outer-Rise Seismicity and Dynamics

Source: GEOPHYSICAL JOURNAL INTERNATIONAL

1996, Vol 125, Iss 1, pp 54-72 
Addresses:

US-GEOL-SURVEY, DENVER FED CTR, NATL EARTHQUAKE INFORMAT CTR, BOX 25046, MS 967, DENVER, CO 80225, USA

UNIV-TOKYO, EARTHQUAKE RES INST, TOKYO 113, JAPAN

120. Authors: Nelson-AR Shennan-I Long-AJ

Title: Identifying Coseismic Subsidence in Tidal-Wetland Stratigraphic Sequences at the Cascadia Subduction Zone of Western North-America

Source: JOURNAL OF GEOPHYSICAL RESEARCH-SOLID EARTH 1996, Vol 101, Iss B3, pp 6115-6135

Addresses:

US-GEOL-SURVEY, BOX 25046, MS 966, DENVER, CO 80225, USA

UNIV-DURHAM, DEPT GEOG, DURHAM DH1-3LE, ENGLAND

UNIV-SOUTHAMPTON, DEPT GEOG, SOUTHAMPTON SO17-1BJ, HANTS, ENGLAND

121. Authors: Drew-LJ Berger-BR Kurbanov-NK

Title: Geology and Structural Evolution of the Muruntau Gold Deposit, Kyzyl-Kum Desert, Uzbekistan

Source: ORE GEOLOGY REVIEWS 1996, Vol 11, Iss 4, pp 175-196

Addresses:

US-GEOL-SURVEY, MS920, RESTON, VA 22092, USA

US-GEOL-SURVEY, DENVER, CO 80225, USA

TSNIGRI, MOSCOW 113545, RUSSIA

122. Authors: Kirby-SH Stein-S Okal-EA Rubie-DC

Title: Metastable Mantle Phase-Transformations and Deep

Earthquakes in Subducting Oceanic Lithosphere

Source: REVIEWS OF GEOPHYSICS 1996, Vol 34, Iss 2, pp 261-306

Addresses:

US-GEOL-SURVEY, 345 MIDDLEFIELD RD, MAIL STOP 977, MENLO-PK, CA 94025, USA

NORTHWESTERN-UNIV, DEPT GEOL SCI, EVANSTON, IL 60208, USA

UNIV-BAYREUTH, BAYER GEOINST, D-95440 BAYREUTH, GERMANY

123. Authors: Hampton-MA Lee-HJ Locat-J

Title: Submarine Landslides

Source: REVIEWS OF GEOPHYSICS

1996, Vol 34, Iss 1, pp 33-59

Addresses:

US-GEOL-SURVEY, BRANCH PACIFIC MARINE GEOL, 345 MIDDLEFIELD

RD, MAIL STOP 99, MENLO-PK, CA 94025, USA

UNIV-LAVAL, DEPT GEOL \& GEOL ENGN, QUEBEC-CITY, PQ G1K-7P4, CANADA

124. Authors: Denis-L Grenz-C Plantecuny-MR

Title: Experimental-Study of Microphytobenthos Resuspension

Source: COMPTES RENDUS DE L ACADEMIE DES SCIENCES SERIE IIISCIENCES DE LA VIE-LIFE SCIENCES

1996, Vol 319, Iss 6, pp 529-535 
Addresses:

UNIV-MEDITERRANEE-AIX-MARSEILLE-2, URA CNRS 41, CTR OCEANOL MARSEILLE, STN MARINE ENDOUME, F-13007 MARSEILLE, FRANCE

US-GEOL-SURVEY, MENLO-PK, CA 94025, USA

\section{7}

1.Authors: Clayton-JL Yang-J King-JD Lillis-PG Warden-A

Title: Geochemistry of Oils from the Junggar Basin, Northwest China

Source: AAPG BULLETIN-AMERICAN ASSOCIATION OF PETROLEUM GEOLOGISTS 1997, Vol 81, Iss 11, pp 1926-1944

Addresses:

US-GEOL-SURVEY, DENVER FED CTR, BOX 25046, DENVER, CO 80225, USA

RES-INST-EXPLORAT-\&-DEV, KARAMAY 834000, XINJIANG, PEOPLES-R-CHINA

2.Authors: Ferrer-I Thurman-EM Barcelo-D

Title: Identification of Ionic Chloroacetanilide-Herbicide Metabolites in Surface-Water and Groundwater by HPLC/MS

Using Negative-Ion Spray

Source: ANALYTICAL CHEMISTRY

1997, Vol 69, Iss 22, pp 4547-4553

Addresses:

CSIC, CID, DEPT ENVIRONM CHEM, ES-08034 BARCELONA, SPAIN

US-GEOL-SURVEY, LAWRENCE, KS 66049, USA

3.Authors: Qi-HP Coplen-TB Wang-QZ Wang-YH

Title: Unnatural Isotopic Composition of Lithium Reagents

Source: ANALYTICAL CHEMISTRY

1997, Vol 69, Iss 19, pp 4076-4078

Addresses:

US-GEOL-SURVEY, RESTON, VA 22092, USA

ACAD-SINICA, QINGHAI INST SALT LAKES, XINING 810008, PEOPLES-R-CHINA

4.Authors: Deichmann-N

Title: Far-Field Pulse Shapes from Circular Sources with Variable Rupture Velocities

Source: BULLETIN OF THE SEISMOLOGICAL SOCIETY OF AMERICA 1997, Vol 87, Iss 5, pp 1288-1296

Addresses:

ETH-HONGGERBERG, INST GEOPHYS, SWISS SEISMOL SERV, CH-8093

ZURICH, SWITZERLAND

US-GEOL-SURVEY, MENLO-PK, CA 94025, USA

5.Authors: Freeman-S Redman-RS Grantham-G Rodriguez-RJ

Title: Characterization of a Linear DNA Plasmid from the

Filamentous Fungal Plant Pathogen Glomerella-Musae

(Anamorph, Colletotrichum-Musae (Berk. and Curt.) Arx.)

Source: CURRENT GENETICS 1997, Vol 32, Iss 2, pp 152-156

Addresses:

US-GEOL-SURVEY, BIOL RESOURCES DIV, NW BIOL SCI CTR, SEATTLE, WA 98115 , USA 
AGR-RES-ORG, VOLCANI CTR, DEPT PLANT PATHOL, IL-50250

BET-DAGAN, ISRAEL

UNIV-CALIF-RIVERSIDE, DEPT PLANT PATHOL, RIVERSIDE, CA 92521, USA

6. Authors: Ozturk-H Hein-JR

Title: Mineralogy and Stable Isotopes of Black Shale-Hosted Manganese Ores, Southwestern Taurides, Turkey

Source: ECONOMIC GEOLOGY AND THE BULLETIN OF THE SOCIETY OF ECONOMIC GEOLOGISTS 1997, Vol 92, Iss 6, pp 733-744

Addresses:

US-GEOL-SURVEY, MENLO-PK, CA 94025, USA

ISTANBUL-UNIV, DEPT GEOL, ISTANBUL, TURKEY

7. Authors: Ebert-SW Rye-RO

Title: Secondary Precious-Metal Enrichment by Steam-Heated Fluids in the Crofoot-Lewis Hot-Spring Gold-Silver Deposit and Relation to Paleoclimate

Source: ECONOMIC GEOLOGY AND THE BULLETIN OF THE SOCIETY OF ECONOMIC GEOLOGISTS 1997, Vol 92, Iss 5, pp 578-600

Addresses:

UNIV-WESTERN-AUSTRALIA, DEPT GEOL \& GEOPHYS, KEY CTR STRATEG MINERAL DEPOSITS, NEDLANDS, WA 6907, AUSTRALIA

US-GEOL-SURVEY, DENVER FED CTR, DENVER, CO 80225, USA

8. Authors: Pankow-JF Thomson-NR Johnson-RL Baehr-AL Zogorski-JS

Title: The Urban Atmosphere as a Nonpoint-Source for the Transport of Mtbe and Other Volatile Organic-Compounds (Vocs) to Shallow Groundwater

Source: ENVIRONMENTAL SCIENCE \& TECHNOLOGY 1997, Vol 31, Iss 10, pp 2821-2828

Addresses:

OREGON-GRAD-INST, DEPT ENVIRONM SCI \& ENGN, POB 91000, PORTLAND, OR 97291, USA

UNIV-WATERLOO, DEPT CIVIL ENGN, WATERLOO, ON N2L-3G1, CANADA US-GEOL-SURVEY, DIV WATER RESOURCES, W-TRENTON, NJ 08628, USA US-GEOL-SURVEY, DIV WATER RESOURCES, RAPID-CITY, SD 57702, USA

9. Authors: Chabaux-F Onions-RK Cohen-AS Hein-JR

Title: U-238 U-234-Th-230 Disequilibrium in Hydrogenous Oceanic Fe-Mn Crusts - Palaeoceanographic Record or Diagenetic Alteration

Source: GEOCHIMICA ET COSMOCHIMICA ACTA 1997, Vol 61, Iss 17, pp 3619-3632

Addresses:

UNIV-CAMBRIDGE, DEPT EARTH SCI, CAMBRIDGE CB2-3EQ, ENGLAND

CNRS, CTR GEOCHIM SURFACE, F-67084 STRASBOURG, FRANCE

UNIV-OXFORD, DEPT EARTH SCI, OXFORD OX1-3PR, ENGLAND

OPEN-UNIV, DEPT EARTH SCI, MILTON-KEYNES MK7-6AA, BUCKS, ENGLAND

US-GEOL-SURVEY, MENLO-PK, CA 94024, USA 
10. Authors: Lipman-P Dungan-M Bachmann-O

Title: Comagmatic Granophyric Granite in the Fish Canyon Tuff,

Colorado - Implications for Magma-Chamber Processes

During a Large Ash-Flow Eruption

Source: GEOLOGY

1997, Vol 25, Iss 10, pp 915-918

Addresses:

US-GEOL-SURVEY, 345 MIDDLEFIELD RD, MENLO-PK, CA 94025, USA

UNIV-GENEVA, DEPT MINERAL, CH-1211 GENEVA 4, SWITZERLAND

11. Authors: Arnet-F Kahle-HG Klingele-E Smith-RB Meertens-CM

Dzurisin-D

Title: Temporal Gravity and Height Changes of the Yellowstone

Caldera, 1977-1994

Source: GEOPHYSICAL RESEARCH LETTERS

1997, Vol 24, Iss 22, pp 2741-2744

Addresses:

ETH-HONGGERBERG, INST GEODESY \& PHOTOGRAMMETRY, CH-8093

ZURICH, SWITZERLAND

UNIV-UTAH, DEPT GEOL \& GEOPHYS, SALT-LAKE-CITY, UT 84112, USA

US-GEOL-SURVEY, CASCADES VOLCANO OBSERV, VANCOUVER, WA 98661, USA

12. Authors: Thatcher-W Massonnet-D

Title: Crustal Deformation at Long Valley Caldera, Eastern

California, 1992-1996 Inferred from Satellite Radar

Interferometry

Source: GEOPHYSICAL RESEARCH LETTERS

1997, Vol 24, Iss 20, pp 2519-2522

Addresses:

US-GEOL-SURVEY, 345 MIDDLEFIELD RD, MS 977, MENLO-PK, CA

94025, USA

CTR-NATL-ETUD-SPATIALES, F-31400 TOULOUSE, FRANCE

13. Authors: Loveland-TR Belward-AS

Title: The Igbp-Dis Global 1 km Land-Cover Data Set, Discover First Results

Source: INTERNATIONAL JOURNAL OF REMOTE SENSING

1997, Vol 18, Iss 15, pp 3291-3295

Addresses:

US-GEOL-SURVEY, EROS DATA CTR, SIOUX-FALLS, SD 57198, USA

COMMISS-EUROPEAN-COMMUNITIES, JOINT RES CTR, SPACE APPLICAT

INST, I-21020 ISPRA, VA, ITALY

14. Authors: Thrush-SF Pridmore-RD Bell-RG Cummings-VJ

Dayton-PK Ford-R Grant-J Green-MO Hewitt-JE

Hines-AH Hume-TM Lawrie-SM Legendre-P Mcardle-BH

Morrisey-D Schneider-DC Turner-SJ Walters-RA

Whitlatch-RB Wilkinson-MR

Title: The Sandflat Habitat - Scaling from Experiments to

Conclusions

Source: JOURNAL OF EXPERIMENTAL MARINE BIOLOGY AND ECOLOGY 1997, Vol 216, Iss 1-2, pp 1-9 
Addresses:

NATL-INST-WATER-\&-ATMOSPHER-RES, POB 11-115, HAMILTON, NEW-ZEALAND

UNIV-CALIF-SAN-DIEGO, SCRIPPS INST OCEANOG, LA-JOLLA, CA 92093, USA

UNIV-OTAGO, DEPT MARINE SCI, DUNEDIN, NEW-ZEALAND

DALHOUSIE-UNIV, DEPT OCEANOG, HALIFAX, NS B3H-4J1, CANADA

SMITHSONIAN-ENVIRONM-RES-CTR, EDGEWATER, MD 21037, USA

UNIV-ABERDEEN, CULTERTY FILED STN, NEWBURGH AB4-0AA, SCOTLAND

UNIV-MONTREAL, DEPT SCI BIOL, MONTREAL, PQ H3C-3J7, CANADA

UNIV-AUCKLAND, SCH BIOL SCI, BIOSTAT UNIT, AUCKLAND 1, NEW-ZEALAND

MEM-UNIV-NEWFOUNDLAND, CTR OCEAN SCI, ST-JOHNS, NF-ALC 5ST, CANADA

UNIV-CONNECTICUT, DEPT MARINE SCI, GROTON, CT 06340, USA

US-GEOL-SURVEY, TACOMA, WA 98402, USA

15. Authors: Bell-RG Hume-TM Dolphin-TJ Green-MO Walters-RA

Title: Characterization of Physical Environmental-Factors on an Intertidal Sandflat, Manukau Harbor, New-Zealand

Source: JOURNAL OF EXPERIMENTAL MARINE BIOLOGY AND ECOLOGY 1997, Vol 216, Iss 1-2, pp 11-31

Addresses:

NATL-INST-WATER-\&-ATMOSPHER-RES, POB 11-115, HAMILTON, NEW-ZEALAND

UNIV-WAIKATO, DEPT EARTH SCI, HAMILTON, NEW-ZEALAND

US-GEOL-SURVEY, TACOMA, WA 98402, USA

16. Authors: Schneider-DC Walters-R Thrush-S Dayton-P

Title: Scale-Up of Ecological Experiments - Density Variation in the Mobile Bivalve Macomona-Liliana

Source: JOURNAL OF EXPERIMENTAL MARINE BIOLOGY AND ECOLOGY 1997, Vol 216, Iss 1-2, pp 129-152

Addresses:

MEM-UNIV-NEWFOUNDLAND, CTR OCEAN SCI, ST-JOHNS, NF A1B-3X7, CANADA

US-GEOL-SURVEY, TACOMA, WA 98402, USA

NATL-INST-WATER-\&-ATMOSPHER-RES, HAMILTON, NEW-ZEALAND

UNIV-CALIF-SAN-DIEGO, SCRIPPS INST OCEANOG, LA-JOLLA, CA 92093, USA

17. Authors: Cole-F Bird-KJ Toro-J Roure-F Osullivan-PB Pawlewicz-M Howell-DG

Title: An Integrated Model for the Tectonic Development of the Frontal Brooks Range and Colville Basin $250 \mathrm{~km}$ West of the Trans-Alaska Crustal Transect

Source: JOURNAL OF GEOPHYSICAL RESEARCH-SOLID EARTH 1997, Vol 102, Iss B9, pp 20685-20706

Addresses:

US-GEOL-SURVEY, 345 MIDDLEFIELD RD, MS 969, MENLO-PK, CA 94025, USA

LA-TROBE-UNIV, SCH EARTH SCI, DEPT GEOL, BUNDOORA, VIC 3083, AUSTRALIA

US-GEOL-SURVEY, DENVER, CO 80225, USA 
INST-FRANCAIS-PETR, F-92506 RUEIL-MALMAISON, FRANCE

STANFORD-UNIV, DEPT GEOG \& ENVIRONM SCI, STANFORD, CA 94305, USA

18. Authors: Osullivan-PB Murphy-JM Blythe-AE

Title: Late Mesozoic and Cenozoic Thermotectonic Evolution of the Central Brooks Range and Adjacent North Slope Foreland Basin, Alaska - Including Fission-Track Results from the Trans-Alaska Crustal Transect (Tact)

Source: JOURNAL OF GEOPHYSICAL RESEARCH-SOLID EARTH 1997, Vol 102, Iss B9, pp 20821-20845

Addresses:

LA-TROBE-UNIV, SCH EARTH SCI, BUNDOORA, VIC 3083, AUSTRALIA

UNIV-SO-CALIF, DEPT EARTH SCI, LOS-ANGELES, CA 90089, USA

UNIV-WYOMING, DEPT GEOL \& GEOPHYS, LARAMIE, WY 82071, USA

US-GEOL-SURVEY, MENLO-PK, CA 94025, USA

19. Authors: Tenbrink-US Hackney-RI Bannister-S Stern-TA Makovsky-Y

Title: Uplift of the Transantarctic Mountains and the Bedrock Beneath the East Antarctic Ice-Sheet

Source: JOURNAL OF GEOPHYSICAL RESEARCH-SOLID EARTH 1997, Vol 102, Iss B12, pp 27603-27621

Addresses:

US-GEOL-SURVEY, QUISSETT CAMPUS, WOODS-HOLE, MA 02543, USA

INST-GEOL-\&-NUCL-SCI, WELLINGTON, NEW-ZEALAND

UNIV-WESTERN-AUSTRALIA, DEPT GEOL \& GEOPHYS, NEDLANDS, WA 6009, AUSTRALIA

STANFORD-UNIV, DEPT GEOPHYS, STANFORD, CA 94305, USA

VICTORIA-UNIV-WELLINGTON, INST GEOPHYS, WELLINGTON, NEW-ZEALAND

20. Authors: Walker-JF Wang-DP

Title: Measurement of Flow Under-Ice Covers in North-America

Source: JOURNAL OF HYDRAULIC ENGINEERING-ASCE 1997, Vol 123, Iss 11, pp 1037-1040

Addresses:

US-GEOL-SURVEY, 8505 RES WAY, MIDDLETON, WI 53562, USA

ENVIRONM-CANADA, WATER SURVEY CANADA DIV, OTTAWA, ON K1A-0H3, CANADA

21. Authors: Dickens-GR Barron-JA

Title: A Rapidly Deposited Pennate Diatom Ooze in Upper Miocene Lower Pliocene Sediment Beneath the North Pacific Polar Front

Source: MARINE MICROPALEONTOLOGY 1997, Vol 31, Iss 3-4, pp 177-182

Addresses:

JAMES-COOK-UNIV-N-QUEENSLAND, DEPT EARTH SCI, TOWNSVILLE, QLD 4811, AUSTRALIA

US-GEOL-SURVEY, MENLO-PK, CA 94025, USA

UNIV-MICHIGAN, DEPT GEOL SCI, ANN-ARBOR, MI 48109, USA

22. Authors: Campbell-JL Czamanske-GK Macdonald-L Teesdale-WJ

Title: Quantitative-Analysis of Major Elements in Silicate

Minerals and Glasses by Micro-PIXE 
Source: NUCLEAR INSTRUMENTS \& METHODS IN PHYSICS RESEARCH SECTION B-BEAM INTERACTIONS WITH MATERIALS AND ATOMS 1997, Vol 130, Iss 1-4, pp 608-616

Addresses:

UNIV-GUELPH, GUELPH WATERLOO PROGRAM GRAD WORK PHYS, GUELPH, ON N1G-2W1, CANADA

US-GEOL-SURVEY, MENLO-PK, CA 94025, USA

23. Authors: Golombek-MP Cook-RA Economou-T Folkner-WM

Haldemann-AFC Kallemeyn-PH Knudsen-JM Manning-RM

Moore-HJ Parker-TJ Rieder-R Schofield-JT Smith-PH

Vaughan-RM

Title: Overview of the Mars Pathfinder Mission and Assessment of Landing Site Predictions

Source: SCIENCE 1997, Vol 278, Iss 5344, pp 1743-1748

Addresses:

CALTECH, JET PROP LAB, 4800 OAK GROVE DR, PASADENA, CA 91109, USA

UNIV-CHICAGO, ENRICO FERMI INST, CHICAGO, IL 60637, USA

UNIV-COPENHAGEN, NIELS BOHR INST, DK-1168 COPENHAGEN, DENMARK

US-GEOL-SURVEY, ASTROGEOL TEAM, MENLO-PK, CA 94025, USA

MAX-PLANCK-INST-CHEM, D-6500 MAINZ, GERMANY

UNIV-ARIZONA, LUNAR \& PLANETARY LAB, TUCSON, AZ 85721, USA

24. Authors: Smith-PH Bell-JF Bridges-NT Britt-DT Gaddis-L

Greeley-R Keller-HU Herkenhoff-KE Jaumann-R

Johnson-JR Kirk-RL Lemmon-M Maki-JN Malin-MC

Murchie-SL Oberst-J Parker-TJ Reid-RJ Sablotny-R

Soderblom-LA Stoker-C Sullivan-R Thomas-N

Tomasko-MG Ward-W Wegryn-E

Title: Results from the Mars Pathfinder Camera

Source: SCIENCE 1997, Vol 278, Iss 5344, pp 1758-1765

Addresses:

UNIV-ARIZONA, LUNAR \& PLANETARY LAB, TUCSON, AZ 85721, USA

CORNELL-UNIV, ITHACA, NY 14853, USA

CALTECH, JET PROP LAB, PASADENA, CA 91109, USA

US-GEOL-SURVEY, FLAGSTAFF, AZ 86001, USA

ARIZONA-STATE-UNIV, TEMPE, AZ 85287, USA

MAX-PLANCK-INST-AERON, KATHENBURG-LINDAU, GERMANY

DLR, BERLIN, GERMANY

MALIN-SPACE-SCI-SYST, SAN-DIEGO, CA, USA

NASA, AMES RES CTR, MOFFETT-FIELD, CA 94035, USA

JOHNS-HOPKINS-UNIV, APPL PHYS LAB, BALTIMORE, MD 21218, USA

25. Authors: Katupitiya-A Eisenhauer-DE Ferguson-RB Spalding-RF

Roeth-FW Bobier-MW

Title: Long-Term Tillage and Crop-Rotation Effects on Residual

Nitrate in the Crop Root-Zone and Nitrate Accumulation

in the Intermediate Vadose Zone

Source: TRANSACTIONS OF THE ASAE

1997, Vol 40, Iss 5, pp 1321-1327 
Addresses:

UNIV-NEBRASKA, LINCOLN, NE 68583, USA

US-GEOL-SURVEY, IOWA-CITY, IA, USA

CHARLES-STURT-UNIV, BATHURST, NSW 2795, AUSTRALIA

26. Authors: Ponomarev-AV Zavyalov-AD Smirnov-VB Lockner-DA

Title: Physical Modeling of the Formation and Evolution of

Seismically Active Fault Zones

Source: TECTONOPHYSICS

1997, Vol 277, Iss 1-3, pp 57-81

Addresses:

RUSSIAN-ACAD-SCI, UIPE, INST SEISMOL, BOL GRUZINSKAYA 10,

MOSCOW 123810, RUSSIA

MOSCOW-MV-LOMONOSOV-STATE-UNIV, FAC PHYS, MOSCOW, RUSSIA

US-GEOL-SURVEY, MENLO-PK, CA 91425, USA

27. Authors: Ritter-JRR Evans-JR

Title: Deep-Structure of Medicine-Lake Volcano, California

Source: TECTONOPHYSICS 1997, Vol 275, Iss 1-3, pp 221-241

Addresses:

UNIV-KARLSRUHE, INST GEOPHYS, HERTZSTR 16, D-76187 KARLSRUHE, GERMANY

US-GEOL-SURVEY, MENLO-PK, CA 94025, USA

28. Authors: Huber-K Fuchs-K Palmer-J Roth-F Khakhaev-BN

Vankin-LE Pevzner-LA Hickman-S Moos-D Zoback-MD

Schmitt-D

Title: Analysis of Borehole Televiewer Measurements in the Vorotilov Drillhole, Russia - First Results

Source: TECTONOPHYSICS

1997, Vol 275, Iss 1-3, pp 261-272

Addresses:

UNIV-KARLSRUHE, INST GEOPHYS, D-76187 KARLSRUHE, GERMANY

GEOFORSCHUNGSZENTRUM-POTSDAM, DIV DISASTER RES, SECT BOREHOLE

LOGGING, D-14473 POTSDAM, GERMANY

GNPP, YAROSLAVL 150013, RUSSIA

US-GEOL-SURVEY, MENLO-PK, CA 94025, USA

STANFORD-UNIV, DEPT GEOPHYS, STANFORD, CA 94305, USA

UNIV-ALBERTA, DEPT PHYS, EDMONTON, AB T6G-2J1, CANADA

29. Authors: Takada-H Satoh-F Bothner-MH Tripp-BW Johnson-CG Farrington-JW

Title: Anthropogenic Molecular Markers - Tools to Identify the Sources and Transport Pathways of Pollutants

Source: ACS SYMPOSIUM SERIES 1997, Vol 671, pp 178-195

Addresses:

TOKYO-UNIV-AGR-\&-TECHNOL, FAC AGR, FUCHU, TOKYO 183, JAPAN

US-GEOL-SURVEY, WOODS-HOLE, MA 02543, USA

WOODS-HOLE-OCEANOG-INST, WOODS-HOLE, MA 02543, USA

30. Authors: Conroy-MJ Nichols-JD Asanza-ER

Title: Contemporary Quantitative Methods to Understand and

Manage Animal Populations and Communities 


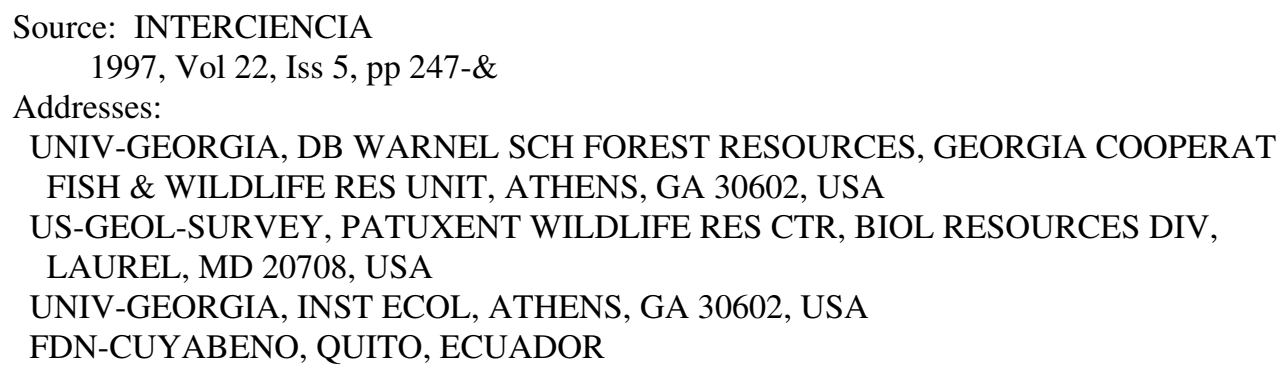

31. Authors: Queralt-I Julia-R Plana-F Bischoff-JL

Title: A Hydrous Ca-Bearing Magnesium Carbonate from Playa LakeSediments, Salines Lake, Spain

Source: AMERICAN MINERALOGIST 1997, Vol 82, Iss 7-8, pp 812-819

Addresses:

CSIC, INST EARTH SCI JAUME ALMERA, SOLE SABARIS S-N, BARCELONA 08028, SPAIN

US-GEOL-SURVEY, MENLO-PK, CA 94025, USA

32. Authors: Dyke-AS England-J Reimnitz-E Jette-H

Title: Changes in Driftwood Delivery to the Canadian Arctic Archipelago - The Hypothesis of Postglacial Oscillations of the Transpolar Drift

Source: ARCTIC 1997, Vol 50, Iss 1, pp 1-16

Addresses:

GEOL-SURVEY-CANADA, TERRAIN SCI DIV, 601 BOOTH ST, OTTAWA, ON K1A-0E8, CANADA UNIV-ALBERTA, DEPT EARTH \& ATMOSPHER SCI, EDMONTON, AB T6G-2E3, CANADA US-GEOL-SURVEY, MENLO-PK, CA 94025, USA

33. Authors: Sulak-KJ Shcherbachev-YN

Title: Zoogeography and Systematics of 6 Deep-Living Genera of Synaphobranchid EELS, with a Key to Taxa and Description of 2 New Species of Ilyophis

Source: BULLETIN OF MARINE SCIENCE 1997, Vol 60, Iss 3, pp 1158-1194

Addresses:

US-GEOL-SURVEY, FLORIDA CARIBBEAN SCI CTR, 7920 NW 71ST ST, GAINESVILLE, FL 32615, USA

RUSSIAN-ACAD-SCI, INST OCEANOL, MOSCOW 117519, RUSSIA

34. Authors: Bodin-P Gomberg-J Singh-SK Santoyo-M

Title: Dynamic Deformations of Shallow Sediments in the Valley of Mexico .1. 3-Dimensional Strains and Rotations Recorded on a Seismic Array

Source: BULLETIN OF THE SEISMOLOGICAL SOCIETY OF AMERICA 1997, Vol 87, Iss 3, pp 528-539

Addresses:

UNIV-MEMPHIS, CTR EARTHQUAKE RES \& INFORMAT, MEMPHIS, TN 38152, USA

US-GEOL-SURVEY, MEMPHIS, TN 38152, USA 


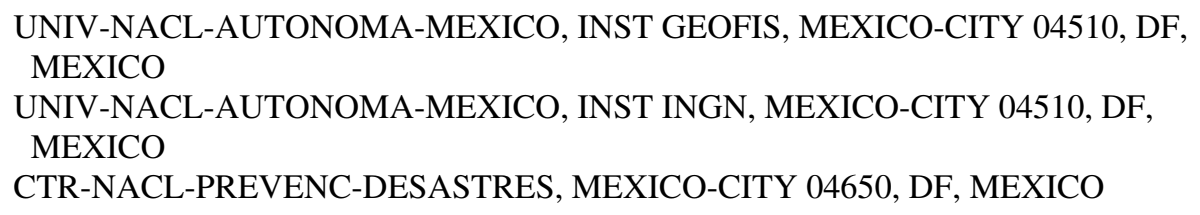

35. Authors: Ohminato-T Chouet-BA

Title: A Free-Surface Boundary-Condition for Including 3D

Topography in the Finite-Difference Method

Source: BULLETIN OF THE SEISMOLOGICAL SOCIETY OF AMERICA 1997, Vol 87, Iss 2, pp 494-515

Addresses:

GEOL-SURVEY-JAPAN, 1-1-3 HIGASHI, TSUKUBA, IBARAKI 305, JAPAN

US-GEOL-SURVEY, MENLO-PK, CA 94025, USA

36. Authors: Mcknight-DM Harnish-R Wershaw-RL Baron-JS Schiff-S

Title: Chemical Characteristics of Particulate, Colloidal, and Dissolved Organic Material in Loch Vale Watershed, Rocky-

Mountain-National-Park

Source: BIOGEOCHEMISTRY

1997, Vol 36, Iss 1, pp 99-124

Addresses:

US-GEOL-SURVEY, 3215 MARINE ST, BOULDER, CO 80303, USA

COLORADO-STATE-UNIV, NATL BIOL SERV, FT-COLLINS, CO 80523, USA

COLORADO-STATE-UNIV, NAT RESOURCE ECOL LAB, FT-COLLINS, CO

80523, USA

UNIV-WATERLOO, WATERLOO CTR GROUNDWATER RES, WATERLOO, ON

N2L-3G1, CANADA

37. Authors: Clague-JJ Naesgaard-E Nelson-AR

Title: Age and Significance of Earthquake-Induced Liquefaction

Near Vancouver, British-Columbia, Canada

Source: CANADIAN GEOTECHNICAL JOURNAL 1997, Vol 34, Iss 1, pp 53-62

Addresses:

GEOL-SURVEY-CANADA, 101-605 ROBSON ST, VANCOUVER, BC V6B-5J3, CANADA

MACLEOD-GEOTECH-LTD, W-VANCOUVER, BC V7T-1B8, CANADA

US-GEOL-SURVEY, DENVER, CO 80225, USA

38. Authors: Barnes-SJ Zientek-ML Severson-MJ

Title: $\mathrm{Ni}, \mathrm{Cu}, \mathrm{Au}$, and Platinum-Group Element Contents of

Sulfides Associated with Intraplate Magmatism - A

Synthesis

Source: CANADIAN JOURNAL OF EARTH SCIENCES 1997, Vol 34, Iss 4, pp 337-351

Addresses:

UNIV-QUEBEC, CHICOUTIMI, PQ G7H-2B1, CANADA

US-GEOL-SURVEY, SPOKANE, WA 99201, USA

UNIV-MINNESOTA, NAT RESOURCES RES INST, DULUTH, MN 55811, USA

39. Authors: Goldstein-S Arndt-NT Stallard-RF

Title: The History of a Continent from U-Pb Ages of Zircons

from Orinoco River Sand and Sm-Nd Isotopes in Orinoco

Basin River Sediments 
Source: CHEMICAL GEOLOGY

1997, Vol 139, Iss 1-4, pp 271-286

Addresses:

MAX-PLANCK-INST-CHEM, D-50220 MAINZ, GERMANY

UNIV-RENNES, INST GEOL, F-35042 RENNES, FRANCE

US-GEOL-SURVEY, BOULDER, CO 80303, USA

40. Authors: Drits-V Srodon-J Eberl-DD

Title: XRD Measurement of Mean Crystalline Thickness of Illite and Illite/Smectite - Reappraisal of the Kubler Index and the Scherrer Equation

Source: CLAYS AND CLAY MINERALS 1997, Vol 45, Iss 3, pp 461-475

Addresses:

RUSSIAN-ACAD-SCI, INST GEOG, PYZHEVSKY ST 7, MOSCOW 109017, RUSSIA

PAN, INST GEOL SCI, PL-31002 KRAKOW, POLAND

US-GEOL-SURVEY, BOULDER, CO 80303, USA

41. Authors: Eicken-H Reimnitz-E Alexandrov-V Martin-T

Kassens-H Viehoff-T

Title: Sea-Ice Processes in the Laptev Sea and Their Importance for Sediment Export

Source: CONTINENTAL SHELF RESEARCH 1997, Vol 17, Iss 2, pp 205-\&

Addresses:

ALFRED-WEGENER-INST-POLAR-\&-MARINE-RES, POSTFACH 120161, D-27515 BREMERHAVEN, GERMANY

US-GEOL-SURVEY, MENLO-PK, CA 94025, USA

ARCTIC-\&-ANTARCTIC-RES-INST, ST-PETERSBURG, RUSSIA

GEOMAR, D-24148 KIEL, GERMANY

42. Authors: Brophy-JG Dorais-MJ Donnellynolan-J Singer-BS

Title: Plagioclase Zonation Styles in Hornblende Gabbro Inclusions from Little Glass Mountain, Medicine Lake Volcano, California - Implications for Fractionation Mechanisms and the Formation of Composition Gaps

Source: CONTRIBUTIONS TO MINERALOGY AND PETROLOGY 1996, Vol 126, Iss 1-2, pp 121-136

Addresses:

INDIANA-UNIV, DEPT GEOL SCI, BLOOMINGTON, IN 47405, USA

US-GEOL-SURVEY, MENLO-PK, CA 94025, USA

UNIV-GENEVA, DEPT MINERAL, GENEVA, SWITZERLAND

43. Authors: Amelin-YV Ritsk-EY Neymark-LA

Title: Effects of Interaction Between Ultramafic Tectonite and Mafic Magma on Nd-Pb-Sr Isotopic Systems in the Neoproterozoic Chaya Massif, Baikal-Muya Ophiolite Belt

Source: EARTH AND PLANETARY SCIENCE LETTERS 1997, Vol 148, Iss 1-2, pp 299-316

Addresses:

ROYAL-ONTARIO-MUSEUM, GEOCHRONOL LAB, 100 QUEENS PK, TORONTO, ON M5S-2C6, CANADA

INST-PRECAMBRIAN-GEOL-\&-GEOCHRONOL, ST-PETERSBURG 199034, RUSSIA

US-GEOL-SURVEY, DENVER FED CTR, DENVER, CO 80225, USA 
44. Authors: Ling-HF Burton-KW Onions-RK Kamber-BS

Vonblanckenburg-F Gibb-AJ Hein-JR

Title: Evolution of $\mathrm{Nd}$ and $\mathrm{Pb}$ Isotopes in Central Pacific

Seawater from Ferromanganese Crusts

Source: EARTH AND PLANETARY SCIENCE LETTERS 1997, Vol 146, Iss 1-2, pp 1-12

Addresses:

UNIV-OXFORD, DEPT EARTH SCI, OXFORD OX1-3PR, ENGLAND

INST-PHYS-GLOBE, CNRS, LAB GEOCHIM \& COSMOCHIM, F-75252 PARIS 05, FRANCE

US-GEOL-SURVEY, MENLO-PK, CA 94025, USA

45. Authors: Wang-J Cheng-RT Smith-PC

Title: Seasonal Sea-Level Variations in San-Francisco Bay in Response to Atmospheric Forcing, 1980

Source: ESTUARINE COASTAL AND SHELF SCIENCE 1997, Vol 45, Iss 1, pp 39-52

Addresses:

UNIV-MIAMI, ROSENSTIEL SCH MARINE \& ATMOSPHER SCI, 4600

RICKENBACKER CAUSEWAY, MIAMI, FL 33149, USA

US-GEOL-SURVEY, MENLO-PK, CA 94025, USA

FISHERIES-\&-OCEANS-CANADA, BEDFORD INST OCEANOG, COASTAL

OCEANOG DIV, DARTMOUTH, NS B2Y-4A2, CANADA

46. Authors: Belkin-HE Devivo-B Lima-A Torok-K

Title: Magmatic (Silicates/Saline/Sulfur-Rich/CO2) Immiscibility and Zirconium and Rare-Earth Element Enrichment from Alkaline Magma Chamber Margins -

Evidence from Ponza Island, Pontine Archipelago, Italy

Source: EUROPEAN JOURNAL OF MINERALOGY 1996, Vol 8, Iss 6, pp 1401-1420

Addresses:

US-GEOL-SURVEY, MS 959, RESTON, VA 22092, USA

UNIV-NAPLES-FEDERICO-II, DIPARTIMENTO GEOFIS \& VULCANOL, I-80134 NAPLES, ITALY

LORAND-EOTVOS-UNIV, DEPT PETROG \& GEOCHEM, H-1088 BUDAPEST, HUNGARY

47. Authors: Novarino-G Warren-A Butler-H Lambourne-G

Boxshall-A Bateman-J Kinner-NE Harvey-RW Mosse-RA

Teltsch-B

Title: Protistan Communities in Aquifers - A Review

Source: FEMS MICROBIOLOGY REVIEWS 1997, Vol 20, Iss 3-4, pp 261-275

Addresses:

NAT-HIST-MUSEUM, DEPT ZOOL, CROMWELL RD, LONDON SW7-5BD, ENGLAND

UNIV-MARINE-BIOL-STN, MILLPORT, ISLE-CUMBRAE, SCOTLAND

UNIV-NEW-HAMPSHIRE, DEPT CIVIL ENGN, ENVIRONM RES GRP, DURHAM, NH 03824, USA

US-GEOL-SURVEY, DIV WATER RESOURCES, BOULDER, CO, USA

MEKOROT-WATER-CO-LTD, NAZARETH, ISRAEL 
48. Authors: Pronin-NM Fleischer-GW Baldanova-DR Pronina-SV

Title: Parasites of the Recently Established Round Goby

(Neogobius-Melanostomus) and Tubenose Goby

(Proterorhinus-Marmoratus) (Cottidae) from the St-Clair

River and Lake St-Clair, Michigan, USA

Source: FOLIA PARASITOLOGICA

1997, Vol 44, Iss 1, pp 1-6

Addresses:

RUSSIAN-ACAD-SCI, BURYAT INST BIOL, SIBERIAN DIV, 6 SAKHYANOVA

STR, ULAN-UDE 670042, RUSSIA

US-GEOL-SURVEY, GREAT LAKES SCI CTR, BIOL RESOURCES DIV, ANN-ARBOR, MI 48105, USA

49. Authors: Cobban-WA Kennedy-WJ Scott-GR

Title: Didymoceras Puebloense, a New Species of Heteromorph

Ammonite from the Upper Campanian of Colorado and Wyoming

Source: GEOBIOS

1997, Vol 30, Iss 2, pp 225-230

Addresses:

US-GEOL-SURVEY, DENVER FED CTR, BOX 25046, MAIL STOP 913,

DENVER, CO 80225, USA

UNIV-MUSEUM, OXFORD OX1-3PW, ENGLAND

50. Authors: Koopmans-MP Schaefferreiss-C Deleeuw-JW Lewan-MD

Maxwell-JR Schaeffer-P Damste-JSS

Title: Sulfur and Oxygen Sequestration of N-C-37 and N-C-38

Unsaturated-Ketones in an Immature Kerogen and the

Release of Their Carbon Skeletons During Early Stages of

Thermal Maturation

Source: GEOCHIMICA ET COSMOCHIMICA ACTA

1997, Vol 61, Iss 12, pp 2397-2408

Addresses:

NETHERLANDS-INST-SEA-RES, NIOZ, DEPT MARINE BIOGEOCHEM \&

TOXICOL, NL-1790-AB DEN-BURG, NETHERLANDS

UNIV-BRISTOL, SCH CHEM, ORGAN GEOCHEM UNIT, BRISTOL BS8-1TS, AVON, ENGLAND

US-GEOL-SURVEY, DENVER FED CTR, DENVER, CO 80225, USA

51. Authors: Ozima-M Tatsumoto-M

Title: Radiation-Induced Diamond Crystallization - Origin of

Carbonados and Its Implications on Meteorite Nano-

Diamonds

Source: GEOCHIMICA ET COSMOCHIMICA ACTA

1997, Vol 61, Iss 2, pp 369-376

Addresses:

WASHINGTON-UNIV, DEPT EARTH \& PLANETARY SCI, ST-LOUIS, MO

63130, USA

UNIV-TOKYO, DEPT EARTH \& PLANETARY PHYS, BUNKYO KU, TOKYO 113,

JAPAN

US-GEOL-SURVEY, DENVER, CO 80225, USA

52. Authors: Vonblanckenburg-F Onions-RK Hein-JR

Title: Distribution and Sources of Pre-Anthropogenic Lead

Isotopes in Deep-Ocean Water from Fe-Mn Crusts

Source: GEOCHIMICA ET COSMOCHIMICA ACTA

1996, Vol 60, Iss 24, pp 4957-4963 
Addresses:

UNIV-OXFORD, DEPT EARTH SCI, PARKS RD, OXFORD OX1-3PR, ENGLAND

US-GEOL-SURVEY, MENLO-PK, CA 94025, USA

53. Authors: Matsumoto-A Hinkley-TK

Title: Determination of Lead, Cadmium, Indium, Thallium and

Silver in Ancient Ices from Antarctica by Isotope-

Dilution Thermal Ionization Mass-Spectrometry

Source: GEOCHEMICAL JOURNAL

1997, Vol 31, Iss 3, pp 175-181

Addresses:

GEOL-SURVEY-JAPAN, 1-1-3 HIGASHI, TSUKUBA, IBARAKI 305, JAPAN

US-GEOL-SURVEY, DENVER FED CTR, DENVER, CO 80225, USA

54. Authors: Moore-TC Klitgord-KD Golmshtok-AJ Weber-E

Title: Sedimentation and Subsidence Patterns in the Central and North Basins of Lake-Baikal from Seismic Stratigraphy

Source: GEOLOGICAL SOCIETY OF AMERICA BULLETIN 1997, Vol 109, Iss 6, pp 746-766

Addresses:

UNIV-MICHIGAN, DEPT GEOL SCI, 1006 CC LITTLE BLDG, ANN-ARBOR, MI 48109, USA

US-GEOL-SURVEY, PALO-ALTO, CA 94304, USA

PP-SHIRSHOV-OCEANOL-INST, SO BRANCH, GELENDZHIK, RUSSIA

55. Authors: Singer-BS Thompson-RA Dungan-MA Feeley-TC

Nelson-ST Pickens-JC Brown-LL Wulff-AW Davidson-JP

Metzger-J

Title: Volcanism and Erosion During the Past $930 \mathrm{Ky}$ at the

Tatara-San-Pedro Complex, Chilean Andes

Source: GEOLOGICAL SOCIETY OF AMERICA BULLETIN 1997, Vol 109, Iss 2, pp 127-142

Addresses:

UNIV-GENEVA, DEPT MINERAL, CH-1211 GENEVA 4, SWITZERLAND

US-GEOL-SURVEY, DENVER, CO 80225, USA

WOODWARD-CLYDE-FED-SERV, LAS-VEGAS, NV 89109, USA

UNIV-MASSACHUSETTS, DEPT GEOSCI, AMHERST, MA 01003, USA

UNIV-CALIF-LOS-ANGELES, DEPT EARTH \& SPACE SCI, LOS-ANGELES, CA 90024, USA

56. Authors: Vangeen-A Adkins-JF Boyle-EA Nelson-CH Palanques-A

Title: A 120-Year Record of Widespread Contamination from

Mining of the Iberian Pyrite Belt

Source: GEOLOGY

1997, Vol 25, Iss 4, pp 291-294

Addresses:

COLUMBIA-UNIV, LAMONT DOHERTY EARTH OBSERV, PALISADES, NY

10964, USA

MIT, DEPT EARTH ATMOSPHER \& PLANETARY SCI, CAMBRIDGE, MA

02139, USA

US-GEOL-SURVEY, MENLO-PK, CA 94025, USA

INST-CIENCIAS-MAR, E-08039 BARCELONA, SPAIN

57. Authors: Fuenzalida-H Rivera-L Haessler-H Legrand-D

Philip-H Dorbath-L Mccormack-D Arefiev-S Langer-C

Cisternas-A 
Title: Seismic Source Study of the Racha-Dzhava (Georgia)

Earthquake from Aftershocks and Broad-Band Teleseismic

Body-Wave Records - An Example of Active Nappe Tectonics

Source: GEOPHYSICAL JOURNAL INTERNATIONAL

1997, Vol 130, Iss 1, pp 29-46

Addresses:

INST-PHYS-GLOBE-STRASBOURG, 5 RUE RENE DESCARTES, F-67084

STRASBOURG, FRANCE

UNIV-MONTPELLIER-2, LAB GEOL STRUCT, F-34060 MONTPELLIER,

FRANCE

UNIV-CAMBRIDGE, BULLARD LABS, CAMBRIDGE, ENGLAND

RUSSIAN-ACAD-SCI, INST PHYS EARTH, MOSCOW 123810, RUSSIA

US-GEOL-SURVEY, DENVER FED CTR, DENVER, CO 80225, USA

ORSTOM, F-75480 PARIS 10, FRANCE

58. Authors: Stein-RS Barka-AA Dieterich-JH

Title: Progressive Failure on the North Anatolian Fault Since

1939 by Earthquake Stress Triggering

Source: GEOPHYSICAL JOURNAL INTERNATIONAL 1997, Vol 128, Iss 3, pp 594-604

Addresses:

US-GEOL-SURVEY, MS 977, 345 MIDDLEFIELD RD, MENLO-PK, CA

94025, USA

ISTANBUL-TECH-UNIV, DEPT GEOL, TR-80626 ISTANBUL, TURKEY

59. Authors: Hinkley-T Pertsiger-F Zavjalova-L

Title: The Modern Atmospheric Background Dust Load -

Recognition in Central-Asian Snowpack, and Compositional

Constraints

Source: GEOPHYSICAL RESEARCH LETTERS

1997, Vol 24, Iss 13, pp 1607-1610

Addresses:

US-GEOL-SURVEY, DENVER FED CTR, BOX 25046, DENVER, CO 80225, USA

GLAVGIDROMET, CABINET MINISTERS, MAIN ADM HYDROMETEOROL, TASHKENT 700052, UZBEKISTAN

SANIGMI, CENT ASIAN RES HYDROMETEOROL INST, MAIN ADM

HYDROMETEOROL, TASHKENT 700052, UZBEKISTAN

60. Authors: Massonnet-D Holzer-T Vadon-H

Title: Land Subsidence Caused by the East Mesa Geothermal-

Field, California, Observed Using SAR Interferometry

Source: GEOPHYSICAL RESEARCH LETTERS

1997, Vol 24, Iss 8, pp 901-904

Addresses:

CTR-NATL-ETUD-SPATIALES, 18 AVE EDOUARD BELIN, F-31055

TOULOUSE, FRANCE

US-GEOL-SURVEY, MENLO-PK, CA 94025, USA

61. Authors: Julian-BR Miller-AD Foulger-GR

Title: Non-Double-Couple Earthquake Mechanisms at the Hengill-

Grensdalur Volcanic Complex, Southwest Iceland

Source: GEOPHYSICAL RESEARCH LETTERS

1997, Vol 24, Iss 7, pp 743-746 
Addresses:

US-GEOL-SURVEY, MS 977, 345 MIDDLEFIELD RD, MENLO-PK, CA

94025, USA

UNIV-DURHAM, DEPT GEOL SCI, DURHAM DH1-3LE, ENGLAND

62. Authors: Melbourne-T Carmichael-I Demets-C Hudnut-K

Sanchez-O Stock-J Suarez-G Webb-F

Title: The Geodetic Signature of the M8.0-October-9,-1995, Jalisco Subduction Earthquake

Source: GEOPHYSICAL RESEARCH LETTERS

1997, Vol 24, Iss 6, pp 715-718

Addresses:

CALTECH, SEISMOL LAB 252 21, PASADENA, CA 91125, USA

UNIV-CALIF-BERKELEY, DEPT GEOL \& GEOPHYS, BERKELEY, CA 94270, USA

UNIV-WISCONSIN, DEPT GEOL \& GEOPHYS, MADISON, WI 53076, USA

US-GEOL-SURVEY, PASADENA, CA 91106, USA

NATL-AUTONOMOUS-UNIV-MEXICO, INST GEOFIS, MEXICO-CITY 04510, DF, MEXICO

CALTECH, JET PROP LAB, PASADENA, CA 91109, USA

63. Authors: Foulger-GR Grant-CC Ross-A Julian-BR

Title: Industrially Induced Changes in Earth Structure at the Geysers Geothermal Area, California

Source: GEOPHYSICAL RESEARCH LETTERS 1997, Vol 24, Iss 2, pp 135-137

Addresses:

UNIV-DURHAM, DEPT GEOL SCI, SOUTH RD, DURHAM DH1-3LE, ENGLAND

US-GEOL-SURVEY, SEISMOL SECT, MENLO-PK, CA 94025, USA

64. Authors: Stute-M Deak-J Revesz-K Bohlke-JK Deseo-E Weppernig-R Schlosser-P

Title: Tritium/He-3 Dating of River Infiltration - An Example from the Danube in the Szigetkoz Area, Hungary

Source: GROUND WATER 1997, Vol 35, Iss 5, pp 905-911

Addresses:

COLUMBIA-UNIV, LAMONT DOHERTY EARTH OBSERV, PALISADES, NY 10964, USA

VITUKI, WATER RESOURCES RES CTR, H-1095 BUDAPEST, HUNGARY

US-GEOL-SURVEY, RESTON, VA 22092, USA

COLUMBIA-UNIV, DEPT EARTH \& ENVIRONM SCI, NEW-YORK, NY 10027, USA

COLUMBIA-UNIV-BARNARD-COLL, NEW-YORK, NY 10027, USA

65. Authors: Morin-RH Carleton-GB Poirier-S

Title: Fractured-Aquifer Hydrogeology from Geophysical Logs -

The Passaic Formation, New-Jersey

Source: GROUND WATER

1997, Vol 35, Iss 2, pp 328-338

Addresses:

US-GEOL-SURVEY, BOX 25046, DENVER, CO 80225, USA

US-GEOL-SURVEY, W-TRENTON, NJ 08628, USA

UNIV-QUEBEC, CHICOUTIMI, PQ G7H-2B1, CANADA 
66. Authors: Hogenboom-DL Kargel-JS Consolmagno-GJ Holden-TC

Lee-L Buyyounouski-M

Title: The Ammonia-Water System and the Chemical

Differentiation of Icy Satellites

Source: ICARUS

1997, Vol 128, Iss 1, pp 171-180

Addresses:

LAFAYETTE-COLL, DEPT PHYS, EASTON, PA 18042, USA

US-GEOL-SURVEY, FLAGSTAFF, AZ 86001, USA

VATICAN-OBSERV, V-00120 VATICAN-CITY, VATICAN

67. Authors: Finster-K Coates-JD Liesack-W Pfennig-N

Title: Desulfuromonas Thiophila Sp-Nov, a New Obligately Sulfur-

Reducing Bacterium from Anoxic Fresh-Water Sediment

Source: INTERNATIONAL JOURNAL OF SYSTEMATIC BACTERIOLOGY 1997, Vol 47, Iss 3, pp 754-758

Addresses:

AARHUS-UNIV, DEPT MICROBIAL ECOL, INST BIOL SCI, NY MUNKEGADE, DK-8000 AARHUS, DENMARK

US-GEOL-SURVEY, DIV WATER RESOURCES, RESTON, VA 22092, USA

MAX-PLANCK-INST-TERR-MIKROBIOL, D-35043 MARBURG, GERMANY

68. Authors: Hedrick-JB Sinha-SP Kosynkin-VD

Title: Loparite, a Rare-Earth Ore (Ce, $\mathrm{Na}, \mathrm{Sr}, \mathrm{Ca})(\mathrm{Ti}, \mathrm{Nb}, \mathrm{Ta}$, $\mathrm{Fe}+3) \mathrm{O}-3$

Source: JOURNAL OF ALLOYS AND COMPOUNDS

1997, Vol 250, Iss 1-2, pp 467-470

Addresses:

US-GEOL-SURVEY, 983 NATL CTR, RESTON, VA 20192, USA

SCI-RES-\&-DEV, DAYTON, OH 45459, USA

ALL-UNION-RES-INST-CHEM-TECHNOL, MOSCOW 115230, RUSSIA

69. Authors: Chen-TH Hendersonsellers-A Milly-PCD Pitman-AJ

Beljaars-ACM Polcher-J Abramopoulos-F Boone-A

Chang-S Chen-F Dai-Y Desborough-CE Dickinson-RE

Dumenil-L Ek-M Garratt-JR Gedney-N Gusev-YM Kim-J

Koster-R Kowalczyk-EA Laval-K Lean-J Lettenmaier-D

Liang-X Mahfouf-JF Mengelkamp-HT Mitchell-K

Nasonova-ON Noilhan-J Robock-A Rosenzweig-C

Schaake-J Schlosser-CA Schulz-JP Shao-Y Shmakin-AB

Verseghy-DL Wetzel-P Wood-EF Xue-Y Yang-ZL Zeng-Q

Title: Cabauw Experimental Results from the Project for Intercomparison of Land-Surface Parameterization Schemes

Source: JOURNAL OF CLIMATE 1997, Vol 10, Iss 6, pp 1194-1215

Addresses:

MACQUARIE-UNIV, CLIMAT IMPACTS CTR, SYDNEY, NSW 2109, AUSTRALIA

US-GEOL-SURVEY, PRINCETON, NJ, USA

NOAA, GEOPHYS FLUID DYNAM LAB, PRINCETON, NJ, USA

ROYAL-NETHERLANDS-METEOROL-INST, NL-3730-AE DE-BILT, NETHERLANDS

CNRS, METEOROL DYNAM LAB, PARIS, FRANCE

SCI-SYST-\&-APPLICAT-INC, NEW-YORK, NY, USA

NASA, GODDARD SPACE FLIGHT CTR, MESOSCALE DYNAM \& PRECIPITAT

BRANCH, GREENBELT, MD 20771, USA

PHILLIPS-LAB, GPAB, HANSCOM-AFB, MA, USA 
UNIV-MARYLAND, DEPT METEOROL, COLLEGE-PK, MD 20742, USA

INST-GEOG, MOSCOW, RUSSIA

LAWRENCE-LIVERMORE-NATL-LAB, LIVERMORE, CA, USA

UNIV-ARIZONA, INST ATMOSPHER PHYS, TUCSON, AZ 85721, USA

ECMWF, READING, BERKS, ENGLAND

ATMOSPHER-ENVIRONM-SERV, CLIMATE RES BRANCH, DOWNSVIEW, ON, CANADA

MAX-PLANCK-INST-METEOROL, HAMBURG, GERMANY

NATL-WEATHER-SERV, NOAA, OFF HYDROL, SILVER-SPRING, MD, USA

UNIV-READING, DEPT METEOROL, READING, BERKS, ENGLAND

CSIRO, DIV ATMOSPHER RES, ASPENDALE, VIC 3195, AUSTRALIA

RUSSIAN-ACAD-SCI, INST WATER PROBLEMS, MOSCOW 103064, RUSSIA

PRINCETON-UNIV, DEPT CIVIL ENGN \& OPERAT RES, PRINCETON, NJ

08544, USA

GKSS-FORSCHUNGSZENTRUM-GEESTHACHT-GMBH, INST ATMOSPHER PHYS,

GEESTHACHT, GERMANY

UNIV-WASHINGTON, DEPT CIVIL ENGN, SEATTLE, WA 98195, USA

METEOROL-OFF, HADLEY CTR CLIMATE PREDICT \& RES, BRACKNELL, BERKS, ENGLAND

UNIV-NEW-S-WALES, CTR ADV NUMER COMPUTAT ENGN \& SCI, SYDNEY, NSW, AUSTRALIA

CTR-OCEAN-LAND-ATMOSPHERE-STUDIES, CALVERTON, MD, USA

METEO-FRANCE, CNRM, TOULOUSE, FRANCE

NOAA, DEV DIV, NATL CTR ENVIRONM PREDICT, CAMP-SPRINGS, MD, USA

NASA, GODDARD SPACE FLIGHT CTR, HYDROL SCI BRANCH, GREENBELT, MD 20771, USA

CHINESE-ACAD-SCI, INST ATMOSPHER PHYS, BEIJING, PEOPLES-R-CHINA

70. Authors: Smith-PH Tomasko-MG Britt-D Crowe-DG Reid-R

Keller-HU Thomas-N Gliem-F Rueffer-P Sullivan-R

Greeley-R Knudsen-JM Madsen-MB Gunnlaugsson-HP

Hviid-SF Goetz-W Soderblom-LA Gaddis-L Kirk-R

Title: The Imager for Mars Pathfinder Experiment

Source: JOURNAL OF GEOPHYSICAL RESEARCH-PLANETS

1997, Vol 102, Iss E2, pp 4003-4025

Addresses:

UNIV-ARIZONA, LUNAR \& PLANETARY LAB, TUCSON, AZ 85721, USA

US-GEOL-SURVEY, FLAGSTAFF, AZ 86001, USA

TECH-UNIV-CAROLO-WILHELMINA-BRAUNSCHWEIG, D-3300 BRAUNSCHWEIG, GERMANY

ARIZONA-STATE-UNIV, DEPT GEOL, TEMPE, AZ 85287, USA

MAX-PLANCK-INST-AERON, D-3411 KATLENBURG-DUHM, GERMANY

NIELS-BOHR-INST, DK-2100 COPENHAGEN, DENMARK

71. Authors: Miller-KC Keller-GR Gridley-JM Luetgert-JH

Mooney-WD Thybo-H

Title: Crustal Structure Along the West Flank of the Cascades, Western Washington

Source: JOURNAL OF GEOPHYSICAL RESEARCH-SOLID EARTH 1997, Vol 102, Iss B8, pp 17857-17873

Addresses:

UNIV-TEXAS, DEPT GEOL SCI, EL-PASO, TX 79968, USA

US-GEOL-SURVEY, MENLO-PK, CA 94025, USA

UNIV-COPENHAGEN, INST GEOL, DK-1350 COPENHAGEN, DENMARK 
72. Authors: Forte-AM Woodward-RL

Title: Seismic-Geodynamic Constraints on 3-Dimensional Structure, Vertical Flow, and Heat-Transfer in the Mantle

Source: JOURNAL OF GEOPHYSICAL RESEARCH-SOLID EARTH 1997, Vol 102, Iss B8, pp 17981-17994

Addresses:

INST-PHYS-GLOBE, CNRS, URA 195, DEPT SISMOL, 4 PL JUSSIEU, TOUR 24, ETG 4, F-75252 PARIS 05, FRANCE

US-GEOL-SURVEY, ALBUQUERQUE SEISMOL LAB, ALBUQUERQUE, NM 87115, USA

73. Authors: Chouet-B Saccorotti-G Martini-M Dawson-P Deluca-G Milana-G Scarpa-R

Title: Source and Path Effects in the Wave-Fields of Tremor and Explosions at Stromboli-Volcano, Italy

Source: JOURNAL OF GEOPHYSICAL RESEARCH-SOLID EARTH 1997, Vol 102, Iss B7, pp 15129-15150

Addresses:

US-GEOL-SURVEY, 345 MIDDLEFIELD RD, MS 977, MENLO-PK, CA 94025, USA

UNIV-AQUILA, DIPARTIMENTO FIS, I-67010 COPPITO, ITALY

OSSERV-VESUVIANO, I-80123 NAPLES, ITALY

SERV-SISMICO-NAZL, I-00185 ROME, ITALY

UNIV-NAPLES-FEDERICO-II, DIPARTIMENTO GEOFIS \& VULCANOL, NAPLES, ITALY

74. Authors: Gerlach-TM Delgado-H Mcgee-KA Doukas-MP Venegas-JJ Cardenas-L

Title: Application of the Li-Cor CO2 Analyzer to Volcanic Plumes - A Case-Study, Volcan-Popocatepetl, Mexico, June 7-10, 1995

Source: JOURNAL OF GEOPHYSICAL RESEARCH-SOLID EARTH 1997, Vol 102, Iss B4, pp 8005-8019

Addresses:

US-GEOL-SURVEY, CASCADES VOLCANO OBSERV, 5400 MACARTHUR BLVD, VANCOUVER, WA 98661, USA

CTR-NACL-PREVENC-DESASTRES, MEXICO-CITY, DF, MEXICO

NATL-AUTONOMOUS-UNIV-MEXICO, INST GEOFIS, MEXICO-CITY 04510, DF, MEXICO

75. Authors: Bischoff-JL Fitzpatrick-JA Leon-L Arsuaga-JL Falgueres-C Bahain-JJ Bullen-T

Title: Geology and Preliminary Dating of the Hominid-Bearing Sedimentary Fill of the Sima-de-Los-Huesos Chamber, Cueva-Mayor of the Sierra-de-Atapuerca, Burgos, Spain

Source: JOURNAL OF HUMAN EVOLUTION 1997, Vol 33, Iss 2-3, pp 129-154

Addresses:

US-GEOL-SURVEY, 345 MIDDLEFIELD RD, MENLO-PK, CA 94025, USA UNIV-COMPLUTENSE-MADRID, DEPT PALEONTOL, E-28040 MADRID, SPAIN INST-PALEONTOL-HUMAINE, F-75013 PARIS, FRANCE

76. Authors: Bazuhair-AS Wood-WW

Title: Chloride Mass-Balance Method for Estimating Ground-Water

Recharge in Arid Areas - Examples from Western Saudi-

Arabia 
Source: JOURNAL OF HYDROLOGY

1996, Vol 186, Iss 1-4, pp 153-159

Addresses:

KING-ABDULAZIZ-UNIV, FAC EARTH SCI, JEDDAH 21441, SAUDI-ARABIA

US-GEOL-SURVEY, NATL CTR, RESTON, VA 22091, USA

77. Authors: Kennedy-WJ Cobban-WA

Title: Upper Campanian (Upper Cretaceous) Ammonites from the Marshalltown Formation Mount-Laurel Boundary Beds in Delaware

Source: JOURNAL OF PALEONTOLOGY 1997, Vol 71, Iss 1, pp 62-73

Addresses:

UNIV-OXFORD, UNIV MUSEUM, S PARKS RD, OXFORD OX1-3PW, ENGLAND

US-GEOL-SURVEY, DENVER, CO 80225, USA

78. Authors: Chernykh-VV Ritter-SM Wardlaw-BR

Title: Streptognathodus Isolatus New Species (Conodonta) -

Proposed Index for the Carboniferous-Permian Boundary

Source: JOURNAL OF PALEONTOLOGY

1997, Vol 71, Iss 1, pp 162-164

Addresses:

RUSSIAN-ACAD-SCI, URALIAN BRANCH, POCHTOVYI PER 7,

EKATERINBURG 620219, RUSSIA

BRIGHAM-YOUNG-UNIV, DEPT GEOL, PROVO, UT 84602, USA

US-GEOL-SURVEY, NATL CTR MS 970, RESTON, VA 22092, USA

79. Authors: Newman-J Price-LC Johnston-JH

Title: Hydrocarbon Source Potential and Maturation in Eocene

New-Zealand Vitrinite-Rich Coals - Insights from

Traditional Coal Analyses, and Rock-Eval and Biomarker

Studies

Source: JOURNAL OF PETROLEUM GEOLOGY

1997, Vol 20, Iss 2, pp 137-163

Addresses:

UNIV-CANTERBURY, DEPT GEOL, CRL LTD, PB4800, CHRISTCHURCH 1, NEW-ZEALAND

US-GEOL-SURVEY, ENERGY TEAM, DENVER, CO 80225, USA

UNIV-VICTORIA, RES SCH EARTH SCI, WELLINGTON, NEW-ZEALAND

80. Authors: Williamsstroud-SC Paul-J

Title: Initiation and Growth of Gypsum Piercement Structures in the Zechstein Basin

Source: JOURNAL OF STRUCTURAL GEOLOGY 1997, Vol 19, Iss 7, pp 897-907

Addresses:

US-GEOL-SURVEY, BOX 25046, MS 911, DENVER, CO 80225, USA

UNIV-GOTTINGEN, INST GEOL \& PALAONTOL, D-37077 GOTTINGEN, GERMANY

81. Authors: Cruz-FG Chouet-BA

Title: Long-Period Events, the Most Characteristic Seismicity Accompanying the Emplacement and Extrusion of a Lava Dome in Galeras-Volcano, Colombia, in 1991

Source: JOURNAL OF VOLCANOLOGY AND GEOTHERMAL RESEARCH 1997, Vol 77, Iss 1-4, pp 121-158 
Addresses:

INGEOMINAS, OBSERV VULCANOL \& SISMOL MANIZALES, AVE 12 OCTUBRE 15-47, MANIZALES, COLOMBIA

US-GEOL-SURVEY, MENLO-PK, CA 94025, USA

82. Authors: Fierstein-J Houghton-BF Wilson-CJN Hildreth-W

Title: Complexities of Plinian Fall Deposition at Vent - An

Example from the 1912 Novarupta Eruption (Alaska)

Source: JOURNAL OF VOLCANOLOGY AND GEOTHERMAL RESEARCH 1997, Vol 76, Iss 3-4, pp 215-227

Addresses:

INST-GEOL-\&-NUCL-SCI, TAUPO 2730, NEW-ZEALAND

US-GEOL-SURVEY, MENLO-PK, CA 94025, USA

83. Authors: Samuel-MD Goldberg-DR Shadduck-DJ Price-JI Cooch-EG

Title: Pasteurella-Multocida Serotype-1 Isolated from a Lesser

Snow Goose - Evidence of a Carrier State

Source: JOURNAL OF WILDLIFE DISEASES 1997, Vol 33, Iss 2, pp 332-335

Addresses:

US-GEOL-SURVEY, BIOL RESOUCES DIV, NATL WILDLIFE HLTH CTR, 6006 SCHROEDER RD, MADISON, WI 53711, USA

SIMON-FRASER-UNIV, DEPT BIOL SCI, BURNABY, BC V5A-1S6, CANADA

84. Authors: Callender-E Granina-L

Title: Geochemical Mass Balances of Major Elements in Lake Baikal

Source: LIMNOLOGY AND OCEANOGRAPHY 1997, Vol 42, Iss 1, pp 148-155

Addresses:

US-GEOL-SURVEY, NATL CTR 432, RESTON, VA 22092, USA

RUSSIAN-ACAD-SCI, INST LIMNOL, IRKUTSK 664033, RUSSIA

85. Authors: Lyons-WB Lent-RM Burnett-WC Chin-P Landing-WM Orem-WH Mcarthur-JM

Title: Jellyfish Lake, Palau - Regeneration of C, N, Si, and P in Anoxic Marine Lake-Sediments

Source: LIMNOLOGY AND OCEANOGRAPHY 1996, Vol 41, Iss 7, pp 1394-1403

Addresses:

UNIV-NEW-HAMPSHIRE, BIOGEOCHEM SYST CTR, INST STUDY EARTH OCEANS \& SPACE, DURHAM, NH 03824, USA

FLORIDA-STATE-UNIV, DEPT OCEANOG, TALLAHASSEE, FL 32306, USA

US-GEOL-SURVEY, RESTON, VA 22092, USA

UNIV-COLL-LONDON, DEPT GEOL SCI, LONDON, ENGLAND

86. Authors: Callender-E Granina-L

Title: Biogeochemical Phosphorus Mass-Balance for Lake Baikal,

Southeastern Siberia, Russia

Source: MARINE GEOLOGY

1997, Vol 139, Iss 1-4, pp 5-19

Addresses:

US-GEOL-SURVEY, NATL CTR 432, RESTON, VA 22092, USA

RUSSIAN-ACAD-SCI, INST LIMNOL, IRKUTSK 664003, RUSSIA 
87. Authors: Deike-RG Granina-L Callender-E Mcgee-JJ

Title: Formation of Ferric Iron Crusts in Quaternary Sediments of Lake Baikal, Russia, and Implications for Paleoclimate

Source: MARINE GEOLOGY 1997, Vol 139, Iss 1-4, pp 21-46

Addresses:

US-GEOL-SURVEY, RESTON, VA 22092, USA

RUSSIAN-ACAD-SCI, INST LIMNOL, IRKUTSK 664003, RUSSIA

88. Authors: Andreassen-K Hart-PE Mackay-M

Title: Amplitude Versus Offset Modeling of the Bottom Simulating Reflection Associated with Submarine Gas Hydrates

Source: MARINE GEOLOGY 1997, Vol 137, Iss 1-2, pp 25-40

Addresses:

UNIV-TROMSO, IBG, DEPT GEOL, N-9037 TROMSO, NORWAY

US-GEOL-SURVEY, MENLO-PK, CA 94025, USA

UNIV-HAWAII, SCH OCEAN \& EARTH SCI \& TECHNOL, HAWAII INST

GEOPHYS \& PLANETOL, HONOLULU, HI 96822, USA

89. Authors: Singer-DA Kouda-R

Title: Application of a Feedforward Neural-Network in the Search for Kuroho Deposits in the Hokuroku District, Japan

Source: MATHEMATICAL GEOLOGY 1996, Vol 28, Iss 8, pp 1017-1023

Addresses:

US-GEOL-SURVEY, 345 MIDDLEFIELD RD, MENLO-PK, CA 94025, USA

GEOL-SURVEY-JAPAN, TSUKUBA, IBARAKI 305, JAPAN

90. Authors: Haig-SM Grattotrevor-CL Mullins-TD Colwell-MA

Title: Population-Identification of Western-Hemisphere

Shorebirds Throughout the Annual Cycle

Source: MOLECULAR ECOLOGY

1997, Vol 6, Iss 5, pp 413-427

Addresses:

US-GEOL-SURVEY, FOREST \& RANGELAND ECOSYST SCI CTR, BIOL RESOURCES DIV, 3200 SW JEFFERSON WAY, CORVALLIS, OR 97331, USA

ENVIRONM-CANADA, CANADIAN WILDLIFE SERV, SASKATOON, SK S7N-0X4, CANADA

HUMBOLDT-STATE-UNIV, DEPT WILDLIFE, ARCATA, CA 95521, USA

91. Authors: Pamic-J Lanphere-M Belak-M

Title: Hercynian I-Type and S-Type Granitoids from the

Slavonian Mountains (Southern Pannonian Basin, Northern

Croatia)

Source: NEUES JAHRBUCH FUR MINERALOGIE-ABHANDLUNGEN

1996, Vol 171, Iss 2, pp 155-186

Addresses:

CROATIAN-ACAD-SCI-\&-ARTS, CROATIAN NATL IGCP COMM, ANTE

KOVACICA 5, ZAGREB 10000, CROATIA

US-GEOL-SURVEY, MENLO-PK, CA 94025, USA

INST-GEOL, ZAGREB 10000, CROATIA 
92. Authors: Vanderhilst-RD Widiyantoro-S Engdahl-ER

Title: Evidence for Deep Mantle Circulation from Global Tomography

Source: NATURE

1997, Vol 386, Iss 6625, pp 578-584

Addresses:

MIT, DEPT EARTH ATMOSPHER \& PLANETARY SCI, RM 54-514,

CAMBRIDGE, MA 02139, USA

AUSTRALIAN-NATL-UNIV, RES SCH EARTH SCI, CANBERRA, ACT 0200,

AUSTRALIA

US-GEOL-SURVEY, DENVER FED CTR, DENVER, CO 80225, USA

93. Authors: Abbott-LD Silver-EA Anderson-RS Smith-R Ingle-JC

Kling-SA Haig-D Small-E Galewsky-J Sliter-W

Title: Measurement of Tectonic Surface Uplift Rate in a Young

Collisional Mountain Belt

Source: NATURE

1997, Vol 385, Iss 6616, pp 501-507

Addresses:

UNIV-CALIF-SANTA-CRUZ, DEPT EARTH SCI, SANTA-CRUZ, CA 95064, USA

GEOINFORMAT-TECHNOL, FRESNO, CA 93710, USA

STANFORD-UNIV, DEPT GEOG \& ENVIRONM SCI, STANFORD, CA 94305, USA

UNIV-WESTERN-AUSTRALIA, DEPT GEOL \& GEOPHYS, PERTH, WA 6009, AUSTRALIA

US-GEOL-SURVEY, WESTERN GEOL MAPPING TEAM, MENLO-PK, CA 94025, USA

94. Authors: Paul-M Berkovits-D Cecil-LD Feldstein-H

Hershkowitz-A Kashiv-Y Vogt-S

Title: Environmental Sr-90 Measurements

Source: NUCLEAR INSTRUMENTS \& METHODS IN PHYSICS RESEARCH SECTION B-BEAM INTERACTIONS WITH MATERIALS AND ATOMS 1997, Vol 123, Iss 1-4, pp 394-399

Addresses:

HEBREW-UNIV-JERUSALEM, RACAH INST PHYS, IL-91904 JERUSALEM, ISRAEL

SOREQ-NRC, IL-81800 YAVNE, ISRAEL

US-GEOL-SURVEY, IDAHO-FALLS, ID 83403, USA

PURDUE-UNIV, DEPT CHEM, W-LAFAYETTE, IN 47907, USA

95. Authors: Koopmans-MP Deleeuw-JW Lewan-MD Damste-JSS

Title: Impact of Diagenesis and Catagenesis on Sulfur and

Oxygen Sequestration of Biomarkers as Revealed by

Artificial Maturation of an Immature Sedimentary-Rock

Source: ORGANIC GEOCHEMISTRY

1996, Vol 25, Iss 5-7, pp 391-426

Addresses:

NETHERLANDS-INST-SEA-RES, INST MARINE BIOGEOCHEM \& TOXICOL,

POB 59, NL-1790-AB DEN-BURG, NETHERLANDS

US-GEOL-SURVEY, DENVER FED CTR, DENVER, CO 80225, USA 
96. Authors: White-JM Ager-TA Adam-DP Leopold-EB Liu-G Jette-H Schweger-CE

Title: An 18 Million Year Record of Vegetation and ClimateChange in Northwestern Canada and Alaska - Tectonic and Global Climatic Correlates

Source: PALAEOGEOGRAPHY PALAEOCLIMATOLOGY PALAEOECOLOGY 1997, Vol 130, Iss 1-4, pp 293-306

Addresses:

GEOL-SURVEY-CANADA, 3303 33RD ST NW, CALGARY, AB T2L-2A7, CANADA

US-GEOL-SURVEY, DENVER, CO 80225, USA

US-GEOL-SURVEY, MENLO-PK, CA 94025, USA

UNIV-WASHINGTON, DEPT BOT, SEATTLE, WA 98195, USA

ACAD-SINICA, NANJING INST GEOL \& PALEONTOL, NANJING 210008, PEOPLES-R-CHINA

GEOL-SURVEY-CANADA, OTTAWA, ON K1A-0E8, CANADA

UNIV-ALBERTA, DEPT ANTHROPOL, EDMONTON, AB T6G-2H4, CANADA

97. Authors: Krouse-HR Coplen-TB

Title: Reporting of Relative Sulfur Isotope-Ratio Data (Technical Report)

Source: PURE AND APPLIED CHEMISTRY 1997, Vol 69, Iss 2, pp 293-295

Addresses:

UNIV-CALGARY, CALGARY, AB T2N-1N4, CANADA

US-GEOL-SURVEY, RESTON, VA 22092, USA

98. Authors: Lyons-PC Zodrow-EL Millay-MA Dolby-G Gillis-KS Cross-AT

Title: Coal-Ball Floras of Maritime Canada and Palynology of the Foord Seam - Geologic, Paleobotanical and Paleoecological Implications

Source: REVIEW OF PALAEOBOTANY AND PALYNOLOGY 1997, Vol 95, Iss 1-4, pp 31-50

Addresses:

US-GEOL-SURVEY, NATL CTR, MS 956, RESTON, VA 20192, USA

UNIV-COLL-CAPE-BRETON, SYDNEY, NS B1P-6L2, CANADA

OHIO-UNIV-SO-CAMPUS, DEPT ENVIRONM \& PLANT BIOL, IRONTON, OH 45638, USA

NOVA-SCOTIA-DEPT-NAT-RESOURCES, STELLARTON, NS B0K-1SO, CANADA

MICHIGAN-STATE-UNIV, DEPT GEOL SCI, E-LANSING, MI 48824, USA

99. Authors: Wagner-RH Lyons-PC

Title: A Critical Analysis of the Higher Pennsylvanian Megafloras of the Appalachian Region

Source: REVIEW OF PALAEOBOTANY AND PALYNOLOGY 1997, Vol 95, Iss 1-4, pp 255-283

Addresses:

JARDIN-BOT-CORDOBA, AVE LINNEO S-N, CORDOBA 14004, SPAIN

US-GEOL-SURVEY, RESTON, VA 20192, USA

100. Authors: Moscariello-A Costa-F

Title: The Upper Laacher See Tephra in Lake Geneva Sediments -

Paleoenvironmental and Paleoclimatological Implications 
Source: SCHWEIZERISCHE MINERALOGISCHE UND PETROGRAPHISCHE MITTEILUNGEN

1997, Vol 77, Iss 2, pp 175-185

Addresses:

UNIV-GENEVA, SECT SCI TERRE, CH-1211 GENEVA 4, SWITZERLAND

US-GEOL-SURVEY, CENT REG GEOL HAZARDS TEAM, GOLDEN, CO 80401, USA

101. Authors: Ogorman-R Johannsson-OE Schneider-CP

Title: Age and Growth of Alewives in the Changing Pelagia of Lake Ontario, 1978-1992

Source: TRANSACTIONS OF THE AMERICAN FISHERIES SOCIETY 1997, Vol 126, Iss 1, pp 112-126

Addresses:

US-GEOL-SURVEY, BIOL RESOURCES DIV, GREAT LAKES SCI CTR, LAKE

ONTARIO BIOL STN, 17 LAKE ST, OSWEGO, NY 13126, USA

ENVIRONM-CANADA, CANADA CTR INLAND WATERS, GREAT LAKES LAB

FISHERIES \& AQUAT SCI, BURLINGTON, ON L7R-4A6, CANADA

NEW-YORK-DEPT-ENVIRONM-CONSERVAT, CAPE VINCENT FISHERIES RES

STN, CAPE-VINCENT, NY 13618, USA

102. Authors: Mueller-PA Heatherington-AL Darcy-KA Wooden-JL Nutman-AP

Title: Contrasts Between Sm-Nd Whole-Rock and U-Pb Zircon Systematics in the Tobacco Root Batholith, Montana Implications for the Determination of Crustal Age Provinces

Source: TECTONOPHYSICS 1996, Vol 265, Iss 1-2, pp 169-179

Addresses:

UNIV-FLORIDA, DEPT GEOL, GAINESVILLE, FL 32611, USA

US-GEOL-SURVEY, MENLO-PK, CA 94025, USA

AUSTRALIAN-NATL-UNIV, RES SCH EARTH SCI, CANBERRA, ACT, AUSTRALIA

103. Authors: Edgington-DN Robbins-JA Colman-SM Orlandini-KA Gustin-MP Klump-JV Granina-LZ

Title: Comment on Uranium-Series Disequilibrium, Sedimentation, Diatom Frustules, and Paleoclimate Change in Lake Baikal - Reply

Source: EARTH AND PLANETARY SCIENCE LETTERS 1997, Vol 148, Iss 1-2, pp 399-404

Addresses:

UNIV-WISCONSIN, CTR GREAT LAKES STUDIES, MILWAUKEE, WI 53204, USA

NOAA, GREAT LAKES ENVIRONM RES LAB, ANN-ARBOR, MI 48105, USA

US-GEOL-SURVEY, WOODS-HOLE, MA 02543, USA

ARGONNE-NATL-LAB, DIV ENVIRONM RES, ARGONNE, IL 60439, USA

RUSSIAN-ACAD-SCI, INST LIMNOL, SIBERIAN DIV, IRKUTSK 664003, RUSSIA

104. Authors: Bargar-JR Persson-P Brown-GE

Title: Characterization of Outer-Sphere EDTA and Pb(II)EDTA

Surface Complexes on Goethite by FTIR and XAFS

Spectroscopy 
Source: ABSTRACTS OF PAPERS OF THE AMERICAN CHEMICAL SOCIETY

1997, Vol 213, Iss APR, pp 135-GEOC

Addresses:

US-GEOL-SURVEY, DIV WATER RESOURCES, MENLO-PK, CA 94025, USA

UMEA-UNIV, DEPT INORGAN CHEM, S-90187 UMEA, SWEDEN

STANFORD-UNIV, DEPT GEOL \& ENVIRONM SCI, STANFORD, CA 94305, USA

105. Authors: Lehnert-O Miller-JF Repetski-JE

Title: Paleogeographic Significance of Clavohamulus-Hintzei

Miller (Conodonta) and Other Ibexian Conodonts in an

Early Paleozoic Carbonate Platform Facies of the

Argentine Precordillera

Source: GEOLOGICAL SOCIETY OF AMERICA BULLETIN

1997, Vol 109, Iss 4, pp 429-443

Addresses:

UNIV-ERLANGEN-NURNBERG, INST GEOL \& MINERAL, SCHLOSSGARTEN 5, D-91054 ERLANGEN, GERMANY

SW-MISSOURI-STATE-UNIV, DEPT GEOG GEOL \& PLANNING,

SPRINGFIELD, MO 65804, USA

US-GEOL-SURVEY, RESTON, VA 22092, USA

106. Authors: Grachev-MA Likhoshvai-EV Kolman-SM Kuzmina-AE

Title: Studies of the Flux of Sedimentation of Diatom Algae in

Lake Baikal by Means of Automatic Traps

Source: DOKLADY AKADEMII NAUK

1996, Vol 350, Iss 1, pp 87-91

Addresses:

RUSSIAN-ACAD-SCI, INST LIMNOL, SIBERIAN DIV, IRKUTSK 664003 , RUSSIA

US-GEOL-SURVEY, WOODS-HOLE, MA 02543, USA

\section{8}

1.Authors: Baron-JS Hartman-MD Kittel-TGF Band-LE Ojima-DS

Lammers-RB

Title: Effects of Land-Cover, Water Redistribution, and

Temperature on Ecosystem Processes in the South Platte

Basin

Source: ECOLOGICAL APPLICATIONS

1998, Vol 8, Iss 4, pp 1037-1051

Addresses:

US-GEOL-SURVEY, BIOL RESOURCES DIV, MID CONTINENT ECOL SCI

CTR, FT-COLLINS, CO 80523, USA

COLORADO-STATE-UNIV, NAT RESOURCE ECOL LAB, FT-COLLINS, CO

80523, USA

NATL-CTR-ATMOSPHER-RES, BOULDER, CO 80307, USA

UNIV-TORONTO, DEPT GEOG, TORONTO, ON M5S-1A1, CANADA

UNIV-NEW-HAMPSHIRE, COMPLEX SYST RES CTR, DURHAM, NH 03824, USA

2. Authors: Fynnaikins-K Bowser-PR Honeyfield-DC Fitzsimons-JD

Ketola-HG

Title: Effect of Dietary Amprolium on Tissue Thiamin and Cayuga-

Syndrome in Atlantic Salmon 


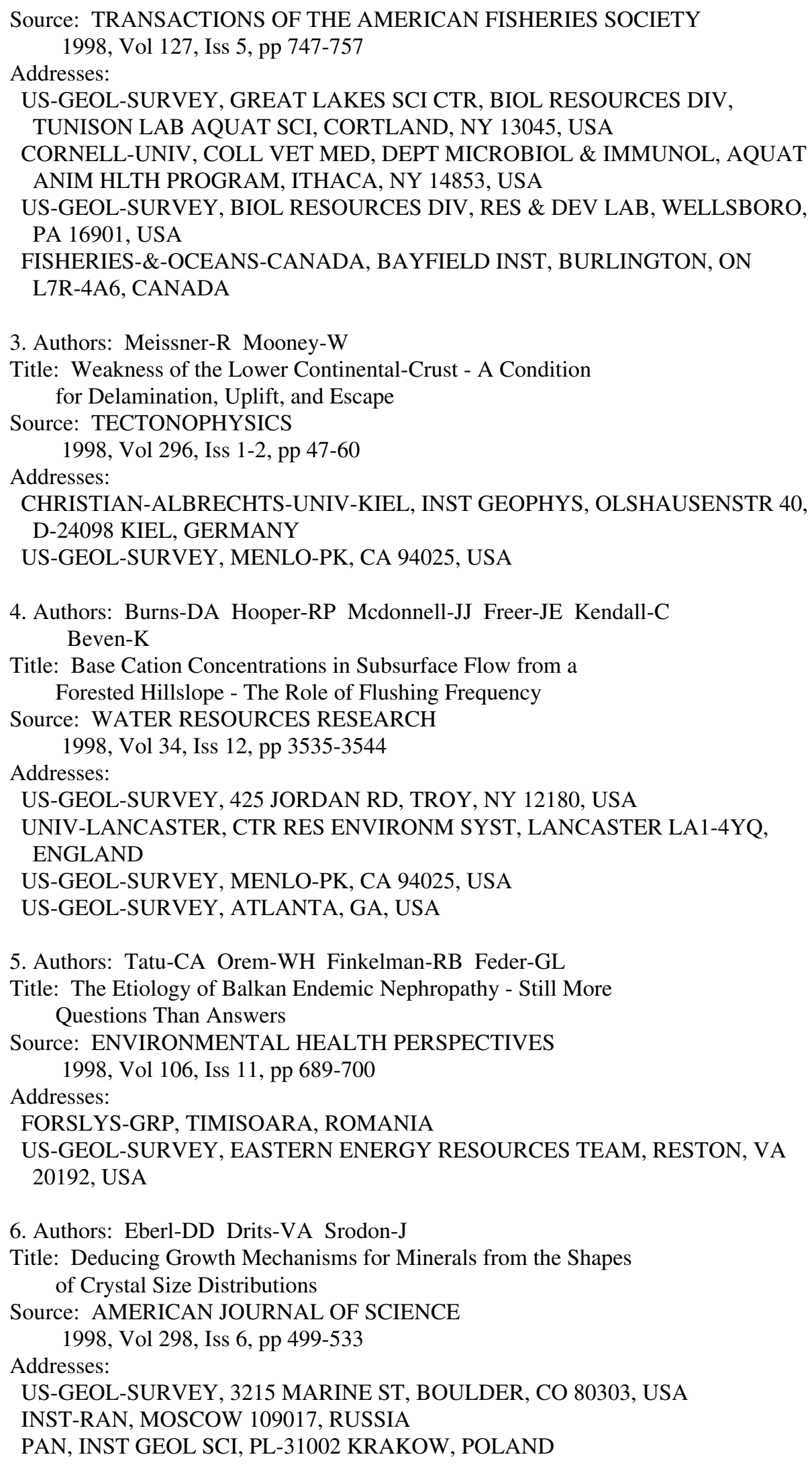


7. Authors: Nasdala-L Witzke-T Ullrich-B Brett-R

Title: Gordaite (Zn4Na(Oh)(6)(SO4)Cl-Center-Dot-6H(2)O) - 2nd

Occurrence in the Juan-de-Fuca Ridge, and New Data

Source: AMERICAN MINERALOGIST

1998, Vol 83, Iss 9-10, pp 1111-1116

Addresses:

TU-BERGAKAD-FREIBERG, INST THEORET PHYS, D-09596 FREIBERG, GERMANY

UNIV-HALLE-WITTENBERG, INST GEOSCI, D-06108 HALLE, GERMANY

TU-BERGAKAD-FREIBERG, INST CERAM ENGN, D-09596 FREIBERG, GERMANY

US-GEOL-SURVEY, RESTON, VA 20192, USA

8. Authors: Salje-EKH Buckley-A Vantendeloo-G Ishibashi-Y Nord-GL

Title: Needle Twins and Right-Angled Twins in Minerals Comparison Between Experiment and Theory

Source: AMERICAN MINERALOGIST 1998, Vol 83, Iss 7-8, pp 811-822

Addresses:

UNIV-CAMBRIDGE, DEPT EARTH SCI, DOWNING ST, CAMBRIDGE CB2-3EQ, ENGLAND

UNIV-ANTWERP, RUCA, EMAT, B-2020 ANTWERP, BELGIUM

NAGOYA-UNIV, SCH ENGN, DEPT APPL PHYS, CHIKUSA KU, NAGOYA, AICHI 46401, JAPAN

US-GEOL-SURVEY, NATL CTR 956, RESTON, VA 20192, USA

9. Authors: Hemingway-BS Bohlen-SR Hankins-WB Westrum-EF Kuskov-OL

Title: Heat-Capacity and Thermodynamic Properties for Coesite and Jadeite, Reexamination of the Quartz-Coesite Equilibrium Boundary

Source: AMERICAN MINERALOGIST 1998, Vol 83, Iss 5-6, pp 409-418

Addresses:

US-GEOL-SURVEY, 959 NATL CTR, RESTON, VA 20192, USA

US-GEOL-SURVEY, MENLO-PK, CA 94025, USA

UNIV-MICHIGAN, DEPT CHEM, ANN-ARBOR, MI 48109, USA

RUSSIAN-ACAD-SCI, VERNADSKY INST GEOCHEM \& ANALYT CHEM, MOSCOW 117975, RUSSIA

10. Authors: Sucha-V Elsass-F Eberl-DD Kuchta-L Madejova-J Gates-WP Komadel-P

Title: Hydrothermal Synthesis of Ammonium Illite

Source: AMERICAN MINERALOGIST 1998, Vol 83, Iss 1-2, pp 58-67

Addresses:

COMENIUS-UNIV, DEPT GEOL MINERAL DEPOSITS, MLYNSKA DOLINA G, BRATISLAVA 84215, SLOVAKIA

INRA, SCI SOL STN, F-78026 VERSAILLES, FRANCE

US-GEOL-SURVEY, BOULDER, CO 80303, USA

COMENIUS-UNIV, DEPT INORGAN CHEM, BRATISLAVA 84215, SLOVAKIA

SLOVAK-ACAD-SCI, INST INORGAN CHEM, BRATISLAVA 84236, SLOVAKIA

UNIV-GEORGIA, SAVANNAH RIVER ECOL LAB, DIV BIOGEOCHEM, AIKEN, SC 29802, USA 
11. Authors: Lanctot-RB Weatherhead-PJ Kempenaers-B Scribner-KT

Title: Male Traits, Mating Tactics and Reproductive Success in the Buff-Breasted Sandpiper, Tryngites Subruficollis

Source: ANIMAL BEHAVIOUR 1998, Vol 56, Iss AUG, pp 419-432

Addresses:

US-GEOL-SURVEY, BIOL RESOURCES DIV, ALASKA BIOL SCI CTR, 1011

E TUDOR RD, ANCHORAGE, AK 99503, USA

CARLETON-UNIV, DEPT BIOL, OTTAWA, ON K1S-5B6, CANADA

KONRAD-LORENZ-INST-COMPARAT-ETHOL, A-1160 VIENNA, AUSTRIA

12. Authors: Kinner-NE Harvey-RW Blakeslee-K Novarino-G

Meeker-LD

Title: Size-Selective Predation on Groundwater Bacteria by

Nanoflagellates in an Organic-Contaminated Aquifer

Source: APPLIED AND ENVIRONMENTAL MICROBIOLOGY

1998, Vol 64, Iss 2, pp 618-625

Addresses:

UNIV-NEW-HAMPSHIRE, ENVIRONM RES GRP, KINGSBURY HALL, DURHAM, NH 03824, USA

UNIV-NEW-HAMPSHIRE, DEPT MATH, DURHAM, NH 03824, USA

US-GEOL-SURVEY, DIV WATER RESOURCES, BOULDER, CO, USA

NAT-HIST-MUSEUM, PROTOZOOL SECT, LONDON SW7-5BD, ENGLAND

13. Authors: Piersma-T Gill-RE

Title: Guts Dont Fly - Small Digestive Organs in Obese Bar-

Tailed Godwits

Source: AUK

1998, Vol 115, Iss 1, pp 196-203

Addresses:

NETHERLANDS-INST-SEA-RES, POB 59, NL-1790-AB DEN-BURG, TEXEL, NETHERLANDS

UNIV-GRONINGEN, CTR ECOL \& EVOLUTIONARY STUDIES, NL-9750-AA

HAREN, NETHERLANDS

US-GEOL-SURVEY, ALASKA SCI CTR, ANCHORAGE, AK 99503, USA

14. Authors: Saccorotti-G Chouet-B Martini-M Scarpa-R

Title: Bayesian Statistics Applied to the Location of the

Source of Explosions at Stromboli Volcano, Italy

Source: BULLETIN OF THE SEISMOLOGICAL SOCIETY OF AMERICA 1998, Vol 88, Iss 5, pp 1099-1111

Addresses:

UNIV-NAPLES-FEDERICO-II, DIPARTIMENTO GEOFIS \& VULCANOL, LARGO

SAN MARCELINO 10, I-80138 NAPLES, ITALY

US-GEOL-SURVEY, MENLO-PK, CA 94025, USA

OSSERV-VESUVIANO, I-80123 NAPLES, ITALY

UNIV-AQUILA, DIPARTIMENTO FIS, I-67010 COPPITO, LAQUILA, ITALY

15. Authors: Thywissen-K Boatwright-J

Title: Using Safety Inspection Data to Estimate Shaking

Intensity for the 1994 Northridge Earthquake

Source: BULLETIN OF THE SEISMOLOGICAL SOCIETY OF AMERICA 1998, Vol 88, Iss 5, pp 1243-1253

Addresses:

GEOFORSCHUNGSZENTRUM-POTSDAM, D-14473 POTSDAM, GERMANY

US-GEOL-SURVEY, MENLO-PK, CA 94025, USA 
16. Authors: Atkinson-GM Boore-DM

Title: Evaluation of Models for Earthquake Source Spectra in Eastern North-America

Source: BULLETIN OF THE SEISMOLOGICAL SOCIETY OF AMERICA 1998, Vol 88, Iss 4, pp 917-934

Addresses:

CARLETON-UNIV, DEPT EARTH SCI, 1125 COLONEL DR, OTTAWA, ON

K1S-5B6, CANADA

US-GEOL-SURVEY, MENLO-PK, CA 94025, USA

17. Authors: Chouet-B Deluca-G Milana-G Dawson-P Martini-M Scarpa-R

Title: Shallow Velocity Structure of Stromboli Volcano, Italy,

Derived from Small-Aperture Array Measurements of Strombolian Tremor

Source: BULLETIN OF THE SEISMOLOGICAL SOCIETY OF AMERICA 1998, Vol 88, Iss 3, pp 653-666

Addresses:

US-GEOL-SURVEY, 345 MIDDLEFIELD RD, MS 977, MENLO-PK, CA

94025, USA

UNIV-AQUILA, DIPARTIMENTO FIS, I-67010 COPPITO, LAQUILA, ITALY

SERV-SISM-NAZL, ROME, ITALY

OSSERV-VESUVIANO, I-80123 NAPLES, ITALY

18. Authors: Spudich-P Guatteri-M Otsuki-K Minagawa-J

Title: Use of Fault Striations and Dislocation Models to Infer

Tectonic Shear-Stress During the 1995 Hyogo-Ken-Nanbu

(Kobe) Earthquake

Source: BULLETIN OF THE SEISMOLOGICAL SOCIETY OF AMERICA 1998, Vol 88, Iss 2, pp 413-427

Addresses:

US-GEOL-SURVEY, MS977, MENLO-PK, CA 94025, USA

STANFORD-UNIV, DEPT GEOPHYS, STANFORD, CA 94305, USA

TOHOKU-UNIV, DEPT GEOENVIRONM SCI, SENDAI, MIYAGI 980, JAPAN

DIA-CONSULTANTS-CO-LTD, TOSHIMA KU, TOKYO 171, JAPAN

19. Authors: Margaris-BN Boore-DM

Title: Determination of Delta-Sigma and Kappa(0) from Response

Spectra of Large Earthquakes in Greece

Source: BULLETIN OF THE SEISMOLOGICAL SOCIETY OF AMERICA 1998, Vol 88, Iss 1, pp 170-182

Addresses:

INST-ENGN-SEISMOL-\&-EARTHQUAKE-ENGN, ITSAK, POB 53, GR-55102 THESSALONIKI, GREECE

US-GEOL-SURVEY, MENLO-PK, CA 94025, USA

20. Authors: Milia-A Mirabile-L Torrente-MM Dvorak-JJ

Title: Volcanism Offshore of Vesuvius Volcano in Naples Bay

Source: BULLETIN OF VOLCANOLOGY 1998, Vol 59, Iss 6, pp 404-413

Addresses:

UNIV-NAPLES-FEDERICO-II, DIPARTIMENTO SCI TERRA, I-80138

NAPLES, ITALY

IST-UNIV-NAVALE, IST OCEANOL, I-80133 NAPLES, ITALY 
US-GEOL-SURVEY, CASCADES VOLCANO OBSERV, VANCOUVER, WA 98661, USA

21. Authors: Mothes-PA Hall-ML Janda-RJ

Title: The Enormous Chillos Valley Lahar - An Ash-Flow-

Generated Debris Flow from Cotopaxi Volcano, Ecuador

Source: BULLETIN OF VOLCANOLOGY

1998, Vol 59, Iss 4, pp 233-244

Addresses:

ESCUELA-POLITEC-NACL, INST GEOFIS, QUITO, ECUADOR

US-GEOL-SURVEY, VANCOUVER, WA, USA

22. Authors: Minissale-A Evans-WC Magro-G Vaselli-O

Title: Multiple Source Components in Gas Manifestations from North-Central Italy

Source: CHEMICAL GEOLOGY 1997, Vol 142, Iss 3-4, pp 175-192

Addresses:

CNR, CTR STUDIO MINEROGENESI \& GEOCHIM APPLICATA, VIA PIRA 4, I-50121 FLORENCE, ITALY

US-GEOL-SURVEY, MENLO-PK, CA 94025, USA

CNR, IST GEOCRONOL \& GEOCHIM ISOTOP, I-56100 PISA, ITALY

UNIV-FLORENCE, DIPARTIMENTO SCI TERRA, I-50121 FLORENCE, ITALY

23. Authors: Drits-VA Eberl-DD Srodon-J

Title: XRD Measurement of Mean Thickness, Thickness

Distribution and Strain for Illite and Illite-Smectite

Crystallites by the Bertaut-Warren-Averbach Technique

Source: CLAYS AND CLAY MINERALS

1998, Vol 46, Iss 1, pp 38-50

Addresses:

RAN, INST GEOL, PYZHEVSKY 7, MOSCOW 109017, RUSSIA

US-GEOL-SURVEY, BOULDER, CO 80303, USA

PAN, INST GEOL SCI, PL-31002 KRAKOW, POLAND

24. Authors: Eberl-DD Nuesch-R Sucha-V Tsipursky-S

Title: Measurement of Fundamental Illite Particle Thicknesses

by X-Ray-Diffraction Using PVP-10 Intercalation

Source: CLAYS AND CLAY MINERALS

1998, Vol 46, Iss 1, pp 89-97

Addresses:

US-GEOL-SURVEY, 3215 MARINE ST, BOULDER, CO 80303, USA

ETH-ZURICH, IGT, CLAYLAB, INST GEOTECH, CH-8092 ZURICH, SWITZERLAND

COMENIUS-UNIV, DEPT GEOL MINERAL DEPOSITS, BRATISLAVA 84215, SLOVAKIA

NANOCOR, CHICAGO, IL, USA

25. Authors: Ralph-CJ Fancy-SG Male-TM

Title: Demography of an Introduced Red-Billed Leiothrix

Record 186 of 723.

Authors: Huh-CA Pisias-NG Kelley-JM Maiti-TC Grantz-A

Title: Natural Radionuclides and Plutonium in Sediments from

the Western Arctic-Ocean - Sedimentation-Rates and

Pathways of Radionuclides 
Source: DEEP-SEA RESEARCH PART II-TOPICAL STUDIES IN OCEANOGRAPHY 1997, Vol 44, Iss 8, pp 1725+

Addresses:

ACAD-SINICA, INST EARTH SCI, TAIPEI 115, TAIWAN

OREGON-STATE-UNIV, COLL OCEAN \& ATMOSPHER SCI, CORVALLIS, OR 97331, USA

BATTELLE-PACIFIC-NW-LABS, RICHLAND, WA 99352, USA

US-GEOL-SURVEY, MENLO-PK, CA 94025, USA

26. Authors: Nose-M Iyemori-T Takeda-M Kamei-T Milling-DK

Orr-D Singer-HJ Worthington-EW Sumitomo-N

Title: Automated Detection of Pi-2 Pulsations Using Wavelet

Analysis - 1 - Method and an Application for Substorm

Monitoring

Source: EARTH PLANETS AND SPACE

1998, Vol 50, Iss 9, pp 773-783

Addresses:

KYOTO-UNIV, FAC SCI, KYOTO 6068502, JAPAN

UNIV-YORK, DEPT PHYS, YORK Y010-5DD, N-YORKSHIRE, ENGLAND

NOAA, SPACE ENVIRONM CTR, BOULDER, CO 80303, USA

US-GEOL-SURVEY, COLL OBSERV, FAIRBANKS, AK 99775, USA

KYOTO-UNIV, DISASTER PREVENT RES INST, KYOTO 6110011, JAPAN

27. Authors: Brown-ET Stallard-RF Larsen-MC Bourles-DL Raisbeck-GM Yiou-F

Title: Determination of Predevelopment Denudation Rates of an Agricultural Watershed (Cayaguas River, Puerto-Rico)

Using in-Situ-Produced Be-10 in River-Borne Quartz

Source: EARTH AND PLANETARY SCIENCE LETTERS 1998, Vol 160, Iss 3-4, pp 723-728

Addresses:

UNIV-MINNESOTA, LARGE LAKES OBSERV, DULUTH, MN 55812, USA

US-GEOL-SURVEY, BOULDER, CO 80303, USA

US-GEOL-SURVEY, GSA CTR, GUAYNABO, PR 00965, USA

IN2P3, CTR SPECTROMETRIE NUCL \& SPECTROMETRIE MASSE, CNRS, F-91405 ORSAY, FRANCE

28. Authors: Tucker-RD Bradley-DC Straeten-CAV Harris-AG

Ebert-JR Mccutcheon-SR

Title: New U-Pb Zircon Ages and the Duration and Division of Devonian Time

Source: EARTH AND PLANETARY SCIENCE LETTERS 1998, Vol 158, Iss 3-4, pp 175-186

Addresses:

WASHINGTON-UNIV, DEPT EARTH \& PLANETARY SCI, 1 BROOKINGS DR, ST-LOUIS, MO 63130, USA

US-GEOL-SURVEY, ANCHORAGE, AK 99508, USA

NORTHWESTERN-UNIV, DEPT GEOL SCI, EVANSTON, IL 60201, USA

US-GEOL-SURVEY, RESTON, VA 20192, USA

SUNY-COLL-ONEONTA, DEPT EARTH SCI, ONEONTA, NY 13820, USA

NEW-BRUNSWICK-DEPT-NAT-RESOURCES-\&-ENERGY, BATHURST, NB E2A-3Z1, CANADA

29. Authors: Jackson-M Moskowitz-B Rosenbaum-J Kissel-C

Title: Field-Dependence of AC Susceptibility in Titanomagnetites 
Source: EARTH AND PLANETARY SCIENCE LETTERS

1998, Vol 157, Iss 3-4, pp 129-139

Addresses:

UNIV-MINNESOTA, DEPT GEOL \& GEOPHYS, MINNEAPOLIS, MN 55455, USA

UNIV-MINNESOTA, INST ROCK MAGNETISM, MINNEAPOLIS, MN 55455, USA

US-GEOL-SURVEY, DENVER, CO 80225, USA

CEA, CTR FAIBLES RADIOACT, LAB MIXTE, CNRS, GIF-SUR-YVETTE,

FRANCE

30. Authors: Langtimm-CA Oshea-TJ Pradel-R Beck-CA

Title: Estimates of Annual Survival Probabilities for Adult

Florida Manatees (Trichechus-Manatus-Latirostris)

Source: ECOLOGY

1998, Vol 79, Iss 3, pp 981-997

Addresses:

US-GEOL-SURVEY, FLORIDA CARIBBEAN SCI CTR, SIRENIA PROJECT,

GAINESVILLE, FL 32601, USA

CNRS, CTR ECOL FONCT \& EVOLUT, F-34293 MONTPELLIER 5, FRANCE

31. Authors: Lambert-DD Foster-JG Frick-LR Ripley-EM Zientek-ML

Title: Geodynamics of Magmatic Cu-Ni-PGE Sulfide Deposits - New Insights from the Re-Os Isotope System

Source: ECONOMIC GEOLOGY AND THE BULLETIN OF THE SOCIETY OF ECONOMIC GEOLOGISTS 1998, Vol 93, Iss 2, pp 121-136

Addresses:

MONASH-UNIV, DEPT EARTH SCI, VICTORIAN INST EARTH \& PLANETARY

SCI, CLAYTON, VIC 3168, AUSTRALIA

INDIANA-UNIV, DEPT GEOL SCI, BLOOMINGTON, IN 47405, USA

US-GEOL-SURVEY, SPOKANE, WA 99201, USA

32. Authors: Jiang-SY Palmer-MR Slack-JF Shaw-DR

Title: Paragenesis and Chemistry of Multistage Tourmaline

Formation in the Sullivan Pb-Zn-Ag Deposit, British-

Columbia

Source: ECONOMIC GEOLOGY AND THE BULLETIN OF THE SOCIETY OF ECONOMIC GEOLOGISTS

1998, Vol 93, Iss 1, pp 47-67

Addresses:

UNIV-BRISTOL, DEPT GEOL, BRISTOL BS8-1RJ, AVON, ENGLAND

US-GEOL-SURVEY, NATL CTR, RESTON, VA 22092, USA

HYCAL-ENERGY-RES-LABS, CALGARY, AB T2E-6T6, CANADA

33. Authors: Kalkhoff-SJ Kolpin-DW Thurman-EM Ferrer-I Barcelo-D

Title: Degradation of Chloroacetanilide Herbicides - The

Prevalence of Sulfonic and Oxanilic Acid Metabolites in

Iowa Groundwaters and Surface Waters

Source: ENVIRONMENTAL SCIENCE \& TECHNOLOGY

1998, Vol 32, Iss 11, pp 1738-1740

Addresses:

US-GEOL-SURVEY, BOX 1230, IOWA-CITY, IA 52244, USA

US-GEOL-SURVEY, LAWRENCE, KS 66046, USA

CSIC, CID, DEPT ENVIRONM CHEM, ES-08034 BARCELONA, SPAIN 
34. Authors: Long-ER Macdonald-DD Cubbage-JC Ingersoll-CG

Title: Predicting the Toxicity of Sediment-Associated Trace-

Metals with Simultaneously Extracted Trace-Metal - Acid-

Volatile Sulfide Concentrations and Dry Weight-

Normalized Concentrations - A Critical Comparison

Source: ENVIRONMENTAL TOXICOLOGY AND CHEMISTRY

1998, Vol 17, Iss 5, pp 972-974

Addresses:

NOAA, ORCA, COASTAL MONITORING \& BIOEFFECTS ASSESSMENT DIV, 7600 SAND POINT WAY NE, SEATTLE, WA 98115, USA

MACDONALD-ENVIRONM-SCI-LTD, NANAIMO, BC V9X-1C6, CANADA

WASHINGTON-DEPT-ECOL, OLYMPIA, WA 98504, USA

US-GEOL-SURVEY, ENVIRONM \& CONTAMINANTS RES CTR, COLUMBIA, MO 65201, USA

35. Authors: Roman-CT Peck-JA Allen-JR King-JW Appleby-PG

Title: Accretion of a New-England (USA) Salt-Marsh in Response to Inlet Migration, Storms, and Sea-Level Rise

Source: ESTUARINE COASTAL AND SHELF SCIENCE 1997, Vol 45, Iss 6, pp 717-727

Addresses:

UNIV-RHODE-ISL, GRAD SCH OCEANOG, BIOL RESOURCES DIV, US GEOL

SURVEY, NARRAGANSETT, RI 02882, USA

US-GEOL-SURVEY, DIV BIOL RESOURCES, BOSTON, MA 02109, USA

UNIV-LIVERPOOL, DEPT APPL MATH \& THEORET PHYS, LIVERPOOL

L69-3BX, MERSEYSIDE, ENGLAND

36. Authors: Blanc-EJP Blancaletru-MC Mojon-PO

Title: Soft-Sediment Deformation Structures Interpreted as

Seismites in the Uppermost Aptian to Lowermost Albian

Transgressive Deposits of the Chihuahua Basin (Mexico)

Source: GEOLOGISCHE RUNDSCHAU

1998, Vol 86, Iss 4, pp 875-883

Addresses:

INST-DOLOMIEU, F-38031 GRENOBLE, FRANCE

US-GEOL-SURVEY, MENLO-PK, CA 94025, USA

37. Authors: Iverson-RM Schilling-SP Vallance-JW

Title: Objective Delineation of Lahar-Inundation Hazard Zones

Source: GEOLOGICAL SOCIETY OF AMERICA BULLETIN 1998, Vol 110, Iss 8, pp 972-984

Addresses:

US-GEOL-SURVEY, CASCADES VOLCANO OBSERV, 5400 MACARTHUR BLVD, VANCOUVER, WA 98661, USA

MCGILL-UNIV, DEPT CIVIL ENGN \& APPL MECH, MONTREAL, PQ

H3A-2K6, CANADA

38. Authors: Goff-F Janik-CJ Delgado-H Werner-C Counce-D

Stimac-JA Siebe-C Love-SP Williams-SN Fischer-T

Johnson-L

Title: Geochemical Surveillance of Magmatic Volatiles at

Popocatepetl Volcano, Mexico

Source: GEOLOGICAL SOCIETY OF AMERICA BULLETIN

1998, Vol 110, Iss 6, pp 695-710 
Addresses:

LOS-ALAMOS-NATL-LAB, POB 1663, LOS-ALAMOS, NM 87545, USA

US-GEOL-SURVEY, MENLO-PK, CA 94025, USA

NATL-AUTONOMOUS-UNIV-MEXICO, INST GEOFIS, MEXICO-CITY 04510, DF, MEXICO

UNIV-MANITOBA, WINNIPEG, MB R3T-2N2, CANADA

ARIZONA-STATE-UNIV, TEMPE, AZ 85287, USA

39. Authors: Grantz-A Clark-DL Phillips-RL Srivastava-SP Blome-CD Gray-LB Haga-H Mamet-BL Mcintyre-DJ Mcneil-DH Mickey-MB Mullen-MW Murchey-BI Ross-CA Stevens-CH Silberling-NJ Wall-JH Willard-DA

Title: Phanerozoic Stratigraphy of Northwind Ridge, MagneticAnomalies in the Canada Basin, and the Geometry and Timing of Rifting in the Amerasia Basin, Arctic-Ocean

Source: GEOLOGICAL SOCIETY OF AMERICA BULLETIN 1998, Vol 110, Iss 6, pp 801-820

Addresses:

UNIV-WISCONSIN, DEPT GEOL \& GEOPHYS, MADISON, WI 53706, USA

GEOL-SURVEY-CANADA, DARTMOUTH, NS B2Y-4AZ, CANADA

US-GEOL-SURVEY, DENVER, CO 80225, USA

US-GEOL-SURVEY, MENLO-PK, CA 94025, USA

MICROPALEO-CONSULTANTS-INC, ENCINITAS, CA 92094, USA

UNIV-MONTREAL, DEPT GEOL, MONTREAL, PQ H3C-3J7, CANADA

GEOL-SURVEY-CANADA, CALGARY, AB T2L-2A7, CANADA

US-GEOL-SURVEY, RESTON, VA 20192, USA

GEOBIOSTRAT, BELLINGHAM, WA 98225, USA

SAN-JOSE-STATE-UNIV, DEPT GEOL, SAN-JOSE, CA 95102, USA

40. Authors: Dunne-T Mertes-LAK Meade-RH Richey-JE Forsberg-BR

Title: Exchanges of Sediment Between the Flood-Plain and

Channel of the Amazon River in Brazil

Source: GEOLOGICAL SOCIETY OF AMERICA BULLETIN

1998, Vol 110, Iss 4, pp 450-467

Addresses:

UNIV-CALIF-SANTA-BARBARA, DONALD BREN SCH ENVIRONM SCI \& MANAGEMENT, SANTA-BARBARA, CA 93106, USA

UNIV-CALIF-SANTA-BARBARA, DEPT GEOG, SANTA-BARBARA, CA 93106, USA

UNIV-CALIF-SANTA-BARBARA, INST COMPUTAT EARTH SYST SCI,

SANTA-BARBARA, CA 93106, USA

US-GEOL-SURVEY, DENVER FED CTR, DIV WATER RESOURCES, DENVER, CO 80225, USA

UNIV-WASHINGTON, SCH OCEANOG, SEATTLE, WA 98195, USA

INST-NACL-PESQUISAS, MANAUS, AMAZONAS, BRAZIL

41. Authors: Ramo-OT Calzia-JP

Title: Nd Isotopic Composition of Cratonic Rocks in the

Southern Death-Valley Region - Evidence for a

Substantial Archean Source Component in Mojavia

Source: GEOLOGY

1998, Vol 26, Iss 10, pp 891-894

Addresses:

UNIV-HELSINKI, DEPT GEOL, POB 11, FIN-00014 HELSINKI, FINLAND

US-GEOL-SURVEY, MENLO-PK, CA 94025, USA 
42. Authors: Moore-JC Klaus-A Bangs-NL Bekins-B Bucker-CJ

Bruckmann-W Erickson-SN Hansen-O Horton-T

Ireland-P Major-CO Moore-GF Peacock-S Saito-S

Screaton-EJ Shimeld-JW Stauffer-PH Taymaz-T

Teas-PA Tokunaga-T

Title: Consolidation Patterns During Initiation and Evolution of a Plate-Boundary Decollement Zone - Northern Barbados Accretionary Prism

Source: GEOLOGY

1998, Vol 26, Iss 9, pp 811-814

Addresses:

UNIV-CALIF-SANTA-CRUZ, DEPT EARTH SCI, SANTA-CRUZ, CA 95064, USA

TEXAS-A\&M-UNIV, OCEAN DRILLING PROGRAM, COLLEGE-STN, TX 77845, USA

UNIV-TEXAS, INST GEOPHYS, AUSTIN, TX 78759, USA

US-GEOL-SURVEY, MENLO-PK, CA 94025, USA

NIEDERSAECHS-LANDESAMT-BODENFORSCH-GEOWISSENSCH-G, D-30631

HANNOVER, GERMANY

CHRISTIAN-ALBRECHTS-UNIV-KIEL, GEOMAR, D-24148 KIEL, GERMANY

UNIV-UTAH, DEPT GEOL \& GEOPHYS, SALT-LAKE-CITY, UT 84112, USA

ANADRILL-SCHLUMBERGER, YOUNGSVILLE, LA, USA

LAMONT-DOHERTY-EARTH-OBSERV, PALISADES, NY 10964, USA

UNIV-BIRMINGHAM, SCH EARTH SCI, BIRMINGHAM B15-2TT,

W-MIDLANDS, ENGLAND

UNIV-TOKYO, OCEAN RES INST, TOKYO 164, JAPAN

UNIV-FLORIDA, DEPT GEOL, GAINESVILLE, FL 32611, USA

UNIV-TOKYO, DEPT GEOSYST ENGN, TOKYO 113, JAPAN

ISTANBUL-TECH-UNIV, JEOFIZ MUHENDISLIGI BOLUMU, TR-80626

ISTANBUL, TURKEY

UNIV-HAWAII, SCH OCEAN \& EARTH SCI \& TECHNOL, HONOLULU, HI

96822, USA

DALHOUSIE-UNIV, DEPT EARTH SCI, HALIFAX, NS B3H-3J5, CANADA

43. Authors: Slack-JF Turner-RJW Ware-PLG

Title: Boron-Rich Mud Volcanos of the Black-Sea Region - Modern Analogs to Ancient Sea-Floor Tourmalinites Associated with Sullivan-Type $\mathrm{Pb}-\mathrm{Zn}$ Deposits

Source: GEOLOGY 1998, Vol 26, Iss 5, pp 439-442

Addresses:

US-GEOL-SURVEY, NATL CTR, MS 954, RESTON, VA 20192, USA

GEOL-SURVEY-CANADA, VANCOUVER, BC V6B-5J3, CANADA

UNOCAL-CORP, SUGAR-LAND, TX 77478, USA

44. Authors: Parsons-T Trehu-AM Luetgert-JH Miller-K Kilbride-F

Wells-RE Fisher-MA Flueh-E Tenbrink-US

Christensen-NI

Title: A New View into the Cascadia Subduction Zone and Volcanic Arc - Implications for Earthquake Hazards Along the Washington Margin

Source: GEOLOGY 1998, Vol 26, Iss 3, pp 199-202 
Addresses:

US-GEOL-SURVEY, MS 999, 345 MIDDLEFIELD RD, MENLO-PK, CA

94025, USA

OREGON-STATE-UNIV, COLL OCEAN \& ATMOSPHER SCI, CORVALLIS, OR

97331, USA

UNIV-TEXAS, DEPT GEOL SCI, EL-PASO, TX 79968, USA

GEOMAR-RES-CTR-MARINE-SCI, D-24148 KIEL, GERMANY

US-GEOL-SURVEY, WOODS-HOLE, MA 02543, USA

UNIV-WISCONSIN, DEPT GEOL \& GEOPHYS, MADISON, WI 53706, USA

45. Authors: Wilson-CJN Hildreth-W

Title: Hybrid Fall Deposits in the Bishop-Tuff, California - A

Novel Pyroclastic Depositional Mechanism

Source: GEOLOGY

1998, Vol 26, Iss 1, pp 7-10

Addresses:

INST-GEOL-\&-NUCL-SCI, PRIVATE BAG 2000, TAUPO 2730, NEW-ZEALAND

US-GEOL-SURVEY, MENLO-PK, CA 94025, USA

46. Authors: Miller-AD Julian-BR Foulger-GR

Title: 3-Dimensional Seismic Structure and Moment Tensors of

Non-Double-Couple Earthquakes at the Hengill-Grensdalur

Volcanic Complex, Iceland

Source: GEOPHYSICAL JOURNAL INTERNATIONAL

1998, Vol 133, Iss 2, pp 309-325

Addresses:

UNIV-DURHAM, DEPT GEOL SCI, SCI LABS, SOUTH RD, DURHAM

DH1-3LE, ENGLAND

US-GEOL-SURVEY, MENLO-PK, CA 94025, USA

47. Authors: Julian-BR Pitt-AM Foulger-GR

Title: Seismic Image of a CO2 Reservoir Beneath a Seismically

Active Volcano

Source: GEOPHYSICAL JOURNAL INTERNATIONAL 1998, Vol 133, Iss 1, pp F7-F10

Addresses:

US-GEOL-SURVEY, 345 MIDDLEFIELD RD, MS977, MENLO-PK, CA 94025, USA

UNIV-DURHAM, DEPT GEOL SCI, DURHAM DH1-3LE, ENGLAND

48. Authors: White-RA Miller-AD Lynch-L Power-J

Title: Observations of Hybrid Seismic Events at Soufriere Hills

Volcano, Montserrat - July 1995 to September 1996

Source: GEOPHYSICAL RESEARCH LETTERS

1998, Vol 25, Iss 19, pp 3657-3660

Addresses:

US-GEOL-SURVEY, MAILSTOP 910, 345 MIDDLEFIELD RD, MENLO-PK, CA

94025, USA

BRITISH-GEOL-SURVEY, EDINBURGH EH9-3LA, MIDLOTHIAN, SCOTLAND

UNIV-W-INDIES, SEISM RES UNIT, ST-AUGUSTINE, TRINID-\&-TOBAGO

US-GEOL-SURVEY, ANCHORAGE, AK 99508, USA

49. Authors: Aspinall-WP Miller-AD Lynch-LL Latchman-JL

Stewart-RC White-RA Power-JA

Title: Soufriere Hills Eruption, Montserrat, 1995-1997 -

Volcanic Earthquake Locations and Fault Plane Solutions 
Source: GEOPHYSICAL RESEARCH LETTERS

1998, Vol 25, Iss 18, pp 3397-3400

Addresses:

ASPINALL-\&-ASSOC, 5 WOODSIDE CLOSE, BEACONSFIELD HP9-1JQ, BUCKS, ENGLAND

GEOWALKS, EDINBURGH EH9-1JJ, MIDLOTHIAN, SCOTLAND

AWE-BLACKNEST, BRIMPTON RG7-4RS, BUCKS, ENGLAND

UNIV-W-INDIES, SEISM RES UNIT, ST-AUGUSTINE, TRINID-\&-TOBAGO

US-GEOL-SURVEY, VOLCANO CRISIS ASSISTANCE TEAM, MENLO-PK, CA 94025, USA

US-GEOL-SURVEY, AVO, ANCHORAGE, AK 99508, USA

50. Authors: Miller-AD Stewart-RC White-RA Luckett-R Baptie-BJ

Aspinall-WP Latchman-JL Lynch-LL Voight-B

Title: Seismicity Associated with Dome Growth and Collapse at the Soufriere Hills Volcano, Montserrat

Source: GEOPHYSICAL RESEARCH LETTERS 1998, Vol 25, Iss 18, pp 3401-3404

Addresses:

BRITISH-GEOL-SURVEY, W MAINS RD, EDINBURGH EH9-3LA, MIDLOTHIAN, SCOTLAND

AWE-BLACKNEST, BRIMPTON RG7-4RS, BERKS, ENGLAND

US-GEOL-SURVEY, MENLO-PK, CA 94025, USA

ASPINALL-\&-ASSOCIATES, BEACONSFIELD HP9-1JQ, BUCKS, ENGLAND

UNIV-W-INDIES, SEISM RES UNIT, ST-AUGUSTINE, TRINID-\&-TOBAGO

PENN-STATE-UNIV, UNIVERSITY-PK, PA 16802, USA

51. Authors: Cole-PD Calder-ES Druitt-TH Hoblitt-R Robertson-R Sparks-RSJ Young-SR

Title: Pyroclastic Flows Generated by Gravitational-Instability of the 1996-97 Lava Dome of Soufriere Hills Volcano, Montserrat

Source: GEOPHYSICAL RESEARCH LETTERS 1998, Vol 25, Iss 18, pp 3425-3428

Addresses:

UNIV-LUTON, DEPT GEOL, LUTON LU1-3JU, BEDS, ENGLAND

UNIV-BRISTOL, BRISTOL, AVON, ENGLAND

UNIV-CLERMONT-FERRAND, CLERMONT-FERRAND, FRANCE

US-GEOL-SURVEY, CVO, VANCOUVER, WA 98661, USA

UNIV-W-INDIES, SRU, PORT-OF-SPAIN, TRINID-\&-TOBAGO

BRITISH-GEOL-SURVEY, EDINBURGH, MIDLOTHIAN, SCOTLAND

52. Authors: Voros-Z Kovacs-P Juhasz-A Kormendi-A Green-AW

Title: Scaling Laws from Geomagnetic Time-Series

Source: GEOPHYSICAL RESEARCH LETTERS

1998, Vol 25, Iss 14, pp 2621-2624

Addresses:

GEOPHYS-INST-SAS, HURBANOVO 94701, SLOVAKIA

US-GEOL-SURVEY, DENVER, CO 80225, USA

LORAND-EOTVOS-UNIV, DEPT GEOPHYS, H-1083 BUDAPEST, HUNGARY

EOTVOS-LORAND-GEOPHYS-INST, H-1440 BUDAPEST, HUNGARY

53. Authors: Villasenor-A Benz-HM Filippi-L Deluca-G Scarpa-R

Patane-G Vinciguerra-S

Title: 3-Dimensional P-Wave Velocity Structure of Mt-Etna, Italy 
Source: GEOPHYSICAL RESEARCH LETTERS

1998, Vol 25, Iss 11, pp 1975-1978

Addresses:

US-GEOL-SURVEY, DENVER, CO 80225, USA

UNIV-AQUILA, DIPARTIMENTO FIS, I-67010 COPPITO, ITALY

UNIV-CATANIA, IST GEOL \& GEOFIS, I-95129 CATANIA, ITALY

54. Authors: Arnadottir-T Sigmundsson-F Delaney-PT

Title: Sources of Crustal Deformation Associated with the

Krafla, Iceland, Eruption of September 1984

Source: GEOPHYSICAL RESEARCH LETTERS

1998, Vol 25, Iss 7, pp 1043-1046

Addresses:

PRINCETON-UNIV, DEPT GEOSCI, PRINCETON, NJ 08544, USA

UNIV-ICELAND, NORD VOLCANOL INST, IS-108 REYKJAVIK, ICELAND

US-GEOL-SURVEY, FLAGSTAFF, AZ 86001, USA

55. Authors: Johnston-CT Cook-PG Frape-SK Plummer-LN

Busenberg-E Blackport-RJ

Title: Ground-Water Age and Nitrate Distribution Within a

Glacial Aquifer Beneath a Thick Unsaturated Zone

Source: GROUND WATER

1998, Vol 36, Iss 1, pp 171-180

Addresses:

UNIV-WATERLOO, DEPT EARTH SCI, WATERLOO CTR GROUNDWATER RES, WATERLOO, ON N2L-3G1, CANADA

CSIRO, DIV WATER RESOURCES, GLEN-OSMOND, SA 5064, AUSTRALIA

US-GEOL-SURVEY, DIV WATER RESOURCES, NATL CTR 432, RESTON, VA 22092, USA

56. Authors: Martinelli-TL Hansel-HC Shively-RS

Title: Growth and Physiological-Responses to Surgical and

Gastric Radio Transmitter Implantation Techniques in

Subyearling Chinook Salmon (Oncorhynchus-Tshawytscha)

Source: HYDROBIOLOGIA

1998, Vol 372, pp 79-87

Addresses:

US-GEOL-SURVEY, BIOL RESOURCES DIV, COLUMBIA RIVER RES LAB, 5501A COOK UNDERWOOD RD, COOK, WA 98605, AUSTRALIA

57. Authors: Greeley-R Sullivan-R Klemaszewski-J Homan-K

Head-JW Pappalardo-RT Veverka-J Clark-BE

Johnson-TV Klaasen-KP Belton-M Moore-J Asphaug-E

Carr-MH Neukum-G Denk-T Chapman-CR Pilcher-CB

Geissler-PE Greenberg-R Tufts-R

Title: Europa - Initial Galileo Geological Observations

Source: ICARUS

1998, Vol 135, Iss 1, pp 4-24

Addresses:

ARIZONA-STATE-UNIV, DEPT GEOL, BOX 871404, TEMPE, AZ 85287, USA

BROWN-UNIV, DEPT GEOL SCI, PROVIDENCE, RI 02912, USA

CORNELL-UNIV, ITHACA, NY 14853, USA

CALTECH, JET PROP LAB, PASADENA, CA 91109, USA

NATL-OPT-ASTRON-OBSERV, TUCSON, AZ 85726, USA 
NASA, AMES RES CTR, MOFFETT-FIELD, CA 94035, USA

US-GEOL-SURVEY, BRANCH ASTROGEOL STUDIES, MENLO-PK, CA 94025, USA

DLR, INST PLANETARY EXPLORAT, D-12489 BERLIN, GERMANY

UNIV-ARIZONA, LUNAR \& PLANETARY LAB, TUCSON, AZ 85271, USA

SW-RES-INST, BOULDER, CO 80302, USA

NASA, WASHINGTON, DC 20546, USA

58. Authors: Carr-MH Mcewen-AS Howard-KA Chuang-FC Thomas-P

Schuster-P Oberst-J Neukum-G Schubert-G

Title: Mountains and Calderas on Io - Possible Implications for

Lithosphere Structure and Magma Generation

Source: ICARUS

1998, Vol 135, Iss 1, pp 146-165

Addresses:

US-GEOL-SURVEY, 345 MIDDLEFIELD RD, MENLO-PK, CA 94025, USA

UNIV-ARIZONA, LUNAR \& PLANETARY LAB, TUCSON, AZ 85721, USA

US-GEOL-SURVEY, MENLO-PK, CA 94025, USA

ARIZONA-STATE-UNIV, DEPT GEOL, TEMPE, AZ 85287, USA

CORNELL-UNIV, ITHACA, NY 14853, USA

DLR, INST PLANETENERKUNDUNG, D-12489 BERLIN, GERMANY

UNIV-CALIF-LOS-ANGELES, DEPT EARTH \& SPACE SCI, LOS-ANGELES,

CA 90095, USA

59. Authors: Pappalardo-RT Head-JW Collins-GC Kirk-RL Neukum-G

Oberst-J Giese-B Greeley-R Chapman-CR

Helfenstein-P Moore-JM Mcewen-A Tufts-BR Senske-DA

Breneman-HH Klaasen-K

Title: Grooved Terrain on Ganymede - First Results from Galileo

High-Resolution Imaging

Source: ICARUS

1998, Vol 135, Iss 1, pp 276-302

Addresses:

BROWN-UNIV, DEPT GEOL SCI, PROVIDENCE, RI 02912, USA

US-GEOL-SURVEY, FLAGSTAFF, AZ 86001, USA

DLR, INST PLANETARY EXPLORAT, D-12489 BERLIN, GERMANY

SW-RES-INST, BOULDER, CO 80302, USA

CORNELL-UNIV, LAB PLANETARY SCI, ITHACA, NY 14853, USA

NASA, AMES RES CTR, MOFFETT-FIELD, CA 94035, USA

UNIV-ARIZONA, LUNAR \& PLANETARY LAB, TUCSON, AZ 85721, USA

CALTECH, JET PROP LAB, PASADENA, CA 91109, USA

60. Authors: Erard-S Calvin-W

Title: New Composite Spectra of Mars, 0.4-5.7 Mu-M

Source: ICARUS

1997, Vol 130, Iss 2, pp 449-460

Addresses:

UNIV-PARIS-11, CNRS, INST ASTROPHYS SPATIALE, BATIMENT 121,

F-91405 ORSAY, FRANCE

US-GEOL-SURVEY, FLAGSTAFF, AZ 86001, USA

61. Authors: Thome-K Arai-K Hook-S Kieffer-H Lang-H

Matsunaga-T Ono-A Palluconi-F Sakuma-H Slater-P

Takashima-T Tonooka-H Tsuchida-S Welch-RM

Zalewski-E 
Title: Aster Preflight and Inflight Calibration and the Validation of Level-2 Products

Source: IEEE TRANSACTIONS ON GEOSCIENCE AND REMOTE SENSING 1998, Vol 36, Iss 4, pp 1161-1172

Addresses:

UNIV-ARIZONA, CTR OPT SCI, TUCSON, AZ 85721, USA

SAGA-UNIV, FAC SCI ENGN, SAGA 840, JAPAN

JET-PROP-LAB, PASADENA, CA 91109, USA

US-GEOL-SURVEY, FLAGSTAFF, AZ 86001, USA

GEOL-SURVEY-JAPAN, TSUKUBA, IBARAKI 305, JAPAN

NATL-RES-LAB-METROL, TSUKUBA, IBARAKI 305, JAPAN

NASDA, EARTH OBSERVAT RES CTR, TOKYO 106, JAPAN

IBARAKI-UNIV, HITACHI, IBARAKI 316, JAPAN

UNIV-ALABAMA, HUNTSVILLE, AL 35807, USA

62. Authors: Barker-CE Bone-Y Lewan-MD

Title: Fluid Inclusion and Vitrinite Reflectance Geothermometry Compared to Heat-Flow Models of Maximum Paleotemperature Next to Dikes, Western Onshore Gippsland Basin, Australia

Source: INTERNATIONAL JOURNAL OF COAL GEOLOGY 1998, Vol 37, Iss 1-2, pp 73-111

Addresses:

US-GEOL-SURVEY, BOX 25046, DENVER, CO 80225, USA

UNIV-ADELAIDE, DEPT GEOL, ADELAIDE, SA 5005, AUSTRALIA

63. Authors: Schwartz-DP Pantosti-D Okumura-K Powers-TJ Hamilton-JC

Title: Paleoseismic Investigations in the Santa-Cruz Mountains, California - Implications for Recurrence of LargeMagnitude Earthquakes on the San-Andreas Fault

Source: JOURNAL OF GEOPHYSICAL RESEARCH-SOLID EARTH 1998, Vol 103, Iss B8, pp 17985-18001

Addresses:

US-GEOL-SURVEY, WESTERN EARTHQUAKE HAZARD TEAM, 345

MIDDLEFIELD RD, MENLO-PK, CA 94025, USA

HIROSHIMA-UNIV, DEPT GEOG, HIROSHIMA 7398522, JAPAN

IST-NAZL-GEOFIS, I-00143 ROME, ITALY

64. Authors: Alt-JC Teagle-DAH Brewer-T Shanks-WC Halliday-A

Title: Alteration and Mineralization of an Oceanic Fore-Arc and the Ophiolite-Ocean Crust Analogy

Source: JOURNAL OF GEOPHYSICAL RESEARCH-SOLID EARTH 1998, Vol 103, Iss B6, pp 12365-12380

Addresses:

UNIV-MICHIGAN, DEPT GEOL SCI, 2534 CC LITTLE BLDG, ANN-ARBOR, MI 48109, USA

UNIV-LEICESTER, DEPT GEOL, LEICESTER LE1-7RH, LEICS, ENGLAND

US-GEOL-SURVEY, DENVER FED CTR, DENVER, CO, USA

65. Authors: Nakano-M Kumagai-H Kumazawa-M Yamaoka-K Chouet-BA

Title: The Excitation and Characteristic Frequency of the Long-

Period Volcanic Event - An Approach Based on an

Inhomogeneous Autoregressive Model of a Linear Dynamic

System 
Source: JOURNAL OF GEOPHYSICAL RESEARCH-SOLID EARTH 1998, Vol 103, Iss B5, pp 10031-10046

Addresses:

NAGOYA-UNIV, DEPT EARTH \& PLANETARY SCI, NAGOYA, AICHI 4648602, JAPAN

US-GEOL-SURVEY, MENLO-PK, CA 94025, USA

NAGOYA-UNIV, RES CTR SEISMOL \& VOLCANOL, NAGOYA, AICHI 4648602, JAPAN

TONO-GEOSCI-CTR, GIFU 5095102, JAPAN

66. Authors: Ohminato-T Chouet-BA Dawson-P Kedar-S

Title: Wave-Form Inversion of Very Long-Period Impulsive Signals Associated with Magmatic Injection Beneath Kilauea Volcano, Hawaii

Source: JOURNAL OF GEOPHYSICAL RESEARCH-SOLID EARTH 1998, Vol 103, Iss B10, pp 23839-23862

Addresses:

GEOL-SURVEY-JAPAN, TSUKUBA, IBARAKI 305, JAPAN

US-GEOL-SURVEY, MENLO-PK, CA 94025, USA

67. Authors: Nostro-C Stein-RS Cocco-M Belardinelli-ME Marzocchi-W

Title: 2-Way Coupling Between Vesuvius Eruptions and Southern Apennine Earthquakes, Italy, by Elastic Stress Transfer

Source: JOURNAL OF GEOPHYSICAL RESEARCH-SOLID EARTH 1998, Vol 103, Iss B10, pp 24487-24504

Addresses:

IST-NAZL-GEOFIS, VIA VIGNA MURATA 605, I-00143 ROME, ITALY

US-GEOL-SURVEY, MENLO-PK, CA 94025, USA

UNIV-BOLOGNA, DIPARTIMENTO FIS, SETTORE GEOFIS, I-40127

BOLOGNA, ITALY

68. Authors: Toda-S Stein-RS Reasenberg-PA Dieterich-JH Yoshida-A

Title: Stress Transferred by the $1995 \mathrm{M}-\mathrm{W}=6.9-\mathrm{Obe}$, Japan, Shock - Effect on Aftershocks and Future Earthquake Probabilities

Source: JOURNAL OF GEOPHYSICAL RESEARCH-SOLID EARTH 1998, Vol 103, Iss B10, pp 24543-24565

Addresses:

CENT-RES-INST-ELECT-POWER-IND, 1646 ABIKO, CHIBA 27011, JAPAN

US-GEOL-SURVEY, MENLO-PK, CA 94025, USA

JAPAN-METEOROL-AGCY, CHIYODA KU, TOKYO 100, JAPAN

69. Authors: Thatcher-W England-PC

Title: Ductile Shear Zones Beneath Strike-Slip Faults Implications for the Thermomechanics of the San-Andreas Fault Zone

Source: JOURNAL OF GEOPHYSICAL RESEARCH-SOLID EARTH 1998, Vol 103, Iss B1, pp 891-905

Addresses:

US-GEOL-SURVEY, 345 MIDDLEFIELD RD, MS 977, MENLO-PK, CA 94025, USA

UNIV-OXFORD, DEPT EARTH SCI, OXFORD OX1-3PR, ENGLAND 
70. Authors: Norman-JM Kucharik-CJ Gower-ST Baldocchi-DD

Crill-PM Rayment-M Savage-K Striegl-RG

Title: A Comparison of 6 Methods for Measuring Soil-Surface Carbon-Dioxide Fluxes

Source: JOURNAL OF GEOPHYSICAL RESEARCH-ATMOSPHERES 1997, Vol 102, Iss D24, pp 28771-28777

Addresses:

UNIV-WISCONSIN, DEPT SOIL SCI, 1525 OBSERV DR, MADISON, WI

53706, USA

NOAA, ARL, ATMOSPHER TURBULENCE \& DIFFUS DIV, OAK-RIDGE, TN 37831, USA

UNIV-NEW-HAMPSHIRE, EOS, MORSE, DURHAM, NH 03824, USA

UNIV-WISCONSIN, DEPT FORESTRY, MADISON, WI 53706, USA

UNIV-EDINBURGH, INST ECOL \& RESOURCE MANAGEMENT, EDINBURGH

EH9-3JU, MIDLOTHIAN, SCOTLAND

MCGILL-UNIV, DEPT GEOG, QUEBEC-CITY, PQ H3A-2K6, CANADA

US-GEOL-SURVEY, DENVER, CO 80225, USA

71. Authors: Harden-JW Oneill-KP Trumbore-SE Veldhuis-H

Stocks-BJ

Title: Moss and Soil Contributions to the Annual Net Carbon

Flux of a Maturing Boreal Forest

Source: JOURNAL OF GEOPHYSICAL RESEARCH-ATMOSPHERES

1997, Vol 102, Iss D24, pp 28805-28816

Addresses:

US-GEOL-SURVEY, 345 MIDDLEFIELD RD, MENLO-PK, CA 94025, USA

FORESTRY-CANADA, SAULT-ST-MARIE, ON P6A-5M7, CANADA

UNIV-CALIF-IRVINE, IRVINE, CA 92717, USA

AGR-CANADA, WINNIPEG, MB R3T-2M9, CANADA

72. Authors: Lavigne-MB Ryan-MG Anderson-DE Baldocchi-DD

Crill-PM Fitzjarrald-DR Goulden-ML Gower-ST

Massheder-JM Mccaughey-JH Rayment-M Striegl-RG

Title: Comparing Nocturnal Eddy Covariance Measurements to

Estimates of Ecosystem Respiration Made by Scaling

Chamber Measurements at 6 Coniferous Boreal Sites

Source: JOURNAL OF GEOPHYSICAL RESEARCH-ATMOSPHERES

1997, Vol 102, Iss D24, pp 28977-28985

Addresses:

CANADIAN-FOREST-SERV-ATLANTIC, POB 4000, FREDERICTON, NB

E3B-5P7, CANADA

US-GEOL-SURVEY, DENVER, CO 80225, USA

NOAA, ARL, ATMOSPHER TURBULENCE \& DIFFUS DIV, OAK-RIDGE, TN 37831, USA

UNIV-NEW-HAMPSHIRE, DURHAM, NH 03824, USA

SUNY-ALBANY, ATMOSPHER SCI RES CTR, ALBANY, NY 12203, USA

HARVARD-UNIV, ENGN SCI LAB, CAMBRIDGE, MA 02138, USA

UNIV-WISCONSIN, DEPT FORESTRY, MADISON, WI 53706, USA

UNIV-EDINBURGH, INST ECOL \& RESOURCE MANAGEMENT, EDINBURGH

EH8-9YL, MIDLOTHIAN, SCOTLAND

QUEENS-UNIV, DEPT GEOG, KINGSTON, ON K7L-3N6, CANADA

US-FOREST-SERV, FT-COLLINS, CO 80526, USA 
73. Authors: Hoff-MH Pronin-NM Baldanova-DR

Title: Parasites of Lake Herring (Coregonus-Artedi) from Lake Superior, with Special Reference to Use of Parasites as Markers of Stock Structure

Source: JOURNAL OF GREAT LAKES RESEARCH 1997, Vol 23, Iss 4, pp 458-467

Addresses:

US-GEOL-SURVEY, GREAT LAKES SCI CTR, LAKE SUPER BIOL STN, 2800 LAKE SHORE DR E, ASHLAND, WI, USA

RUSSIAN-ACAD-SCI, BURYAT INST BIOL, ULAN-UDE, RUSSIA

74. Authors: Henny-CJ Galushin-VM Dudin-PI Khrustov-AV Mischenko-AL Moseikin-VN Sarychev-VS Turchin-VG

Title: Organochlorine Pesticides, PCB and Mercury in Hawk, Falcon, Eagle and Owl Eggs from the Lipetsk, Voronezh, Novgorod and Saratov Regions, Russia, 1992-1993

Source: JOURNAL OF RAPTOR RESEARCH 1998, Vol 32, Iss 2, pp 143-150

Addresses:

US-GEOL-SURVEY, FOREST \& RANGELAND ECOSYST SCI CTR, 3080 SE

CLEARWATER DR, CORVALLIS, OR 97333, USA

RUSSIAN-BIRD-CONSERVAT-UNION, MOSCOW 129278, RUSSIA

75. Authors: Brown-SJA Wilson-CJN Cole-JW Wooden-J

Title: The Whakamaru Group Ignimbrites, Taupo Volcanic Zone, New-Zealand - Evidence for Reverse Tapping of a Zoned Silicic Magmatic System

Source: JOURNAL OF VOLCANOLOGY AND GEOTHERMAL RESEARCH 1998, Vol 84, Iss 1-2, pp 1-37

Addresses:

UNIV-CANTERBURY, DEPT GEOL SCI, PRIVATE BAG 4800, CHRISTCHURCH 1, NEW-ZEALAND

UNIV-WESTERN-AUSTRALIA, DEPT GEOL \& GEOPHYS, PERTH, WA 6907, AUSTRALIA

INST-GEOL-\&-NUCL-SCI, TAUPO, NEW-ZEALAND

US-GEOL-SURVEY, BRANCH ISOTOPE GEOL, MENLO-PK, CA 94025, USA

76. Authors: Rolandi-G Petrosino-P Mcgeehin-J

Title: The Interplinian Activity at Somma-Vesuvius in the Last 3500 Years

Source: JOURNAL OF VOLCANOLOGY AND GEOTHERMAL RESEARCH 1998, Vol 82, Iss 1-4, pp 19-52

Addresses:

UNIV-NAPLES, DIPARTIMENTO GEOFIS \& VULCANOL, LARGO S MARCELLINO 10, I-80138 NAPLES, ITALY

US-GEOL-SURVEY, RESTON, VA 22092, USA

77. Authors: Ayuso-RA Devivo-B Rolandi-G Seal-RR Paone-A

Title: Geochemical and Isotopic (Nd-Pb-Sr-O) Variations Bearing on the Genesis of Volcanic-Rocks from Vesuvius, Italy

Source: JOURNAL OF VOLCANOLOGY AND GEOTHERMAL RESEARCH 1998, Vol 82, Iss 1-4, pp 53-78

Addresses:

US-GEOL-SURVEY, NATL CTR, MAIL STOP 954, RESTON, VA 22092, USA

UNIV-NAPLES, DIPARTIMENTO GEOFIS \& VULCANOL, I-80138 NAPLES, ITALY 
78. Authors: Belkin-HE Devivo-B Torok-K Webster-JD

Title: Pre-Eruptive Volatile Content, Melt-Inclusion Chemistry, and Microthermometry of Interplinian Vesuvius Lavas (PreAd1631)

Source: JOURNAL OF VOLCANOLOGY AND GEOTHERMAL RESEARCH 1998, Vol 82, Iss 1-4, pp 79-95

Addresses:

US-GEOL-SURVEY, MS 956, RESTON, VA 22092, USA

DIPARTIMENTO-GEOFIS-\&-VULCANOL, I-80134 NAPLES, ITALY

LORAND-EOTVOS-UNIV, DEPT GEOPHYS, H-1083 BUDAPEST, HUNGARY

AMER-MUSEUM-NAT-HIST, DEPT EARTH \& PLANETARY SCI, NEW-YORK, NY 10024, USA

79. Authors: Tyler-SW Cook-PG Butt-AZ Thomas-JM Doran-PT

Lyons-WB

Title: Evidence of Deep Circulation in 2 Perennially IceCovered Antarctic Lakes

Source: LIMNOLOGY AND OCEANOGRAPHY 1998, Vol 43, Iss 4, pp 625-635

Addresses:

UNIV-NEVADA, DESERT RES INST, RENO, NV 89506, USA

CSIRO, CTR GROUNDWATER STUDIES, ADELAIDE, SA 5064, AUSTRALIA

UNIV-NEVADA, RENO, NV 89512, USA

US-GEOL-SURVEY, CARSON-CITY, NV 89706, USA

UNIV-ALABAMA, TUSCALOOSA, AL 35487, USA

80. Authors: Allen-CM Wooden-JL Chappell-BW

Title: Late Paleozoic Crustal History of Central Coastal Queensland Interpreted from Geochemistry of Mesozoic Plutons - The Effects of Continental Rifting

Source: LITHOS 1997, Vol 42, Iss 1-2, pp 67-88

Addresses:

AUSTRALIAN-NATL-UNIV, DEPT GEOL, KEY CTR GEOCHEM EVOLUT \& METALLOGENY CONTINENTS, CANBERRA, ACT 0200, AUSTRALIA

US-GEOL-SURVEY, MENLO-PK, CA 94025, USA

81. Authors: Caffrey-JM Cloern-JE Grenz-C

Title: Changes in Production and Respiration During a Spring Phytoplankton Bloom in San-Francisco Bay, California, USA - Implications for Net Ecosystem Metabolism

Source: MARINE ECOLOGY-PROGRESS SERIES 1998, Vol 172, pp 1-12

Addresses:

UNIV-CALIF-SANTA-CRUZ, INST MARINE SCI, SANTA-CRUZ, CA 95064, USA

US-GEOL-SURVEY, MENLO-PK, CA 94025, USA

UNIV-MEDITERRANEE, CNRS, UMR 6535, F-13007 MARSEILLE, FRANCE

82. Authors: Qiang-J Mccabe-PJ

Title: Genetic Features of Petroleum Systems in Rift Basins of Eastern China

Source: MARINE AND PETROLEUM GEOLOGY

1998, Vol 15, Iss 4, pp 343-358 
Addresses:

UNIV-PETR, DEPT RESOURCES, SHANDONG 257062, PEOPLES-R-CHINA

US-GEOL-SURVEY, DENVER, CO 80225, USA

83. Authors: Goswami-JN Sahijpal-S Kehm-K Hohenberg-CM

Swindle-T Grossman-JN

Title: In-Situ Determination of Iodine Content and Iodine-Xenon

Systematics in Silicates and Troilite Phases in

Chondrules from the L13 Chondrite Semarkona

Source: METEORITICS \& PLANETARY SCIENCE

1998, Vol 33, Iss 3, pp 527-534

Addresses:

PHYS-RES-LAB, AHMEDABAD 380009, GUJARAT, INDIA

WASHINGTON-UNIV, MCDONNELL CTR SPACE SCI, ST-LOUIS, MO 63130, USA

UNIV-ARIZONA, LUNAR \& PLANETARY LAB, TUCSON, AZ 85721, USA

US-GEOL-SURVEY, RESTON, VA 22092, USA

84. Authors: Jiang-SY Palmer-MR Slack-JF

Title: Alkali-Deficient Tourmaline from the Sullivan $\mathrm{Pb}-\mathrm{Zn}-\mathrm{Ag}$

Deposit, British-Columbia

Source: MINERALOGICAL MAGAZINE

1997, Vol 61, Iss 6, pp 853-860

Addresses:

UNIV-BRISTOL, DEPT GEOL, WILLS MEM BLDG, BRISTOL BS8-1RJ, AVON, ENGLAND

MAX-PLANCK-INST-CHEM, ABT GEOCHEM, D-55020 MAINZ, GERMANY

US-GEOL-SURVEY, NATL CTR, RESTON, VA 22092, USA

85. Authors: Chin-K Tokaryk-TT Erickson-GM Calk-LC

Title: A King-Sized Theropod Coprolite

Source: NATURE 1998, Vol 393, Iss 6686, pp 680-682

Addresses:

US-GEOL-SURVEY, 345 MIDDLEFIELD RD, MS 975, MENLO-PK, CA 94025, USA

ROYAL-SASKATCHEWAN-MUSEUM, EASTEND FOSSIL RES STN, EASTEND, SK SON-OT0, CANADA

UNIV-CALIF-BERKELEY, DEPT INTEGRAT BIOL, BERKELEY, CA 94720 , USA

UNIV-CALIF-BERKELEY, MUSEUM VERTEBRATE ZOOL, BERKELEY, CA

94720, USA

UNIV-CALIF-BERKELEY, MUSEUM PALEONTOL, BERKELEY, CA 94720, USA

86. Authors: Zierenberg-RA Fouquet-Y Miller-DJ Bahr-JM Baker-PA

Bjerkgard-T Brunner-CA Duckworth-RC Gable-R

Gieskes-J Goodfellow-WD Groschelbecker-HM Guerin-G

Ishibashi-J Iturrino-G James-RH Lackschewitz-KS

Marquez-LL Nehlig-P Peter-JM Rigsby-CA

Schultheiss-P Shanks-WC Simoneit-BRT Summit-M

Teagle-DAH Urbat-M Zuffa-GG

Title: The Deep-Structure of a Sea-Floor Hydrothermal Deposit

Source: NATURE

1998, Vol 392, Iss 6675, pp 485-488 


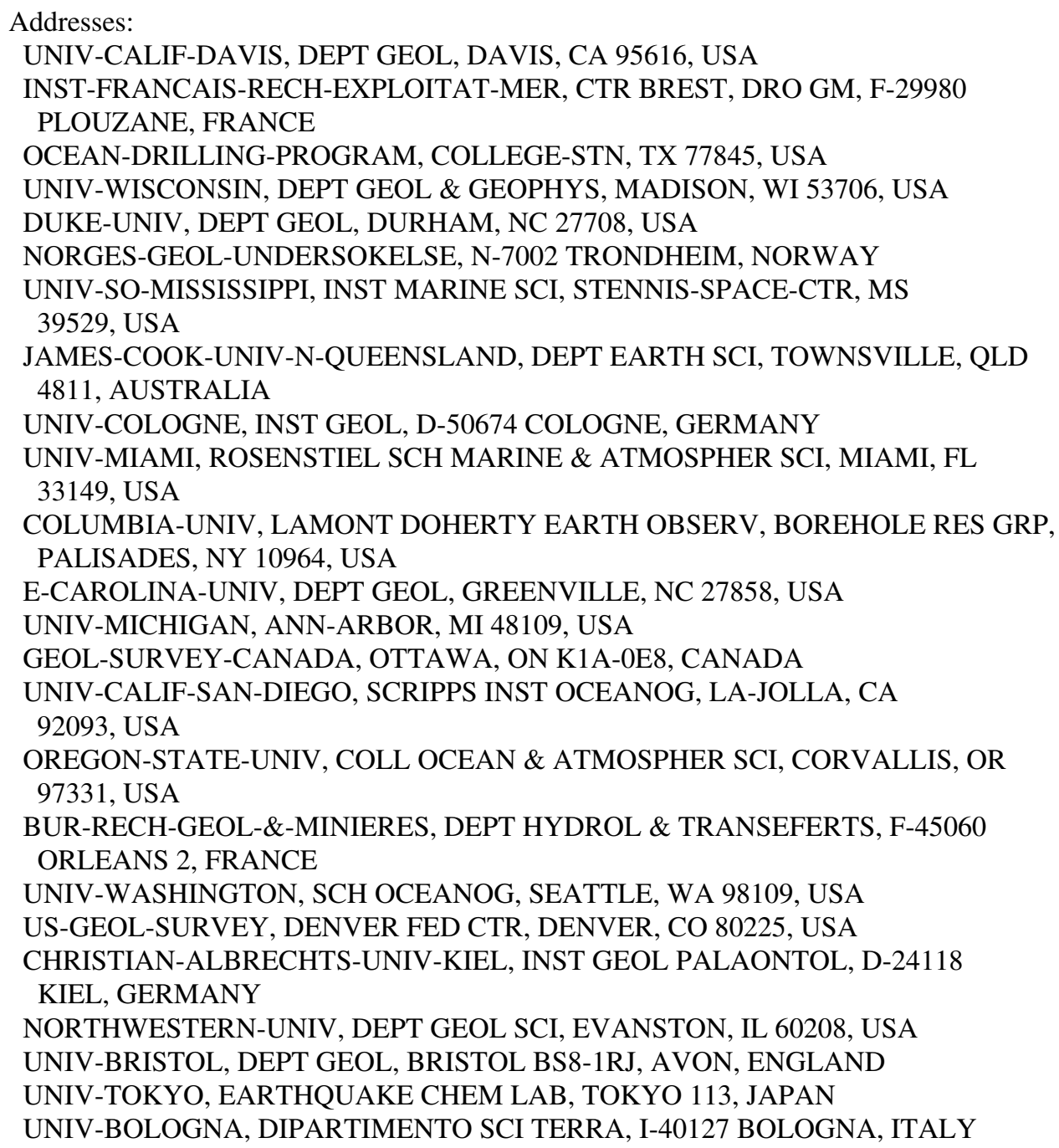


RAND-CORP, SANTA-MONICA, CA 90406, USA

UNIV-ARIZONA, LUNAR \& PLANETARY LAB, TUCSON, AZ 85721, USA

ARIZONA-STATE-UNIV, DEPT GEOL, TEMPE, AZ 85287, USA

CORNELL-UNIV, ITHACA, NY 14853, USA

BROWN-UNIV, DEPT GEOL, PROVIDENCE, RI 02912, USA

JET-PROP-LAB, PASADENA, CA 91909, USA

DLR, INST PLANETENERKUNDUNG, D-12489 BERLIN, GERMANY

NASA, AMES RES CTR, MOFFETT-FIELD, CA 94035, USA

UNIV-CALIF-LOS-ANGELES, DEPT EARTH \& SPACE SCI, LOS-ANGELES,

CA 90095, USA

89. Authors: Groves-DI Goldfarb-RJ Gebremariam-M Hagemann-SG

Robert-F

Title: Orogenic Gold Deposits - A Proposed Classification in

the Context of Their Crustal Distribution and

Relationship to Other Gold Deposit Types

Source: ORE GEOLOGY REVIEWS

1998, Vol 13, Iss 1-5, pp 7-27

Addresses:

UNIV-WESTERN-AUSTRALIA, CTR TEACHING \& RES STRATEG MINERAL

DEPOSITS, DEPT GEOL \& GEOPHYS, NEDLANDS, WA 6907, AUSTRALIA

US-GEOL-SURVEY, DENVER FED CTR, DENVER, CO 80225, USA

WILUNA-GOLD-MINES-LTD, PERTH, WA 6005, AUSTRALIA

GEOL-SURVEY-CANADA, OTTAWA, ON K1A-0E8, CANADA

90. Authors: Goldfarb-RJ Phillips-GN Nokleberg-WJ

Title: Tectonic Setting of Synorogenic Gold Deposits of the Pacific-rim

Source: ORE GEOLOGY REVIEWS

1998, Vol 13, Iss 1-5, pp 185-218

Addresses:

US-GEOL-SURVEY, DENVER FED CTR, BOX 25046, DENVER, CO 80225, USA

GREAT-CENT-MINES-LTD, MALVERN, VIC 3145, AUSTRALIA

US-GEOL-SURVEY, MENLO-PK, CA 94025, USA

91. Authors: Spotl-C Houseknecht-DW Jaques-RC

Title: Kerogen Maturation and Incipient Graphitization of Hydrocarbon Source Rocks in the Arkoma Basin, Oklahoma and Arkansas - A Combined Petrographic and Raman Spectrometric Study

Source: ORGANIC GEOCHEMISTRY

1998, Vol 28, Iss 9-10, pp 535-542

Addresses:

INNSBRUCK-UNIV, INST GEOL \& PALAONTOL, INNRAIN 52, A-6020

INNSBRUCK, AUSTRIA

US-GEOL-SURVEY, RESTON, VA 20192, USA

UNIV-MISSOURI, DEPT GEOL SCI, COLUMBIA, MO 65211, USA

92. Authors: Koopmans-MP Rijpstra-WIC Deleeuw-JW Lewan-MD Damste-JSS

Title: Artificial Maturation of an Immature Sulfur and OrganicMatter Rich Limestone from the Ghareb Formation, Jordan

Source: ORGANIC GEOCHEMISTRY 1998, Vol 28, Iss 7-8, pp 503-521 
Addresses:

NETHERLANDS-INST-SEA-RES, DEPT MARINE BIOGEOCHEM \& TOXICOL, NL-1790-AB DEN-BURG, NETHERLANDS

US-GEOL-SURVEY, DENVER FED CTR, DENVER, CO 80225, USA

93. Authors: Mei-SL Zhang-KX Wardlaw-BR

Title: A Refined Succession of Changhsingian and Griesbachian

Neogondolellid Conodonts from the Meishan Section,

Candidate of the Global Stratotype Section and Point of

the Permian-Triassic Boundary

Source: PALAEOGEOGRAPHY PALAEOCLIMATOLOGY PALAEOECOLOGY 1998, Vol 143, Iss 4, pp 213-226

Addresses:

CHINA-UNIV-GEOSCI, BEIJING 100083, PEOPLES-R-CHINA

CHINA-UNIV-GEOSCI, WUHAN 430074, PEOPLES-R-CHINA

US-GEOL-SURVEY, RESTON, VA 22092, USA

94. Authors: Warwick-PD Johnson-EA Khan-IH

Title: Collision-Induced Tectonism Along the Northwestern

Margin of the Indian Subcontinent as Recorded in the

Upper Paleocene to Middle Eocene Strata of Central

Pakistan (Kirthar and Sulaiman Ranges)

Source: PALAEOGEOGRAPHY PALAEOCLIMATOLOGY PALAEOECOLOGY 1998, Vol 142, Iss 3-4, pp 201-216

Addresses:

US-GEOL-SURVEY, NATL CTR, MS 956, 12201 SUNRISE VALLEY DR,

RESTON, VA 22091, USA

US-GEOL-SURVEY, DENVER FED CTR, DENVER, CO 80225, USA

GEOL-SURVEY-PAKISTAN, QUETTA 7300, PAKISTAN

95. Authors: Scholz-CA Moore-TC Hutchinson-DR Golmshtok-AJ

Klitgord-KD Kurotchkin-AG

Title: Comparative Sequence Stratigraphy of Low-Latitude Versus

High-Latitude Lacustrine Rift Basins - Seismic Data

Examples from the East-African and Baikal Rifts

Source: PALAEOGEOGRAPHY PALAEOCLIMATOLOGY PALAEOECOLOGY 1998, Vol 140, Iss 1-4, pp 401-420

Addresses:

UNIV-MIAMI, ROSENSTIEL SCH MARINE \& ATMOSPHER SCI, MIAMI, FL 33149, USA

UNIV-MICHIGAN, DEPT GEOL SCI, ANN-ARBOR, MI 48109, USA

US-GEOL-SURVEY, WOODS-HOLE, MA 02543, USA

INST-OCEANOL, SO BRANCH, GELENDZHIK, RUSSIA

US-GEOL-SURVEY, MENLO-PK, CA 94025, USA

KUBAN-STATE-UNIV, KRASNODAR 350751, RUSSIA

96. Authors: Karabanov-EB Prokopenko-AA Williams-DF Colman-SM

Title: Evidence from Lake-Baikal for Siberian Glaciation During

Oxygen-Isotope Substage 5D

Source: QUATERNARY RESEARCH

1998, Vol 50, Iss 1, pp 46-55

Addresses:

UNIV-S-CAROLINA, DEPT GEOL SCI, BAIKAL DRILLING PROJECT,

COLUMBIA, SC 29208, USA

RUSSIAN-ACAD-SCI, INST GEOCHEM, IRKUTSK 664003, RUSSIA 
RUSSIAN-ACAD-SCI, UNITED INST GEOL GEOPHYS \& GEOCHEM, NOVOSIBIRSK, RUSSIA

US-GEOL-SURVEY, WOODS-HOLE, MA 02543, USA

97. Authors: Lin-JC Broecker-WS Hemming-SR Hajdas-I Anderson-RF Smith-GI Kelley-M Bonani-G

Title: A Reassessment of U-Th and C-14 Ages for Late-Glacial High-Frequency Hydrological Events at Searles Lake, California

Source: QUATERNARY RESEARCH 1998, Vol 49, Iss 1, pp 11-23

Addresses:

COLUMBIA-UNIV, LAMONT DOHERTY EARTH OBSERV, PALISADES, NY 10964, USA

ETH-HONGGERBERG, INST TEILCHENPHYS, CH-8093 ZURICH, SWITZERLAND

US-GEOL-SURVEY, MENLO-PK, CA 94025, USA

98. Authors: Dafiljensen-D Mosegaard-K Gundestrup-N Clow-GD Johnsen-SJ Hansen-AW Balling-N

Title: Past Temperatures Directly from the Greenland Ice-Sheet

Source: SCIENCE 1998, Vol 282, Iss 5387, pp 268-271

Addresses:

NIELS-BOHR-INST-ASTRON-PHYS-\&-GEOPHYS, DEPT GEOPHYS, JULIANE MARLES VEJ 30, DK-2100 COPENHAGEN OE, DENMARK

US-GEOL-SURVEY, DENVER FED CTR, CLIMATE PROGRAM, DENVER, CO 80225, USA

UNIV-AARHUS, DEPT EARTH SCI, GEOPHYS LAB, DK-8200 AARHUS, DENMARK

99. Authors: Farley-KA Montanari-A Shoemaker-EM Shoemaker-CS

Title: Geochemical Evidence for a Comet Shower in the Late Eocene

Source: SCIENCE 1998, Vol 280, Iss 5367, pp 1250-1253

Addresses:

CALTECH, DIV GEOL \& PLANETARY SCI, MS 170-25, PASADENA, CA 91125, USA

ECOLE-MINES, PARIS, FRANCE

OSSERVATORIO-GEOL-COLDIGIOCO, I-62020 FRONTALE-DI-APIRO, APIRO, ITALY

US-GEOL-SURVEY, FLAGSTAFF, AZ 86001, USA

100. Authors: Christensen-PR Anderson-DL Chase-SC Clancy-RT Clark-RN Conrath-BJ Kieffer-HH Kuzmin-RO Malin-MC Pearl-JC Roush-TL

Title: Results from the Mars Global Surveyor Thermal Emission Spectrometer

Source: SCIENCE 1998, Vol 279, Iss 5357, pp 1692-1698

Addresses:

ARIZONA-STATE-UNIV, TEMPE, AZ 85287, USA

UNIV-COLORADO, GOLETA, CA 93017, USA

US-GEOL-SURVEY, DENVER, CO 80225, USA

CORNELL-UNIV, ITHACA, NY 14850, USA

US-GEOL-SURVEY, FLAGSTAFF, AZ 86001, USA

VI-VERNADSKII-INST-GEOCHEM-\&-ANALYT-CHEM, MOSCOW 117975, RUSSIA 
MALIN-SPACE-SCI-SYST, SAN-DIEGO, CA, USA

NASA, GODDARD SPACE FLIGHT CTR, GREENBELT, MD 20771, USA

SANTA-BARBARA-REMOTE-SENSING, GOLETA, CA 93017, USA

NASA, AMES RES CTR, MOFFETT-FIELD, CA 94035, USA

101. Authors: Normark-WR Piper-DJW Hiscott-RN

Title: Sea-Level Controls on the Textural Characteristics and Depositional Architecture of the Hueneme and Associated Submarine Fan Systems, Santa-Monica Basin, California

Source: SEDIMENTOLOGY 1998, Vol 45, Iss 1, pp 53-70

Addresses:

US-GEOL-SURVEY, MS-999, 345 MIDDLEFIELD RD, MENLO-PK, CA 94025, USA

GEOL-SURVEY-CANADA, ATLANTIC GEOSCI CTR, DARTMOUTH, NS

B2Y-4A2, CANADA

MEM-UNIV-NEWFOUNDLAND, DEPT EARTH SCI, ST-JOHNS, NF A1B-3X5, CANADA

102.Authors: Counihan-TD Miller-AI Mesa-MG Parsley-MJ

Title: The Effects of Dissolved-Gas Supersaturation on White Sturgeon Larvae

Source: TRANSACTIONS OF THE AMERICAN FISHERIES SOCIETY 1998, Vol 127, Iss 2, pp 316-322

Addresses:

US-GEOL-SURVEY, BIOL RESOURCES DIV, NW BIOL SCI CTR, COLUMBIA RIVER RES LAB, 5501A COOK UNDERWOOD R, COOK, WA 98605, USA

103. Authors: Adams-NS Rondorf-DW Evans-SD Kelly-JE

Title: Effects of Surgically and Gastrically Implanted Radio

Transmitters on Growth and Feeding-Behavior of Juvenile

Chinook Salmon

Source: TRANSACTIONS OF THE AMERICAN FISHERIES SOCIETY 1998, Vol 127, Iss 1, pp 128-136

Addresses:

US-GEOL-SURVEY, BIOL RESOURCES DIV, NW BIOL SCI CTR, COLUMBIA RIVER RES LAB, 5501A COOK UNDERWOOD R, COOK, WA 98605, USA

104. Authors: Flueh-ER Fisher-MA Bialas-J Jonathan-R Klaeschen-D Kukowski-N Parsons-T Scholl-DW Tenbrink-U Trehu-AM Vidal-N

Title: New Seismic Images of the Cascadia Subduction Zone from Cruise So108-Orwell

Source: TECTONOPHYSICS 1998, Vol 293, Iss 1-2, pp 69-84

Addresses:

CHRISTIAN-ALBRECHTS-UNIV-KIEL, GEOMAR, WISCHHOFSTR 1-3,

D-24148 KIEL, GERMANY

US-GEOL-SURVEY, MENLO-PK, CA 94025, USA

STANFORD-UNIV, STANFORD, CA 94305, USA

US-GEOL-SURVEY, WOODS-HOLE, MA 02543, USA

OREGON-STATE-UNIV, CORVALLIS, OR 97331, USA 
105. Authors: Ryberg-T Fuis-GS

Title: The San-Gabriel Mountains Bright Reflective Zone -

Possible Evidence of Young Midcrustal Thrust Faulting in

Southern California

Source: TECTONOPHYSICS

1998, Vol 286, Iss 1-4, pp 31-46

Addresses:

GEOFORSCHUNGSZENTRUM-POTSDAM, TELEGRAFENBERG, D-14473 POTSDAM, GERMANY

US-GEOL-SURVEY, MENLO-PK, CA 94025, USA

106. Authors: Peters-NE Ratcliffe-EB

Title: Tracing Hydrologic Pathways Using Chloride at the Panola

Mountain Research Watershed, Georgia, USA

Source: WATER AIR AND SOIL POLLUTION

1998, Vol 105, Iss 1-2, pp 263-275

Addresses:

US-GEOL-SURVEY, 3039 AMWILER RD, SUITE 130, ATLANTA, GA 30360 , USA

UNIV-BRISTOL, SCH GEOG SCI, BRISTOL BS8-1SS, AVON, ENGLAND

107. Authors: Naff-RL Haley-DF Sudicky-EA

Title: High-Resolution Monte-Carlo Simulation of Flow and Conservative Transport in Heterogeneous Porous-Media 1 Methodology and Flow Results

Source: WATER RESOURCES RESEARCH 1998, Vol 34, Iss 4, pp 663-677

Addresses:

US-GEOL-SURVEY, DIV WATER RESOURCES, MS 413, BOX 25046 , DENVER, CO 80235, USA

UNIV-WATERLOO, WATERLOO CTR GROUNDWATER RES, WATERLOO, ON N2L-3G1, CANADA

108. Authors: Naff-RL Haley-DF Sudicky-EA

Title: High-Resolution Monte-Carlo Simulation of Flow and Conservative Transport in Heterogeneous Porous-Media 2 Transport Results

Source: WATER RESOURCES RESEARCH 1998, Vol 34, Iss 4, pp 679-697

Addresses:

US-GEOL-SURVEY, DIV WATER RESOURCES, MS 413, BOX 25046, DENVER, CO 80235, USA

UNIV-WATERLOO, WATERLOO CTR GROUNDWATER RES, WATERLOO, ON N2L-3G1, CANADA

109. Authors: Ellis-DH Hjertaas-D Johns-BW Urbanek-RP

Title: Use of a Helicopter to Capture Flighted Cranes

Source: WILDLIFE SOCIETY BULLETIN 1998, Vol 26, Iss 1, pp 103-107

Addresses:

US-GEOL-SURVEY, PATUXENT WILDLIFE RES CTR, HCR 1 BOX 4420, ORACLE, AZ 85623, USA

SASKATCHEWAN-ENVIRONM-\&-RESOURCE-MANAGEMENT, REGINA, SK S4S-5W6, CANADA 
CANADIAN-WILDLIFE-SERV, SASKATOON, SK S7N-0X4, CANADA

US-FISH-\&-WILDLIFE-SERV, SENEY NATL WILDLIFE REFUGE, SENEY, MI

49883, USA

110. Authors: Parhar-IS Nagahama-Y Grau-EG Ross-RM

Title: Immunocytochemical and Ultrastructural Identification of

Pituitary Cell-Types in the Protogynous Thalassoma

Duperrey During Adult Sexual Ontogeny

Source: ZOOLOGICAL SCIENCE

1998, Vol 15, Iss 2, pp 263-276

Addresses:

NIPPON-MED-COLL, DEPT PHYSIOL, 1-1-5 SENDAGI, TOKYO 113, JAPAN

NATL-INST-BASIC-BIOL, REPROD BIOL LAB, OKAZAKI, AICHI 444,

JAPAN

UNIV-HAWAII, DEPT ZOOL, KANEOHE, HI 96744, USA

UNIV-HAWAII, HAWAII INST MARINE BIOL, KANEOHE, HI 96744, USA

US-GEOL-SURVEY, RES \& DEV LAB, WELLSBORO, PA 16901, USA

111. Authors: Massonnet-D Holzer-T Vadon-H

Title: Land Subsidence Caused by the East Mesa Geothermal-

Field, California, Observed Using SAR Interferometry

(Vol 24, Pg 901, 1997)

Source: GEOPHYSICAL RESEARCH LETTERS

1998, Vol 25, Iss 16, pp 3213-3213

Addresses:

CNES, 18 AVE E BELIN, F-31401 TOULOUSE 4, FRANCE

US-GEOL-SURVEY, MENLO-PK, CA 94025, USA

112. Authors: Lenz-AC Edwards-LE Pratt-BR

Title: Revision of the Biostratigraphic Units Section of the North-American Stratigraphic Code

Source: EPISODES 1997, Vol 20, Iss 3, pp 203-204

Addresses:

UNIV-WESTERN-ONTARIO, DEPT EARTH SCI, LONDON, ON N6A-5B7, CANADA

US-GEOL-SURVEY, NATL CTR 970, RESTON, VA 22092, USA

UNIV-SASKATCHEWAN, DEPT GEOL SCI, SASKATOON, SK S7N-0W0, CANADA

113. Authors: Aspinall-WP Lynch-LL Robertson-REA Rowley-K Sparks-RSJ Voight-B Young-SR

Title: The Soufriere Hills Eruption, Montserrat, British-WestIndies - Introduction to Special Section, Part 1

Source: GEOPHYSICAL RESEARCH LETTERS 1998, Vol 25, Iss 18, pp 3387-3387

Addresses:

ASPINALL-ASSOC, KEYWORTH NG12-5GG, NOTTS, ENGLAND

BRITISH-GEOL-SURVEY, KEYWORTH NG12-5GG, NOTTS, ENGLAND

UNIV-W-INDIES, SEISM RES UNIT, ST-AUGUSTINE, TRINID-\&-TOBAGO

UNIV-BRISTOL, DEPT GEOSCI, BRISTOL BS8-1RJ, AVON, ENGLAND

PENN-STATE-UNIV, UNIVERSITY-PK, PA 16802, USA

US-GEOL-SURVEY, VOLCANO HAZARDS PROGRAM, RESTON, VA, USA 
114. Authors: Seltmann-R Lehmann-B Lowenstern-JB Candela-PA

Title: High-Level Silicic Magmatism and Related Hydrothermal Systems

Source: JOURNAL OF PETROLOGY 1997, Vol 38, Iss 12, pp 1617-1618

Addresses:

GEOFORSCHUNGSZENTRUM-POTSDAM, D-14473 POTSDAM, GERMANY

TECH-UNIV-CLAUSTHAL, INST MINERAL \& MINERAL ROHSTOFFE, D-38678

CLAUSTHAL-ZELLERFELD, GERMANY

US-GEOL-SURVEY, MENLO-PK, CA 94025, USA

UNIV-MARYLAND, DEPT GEOL, COLLEGE-PK, MD 20742, USA

115. Authors: Syvitski-JPM Stoker-MS Cooper-AK

Title: Preface - Seismic Facies of Glacial Deposits from Marine and Lacustrine Environments

Source: MARINE GEOLOGY

1997, Vol 143, Iss 1-4, pp 1-4

Addresses:

UNIV-COLORADO, INSTAAR, 1560 30TH ST, BOULDER, CO 80309, USA

BRITISH-GEOL-SURVEY, EDINBURGH EH9-3LA, MIDLOTHIAN, SCOTLAND

US-GEOL-SURVEY, MENLO-PK, CA 94025, USA

116. Authors: Atkinson-GM Boore-DM Boatwright-J

Title: Comment on Earthquake Source Spectra in Eastern NorthAmerica by Haddon,R.A.W

Source: BULLETIN OF THE SEISMOLOGICAL SOCIETY OF AMERICA 1997, Vol 87, Iss 6, pp 1697-1702

Addresses:

CARLETON-UNIV, DEPT EARTH SCI, 1125 COLONEL DR, OTTAWA, ON

K1S-5B6, CANADA

US-GEOL-SURVEY, MENLO-PK, CA 94025, USA

117. Authors: Coplen-TB Krouse-HR

Title: Sulfur Isotope Data Consistency Improved

Source: NATURE 1998, Vol 392, Iss 6671, pp 32-32

Addresses:

US-GEOL-SURVEY, 431 NATL CTR, RESTON, VA 20192, USA

UNIV-CALGARY, DEPT PHYS \& ASTRON, CALGARY, AB T2N-1N4, CANADA

118. Authors: Hofstetter-A Rybakov-M Tenbrink-U

Title: Comment on New Evidence of Magmatic Diapirs in the Intermediate Crust Under the Dead-Sea, Israel by Rabinowitz,Nitzan, Steinberg,Jean, and Mart,Yossi

Source: TECTONICS 1998, Vol 17, Iss 5, pp 819-820

Addresses:

GEOPHYS-INST-ISRAEL, POB 2286, IL-58122 HOLON, ISRAEL

US-GEOL-SURVEY, WOODS-HOLE, MA 02543, USA

119. Authors: Kotarba-MJ Lewan-MD

Title: Thermogenic Gases from the Polish Bituminous and Brown Coals - Hydrous Pyrolysis and Isotopic Approach

Source: ABSTRACTS OF PAPERS OF THE AMERICAN CHEMICAL SOCIETY 1998, Vol 215, Iss APR, pp 12-GEOC 
Addresses:

STANISLAW-STASZIC-UNIV-MIN-\&-MET, DEPT FOSSIL FUELS, PL-30059

KRAKOW, POLAND

US-GEOL-SURVEY, DENVER FED CTR, DENVER, CO 80225, USA

120. Authors: Grossman-JN Alexander-CMO Wang-J Zanda-B

Bourotdenise-M Hewins-RH Yu-Y

Title: The Lack of Potassium-Isotopic Fractionation in Bishunpur Chondrules

Source: METEORITICS \& PLANETARY SCIENCE 1998, Vol 33, Iss 4, pp A64-A65

Addresses:

US-GEOL-SURVEY, RESTON, VA 20192, USA

CARNEGIE-INST-WASHINGTON, DEPT TERR MAGNETISM, WASHINGTON, DC 20015, USA

RUTGERS-STATE-UNIV, DEPT GEOL SCI, PISCATAWAY, NJ 08854, USA

MUSEUM-NATL-HIST-NAT, F-7500 PARIS, FRANCE

121. Authors: Laurs-BM Dilles-JH Wairrach-Y Kausar-AB Snee-LW

Title: Geological Setting and Petrogenesis of Symmetrically

Zoned, Miarolitic Granitic Pegmatites at Stak Nala,

Nanga Parbat Haramosh Massif, Northern Pakistan

Source: CANADIAN MINERALOGIST

1998, Vol 36, Iss FEB, pp 1-47

Addresses:

OREGON-STATE-UNIV, DEPT GEOSCI, CORVALLIS, OR 97331, USA

GEOL-SURVEY-PAKISTAN, GEOSCI LAB, ISLAMABAD, PAKISTAN

US-GEOL-SURVEY, DENVER FED CTR, DENVER, CO 80225, USA

122. Authors: Coombs-DS Alberti-A Armbruster-T Artioli-G

Colella-C Galli-E Grice-JD Liebau-F Mandarino-JA

Minato-H Nickel-EH Passaglia-E Peacor-DR

Quartieri-S Rinaldi-R Ross-M Sheppard-RA

Tillmanns-E Vezzalini-G

Title: Recommended Nomenclature for Zeolite Minerals - Report

of the Subcommittee on Zeolites of the International-

Mineralogical-Association, Commission on New Minerals

and Mineral Names

Source: CANADIAN MINERALOGIST

1997, Vol 35, Iss DEC, pp 1571-1606

Addresses:

UNIV-OTAGO, DEPT GEOL, POB 56, DUNEDIN, NEW-ZEALAND

UNIV-FERRARA, IST MINERAL, I-44100 FERRARA, ITALY

UNIV-BERN, LAB CHEM \& MINERAL KRISTALLOG, CH-3012 BERN, SWITZERLAND

UNIV-MILAN, DIPARTIMENTO SCI TERRA, I-20133 MILAN, ITALY

UNIV-NAPLES-FEDERICO-II, DIPARTIMENTO INGN MAT \& PROD, I-80125

NAPLES, ITALY

UNIV-MODENA, DIPARTIMENTO SCI TERRA, I-41100 MODENA, ITALY

CANADIAN-MUSEUM-NAT, MINERAL SCI DIV, OTTAWA, ON K1P-6P4,

CANADA

CHRISTIAN-ALBRECHTS-UNIV-KIEL, MINERAL PETROG INST, D-2300

KIEL, GERMANY

ROYAL-ONTARIO-MUSEUM, DEPT MINERAL, TORONTO, ON M5S-2C6, CANADA

UNIV-PERUGIA, DIPARTIMENTO SCI TERRA, I-06100 PERUGIA, ITALY

US-GEOL-SURVEY, RESTON, VA 20192, USA 
UNIV-VIENNA, INST KRISTALLOG \& MINERAL, A-1090 VIENNA, AUSTRIA

UNIV-MICHIGAN, DEPT GEOL SCI, ANN-ARBOR, MI 48109, USA

US-GEOL-SURVEY, DENVER FED CTR, DENVER, CO 80225, USA

CSIRO, DIV EXPLORAT \& MIN, WEMBLY, WA 6014, AUSTRALIA

123. Authors: Coombs-DS Alberti-A Armbruster-T Artioli-G

Colella-C Galli-E Grice-JD Liebau-F Mandarino-JA

Minato-H Nickel-EH Passaglia-E Peacor-DR

Quartieri-S Rinaldi-R Ross-M Sheppard-RA

Tillmanns-E Vezzalini-G

Title: Recommended Nomenclature for Zeolite Minerals - Report

of the Subcommittee on Zeolites of the International

Mineralogical Association, Commission on New Minerals and Mineral Names

Source: EUROPEAN JOURNAL OF MINERALOGY

1998, Vol 10, Iss 5, pp 1037-1081

Addresses:

UNIV-OTAGO, DEPT GEOL, POB 56, DUNEDIN, NEW-ZEALAND

UNIV-FERRARA, IST MINERAL, I-44100 FERRARA, ITALY

UNIV-BERN, LAB CHEM \& MINERAL KRISTALLOG, CH-3012 BERN,

SWITZERLAND

UNIV-MILAN, DIPARTIMENTO SCI TERRA, I-20133 MILAN, ITALY

UNIV-NAPLES-FEDERICO-II, DIPARTIMENTO INGN MAT \& PROD, I-80125

NAPLES, ITALY

UNIV-MODENA, DIPARTIMENTO SCI TERRA, I-41100 MODENA, ITALY

CANADIAN-MUSEUM-NAT, DIV MINERAL SCI, OTTAWA, ON K1P-6P4,

CANADA

CHRISTIAN-ALBRECHTS-UNIV-KIEL, MINERAL PETROG INST, D-24098

KIEL, GERMANY

CSIRO, DIV EXPLORAT \& MIN, WEMBLY, WA 6014, AUSTRALIA

UNIV-VIENNA, INST MINERAL \& KRISTALLOG, A-1090 VIENNA, AUSTRIA

US-GEOL-SURVEY, FED CTR, DENVER, CO 80225, USA

UNIV-PERUGIA, DIPARTIMENTO SCI TERRA, I-06100 PERUGIA, ITALY

US-GEOL-SURVEY, RESTON, VA 20192, USA

UNIV-MICHIGAN, DEPT GEOL SCI, ANN-ARBOR, MI 48109, USA

ROYAL-ONTARIO-MUSEUM, DEPT MINERAL, TORONTO, ON M5S-2C6, CANADA

124. Authors: Heath-E Macdonald-R Belkin-H Hawkesworth-C

Sigurdsson-H

Title: Magmagenesis at Soufriere Volcano, St-Vincent, Lesser-

Antilles Arc

Source: JOURNAL OF PETROLOGY

1998, Vol 39, Iss 10, pp 1721-1764

Addresses:

UNIV-LANCASTER, INST ENVIRONM \& BIOL SCI, DIV ENVIRONM SCI,

LANCASTER LA1-4YQ, ENGLAND

US-GEOL-SURVEY, RESTON, VA 22092, USA

OPEN-UNIV, DEPT EARTH SCI, MILTON-KEYNES MK7-6AA, BUCKS,

ENGLAND

UNIV-RHODE-ISL, GRAD SCH OCEANOG, NARRAGANSETT, RI 02882, USA

125. Authors: Stein-HJ Sundblad-K Markey-RJ Morgan-JW Motuza-G

Title: Re-Os Ages for Archean Molybdenite and Pyrite, Kuittila-

Kivisuo, Finland and Proterozoic Molybdenite, Kabeliai,

Lithuania - Testing the Chronometer in a Metamorphic and

Metasomatic Setting 
Source: MINERALIUM DEPOSITA

1998, Vol 33, Iss 4, pp 329-345

Addresses:

COLORADO-STATE-UNIV, DEPT EARTH RESOURCES, AIRIE GRP,

FT-COLLINS, CO 80523, USA

US-GEOL-SURVEY, NATL CTR, RESTON, VA 20192, USA

UNIV-STOCKHOLM, DEPT GEOL \& GEOCHEM, S-10691 STOCKHOLM, SWEDEN

GEOL-SURVEY-LITHUANIA, VILNIUS, LITHUANIA

126. Authors: Coombs-DS Alberti-A Armbruster-T Artioli-G

Colella-C Galli-E Grice-JD Liebau-F Mandarino-JA

Minato-H Nickel-EH Passaglia-E Peacor-DR

Quartieri-S Rinaldi-R Ross-M Sheppard-RA

Tillmanns-E Vezzalini-G

Title: Recommended Nomenclature for Zeolite Minerals - Report

of the Subcommittee on Zeolites of the International-

Mineralogical-Association, Commission on New Minerals

and Mineral Names

Source: MINERALOGICAL MAGAZINE

1998, Vol 62, Iss 4, pp 533-571

Addresses:

UNIV-OTAGO, DEPT GEOL, POB 56, DUNEDIN, NEW-ZEALAND

UNIV-FERRARA, IST MINERAL, I-44100 FERRARA, ITALY

UNIV-BERN, LAB CHEM \& MINERAL KRISTALLOG, CH-3012 BERN,

SWITZERLAND

UNIV-MILAN, DIPARTIMENTO SCI TERRA, I-20133 MILAN, ITALY

UNIV-NAPLES-FEDERICO-II, DIPARTIMENTO INGN MAT \& PROD, I-80125

NAPLES, ITALY

UNIV-MODENA, DIPARTIMENTO SCI TERRA, I-41100 MODENA, ITALY

CANADIAN-MUSEUM-NAT, MINERAL SCI DIV, OTTAWA, ON K1P-6P4,

CANADA

CHRISTIAN-ALBRECHTS-UNIV-KIEL, MINERAL PETROG INST, D-24098

KIEL, GERMANY

US-GEOL-SURVEY, FED CTR, LAKEWOOD, CO 80225, USA

ROYAL-ONTARIO-MUSEUM, DEPT MINERAL, TORONTO, ON M5S-2C6, CANADA

US-GEOL-SURVEY, RESTON, VA 20192, USA

CSIRO, DIV EXPLORAT \& MIN, WEMBLY, WA 6014, AUSTRALIA

UNIV-VIENNA, INST KRISTALLOG \& MINERAL, A-1090 VIENNA, AUSTRIA

UNIV-PERUGIA, DIPARTIMENTO SCI TERRA, I-06100 PERUGIA, ITALY

UNIV-MICHIGAN, DEPT GEOL SCI, ANN-ARBOR, MI 48109, USA

127. Authors: Seaman-DE Griffith-B Powell-RA

Title: Kernelhr - A Program for Estimating Animal Home Ranges

Source: WILDLIFE SOCIETY BULLETIN

1998, Vol 26, Iss 1, pp 95-100

Addresses:

US-GEOL-SURVEY, BIOL RESOURCES DIV, FOREST \& RANGELAND ECOSYST

SCI CTR, OLYMP FIELD STN, PORT-ANGELES, WA 98362, AUSTRALIA

UNIV-ALASKA-FAIRBANKS, ALASKA COOPERAT FISH \& WILDLIFE RES

UNIT, FAIRBANKS, AK 99775, USA

N-CAROLINA-STATE-UNIV, DEPT ZOOL, RALEIGH, NC 27695, USA

128. Authors: Coplen-TB Ramendik-GI

Title: Summary of the IUPAC Recommendations for the Publication 
of Delta-Value for $\mathrm{H}, \mathrm{C}$, and O Stable-Isotope Ratios Source: GEOKHIMIYA

1998, Iss 3, pp 334-336

Addresses:

US-GEOL-SURVEY, NATL CTR 431, RESTON, VA 20192, USA

NS-KURNAKOV-GEN-\&-INORGAN-CHEM-INST, MOSCOW 117907, RUSSIA 